Escuela Técnica Superior de Ingeniería Industrial, Informática y de Telecomunicación

Departamento de Ingeniería Eléctrica, Electrónica y de Comunicación

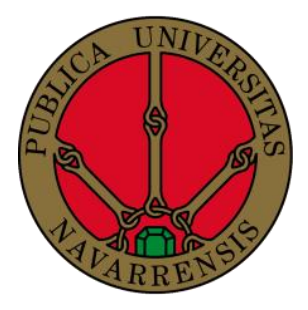

Doctor of Philosophy dissertation

\title{
Estimation of the motor unit structure from scanning electromyography and surface electromyography recordings
}

\author{
by \\ Íñigo Corera Orzanco
}

Supervisors: Javier Navallas Irujo, Javier Rodríguez Falces

Pamplona, 2020 
upna

Universidad Púbilica de Navara
Natarmaako Unibersitate Publikoa 
Quiero dar las gracias a todas las personas que han estado apoyándome durante todos estos años de trabajo.

En primer lugar, quiero dar las gracias a mi familia. A mi madre Begoña, a mi hermano Sergio, y a mi abuela María. También a mi padre Carlos, que falleció cuando yo era pequeño. A mi tío Javier, a mi tía María Ángeles, y a mis primos, Erik, Ander, Olaia, así como a mis tíos y primos por parte de mi padre.

Quiero también agradecer el apoyo que he recibido por parte de mi novia Vanessa, así como de mis suegros Pedro y María África, y de mi cuñado Mikel.

También quisiera dar las gracias a todos mis amigos: Alex, Aritz, Claudia, Ekaitz, Egoitz, Iñaki, Jon, Leire, Marta y Mikel (Lucky).

Por supuesto, también quiero decir gracias a mi director de tesis Javier (Navallas). También a mi segundo director de tesis Javier (Rodríguez), así como a otros profesores con los que he convivido estos años de investigación: Armando, Rafa, Arantxa y Sonia.

Por último, no me olvido de incluir en los agradecimientos a los compañeros que han pasado por el laboratorio: Andoni, Gonzalo, Fran, Aritz, Tunca, Davide, Mattia, Adrián y Oliver.

A todos vosotros, gracias. 
upna

Universidad Púbilica de Navara
Natarmaako Unibersitate Publikoa 


\section{Summary}

The motor unit (MU) is the minimum functional unit of the neuromuscular system and it is composed by a motoneuron and the set of muscle fibers that it innervates. The knowledge of the anatomical and physiological structure of the MUs allows to detect and analyze changes occurring in the neuromuscular system, e.g., between normal and pathological conditions, or in the processes of fatigue or aging. Therefore, the estimation of the fundamental parameters that define the structure and physiology of the MU has important applications in the detection and follow up of all these changes.

Different electromyographic (EMG) techniques allow to record the motor unit potential (MUP), whose waveform is closely related to the specific values taken by the parameters characterizing the MU. The MU parameters having more influence on the MUP waveform characteristics are: the positions of the fibers constituting the MU, the position of the corresponding neuromuscular junctions, the fiber diameters, and the potential propagation velocity of the fibers. There are several mathematical models that allow to simulate a recorded MUP waveform from the set of anatomical and physiological MU parameters. We will refer to this model-based simulation as the forward problem.

The reverse process is called the inverse problem; i.e., estimating the MU parameters from the information provided by a recorded MUP waveform. The inverse problem can be tackled with the use of the MUP models together with optimization algorithms. Prior attempts to address the MUP inverse problem are based on the recording of the MUP in a single position. However, in this scenario the MUP does not provide enough information to achieve a univocal MU parameter estimation. This thesis aims to reduce the inverse problem solution ambiguity under the hypothesis that the use of MUPs recorded from several positions allows to enhance significantly the MU parameter estimation.

In this thesis, several algorithmic procedures to estimate the MU fiber parameters from the waveform of a scanning-EMG signal (i.e., from the recording of a MUP in multiple positions along a linear corridor) have been developed. The different procedures correspond to different modifications of the scanning-EMG technique, which differ in the number of scanning needles and recording ports used to record the signal. The three proposed variants are: the 1-port recording, based on a single scanning needle with a single recording port placed at one side of the needle (i.e., a single fiber EMG needle); the 2-port recording, based on a single scanning needle, with two recording ports, placed at opposite sides of the needle; and the 4-port recording, based on two scanning needles, each one with two recording ports. The estimation system also uses a linear array of surface-EMG recordings to obtain complementary information about the MU. In this way, the recording setup to achieve the MU parameter estimation consists on a simultaneous surface- and scanning-EMG signal recording of the MUP. 
The estimation system has been evaluated for the three scanning-EMG recording configurations (1-port, 2-port, and 4-port), and compared to the case in which the estimation is performed from a MUP recorded at a single position. The evaluation has been done in a simulation framework, using state of the art models of the muscle, MUs, recruitment, and needles, and developing specific models for the simultaneous surfaceand scanning-EMG recording process. This provides a controlled environment in which the performance of the system can be objectively quantified and evaluated.

The results evidence that MU parameters are estimated much more accurately when using the scanning-EMG technique than when using a MUP recorded at a single position, corroborating the hypothesis that the use of signals recorded at multiple positions enhances the parameter estimation. Among the three proposed recording configurations, the poorest estimation results have been obtained for the 1-port configuration which, moreover, is only capable of estimating the MU fibers at one side of the needle. The 2-port configuration gives better results, and allows to estimate the MU fibers at both sides of the needle. The 4-port configuration is the one that provides the best performance, but it has the disadvantage of being the most difficult configuration to be physically implemented. In the view of these results, a deeper evaluation of the 2-port recording configuration is done. This is because it combines a good estimation performance with a relative ease to be physically implemented.

An additional effort is done to calculate several global MU parameters, such as the MU fiber density, the average potential propagation velocity of the fibers, and the width of the innervation zone, from the resulting set of estimated fibers. These global MU parameters provide relevant physiological information from a clinical point of view. Hence global parameters connect the estimation system developed in this thesis with a future application in the diagnosis and follow-up of neuromuscular pathologies. 


\section{Thesis overview}

Chapter 1 describes the theoretical basis, including the muscle and the motor unit, the generation of the electric potentials, the electromyography of the motor unit, the MU structure estimation, and the mathematical modeling of the EMG signals used in this thesis.

Chapter 2 describes different algorithms developed during this thesis to process and analyze EMG signals. Some of these algorithms are key pieces of the final estimation system developed to solve the MUP inverse problem.

Chapter 3 describes the theoretical framework in which the MUP inverse problem has been addressed. The chapter starts stating the objective of the thesis, i.e., the estimation of the MU fiber parameters. Then, it analyzes the MUP forward problem to study the potential ability of the proposed recording configurations to estimate MU fiber parameters. The chapter continues describing the basis of the estimation system developed during this thesis to solve the MUP inverse problem, and it finishes describing the mathematical framework used to evaluate the estimation system performance.

Chapter 4 analyzes the most relevant problems that occur when a realistic recording scenario is taken into account, and describes the pertinent modifications implemented in the estimation system to overcome these problems. The particular problems taken into account include: the effect of the indetermination of the potential initiation instant; the effect of the noise in the recording of the scanning-EMG signal; the effect of the cannula in needle recordings; and the effect of using other needle electrodes, instead of single fiber ones.

Chapter 5 describes the evaluation of the estimation system. The design of the simulation experiments implemented to evaluate the system, the corresponding results, and the discussion of these results are presented in this chapter. The chapter also shows and briefly discusses the result of applying the estimation system in a real environment, after several cases of study.

Chapter 6 presents the final conclusions of the thesis and the future lines of this research. 
upna

Universidad Púbilica de Navara
Natarmaako Unibersitate Publikoa 


\section{Contents}

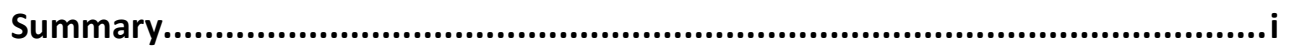

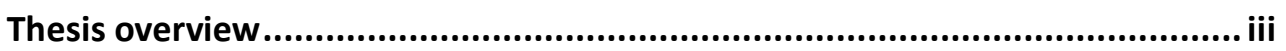

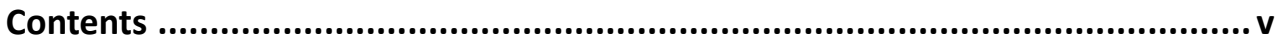

$1 \quad$ Basis.......................................................................................

1.1 Anatomy and physiology of the muscle and the motor unit ...........................3

1.1.1 Anatomy and physiology of muscle fiber..............................................

1.1.2 Anatomy and physiology of the motor unit ...........................................

1.1.3 Anatomy and physiology of the muscle .................................................. 8

$1.2 \quad$ Electromyography of the motor unit ............................................................12

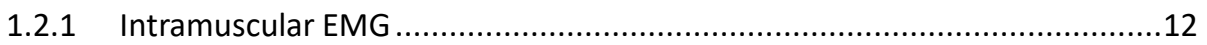

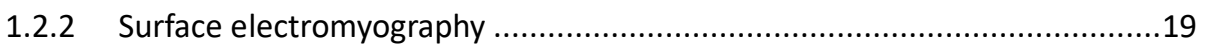

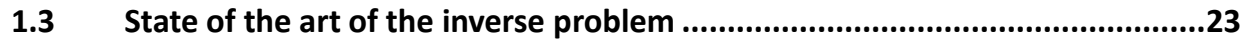

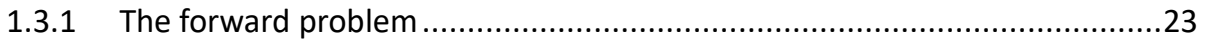

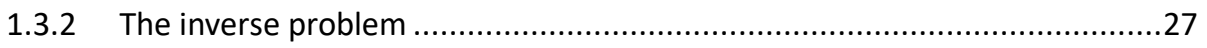

1.3.3 First attempt of a complete MUP decomposition ....................................32

1.3.4 Second attempt of a complete MUP decomposition .................................34

1.3.5 Limitations due to the single position recording .......................................37

1.4 Models for the simulation of electromyographic signals ...............................40

1.4.1 Modeling the Muscle and MU ..................................................................40

1.4.2 Modeling the MUP .........................................................................43

1.4.3 Modeling the needle electrode .........................................................43

1.4.4 Modeling recruitment and firing pattern ...................................................45

1.4.5 Modeling the EMG interference pattern ...............................................46

1.4.6 Modeling the scanning-EMG recording .................................................46

1.4.7 Modeling the surface-EMG recording ..................................................48

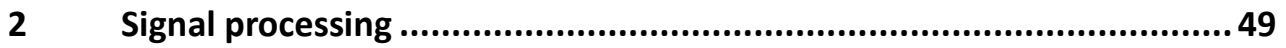

2.1 Processing of the scanning-EMG signal ....................................................51

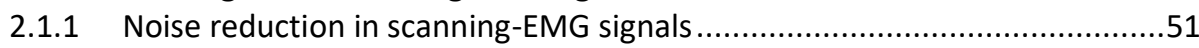

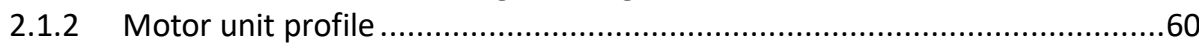

2.1.3 Detection of the scanning-EMG signal active region................................67

2.2 Processing of the surface-EMG signal......................................................70

2.2.1 Spike-trigger averaging ................................................................... 70

2.2.2 Estimation of the $I Z$, the MFCV, and the potential initiation instant............70

3 Theoretical framework of the MUP inverse problem .........................77

B.1 Restating the objective of the thesis ..........................................................79

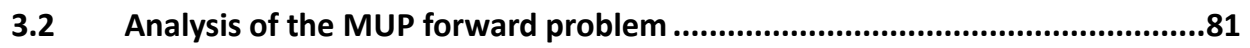


3.2.1 MU fiber parameters influence on MUP waveform ................................ 81

3.2.2 Analysis of the error function in the parameter space ............................ 82

3.2.3 Analysis of the dispersion region of a single MU fiber................................ 87

3.2.4 Analysis of the dispersion region of two MU fibers ................................. 93

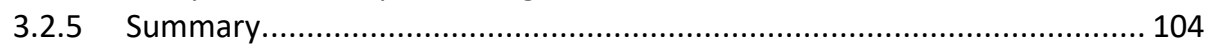

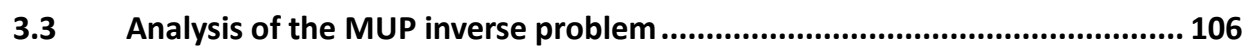

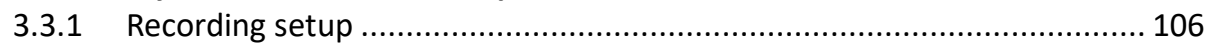

3.3.2 Probabilistic interpretation of the MUP inverse problem ......................... 112

3.3.3 General outline of the estimation system ............................................ 114

3.4 Evaluation framework of the estimation system. ..................................... 126

3.4.1 Error of the individual MU fiber parameters ....................................... 127

3.4.2 Error of MU global parameters........................................................... 132

$4 \quad$ Design of the estimation system ........................................................ 139

4.1 The indetermination of the potential initiation instant ................................ 141

4.1.1 Estimation error analysis of the potential initiation instant .................... 142

4.1.2 Effect of the potential initiation error on the fiber parameter estimation. 143

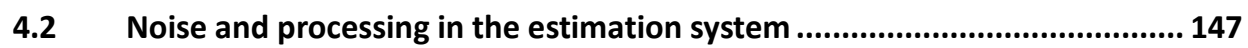

4.2.1 Metric adapted to the noise present in the scanning-EMG signal ............. 147

4.2.2 Relationship between the Mahalanobis metric and the signal filtering ..... 148

4.2.3 Processing of the scanning-EMG signal noise ..................................... 149

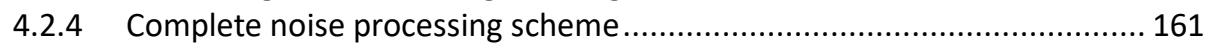

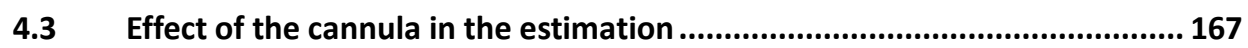

4.3.1 Effect of the cannula in the 1-port scanning-EMG configuration ................ 167

4.3.2 Effect of the cannula in the 2-port scanning-EMG configuration ................ 173

4.3.3 Effect of the cannula in the 4-port scanning-EMG configuration ................ 174

4.4 Considerations about the type of electrode used in the estimation............. 176

4.4.1 The monopolar needle electrode ....................................................... 176

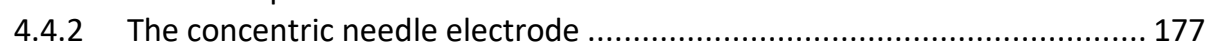

$5 \quad$ Evaluation of the estimation system.......................................... 183

5.1 Description of the simulation experiments................................................ 185

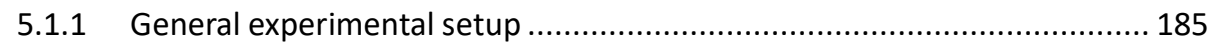

5.1.2 Estimation with the different recording configurations ........................... 187

5.1.3 Estimation with increased muscle contraction...................................... 188

5.1.4 Estimation with variability in the IAP parameters .................................. 188

5.1.5 Estimation varying the global properties of the MUs ............................... 190

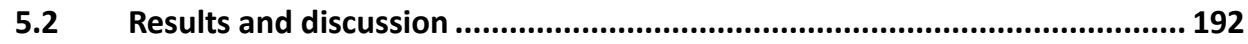

5.2.1 Estimation with different recording configurations ................................ 192

5.2.2 Estimation with an increased muscle contraction .................................. 211

5.2.3 Estimation with variability in the IAP parameters .................................. 215

5.2.4 Estimation varying the global properties of the MUs .............................. 217 
5.3 Testing the estimation system with real EMG signals ................................237

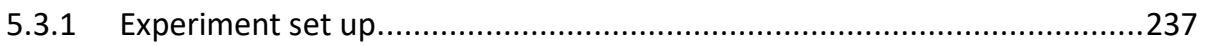

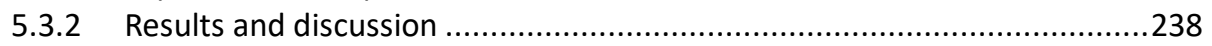

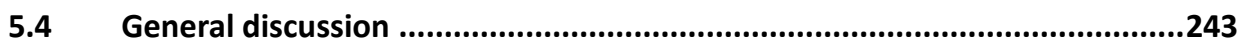

6 Final conclusions and future lines............................................... 247

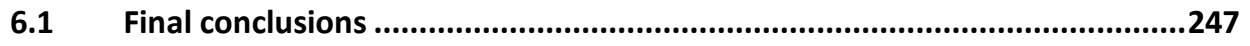

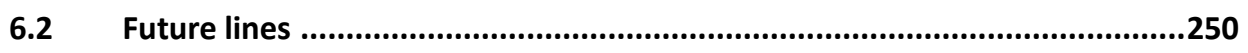

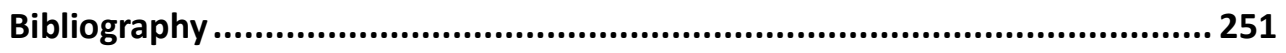

List of Abbreviations ........................................................................ 265

List of Publications............................................................................. 266 
upna

Universidad Púbilica de Navara
Natarmaako Unibersitate Publikoa 


\section{Basis}

In this chapter, the theoretical basis used to develop this thesis is exposed. The chapter starts introducing the muscle, the motor unit, and the production of electric potentials during muscle contraction. Then, a general overview about the electromyography of the motor unit is presented. The chapter continues describing the state of the art of the inverse problem, including the mathematical models used in these previous attempts of estimating the MU structure. It finishes with a complete and detailed presentation of all the mathematical models incorporated in the simulation of the electromyographic signals used in this thesis. 
upna

Universidad Púbilica de Navara
Natarmaako Unibersitate Publikoa 


\subsection{Anatomy and physiology of the muscle and the motor unit}

\subsubsection{Anatomy and physiology of muscle fiber}

\section{Anatomy of the muscle fiber}

The skeletal muscle fibers are cells specialized to produce force and movement during contraction. The shape of muscle fibers is approximately cylindrical, with a size that varies depending on the type of muscle. The fiber length can range from $10 \mathrm{~mm}$ to $400 \mathrm{~mm}$, and the diameter from $10 \mu \mathrm{m}$ to $100 \mu \mathrm{m}$. The main anatomical parts of the muscle fiber are:

- Myofibrils: the muscle fiber is constituted by bundles of these structures, which are responsible of the contraction and relaxation of the fiber. The myofibrils are in turn constituted by bundles of contractile proteins called myofilaments.

- Sarcolemma: this is the membrane that surrounds the muscle fiber. It enables the cytoplasm to have a markedly different chemical composition than the fluid surrounding the fiber. In this way, it actively maintains a constant potential difference between the interior of the muscle fiber and extracellular environment. This potential depends on the activity of the sodium-potassium pumps, which work against the concentration gradients of ions flowing through the plasmalemma. The value of this potential difference ranges from $-70 \mathrm{mV}$ to -90 $\mathrm{mV}$. In addition to this function, the plasmalemma enables the transmission of electrical impulses.

- Sarcoplasmic reticulum: this structure is a network of channels surrounding the individual myofibrils whose function is to release sodium ions during contraction.

\section{Intracellular action potential}

In order to produce the contraction of the muscle fiber, the motoneuron sends an electric impulse that is propagated along the axon (Fig. 1-1). This impulse eventually arrives to the neuromuscular junction of the muscle fiber producing the release of acetylcholine in the gap between the axon terminal and the muscle fiber membrane. This process excites the membrane in the neuromuscular junction producing an inversion of the potential in this part of the muscle fiber. This potential, called intracellular action potential (IAP), is propagated alongthe muscle fiber in both directions from the neuromuscular junction to the tendon endings $[65,21]$, inducing the contraction of the muscle fiber. The IAP is not only function of time but also of space, as it spreads out along the muscle fiber. The shape of the IAP can be characterized by a depolarization phase, a repolarization phase, and a hyperpolarizing long after potential [77]. The velocity with which the IAP is propagated is called 


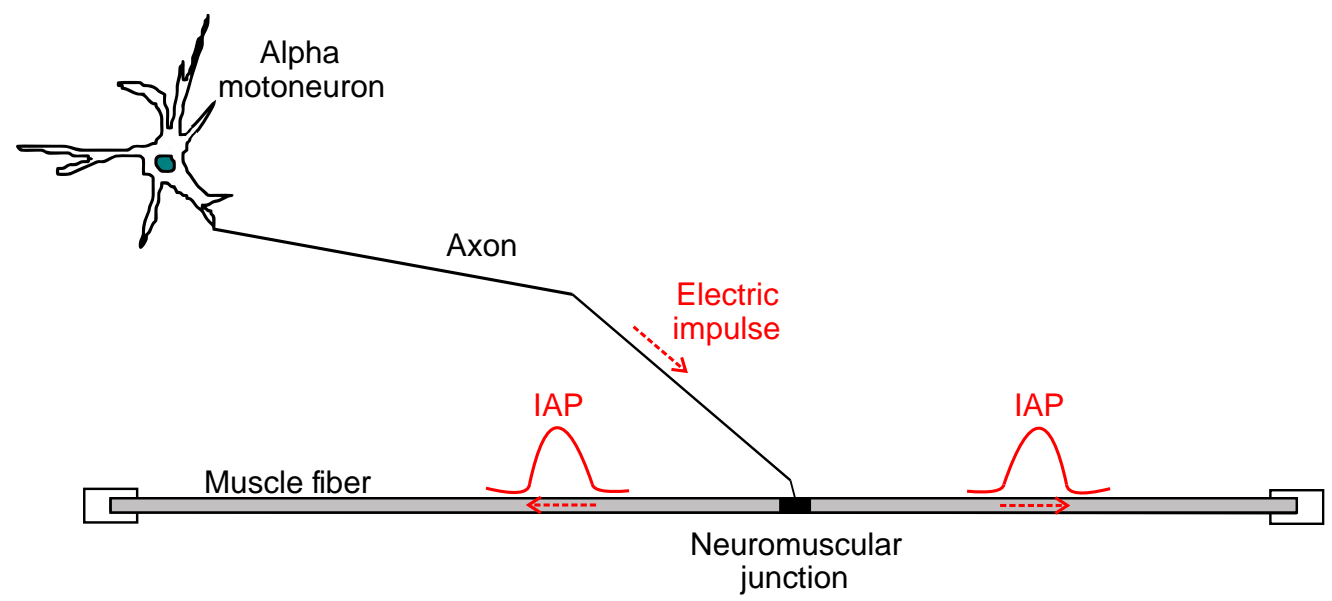

Fig. 1-1. Schematic representation of a motoneuron, its axon, and an innervated muscle fiber. An electric impulse sent by the motoneuron propagates through the axon. After neuromuscular transmission, this impulse produces an action potential in the muscle fiber, the IAP, which is propagated in both directions towards the two fiber endings.

muscle fiber conduction velocity (MFCV), which mainly depends on the muscle fiber diameter, the higher the MFCV the larger the diameter [47]. The value of the MFCV in normal adult muscles ranges from 1.5 to $6.5 \mathrm{~m} / \mathrm{s}$ [113].

\section{Single fiber action potential}

The IAP propagating along the muscle fiber generates an electric potential in the extracellular medium: the single fiber action potential (SFAP). The generation of the SFAP strongly depends on the biological tissue existing outside the muscle fiber. This tissue is usually referred as volume conductor due to its properties as electric conductor [96]. This implies that the electric potential existing at a certain point outside the fiber decreases with increasing distance to the front of propagation [81]. The amplitude of the SFAP is also dependent to the muscle fiber diameter, the larger the diameter the larger the amplitude [81]. The time delay between the firing of the motoneuron and the arrival of the SFAP spike at a certain point depends on several factors. This delay can be divided into two components: the delay in the initiation of depolarization, which depends on the length and propagation velocity of the axonal terminal branch [114], plus the neuromuscular junction delay; and the propagation delay between the initiation of depolarization and the arrival of the main potential contribution to the point under consideration, being inversely proportional to the MFCV and proportional to the axial distance between such point and the neuromuscular junction [125]. The latter is generally larger than the former. Regarding the duration of the SFAP, it is mainly affected by two factors: the distance between the fiber and the point at which the potential is being considered [139], and the fiber diameter [139]. The SFAP duration increases with increasing distance, and decreases with increasing diameter. 


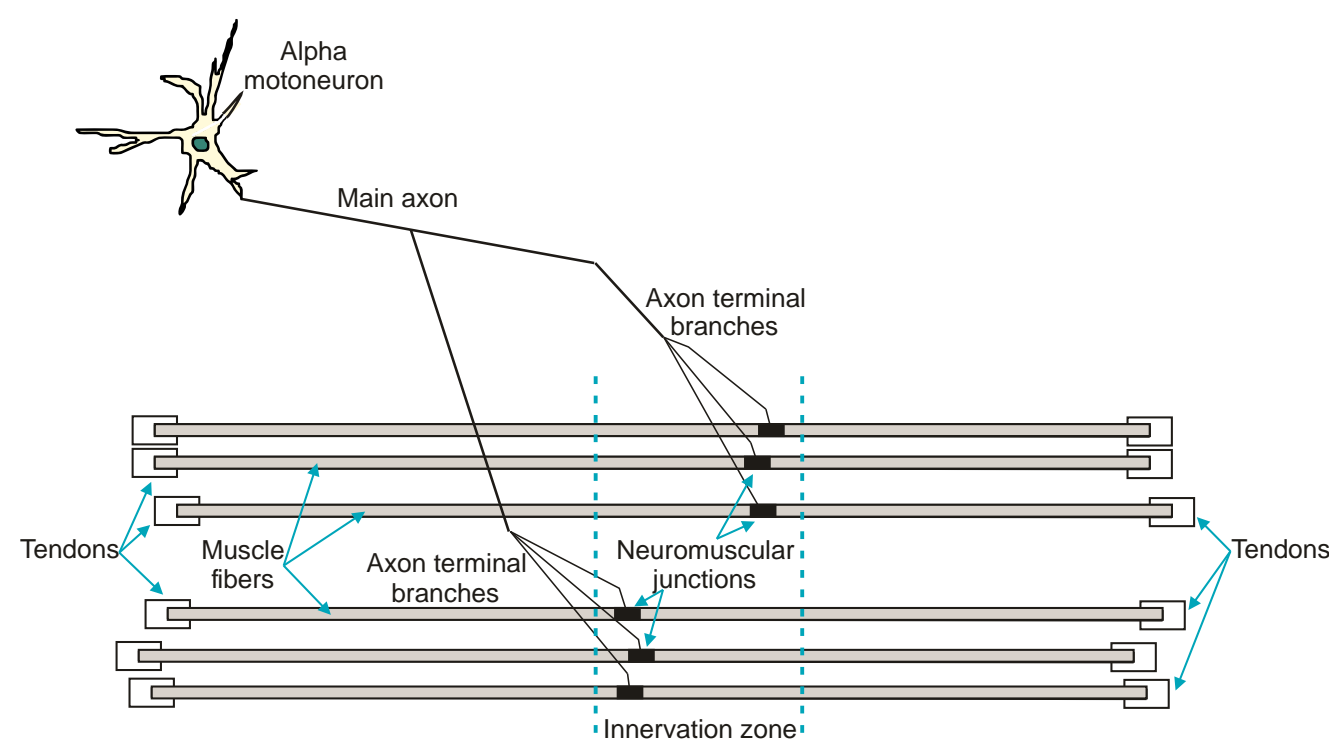

Fig. 1-2. Schematic representation of the MU, constituted by an alpha motoneuron, the axon with its ramifications, and the innervated muscle fibers. The innervation zone is the region in which the neuromuscular junctions are located. Note that the neuromuscular junctions are arranged forming two differentiated sub-bands within the innervation zone, corresponding to two different axon ramifications.

\subsubsection{Anatomy and physiology of the motor unit}

\section{Motor unit}

The motor unit (MU) is the smallest functional entity of the neuromuscular system in the production of force and movement. The MU is constituted by an alpha motoneuron, the axon with its ramifications, and the set of muscle fibers innervated by the axon [112] (Fig. 1-2). A control command from the motoneuron produces a synchronous contraction of all the muscle fibers belonging to the MU. This command consists on an electric impulse that propagates through the axon, then through the axonal ramifications, and reaches the neuromuscular junctions of the different MU fibers.

\section{Motoneuron}

The alpha motoneurons are the neurons of the central nervous system that innervate the muscle fibers. Their somas are located in longitudinal columns in the ventral horn of the spinal cord forming a complex structure denominated motoneuron pool. From there, axons are projected in bundles. These axons eventually reach the muscle belly, where they undergo a branching process so that each of them innervates 
a certain set of muscle fibers, i.e., the muscle fibers of a MU. Axonal branching pattern is important to understand the MU topography, as it is intimately related to the dispersion of the muscle fibers within the MU territory [94, 9], as well as to the synchronization pattern of the initiation of the depolarization of the muscle fibers belonging to the MU.

\section{Neuromuscular junction}

The neuromuscular junction is the synapse formed in the connection between the axon and the muscle fiber. The region of the MU where the neuromuscular junctions of the MU fibers are located is called innervation zone (IZ). This region usually tends to be a band located in the mid-length of the muscle fibers; e.g., in the biceps brachii, the IZ comprises a thin region about $6-10 \mathrm{~mm}$ width $[4,3]$. Many researchers point out that the neuromuscular junctions associated to the muscle fibers of each $\mathrm{MU}$ are organized forming several narrow sub-bands within the IZ [120, 122, 87]. A working hypothesis is that each of these sub-bands corresponds to a specific set of muscle fibers innervated by a common axonal branch [120, 87] (Fig. 1-2). This is consistent with the idea that MU fibers tend to be arranged in clusters due to this branching pattern [9].

\section{Spatial distribution of the motor unit muscle fibers}

In humans, the number of muscle fibers of a MU can vary from approximately 10 fibers in the case of the extraocular musculature to even thousands for the lower limb muscles $[24,34,109]$. The muscle fibers of a MU are scattered within a certain region of the muscle cross-section called MU territory [58]. These territories have been described as irregular, mainly rounded, and oval shaped [8]. Within the territory of a MU, its corresponding fibers are intermingled with fibers from other MUs [14], implying that several MU territories are usually overlapped in the same region of the muscle cross-section (Fig. 1-3).

There is no clear consensus about the spatial distribution of MU fibers within its territory. This distribution has been described as uniform without localized collections [14], evenly distributed [137], quasi-Gaussian [79], uniform [40], and random [36]. To shed some light into this subject, different quantitative statistical analysis have been applied to glycogen-depleted MUs data [8,9]. These studies suggest that the MU fibers are not homogeneously distributed within the MU territory, but they show regions of high fiber density (fractions) and regions with very low fiber density (holes).

The MU fiber density is the ratio of the number of MU fibers to the area of the MU territory. This parameter is found to have different ranges depending on the MU type, and also to be somewhat independent to the number of MU fibers within each MU type [55]. 


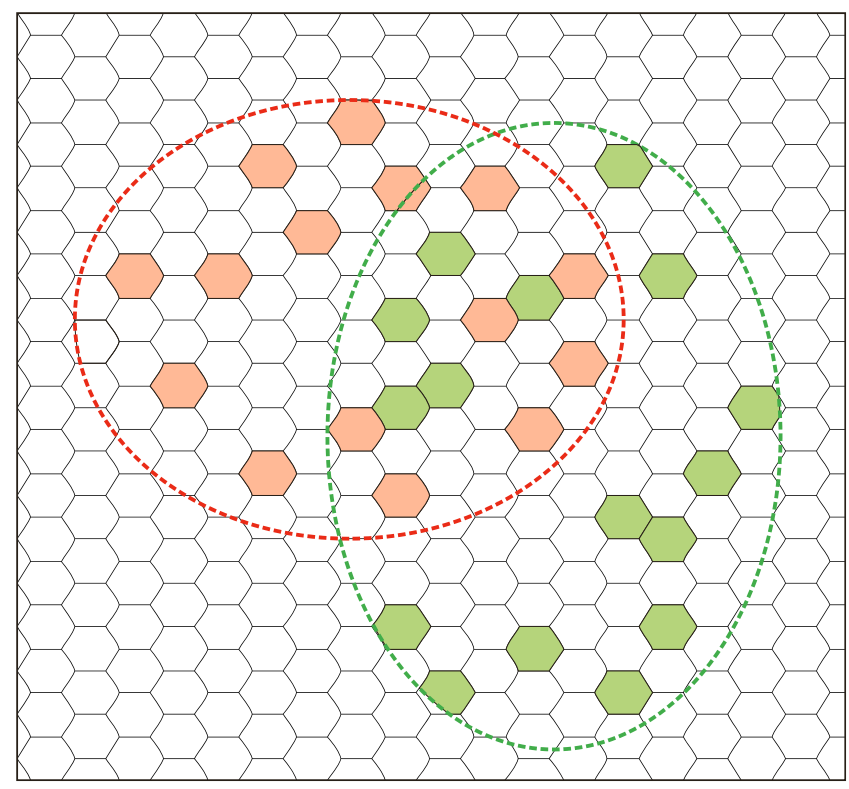

Fig. 1-3. Schematic representation of two MUs whose territories are overlapping within the muscle cross-section. The muscle fibers are represented by hexagons, where the ones colored in red corresponds to one MU, and that of colored in green to the other MU.

\section{Motor unit under pathology}

The motor unit undergoes significant changes during pathology. These changes can affect to the muscle fiber membrane, the neuromuscular junction, as well as to the microanatomy of the MU. Changes in the muscle fiber membrane and neuromuscular junction lead to abnormal impulse conduction across the muscle fibers or the neuromuscular junctions. Regarding the anatomical changes produced by pathologies, it is necessary to differentiate between myopathic and neuropathic conditions. Under myopathy muscle fiber diameters become in general smaller, but with an increased dispersion in the diameter sizes. The MU tends to be smaller with fewer fibers than in normal conditions. This is due to processes such as fiber degeneration, regeneration, segmental necrosis, splitting, fibrosis, etc. Muscle fibers that are not innervated may become atrophic. This can occur for instance with fibers that have suffered segmental necrosis or with regenerating fibers. Gradually, these atrophic fibers are reinnervated by collateral sprouting from the nerve terminal. Under neuropathy, nerve lesions produce axonal denervation. The surviving axons reinnervate dennervated muscle fibers by collateral sprouting [58]. As a consequence of reinnervation, the surviving MUs are more compact and denser, with an increased number of muscle fibers. The territory of these MUs remains practically unchanged, as reinnervation is usually restricted to the originally innervated fascicles. Another consequence of reinnervation is an increase in the dispersion of motor end plates and in the fiber diameter variability. 


\section{Motor unit potential}

The motor unit potential (MUP) is the summation of the SFAPs from the muscle fibers of the MU [125] (Fig. 1-4). The shape of the MUP mainly depends on the amplitudes and time delays of its constituent SFAPs [125]. If we consider the MUP at a certain point, $\mathrm{MU}$ fibers closer to this point will contribute with higher amplitudes than distant fibers [125] (Fig. 1-4 (a)). The MU fiber diameters are also important to understand the shape of the MUP. The diameters influence not only in the amplitude of the individual SFAPs but also in their time delays [81]. Note that the larger the fiber diameters the higher the MFCVs of the MU fibers, and therefore the lower the time delays of the SFAPs (Fig. 1-4 (c)). The longitudinal distribution of the neuromuscular junctions within the IZ also affects to the MUP shape, as these positions condition the time delay of the SFAPs (Fig. 1-4 (b)). In general, a high variability in the MU fiber diameters or in the neuromuscular junction positions implies that the individual SFAPs are summed with very different time delays, which in turn implies that the shape of the MUP will be complex, with many turns and phases [125].

\subsubsection{Anatomy and physiology of the muscle}

\section{Anatomy of the skeletal muscle}

The muscle is a tissue with contractile properties so that it can produce force and motion. There are three general types of muscle in the human body: the cardiac muscle, the smooth muscle and the skeletal muscle. Of these, only the skeletal muscle is contracted voluntarily. The skeletal muscle is formed by bundles of striated muscle fibers denominated fascicles, and is anchored to the bones by tendons.

Physiologically, each muscle is constituted by several MUs, each of them occupying a fraction of the total muscle cross-section. The number of these MUs and the area they occupy within the muscle cross-section vary in function of the size of the muscle itself. Note that, territories of different MUs are usually overlapping each other within the muscle. The degree of overlapping may range from 10 to 25 units in some human muscles [74].

The twitch force exerted by the different MUs within a single muscle presents a large range of variation, with a highly skewed distribution with a lot of MUs of small twitch force and few MUs of large twitch force [72, 35]. As the force produced by a single MU is also proportional to the number of MU fibers [35], it can be assumed that the same kind of statistical distribution governs the number of fibers of the MUs within a single muscle [25]. The area of the MU territories is strongly correlated with the number of MU fibers [55], and also with the maximum tension exerted by the MU [55, 7]. Therefore, the motor unit territory areas also present a skewed statistical distribution similar than for the number of MU fibers, with a lot of small MUs and few big MUs existing in a single muscle. The MU fiber diameter also varies for the 

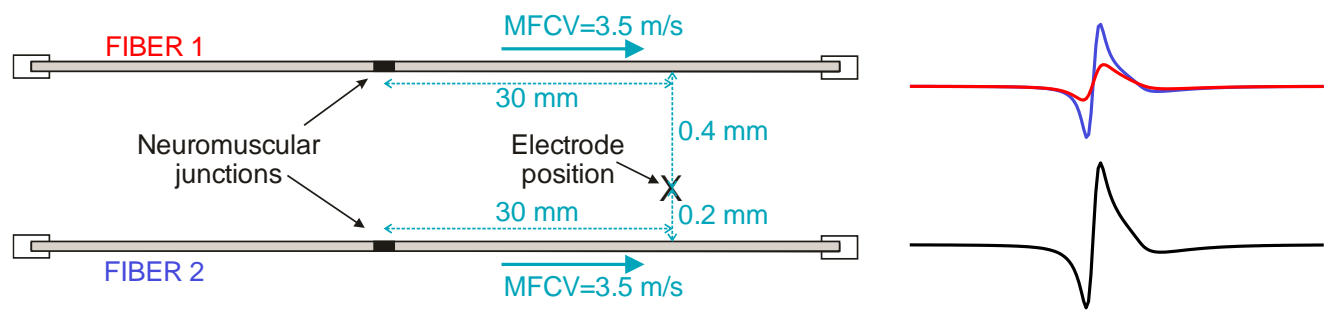

(a)
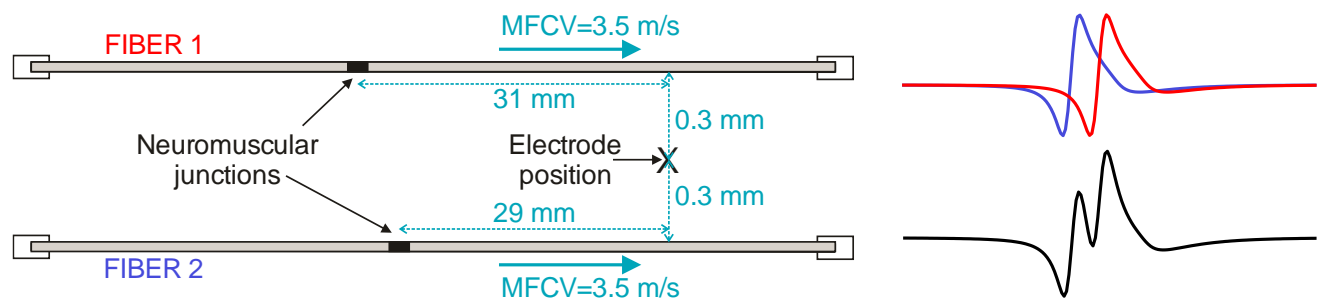

(b)
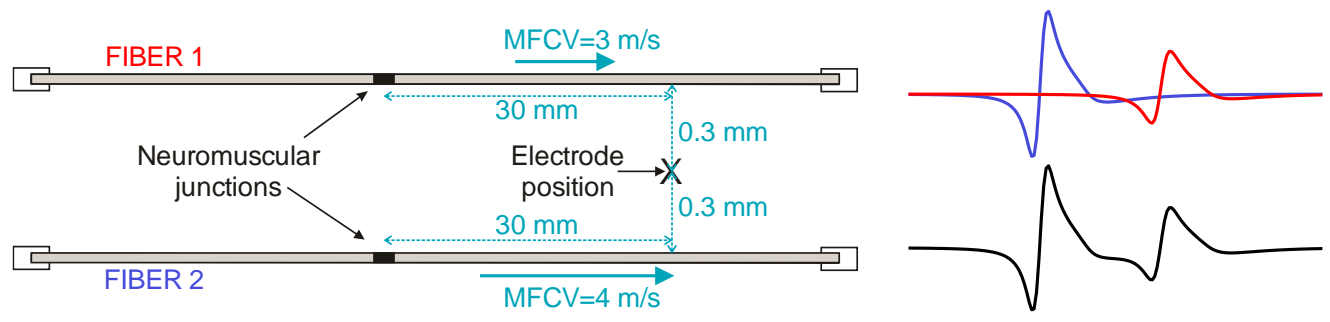

(c)

Fig. 1-4. Schematic representation of the generation of the MUP from the SFAP contributions of the MU fibers. In this example, the contributions of only two fibers are considered. On the left, representations of the two fibers together with its parameters, and the electrode position (black cross). On the right, representation of the SFAPs corresponding to each fiber (fiber 1, in red; and fiber 2, in blue), and the MUP resulting of summing the two SFAPs (in black). The generation of the MUP has been represented for three different scenarios: (a) the two fibers are located at different radial distances respect to the electrode, while they share the remaining fiber parameters. Note that, different distances imply different SFAP amplitudes; (b) the two fibers have different positions of the neuromuscular junction, while they share the remaining parameters. Note that, this implies that the SFAPs present different time delays, which means that the resulting MUP present a complex shape; (c) the two fibers have different MFCVs (which implies also different fiber diameters), while they share the remaining parameters. Note that this implies that the SFAPs presents different time delays, and different amplitudes.

different MUs within a single muscle. They are considered on average larger for the MUs having larger number of fibers [48]. Note that in smaller MUs type I fibers predominate $[51,52]$, which usually have smaller diameters than the type II fibers [25, 11]. The latter fibers are more common in larger MUs. 


\section{Motor unit recruitment and firing pattern}

In order to produce muscle movement, the motoneuron pool receives an order from the motor cortex modulated by the cerebellum and different peripheral receptors. The motoneuron pool responds to these inputs producing the firing of the different MUs that finally command muscle activation. To produce a contraction sustained over a period of time, it is necessary that the motoneurons fire repeatedly with a certain firing rate.

The force exerted by a muscle is mainly modulated by two factors: the recruitment of the MUs and its corresponding firing rates [59, 78, 80]. The larger the number of recruited MUs, the higher the force; the higher the firing rates, the higher the force. MUs are recruited by size in ascending order, which is usually denominated "the size principle" [51]. At a low level of muscle contraction only the smallest MUs are recruited, which are constituted by a small number of fibers. This allows the muscle to perform smoother and more precise movements than at higher contraction levels. As more force is required, more MUs are recruited being the larger ones the last ones to be recruited. The firing rate of a recruited MU will be higher the higher the intensity of the muscle contraction. The firing rate of a MU also depends on its size. MUs which have been recruited at a lower contraction level (and therefore which are smaller) tend to have higher firing rates $[35,20]$.

MU 1
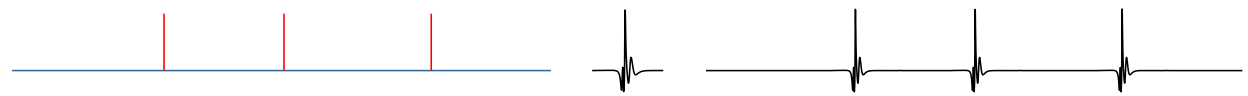

MU 2
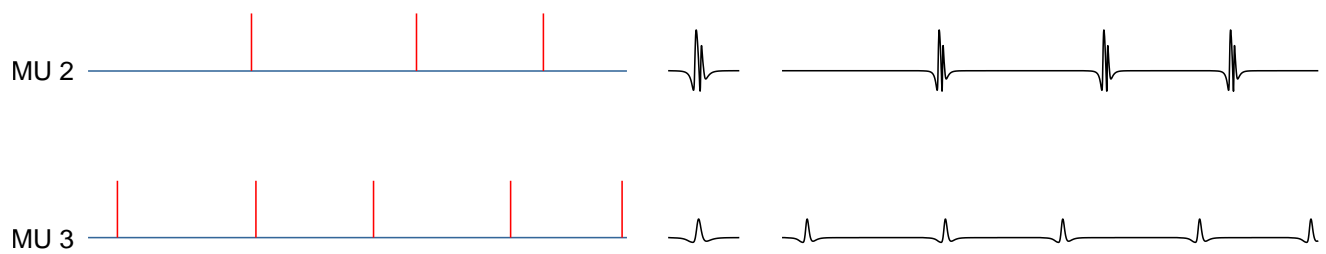

(a)

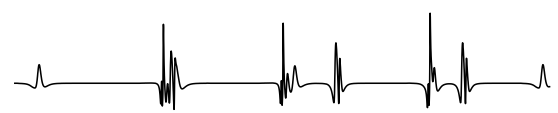

(b)

(c)

Fig. 1-5. Schematic representation of the generation of the EMG pattern; (a) firing patterns corresponding to each $\mathrm{MU}$, where the different discharge instants are represented by vertical red lines; (b) MUPs corresponding to each MU; (c) each discharge of the firing patterns takes the form of the MUP of the corresponding MU, resulting in the corresponding MUP trains. The interference pattern or EMG signal corresponding to the summation of the different MUP trains is represented on the bottom. 


\section{Generation of the EMG pattern}

During the contraction of the muscle, each recruited MU generates a train of discharges called firing pattern. For each discharge of a MU firing pattern, a MUP is generated, forming a MUP train (Fig. 1-5). The electric signal produced within the muscle as the result of summing all MUP trains is called interference pattern or simply EMG signal (Fig. 1-5). If the force exerted by the muscle is low, individual MUPs can be visually identified in the EMG when it is recorded by a needle electrode [128]. This is because at low contraction levels only a few MUs are recruited, and furthermore, they are firing at a low rate. Therefore, in this situation it is rare for MUPs from different MUs to be superimposed in the same time interval. When the contraction level increases, MU firing rates also increase, and previously inactive MUs are recruited [128]. This implies that a larger number of MUP superimpositions is present in the EMG, and that therefore it is increasingly difficult to distinguish individual MUPs. When the contraction level is so high that the EMG baseline can no longer be distinguished the interference pattern is said to be "full" [128]. 


\subsection{Electromyography of the motor unit}

Different electromyographical techniques can be employed to study the electrical activity produced by the skeletal muscle. Specifically, we are interested in those methods focused on the study of the MU. Consequently, these are the methods that are described in this section.

In pathological processes, the architecture of the MU changes. This, in turn, causes characteristic changes in the recorded EMG signals, as there is a neat relationship between the MU structure and the EMG signal waveform [125]. This makes the EMG a very powerful technique both in research and in the diagnosis and follow up of neuromuscular diseases.

The EMG signal can be recorded by using different types of electrodes, which enable different electromyographic techniques. These can be divided according to whether the recording is made in the surface of the skin, or by inserting a needle electrode within the muscle. The former is called surface-EMG, and the latter intramuscular-EMG.

\subsubsection{Intramuscular EMG}

There are various types of needle electrodes to be used in intramuscular-EMG: the single fiber needle electrode (SF electrode), the concentric needle electrode (CN electrode), the monopolar needle electrode (MN electrode), and the macro needle electrode among them. The specific application of these needle electrodes mainly depends on the size of the leading-off electrode surface, as it determines the region of the MU in which the muscle fiber electrical activity is detected (the so-called uptake area). The smaller the active surface of the electrode, the larger the rate at which the amplitude of a SFAP decreases with increasing fiber-electrode distance. Therefore, the smaller the active surface, the smaller the electrode uptake area [128]. In this section, the most commonly used intramuscular electrodes together with the most relevant EMG techniques are described.

\section{Single fiber EMG}

The standard SF electrode consists of a $0.5 \mathrm{~mm}$ thick needle cannula with a side port, in which the leading-off platinum surface is exposed. The recorded signal is the electric potential difference between the active surface and the cannula. The size of the active surface is very small, with a diameter about $25 \mu \mathrm{m}$. That is the reason why the SF electrode has a very pronounced radial decline and a very small uptake area. The uptake area can be defined as the region where the amplitude of the recorded SFAP is larger than $200 \mu \mathrm{V}$; in practice, it is assumed to be a hemisphere of about $300 \mu \mathrm{m}$ radius [119]. It is therefore a very selective electrode, with a high spatial resolution. 
Consequently, the SF electrode only detects the activity of a few muscle fibers surrounding the active surface. The most relevant neuromuscular features that can be measured using this electrode are fiber density [115, 39, 40], jitter [27] and propagation velocity [113].

\section{Fiber density}

Fiber density is an electrophysiological measure of the average number of MU fibers within a semicircular uptake area of $300 \mu \mathrm{m}$ radius $[115,39,40]$. To obtain this measure, a SF electrode is inserted within the muscle, adjusting its position so that a SFAP is detected with maximum amplitude. This ensures that the electrode is as near as possible to a muscle fiber. At this position, the number of significantly contributing time-locked SFAPs whose amplitude exceeds $200 \mu \mathrm{V}$, and whose rise time is less than $300 \mu \mathrm{s}$, including the triggering SFAP is counted. This procedure is repeated several times by moving the needle to different locations. Also, several needle insertions are made, so that a total of approximately 20 counts per muscle are obtained and averaged to obtain the fiber density value. Fiber density is a measure sensitive to changes in the MU fiber distribution associated to certain pathological processes such as myopathies or neuropathies.

\section{Jitter}

Jitter is a measure of the variability in the neuromuscular transmission time measured through the variability in the propagation delay of the muscle fiber action potential, among different discharges of the MU [27]. Differences in the propagation delay can be caused by variations in the travelling time of the action potential along the nerve, the neuromuscular junction, and the muscle fiber. The jitter can be measured by inserting a SF electrode within a muscle contracted at a very low force [26]. Once inserted, the needle is repositioned until two time-locked SFAPs are recorded. This ensures that these SFAPs come from two different muscle fibers of the same MU. One of the SFAPs is used as a trigger to time align the different discharges. The jitter is shown in the other SFAP as differences in the interpotential delay. To quantify the jitter, the mean of consecutive differences in the interpotential delays of a sequence of 50 100 discharges can be calculated. Jitter analysis is useful in the diagnosis of neuromuscular transmission disorders such as the myasthenia gravis or others myasthenic syndromes.

\section{Muscle fiber conduction velocity}

Conduction velocity along the muscle fiber can be measured by using a specially designed multichannel SF electrode in voluntary activated muscles [113]. The multielectrode consists on several leading-off surfaces arranged in two rows, typically with a surface disposition of $3+11$. To measure the MFCV, the multielectrode is inserted within the muscle and its position is adjusted so that two of the leading-off 
surfaces constituting the multielectrode lie parallel to a nearby muscle fiber. To achieve this, the same SFAP must be recorded at these two leading-off surfaces, and both SFAP recordings must have maximum amplitude and parallel rising phases. Then, the time delay between the SFAPs is measured, and the propagation velocity is calculated from this delay and the interelectrode distance, which is known beforehand. Although this technique has not been commercialized for clinical use, it has been used in research studies [113].

\section{Concentric needle EMG}

One of the most widely used electrodes in clinical examinations is the $\mathrm{CN}$ electrode [125]. In its standard version it consists of a $150 \mu \mathrm{m}$ diameter wire inside a cannula. The tip of the needle is beveled to a $15^{\circ}$ angle, resulting in an elliptical recording surface of $0.07 \mathrm{~mm}^{2}$, with a $580 \mu \mathrm{m}$ mayor axis and $150 \mu \mathrm{m}$ minor axis. The cannula serves as reference electrode. Due to the dimensions of the recording surface, the $\mathrm{CN}$ electrode has a larger uptake area than the SF electrode. The $\mathrm{CN}$ electrode uptake area is complex due to the shape of the active surface and the effect of the cannula. The latter is considered to act as a shield for the muscle fibers located behind the tip, so that the electrical activity is attenuated for those fibers [84]. However, some experiments suggest that the $\mathrm{CN}$ electrode may also capture muscle fibers behind the tip $[50,57]$.

\section{Quantitative EMG}

An important application of this electrode is the quantitative analysis of the MUP, also known as quantitative EMG (QEMG) [124]. It consists on extracting morphological features from a sample of MUPs recorded during slight voluntary contraction activity. Due to the high variability of the MUP shapes, a sufficiently large sample (typically around 20 MUPs) has to be recorded. The extracted features reflect structural changes in the MU associated to certain diseases. Consequently, they allow to assess whether the patient suffers a pathology, and in that case, if it is a neuropathy or a myopathy. The analysis is also useful to determine the degree and progress of the pathology. Some of the most common morphological features to be extracted from the MUP are amplitude, duration, area, rise-time, size-index, turns, phases, or irregularity coefficient [121, 138, 124]. Once the MUP features have been extracted, statistical analysis of the features can be performed [131]. This analysis usually consists on calculating the mean and the standard deviation of the different features. Deviation of these statistics from standard normative values could be indicative of pathology.

As stated above, changes in the MU structure undergoing in pathological processes cause changes in the recorded MUP [124]. Under neuropathy, reinnervation of muscle fibers causes an increase in the number of MU fibers, which in turn implies an increase in the amplitude of the recorded MUPs. Due to an increase in the diameter variability, and an increase in the dispersion of the neuromuscular junction positions, 
reinnervation is also associated to more complex MUP waveforms, with a larger number of turns and phases, and a larger duration than in normal conditions [28]. Under myopathy, fiber atrophy, fiber loss, and fiber splitting tend to reduce the amplitude and duration of the MUPs [123]. However, high amplitude MUPs may also be recorded due to the presence of hypertrophic fibers. The increased fiber diameter variability associated to myopathies implies that the recorded MUP has usually a complex waveform.

\section{Monopolar needle EMG}

Another widely used needle electrode is the MN electrode. It is composed by an insulated needle with a conical recording surface of $0.25 \mathrm{~mm}^{2}$ area. The recording uptake area of this electrode is larger than that of the $\mathrm{CN}$ electrode, and due to its shape, it is approximately omnidirectional. The $\mathrm{MN}$ electrode uses an independent surface electrode as reference, instead of the cannula surface. Therefore, activity from more distant sources is captured with this electrode. The initiation of the potential and its extinction in the tendon can be observed in the MUP recorded with the MN electrode [61]. This is not the case of the CN and SF electrodes, for which distant activity is largely cancelled, as it is captured with similar amplitude in the active surface and in the cannula. The main disadvantage of the MN electrode is that a lot of noise from distant activity is recorded, resulting in a signal with a more unstable baseline.

The MN electrode can also be used in QEMG. However, the statistics obtained in the analysis will differ from that of the $\mathrm{CN}$ electrode, since the recording characteristics of both electrodes are different. This must be taken into account by the electromyographer when it comes to perform quantitative analysis.

\section{Macro needle EMG}

Macro needle electrode is aimed to record the activity from the entire MU [118]. It consists of a modified SF electrode with the cannula insulated except for the $15 \mathrm{~mm}$ closest to the tip. The single fiber leading-off surface is located at $7.5 \mathrm{~mm}$ from the tip. The recording is made in two channels. The first one is derived between the cannula and a surface electrode placed on the skin, and the second one between the cannula and the single fiber leading-off. This second channel is used to identify the firing of the MU under study, acting as a recording trigger for the cannula signal on the first channel. The recording is made under slight voluntary contraction. A macro MUP is obtained by spike-trigger averaging the cannula signal until a smooth baseline and a constant MUP is observed. The signal recorded with the macro EMG technique is nonselective. The very large uptake area of this electrode implies that most of the fibers of the entire MU are significantly contributing to the macro MUP. This makes this technique adequate to study the number of muscle fibers of the MU. Parameters such as the peak-to-peak amplitude, and the area of the macro MUP are correlated with the number of MU fibers [82]. 


\section{Multielectrode EMG}

Electrodes with several leading-off surfaces can be used to perform recordings in different locations simultaneously. Although these multielectrodes have never been commercialized, they have been used in many experimental studies. A 14-port single fiber multielectrode was used to measure the conduction velocity of individual muscle fibers [113] (see section 1.2.1). A 12-port configuration with well-defined distances between leads (about $40 \mu \mathrm{m}$ ) was used to study the volume conduction within the muscle [37], as well as the uptake radius of the SF electrode [38]. Other multielectrode configurations have allowed to investigate the MU territories: a multielectrode of 12 long ports $(1.5 \mathrm{~mm})$ distributed over $25 \mathrm{~mm}$ was used for this purpose [12, 13]; and in another research, the MU territory was investigated by means of a 14-port multielectrode [116]. Multielectrodes were also used in EMG decomposition analysis to achieve a better discrimination between MUPs from different MUs: in [19, 63, 64] a needle consisting on four leads close to each other was used for this purpose; in the same line, in [1] a modified CN which integrated a quadrifillar electrode was used.

\section{Scanning EMG}

Scanning-EMG was developed as an extension of the multielectrode technique. It consists on recording the electrical activity of the MU in multiple locations along a linear corridor [117]. A needle electrode is inserted within the muscle, and it is moved in small steps through the MU territory, recording the MUP at each location. Usually, a CN electrode is used for this purpose. The different MUPs recorded with the scanning electrode must be synchronized with the firing of the MU so that the acquired scanning-EMG signal is equivalent to simultaneously recording the MUP in all the steps taken throughout the linear corridor. To achieve this, a second needle electrode is inserted within the muscle so that the firing pattern of the MU can be extracted. This trigger electrode, usually a SF electrode, detects localized activity of the MU under study, ideally from a single muscle fiber. The scanning-EMG signal obtained in this way is a collection of MUPs recorded from the different positions. Therefore, this signal has two dimensions: the temporal dimension, as each MUP is a signal varying in time; and the spatial dimension, representing the different recording positions taken by the scanning electrode (Fig. 1-6).

\section{Recording procedure}

In order to record a scanning-EMG signal, the first step is to insert the trigger electrode within the muscle to record the activity from a MU. During slight voluntary contraction activity, the electromyographist adjusts the position of the trigger electrode so that a stable waveform, free of interference from other MUs is observed. This position should be maintained during all the recording procedure. Then, the scanning electrode is inserted 20 to $30 \mathrm{~mm}$ away from the trigger electrode along the direction of the MU fibers. The insertion of the scanning electrode must be as perpendicular to 


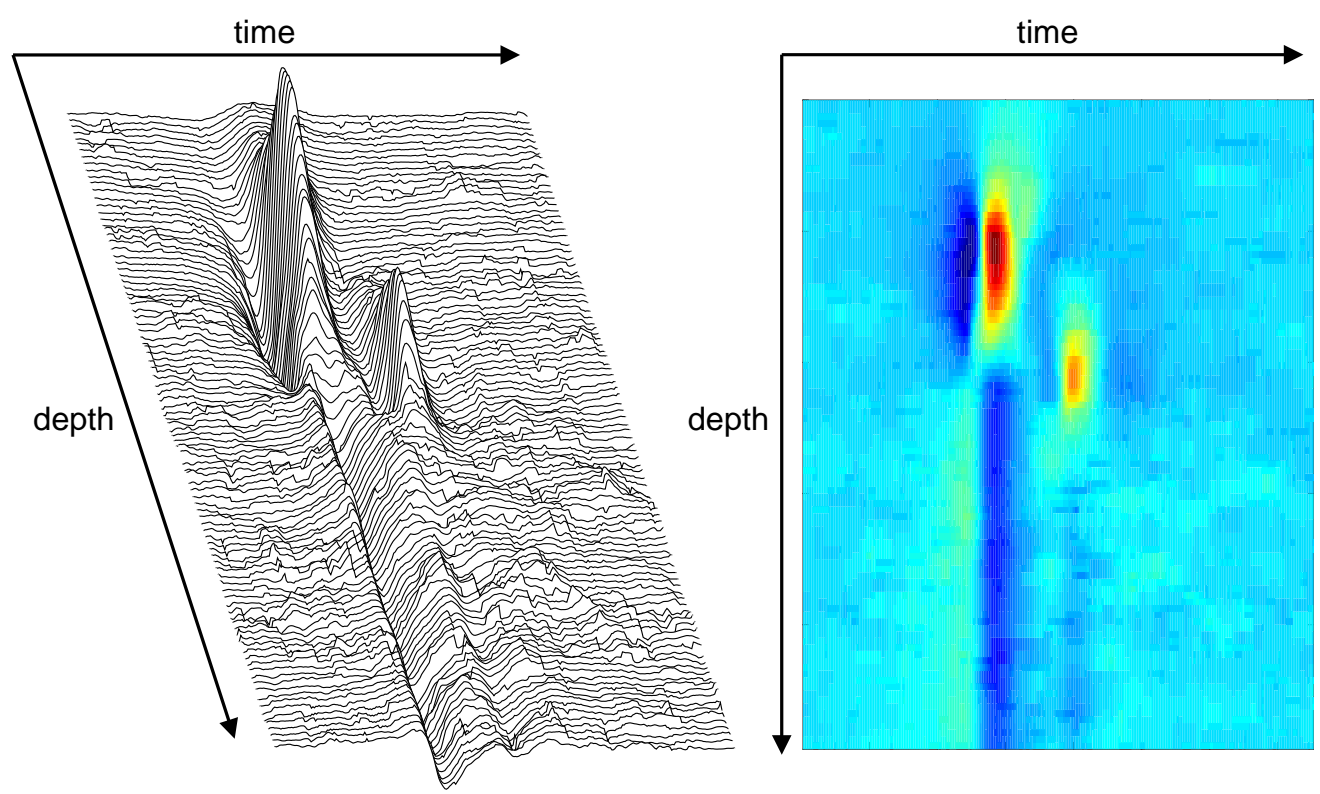

Fig. 1-6. Representation of a scanning-EMG signal; (a) waterfall plot comprising all recorded MUPs associated to the different depths; (b) Color map plot of the two-dimensional potential associated to the scanning-EMG signal. The longitudinal axis represents the temporal dimension, and the vertical one the spatial dimension, i.e., the different depths at which the MUP is recorded. Note that two separated regions of the signal with high amplitude (i.e., two MU fractions) can be observed. Note also that the deepest positions present an inverted MUP waveform associated to the potential recorded with the cannula.

the MU fibers as possible. Once the scanning electrode is inserted, it is moved looking for electrical activity synchronized with the trigger signal. This activity should have high amplitude and a short rise time in order to ensure the scanning electrode is inside the MU territory. Then, the needle is pushed until reaching a position in which electrical activity is barely detected, so that the MU territory has been completely traversed by the needle. After that, the scanning electrode is connected to a step-motor whose function is to manage the movement of the scanning electrode. During the signal recording, the step-motor pulls the scanning electrode in steps of $50 \mu \mathrm{m}$ until the electrode exits the skin. To record the MUP at each step, the following procedure is performed:

1- When the trigger signal exceeds a certain amplitude threshold, a trace of the scanning signal within a temporal window is recorded. The temporal window must be set to ensure that it always contains the entire MUP.

2- The micro-motor advances a step to the next position.

3- The system waits for the occurrence of another trigger event.

The complexity and the invasiveness of the recording setup is the main limitation 
of the scanning-EMG thecnique. Another limitation of this thecnique is that it is biased towards low threshold MUs, as the signal recording is usually made at low muscle contraction levels.

\section{Signal analysis}

The scanning-EMG signal reflects how the electrical activity of the MU changes as the relative geometry of MU fibers respect to the electrode changes. This provides valuable information related to the arrangement of the MU fibers within the $\mathrm{MU}$ territory. This information cannot be provided by any other EMG technique. It is very common to observe activity regions of the scanning-EMG signal clearly separated in time or space called MU fractions (Fig. 1-6), and regions of very low amplitude between fractions called silent zones. Fractions correspond to parts of the MU in which there are MU fibers next to the corridor, while silent zones correspond to parts of the MU depleted of MU fibers. The scanning-EMG signal not only provides spatial information, but also temporal. Different fractions of the same scanning-EMG signal use to present different latencies [120,87]. This suggests that each fraction corresponds to a set of MU fibers innervated by a common axonal branch, and the innervation zone corresponding to this set of fibers would occupy only a narrow sub-band of the overall innervation zone $[120,122,87]$.

Another important characteristic of the scanning-EMG signal is that an inverted MUP waveform can be observed in the deepest positions of the corridor (Fig. 1-6). In these positions the recording electrode is too far away from the MU territory to detect the activity of the MU fibers, and therefore, MU fibers are only detected by the cannula, which is entirely traversing the MU territory.

Quantitative analysis of the scanning-EMG signal can be performed taking individual traces of the scanning-EMG signal. In addition to the QEMG features, the scanning-EMG signal allows to extract other features that exploit the two-dimensional nature of the signal. Some of these features are: the length of the MU territory [42], the number and length of MU fractions [122], the number and length of polyphasic fractions [122], the temporal delay between fractions [87], the number and length of silent zones [122], the maximum amplitude [6], number of peaks [6], spike duration [6], and maximum amplitude $\times$ spike duration [6].

\section{Multi-scanning-EMG}

Multi-scanning-EMG is a modified implementation of the scanning-EMG technique that allows to simultaneously record the electrical activity of several MUs in a single scan $[136,92,60]$, i.e., with a single insertion of the scanning electrode. This provides a great advantage over the conventional scanning-EMG technique, in which the recording procedure would have to be repeated as many times as the number of MUs wanted to be investigated, implying one insertion per MU. In addition to this 
important advantage, the multi-scanning-EMG technique has allowed for the first time to study the overlapping of the MU territories within the muscle. This kind of study cannot be achieved with the conventional scanning-EMG technique.

The recording procedure of the multi-scanning-EMG technique has significant differences over that of conventional scanning-EMG. In the new technique, the aim of the trigger electrode is not to extract the firing pattern of a single MU, but of several MUs. Therefore, the trigger electrode must be suitable to record the activity of a wide region of the volume conductor. In [136], two pairs of intramuscular fine-wire electrodes were used as trigger electrode, while in [60], a two-dimensional grid of 256 surface electrodes placed on the skin was used for this purpose. A decomposition algorithm is used to extract the firing pattern of the MUs from the trigger signal. Another important difference relates to the recording procedure. In conventional scanning-EMG, when the firing of the MU is detected, a short trace containing the MUP is recorded, and then the scanning electrode is moved to the next position. Conversely, in multi-scanning-EMG, the scanning electrode remains at each position a predefined time interval, and the entire EMG segment is recorded with the scanning electrode at each position. Using the firing pattern of the MUs decomposed from the trigger signal, MUPs are extracted from each EMG segment, and the scanning-EMG signals are built from the MUPs obtained at each position. A more advanced technique, the single-needle-multi-scanning-EMG [23, 92], applies EMG decomposition techniques to each EMG segment and builds the scanning MUPs by joining the MUP waveforms from contiguous recording positions according to shape similarity criteria. In this technique, only the scanning needle is needed to perform the multiple scanningEMG recording.

\subsubsection{Surface electromyography}

Surface-EMG consists on recording the electrical activity produced by the muscle fibers from one or several electrodes placed on the surface of the skin. It is therefore a noninvasive method, very useful when the use of intramuscular needles is not desirable or not possible, as it occurs in sports medicine, ergonomics, or clinical examinations of children [77]. Due to the long distances between the muscle fibers and the recording electrodes, signals recorded with surface-EMG are very influenced by the propagation through the muscle tissue. This tissue acts as a low-pass filter [77], making a lot of muscle fibers to contribute with a similar amplitude. In this regard, the skin and the fat layers also contribute to additionally filter the potential contributions of the muscle fibers. For this reason, the scope of the analysis of the surface-EMG is different from that of intramuscular-EMG. While intramuscular-EMG is more suitable to analyze the electrical activity from a few MU fibers, surface-EMG is especially useful when it comes to analyze the activity of the entire MU, or even the whole muscle. A great advantage of surface-EMG is that multiples electrodes can be placed on the skin to record the signal from different spatial locations, so that the spatial evolution of the potential can be analyzed [149]. This provides useful information about some anatomical and physiological characteristics of the MU, such as the location of the IZ, 
the average MFCV, the average fiber length, or the location and size of the MU [149]. This information is difficult to obtain from intramuscular-EMG, especially information related to initiation, propagation and extinction of the potential along the muscle fibers.

\section{Recording procedure}

In order to record the surface-EMG signal, surface electrodes are placed on the skin. The recording can be derived from the potential difference between each of these electrodes and another reference electrode placed far away from the muscle under study [149]. Alternatively, the recording can be obtained from the activity of each pair of adjacent electrodes, without using an external electrode reference [149]. These two configurations are called monopolar and bipolar recording, respectively. There are more complex configurations, in which more than two surrounding electrodes are used to derive the signal recording. These derivations cause a high-pass filtering effect, aimed to increase the spatial selectivity of the recording [149]. Regarding the geometry of the electrode grid, it can be placed forming a linear array either along the direction of the muscle fibers, or perpendicular to it. The type of information obtained about the MU is different in each case [149] (see section 1.2.2). It is also possible to use twodimensional electrode arrays to obtain information in both spatial directions. The grid geometry also determines the number of dimensions of the recorded surface-EMG signal. If a linear array is used, the recording has one spatial and one temporal dimension. Instead, when a two-dimensional array is used, the recording has two spatial and one temporal dimensions.

\section{Signal analysis}

MUPs corresponding to different MUs can be extracted from the recorded surfaceEMG signal. Once a surface-MUP is extracted, its subsequent analysis allows to obtain some relevant characteristics of the MU. However, identifying individual surfaceMUPs from the recorded signal is not always easy. This task is hindered by the presence of distant components from co-activated MUs. To extract a surface-MUP from a certain MU, an intramuscular needle can be inserted within the muscle, so that the firing pattern of this MU is obtained. Then, spike-trigger averaging is applied to extract the surface-MUP from the surface-EMG signal [33].

By using a linear electrode array placed in the direction of the muscle fibers to record the signal, the average MFCV of the MU, the location of the IZ, and the location of the tendon zones can be estimated. The average MFCV can be estimated by detecting the MUP at two or more channels as it propagates along the muscle fibers. These channels must be located between the IZ and one of the tendon regions. The time delay of the propagating MUPs can be measured, and the average MFCV of the MU can be obtained by dividing the inter-electrode distance by the time delay. Based on this rationale, several algorithms to calculate the average MFCV have been 
developed $[10,71,5,148,135,30,31]$.

The location of the IZ is associated with a change in the direction of the MUP propagation. In bipolar recordings, an inversion of the polarity is produced in this region (Fig. 1-7). The width of the IZ can be approximated from the number of electrodes presenting a reduced and/or irregular amplitude. Several methods have been used to estimate the IZ by using linear arrays $[69,70,75,29]$.

The tendon zone can be identified from the attenuation of the potential at the extremes of the array [76] (Fig. 1-7). Information related to the tendon zone can also

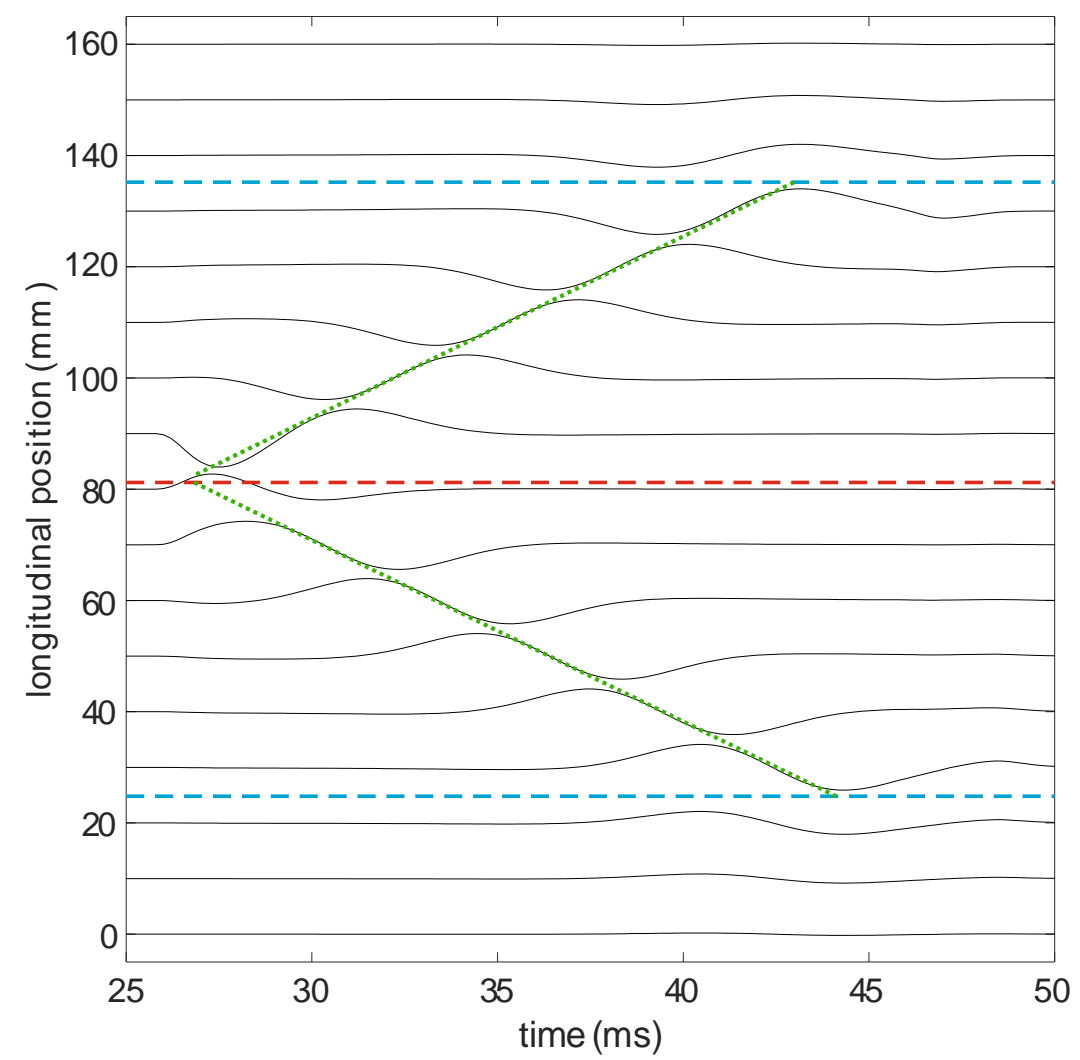

Fig. 1-7. Representation of a bipolar surface-MUP, where the horizontal axis represents the temporal dimension, and the vertical axis represents the different recording positions corresponding to the different channels, which in this example are placed along the direction longitudinal to the muscle fibers. The dashed red line represents the IZ. Note the inversion of the polarity of the recorded MUPs respect to this position. The dashed blue lines represent the extinction of the potential in both tendon zones. Note that, beyond the tendon position, the recorded MUPs are gradually attenuated but stay in the same position (nonpropagating components). The dotted green lines represent the wave front propagation along the different recording positions. Note that the slope of these lines corresponds to the average MFCV. 
be obtained from the interval in which the potentials propagate. This interval in combination to the average MFCV gives an estimation of the mean half length of the MU fibers [149].

Other anatomical properties of the MU can be estimated if the linear electrode array is placed along the direction perpendicular to the muscle fibers. In this case, information about the location, depth, and size of the MU can be obtained. The signal amplitude depends on the MU-electrode distance, and therefore, the MU position can be estimated by analyzing the amplitude profile of the surface-MUP among the different recording electrodes [104]. The location of the MU under the skin corresponds to the electrode with the highest amplitude, and the MU depth can be deduced from the amplitude decline of the surface-MUP. Regarding the size of the MU, a positive correlation between this parameter and the amplitude and area of the monopolar surface-MUP has been observed [105, 106]. 


\subsection{State of the art of the inverse problem}

This section describes the state of the art of the forward and inverse problem of the electromyographic MUP. Other models dealing with MU recruitment, MU firing rate, MU territory placement and overlapping, muscle fiber innervation, etc. are not discussed here, but in the next section (see section 1.4).

The forward problem in MU electromyography models how the underlying anatomical and physiological characteristics of the MUs (e.g., parameters in Table 1-1) influence in the generated MUP signals (e.g., features in Table 1-1). Conversely, the inverse problem analyzes the recorded MUP to infer these anatomical and physiological characteristics.

\subsubsection{The forward problem}

There are several mathematical models that describe how the MUP is related to the structure of the MU. The use of these models has an important role in the understanding and interpretation of the relationship between the MUP waveform and the anatomy and physiology of the MU, not only in normal conditions, but also in pathology. In this section, an unanimously accepted MUP model is described, as well as the most relevant MUP modeling studies.

\section{Modeling the MUP}

MUP models provide a mathematical description of the MUP waveform. These models obtain the simulated MUP by summing the SFAPs corresponding to each MU fiber. The SFAP model, in turn, is determined by a set of parameters describing the muscle fiber. For each muscle fiber of the MU, these parameters are: the position in the muscle cross-section, the position of its neuromuscular junction, the length, the diameter, and the MFVC (Fig. 1-8).

\section{Modeling the SFAP}

There are a lot of models of the SFAP in the literature, such as $[107,66,95,96$, $97,2,81,43,134,21]$. The most widely used are the model developed by Nandedkar and Stålberg [81], and the one developed by Dimitrov and Dimitrova [21], due to their computational efficiency. Here, the Dimitrov-Dimitrova model is described, as it will be the one used throughout the thesis.

The Dimitrov-Dimitrova model describes the SFAP as the convolution of an excitation source, dependent on the IAP, and an impulse response depending on the recording point and on the electrical properties of the volume conductor. This description assumes that the shape of the IAP and the propagation velocity (the MFCV) 


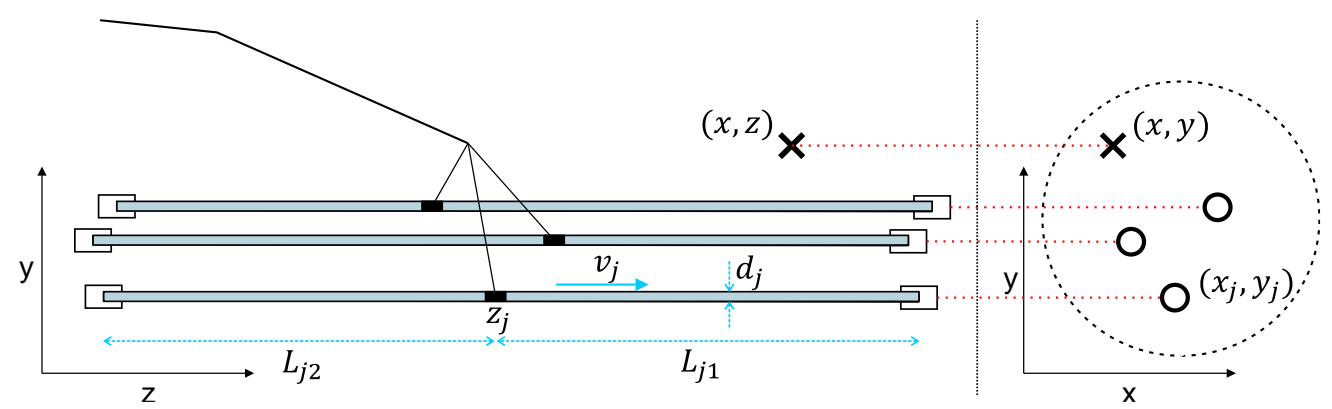

Fig. 1-8. Schematic representation of the parameters characterizing the fibers of the MU in the muscle section plane longitudinal to the MU fibers (left sub-figure), and the muscle crosssection plane, perpendicular to the MU fibers (right sub-figure). Given a certain MU fiber, denoted by index $j$, its parameters are: the position in the muscle cross-section $\left(x_{j}, y_{j}\right)$; the position of its neuromuscular junction $z_{j}$; the two fiber semi-lengths, $L_{j 1}$ and $L_{j 2}$ respectively; the diameter $d_{j}$; and the MFCV $v_{j}$. The recording point $(x, y, z)$ is represented by a black cross.

are not altered along the muscle fiber, so that the system can be considered as linear time-shift invariant.

Let's consider the $j$ th a muscle fiber located at the position $\left(x_{j}, y_{j}\right)$ of the muscle cross-section, with the neuromuscular junction located at position $z_{j}$ of the longitudinal axis, with semi-lengths $L_{j 1}$ and $L_{j 2}$, diameter $d_{j}$, and a MFCV of $v_{j}$ (Fig. 1-8). The SFAP generated by the $j$ th muscle fiber can be calculated as

$$
\operatorname{SFAP}_{j}(t)=C_{e} \cdot d_{j}^{2} \cdot \frac{1}{v_{j}} \cdot \frac{\partial I A P(t)}{\partial t} * I R_{j}(t)
$$

where $C_{e}$ is a proportionality constant depending on the tissue conductivity, and $t$ denotes the temporal dimension. The excitation source is the first derivative in time of the IAP, $\partial I A P(t) / \partial t$. Different mathematical descriptions of the IAP can be found in $[107,81,21,100]$. The impulse response, $I R_{j}(t)$ can be expressed as

$$
I R_{j}(t)=I R_{j 1}(t)+I R_{j 2}(t)
$$

where

$$
I R_{j 1}(t)=C_{1} \frac{\partial}{\partial t}\left\{\frac{1}{\left[\left(z-z_{j}-v_{j} t\right)^{2}+K_{a n}\left(x-x_{j}\right)^{2}+K_{a n}\left(y-y_{j}\right)^{2}\right]^{\frac{1}{2}}}\right\}
$$

and 


$$
I R_{j 2}(t)=C_{2} \frac{\partial}{\partial t}\left\{\frac{1}{\left[\left(z-z_{j}+v_{j} t\right)^{2}+K_{a n}\left(x-x_{j}\right)^{2}+K_{a n}\left(y-y_{j}\right)^{2}\right]^{\frac{1}{2}}}\right\}
$$

where $(x, y, z)$ is the point of the space in which the SFAP is calculated, with $z$ representing the position in the direction longitudinal to the fiber (Fig. 1-8); the coefficient $K_{a n}=\sigma_{z} / \sigma_{r}$, is the anisotropy ratio, where $\sigma_{r}$ and $\sigma_{z}$ are the conductivities in the transversal and longitudinal direction respectively; and

$$
C_{1}= \begin{cases}1 & t \leq L_{j 1} / v_{j} \\ 0 & t>L_{j 1} / v_{j}\end{cases}
$$

and

$$
C_{2}= \begin{cases}1 & t \leq L_{j 2} / v_{j} \\ 0 & t>L_{j 2} / v_{j}\end{cases}
$$

Note that the two components of the impulse response, $I R_{j 1}(t)$ and $I R_{j 2}(t)$, are associated to the propagation in both directions of the muscle fiber. respectively.

It is important to keep in mind that there is a dependency between the MFCV and the muscle fiber diameter, which can be modeled as [81]

$$
v_{j}=3.7+50 \cdot\left(d_{j}-0.055\right)
$$

with $v_{j}$ expressed in $\mathrm{m} / \mathrm{s}$ and the fiber diameter $d_{j}$ in $\mathrm{mm}$.

\section{Calculating the MUP}

The MUP is calculated as the summation of the SFAPs from all MU fibers

$$
\operatorname{MUP}(t)=\sum_{j=1}^{N_{f}} \operatorname{SFAP}_{j}(t)
$$

where $j$ is indexing the different fibers of the MU, and $N_{f}$ is the number of MU fibers.

\section{Modeling studies}

MUP models have been used to study the relationship between the structure of the MU and the waveform of the MUP, as well as to analyze how changes in the MU morphology undergoing in certain pathological processes affect to this waveform [139, 
$126,127,84,85,86]$. These modeling studies basically consist on the following steps. Firstly, the structure of the MU is simulated for a certain number of realizations. In this way a particular set of parameters that characterize the MU is obtained for each realization. The statistical distribution of the MU parameters is different depending if the MU is simulated in normal conditions, or if myopathy or neuropathy is simulated. Some of these parameters are the number and spatial distribution of the MU fibers, fiber diameters, neuromuscular junction positions, etc. Secondly, the position and the recording characteristics of the electrode are established. Thirdly, the MUP is simulated for each realization of the MU parameters. Fourth, morphological features such as amplitude, duration, area, size-index, turns, phases, or irregularity coefficient are extracted from the MUP [121, 138, 124]. And finally, sample statistics of each MUP feature are extracted. This procedure can be repeated to emulate different conditions of the MUs by varying the distribution of the MU parameters, so that the dependency between MU parameters and MUP features can be studied. In Table 1-1, a summary of the most relevant modeling studies in the literature is presented, showing the dependencies between MU parameters and MUP features that have been analyzed.

Table 1-1. Dependencies between MU parameters and MUP features analyzed in the different modeling studies (marked with an X).

\begin{tabular}{|c|c|c|c|c|c|}
\hline \multirow[t]{2}{*}{ Modeling study } & \multirow[t]{2}{*}{ Parameter varied } & \multicolumn{4}{|c|}{ Feature } \\
\hline & & Amplitude & Duration & Area & Size-index \\
\hline \multirow{2}{*}[84]{} & Electrode-IZ distance & $\mathrm{X}$ & $\mathrm{X}$ & $\mathrm{X}$ & \\
\hline & Number of MU fibers $\propto$ Territory area & $\mathrm{X}$ & $\mathrm{X}$ & $\mathrm{X}$ & \\
\hline [85] & Number of MU fibers $\propto$ Fiber density & $\mathrm{X}$ & $\mathrm{X}$ & $\mathrm{X}$ & \\
\hline \multirow{2}{*}{ [86] } & Number of MU fibers $\propto$ Fiber density & $\mathrm{X}$ & $\mathrm{X}$ & $\mathrm{X}$ & \\
\hline & Variability of fiber diameters & $\mathrm{X}$ & $\mathrm{X}$ & $\mathrm{X}$ & \\
\hline \multirow{4}{*}{ [126] } & Number of MU fibers $\propto$ Territory area & $\mathrm{X}$ & $\mathrm{X}$ & $\mathrm{X}$ & $\mathrm{X}$ \\
\hline & Number of MU fibers $\propto$ Fiber density & $\mathrm{X}$ & $\mathrm{X}$ & $\mathrm{X}$ & $\mathrm{X}$ \\
\hline & Electrode-IZ distance & $\mathrm{X}$ & $\mathrm{X}$ & $\mathrm{X}$ & $\mathrm{X}$ \\
\hline & Mean of the fiber diameters & $\mathrm{X}$ & $\mathrm{X}$ & $\mathrm{X}$ & $\mathrm{X}$ \\
\hline \multirow{4}{*}{ [127] } & Number of MU fibers $\propto$ Fiber density & $\mathrm{X}$ & $\mathrm{X}$ & $\mathrm{X}$ & $\mathrm{X}$ \\
\hline & Number of fibers arranged in groups & $\mathrm{X}$ & $\mathrm{X}$ & $\mathrm{X}$ & $\mathrm{X}$ \\
\hline & Reinnervation after loss of neurons & $\mathrm{X}$ & $\mathrm{X}$ & $\mathrm{X}$ & $\mathrm{X}$ \\
\hline & Variability of fiber diameters & $\mathrm{X}$ & $\mathrm{X}$ & $\mathrm{X}$ & $\mathrm{X}$ \\
\hline \multirow{3}{*}{ [139] } & Variability of fiber diameters & $\mathrm{X}$ & $\mathrm{X}$ & $\mathrm{X}$ & $\mathrm{X}$ \\
\hline & Number of MU fibers $\propto$ Fiber density & $\mathrm{X}$ & $\mathrm{X}$ & $\mathrm{X}$ & $\mathrm{X}$ \\
\hline & Electrode-IZ distance & $\mathrm{X}$ & $\mathrm{X}$ & $\mathrm{X}$ & $\mathrm{X}$ \\
\hline
\end{tabular}




\begin{tabular}{|c|c|c|c|c|c|}
\hline & & Rise-time & Turns & Phases & Irreg. Coef \\
\hline \multirow{2}{*}{ [84] } & Electrode-IZ distance & & & $\mathrm{X}$ & \\
\hline & Number of MU fibers $\propto$ Territory area & & & $\mathrm{X}$ & \\
\hline [85] & Number of MU fibers $\propto$ Fiber density & & $\mathrm{X}$ & $\mathrm{X}$ & \\
\hline \multirow{2}{*}{ [86] } & Number of MU fibers $\propto$ Fiber density & & $\mathrm{X}$ & $\mathrm{X}$ & \\
\hline & Variability of fiber diameters & & $\mathrm{X}$ & $\mathrm{X}$ & \\
\hline \multirow{4}{*}{ [126] } & Number of MU fibers $\propto$ Territory area & & $\mathrm{X}$ & $\mathrm{X}$ & \\
\hline & Number of MU fibers $\propto$ Fiber density & & $\mathrm{X}$ & $X$ & \\
\hline & Electrode-IZ distance & & $\mathrm{X}$ & $\mathrm{X}$ & \\
\hline & Mean of the fiber diameters & & $\mathrm{X}$ & $\mathrm{X}$ & \\
\hline \multirow{4}{*}{ [127] } & Number of MU fibers $\propto$ Fiber density & $\mathrm{X}$ & $\mathrm{X}$ & $\mathrm{X}$ & \\
\hline & Number of fibers arranged in groups & $\mathrm{X}$ & $\mathrm{X}$ & $\mathrm{X}$ & \\
\hline & Reinnervation after loss of neurons & $\mathrm{X}$ & $\mathrm{X}$ & $\mathrm{X}$ & \\
\hline & Variability of fiber diameters & $\mathrm{X}$ & $\mathrm{X}$ & $\mathrm{X}$ & \\
\hline \multirow{3}{*}{ [139] } & Variability of fiber diameters & & $\mathrm{X}$ & $\mathrm{X}$ & $\mathrm{X}$ \\
\hline & Number of MU fibers $\propto$ Fiber density & & $\mathrm{X}$ & $\mathrm{X}$ & $\mathrm{X}$ \\
\hline & Electrode-IZ distance & & $\mathrm{X}$ & $X$ & $X$ \\
\hline
\end{tabular}

\subsubsection{The inverse problem}

The inverse problem consists on determining the structure of the MU from the information provided by a recorded MUP. Hence, the objective is to provide a set of parameters describing the individual muscle fibers of the MU. This problem can also be called MUP decomposition, as the solution requires the MUP to be decomposed in terms of the different SFAP contributions to the potential [67]. Given the available models $[81,21]$, some of the most relevant parameters to be estimated for each MU fiber are: the position in the muscle cross-section, $\left(x_{j}, y_{j}\right)$, the neuromuscular junction position, $z_{j}$, the fiber semi-lengths $L_{1 j}$ and $L_{2 j}$, the diameter, $d_{j}$, and the MFVC, $v_{j}$.

The importance of the inverse problem resides in that it provides relevant physiological information about the MU. This makes its resolution a promising tool from a clinical point of view, which may lead to future applications in the diagnosis and follow-up of neuromuscular pathologies. If successfully applied, solving the inverse problem could provide some of the information of a standard biopsy, although with some differences. The first one is that a biopsy is not specific of a single MU, whereas inverse modeling is; and the second one is that inverse modeling is much less invasive [140]. On the other hand, the resolution of the inverse problem is subjected to estimation errors in parameters that in the biopsy would be directly measured.

In routine QEMG, the MUP is characterized by extracting certain features such as 
amplitude, duration, area, size-index, etc. (see section 1.2.1). Deviation from normal values of some of these features can be indicative of changes in the MU structure associated to pathological processes [124]. The great advantage of solving the inverse problem over QEMG is that the former provides a precise description of the MU in terms of its structural parameters. Because of this, it is believed that the parameters obtained from the resolution of the inverse problem could be more sensitive to changes in the MU structure than the features extracted in QEMG.

The different attempts in which the inverse problem has been partially or completely addressed are described below.

\section{Estimation of the parameters of a single muscle fiber}

There are several methods aiming to estimate certain parameters describing a single muscle fiber, and not the entire MU. In [45], a method that allows to estimate some parameters of a muscle fiber from the SFAP recorded with an 8-lead SF multielectrode was presented. The estimated parameters were: the radial distance between the fiber and the needle, the position of the muscle fiber along the needle direction, a factor related with the anisotropy tissue, and another factor related to the fiber diameter and the tissue conductivity. In order to estimate these parameters, the SFAP model described in [134] was inverted by using an optimization procedure. Recordings were performed with an 8-port multielectrode with leads disposed in a linear array along the main axis of the needle. The distance between adjacent leads was $100 \mu \mathrm{m}$. In this way, the SFAP is recorded at 8 different positions simultaneously, which considerably reduces the degrees of freedom of the problem respect to the case where only a single position is recorded. For each of the 8 recorded SFAPs, the peaks of maximum and minimum amplitude are calculated and used as the input for the optimization algorithm. This algorithm finds a set of parameters that minimizes the discrepancies between the peak values in the recording and that in the simulation. The optimization method used for this purpose was the Levenberg-Marquardt algorithm. The error function to be minimized was the relative residual

$$
\varepsilon=\sqrt{\frac{\sum_{k=1}^{K}\left(\hat{p}_{k}-p_{k}\right)^{2}}{\sum_{k=1}^{K} p_{k}{ }^{2}}}
$$

where $p_{k}$ and $\hat{p}_{k}$ are the peak values $(K=16,8$ maximum and 8 minimum) corresponding to the recorded and simulated SFAPs, respectively.

Another method to estimate parameters from a single muscle fiber was proposed by $[102,103]$. These parameters were the fiber diameter and the radial fiber-electrode distance, as well as the IAP parameters given by the model described in [100]. In this method, SFAPs recorded with a $\mathrm{CN}$ electrode are subjected to a model inversion procedure using the Dimitrov-Dimitrova model [21]. The method finds the value of 
radial distance and fiber diameter that maximizes the waveform similarity between the recorded and the modeled SFAPs. This similarity is quantified by the signal-to-error ratio (SER)

$$
S E R=\sqrt{\frac{\sum_{n=1}^{N} S F A P\left(t_{n}\right)^{2}}{\sum_{n=1}^{N}\left(\widehat{S F A P}\left(t_{n}\right)-\operatorname{SFAP}\left(t_{n}\right)\right)^{2}}}
$$

where $\operatorname{SFAP}\left(t_{n}\right)$ and $\widehat{S F A P}\left(t_{n}\right)$ are the recorded and modeled fiber potentials, respectively, and $n$ indexes the samples of the potential in the time dimension. This procedure is repeated for different combinations of IAP parameters, so that a specific value of diameter and radial distance is obtained for each combination. Only the pairs diameter-distance whose corresponding SER is higher than a certain threshold, are accepted as valid. The diameter and radial distance pairs obtained in this way are used to estimate a bi-variate Gaussian distribution. The peak of the distribution (mean vector) gives the final estimated values for the diameter and the radial distance.

In [142], a method to simultaneously estimate the muscle fiber diameter and the radial fiber-electrode distance from an SFAP recorded with an SF electrode was developed. These two parameters are estimated from two morphological features of the SFAP: the peak-to-peak amplitude, and the negative peak duration. Both amplitude and duration depend on the fiber diameter and the radial distance. The authors studied these dependences by using the Nandedkar-Stålberg model [81]. In this way the dependencies between the parameters and the features were approximated by means two bi-quadratic polynomials. The coefficients of these polynomials were calculated from the mapping between fiber parameters and SFAP features obtained in the simulation study, by means of a least-squares fitting. Once these coefficients are obtained, the bi-quadratic equations can be used to calculate the diameter and the radial distance of a muscle fiber from the amplitude and duration of its corresponding SFAP. This method was applied in [144], to estimate the diameter of muscle fibers from SFAPs recorded in healthy subjects.

In [147] a modification of the method was proposed. Instead of using the amplitude and duration as the features employed to calculate the fiber diameter, the authors proposed to use the ratio of the first minimum to the maximum, and the ratio of the right minimum to the left minimum as features. The authors also proposed to use both versions of the method simultaneously as a way to detect if the SFAP is contaminated with activity from other muscle fibers. If the SFAP is contaminated, the two versions should give contradictory values of the muscle fiber diameter.

\section{Estimation of the MU global parameters}

There are several methods in which some global parameters of the MU have been estimated. Among these parameters, an important one is the number of MU fibers per 
unit area. Information about this parameter can be obtained from the electrophysiological measurement of the fiber density [115, 39, 40], already described in section 1.2.1. This measure gives an estimation of the average number of SFAPs from the same MU significantly contributing to the recorded signal. The electrophysiological fiber density is directly related with the theoretical average number of MU fibers within the electrode uptake area. Under the assumption that the MU fibers are uniformly distributed within the MU territory, the electrophysiological fiber density and theoretical MU fiber number in the uptake area can be related by [40]

$$
N_{e l}-1 \cong N_{t h}
$$

where $N_{e l}$ and $N_{t h}$ are the electrophysiological fiber density, and theoretical MU fiber number, respectively.

The reason why these two parameters differ in one unit is because the electrophysiological fiber density is measured using a muscle fiber as trigger, which is included in the count. Note that these parameters are referred to a specific electrode uptake area, which for the SF electrode is approximately a semicircle of $300 \mu \mathrm{m}$ radius. To calculate the average number of MU fibers per $\mathrm{mm}^{2}$, we can use the following expression

$$
D=\frac{N_{t h}}{\pi R^{2} / 2} \cong \frac{N_{e l}-1}{\pi R^{2} / 2}
$$

where $D$ is the average number of MU fibers per $\mathrm{mm}^{2}$, and $R$ is the radius of the semicircular uptake area. An important consideration to keep in mind about this method is that this fiber density measurement is not representative of a single MU, but is an average value obtained from different MUs of the same muscle.

To quantify the number of SFAPs significantly contributing to the MUP, the number of contributions whose amplitude exceeds $200 \mu \mathrm{V}$, and whose rise time is less than $300 \mu \mathrm{s}$ is counted. This is the protocol used in fiber density measurements [115]. In [130], an alternative and more accurate procedure was proposed. The authors demonstrated that the number of significant SFAP contributions is strongly correlated to the number of peaks in the MUP acceleration signal, i.e., in the second order difference of the MUP. Thus, the number of significant SFAPs can be estimated as the number of peaks exceeding certain threshold in the MUP acceleration signal. As in the case of the fiber density measurements, the identification of the individual peaks is limited by the temporal separation of the SFAP contributions.

In the study presented in [61], several architectural parameters of the MU were obtained from MUPs recorded with a MN electrode. These parameters were the axial distance between the IZ and the electrode, and the distance between the electrode and the tendon location (Fig. 1-9). To obtain these parameters, some morphological 

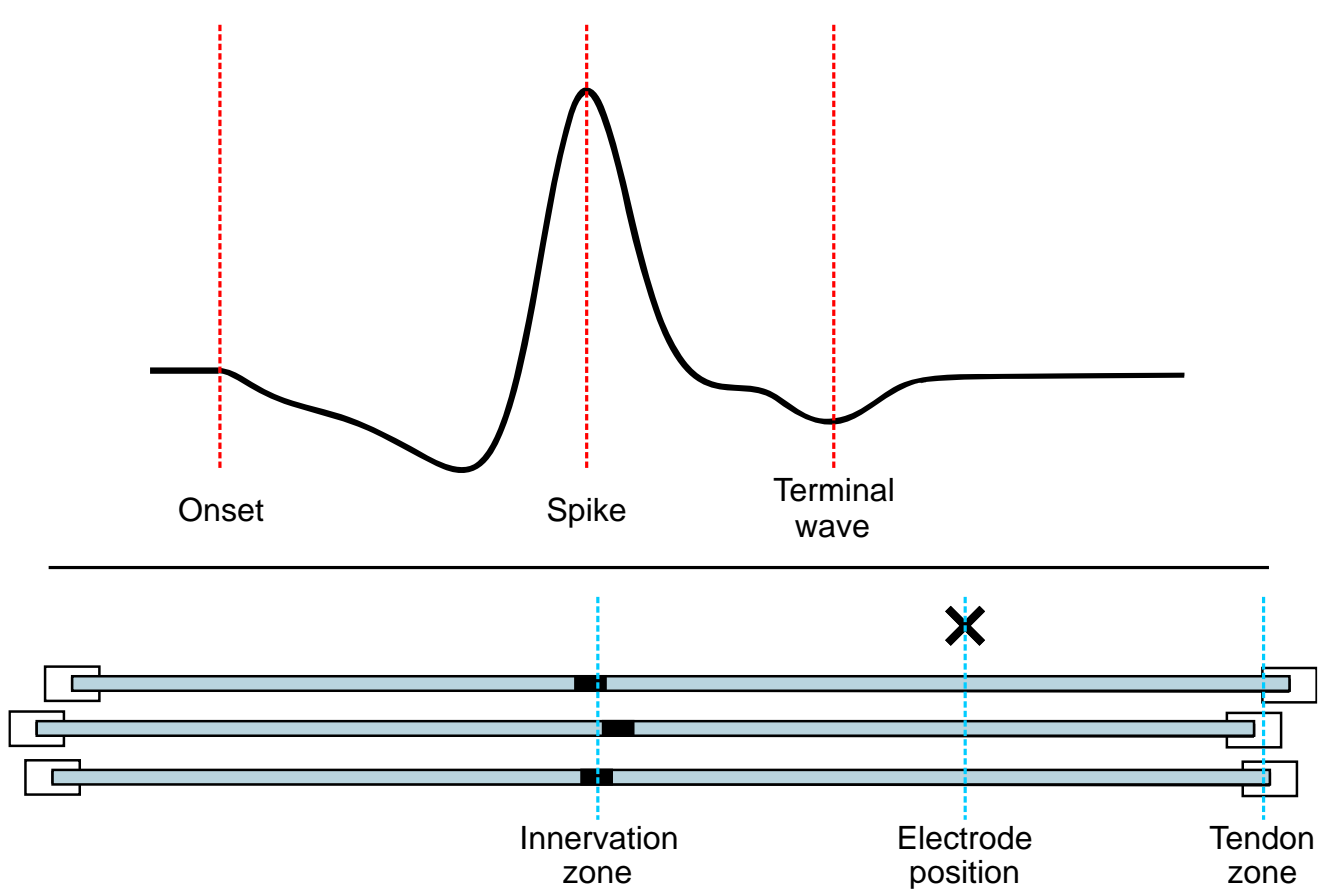

Fig. 1-9. Schematic representation of the MUP features (upper subfigure) and the global MU parameters (lower subfigure) involved in the study of [61].

features of the MUP were extracted: the onset, the spike and the terminal wave of the MUP (Fig. 1-9). The IZ-electrode distance was calculated from the time delay between the onset and the spike, and the electrode-tendon distance from the time delay between the spike and the terminal wave. These distances were calculated by multiplying the corresponding time delays by the average muscle fiber propagation velocity, which was assumed to be $4 \mathrm{~m} / \mathrm{s}$. The reason why a MN electrode was used in this analysis to record the MUPs is because this is the most suitable electrode to distinguish non propagating components (i.e., the onset and the terminal wave). Note that these components are barely noticeable in other electrodes due to the cancellation effect produced by the cannula.

Global information about the MU can also be obtained from multi-electrode surface-EMG recordings, as it has been detailed in section 1.2.2. If a linear electrode array is placed along the muscle fiber direction, the surface-EMG recording allows to estimate the IZ [69, 70, 75, 29], the average MFCV [10, 71, 5, 148, 135, 30, 31], and the tendon zone [76]. When the linear array is placed perpendicularly to the muscle fibers, estimations about location [104], depth [104], and size of the MU [105, 106] can be obtained. Additional information can be obtained if a two-dimensional array is used. As an example, an algorithm that simultaneously estimates the average MFCV, and the muscle fiber orientation from a two-dimensional recording has been developed by [46]. 


\subsubsection{First attempt of a complete MUP decomposition}

So far, only partial approximations to the estimation of the structure of the MU have been described. The first attempt to fully address the estimation of the MU structure was proposed in [67,68]. Lööf developed an algorithm that decomposes the MUP into its constituting SFAPs. In this work, the MUP model was inverted by fitting the MUP as a combination of SFAPs from a pool of pre-simulated SFAP waveforms. The model used to simulate the SFAPs was a modified version of the NandedkarStålberg model [81], adapted to simulate $\mathrm{MN}$ electrode recordings. With this algorithm, parameters such as the number of contributing MU fibers, the MU fiber density, and the temporal dispersion of the SFAPs can be estimated.

\section{Algorithm description}

The first step of the algorithm is to simulate a set of 4 SFAPs, each of them corresponding to a different distance between the electrode and the muscle fiber: 0.15 , $0.30,0.60$ and $1.20 \mathrm{~mm}$. All SFAPs are simulated with a fiber diameter of $55 \mu \mathrm{m}$, and an MFCV of $3.7 \mathrm{~m} / \mathrm{s}$. These simulated SFAPs are used as templates to fit the MUP, so that the MUP is represented by a variable set of templates, each of them adequately time-shifted and scaled. To decompose the MUP into its constituent templates, an iterative procedure is performed.

In the first iteration, each of the 4 templates are cross-correlated with the MUP. The template and the corresponding time delay producing the highest cross-correlation (we will refer to it as a factor) is selected. Then, the selected factor is used to reconstruct the MUP, by applying an adequate amplitude scale. Thus, the reconstructed MUP in this first iteration can be expressed as

$$
\widehat{\operatorname{MUP}}_{1}\left(t_{n}\right)=a_{1} \cdot f_{1}\left(t_{n}\right)
$$

where, $n$ indexes the temporal samples, $a_{1}$ is the amplitude scale, and $f_{1}\left(t_{n}\right)$ is the factor obtained in the first iteration. In order to determine the value of the amplitude scale, $a_{1}$, a least squares criterion over the residual between the recorded and reconstructed MUP is applied, where the residual is

$$
\varepsilon_{1}\left(t_{n}\right)=\operatorname{MUP}\left(t_{n}\right)-\widehat{M U P}_{1}\left(t_{n}\right)
$$

This procedure is repeated in the following iterations. Now, the 4 templates are cross-correlated with the residual obtained in the last iteration. Again, the template with the highest cross-correlation is selected and its corresponding delay is calculated, giving a new factor. This new factor is added to the set of factors already obtained in previous iterations. The updated set of factors is used to reconstruct the MUP, by determining the most adequate amplitude scales 


$$
\widehat{\operatorname{MUP}}_{r}\left(t_{n}\right)=\left[\begin{array}{lll}
a_{1}, & \ldots, & a_{r}
\end{array}\right] \cdot\left[\begin{array}{c}
f_{1}\left(t_{n}\right) \\
\vdots \\
f_{r}\left(t_{n}\right)
\end{array}\right]
$$

where $\left[f_{1}\left(t_{n}\right), \quad \ldots, \quad f_{r}\left(t_{n}\right)\right]^{T}$ is the current set of factors, and $r$ the current iteration. The amplitude scales $\left[a_{1}, \ldots, a_{r}\right]$ associated to each factor are calculated by minimizing the residual over a least-squares criterion, this residual being

$$
\varepsilon_{r}\left(t_{n}\right)=\operatorname{MUP}\left(t_{n}\right)-\widehat{M U P}_{r}\left(t_{n}\right)
$$

As the number of iterations increases the residual decreases, and, therefore, the MUP reconstruction becomes more accurate. The iterative procedure stops when the root mean square of the residual does not decrease more than a certain preset value between two consecutive iterations, or when the maximum number of factors (set to $16)$ is reached.

\section{Parameter estimation}

Relevant information about the MU can be obtained from the arrangement of factors calculated with this algorithm. The author assumed that the sum of the amplitude scales values of all factors gives an estimation of the total number of muscle fibers in the MU. The author also assumed that the factors obtained from the template simulated at $0.15 \mathrm{~mm}$ respect to the electrode are correlated with the activity of the actual muscle fibers located within a certain distance to the electrode (approximately between 0.15 and $0.3 \mathrm{~mm}$ ). Under this assumption, MU fiber density can be calculated by summing the scale amplitude values of all these factors, and dividing it by the corresponding expected area. If a homogeneous distribution of the MU fibers within the MU territory is assumed, the area of this territory can be calculated multiplying the estimated fiber density by the estimated number of MU fibers. The algorithm also estimates the arrival times of the different factors. From these, a measure of the temporal dispersion of the factors can be obtained.

\section{Experiments and results}

The author used simulated MUPs to evaluate the algorithm performance in a controlled environment. The parameters of the MUs were selected to mimic normal and myopathic conditions. In this way, parameters such as the number of MU fibers, the diameter of the MU territory, the width of the IZ or MU fiber diameters were randomly generated within the appropriate ranges to emulate these conditions. The electrode was located at a distance of $40 \mathrm{~mm}$ from the IZ. From these synthetic MUs, the corresponding MUPs were simulated using the Nandedkar-Stålberg model [81]. The estimation algorithm was applied to these simulated MUPs. In addition, MUP features such as amplitude, duration, phases, area, etc., were extracted from the simulated waveforms. The estimated parameters obtained in this analysis were 
compared to the actual input parameters used to simulate the MUs. In order to do it, the correlation coefficients between the input and output parameters were calculated.

An important conclusion of this study is that the actual parameters of the MU correlate better with some of the estimated parameters than with the MUP features. This is especially evident in the case of the number of MU fibers. This input parameter correlates well with the MUP duration (correlation coefficient of 0.47). However, if the actual number of MU fibers is confronted with the estimated number of MU fibers, this results in a correlation coefficient of 0.81 . When it comes to compare the actual MU parameters with its corresponding estimated parameters, correlations are statistically significant. However, the corresponding regression slopes noticeable differ from 1 (e.g., 0.35 for the actual and estimated number of MU fibers), which means that these parameters are not being correctly estimated. In the case of the number of MU fibers, a slope of 0.35 means that, on average, only one-third of the total number of fibers are being detected.

\section{Limitations of the algorithm}

Limitations of this estimation algorithm are mainly caused by the limited computing capacity of the computers at the time in which the algorithm was developed (1986). The main drawback of the algorithm is that it uses a limited number of templates, simulated at fixed distances and with a predefined fiber diameter value. This implies that the algorithm is not able to estimate neither the exact distances of the MU fibers to the electrode, nor its diameters and MFCVs. Furthermore, the neuromuscular positions of the MU fibers are also not estimated with this algorithm. However, all these simplifications may help in the stability of the algorithm and unicity of the solutions.

\subsubsection{Second attempt of a complete MUP decomposition}

The second attempt to perform the estimation of the MU structure was made by Zalewska and Hausmanowa-Petrusewicz [140]. The method, called the approximation method, consists on inverting the model of the MUP by using an optimization algorithm. The algorithm tries to find a set of parameters describing the MU such that the corresponding simulated MUP waveform is as similar as possible to that of the real MUP. The model developed by Nandedkar and Stålberg [81, 84], and modified by Stålberg and Karlson [126, 127], was used to simulate the MUP. The approximation method allows to estimate relevant parameters of the MU such as the number of fibers contributing to the MUP, the fiber distances to the electrode, the fiber diameters and the distance of the electrode to the IZ.

\section{Algorithm description}

In the algorithm, the simulated SFAP is considered to be a function of the 
following parameters

$$
\widehat{\operatorname{SFAP}}_{J}\left(t_{n}\right)=\widehat{\operatorname{SFAP}}_{J}\left(t_{n}, d_{j}, r_{j}, \Delta_{Z}, \Delta t_{0}\right)
$$

where $d_{j}$ is the muscle fiber diameter, $r_{j}$ is the radial fiber-electrode distance, $\Delta_{Z}$ is the axial distance between the IZ and the electrode position, and $\Delta t_{0}$ is the unknown time offset between the origin of the time axis in the recording and the actual potential initiation instant. Note that $d_{j}$ and $r_{j}$ are specific parameters of each MU fiber, while $\Delta_{Z}$ and $\Delta t_{0}$ are global parameters. The simulated MUP can therefore be expressed as

$$
\begin{gathered}
\widehat{\operatorname{MUP}}\left(t_{n}\right)=\widehat{\operatorname{MUP}}\left(t_{n},\left[d_{1}, \ldots, d_{J}\right],\left[r_{1}, \ldots, r_{J}\right], \Delta_{Z}, \Delta t_{0}\right) \\
=\sum_{j=1}^{J} \widehat{\operatorname{SFAP}}\left(t_{n}, d_{j}, r_{j}, \Delta_{Z}, \Delta t_{0}\right)
\end{gathered}
$$

where $J$ is the number of MU fibers. The goal of the algorithm is to find the set of parameters $\left(\left[d_{1}, \ldots, d_{J}\right],\left[r_{1}, \ldots, r_{J}\right], J, \Delta_{Z}, \Delta t_{0}\right)$ such that the corresponding simulated MUP is as close as possible as to the recorded MUP. In order to achieve this, the sum of the quadratic error between the simulated and recorded MUP (the energy of the error) is minimized

$$
\varepsilon=\sum_{n=1}^{N}\left(\widehat{\operatorname{MUP}}\left(t_{n}\right)-M U P\left(t_{n}\right)\right)^{2}
$$

where $N$ is the length in samples of the signals. The minimization of this error is driven by standard Powel optimization method [98]. The algorithm is initialized with a random solution of the MU parameters to be estimated. Regarding the number of MU fibers, the algorithm starts with a sufficiently large number of them (typically from 40 to 80 ). Then, during the algorithm execution, fibers that do not significantly contribute to the potential are eliminated. The criterion to eliminate MU fibers is

$$
\max (\widehat{S F A P}) / \max (M U P) \leq c \cdot d_{j}
$$

where $c=200 \mathrm{~m}^{-1}$, and $d_{j}$ is expressed in $\mathrm{m}$. The algorithm is run several times in order to select the solution that provides the lowest error.

\section{Experiments and results}

The authors tested the algorithm on MUPs recorded with CN electrodes in healthy and diseased subjects [140]. They claimed that the range of diameters and fiber densities obtained in nonpathological conditions were consistent with literature data of the morphological characteristics of the investigated muscle. The authors also stated 
that the results obtained in pathological cases were, in the same way, coherent with changes associated to these processes. However, the rigor of these claims is questionable. Keep in mind that the testing procedure shown in the published article, only consisted on three cases of use of the approximation method [140] in which the estimation is performed from MUPs recorded from a healthy muscle, a myopathic muscle, and a neuropathic muscle respectively. Thus, the sample size was not significant enough as to draw conclusions about the plausibility of the estimated parameters. There is another reason to doubt the validity of these conclusions. Note that the paper presents discrepancies between the variation ranges of estimated fiber diameters indicated in the text, and that of derived from their corresponding histograms (from which it is assumed that these ranges have been obtained). In the case of the healthy subject, the diameter range indicated in the text is much narrower than the one actually observed on the corresponding histogram. This data was used to conclude that the diameter variability was consistent with what is expected for healthy MUs.

Another important issue to note about the algorithm testing is that it has been performed without any knowledge of the actual values of the MUs' parameters, so that there is not an available ground truth to validate the results. The only information available is a certain prior knowledge about the fiber parameter distributions in normal or pathological conditions. All these issues suggest that the tests do not provide evidence that the algorithm is correctly estimating the MU fiber parameters.

The approximation method proposed in [140] has been also used in posterior publications. It was used to find a biological interpretation of satellite components in MUPs recorded from subjects suffering amyotrophic lateral sclerosis (ALS), and spinal muscular atrophy (SMA) [141]. Results of this study indicate that satellite potentials in ALS are usually originated from a single muscle fiber. In the case of SMA, results indicate that satellites are originated from a group of fibers with small fiber diameters. In another study, the method was used also to analyze MUPs with satellites in patients with dystrophinopathies [143]. As in the previous study, results suggest that satellites are originated by small muscle fibers. In [145], the approximation method was used to determine the physiological origin of MUPs recorded under several neuromuscular disorders. In this study, the distribution of muscle fiber diameters and fiber-electrode distances were obtained and analyzed for several MUPs.

In [146], the muscle fiber distribution obtained using the estimation method was compared to that obtained from a standard biopsy. In this study, different subjects diagnosed with muscle pain or weakness were subjected to a muscle biopsy and an EMG examination. For each subject, a set of about 20 MUPs was recorded, and muscle fiber diameters were estimated. Also, the diameter of 200 muscle fibers was determined from muscle biopsy. The MUP recording and the biopsy was performed for each subject in opposite muscles. The results obtained in this study indicate a strong correlation between the muscle fiber diameter distribution obtained from the approximation method, and that of the muscle biopsy. On the other hand, when 
interpreting this result, it must be taken into account that the statistical analysis has been performed at the muscle level, without taking into account to which MU each muscle fiber belongs (note that biopsy is not specific to a single MU). Therefore, this study does not provide evidence that the method is correctly estimating the MU fiber parameters.

\section{Limitations of the algorithm}

The Zalewska and Hausmanowa-Petrusewicz method is, at least theoretically, capable of estimating parameters that the method proposed by Lööf $[67,68]$ is not. This is the case of the fiber diameter and the fiber to electrode radial distance. These parameters can be estimated for those MU fibers close to the recording electrode. However, the approximation method cannot estimate other parameters of the MU fibers. The coordinates $\left(x_{j}, y_{j}\right)$ of the MU fibers in the muscle cross-section cannot be univocally estimated. Since the employed SFAP model assumes a point-size leadingoff surface, the recording electrode has radial symmetry; i.e., given a muscle fiber located at a certain distance of the electrode, $r_{j}$, the same SFAP is recorded independently of the actual position of the fiber. All positions $\left(x_{j}, y_{j}\right)$ of the fiber such that

$$
r_{j}=\sqrt{\left(x_{j}-x_{e}\right)^{2}+\left(y_{j}-y_{e}\right)^{2}}
$$

where $\left(x_{e}, y_{e}\right)$ is the electrode position within the muscle cross-section, give rise to the same SFAP.

The neuromuscular junction positions $\left(z_{j}\right)$ are not estimated either. Instead, the longitudinal distance between the overall IZ and the electrode position, $\Delta_{Z}$, are estimated. However, this distance cannot be correctly estimated if there is not prior knowledge about the actual initiation instant of the potential, which in fact is assumed to be unknown. Therefore, it is arguable that the parameter $\Delta_{Z}$ can be correctly estimated by the algorithm.

Another limitation of the approximation method is that the effect of the leadingoff surface of the electrode has not been taken into account. MUPs are assumed to be recorded with the $\mathrm{CN}$ electrode, while the modeled MUP is simulated assuming a point-size leading-off recording surface. This discrepancy between the real recording and the modeled one will undoubtedly induce additional errors in the estimation of the MU fiber parameters.

\subsubsection{Limitations due to the single position recording}

Both attempts to estimate the parameters of the MU have something in common: the recording of the MUP has been performed in a single position. Under this scenario 
the estimation of the parameters of individual MU fibers is an ill-posed problem. There are some parameters that cannot be univocally estimated. Other parameters, although they can be estimated, give rise to imprecise estimations. This is because different sets of parameters can generate simulated MUPs with very similar shapes, making it difficult or impossible to know which of those parameter sets actually correspond to that of the recorded MUP.

As it has been stated in the previous section, the position of the MU fibers cannot be univocally determined. When the recording is performed in a single position, the radial symmetry of the problem implies that only the radial distances of the MU fibers to the electrode can be determined [67, 140]. However, the correct estimation of these distances is not simple either. Given a muscle fiber, there is a compensation effect between its diameter and its radial distance so that similar simulated SFAPs can be obtained adequately varying these two parameters [45, 101, 102]. Note that the diameter and the MFCV of a muscle fiber have a direct relationship [81], and therefore the estimation of both parameters is totally linked. Thus, the limitations inherent to the estimation of the fiber diameter also arise for the MFCV. That is, the same compensation effect exists between the MFCV and the radial distance. This implies that all the radial distance, the diameter, and the MFCV are parameters difficult to be accurately estimated.

The exposed limitations occur even in the simplest case, when the parameters of a single fiber are being estimated. When the inverse problem involves the estimation of more than one MU fiber, additional compensation effects arise. Note that the superposition of different combinations of simulated SFAPs can result in very similar MUPs when they are summated. This effect further increases the difficulty to correctly estimate the MU parameters.

Another limitation inherent to the recording of the MUP in a single position is that the parameter estimation is limited to those MU fibers located sufficiently close to the electrode as to be contributing significantly to the MUP [120]. Fibers located far from the recording point cannot be correctly characterized.

To overcome these limitations, the estimation of the MU parameters must be performed by recording the MUP not only in a single position, but in multiple ones. This idea was already exposed by Gootzen [45], who indicated that a complete estimation of the muscle fiber parameters requires to record the SFAP in multiple points. Gootzen estimated the parameters of a single fiber by using a multi-electrode of 8 recording positions disposed in the vertical axis (the direction of the needle insertion). The hypothesis of this thesis is that this idea can be extended to estimate the parameters of several MU fibers.

This thesis aims to improve the inverse problem solution by taking into account these considerations. The algorithms developed during this thesis estimate the MU 
parameters from signals recorded with the scanning-EMG technique (details about this technique can be found in section 1.2.1). Using this technique, a recording of the MUP in multiple positions along a linear corridor through the MU territory is available. This provides much more information than that provided by a single-point recording, which considerably reduces the degrees of freedom of the inverse problem. Therefore, as it will be presented, a noticeably more accurate parameter estimation can be achieved. 


\subsection{Models for the simulation of electromyographic signals}

The development and testing of the algorithms and methods implemented during this thesis has required the use of synthetic EMG signals, generated in a controlled environment. In this way, the exact a priori knowledge about these signals and its generating parameters is available, allowing to objectively evaluate the performance of the algorithms.

Specifically, realistic scanning-EMG and surface-EMG signals has been generated. In order to do that, a simulation toolbox implemented on Matlab by the Biomedical Engineering Group of the UPNA has been used. The toolbox consists of the necessary models for the complete simulation of EMG signals. The models implemented in the toolbox comprise the following aspects (Fig. 1-10): muscle architecture, including MUs and recreation of muscle fiber innervation; generation of the MUP, including IAP and SFAP models; recording in the needle electrode; recruitment and firing pattern of the MUs; recording procedure of the scanning-EMG signal; and recording of the surface-EMG signal. In this section, the different models constituting the toolbox are individually described.

\subsubsection{Modeling the Muscle and MU}

The simulated muscles have a circular cross-section of a given radius. Each muscle is composed by a fixed number of MUs. The cross-sectional areas of MU territories were modeled fitting an exponential function [35] between the area of the smallest MU, and the area of the largest MU. MU territories were circular in shape except when constrained by the muscle boundary, in which case the MU territory was cut to fit within the muscle limits, and the radius was regrown so as to keep the territory area unchanged $[56,88,89]$. MU territories were placed within the muscle in such a way that the spatial variance of the overlapping between MU territories within the muscle was minimized $[110,89]$. The muscle has a given length along the direction longitudinal to the muscle fibers.

To generate the muscle fibers, a hexagonal grid is created so that the center of each hexagon represents the position of a muscle fiber. To recreate innervation, each muscle fiber is randomly assigned to a MU of those whose territory covers the fiber position [89]. With this random innervation, the MU fiber density is practically the same for all MUs of the muscle. In this way, the number of muscle fibers in each MU depends linearly on the area of the MU, and therefore, it follows an exponential distribution. The conduction velocity of muscle fibers within the MU is given by a Gaussian distribution, whose mean is modeled by an exponential function [35] between the MU conduction velocity of the smallest MU, and the MU conduction velocity of the largest $\mathrm{MU}$, and whose coefficient of variation is set to a fixed value, common for all MUs. 


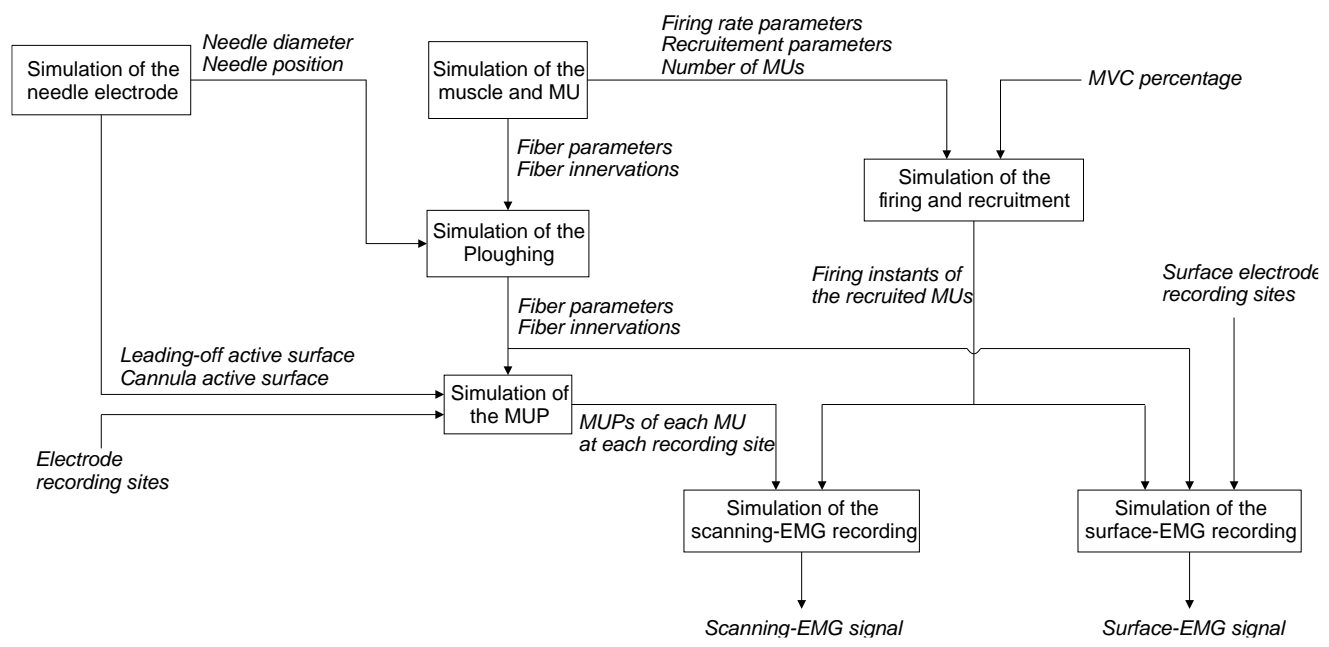

Fig. 1-10. Schematic representation of all models involved in the generation of the EMG signals.

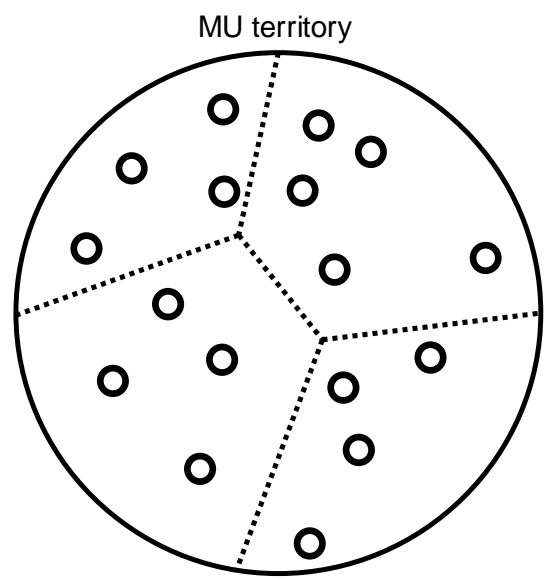

(a)

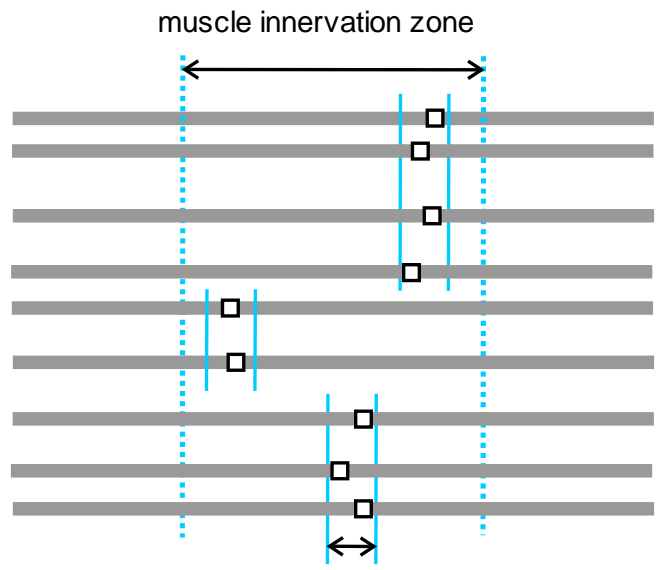

MU fraction innervation zone

(b)

Fig. 1-11. (a) Schematic representation of a simulated MU cross-section. The MU is divided in different Voronoi cells (dotted lines), which correspond to the different fractions of the MU. The fibers of the MU are represented with holed black circles; (b) schematic representation of the innervation zone of a MU of the simulated muscle. The horizontal gray lines represent the different fibers of the MU. The innervation position of the fibers (represented by squares) follow a uniform distribution within the limits of each fraction zone (vertical solid lines). The different MU fraction zones follow a uniform distribution within the muscle innervation zone (vertical dashed lines). 
The cross-section of each muscle is divided into regions called fractions (Fig. 1-11). Given a specific MU, each fraction located within the MU territory represents the set of muscle fibers innervated by a common axonal branch [119, 22, 87]. A set of points is created within the muscle cross-section at random positions following a uniform spatial distribution. From these points, a Voronoi tessellation of the MU territories is performed [93], each Voronoi cell corresponding to a different fraction (Fig. 1-11 (a)). Muscle fibers are assigned to fractions on the basis of position. The innervation zone center of the muscle was located in the middle, lengthwise, of the muscle fibers. Innervation zone centers of the different fractions within each MU are positioned according to a uniform distribution scattered in a certain width around the muscle innervation zone center (Fig. 1-11 (b)). Innervation positions for muscle fibers follows a uniform distribution of a certain width around the innervation zone center of the fraction of the MU in which the muscle fiber was located (Fig. 1-11 (b)).

In addition to the muscle, a layer of skin and a layer of fat are simulated. The simulation of these layers will be necessary for the correct simulation of the electrode due to the cannula effect in the most superficial MUs.

The values used during this thesis for the parameters of the muscle and MU are shown in Table 1-2.

Table 1-2. Parameter values used to simulate a muscle, its MUs, and its muscle fibers.

\begin{tabular}{ll}
\hline Parameter & Value \\
\hline Number of MUs in the muscle & 120 \\
Radius of the muscle cross-section & $4 \mathrm{~mm}$ \\
Area of the smallest MU & $3.125 \mathrm{~mm}^{2}$ \\
Area of the biggest MU & $25 \mathrm{~mm}^{2}$ \\
Fiber density of the MUs & $8 \mathrm{fib} / \mathrm{mm}^{2}$ \\
Number of fibers of the smallest MU & 25 \\
Number of fibers of the biggest MU & 200 \\
Length of the muscle fibers & $140 \mathrm{~mm}$ \\
Mean MFCV of the smallest MU & $3.25 \mathrm{~m} / \mathrm{s}$ \\
Mean MFCV of the biggest MU & $6.25 \mathrm{~m} / \mathrm{s}$ \\
Coefficient of variation of the MFCVs & 0.03 \\
Number of fractions of the muscle & 90 \\
Width of the overall IZ & $10 \mathrm{~mm}$ \\
Width of a fraction of the IZ & $1 \mathrm{~mm}$ \\
Width of the fat layer & $2 \mathrm{~mm}$ \\
Width of the skin layer & $1 \mathrm{~mm}$ \\
\hline
\end{tabular}




\subsubsection{Modeling the MUP}

The MUP generated at a certain point within the muscle is calculated as the summation of the SFAPs associated to the different muscle fibers of the MU. The SFAP is calculated as the convolution of the IAP and the impulse response of the corresponding muscle fiber by using the Dimitrov-Dimitrova model [21]. A complete mathematical description of this model can be found in section 1.3.1. The IAP model used in the simulations is the one proposed by Rodriguez-Falces [100].

The simulated MUP is dependent on the spatial positions, the neuromuscular junction positions, and MFCVs of the MU fibers, and on the position in which the MUP is calculated. However, the MUP is also dependent on other model parameters, which are related to the sampling frequency, the muscle tissue, and the shape of the modeled IAP (Table 1-3).

Table 1-3. Parameter values used to simulate the MUP.

\begin{tabular}{ll}
\hline Parameter & Value \\
\hline Temporal sampling frequency & $20 \mathrm{kHz}$ \\
Proportionality constant, $C_{e}$ & 0.9375 \\
Anisotropy ratio of the tissue, $K_{a n}$ & 5 \\
IAP parameter, $A_{1}[100]$ & 9000000 \\
IAP parameter, $A_{2}[100]$ & 4,5 \\
IAP parameter, $A_{3}[100]$ & 14 \\
IAP parameter, $T_{s p l}[100]$ & 2 \\
\hline
\end{tabular}

\subsubsection{Modeling the needle electrode}

The MUP model described so far assumes that the MUP is recorded by an electrode with a point-size leading-off surface. It is therefore necessary to include the effect produced by the shape of the active surface and the cannula. In order to simulate the MUP recorded with a CN electrode, the MUP (obtained from the point-size recording model) is calculated and averaged for a grid of points over the $\mathrm{CN}$ leadingoff surface area [84]. This area is an elliptical recording surface of $0.07 \mathrm{~mm}^{2}$, with a $580 \mu \mathrm{m}$ mayor axis and a $150 \mu \mathrm{m}$ minor axis. In the case of a facial $\mathrm{CN}$ electrode, the recording area is $0.02 \mathrm{~mm}^{2}$ with a $300 \mu \mathrm{m}$ mayor axis and an $80 \mu \mathrm{m}$ minor axis (Fig. 1-12). When it comes to simulate the MUP recorded with the SF electrode, a pointsize recording surface is assumed, as the size of the SF electrode active surface is very small (with a diameter of about $25 \mu \mathrm{m}$ ). However, due to the hemispherical uptake area of this electrode, the MUP is calculated only taking into consideration the fibers of the MU placed at one side of the needle, this side being the one in which the leadingoff surface is located. For both the $\mathrm{CN}$ electrode and the SF electrode, the cannula 

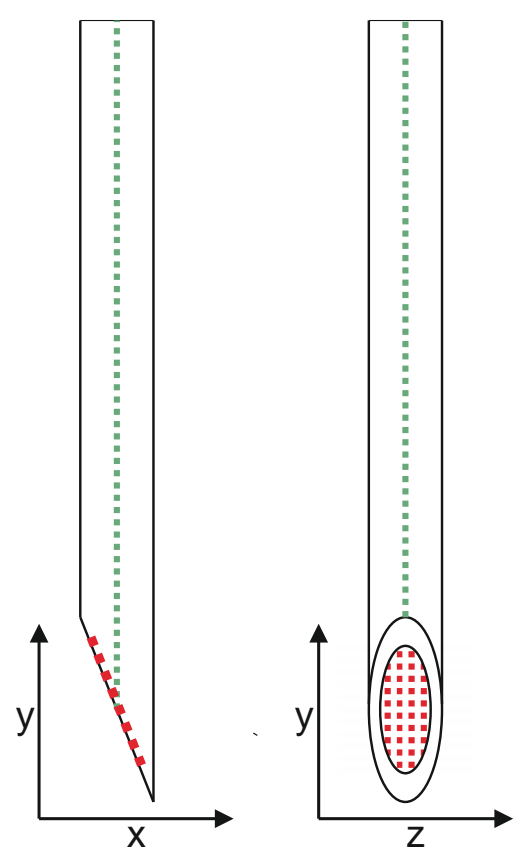

Fig. 1-12. Schematic representation of the $\mathrm{CN}$ electrode model. The leading-off is modeled as a discretized elliptical recording surface (set of red points). The cannula is modeled as a set of points along the central axis of the needle (green points).

acts as the electrical reference. Therefore, in both cases, the cannula signal is subtracted to the MUP in the active surface. The cannula is modeled by a set of points arranged in a straight line along the central axis of the needle (Fig. 1-12). In this way, the simulated MUP in the cannula is the result of calculating and averaging the MUP at these points. Note that the potential does not propagate through the air, and therefore, the averaging includes such cannula points within the muscle, or in the skin and fat layers, but not the points above the skin.

Besides the electrical simulation of the needle electrode, it is important to consider the mechanical effect that the needle electrode produces in the muscle fibers. Note that the muscle fibers are moved as the needle electrode is inserted within the muscle, what is known as ploughing effect [126]. In this thesis, a model of this effect for scanningEMG recordings has been implemented. In a scanning-EMG recording, the scanning needle is completely inserted in the muscle at first, and then, it is retracted step by step during the recording procedure. To model the ploughing accordingly, the muscle fibers are assumed to be moved during the needle insertion, and during the recording procedure they remain in their new positions without moving. Given a needle electrode inserted along the $y$-axis, with its center located at position $x_{0}$, and with a radius $r_{n d l}$, and with the needle tip located either on the right side (i.e., at $x_{0}-r_{n d l}$ ) or on the left side (i.e., at $x_{0}+r_{n d l}$ ), then the needle insertion displaces the muscle fibers along the $\mathrm{x}$-axis, to the right if the tip is located on the left, and to the left direction if the tip is on the right. Given a muscle fiber whose position in the muscle cross-section before 
the ploughing is $(x, y)$, the fiber is displaced by the needle insertion if and only if $x_{0}-$ $r_{n d l} \cdot s \leq x \leq x_{0}+r_{n d l} \cdot s$. If this is the case, the new fiber location is

$$
x^{\prime}=x+\varphi \cdot 2 r_{n d l}-\frac{x-\left(x_{0}-\varphi \cdot r_{n d l}\right)}{s}
$$

where $r_{n d l}=0.275 \mathrm{~mm}, s=2$, and $\varphi$ takes the value 1 if the needle tip is located right, and -1 if the tip is on the left.

\subsubsection{Modeling recruitment and firing pattern}

Recruitment and firing pattern of the MUs is modeled following the works of DeLuca [20] and Fuglevand [35]. The recruitment threshold excitation of the MUs during constant isometric contraction is modeled by an exponential function, with the full recruitment reached for a given percentage of maximum voluntary contraction (MVC) [35]. For the simulation of the firing rate of the MUs, the model established and adjusted by DeLuca [20] is followed, in which the firing rate of the $i$ th $\mathrm{MU}, F R_{i}$, is modeled as a function of the recruitment threshold, $R T_{i}$, and the MVC

$$
F R_{i}\left(R T_{i}, M V C\right)=D \cdot R T_{i}+\left(C-A e^{-\frac{R T_{i}}{B}}\right) \cdot M V C+E
$$

where the $R T_{i}$ and the $M V C$ are normalized between 0 and 1 , and $F R_{i}$ is expressed in Hz. This model uses a set of parameters depending on the muscle, whose values for the vastus lateralis muscle are shown in Table 1-4. With this firing rate model, the MUs recruited earlier will have a higher firing rate than those recruited later. Also, the higher the value of the MVC, the higher the firing rate.

Table 1-4. Parameter values used to simulate the recruitment and firing trains of the MUs.

\begin{tabular}{ll}
\hline Parameter & Value \\
\hline Maximum recruitment threshold & $70 \%$ \\
Firing rate model parameter, $A$ & 116 \\
Firing rate model parameter, $B$ & 0.15 \\
Firing rate model parameter, $C$ & -21 \\
Firing rate model parameter, $D$ & 8 \\
Firing rate model parameter, $E$ & 19 \\
Firing rate coefficient variation & 0.15 \\
\hline
\end{tabular}

The firing train of each MU is modeled as a renewal point process [91] where the interval between discharges follows a Gaussian distribution with a mean that is the inverse of the firing rate, and with a fixed coefficient of variation. In this way, for the 
$i$ th MU, a set of firing instants $\left\{t_{i, l}\right\}_{l=1}^{L_{i}}$ is generated: the MU firing pattern, where $L_{i}$ is the number if firing instants. A set of firing instants is modeled for each of the recruited MUs. A MU is considered to be recruited if its recruitment threshold excitation is lower than the current MVC.

\subsubsection{Modeling the EMG interference pattern}

To simulate the EMG interference pattern, the MUP trains of the different MUs are calculated and summed. The MUP train of the $i$ th MU is obtained by convoluting the corresponding MUP with its firing pattern

$$
\operatorname{MUPT}_{i}(t)=\operatorname{MUP}_{i}(t) * \sum_{l=1}^{L_{i}} \delta\left(t-t_{i, l}\right)
$$

where $M U P_{i}$ and $M U P T_{i}$ are the MUP and the MUP train of the $i$ th MU respectively, and $t_{i, l}$ represents the $l$ th firing instant corresponding to the $i$ th MU. The complete EMG pattern is calculated as the summation of the MUP trains of all the recruited MUs

$$
\operatorname{EMG}(t)=\sum_{i=1}^{I} \operatorname{MUPT}_{i}(t)=\sum_{i=1}^{I}\left(\operatorname{MUP}(t) * \sum_{l=1}^{L_{i}} \delta\left(t-t_{i, l}\right)\right)
$$

where $I$ is the number of recruited MUs.

\subsubsection{Modeling the scanning-EMG recording}

To simulate the scanning-EMG signal, it is necessary to model the different positions taken by the scanning electrode during the recording. As it will be explained in the following chapters, such positions will depend on the recording configuration used (see section 3.3.1). Here, the scanning-EMG signal is modeled for the standard configuration (1-port scanning-EMG). In this configuration, the simulated scanning electrode is inserted in the middle of the muscle cross-section, along the y-axis (Fig. 1-13 (b)). The electrode is placed at a certain distance (usually $30 \mathrm{~mm}$ ) away from the innervation zone in the direction parallel to the fibers, i.e. the z-axis (Fig. 1-13 (a)). During the recording procedure, the scanning electrode is moved along the y-axis in steps of $0.05 \mathrm{~mm}$, traversing the muscle completely from the deepest part to the surface. As a low percentage of MVC is assumed, the scanning-EMG signal to be simulated is the one corresponding to one of the smallest MUs whose territory is traversed by the corridor. In its physiological (noise-free) version, this signal is simulated as the sequence of MUPs obtained at each position of the scanning electrode.

In order to obtain realistic signals, in which artifacts from other active MUs are present, the entire recording procedure of the scanning-EMG signal is simulated [17]. 


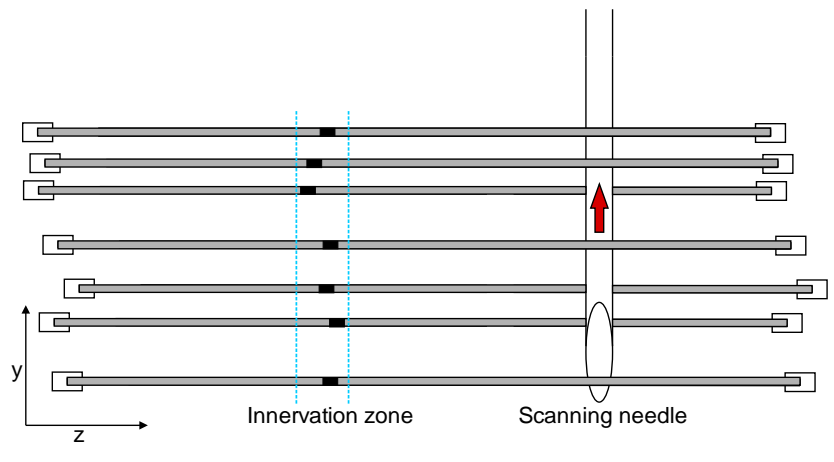

(a)

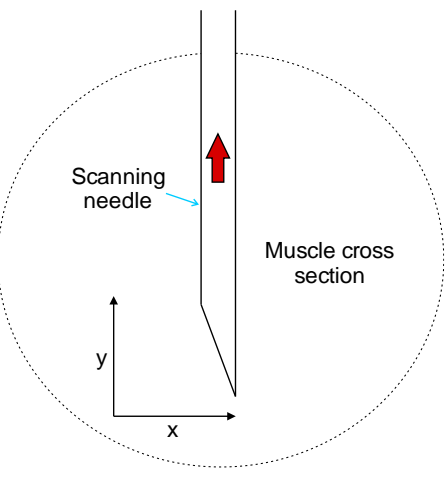

(b)

Fig. 1-13. Schematic representation of the insertion of the scanning needle within the muscle. The red arrow indicates the direction in which the scanning needle is moved during the recording procedure; (a) plane longitudinal to the muscle fibers. The scanning needle electrode is inserted at a certain distance respect to the innervation zone; (b) muscle cross-section plane. The scanning needle is inserted along the $\mathrm{y}$-axis, in the $x$ position corresponding to the middle of the muscle.

At each position of the scanning electrode, the EMG pattern is calculated for a certain percentage of MVC, and a trace of a limited duration is extracted from the EMG for each firing event of the MU. Note that these traces represent different discharges of the MUP under consideration, but may include superimposed contributions of MUPs from nearby MUs. The entire scanning-EMG signal is obtained by repeating this procedure at each recording position. The time interval that the electrode remains at each specific recording position is set to ensure a specific number of MUP discharges, where in the classic scanning-EMG recording this number is 1 .

Table 1-5. Parameter values used to generate the noise in the scanning-EMG signal.

\begin{tabular}{ll}
\hline Parameter & Value \\
\hline Temporal size of the recording traces & $30 \mathrm{~ms}$ \\
Number of discharges per position & 5 \\
AWGN standard deviation & $0.035 \mathrm{mV}$ \\
Baseline standard deviation & $3.5 \mathrm{mV}$ \\
Cut-off frequency of the baseline noise & $50 \mathrm{~Hz}$ \\
Percentage of MVC & $3.5 \%$ \\
\hline
\end{tabular}

Apart from the presence of artifacts from other MUs, the scanning-EMG signal usually presents baseline and instrumentation noise. Baseline noise was modeled as an ARMA process [99] obtained by filtering white Gaussian noise of zero-mean, with a given standard deviation. The filter used was a 5th-order Butterworth low-pass filter with a certain 3 -dB cut-off frequency. Instrumentation noise was simulated as a zero- 
mean, additive, white Gaussian noise (AWGN) process, with a given standard deviation.

\subsubsection{Modeling the surface-EMG recording}

The recording configuration consists on a linear array of several electrodes placed on the surface of the skin, along the direction parallel to the muscle fibers (the z-axis, Fig. 1-14 (a)), separated by certain inter-electrode distance. The array is placed so that the IZ is located in the middle. In the direction perpendicular to the muscle fibers, the $\mathrm{x}$-axis, the electrodes are placed above the center of the muscle belly (Fig. 1-14 (b)). The electrodes have circular shape with a specific diameter.

Table 1-6. Parameter values used to simulate the surface-EMG signal recordings.

\begin{tabular}{ll}
\hline Parameter & Value \\
\hline Number of surface electrodes & 8 \\
Diameter of the surface electrodes & $5 \mathrm{~mm}$ \\
Inter electrode distance & $10 \mathrm{~mm}$ \\
Temporal sampling frequency & $1 \mathrm{kHz}$ \\
\hline
\end{tabular}

The surface-MUP is simulated by evaluating the potential for each of the electrodes constituting the linear array. It is important to keep in mind that this surfaceMUP is not calculated using the models explained in sections 1.4.2 and 1.4.3. Instead, the surface-MUP is obtained by using model detailed in [32]. The surface EMG pattern is calculated from the surface-MUPs associated to the different recruited MUs, as explained in section 1.4.4.

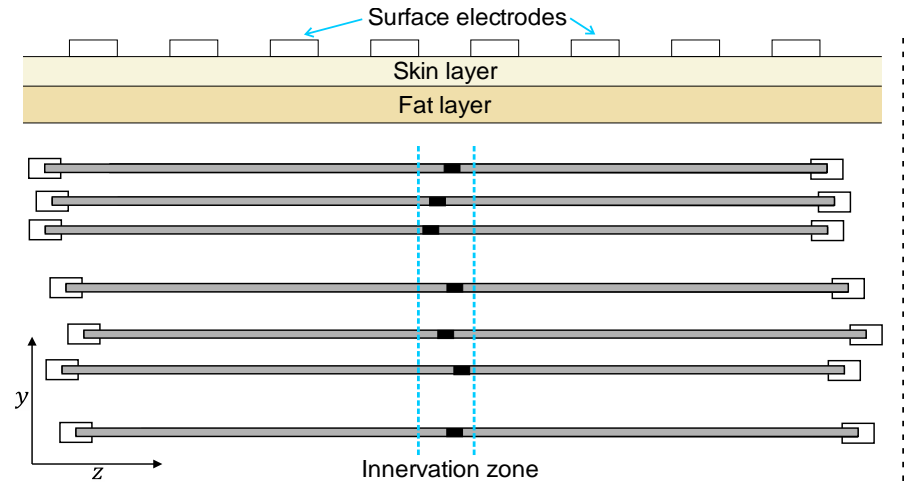

(a)

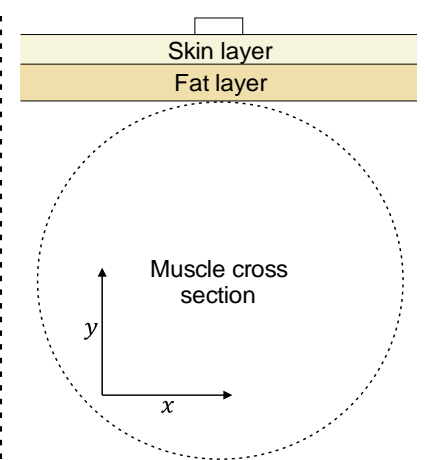

(b)

Fig. 1-14. Schematic representation of the placement of the surface electrode array in the skin; (a) plane longitudinal to the muscle fibers. The surface electrode array is placed parallel to the fiber direction, with the innervation zone in the middle of the array; (b) muscle cross-section plane. The surface electrodes are placed in the $x$ position corresponding to the middle of the muscle. 


\section{Signal processing}

In this chapter, different algorithms developed during this thesis to process and analyze electromyographic signals are described [16, 17, 18]. Some of these algorithms are important pieces of the estimation system developed to solve the inverse problem. The chapter is divided in two sections: the first one is focused in the analysis and processing of the scanning-EMG signal, and the second one is focused in the analysis of the surface-EMG signal. 
upna

Universidad Púbilica de Navara
Natarmaako Unibersitate Publikoa 


\subsection{Processing of the scanning-EMG signal}

The algorithms described in this section include different processing techniques designed to reduce the noise present in the scanning-EMG signal, as well as other techniques devoted to the analysis of the signal, such as the motor unit profile extraction, or the detection of the active region of the signal.

\subsubsection{Noise reduction in scanning-EMG signals}

It is important to remember that the scanning-EMG signal is composed by a collection of MUPs of a MU recorded at different depths within the muscle. The recording can be configured to obtain either one or several discharges of the MUP at each position of the needle. When multiple discharges per position are recorded, the scanning-EMG signal has three dimensions (Fig. 2-1): the temporal dimension, since each MUP is a signal varying in time; the spatial dimension, i.e., the different recording positions; and the discharge dimension, indexing the different discharges recorded at the same recording position.

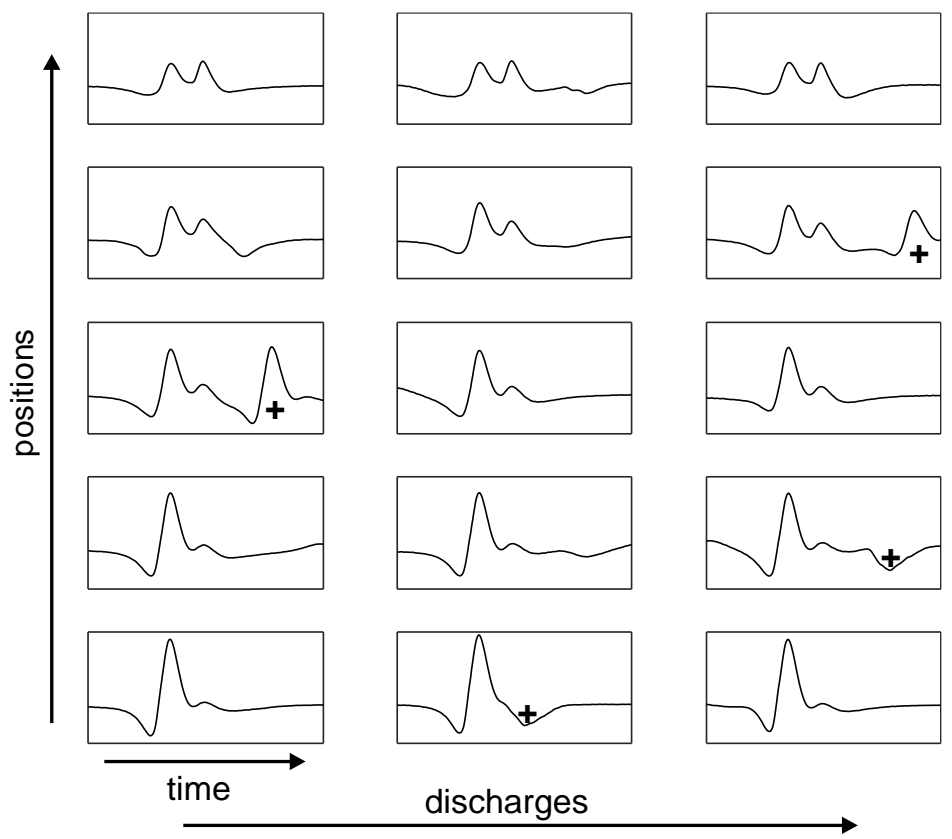

Fig. 2-1. Representation of the MUP discharges recorded at five consecutive positions in a scanning-EMG signal. Note that three discharges per position are being recorded. The horizontal and vertical axis represent the discharge and spatial dimension, respectively. Additionally, each MUP discharge is a signal that varies in time. Note that interfering artifacts are present in some discharges (marked as + ). 


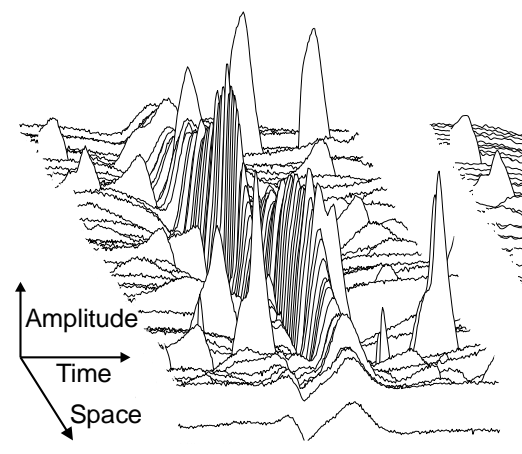

(a)

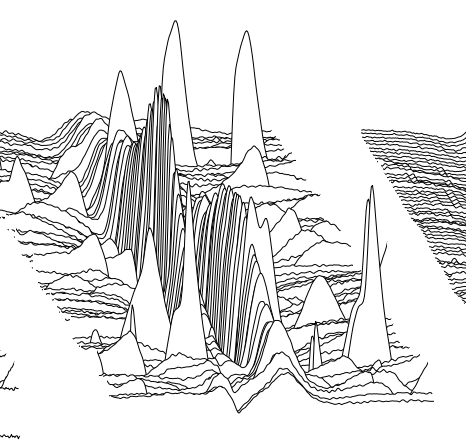

(b)

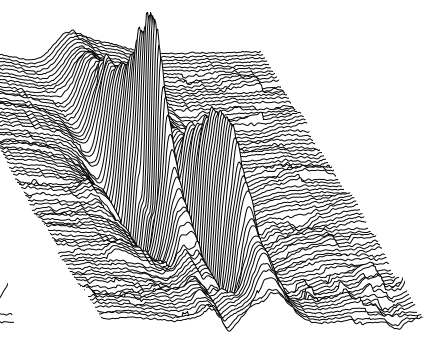

(c)

Fig. 2-2. Three-dimensional representation of a single-discharge scanning-EMG signal for the different steps of a traditional signal processing procedure; (a) raw signal. Note that baseline, as well as the presence of activity not synchronized with the recorded MUP (the artifacts) can be observed; (b) baseline noise is cancelled after applying a temporal band-pass filter; (c) artifacts are eliminated before applying a spatial median filtering.

It is difficult to extract reliable information from a raw scanning-EMG signal as it is contaminated with noise. There are three different types of noise present in scanningEMG recordings. First, there is a slow time varying baseline noise caused by needle and muscle movements $[42,44,90]$; second, there is high frequency noise due to the instrumentation system; and third, and most troublesome, there is interference derived from the coactivation of nearby MUs during the recording of the MUP [42, 44, 90]. This phenomenon results in a large amount of MUP artifacts not synchronized with the firing of the MU being tracked, that are superimposed to the recorded MUP [90] (Fig. 2-1 and Fig. 2-2 (a)). The number of artifacts from these other MUs depends on the level of voluntary contraction exerted by the subject while the recording is being made [62].

\section{State of the art of the noise reduction procedures}

Processing methods must be applied to reduce the noise from the raw scanningEMG signal. Usually, the first step is to apply a temporal band-pass filter to each of the MUP discharges [42, 44, 90] (Fig. 2-2 (b)). This filter removes the baseline noise in the low frequency range of the spectrum and some of the high-frequency noise from the instrumentation system.

The second and most critical step is to reduce the artifacts from other MUs, as this is the most harmful type of noise. A technique used for this purpose must take into account that artifacts are not repeated consistently among the different recorded MUP discharges, due to the lack of synchronization between the artifacts and the MU firing. This means that, if we consider a specific time instant, both in the spatial and in the discharge dimension artifacts are effectively impulsive noise, a fact that can be exploited by a suitable processing technique. 
When only a single discharge of the MUP is recorded at each position, the strategy used to eliminate the artifacts is filtering in the spatial dimension (Fig. 2-2 (c)). A median filter (usually of 3,5 or 7 points) $[42,44,90]$ can be used for this purpose. However, this filter noticeably distorts the waveform of the scanning signal [17]. Phenomena such as peak clipping $[42,44,90]$ or stepping of the amplitude profile in the spatial dimension [17] have been reported when the median filter is used.

When multiple MUP discharges per position are recorded, artifacts can be removed by averaging the different discharges existing at each position using mean or median [119]. If this discharge averaging is followed by a spatial median filtering [136, 60], a much more effective artifact elimination can be achieved.

This is the procedure typically used to eliminate noise in scanning-EMG signals. In the following sections, alternative algorithms developed during this thesis to achieve a more refined noise reduction will be described.

\section{Instrumentation and baseline noise filtering}

As explained in the previous section, to reduce baseline noise it is necessary to remove the low frequency components of the MUP discharges, and to reduce the instrumentation noise level, high frequency components must be removed. For this purpose, a band-pass filter can be used. Regarding baseline removal, it is necessary to keep in mind that only the lowest frequency components must be eliminated in order to distort the signal as little as possible. This implies that the lower cut-off frequency of the band-pass filter must be small. The problem with this requirement is that it can lead to unwanted edge effects. The impulse response of such filters may have a very long size. This, in turn, induces a propagation of the edge discontinuities along a significative number of samples of the signal (Fig. 2-3). This is especially problematic if the length of the recorded MUP discharges is small.

To overcome this problem, the following filtering scheme can be applied to each of the recorded MUP discharge:

1- The signal is artificially extended in both sides by adding $N_{\text {add }}$ samples of a certain constant amplitude value. At each side, this value is calculated by averaging the $N_{\text {ave }}$ samples closest to the edge.

2- The Discrete Fourier Transform (DFT) of the extended signal is calculated.

3- All frequency components lower than $f_{H P F}$, and higher than $f_{L P F}$, are eliminated from the transformed signal by zeroing the corresponding DFT coefficients.

4- The Inverse Discrete Fourier Transform (IDFT) of the transformed signal is calculated.

5- The $N_{\text {add }}$ samples artificially added at each side in step 1 are eliminated.

By artificially extending the signal, the edge discontinuities are moved far enough 


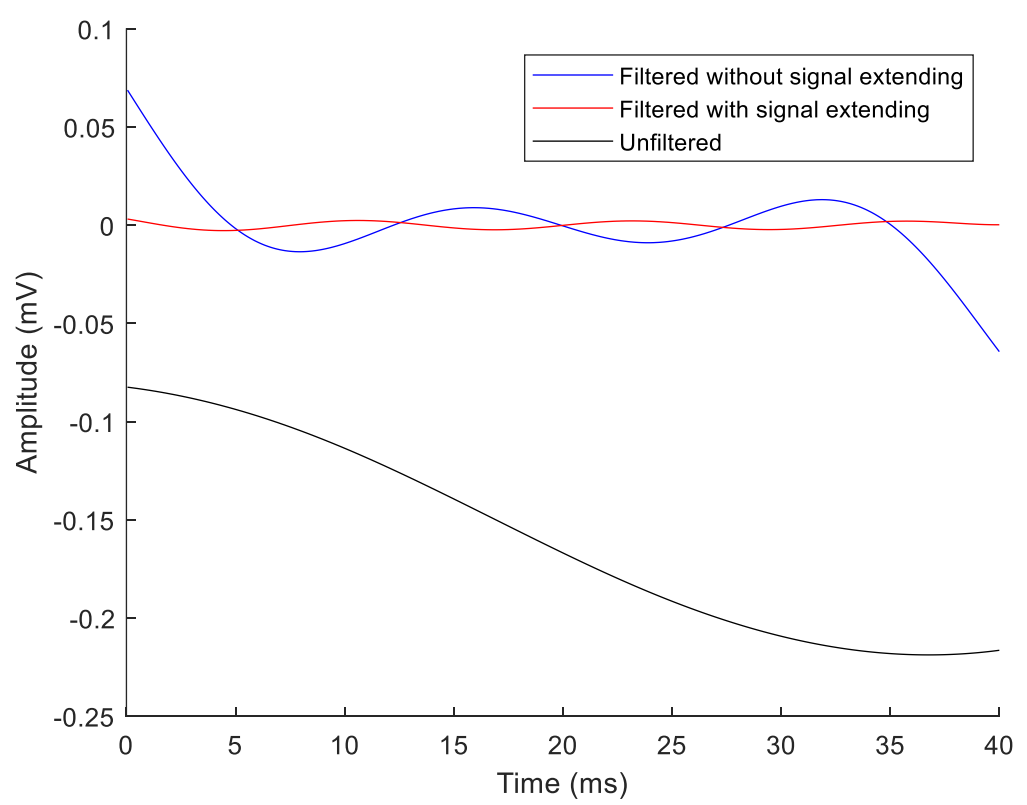

Fig. 2-3. Case of use of the described filtering scheme to an artificially generated baseline signal (black line). When the scheme is applied without extending the signal limits $\left(N_{a d d}=0\right.$, blue line), the resulting filtered signal presents a noticeable edge effect, that is, the amplitude at the bounds is remarkably different from zero. When the scheme is applied with extending the signal limits $\left(N_{\text {add }}=400\right.$, red line $)$, the edge effect is not present.

so that the propagation effect of such discontinuities after the filtering remain out of the original signal limits. In this way, such effect is completely removed in the step 5. In Fig. 2-3, an example is depicted, showing how the edge effects are avoided with the filtering scheme.

This filtering scheme is the one used to eliminate the baseline and instrumentation noise in the estimation system developed during this thesis. Assuming a sample frequency of $20 \mathrm{kHz}$, the parameters used for this purpose are: $N_{\text {add }}=400$ samples; $N_{\text {ave }}=50$ samples; $f_{H P F}=33.3 \mathrm{~Hz} ; f_{L P F}=5000 \mathrm{~Hz}$.

\section{Masked least-squares smoothing algorithm}

As explained in previous sections, a median filter in the spatial dimension is commonly applied to remove artifacts in scanning-EMG recordings. However, this filter has the drawback that it significantly distorts the waveform of the signal $[42,44$, $90,17]$. To overcome this limitation, we developed a new algorithm for artifact removal, the masked least-squares smoothing (MLSS) algorithm [17]. This algorithm recalculates each sample value of the signal using a least-squares smoothing in the spatial dimension, taking into account only those samples that are not contaminated with artifacts. The MLSS algorithm allows to effectively remove the artifacts while it 


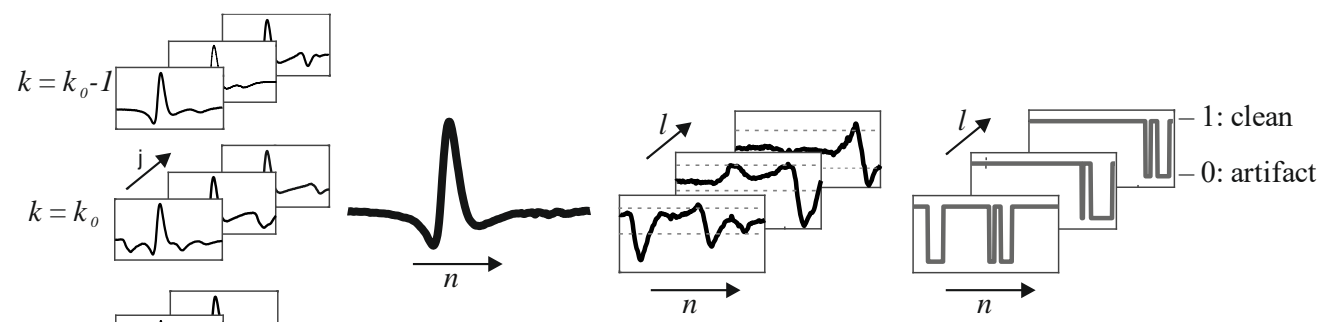

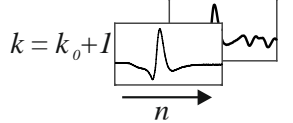

(a) (b) (c) (d)

Fig. 2-4 (a) The scanning-EMG signal at the input of the algorithm is composed by different discharges at each recording position, $k$. In the figure, the discharges corresponding to the positions $k_{0}-1, k_{0}$ and $k_{0}+1$ are shown; (b) median of all discharges contained in the positions $k_{0}-1, k_{0}$ and $k_{0}+1$ (the median filter order is $A=3$ ); (c) difference between the input discharges and the median filtered signal in the position $k_{0}$. The dashed lines represent the positive and negative threshold, respectively; (d) validity mask values associated to the different discharges at the position $k_{0}$. Note that valid samples correspond to 1 , and the invalid ones correspond to 0 .

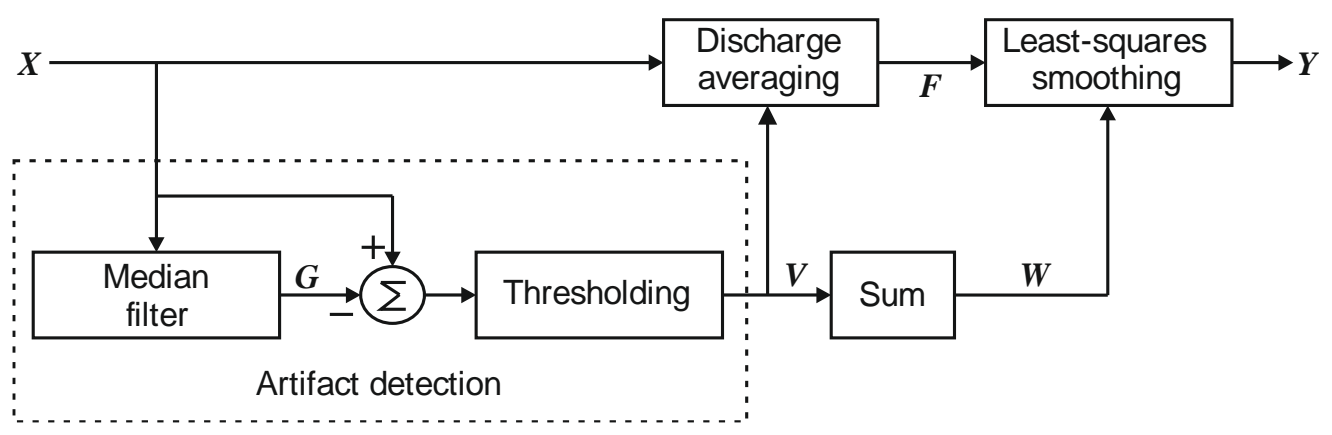

Fig. 2-5. Block diagram describing the different steps of the MMLSS algorithm. In a first step, the artifact-free samples of the input signal $\boldsymbol{X}$ are determined by calculating a validity mask $\boldsymbol{V}$. To calculate $\boldsymbol{V}$ a signal $\boldsymbol{G}$ is prepared by applying a double median filter to the input signal $\boldsymbol{X}$. The signal $\boldsymbol{G}$ is then subtracted from $\boldsymbol{X}$, and this difference is thresholded to give the validity mask $\boldsymbol{V}$. After that, the different discharges contained in $\boldsymbol{X}$ at each position are weight-averaged using the validity mask values $\boldsymbol{V}$ as weights, so that an averaged signal $\boldsymbol{F}$ is obtained from only information of valid samples. Also, an overall validity mask $\boldsymbol{W}$ is obtained by summing at each position the different values of the validity mask $\boldsymbol{V}$ associated to the different discharges. In the last step, the output of the algorithm $\boldsymbol{Y}$ is calculated by applying a least-squares smoothing procedure to the averaged signal $\boldsymbol{F}$ among the spatial dimension, using the overall validity mask values $\boldsymbol{W}$ as weights.

preserves the waveform of the scanning-EMG signal noticeably better than the median filter [17]. 
The main drawback of the MLSS algorithm is that it can only be applied in singledischarge scanning-EMG recordings. In order to adapt it to multiple discharge recordings, a new version of the algorithm has been developed: the multi-masked leastsquares smoothing (MMLSS) algorithm [18]. This algorithm combines the advantages of the MLSS algorithm regarding waveform distortion, with the improved effectiveness provided by the use of multiple MUP discharges.

We assume that several MUP discharges are recorded at each spatial position (Fig. 2-4 (a)). Before applying the algorithm itself, each of these discharges can be processed with a temporal band pass-filter, as presented in the previous section, so as to eliminate the baseline and the instrumentation noise. The MMLSS algorithm has three main steps: artifact detection, discharge averaging, and least-squares smoothing (Fig. 2-5).

\section{Artifact detection}

The first step of the algorithm is to detect for each of the recorded discharges, what samples are contaminated with artifacts (Fig. 2-4). In order to achieve this, artifact noise is estimated by computing the difference between the sample values of the input scanning signal and that of a median filtered version of the input signal. Then, the invalid samples are determined by thresholding the estimated artifact noise.

Let $\boldsymbol{X}=\left\{x_{n, k}^{l}\right\}$ be the scanning-EMG signal at the input of the algorithm, where $1 \leq n \leq N$ and $1 \leq k \leq K$ indicate the temporal and spatial location of the sample respectively, where $N$ and $K$ are the number of temporal and spatial samples of the scanning signal respectively, and where $1 \leq l \leq L(k)$ indexes the discharge to which the sample belongs, where $L(k)$ is the number of discharges recorded in the position $k$. Note that the number of discharges is allowed to vary among the different recording positions.

Computing the median filtered signal $\boldsymbol{G}=\left\{g_{n, k}\right\}$ consists on applying a median filter, a mean subtraction of each trace, and a second median filter. The double median filtering aims to achieve a better artifact removal, while the mean subtraction helps to eliminate the bias of the remaining artifact noise.

The first median filter consists on computing at each space-time position $(n, k)$ the median of all sample values contained within a window of $A$ spatial positions (Fig. 2-4 (a-b)). Note that at each position of the window, several sample values corresponding to the different discharges may exist. Mathematically

$$
g_{n, k}=\operatorname{median}\left\{x_{n, k+a}^{l} \mid 1 \leq l \leq L(k),-(A-1) / 2 \leq a \leq(A-1) / 2\right\}
$$

The signal $\boldsymbol{G}$ size is $N \times K$, as the different discharge values existing at each specific space-time position have been combined into a single value. Once the first median filter is applied, the mean of each trace at each spatial position is subtracted 


$$
g_{n, k}=g_{n, k}-\operatorname{mean}\left\{g_{\eta, k} \mid 1 \leq \eta \leq N\right\}
$$

and then, a second $A$-point median filter is applied among the spatial dimension

$$
g_{n, k}=\operatorname{median}\left\{g_{n, k+a} \mid-(A-1) / 2 \leq a \leq(A-1) / 2\right\}
$$

The validity mask $\boldsymbol{V}=\left\{v_{n, k}^{l}\right\}$ is obtained by thresholding the difference between the input signal $\boldsymbol{X}$ and the median filtered signal $\boldsymbol{G}$ (Fig. 2-4 (c)). The mask is set to 0 when the threshold is exceeded, indicating that an artifact has been detected, and 1 otherwise (Fig. 2-4 (d))

$$
v_{n, k}^{j}= \begin{cases}1 & \left|x_{n, k}^{l}-g_{n, k}\right|<U \cdot(\max (G)-\min (G)) \\ 0 & \left|x_{n, k}^{l}-g_{n, k}\right| \geq U \cdot(\max (G)-\min (G))\end{cases}
$$

where $U$ is the normalized artifact detection threshold. Note that there is a one to one correspondence between the samples of the input scanning signal $\boldsymbol{X}$ and that of the validity mask $\boldsymbol{V}$ (Fig. 2-4 (a) and (d)), thus they have the same size.

\section{Discharge averaging}

The second step of the algorithm is to combine the information of the different discharges at each space-time position. An overall validity mask $\boldsymbol{W}=\left\{w_{n, k}\right\}$ is created by summing at each position $(n, k)$ all the validity mask values associated to the different discharges (Fig. 2-5)

$$
w_{n, k}=\sum_{l=1}^{L(k)} v_{n, k}^{l}
$$

When it comes to build the output signal in the least-squares smoothing step, the overall validity mask will be used to give to each position a weight proportional to the number of valid samples detected at this specific location, which is exactly $w_{n, k}$.

An averaged scanning signal $\boldsymbol{F}=\left\{f_{n, k}\right\}$ is obtained from the input scanning signal by averaging the samples of the different discharges at each position $(n, k)$, weighted by the validity mask (Fig. 2-5)

$$
f_{n, k}=\left\{\begin{array}{cc}
\frac{\sum_{l=1}^{L(k)} v_{n, k}^{l} x_{n, k}^{l}}{w_{n, k}} & w_{n, k}>0 \\
0 & w_{n, k}=0
\end{array}\right.
$$

Note that this ensures that the averaged signal contains only information of 
artifact-free samples, as the invalid samples are multiplied by 0 in the averaging procedure.

\section{Least squares smoothing}

The third and last step of the algorithm is to build the output scanning signal by applying a least-squares smoothing to the averaged signal (Fig. 2-5). For each spacetime position $(n, k)$, a polynomial of order $Q$ is fitted to the amplitude values of the samples contained in a window of length $2 M+1$, selected among the spatial dimension and centered at the position under consideration. The method used for the polynomial fitting is weighed linear least-squares [132], where the weights are the overall validity mask values. In this way, the $N \times K$ output scanning signal $\boldsymbol{Y}=\left\{y_{n, k}\right\}$ is composed by the values of the calculated polynomials at the centre of the windows. Therefore, at each position $(n, k)$, the output value is

$$
y_{n, k}=\beta_{0}
$$

where the coefficients of the polynomial $\boldsymbol{\beta}=\left[\beta_{0}, \cdots, \beta_{Q}\right]^{T}$ are calculated as [132]

$$
\boldsymbol{\beta}=\left(\boldsymbol{S}^{T} \mathbf{H} \boldsymbol{S}\right)^{-1} \boldsymbol{S}^{T} \mathbf{H} \boldsymbol{f}
$$

where $\boldsymbol{f}=\left[f_{n, k-M}, \cdots, f_{n, k}, \cdots, f_{n, k+M}\right]^{T}$ are the samples values within the window, the weight matrix $\mathbf{H}$ is a diagonal matrix built from the overall validity mask values corresponding to the positions within the window

$$
\mathbf{H}=\operatorname{diag}\left\{w_{n, k-M}, \cdots, w_{n, k}, \cdots, w_{n, k+M}\right\}
$$

and the matrix $\boldsymbol{S}$ is defined as

$$
\boldsymbol{S}=\left\{s_{q, m}\right\}, \quad s_{q, m}=\left\{\begin{array}{cc}
m^{q} & q \neq 0 \\
1 & q=0
\end{array}\right.
$$

where $-M \leq m \leq M$ and $0 \leq q \leq Q$.

For spatial positions where there are not $M$ traces on each side, and therefore it is not possible to set the sample window symmetrically, output signal values are taken from the polynomial obtained for the closest entire window which includes the bordering spatial position [17].

Another consideration to keep in mind, is that the value of the polynomial order must be restricted in some space-time positions, in order to avoid overfitted polynomial solutions that can be given if the polynomial order is high relative to the number of samples used. The condition applied is 


$$
Q<\frac{1}{2} \sum_{m=-M}^{M} \mathfrak{u}_{m}
$$

where

$$
\mathfrak{u}_{m}= \begin{cases}1 & w_{n, k+m}>0 \\ 0 & w_{n, k+m}=0\end{cases}
$$

For space-time positions in which this condition is not satisfied, the highest polynomial order $Q$ that satisfies this condition is chosen.

Once the scanning signal $\boldsymbol{Y}$ has been obtained, the mean of each trace at each spatial position is subtracted to eliminate the bias of the remaining noise, as done in $(2-2)$.

A suitable set of parameter values of the MMLSS algorithm would be, for instance: median filter order, $A=5$ samples; artifact detection threshold, $U=3 \cdot 10^{-2}$; polynomial order, $Q=8$; and window semi-length, $W=13$ samples.

\section{Artifact detection adapted to SF electrode recordings}

An important consideration about the artifact detection step of the MMLSS algorithm is that it has been designed to process scanning-EMG signals recorded with a CN electrode. However, the estimation systems presented in the following chapters also require the processing of scanning-EMG signals recorded with the SF electrode. Therefore, in order to incorporate the artifact detection step into these systems, it has been necessary to adapt it to this kind of electrode.

When a SF electrode is used, the recorded scanning-EMG signal may present a very sharp waveform among the spatial dimension, especially if there are MU fibers located close to the scanning corridor. This implies that the algorithm parameters should be different from that used for the $\mathrm{CN}$ electrode, in order to obtain an optimal performance. For the SF electrode, a suitable set of parameters would be: median filter order, $A=5$ samples; artifact detection threshold, $U=4 \cdot 10^{-2}$; polynomial order, $Q=$ 8 ; and window semi-length, $W=11$ samples.

Another consideration is that, when a SF electrode is used, sharp peaks of the recorded scanning-EMG signal may be falsely detected as artifacts. Therefore, it is necessary to discriminate between actual artifacts and sharp peaks of the signal. An artifact usually contaminates a large row of consecutive samples along the temporal dimension, and thus, artifact detection implies that the validity mask is got to 0 also in a relatively large row of samples. On the contrary, a sharp peak usually produces that the validity mask gets to 0 in a short row of samples. Based on that, an additional step 
is applied after the artifact detection step, which consists on eliminating from the validity mask those rows of consecutive 0's along the temporal dimension such that their size is less than 9 samples. This was implemented by applying an image closing operation to the validity mask $\boldsymbol{V}$ using a 9x1 samples linear shape as structural element.

\subsubsection{Motor unit profile}

The analysis of the scanning-EMG signal is usually performed by extracting some features from the signal such as, the length of the MU territory [42], the number and length of fractions [122], the number and length of polyphasic fractions [122], the temporal delay between fractions [87], the number and length of silent zones [122], etc. However, because these features do not make effective provision for the twodimensional information of the scanning-EMG signal, they are insufficient to fully characterize the complexity throughout the whole MU territory. To resolve the above limitation, we proposed a new characterization of the scanning-EMG signal, the motor unit profile [16]. The MU profile is conceived to provide the trajectories of the crests and valleys of the scanning-EMG signal (Fig. 2-6).

In this section, the algorithm developed to extract the MU profile is described in detail. In essence, the algorithm applies point tracking techniques [111, 108] imported from image processing theory to link the turns extracted from the MUP at each recording position in such a way that smooth trajectories are formed. A detailed evaluation of the algorithm performance is available in [16].

\section{Mathematical basis of the MU profile}

In a scanning-EMG signal, the MUP is a two-dimensional continuous bioelectric potential $X(y, t)$, where $y$ is the position of the recording needle along the corridor and $t$ is the time variable (Fig. 2-6 (a)). The MU profile is defined as the mathematical locus of the points within the MUP duration limits satisfying

$$
\frac{\partial X(y, t)}{\partial t}=0
$$

Thus, the MU profile is the set of the crests and valleys of the MUP, where crests and valleys are the set of smooth sequences in the spatial dimension of local maxima and minima of $X(y, t)$ extracted in the temporal dimension (Fig. 2-6 (b)). In practice, a scanning-EMG signal is the recording of the MUP in discrete spatial locations along a linear corridor. This signal, therefore, can be regarded as a spatially and temporally sampled version of $X(y, t)$ (Fig. 2-6 (c)). In this way, the scanning-EMG signal can be expressed as $x(k, n), 1 \leq k \leq K, 1 \leq n \leq N$, where $K$ is the number of recording sites along the scanning corridor, and $N$ is the number of time samples of the recording. When dealing with the discrete-time version of the MUP, the points that satisfy (2-13) are the turns of the MUP extracted for each spatial position, where local maxima 
correspond to positive turns and local minima correspond to negative turns. Hence, the discrete-space-time version definition for the MU profile is the set of trajectories formed by linking through the spatial dimension the turns extracted from each spatial

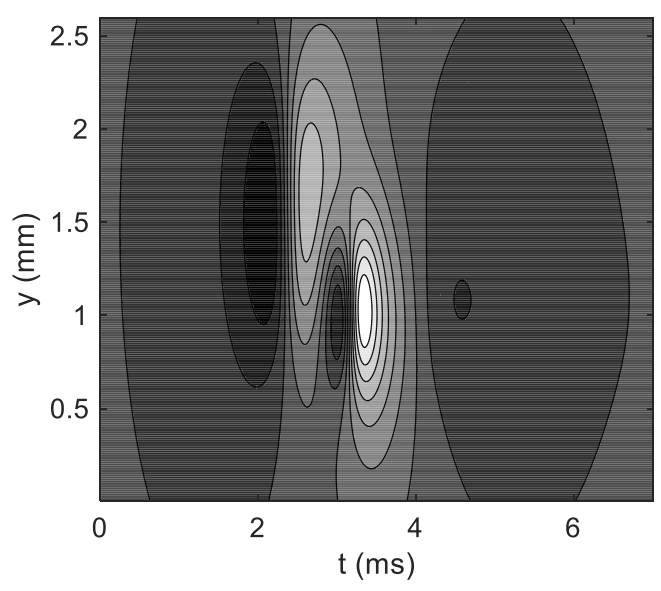

(a)

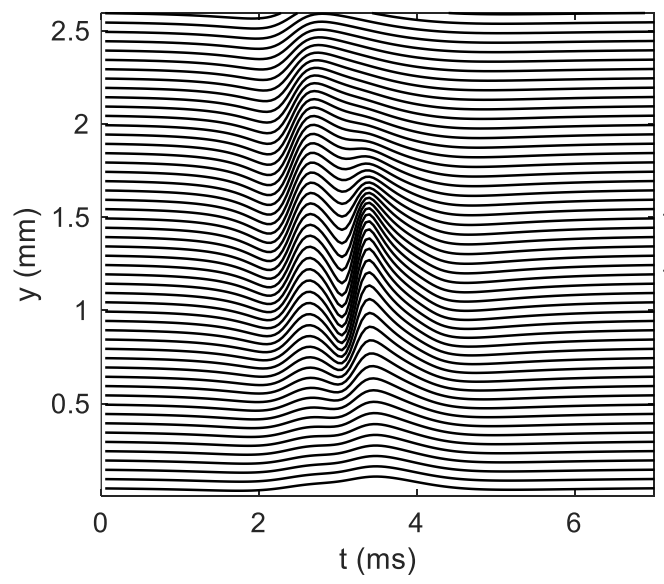

(c)

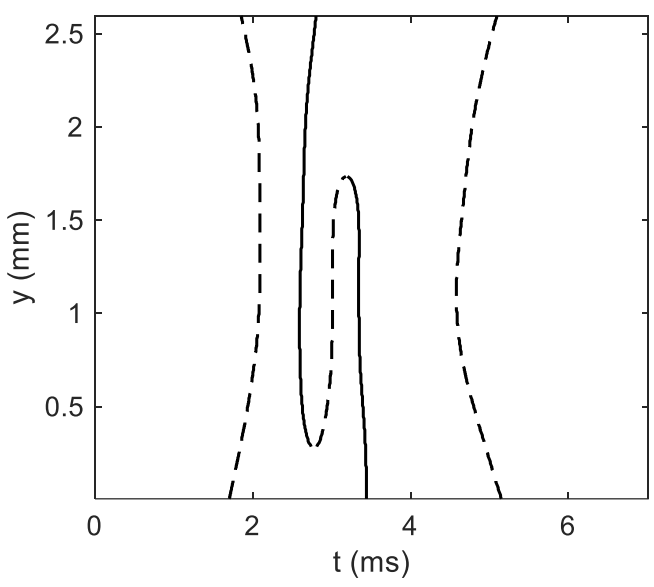

(b)

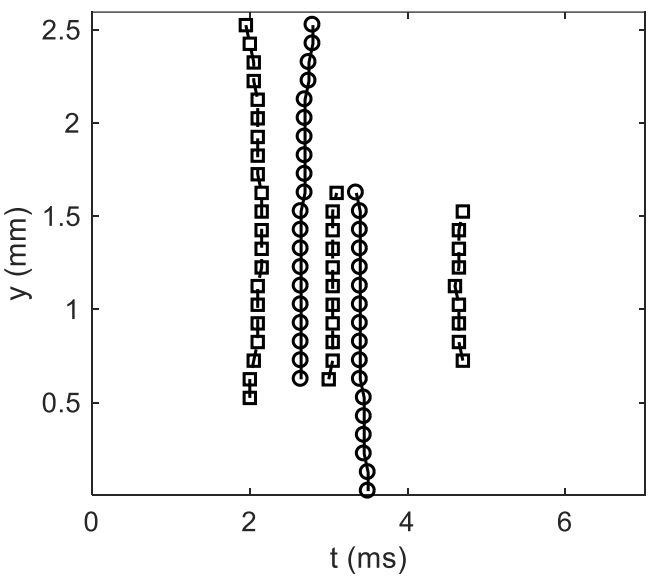

(d)

Fig. 2-6. Representation of a simulated scanning-EMG signal and its MU profile; (a) twodimensional continuous bioelectric potential $X(y, t)$ varying in time and space, with peaks and depressions related to depolarized and repolarized regions of the MUP. Note that the spatial dimension is the depth of the scanning electrode; (b) crests and valleys of the continuous bioelectric potential corresponding to the local maxima (solid lines) and the local minima (dotted lines), i.e., to the set of points satisfying (2-13);(c) the scanning-EMG signal as a discrete version of the two-dimensional continuous bioelectric potential, essentially a MUP observed at different locations along a linear corridor; (d) positive turns (circles) and negative turns (squares) corresponding to local maxima and minima, respectively, extracted at each spatial recording position of the scanning-EMG signal. Observe the direct visual correspondence with the crests and valleys in (b). 


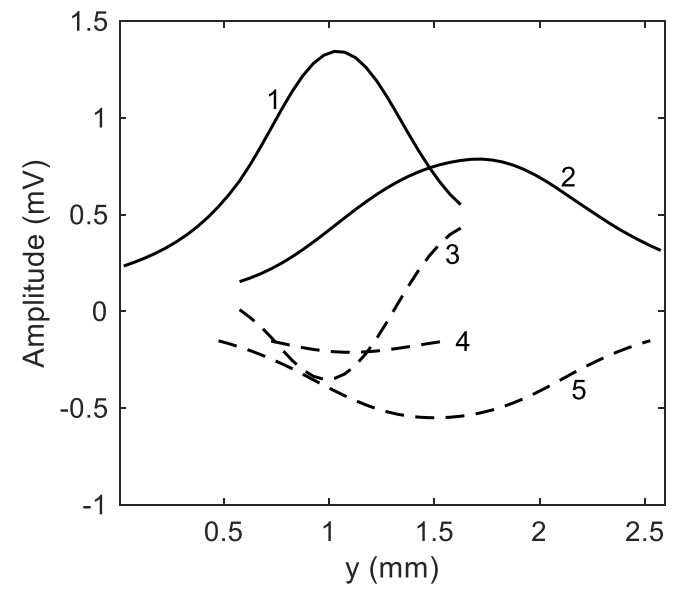

(a)

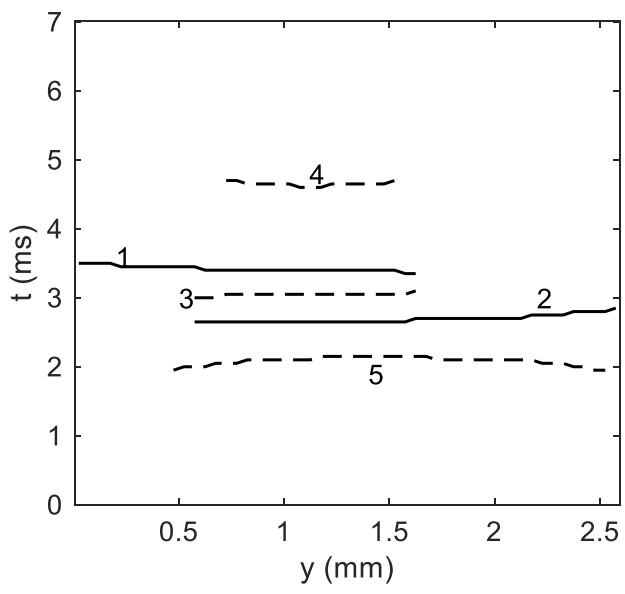

(b)

Fig. 2-7. Representation of the MU profile of the scanning-EMG signal presented in Fig. 2-6; (a) projection of the trajectories in the amplitude-space plane gives a view of the MUP amplitude changes across the scanning corridor; (b) projection of the trajectories in the timespace plane gives a view of the MUP delay and synchronization among MU fractions. Trajectories are numbered from 1 to 5 to show their correspondence in both projections. Note that the trajectories composed of positive turns (solid lines) are associated with the crests, and trajectories composed of negative turns (dotted lines) are associated with the valleys of the scanning-EMG signal.

recording position (Fig. 2-6 (d) and Fig. 2-7).

In each of the recording locations the set of turns of the MUP can be extracted:

$$
T=\left\{t_{k}^{q}\right\}=\left\{k, n_{k}^{q}, x\left(k, n_{k}^{q}\right)\right\}
$$

where $1 \leq q \leq Q_{k}$, with $Q_{k}$ the number of turns in the MUP from the $k$ th recording site; $t_{k}^{q}$ is the $q$ th turn detected in the $k$ th recording site, which is represented by three coordinates, corresponding to the position $k$, the time of occurrence of the turn $n_{k}^{q}$, and the amplitude of the turn $x\left(k, n_{k}^{q}\right)$. Note that at each recording location the MUP may have a different number of turns, $Q_{k}$, since the complexity of the MUP may vary across the recording corridor [22, 90] (Fig. 2-8).

To link the turns into trajectories, point tracking techniques can be used. Hence obtaining the MU profile is equivalent to performing a point tracking over the set of detected turns. The set of trajectories, which is the MU profile, is (Fig. 2-7):

$$
P=\left\{p_{k}^{i}\right\}=\left\{k, m_{k}^{i}, x\left(k, m_{k}^{i}\right)\right\}
$$




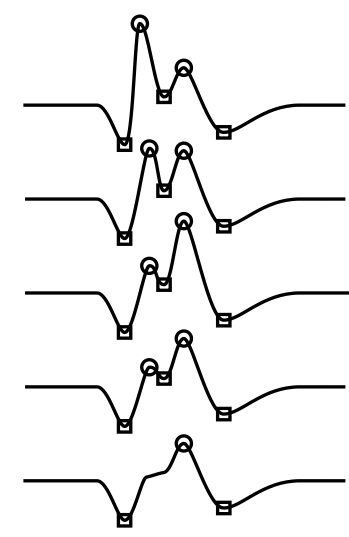

(a)

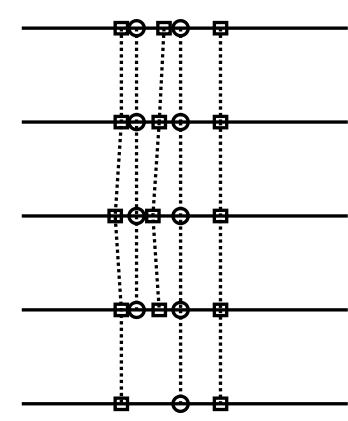

(b)

Fig. 2-8. (a) Five MUPs recorded in successive locations (recording sites), with their positive (circles) and negative (squares) turns identified. The amplitude of a turn can vary from one recording position to the next one. Note that two of the turns disappear in the last recording site; (b) the turns of each frame are represented keeping only their temporal information and are tracked among the five recording sites. Note the sudden disappearance of two trajectories in the last recording site.

where $k_{1}^{i} \leq k \leq k_{2}^{i}$ and $1 \leq i \leq I$, where $k_{1}^{i}$ and $k_{2}^{i}$ are the initial and final positions of the $i$ th trajectory respectively, and $I$ is the number of trajectories found; and $p_{k}^{i}$ is the turn at position $k$ of the $i$ th trajectory, which is represented by three coordinates: the position $k$, the time of occurrence of the turn $m_{k}^{i}$, and the amplitude of the turn $x\left(k, m_{k}^{i}\right)$.

\section{MU profile extraction algorithm}

MU profile extraction is divided into three main steps. The first step is to detect the MUP turns at each recording site of the scanning-EMG signal. The second step is to use a point tracking algorithm to join the detected turns into trajectories. A modified implementation of the greedy exchange algorithm $[111,108]$ has been used for this purpose. The third step is to eliminate the trajectories whose number of turns is smaller than a certain quantity. These steps are detailed as follows.

\section{Turns extraction}

Turns are the local maxima and minima of each MUP. However, in practice, only those turns whose maxima or minima exceed a certain threshold are considered valid turns [121]. This prevents the algorithm from regarding noise in the recorded signal as turns [121].

For each MUP, that is, for each position $k, n_{k}^{q}$ is the time of occurrence of a valid 
positive turn $t_{k}^{q}$ if and only if, some interval $n_{a}<n_{k}^{q}<n_{b}$ exists for which $x\left(k, n_{k}^{q}\right)=\max (x(k, n))$ in that interval and furthermore, $x\left(k, n_{k}^{q}\right) \geq x\left(k, n_{a}\right)+$ $T_{t h r}$, and $x\left(k, n_{k}^{q}\right) \geq x\left(k, n_{b}\right)+T_{t h r}$. Analogously, $n_{k}^{q}$ is the time of occurrence of a valid negative turn $t_{k}^{q}$ if and only if, some interval $n_{a}<n_{k}^{q}<n_{b}$ exists for which $x\left(k, n_{k}^{q}\right)=\min (x(k, n))$ in that interval and furthermore, $x\left(k, n_{k}^{q}\right) \leq x\left(k, n_{a}\right)-$ $T_{t h r}$, and $x\left(k, n_{k}^{q}\right) \leq x\left(k, n_{b}\right)-T_{t h r}$.

Note that $T_{t h r}$ is the turn detection threshold. For each position $k$, turns are ordered in time, satisfying $n_{k}^{q}<n_{k}^{q+1} ; 1 \leq k \leq K$.

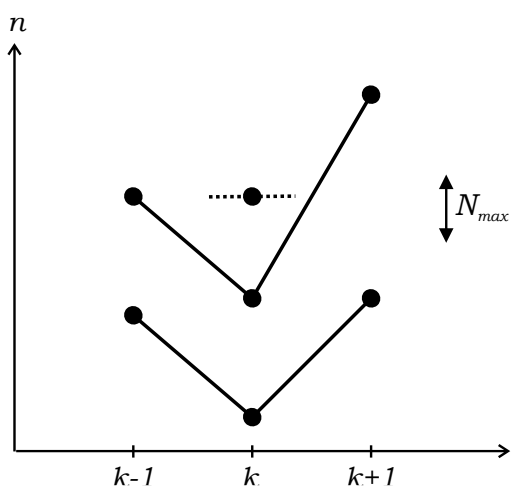

(a)

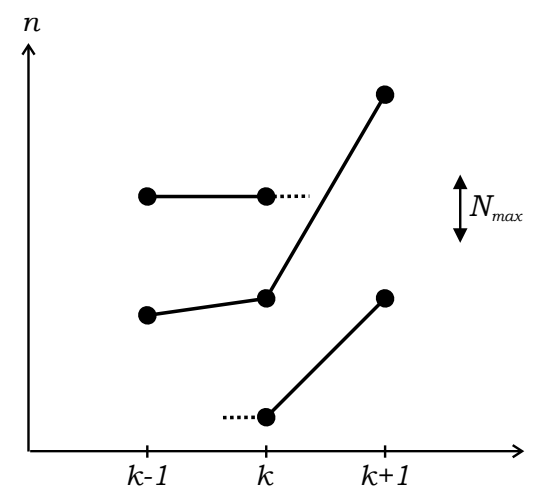

(c)

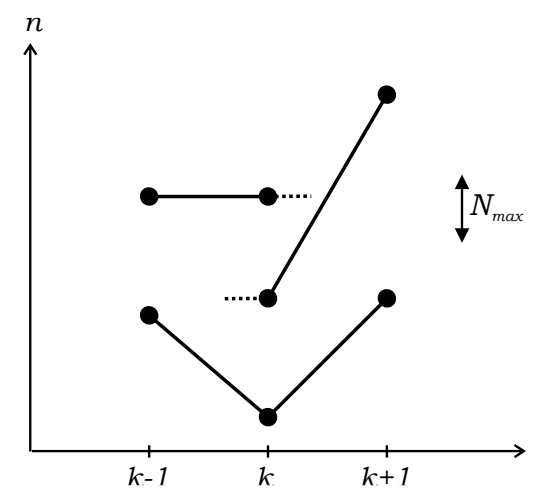

(b)

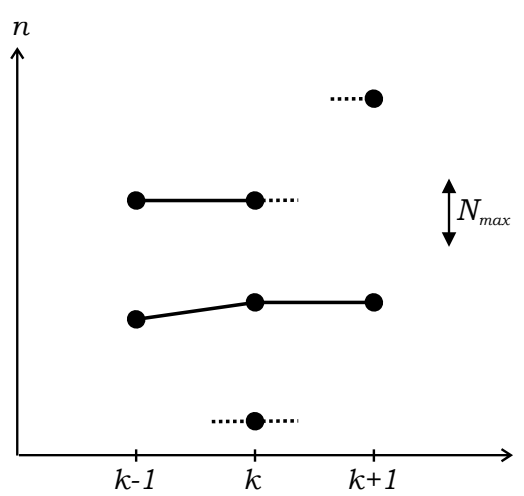

(d)

Fig. 2-9. Evolution of the trajectories in various stages of the algorithm for three consecutive spatial positions. Note that points represent real turns, solid lines represent unions between real turns, and dotted lines represent unions between real and phantom turns; (a) initialization of the trajectories according to the order of occurrence criterion; (b) first exchange in position $k$. The exchange that maximizes the STSR is selected; (c) second exchange in position $k$. After this exchange the STSR cannot increase with any other exchange and the algorithm jumps to the next position;(d) the exchange loop is executed in position $k+1$. Note that the union between two turns does not occur if the time shift is higher than $N_{\max }$. 


\section{Point tracking algorithm}

Point tracking is applied to link the turns forming trajectories. This is done separately to the set of positive turns and the set of negative turns. This precludes the possibility of linking positive and negative turns together, which would result in incorrect trajectories. This has been done by means of a modified implementation of the greedy exchange algorithm [111, 108], adapted to our particular problem. The modified greedy exchange algorithm links the turns using only the temporal information, that is, the time of occurrence of the different turns. In essence the algorithm, by means of an iterative procedure, exchanges turns from different trajectories, refining them, under the criterion of maximizing a certain merit figure (the squared time shift reduction (STSR), which will be mathematically defined later). The iteration procedure stops when there are no turns exchanges that increases such merit figure. The adapted greedy exchange algorithm is described next.

\section{1- Trajectories initialization}

A matrix $\boldsymbol{M}=\left\{m_{k}^{i}\right\}$ containing the time of occurrence of the turns of a set of provisional trajectories is created. Each row of the matrix will contain one or several trajectories that will be modified during the execution of the algorithm. The matrix is initialized following the order of occurrence of the turns for each position (Fig. 2-9 (a)). Thus, in the $k$ th column, the matrix will contain the time of occurrence of the turns of the $k$ th recording position, in ascending order, followed by zeros to complete the column. Note that the zeros in the matrix represent the absence of turn (phantom turns), which serves to allow forming incomplete trajectories comprising real and phantom turns. The number of rows of the matrix will be twice the number of turns at the recording position at which the highest number of turns is found. This ensures that every turn can potentially be exchanged with a phantom turn during the execution of the algorithm. Mathematically

$$
m_{k}^{i}=\left\{\begin{array}{cc}
n_{k}^{i} & i \leq Q_{k} \\
0 & i>Q_{k}
\end{array}\right.
$$

where $1 \leq k \leq K$ and $1 \leq i \leq 2 \max \left(Q_{k}\right)$.

\section{2- Exchange loop}

The initialized trajectories are refined by performing an iterative loop in which turns from the different trajectories are exchanged. According to it, the matrix $\boldsymbol{M}$ is modified during this loop as follows

* $\forall k ; 2 \leq k \leq K$ (for each recording position):

$>$ While some exchange is possible:

- $\forall i, j \in 1, \ldots, 2 \max \left(Q_{k}\right) ; i \neq j$ (for each combination of trajectory 
exchanges at position $k$ ):

- Calculate the STSR of exchanging the elements $m_{k}^{i}$ and $m_{k}^{j}$ of the matrix $\boldsymbol{M}$ as:

$$
\begin{aligned}
& r_{k}^{i j}=\left(s_{k}^{i i}+s_{k}^{j j}\right)-\left(s_{k}^{i j}+s_{k}^{j i}\right) \\
& s_{k}^{a b}=\left\{\begin{array}{cc}
\left|m_{k}^{b}-m_{k-1}^{a}\right|^{2} & m_{k}^{b}, m_{k-1}^{a} \neq 0 \\
N_{\max }^{2} & \text { otherwise }
\end{array}\right.
\end{aligned}
$$

where $N_{\max }$ is the maximum tracking interval threshold.

- Select among all possible exchanges, the exchange (i.e., the $i_{\max }$, $j_{\max }$ pair) with maximum STSR.

- If the STSR for the selected exchange is positive, exchange the elements $m_{k}^{i_{\max }}$ and $m_{k}^{j_{\max }}$ of the matrix $\boldsymbol{M}$. Otherwise, when no possible exchange with positive STSR exists, the loop jumps to the next recording position, $k+1$.

Note that, during execution of the algorithm, exchanges that occur between pairs of trajectories that maximize the STSR (the reduction in squared time-shift) are accepted. The exchange process stops when no further exchange produces a positive STSR.

\section{Trajectory purge}

After running greedy exchange algorithm, it is required to read the trajectories from the processed matrix $\boldsymbol{M}$. Each trajectory will be formed by sets of real turns in consecutive positions of a given row of the matrix. Note that, throughout the entire matrix, there may be more than one trajectory per row.

An additional step is carried out to eliminate any trajectory with a number of turns smaller than a certain quantity. In this way, short trajectories, which are more likely formed by false turn detections due to the presence of noise, are removed. For a trajectory to be considered valid, it must contain a minimum of $L_{\min }$ turns. Therefore, for a given $i$, the set $\left\{k, m_{k}^{i}, x\left(k, m_{k}^{i}\right)\right\} ; k_{1}^{i} \leq k \leq k_{2}^{i}$ is considered a valid trajectory, if it satisfies the following conditions:

- $m_{k}^{i} \neq 0 \quad \forall k ; k_{1}^{i} \leq k \leq k_{2}^{i}$

- $k_{2}^{i}-k_{1}^{i}+1 \geq L_{\min }$

- $\left(m_{k_{1}^{i}-1}^{i}=0\right) \vee\left(k_{1}^{i}=1\right)$

- $\left(m_{k_{2}^{i}+1}^{i}=0\right) \vee\left(k_{2}^{i}=K\right)$ 
where the first two conditions ensure a continuous trajectory of at least $L_{\min }$ turns, and the other two conditions ensure that the trajectory is delimited either by phantom turns or by the recording limits.

\subsubsection{Detection of the scanning-EMG signal active region}

In some situations, it will be necessary to determine the spatio-temporal extent of the MUP within the recording limits of the scanning-EMG signal. This operation can be regarded as a two-dimensional extension of the MUP duration concept [121]; i.e., it can be used to cut the recording so that it only contains the region in which the MUP of the scanned MU is present (the active MUP region), discarding in this way uninformative recording parts.
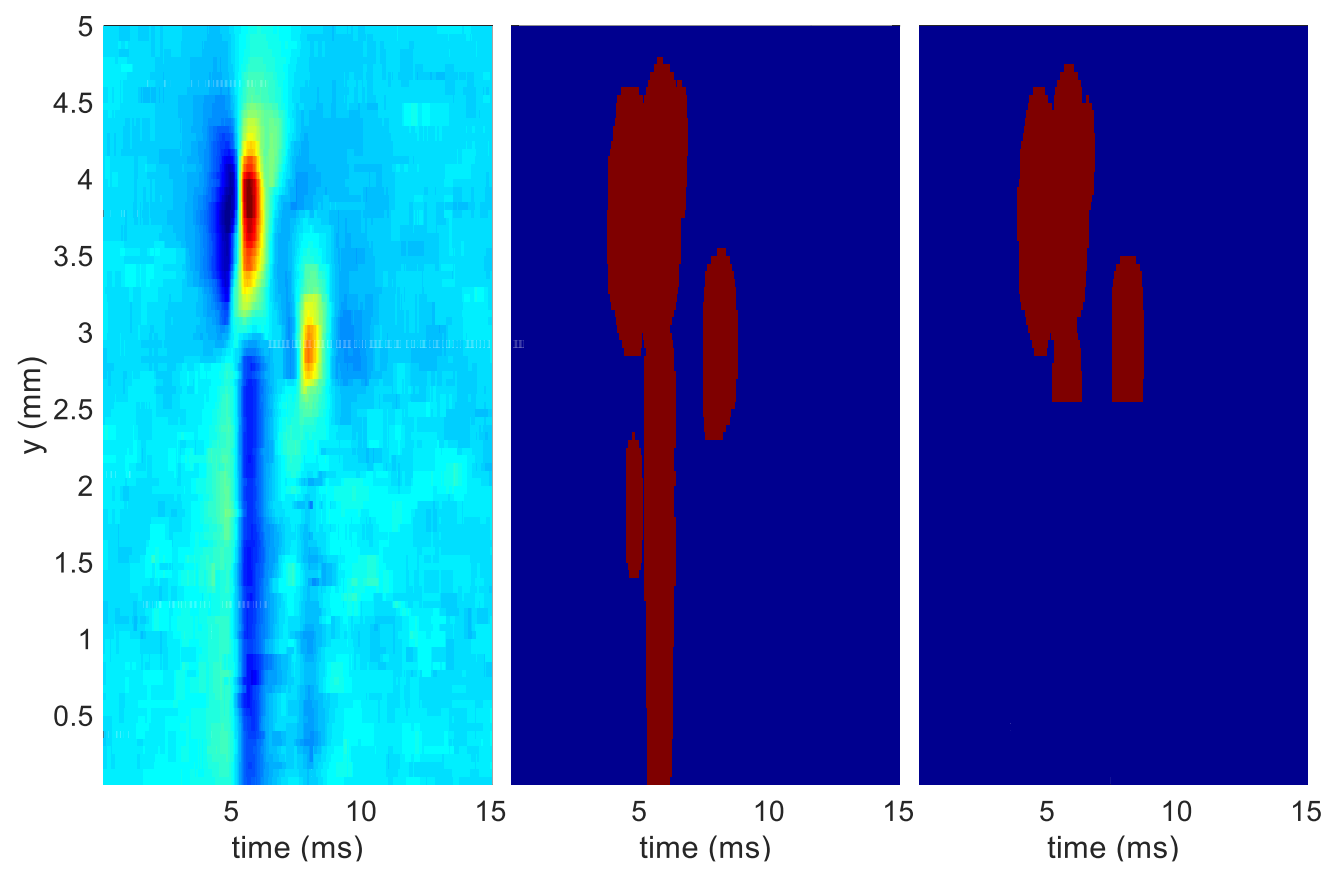

Fig. 2-10. Case of use of the two algorithms for the detection of the active MUP region; (a) scanning-EMG signal for which the active region is detected. Note that, two differentiated parts along the spatial dimension (i.e., the vertical axis) can be observed. The MUP associated to the recording within the MU territory (located between $2.5 \mathrm{~mm}$ and $4.5 \mathrm{~mm}$ approximately), and the cannula potential (located for positions lower than $2.5 \mathrm{~mm}$ approximately). Note that the cannula potential remains almost constant along the spatial dimension; (b) mask of the active MUP region (in red) calculated with the Algorithm 1. Note that the cannula potential has been detected as part of the active region; (c) mask of the active MUP region (in red) calculated with the Algorithm 2. Note that the cannula potential has not been detected as part of the active region. 
Two algorithms have been developed to calculate the active MUP region (Fig. 2-10). Both of them are part of the estimation system developed during this thesis and presented in chapter 3.3. The algorithms include a filtering stage to reduce the noise level, followed an amplitude thresholding stage (binarization), and some morphological operations. The difference between the two algorithms is that the first one considers the cannula signal as a part of the active MUP region, while the second one does not.

\section{Algorithm 1: Detection including the cannula}

Given a raw scanning-EMG signal $\boldsymbol{X}$, the following steps are taken to determine the active MUP region:

1- At each recording position, the median of all MUP discharges of $\boldsymbol{X}$ is calculated. If there is only one discharge per position, then no operation is applied. The output is a two-dimensional signal $\boldsymbol{X}_{1}$.

2- The signal $\boldsymbol{X}_{1}$ is band-pass filtered in the temporal dimension. Lower and higher cut-off frequencies are $f_{d L}$ and $f_{d H}$, respectively. The output of this step is $\boldsymbol{X}_{2}$.

3- The signal $\boldsymbol{X}_{2}$ is filtered with an $A_{d}$-order median filter in the spatial dimension. This filter is applied twice. The output is $\boldsymbol{X}_{3}$.

4- The signal $\boldsymbol{X}_{3}$ is thresholded to obtain a detection mask $\boldsymbol{F}_{4}$. The mask is set to 1 if the absolute value of $\boldsymbol{X}_{3}$, normalized to the maximum amplitude, is higher than $t_{d 1}$. Otherwise, the mask is set to 0 .

5- The two-dimensional detection mask $\boldsymbol{F}_{4}$ is subjected to an image dilatation using a circle of diameter $D_{d 1}$ as structural element. The output of this step and the algorithm is the detection mask $\boldsymbol{F}$.

The active region is the set of spatio-temporal positions where the detection mask value is 1 .

The main drawback of this algorithm is that it detects the inverted cannula potential as part of the active region (Fig. 2-10 (b)). This is an undesired effect, as this potential corresponds to recording positions in which the recording surface in the needle core is deeper than the MU territory. Therefore, this cannula region should not be considered as part of the active region of the recording. The second algorithm overcomes this limitation (Fig. 2-10 (c)).

\section{Algorithm 2: Detection excluding the cannula}

To eliminate the cannula region, the second algorithm takes advantage of how the potential varies in the spatial dimension in the cannula region and in the active region (Fig. 2-10 (a)). While in the cannula region the potential barely varies, in the active MUP region this variation is noticeable. Therefore, the first difference of the signal in the spatial dimension can be used to differentiate the activity in the cannula region. 
The steps of this second algorithm are:

1- At each recording position, the median of all MUP discharges of $\boldsymbol{X}$ is calculated. If there is only one discharge per position, then no operation is applied. The output is a two-dimensional signal $\boldsymbol{X}_{1}$.

2- The signal $\boldsymbol{X}_{1}$ is band-pass filtered in the temporal dimension. Lower and higher cut-off frequencies are $f_{d L}$ and $f_{d H}$, respectively. The output of this step is $\boldsymbol{X}_{2}$.

3- The signal $\boldsymbol{X}_{2}$ is filtered with a $A_{d}$-order median filter in the spatial dimension. This filtering is applied twice. The output is $\boldsymbol{X}_{3}$.

4- The signal $\boldsymbol{X}_{3}$ is filtered with a two-dimensional filter. The impulse response of this filter is a circular disk of a radius $R_{d}$. The output is $\boldsymbol{X}_{4}$.

5- The first difference in the spatial dimension of the signal $\boldsymbol{X}_{4}$ is calculated. The output is $\boldsymbol{d} \boldsymbol{X}_{5}$.

6- A thresholding is applied to $\boldsymbol{d} \boldsymbol{X}_{5}$ to obtain a detection mask $\boldsymbol{F}_{6}$. The mask is set to 1 if the absolute value of $\boldsymbol{d} \boldsymbol{X}_{5}$ is higher than $t_{d 2}$. Otherwise, the mask is set to 0 .

7- The detection mask $\boldsymbol{F}_{6}$ is subjected to an image dilation using a circle of diameter $D_{d 2}$ as structural element. The output of this step is the detection mask $\boldsymbol{F}_{7}$.

8- The Algorithm 1 is applied to the input signal $\boldsymbol{X}$. The output of this step is the detection mask $\boldsymbol{F}_{8}$.

9- For the spatial positions where there are none samples in which $\boldsymbol{F}_{7}$ is 1 , all the samples of $\boldsymbol{F}_{8}$ are set to 0 . The modified $\boldsymbol{F}_{8}$ is the output of the algorithm, $\boldsymbol{F}$.

The parameters used in these algorithms are shown in Table 2-1.

Table 2-1. Parameters used by the Algorithm 1 and the Algorithm 2.

\begin{tabular}{lll}
\hline Symbol & Parameter & Value \\
\hline$f_{d L}$ & BPF low cut-off frequency & $4 / 300$ cycles/sample \\
$f_{d H}$ & BPF high cut-off frequency & 0.5 cycles/sample \\
$A_{d}$ & Median filter order & 15 samples \\
$t_{d 1}$ & Normalized amplitude threshold (Alg1) & 0.2 \\
$D_{d 1}$ & Diameter of the structural element (Alg1) & 10 samples \\
$R_{d}$ & Impulse response radius of the filter (Alg2) & 4 samples \\
$t_{d 2}$ & Absolute amplitude threshold (Alg2) & $0.25 \mathrm{mV}$ \\
$D_{d 1}$ & Diameter of the structural element (Alg2) & 18 samples \\
\hline
\end{tabular}




\subsection{Processing of the surface-EMG signal}

In this section, two processing algorithms applied to surface-EMG recordings are described. Both algorithms have been integrated as part of the estimation system developed during this thesis. The first one, widely used in the literature, is the spike trigger averaging [54]; and the second one is a specifically designed algorithm to estimate the IZ, the average MFCV, and the potential initiation instant from a multichannel surface-MUP signal.

\subsubsection{Spike-trigger averaging}

Let us assume that we have a surface-EMG interference pattern recorded from a linear array of surface electrodes placed on the skin. Let us also assume that the firing pattern of a MU during the entire recording time is known. In that case, spike-trigger averaging can be applied to extract the surface-MUP of this MU from the surfaceEMG pattern.

For the EMG pattern recorded at each electrode, the following two steps are performed:

1- For each firing event of the MU, a windowed section of the surface-EMG is extracted around the firing instant. The size of the window must be large enough to ensure that the surface-MUP is completely inside the window.

2- The different discharges obtained from the previous stage are averaged extracting the median value of each sample after algning the traces.

Using this procedure, a multi-channel surface-MUP is obtained. If the number of MU firing events is large enough, this surface-MUP will be largely free of noise and interference of other MUs.

\subsubsection{Estimation of the IZ, the MFCV, and the potential initiation instant}

An algorithm to estimate some global parameters of the MU (the IZ, the average MFCV, and the potential initiation instant) from a multi-channel surface-MUP has been developed. This algorithm has been inspired in other algorithms to estimate the fiber conduction velocity [30,31]. The developed algorithm is an important piece of the final estimation system, as it will provide a first approximation to the decomposition solution. The algorithm assumes that the surface-MUP has been obtained from a linear array of surface electrodes, in a bipolar configuration, placed on the surface of the skin along the direction of the muscle fibers.

In Fig. 2-11, an example of this kind of surface-MUP is presented. The figure shows how the surface-MUP waveform changes for the different channels, i.e., how this waveform depends on the recording position along the longitudinal direction of 


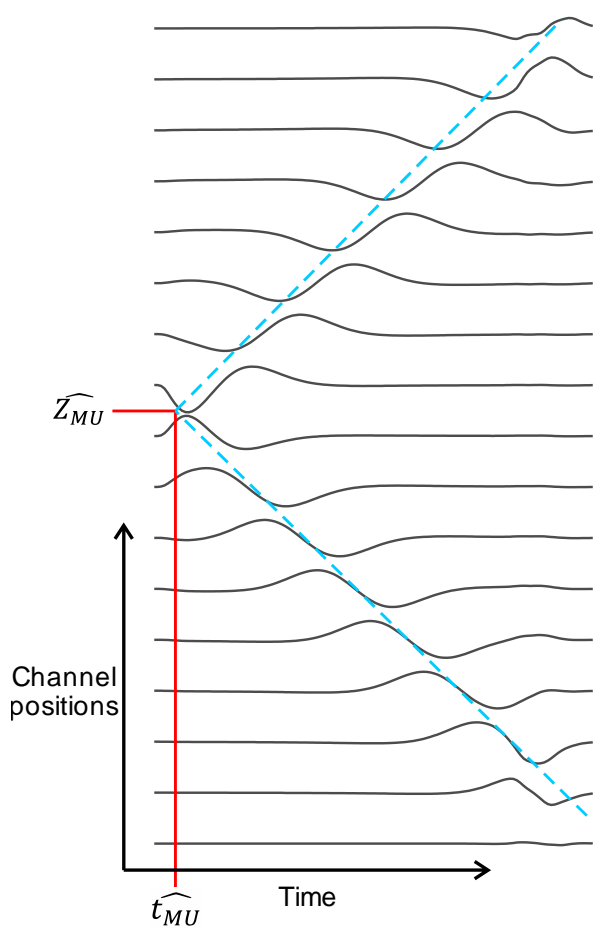

Fig. 2-11.Example of a bipolar surface-MUP recorded from a linear array of surface electrodes placed along the direction of the muscle fibers. The figure also shows the estimation of the IZ position of the MU, $\widehat{Z_{M U}}$, and of the potential initiation instant of the MU, $\widehat{t_{M U}}$. These two parameters (corresponding to the horizontal and vertical red lines, respectively) are estimated as the cut point of the two regression lines traced along the propagation front of the MUP (blue dashed lines).

the fibers. Between the IZ and the tendon zones, the surface-MUP waveform barely changes among consecutive channels; only the time delay does. This change in the time delay is caused by the propagation of the potential through the MU fibers. Note that, at each side of the IZ, the potential propagates in opposite directions. For electrode positions placed at the tendon zone and beyond, there is no change in the time delay of the surface-MUP, as in these positions the potential is no longer propagating. An attenuation of the waveform related to the extinction of the potential in the tendons can be observed standing at these positions.

The algorithm has been designed to estimate the following parameters: the average MFCV of the MU, $V_{M U}$; the approximate IZ position of the MU, $Z_{M U}$; and the potential initiation instant of the MU, $t_{M U}$. The algorithm rationale is the following: the average MFCV can be obtained from the delay between propagating surface-MUPs in consecutive channels and from the distance between electrodes; the position of the IZ can be estimated as the spatial position in which the direction of propagation is inverted; and the potential initiation can be estimated as the time instant in which the waveform is initiated at the IZ position (Fig. 2-11). 


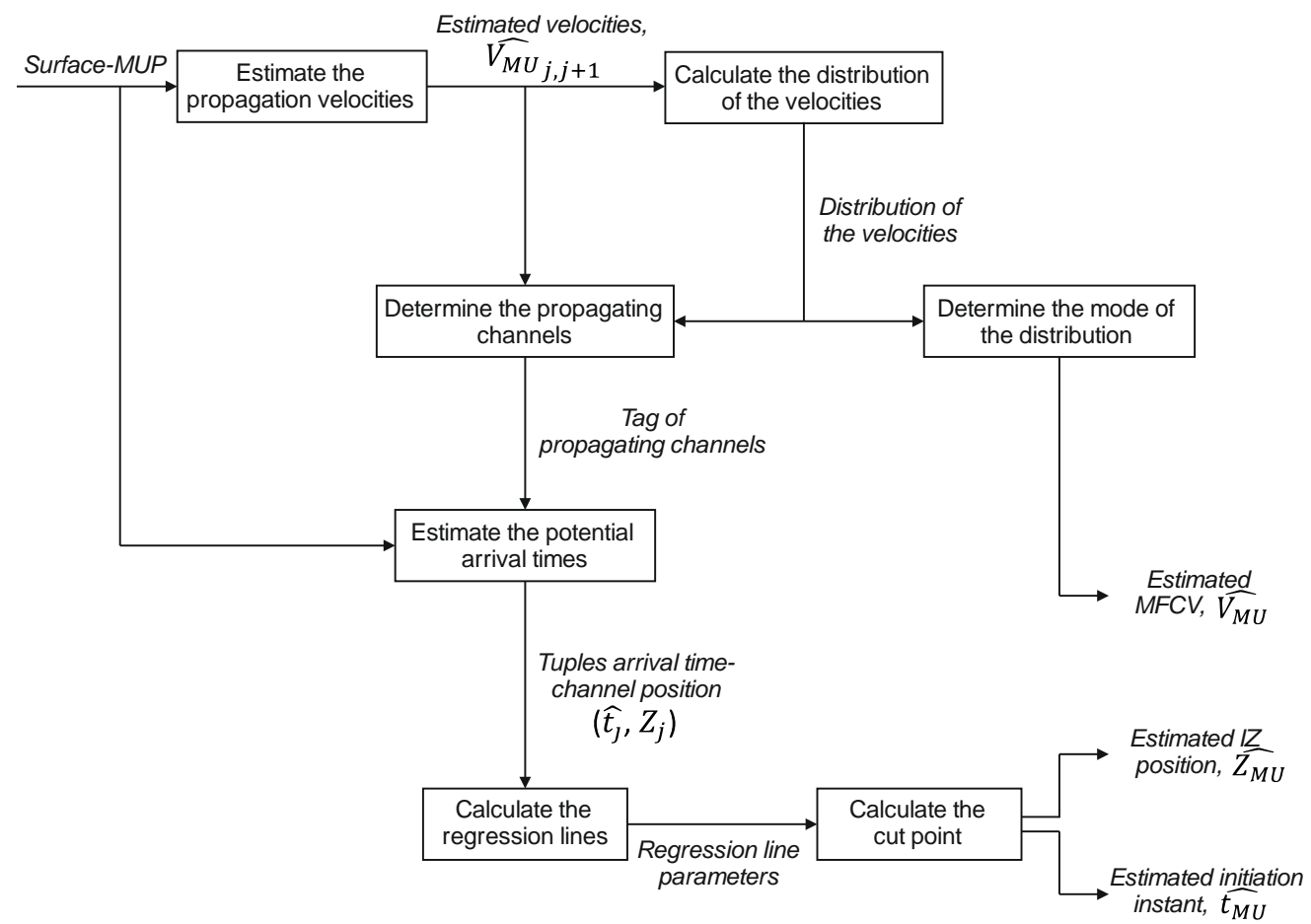

Fig. 2-12. Schematic representation of the processing blocks constituting the algorithm developed to estimate the IZ, the MFCV, and the potential initiation instant.

Fig. 2-12 shows a block diagram describing the different steps of the algorithm. The input of the algorithm is a multi-channel surface-MUP recorded in a bipolar configuration. An important consideration is that in a bipolar configuration, the position representing each channel is not the physical position of the recording electrodes. Instead, as the bipolar signal is calculated from two consecutive electrodes, the channel positions are considered to be represented by the midpoints between each pair of consecutive physical electrodes. The algorithm steps are detailed in the following sections.

\section{Estimation of the propagation velocity for each pair of recording channels}

We can approximate the time, $t$, at which the propagating potential arrives to a certain position along the propagation direction, $z$, as

$$
t=t_{M U}+\frac{z-Z_{M U}}{V_{M U}}
$$

where $V_{M U}$ is the average MFCV of the MU, $Z_{M U}$ the approximate IZ position of the $\mathrm{MU}$ and $t_{M U}$ the potential initiation instant. If we evaluate this expression at a certain 
$j$ th channel we obtain that

$$
t_{j}=t_{M U}+\frac{Z_{j}-Z_{M U}}{V_{M U}}
$$

where $Z_{j}$ is the position of the $j$ th channel, and $t_{j}$ is the time at which the potential arrives to this channel. Then, the potential propagation delay between two consecutive channels can be calculated as

$$
t_{j+1}-t_{j}=\frac{Z_{j+1}-Z_{M U}}{V_{M U}}-\frac{Z_{j}-Z_{M U}}{V_{M U}}=\frac{Z_{j+1}-Z_{j}}{V_{M U}}=\frac{I E D}{V_{M U}}
$$

where IED is the inter-electrode distance, which is known beforehand. In this way, it is possible to obtain an estimation of $V_{M U}$ for each pair of consecutive electrodes as

$$
\widehat{V}_{M U}{ }_{j, j+1}=\frac{I E D}{t_{j+1}-t_{j}}
$$

The first step of the algorithm is to calculate these estimations of the propagation velocity. This requires to compute the delay between consecutive channels, which is obtained by means of the high-resolution alignment algorithm proposed by McGill [73].

\section{Estimation of the MUP propagation velocity}

The second step of the algorithm is to provide a definitive estimation of the average MFCV from the estimated values obtained for each channel. Note that equation (2-20) is only valid when the potential is propagating though the MU fibers, i.e., for those channels placed between the IZ and the tendon zones. This implies that reliable estimations of the $V_{M U}$ can only be obtained for those pair of consecutive channels where the potential is actually propagating. However, this information is not known at first, and a deeper analysis must be performed.

An approximate distribution of the propagation velocity values obtained from each pair of channels is obtained by applying a kernel density estimation. Specifically, the absolute value of the estimated propagation velocities is used as the input to the kernel density estimation. A gaussian kernel of $0.1 \mathrm{~m} / \mathrm{s}$ width is used to calculate the distribution. A consideration to keep in mind is that the values of the estimated propagation velocity higher than $10 \mathrm{~m} / \mathrm{s}$ are discarded beforehand. The rationale is that a high estimated propagation velocity corresponds to a small time delay, which means that the two channels involved in the estimation are possibly located in a region where the MUP is not propagating, like in the tendons. Another consideration is that, those pairs of channels for which an inversion of the waveform has been detected are also discarded for the calculation of the distribution, since this is indicative that the IZ is 
located between these two channels.

The estimated propagation velocity, $\widehat{V_{M U}}$, is calculated as the velocity propagation value for which the distribution reaches the maximum; i.e., the mode of the distribution.

\section{Determination of the propagating channels}

To calculate the other two parameters $\left(Z_{M U}\right.$ and $\left.t_{M U}\right)$, it is firstly necessary to determine the regions corresponding to channels in which the potential is actually propagating. It is important to remember here, that each of these (bipolar) channels actually corresponds to the midpoint between two consecutive physical electrodes.

The range of valid propagation velocity values is calculated from the distribution, as the right and left velocity points from the maximum peak for which the value of the distribution has decreased to $25 \%$ of the peak value. The pairs of channels for which the estimated propagation velocity is within this range are marked as propagating. Otherwise the pairs of channels are marked as non propagating.

For the potential to be propagating between two consecutive channels, both must be placed in the propagating region between the IZ and one of the tendon zones. On the other hand, the propagation direction indicates at which side of the IZ the channels are located. This allows to identify what individual channels are placed within the propagating zones at each side of the IZ. Individual channels are tagged according to the following criterion:

- For each pair of channels, if both have been marked as propagating, both channels are considered to be located between the IZ and the tendon zone, and the tag values associated to these two channels are set to \pm 1 . The sign of the tag is given by the sign of the propagation velocity estimated for the current channel pair. This sign determines the side of the IZ where the electrodes are located.

- The rest of channels are considered to be located either in the IZ or in the tendon zones. For these channels the tag is set to 0 .

In this way, the right propagation zone is associated in the tag to a set of consecutive 1's, and the left propagation zone to a set of consecutive -1's. The IZ is represented by a set of 0 's located in the middle of both propagation zones. If among the 0 's of the IZ there is tag values set to +1 or -1 , then these values are corrected and set to 0 , so that the IZ is actually corresponded to a set of consecutive 0 's. This allows to avoid possible errors regarding to use these channels in the posterior calculations.

\section{Estimation of the arrival time of the potential at each channel}

Given a certain channel whose corresponding tag value is +1 or -1 , the potential 
arrival time is estimated as the instant of occurrence of the first potential peak. This information will be used in the following step to estimate the parameters $Z_{M U}$ and $t_{M U}$. Note that, due to the waveform inversion effect given in the bipolar recordings, the first peak must be positive at one side of the IZ and negative for the other.

\section{Estimation of the IZ position and the potential initiation instant}

Equation (2-19) can be rewritten as

$$
z=\left(V_{M U}\right) \cdot t+\left(-V_{M U} \cdot t_{M U}+Z_{M U}\right)
$$

which expresses the dependency between the arrival time and the position of the propagating potential in the linear form $z=m t+b$. As we have two propagating potentials, we are interested in determining the propagation wave front line for both of them. This information can be obtained from the channels located in the two propagating zones. For each channel, we know its position $Z_{j}$, and we have an estimation of the potential arrival time $\widehat{t}_{j}$, calculated in the previous step. Using the tuples $\left(\widehat{t}_{j}, Z_{j}\right)$ as inputs, we can fit a regression line for each direction. The one corresponding to the right propagation potential is fitted from the tuples $\left(\widehat{t}_{j}, Z_{j}\right)$ associated to channels with tag values set to +1 , obtaining the line parameters, $m_{R}$ and $b_{R}$. Analogously, the regression line corresponding to the left propagation potential is calculated from the tuples for which the tag is set to -1 , obtaining the line parameters, $m_{L}$ and $b_{L}$.

The estimation of the IZ position, $\widehat{Z_{M U}}$, and the potential initiation instant, $\widehat{t_{M U}}$, are given by the cut point of the two regression lines (Fig. 2-11), namely

$$
\begin{aligned}
& \widehat{t_{M U}}=\frac{b_{L}-b_{R}}{m_{R}-m_{L}} \\
& \widehat{Z_{M U}}=m_{R} \frac{b_{L}-b_{R}}{m_{R}-m_{L}}+b_{R}
\end{aligned}
$$


upna

Universidad Púbilica de Navara
Natarmaako Unibersitate Publikoa 


\section{Theoretical framework of the MUP inverse problem}

In this chapter, a theoretical framework to tackle the MUP inverse problem is developed. The chapter starts stating the objective of the thesis, i.e., the estimation of the MU fiber parameters. Then, it continues analyzing the MUP forward problem by studying how the signal waveform is influenced by changes in the MU fiber parameters. When applied to different recording configurations, the forward analysis allows to study the potential ability of these configurations to estimate MU fiber parameters. The chapter continues describing the basis of the estimation systems developed during this thesis to solve the MUP inverse problem, and it finishes describing the mathematical framework used to evaluate the performance of such estimations system. 
upna

Universidad Púbilica de Navara
Natarmaako Unibersitate Publikoa 


\subsection{Restating the objective of the thesis}

The main goal of this thesis is to solve the MUP inverse problem, that is, to estimate the parameters characterizing the muscle fibers constituting the MU from the recording of the MUP. Before establishing the specific parameters to be estimated, it is necessary to keep in mind the following considerations:

- MU fibers are supposed to be infinitely long. The effect of considering fibers of finite length is not especially significant for intramuscular electrodes as the ones considered in this work (SF electrode or $\mathrm{CN}$ electrode). This assumption simplifies the model, favoring the effectiveness of the estimation process.

- In the real world, fibers from the same MU may have different initiation of depolarization instants due to possible differences in axonal transmission delay in the pathways to each of the neuromuscular junctions. However, these differences between initiation of depolarization instants are usually negligible compared to the MUP propagation delays caused by the rest of the fiber parameters [87]. Therefore, in order to reduce the complexity of the problem, it will be considered that all the MU fibers start to depolarize at the same time.

- For each fiber of the MU, its MFCV is assumed to remain unaltered along the entire muscle fiber length.

- A linear dependence between the MFCV and the diameter of the muscle fiber is assumed, as stated in equation (1-7) [81]. Thus, once the MFCV is estimated, the estimation of the fiber diameter is directly obtained by applying (1-7), and therefore, the fiber diameter does not need to be explicitly included as an additional parameter to be estimated.

Given the above considerations, the parameters to be estimated for the $j$ th fiber of the MU, denoted as the vector $\boldsymbol{m}_{\boldsymbol{j}}$, are: the position within the muscle cross-section, $\left(x_{j}, y_{j}\right)$; the longitudinal position of the neuromuscular junction, $z_{j}$; and the MFVC, $v_{j}$. In this way, the objective is to estimate a set of MU parameters $\boldsymbol{M}=\left\{\boldsymbol{m}_{j}\right\} ; 1 \leq j \leq$ $J$, where $J$ is the number of MU fibers, and $\boldsymbol{m}_{j}=\left(x_{j}, y_{j}, z_{j}, v_{j}\right)$.

In order to achieve this, the MUP model is inverted by using optimization algorithms. As explained in section 1.3, this inverse problem approach has already been addressed in previous works $[67,68,140]$. However, these attempts to estimate the MU fiber parameters assume that the MUP is recorded in a single position, which does not provide enough information to estimate the MU fiber parameters univocally. A correct parameter estimation would require the MUP to be recorded in multiple positions (see section 1.3.5). During this thesis, several solutions have been implemented that estimate the MU fiber parameters from the recording of the 
scanning-EMG signal; i.e., from the recording of the MUP in multiple points along a linear corridor. The different solutions implemented correspond to different variants of the scanning-EMG technique that differ in the way the signal is recorded, either using a standard SF needle electrode, using a needle with two SF ports, or using two scanning needles.

Another objective of this thesis is to establish a suitable evaluation framework, so that the quality of the estimation can be analyzed objectively. This evaluation framework is based on the use of synthetic EMG signals obtained from a simulation environment to test the estimation algorithms. In this way, the exact a priori knowledge about the MU fiber parameters used to generate the signals is available, allowing to quantify the error between the estimated MU parameters and the actual ones. 


\subsection{Analysis of the MUP forward problem}

This section aims to study to what extent changes in the MU fiber parameters affect to the MUP waveform. As the resolution of the inverse problem is based on the information provided by the MUP, the way this one is affected by the fiber parameters ultimately determines the ability to achieve a correct parameter estimation. The main problem is that different combinations of the MU fiber parameters may result in simulated MUPs with very similar waveforms. This is what is known as an ill-posed problem. If this is the case, the resolution of the inverse problem cannot be performed correctly, since it is very difficult, or even impossible to know what parameter combination actually corresponds to the recorded MUP waveform. It is therefore important to know to what extent this behavior occurs for different recording configurations, as this allows to determine the potential of each of them to estimate the MU fiber parameters.

\subsubsection{MU fiber parameters influence on MUP waveform}

The relationhship between the MU fiber parameters and the simulated signal waveform has been qualitatively analyzed in a very simple case: the SFAP generated by a single muscle fiber at a single recording point. This aims to provide a preliminary exemplification of how the simulated signal is affected by the fiber parameters. To simulate this signal, the Dimitrov model [21] described in sections 1.3.1 and 1.4.2 has been used. In our approach a muscle fiber is characterized by its position in the muscle cross-section, its neuromuscular junction position, and its MFVC. As the signal is assumed to be recoded with single-point-size leading-off, this problem has radial symmetry, and therefore, the simulated SFAP depends on the following three parameters: the radial distance between the muscle fiber position and the recording point

$$
r_{j}=\sqrt{\left(x_{j}-x_{e}\right)^{2}+\left(y_{j}-y_{e}\right)^{2}}
$$

where $\left(x_{e}, y_{e}\right)$ and $\left(x_{j}, y_{j}\right)$ are the positions in the muscle cross-section of the recording point and the muscle fiber, respectively; the longitudinal distance between the neuromuscular junction position and the recording point

$$
\Delta z_{j}=z_{j}-z_{e}
$$

where $z_{e}$ and $z_{j}$ are respectively the positions in the direction of the muscle fibers of the recording point and of the fiber; and the MFCV, $v_{j}$.

Three sets of SFAP simulations have been performed. In each case, one of the three parameters under study, $r_{j}, \Delta z_{j}$ and $v_{j}$, has been varied (taking the values given 


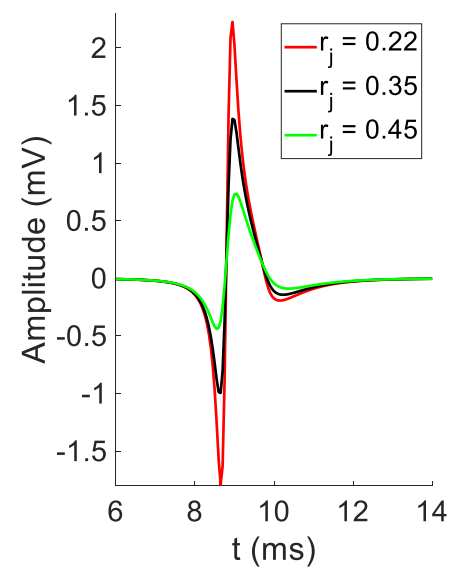

(a)

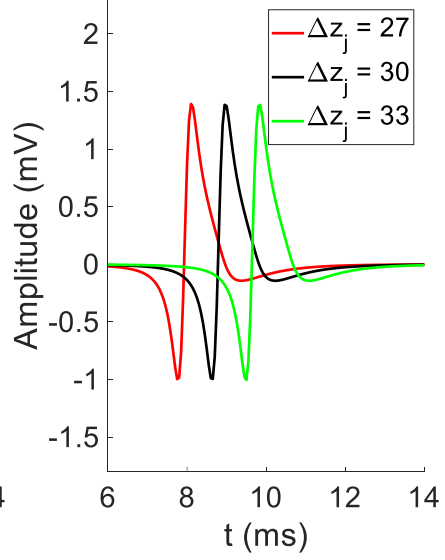

(b)

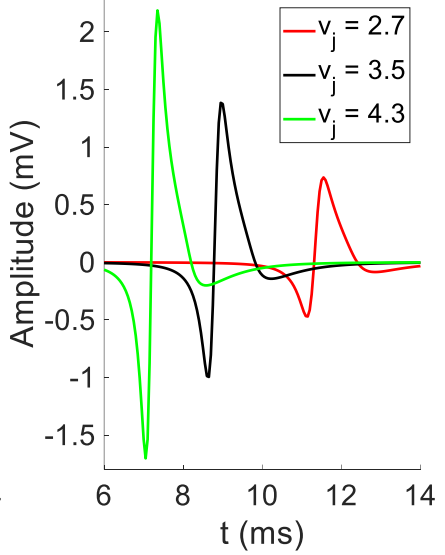

(c)

Fig. 3-1. SFAPs simulated for different parameter values: (a) varying the radial distance, $r_{j}$, mainly affects the SFAP amplitude; (b) varying the longitudinal distance, $\Delta z_{j}$, mainly affects the SFAP delay; (c) varying the MFCV, $v_{j}$, affects both the SFAP amplitude and delay.

in the corresponding column of Table 3-1), while the other two parameters remained constant $\left(r_{j}=0.3 \mathrm{~mm} ; \Delta z_{j}=30 \mathrm{~mm} ; v_{j}=3.5 \mathrm{~m} / \mathrm{s}\right)$.

Table 3-1. Parameters varied in the simulation

\begin{tabular}{ccc}
\hline$r_{j}(\mathrm{~mm})$ & $\Delta z_{j}(\mathrm{~mm})$ & $v_{j}(\mathrm{~m} / \mathrm{s})$ \\
\hline 0.22 & 27 & 2.7 \\
0.3 & 30 & 3.5 \\
0.45 & 33 & 4.3 \\
\hline
\end{tabular}

The results of these simulations are shown in Fig. 3-1, which allow to highlight the following relationships:

- The radial distance between the fiber and the recording point affects to the amplitude of the SFAP. The closer the fiber is to the recording point, the larger the SFAP amplitude [81].

- The longitudinal distance between the neuromuscular junction and the recording point affects to the temporal delay of the SFAP arrival to the recording point. The larger the longitudinal distance, the larger the temporal delay [125].

- The MFCV affects both the amplitude and the temporal delay. The higher the MFCV, the larger the amplitude and the shorter the time delay [125].

\subsubsection{Analysis of the error function in the parameter space}

The region of the parameter space in which the simulated signal waveform 
remains barely unaltered (in the following, the dispersion region) has been studied. This has been done for different recording configurations, and for different arrangements of the MU fibers, with the aim to assess in what conditions the estimation can be potentially made correctly, and in what conditions it cannot. A dispersion region comprising a small hypervolume of the parameter space means that that there is a direct correspondence between the signal waveform and the MU fiber parameters, and therefore such parameters can be correctly determined. On contrary, a wide dispersion region means that the signal waveform lacks the necessary information to be associated with a unique fiber parameter solution; so, in this case, the parameters are expected to be estimated with a great inaccuracy.

Given a certain recording configuration (defined by the set of positions in which the MUP is recorded) and a certain fiber arrangement, the dispersion region is calculated as follows. The first step is to simulate the MUP signal for the MU fiber arrangement under study. We will name this signal reference signal $\boldsymbol{X}$, and the set of MU fiber parameters that generate it reference parameters, $\boldsymbol{M}=\left\{\boldsymbol{m}_{j}\right\}$. Note that the reference signal is constituted by one or several MUPs, depending on the recording configuration considered. Each simulated MUP corresponds to a certain recording position. Note that the MUP can be recorded using $P$ ports (theoretically corresponding to each of the different leading-offs of the needles), and that each port takes $K$ different recording positions. Thus, the positions at which the MUP is recorded are

$$
E=\left\{\left(x_{e_{k, p}}, y_{e_{k, p}}, z_{e_{k, p}}\right)\right\} ; 1 \leq k \leq K ; 1 \leq p \leq P
$$

At each position, a window of $N$ samples (in the time domain) is recorded. Under these assumptions, the reference signal, $\boldsymbol{X}$, can be mathematically expressed as

$$
\boldsymbol{X}(\boldsymbol{M})=\left\{x_{n, k, p}(\boldsymbol{M})\right\}
$$

where

$$
x_{n, k, p}(\boldsymbol{M})=\operatorname{MUP} P_{\boldsymbol{M}}\left(t_{n}, x_{e_{k, p}}, y_{e_{k, p},} z_{e_{k, p}}\right)
$$

where $n$ is denoting the temporal samples; $k$ is denoting the different recording positions taken by the ports; and $p$ is denoting the different ports used by the particular recording configuration. The MUP in (3-5) is calculated as described in section 1.3.1, by applying the equations from (1-1) to (1-8).

The second step is the calculation of the dispersion region. This is done by sampling the parameter space, and checking for all these points if a certain waveform error criterion is fulfilled or not. Only points satisfying such criterion are then considered to be within the dispersion region. This checkup is performed by taking the following steps: 
1- The MUP signal is simulated from the MU fiber parameters associated to the parameter space point under consideration. We will name this signal as candidate signal $\widehat{\boldsymbol{X}}$, and the set of MU fiber parameters that generate it as candidate parameters $\widehat{\boldsymbol{M}}$. Note that both the candidate and the reference signals are simulated at the same recording positions.

2- The discrepancies between the candidate and the reference signal are quantified by computing the normalized mean squared error (NMSE)

$$
\begin{aligned}
\varepsilon(\widehat{\boldsymbol{M}}, \boldsymbol{M})=\frac{\|\widehat{\boldsymbol{X}}(\widehat{\boldsymbol{M}})-\boldsymbol{X}(\boldsymbol{M})\|^{2}}{\|\boldsymbol{X}(\boldsymbol{M})\|^{2}} \\
=\frac{\sum_{n=1}^{N} \sum_{k=1}^{K} \sum_{p=1}^{P}\left(\hat{x}_{n, k, p}(\widehat{\boldsymbol{M}})-x_{n, k, p}(\boldsymbol{M})\right)^{2}}{\sum_{n=1}^{N} \sum_{k=1}^{K} \sum_{p=1}^{P} x_{n, k, p}(\boldsymbol{M})^{2}}
\end{aligned}
$$

3- The candidate parameters are considered to be within the dispersion region if and only if $\varepsilon<5 \cdot 10^{-4}$. The choice of this particular value is heuristic. This has been chosen low enough so that it ensures that the two signals have almost the same waveform.

To perform a suitable sampling of the parameter space, the Metropolis-Hastings algorithm is used [49].

\section{Description of the recording configurations}

The recording configuration determines the sites in which the recording of the MUP is simulated. Here, the dispersion region has been analyzed for three different recording configurations (Fig. 3-2): the single-point recording, the standard scanningEMG recording, and a two-needle scanning-EMG recording.

The single-point recording is the signal recorded with a single port (Fig. 3-2 (a)), at a single position $\left(x_{e}, y_{e}, z_{e}\right)$. This configuration can be described in terms of equation (3-3), where

$$
\begin{aligned}
& x_{e_{1,1}}=x_{e} \\
& y_{e_{1,1}}=z_{e} \\
& z_{e_{1,1}}=z_{e}
\end{aligned}
$$

where the number of scanning steps is $K=1$, and the number of ports is $P=1$. This is the configuration employed in the previous attempts to estimate the MU fiber parameters $[67,68,140]$. For this reason, although this configuration is considered in this thesis as not able to solve the inverse problem, it is analyzed here in order to be compared with the other recording configurations. 

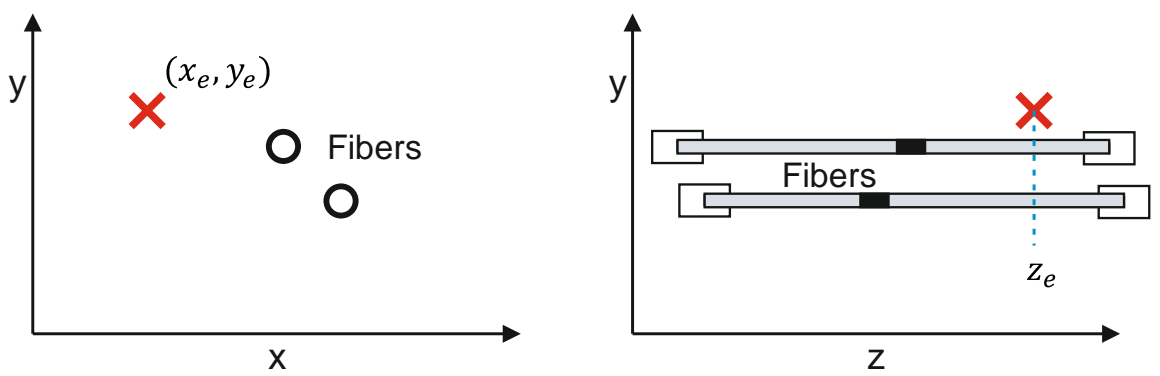

(a)

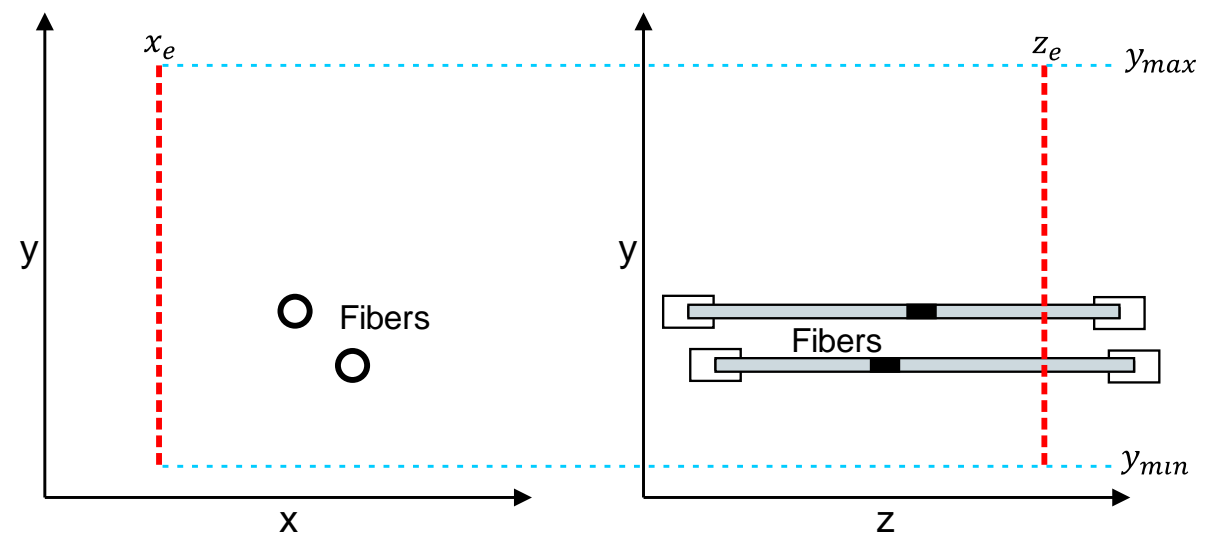

(b)

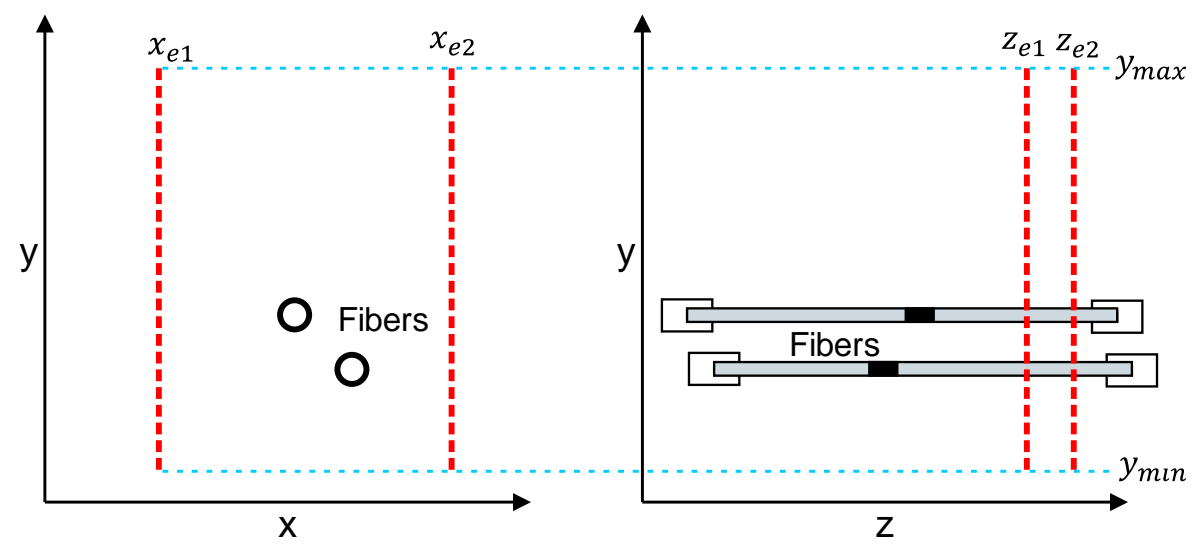

(c)

Fig. 3-2. Schematic representation of the three recording configurations in the cross-section (left subfigures) and longitudinal section (right subfigures) planes. Two hypothetical MU fibers are also depicted in the figure; (a) single-point recording, where the single recording position associated to this configuration is represented by a red cross; (b) standard scanning-EMG recording, where the different recording positions associated to this configuration lie along a linear corridor represented by the red dashed line; (c) two-needle scanning-EMG recording, where the different recording positions associated to this configuration lie along two linear corridors represented by the two red dashed lines, each line corresponding to one of the ports. 
The standard scanning-EMG consists on recording the MUP at different points equally spaced along a linear corridor using a needle electrode with a single port (more details about this technique in section 1.2.1). In the simulations, it is assumed that the recording of this signal is performed with the needle electrode inserted parallel to the $\mathrm{y}$-axis, and positioned at $x=x_{e}$, and at $z=z_{e}$. It is also assumed that the electrode records the MUP along the y-axis, from $y=y_{\min }$ to $y=y_{\max }$, in steps of $0.05 \mathrm{~mm}$ (Fig. 3-2 (b)). Mathematically, these recording positions can be expressed as in equation (3-3), where

$$
\begin{aligned}
& x_{e_{k, 1}}=x_{e}, \quad \forall k \\
& y_{e_{k, 1}}=y_{\min }+0.05 \cdot(k-1) \\
& z_{e_{k, 1}}=z_{e} \quad \forall k
\end{aligned}
$$

where the number of scanning steps is $K=1+\frac{y_{\max }-y_{\min }}{0.05}$, and $P=1$ is the number of ports.

The two-needle scanning-EMG is a modified version of the scanning-EMG technique conceived in this thesis, in which two different needle electrodes are employed to simultaneously record the MUP. To the best of our knowledge, this modified version has never been physically implemented. Here, this configuration is analyzed from a theoretical point of view, in order to assess its potential ability to estimate the MU fiber parameters. The proposed technique would consist on an assembly of two needles spaced by a certain distance. The needle assembly would replace the scanning needle in the original scanning recording technique. Note that, in this configuration, the MUP is simultaneously recorded along two different linear corridors corresponding to the two needles, which are spaced both in the longitudinal and in the perpendicular fiber direction. The motivation of this configuration is that it provides much more information than the standard scanning-EMG, and therefore, it is also expected to provide a more precise MU fiber parameter estimation. In the simulations, it is assumed that the recording is performed with the two needle electrodes inserted along the $y$-axis. One needle electrode is placed at $x=x_{e 1}$ and $z=$ $z_{e 1}$; and the other one at $x=x_{e 2}$ and $z=z_{e 2}$. It is also assumed that the electrodes record the MUP along the y-axis, from $y=y_{\min }$ to $y=y_{\max }$, in steps of $0.05 \mathrm{~mm}$ (Fig. 3-2 (c)). Under these assumptions, the recording positions can be mathematically expressed as in equation (3-3), where

$$
\begin{aligned}
& x_{e_{k, 1}}=x_{e 1}, \quad \forall k \\
& x_{e_{k, 2}}=x_{e 2} \quad \forall k \\
& y_{e_{k, 1}}=y_{\min }+0.05 \cdot(k-1) \\
& y_{e_{k, 2}}=y_{\min }+0.05 \cdot(k-1) \\
& z_{e_{k, 1}}=z_{e 1} \quad \forall k \\
& z_{e_{k, 2}}=z_{e 2} \quad \forall k
\end{aligned}
$$


where the number of scanning steps is $K=1+\frac{y_{\max }-y_{\min }}{0.05}$, and $P=2$ is the number of ports.

In the simulation analysis presented in the next section, the following parameter settings have been used. For the single-point recording, $x_{e}=0, y_{e}=0$, and $z_{e}=0$; for the standard scanning-EMG, $x_{e}=0, z_{e}=0, y_{\min }=-1.2 \mathrm{~mm}$, and $y_{\max }=1.2$ $\mathrm{mm}$; and for the two-needle scanning-EMG, $x_{e 1}=0, z_{e 1}=0, x_{e 2}=0.6 \mathrm{~mm}, z_{e 2}=$ $4 \mathrm{~mm}, y_{\min }=-1.2 \mathrm{~mm}$, and $y_{\max }=1.2 \mathrm{~mm}$.

\subsubsection{Analysis of the dispersion region of a single MU fiber}

The simplest fiber arrangement to be studied is the one composed by a single MU fiber with the following parameters: $x_{1}=0.3 \mathrm{~mm}, y_{1}=0 \mathrm{~mm}, z_{1}=30 \mathrm{~mm}$, and $v_{1}=$ $3.5 \mathrm{~m} / \mathrm{s}$. The dispersion region has been studied for this fiber arrangement, using the three recording configurations. An scheme of each scenario is shown in Fig. 3-3 (a), in Fig. 3-5 (a) and in Fig. 3-7 (a), respectively.

\section{Single-point recording}

Fig. 3-4 shows the dispersion region obtained for the single-point recording configuration. Note that in the muscle cross-section projection (Fig. 3-4 (d)), the dispersion region extends along a halved circular crown. This means that all the points within this ring generate simulated signals below the NMSE threshold (3-6), i.e., almost identical to the reference signal. This implies that the reference signal does not

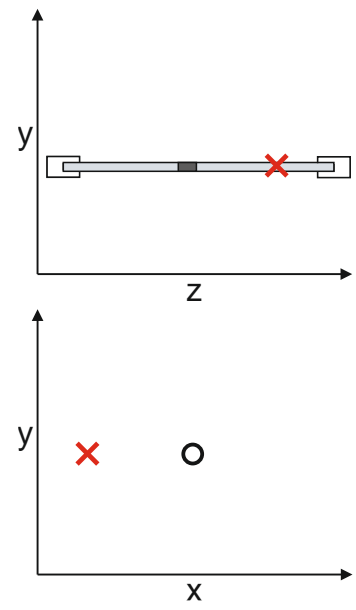

(a)

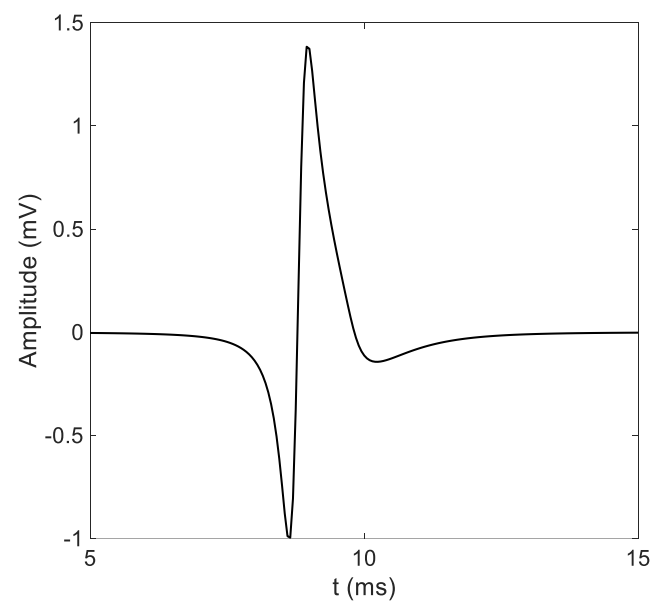

(b)

Fig. 3-3. (a) Schematic representation of the single-point recording for a disposition of one fiber. The recording position is represented by a red cross; (b) corresponding reference signal. Note that this is a MUP recorded at single position. 


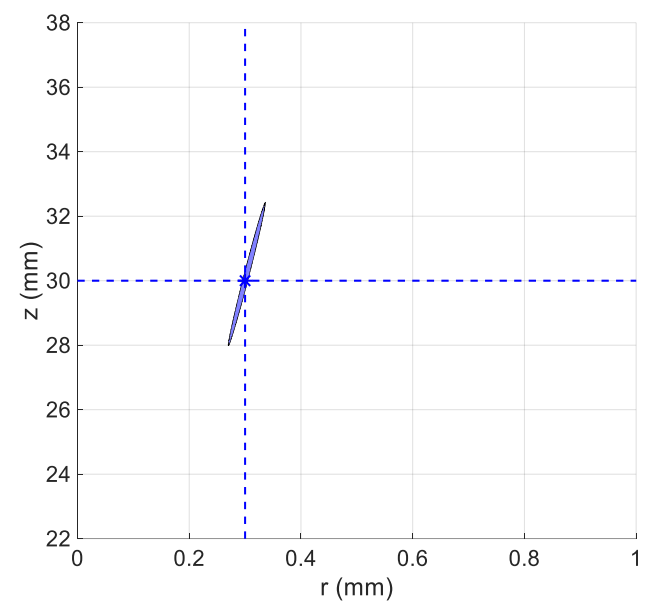

(a)

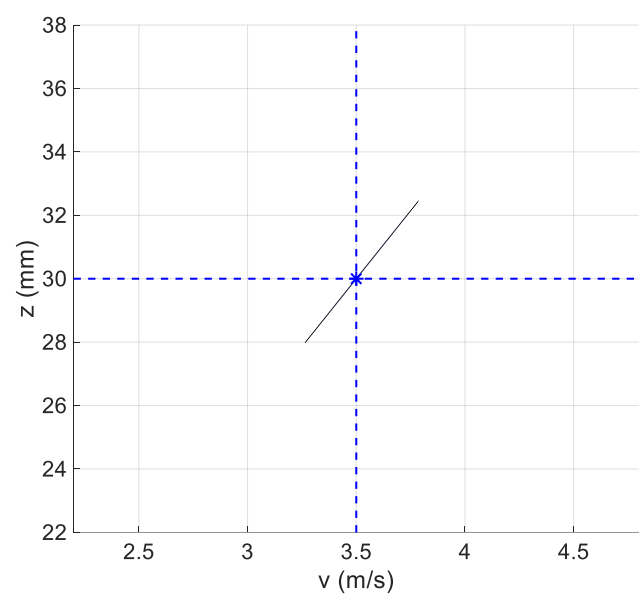

(c)

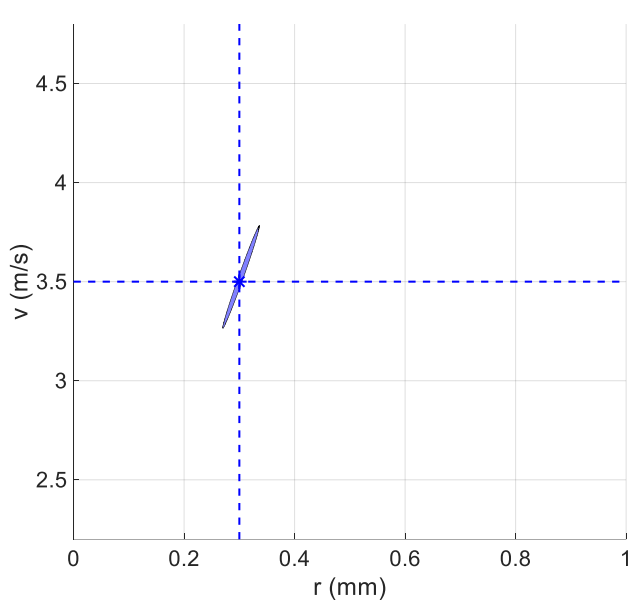

(b)

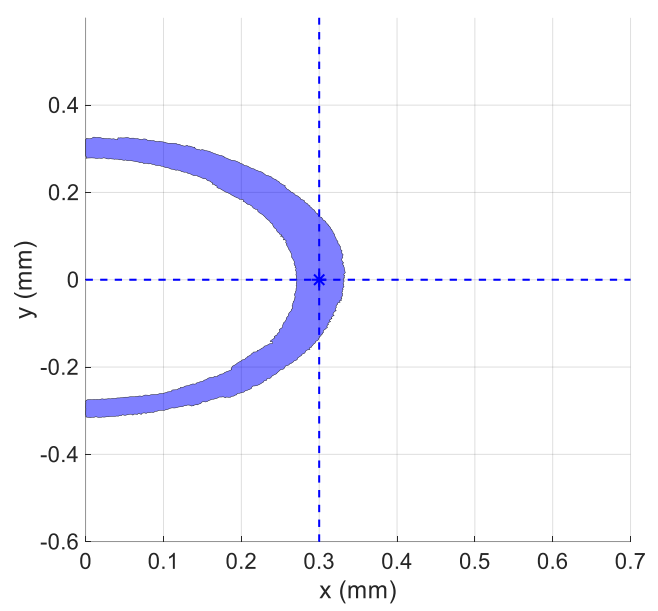

(d)

Fig. 3-4. Dispersion region obtained for the single-point recording configuration and for a single MU fiber. The colored area corresponds to the dispersion region, and the dashed lines and asterisk in which they intersect indicate the reference fiber parameters: (a) projection in the z-r plane; (b) projection in the v-r plane; (c) projection in the z-v plane; (d) projection in the $\mathrm{y}$ $\mathrm{x}$ plane (muscle cross-section).

provide enough information to determine the position $x_{j}$ and the position $y_{j}$ of the MU fiber. Hence, only the radial distance between the fiber and the recording point can potentially be determined

$$
r_{j}=\sqrt{\left(x_{j}-x_{e}\right)^{2}+\left(y_{j}-y_{e}\right)^{2}}
$$


Note that, when a single recording position is considered, the problem has radial symmetry $[67,140]$. If the fiber position is varied so that the radial distance remains constant, the simulated signal waveform will remain unaltered.

Observing the other projections of the parameter space, it can be noticed that the dispersion region also extendeds along the radial distance axis. The same effect occurs with the other two fiber parameters, i.e., with the MFCV, $v_{j}$, and with the neuromuscular junction position, $z_{j}$. In fact, the dispersion of these three parameters is linearly interrelated. Note that the dispersion region extends along a straight line in the $z-r$ projection, in the v-r projection, and in the z-v projection. This implies that the SFAP waveform barely changes if the parameters $r_{j}, v_{j}$ and $z_{j}$, are simulataneously varied so as to stay along this straight line. The rationale of this triple dependence is the following. An increase in the longitudinal distance between the electrode and the neuromuscular junction position, $z_{j}$, implies an increase in the temporal delay of the simulated signal. This increase in the delay can be compensated by increasing the MFCV, $v_{j}$. However, this implies an increase in the signal amplitude which, in turn, can be compensated by increasing the radial distance, $r_{j}$. The result is an SFAP waveform almost identical to the original one. Therefore, it can be concluded that when a single recording position is considered, the parameters $r_{j}, z_{j}$ and $v_{j}$ will be difficult to estimate accurately, even in this simple case, in which there is only a single fiber. This difficulty to estimate the fiber parameters is in agreement with what has already been discussed in previous publications [45, 101, 102] (see details in section 1.3.5).

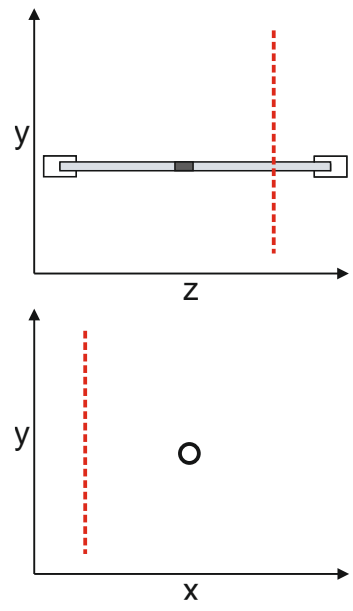

(a)

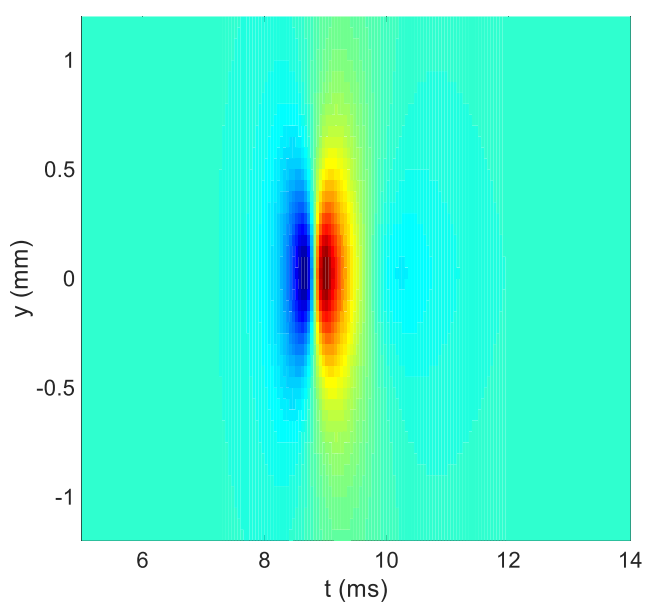

(b)

Fig. 3-5 (a) Schematic representation of the standard scanning-EMG recording for a disposition of one fiber. The recording positions along the corridor are represented by a red dashed line; (b) corresponding reference signal. Note that this is a scanning-EMG signal. 


\section{Standard scanning-EMG recording}

Taking into account the behavior of the dispersion region observed in the previous section, it is clear that the use of a single recording position is not sufficient to correctly estimate the fiber parameters. This issue motivates the use of recordings made at multiple positions. Fig. 3-6 shows the dispersion region obtained for the standard scanning-EMG recording configuration. Note that, in the muscle cross-section projection (Fig. 3-6 (d)), the dispersion region no longer extends over a circular crown,

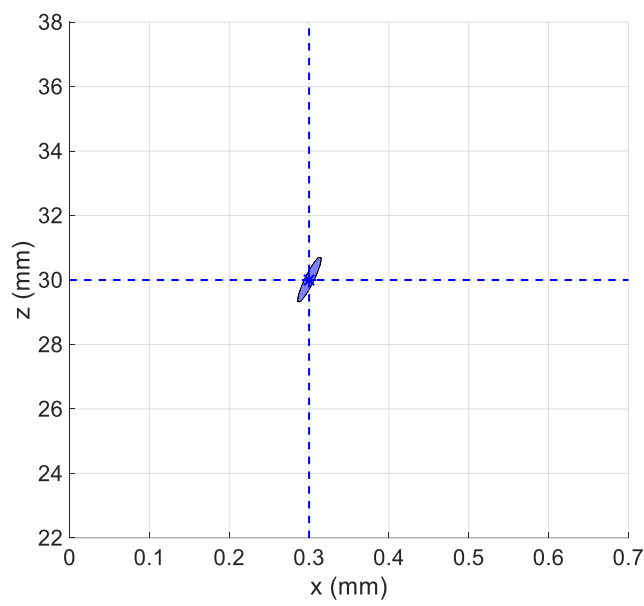

(a)

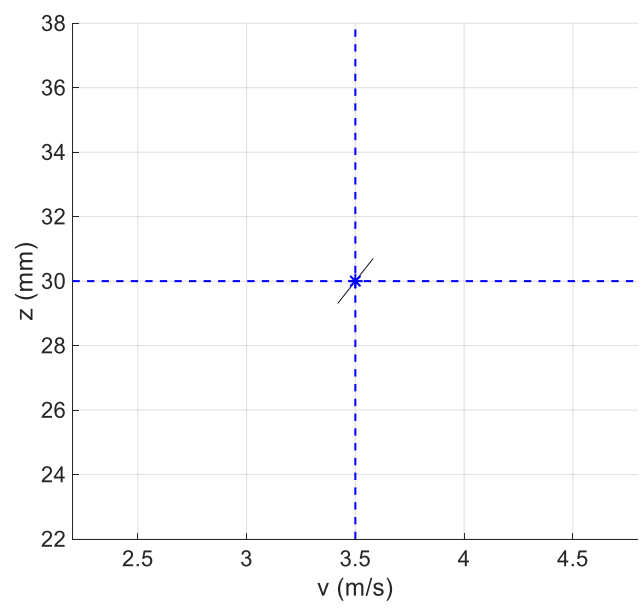

(c)

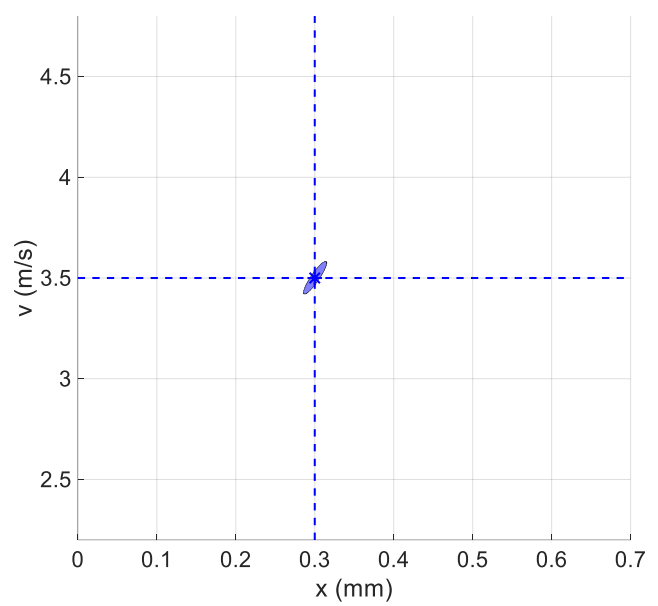

(b)

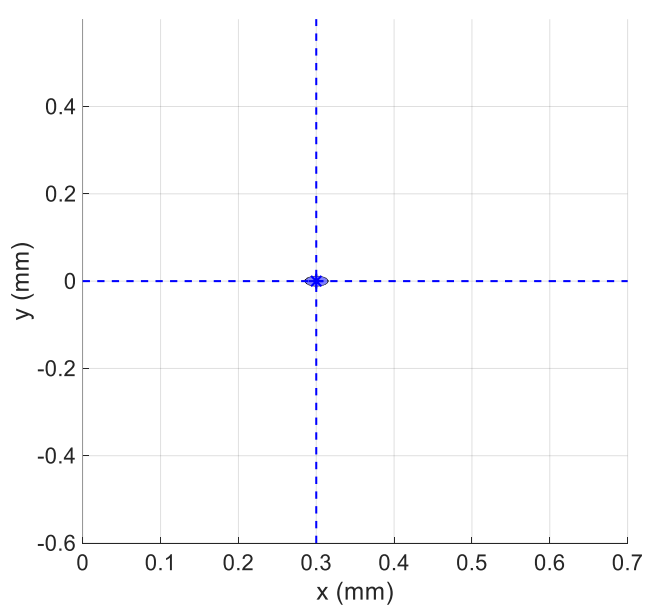

(d)

Fig. 3-6. Dispersion region obtained for the standard scanning-EMG recording configuration, and for a single MU fiber. The colored area corresponds to the dispersion region, and the dashed lines and asterisk in which they intersect indicate the reference fiber parameters: (a) projection in the $\mathrm{z}-\mathrm{x}$ plane; (b) projection in the $\mathrm{v}-\mathrm{x}$ plane; (c) projection in the $\mathrm{z}-\mathrm{v}$ plane; (d) projection in the $y$-x plane (muscle cross-section). 
but it is bounded in a small convex region around the reference parameter position. As the scanning-EMG involves the recording at multiple positions along the $y$-axis, the radial symmetry is broken; hence, it is now possible to determine the fiber position ( $x_{j}$, $\left.y_{j}\right)$. Furthermore, the dispersion of the fiber parameters in the remaining projections is also reduced, and therefore, the potential ability to accurately estimate the parameters $v_{j}$ and $z_{j}$ is increased with respect to the single-point recording configuration.

\section{Two-needle scanning-EMG recording}

Fig. 3-8 shows the dispersion region obtained for the two-needle scanning-EMG recording configuration. It can be observed that the dispersion region has been reduced when compared to that of the standard scanning-EMG. This is evidenced in all the projections of the parameter space. The main explanation of this effect is that this configuration provides much more information than the standard scanning-EMG, due to the inclusion of an additional needle electrode. As both needles are separated by a certain distance in the longitudinal direction (the z-axis) (Fig. 3-7 (a)), the scanningEMG signals recorded with each needle have different propagation delays (Fig. 3-7 (b)). This helps to reduce the degrees of freedom of the valid combinations of those fiber parameters that determine the temporal delay: the MFCV, $v_{j}$, and the neuromuscular junction position, $z_{j}$. Furthermore, both needles are also separated by a certain distance in the horizontal direction perpendicular both to the fibers and the

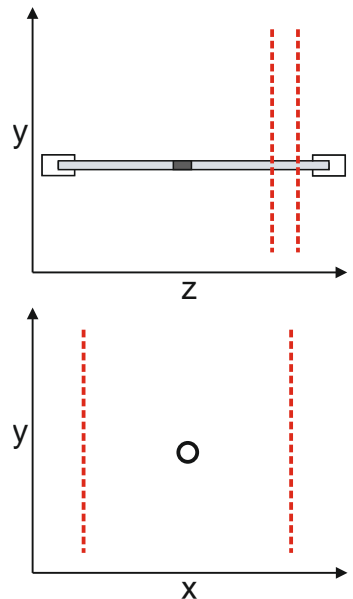

(a)

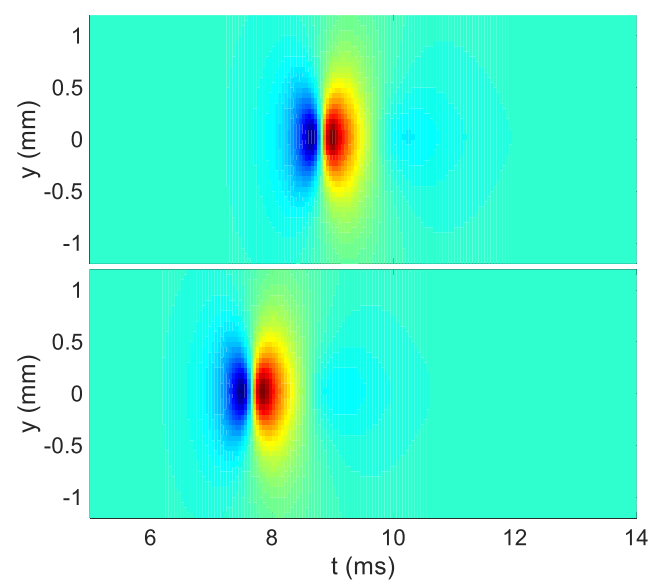

(b)

Fig. 3-7 (a) Schematic representation of the two-needle scanning-EMG recording for a disposition of one fiber. The recording positions along the two corridors (each corridor associated to one needle) are represented by two red dashed lines; (b) corresponding reference signal. Note that this is a double scanning-EMG signal, where each signal corresponds to one needle. 


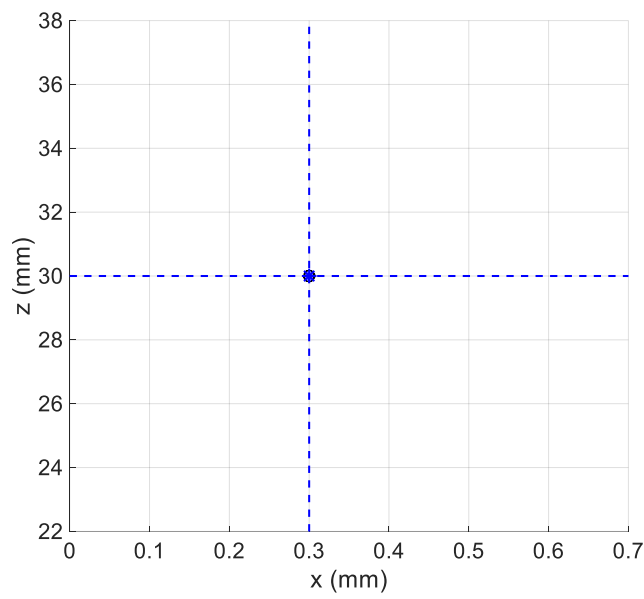

(a)

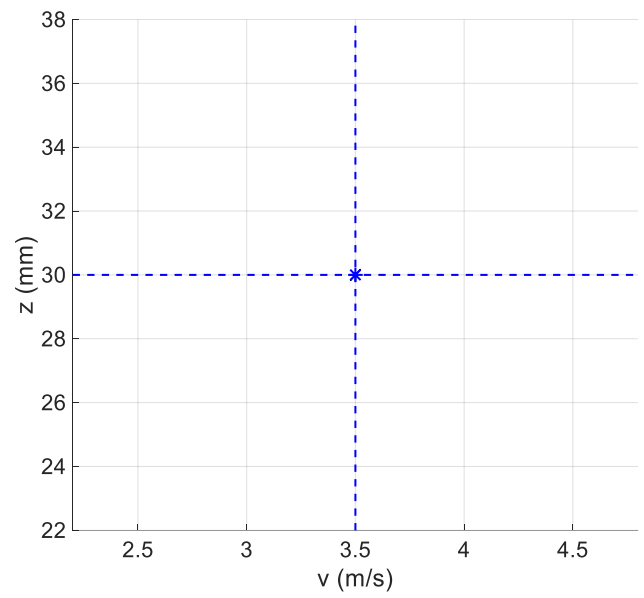

(a)

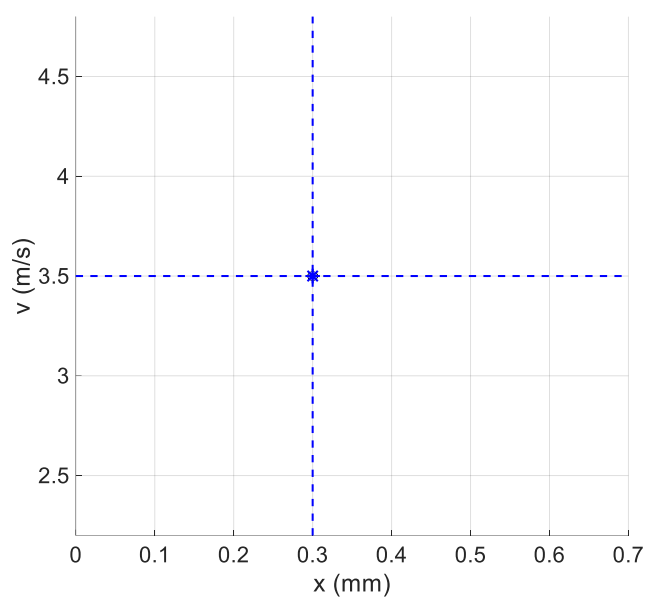

(b)

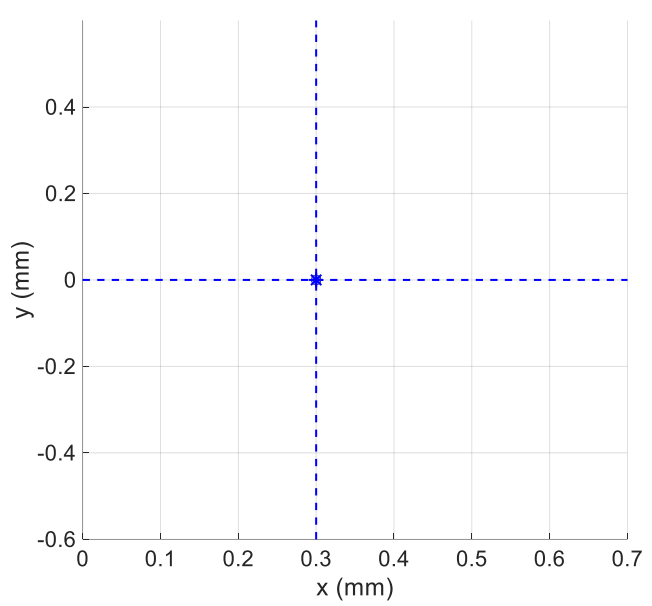

(b)

Fig. 3-8. Dispersion region obtained for the two-needle scanning-EMG recording configuration, and for a single MU fiber. The colored area corresponds to the dispersion region, and the dashed lines and asterisk in which they intersect indicate the reference fiber parameters: (a) projection in the $\mathrm{z}-\mathrm{x}$ plane; (b) projection in the $\mathrm{V}-\mathrm{x}$ plane; (c) projection in the $\mathrm{z}-\mathrm{v}$ plane; (d) projection in the $y$-x plane (muscle cross-section).

scanning corridor (the x-axis) (Fig. 3-7 (a)), which means that the scanning-EMG signals corresponding to each needle are recorded at different distances from the fiber, even if the needles are at the same depth. This provides information about the spatial evolution of the amplitude (usually denoted as radial decline [83]), which helps to reduce the degrees of freedom of the valid combinations of those fiber parameters that determine the amplitude: the MFCV, $v_{j}$, and the fiber position, $x_{j}$. This constraint effect can be understood as follows. A change in the position $x_{j}$ means that the fiber is 
moved away from one of the needles, and gets closer to the other one, which causes the amplitude of the scanning-EMG signal corresponding to the former needle to increase, and that of the latter to decrease. However, a change in $v_{j}$ will cause a simultaneous increase or decrease of the amplitude in both scanning-EMG signals. Therefore, it is no longer possible to properly compensate the effect of a change in $x_{j}$ with a change in $v_{j}$.

\subsubsection{Analysis of the dispersion region of two MU fibers}

The dispersion region has been analyzed, so far, in the case of a single muscle fiber. When considering the presence of more than one fiber, new sources of parameter dispersion arise. This is especially critical when the SFAP contributions of the fibers are superimposed because they lie in the same temporal lapse. In this case, many different fiber combinations may result in a very similar waveform. It is therefore important to study the dispersion regions when more than one fiber is considered. In order to do this, different arrangements composed by two MU fibers have been studied. These arrangements differ in the specific parameters of each fiber.

An important consideration to keep in mind is that now, each point of the parameter space is formed by the parameters of two fibers. The same occurs with the reference parameters, $\boldsymbol{M}=\left\{\boldsymbol{m}_{j}\right\} ; 1 \leq j \leq 2$. To adequately represent the dispersion

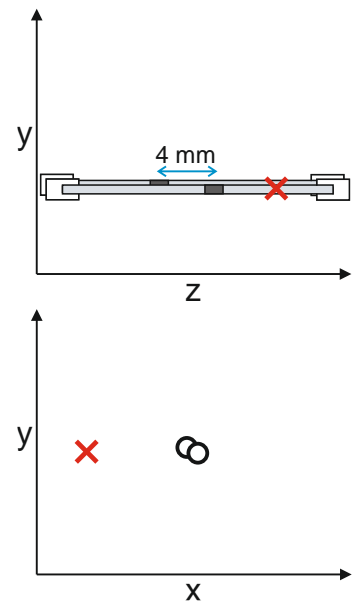

(a)

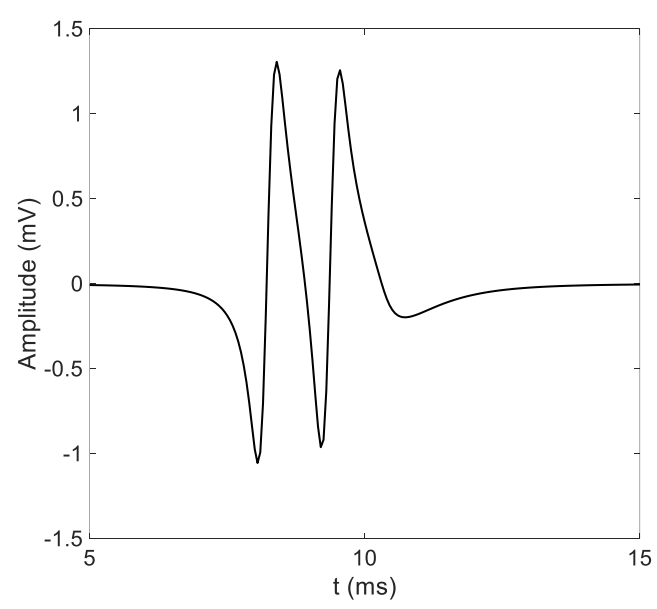

(b)

Fig. 3-9 (a) Schematic representation of the single-point recording for a disposition of two fibers, in which there is a separation of $4 \mathrm{~mm}$ between their neuromuscular junctions. The recording position is represented by a red cross; (b) corresponding reference signal. Note that this is a MUP recorded at single position. 


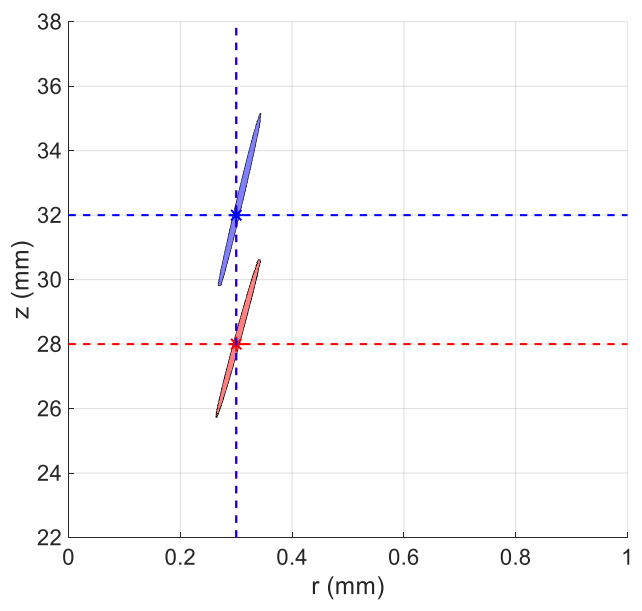

(a)

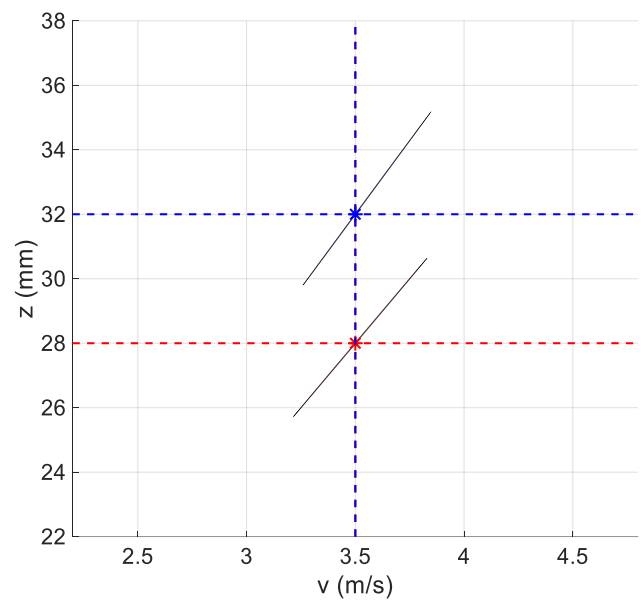

(c)

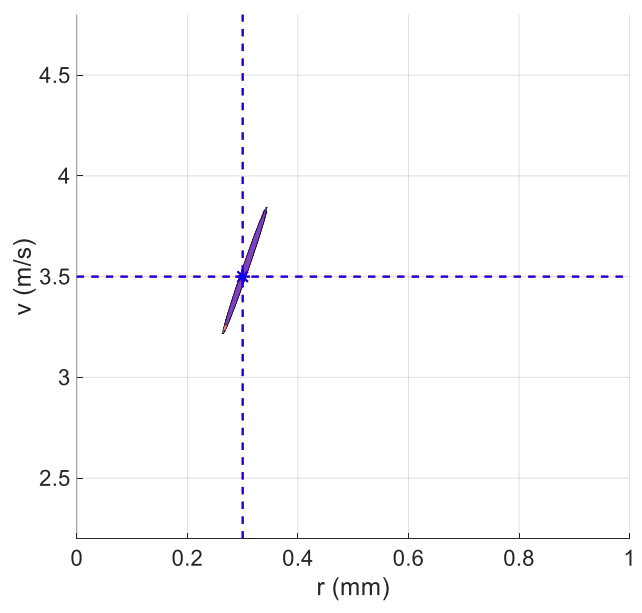

(b)

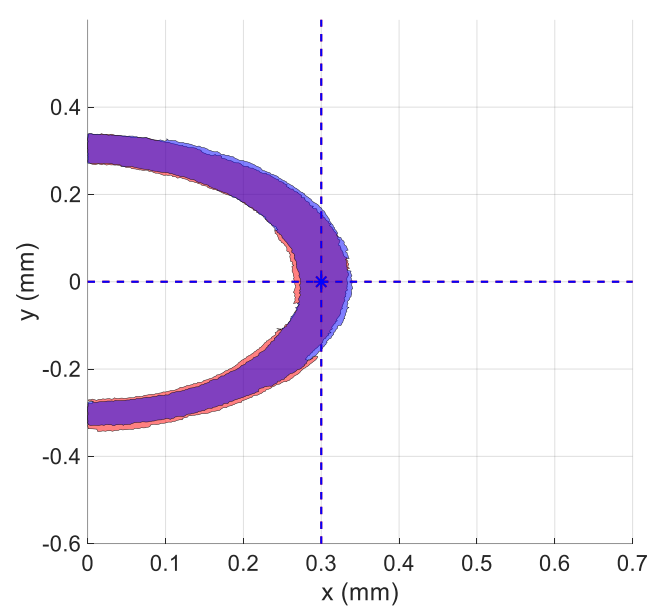

(d)

Fig. 3-10. Dispersion region obtained for the single-point recording configuration, and for two fibers with SFAPs temporally separated. The colored area corresponds to the dispersion region, and the dashed lines and asterisk in which they intersect indicates the reference fiber parameters. The red color is corresponded with one fiber, and the blue one with the other: (a) projection in the z-r plane; (b) projection in the v-r plane; (c) projection in the z-v plane; (d) projection in the $\mathrm{y}-\mathrm{x}$ plane (muscle cross-section).

regions, it is necessary to match the candidate fibers with the reference fibers. The matching procedure follows a waveform similarity criterion. The individual signal contribution (i.e., the SFAP) of each reference and candidate fiber is calculated. Then, the discrepancies between each reference fiber SFAP and each candidate fiber SFAP are quantified by computing the error function given by (3-6). The reference-candidate fibers are matched so as to minimize the computed error. A formal description of the fiber matching, generalized for an arbitrary number of fibers, can be found in section 3.4.1. 


\section{Temporally separated SFAPs and single-point recording}

In this case the dispersion region has been calculated for a single-point recording configuration, and for a reference fiber arrangement consisting of two fibers with the same parameters, except for a separation of $4 \mathrm{~mm}$ between the neuromuscular junctions of each fiber (Fig. 3-9 (a)). This ensures that the SFAPs of the fibers are not superimposed in time (Fig. 3-9 (b)):

$$
\begin{aligned}
& \boldsymbol{m}_{1} \rightarrow x_{1}=0.3 \mathrm{~mm} ; y_{1}=0 \mathrm{~mm} ; z_{1}=28 \mathrm{~mm} ; v_{1}=3.5 \mathrm{~m} / \mathrm{s} \\
& \boldsymbol{m}_{2} \rightarrow x_{2}=0.3 \mathrm{~mm} ; y_{2}=0 \mathrm{~mm} ; z_{2}=32 \mathrm{~mm} ; v_{2}=3.5 \mathrm{~m} / \mathrm{s}
\end{aligned}
$$

The dispersion region obtained in this case is depicted in Fig. 3-10. The results are very similar to the single fiber case (Fig. 3-4), but extended to both fibers. The same compensation effects are observed for this case. Therefore, the single-point recording presents, when it comes to the determine the MU fiber parameters, the same limitations for this fiber arrangement than for the single fiber case: an indetermination of the $x_{j}$ and $y_{j}$ parameters, and a low accuracy when determining $r_{j}, z_{j}$ and $v_{j}$, due to a triple linear dependency.

\section{Temporally separated SFAPs and standard scanning-EMG recording}

In this case, the dispersion region has been calculated for the standard scanningEMG recording configuration, and for the same reference fiber arrangement than in the previous case (Fig. 3-11 (a)), which ensures that the SFAPs of the fibers are not superimposed in time (Fig. 3-11 (b)).

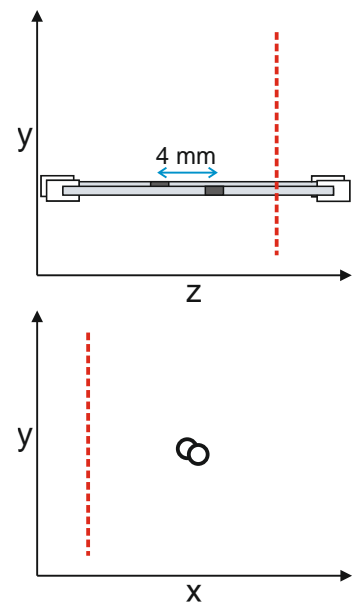

(a)

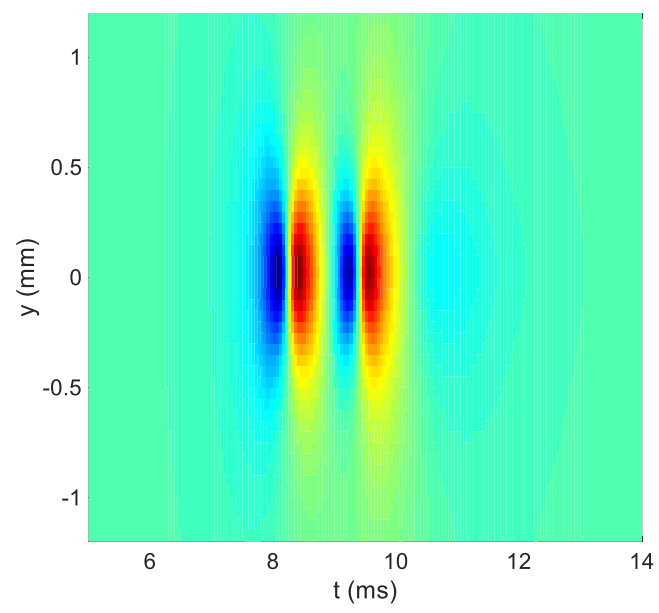

(b)

Fig. 3-11 (a) Schematic representation of the standard scanning-EMG recording for a disposition of two fibers, in which there is a separation of $4 \mathrm{~mm}$ between their neuromuscular junctions. The recording positions along the corridor are represented by a red dashed line; (b) corresponding reference signal. Note that this is a scanning-EMG signal. 
The dispersion region obtained in this case is shown in Fig. 3-12. Note that the dispersion is similar to that of the single fiber case for the same recording configuration, i.e., for the standard scanning-EMG (Fig. 3-6), but extended to both fibers. Since the SFAPs of each fiber are sufficiently separated in time, the dispersion in the parameters corresponding to each fiber is not influenced by the presence of the other fiber. Hence, the dispersion region is relatively small.

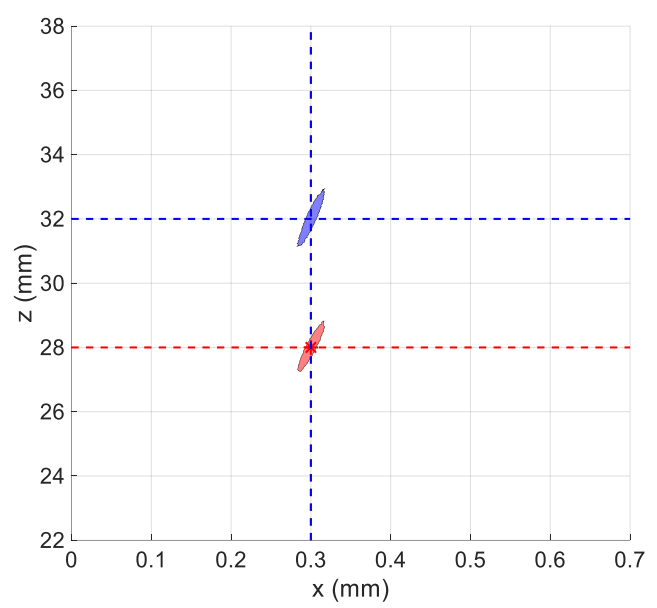

(a)

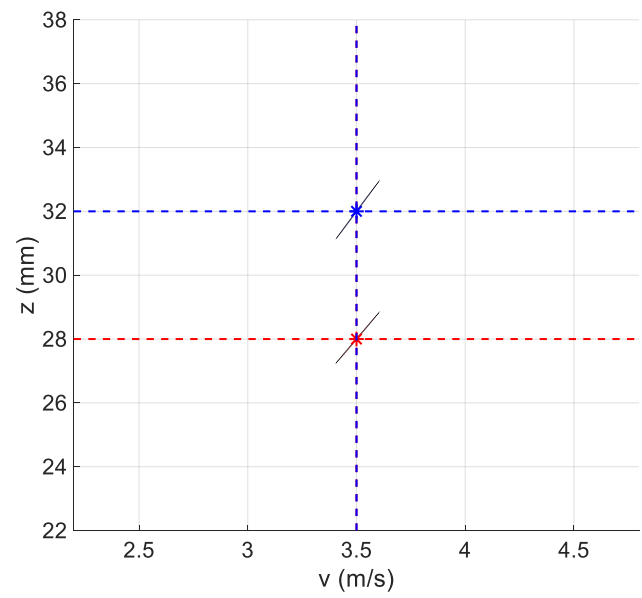

(c)

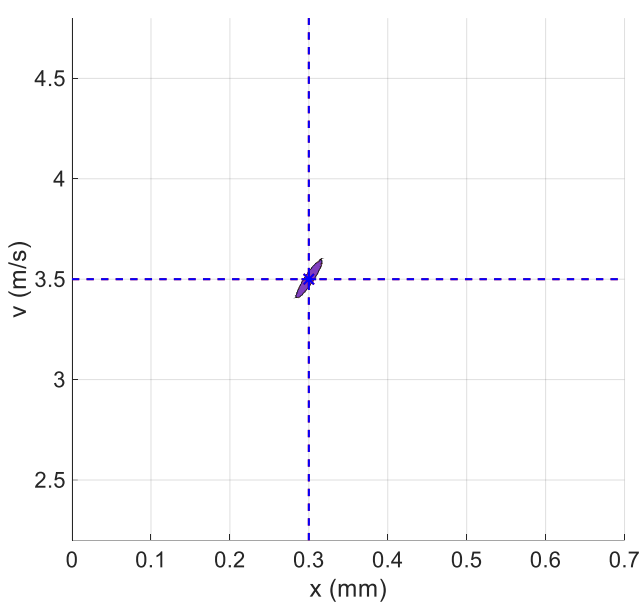

(b)

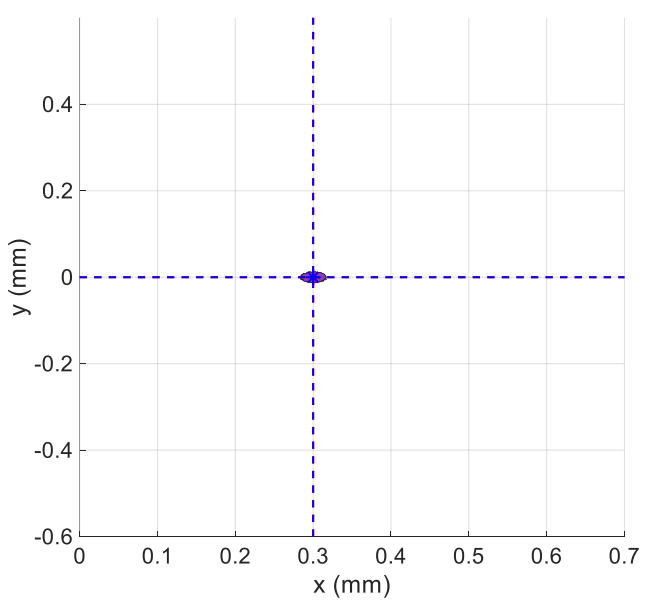

(d)

Fig. 3-12. Dispersion region obtained for the standard scanning-EMG recording configuration, and for two fibers with SFAPs separated in time. The colored area corresponds to the dispersion region, and the dashed lines and asterisk in which they intersect indicate the reference fiber parameters. The red color is corresponded with one fiber, and the blue one with the other: (a) projection in the $\mathrm{z}-\mathrm{x}$ plane; (b) projection in the $\mathrm{v}-\mathrm{x}$ plane; (c) projection in the $\mathrm{z}-\mathrm{v}$ plane; (d) projection in the $\mathrm{y}$-x plane (muscle cross-section). 


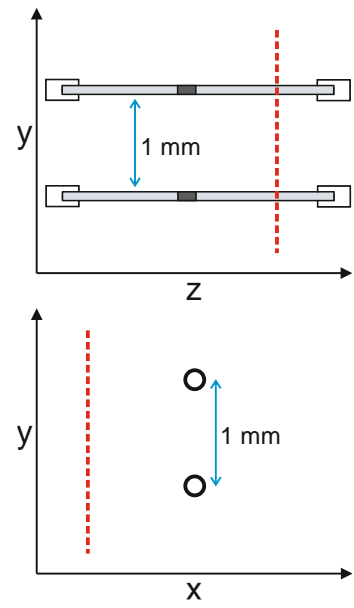

(a)

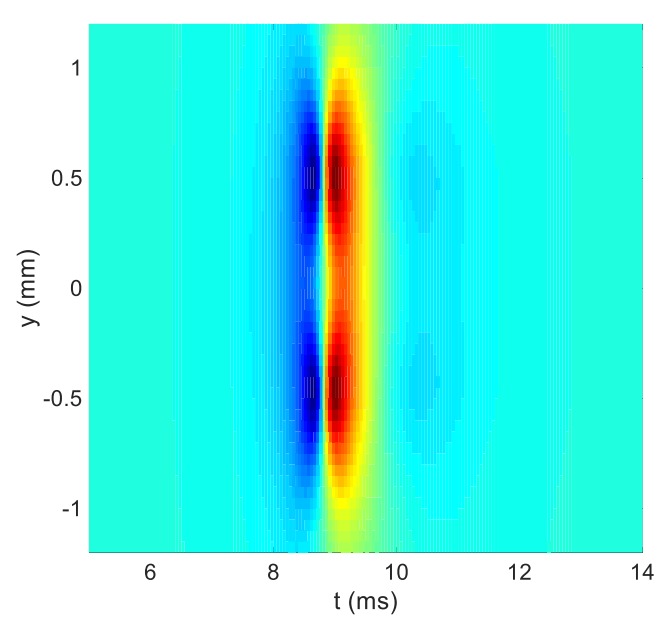

(b)

Fig. 3-13 (a) Schematic representation of the standard scanning-EMG recording for a disposition of two fibers separated $1 \mathrm{~mm}$ in the y-axis. The recording positions along the corridor are represented by a red dashed line; (b) corresponding reference signal. Note that this is a scanning-EMG signal.

\section{Spatially separated SFAPs and standard scanning-EMG recording}

In this case, the dispersion region has been calculated again, for the standard scanning-EMG recording configuration, but now for a new reference fiber arrangement consisting of two fibers separated $1 \mathrm{~mm}$ in the y-axis (Fig. 3-13 (a)). For this fiber arrangement, although the fiber SFAPs are superimposed in time, they are not in the spatial dimension (Fig. 3-13 (b)):

$$
\begin{aligned}
& \boldsymbol{m}_{1} \rightarrow x_{1}=0.3 \mathrm{~mm} ; y_{1}=0.5 \mathrm{~mm} ; z_{1}=30 \mathrm{~mm} ; \text { and } v_{1}=3.5 \mathrm{~m} / \mathrm{s} \\
& \boldsymbol{m}_{2} \rightarrow x_{2}=0.3 \mathrm{~mm} ; y_{2}=-0.5 \mathrm{~mm} ; z_{2}=30 \mathrm{~mm} ; \text { and } v_{2}=3.5 \mathrm{~m} / \mathrm{s}
\end{aligned}
$$

The dispersion region obtained in this case is shown in Fig. 3-14. Note that, the area of the dispersion region projections has increased with respect to the case in which the SFAPs are separated in time (not overlapping). This is so, because in this case the SFAPs are not entirely separated in the spatial dimension (Fig. 3-13 (b)), which results in the appearance of additional combinations of fiber parameters that give rise to similar signal waveforms. The dispersion region suggests that, when the fibers are separated in the y-axis, the scanning-EMG recording configuration can potentially estimate the fiber parameters, although the precision will depend on how much spatially separated the fibers are; i.e., the degree of superposition of the SFAPs determine the ability to make a proper estimation. 


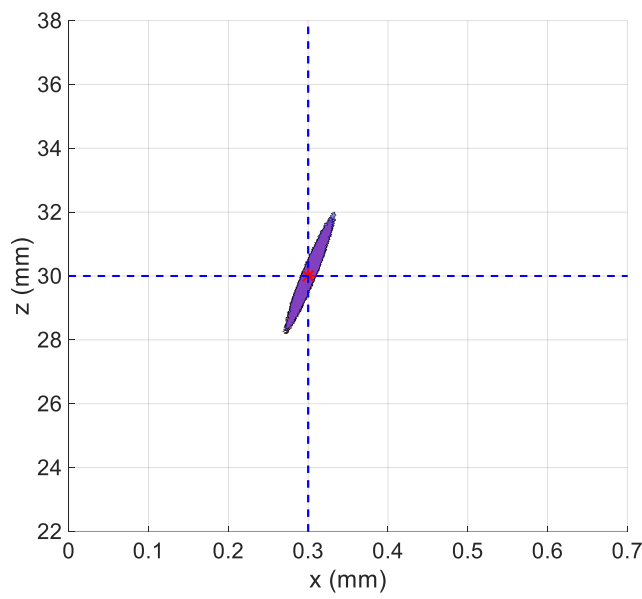

(a)

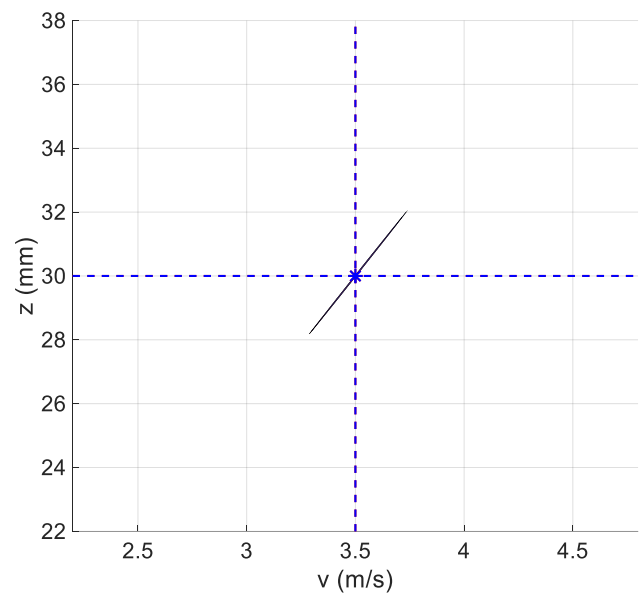

(c)

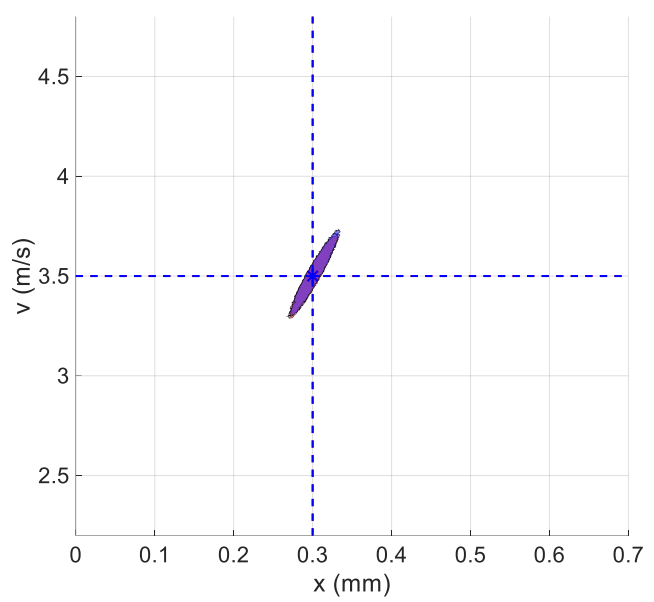

(b)

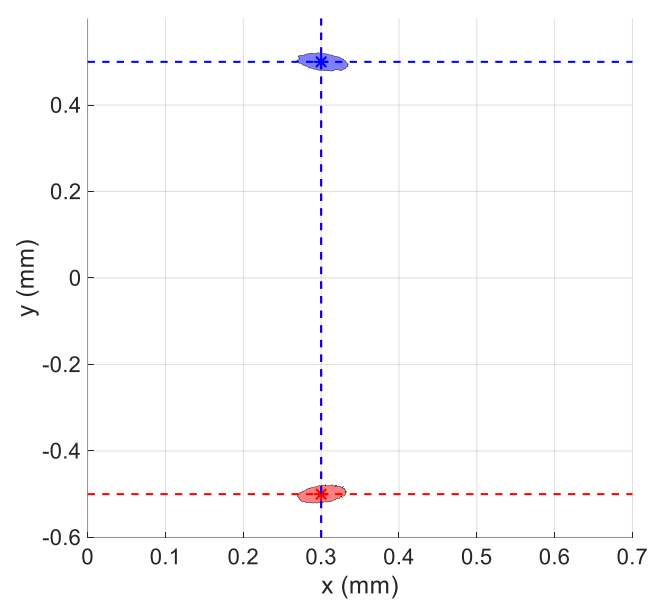

(d)

Fig. 3-14. Dispersion region obtained for the standard scanning-EMG recording configuration, and for two fibers with SFAPs separated in the spatial (y-axis) dimension. The colored area corresponds to the dispersion region, and the dashed lines and asterisk in which they intersect indicate the reference fiber parameters. The red color is corresponded with one fiber, and the blue one with the other: (a) projection in the z-x plane; (b) projection in the v-x plane; (c) projection in the $\mathrm{z}-\mathrm{v}$ plane; (d) projection in the $\mathrm{y}-\mathrm{x}$ plane (muscle cross-section).

\section{Superimposed SFAPs and single-point recording}

In this case the dispersion region has been calculated for a single-point recording configuration, and for a reference fiber arrangement consisting on the two fibers with the same parameters, except for a separation of $0.1 \mathrm{~mm}$ between both fibers in the $\mathrm{x}$ axis (Fig. 3-15 (a)). This ensures that the SFAP of each fiber falls in the same time 
region (Fig. 3-15 (b)). Note that a change in the position along the $\mathrm{x}$-axis only affects to the signal amplitude, and not to the delay:

$\boldsymbol{m}_{1} \rightarrow x_{1}=0.25 \mathrm{~mm} ; y_{1}=0 \mathrm{~mm} ; z_{1}=30 \mathrm{~mm} ;$ and $v_{1}=3.5 \mathrm{~m} / \mathrm{s}$
$\boldsymbol{m}_{2} \rightarrow x_{2}=0.35 \mathrm{~mm} ; y_{2}=0 \mathrm{~mm} ; z_{2}=30 \mathrm{~mm} ;$ and $v_{2}=3.5 \mathrm{~m} / \mathrm{s}$

The dispersion region obtained in this case is shown in Fig. 3-16. Note that for this configuration, the dispersion region is extremely wide in all the projections of the parameter space; i.e., the single-point recording configuration loses its ability to estimate any of the MU fiber parameters, as soon as the SFAPs are superimposed in time. This is because many fiber combinations result in the same waveform when they are summed together (Fig. 3-17). This produces a dramatically increased dispersion, when compared to the dispersion regions studied for the case of a single fiber. These results further evidence that the use of a single recording position is not sufficient to correctly determine the MU fiber parameters.

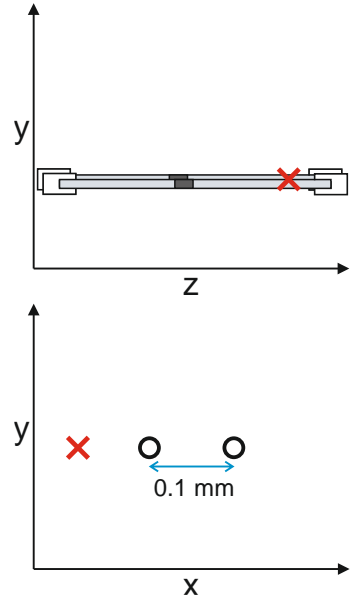

(a)

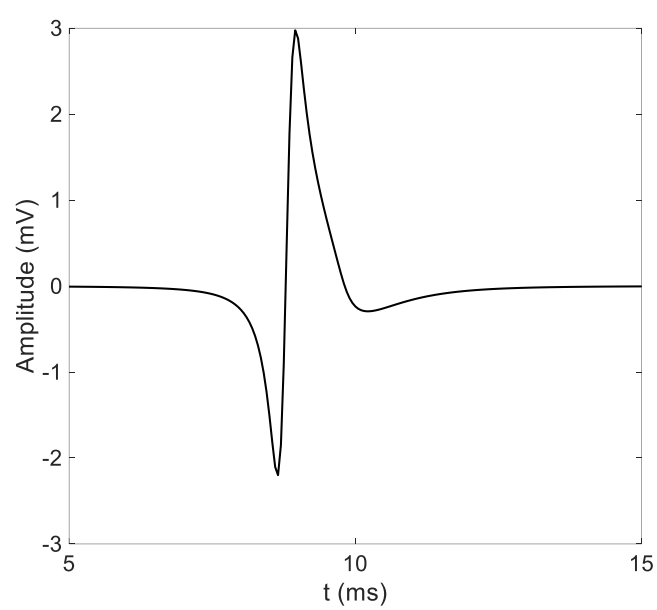

(b)

Fig. 3-15 (a) Schematic representation of the single-point recording for a disposition of two fibers separated $0.1 \mathrm{~mm}$ in the $\mathrm{x}$-axis. The recording position is represented by a red cross; (b) corresponding reference signal. Note that this is a MUP recorded at single position. 


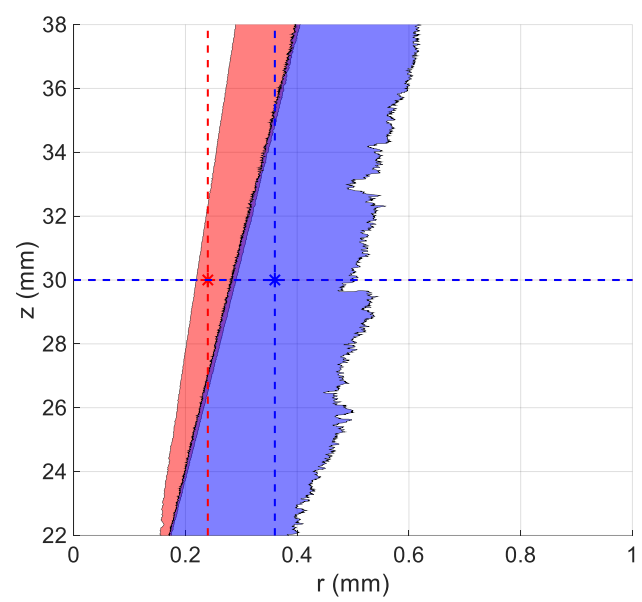

(a)

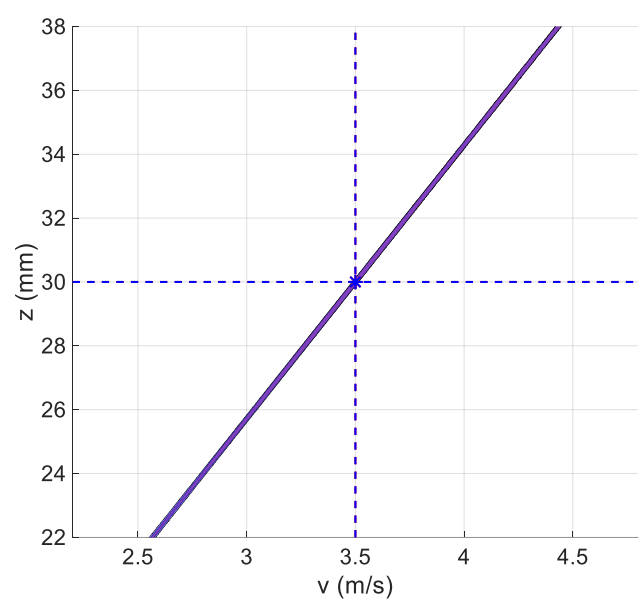

(c)

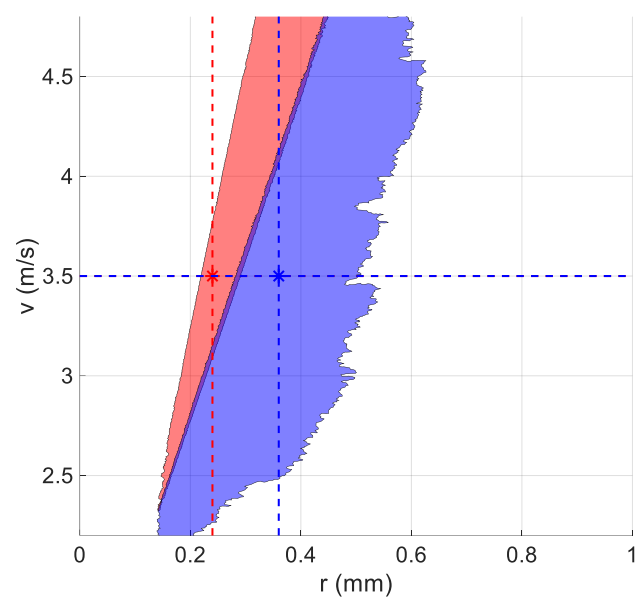

(b)

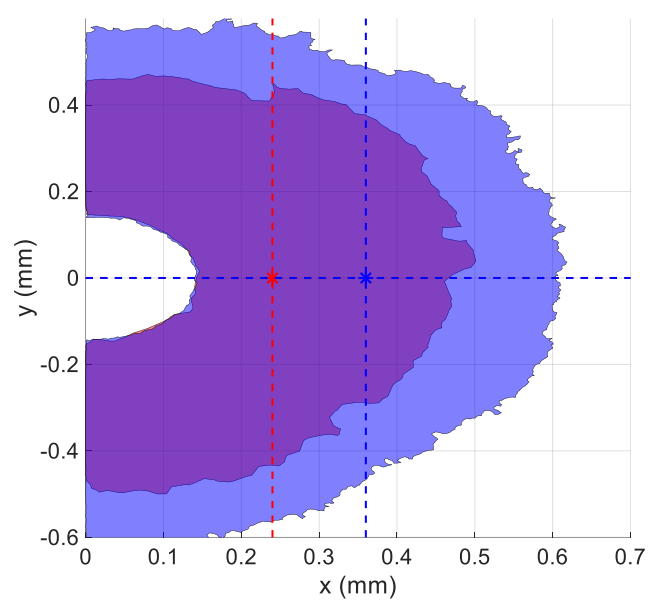

(d)

Fig. 3-16. Dispersion region obtained for the single-point recording configuration, and for two fibers with SFAPs superimposed in the same region. The colored area corresponds to the dispersion region, and the dashed lines and asterisk in which they intersect indicate the reference fiber parameters. The red color is corresponded with one fiber, and the blue one with the other: (a) projection in the z-r plane; (b) projection in the v-r plane; (c) projection in the z$\mathrm{v}$ plane; (d) projection in the $\mathrm{y}$-x plane (muscle cross-section).

\section{Superimposed SFAPs and standard scanning-EMG recording}

In this case the dispersion region has been calculated for the standard scanningEMG recording configuration, and for the same reference fiber arrangement than in the previous case (Fig. 3-18 (a)), which ensures that the SFAPs of the fibers are superimposed both in the time and spatial dimension (Fig. 3-18 (b)). 


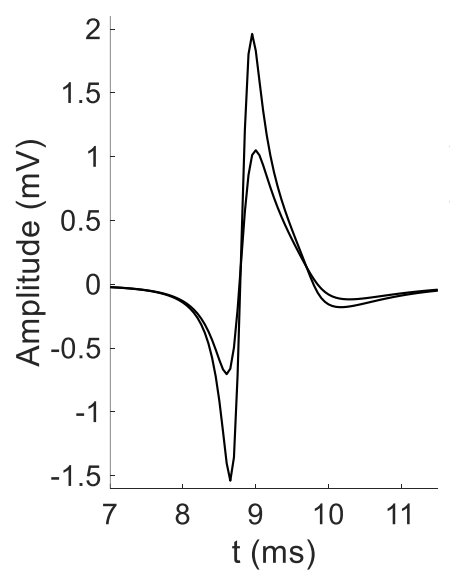

(a)

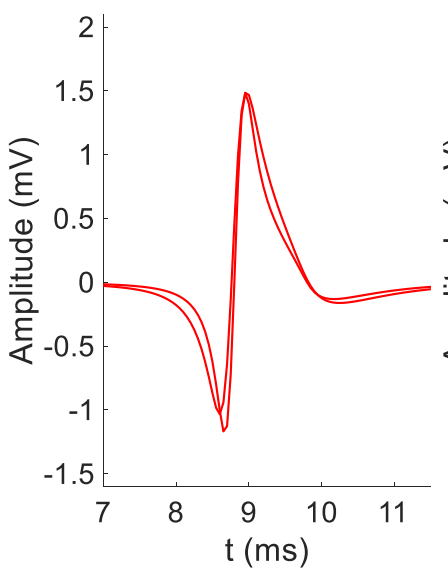

(b)

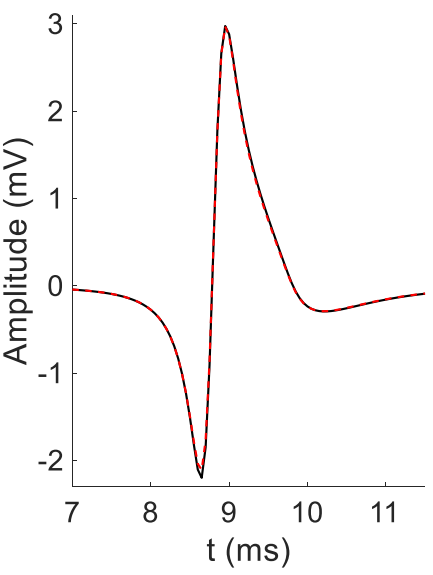

(c)

Fig. 3-17. Simulation example in which the summation of two SFAPs give rise to the same signal waveform, for two completely different fiber parameter combinations: (a) SFAPs corresponding to the first fiber parameters set; (b) SFAPs corresponding to the second fiber parameters set; (c) Resulting waveforms obtained from the summation of the two SFAPs, both for the first (black), and second (red) fiber parameter sets. Note that, although the individual SFAPs of the first and second cases are very different, both give rise to the same waveform.

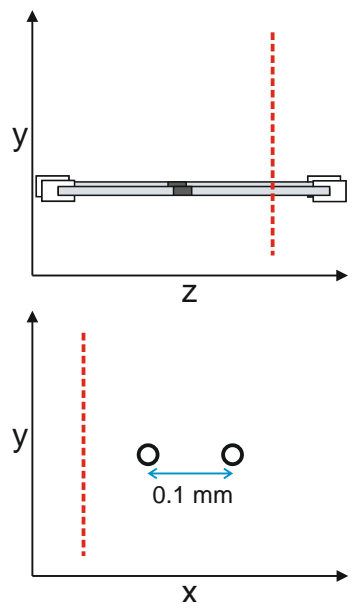

(a)

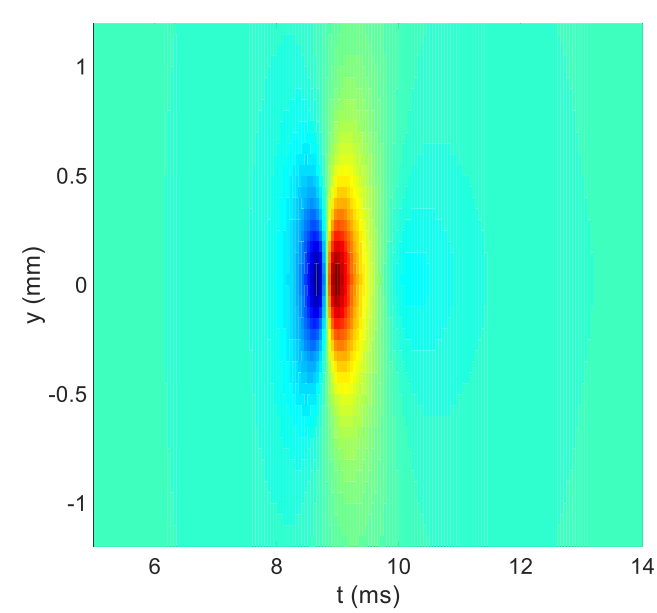

(b)

Fig. 3-18 (a) Schematic representation of the standard scanning-EMG recording for a disposition of two fibers separated $0.1 \mathrm{~mm}$ in the $\mathrm{x}$-axis. The recording positions along the corridor are represented by a red dashed line; (b) corresponding reference signal. Note that this is a scanning-EMG signal.

The dispersion region obtained in this case is shown in Fig. 3-19. Note that for this configuration, the dispersion region is very wide. This means that, when the fiber SFAPs are superimposed in the same space-time region, the scanning-EMG recording 
configuration worsens its ability to estimate the fiber parameters respect to the case where there are no SFAP superimpositions. Nonetheless, there has been an improvement over the single-point recording configuration, as this one presented a significantly larger dispersion (Fig. 3-16). This means that the potential ability to estimate the MU fiber parameters for the scanning-EMG recording is better than for the single-point recording.

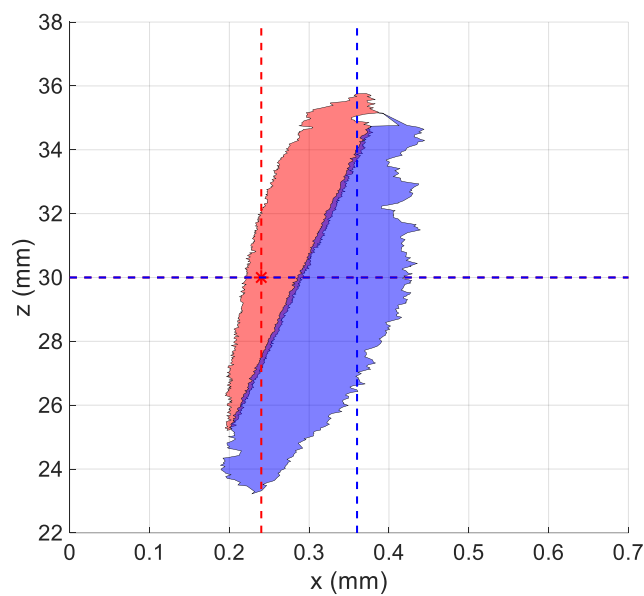

(a)

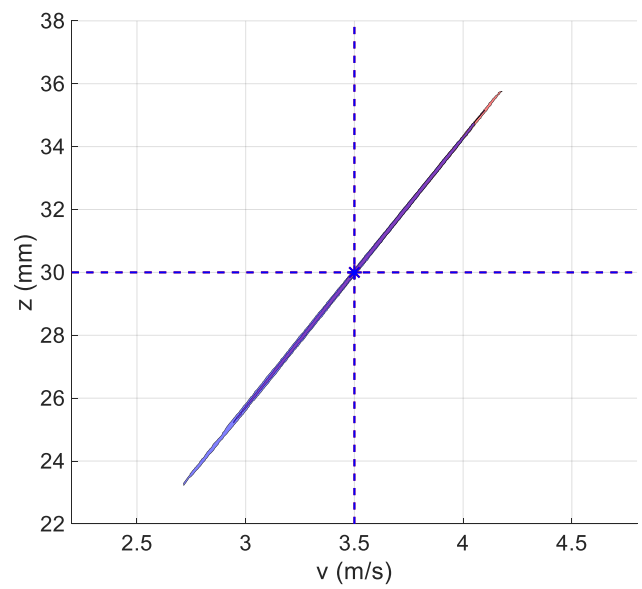

(c)

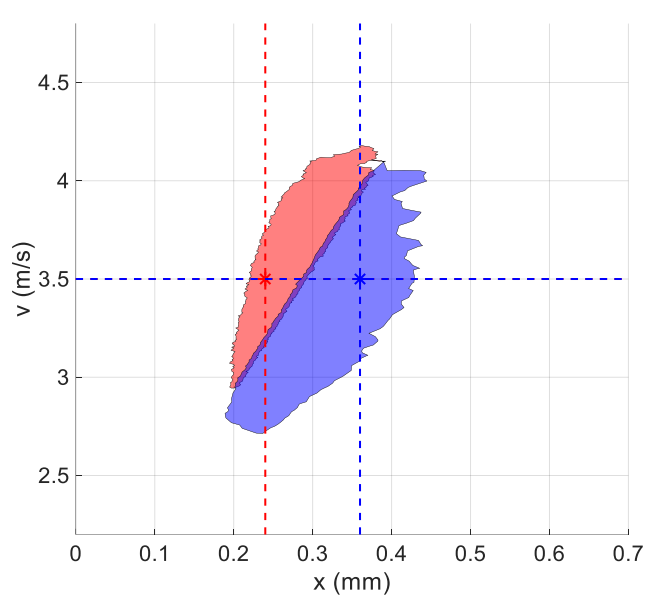

(b)

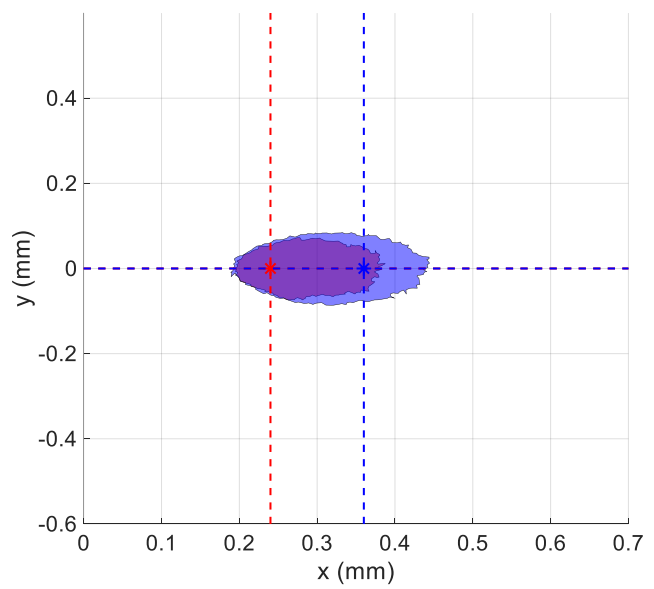

(d)

Fig. 3-19. Dispersion region obtained for the standard scanning-EMG recording configuration, and for two fibers with SFAPs superimposed in the same region. The colored area corresponds to the dispersion region, and the dashed lines and asterisk in which they intersect indicate the reference fiber parameters. The red color is corresponded with one fiber, and the blue one with the other: (a) projection in the z-x plane; (b) projection in the v-x plane; (c) projection in the z$\mathrm{v}$ plane; (d) projection in the $\mathrm{y}-\mathrm{x}$ plane (muscle cross-section). 


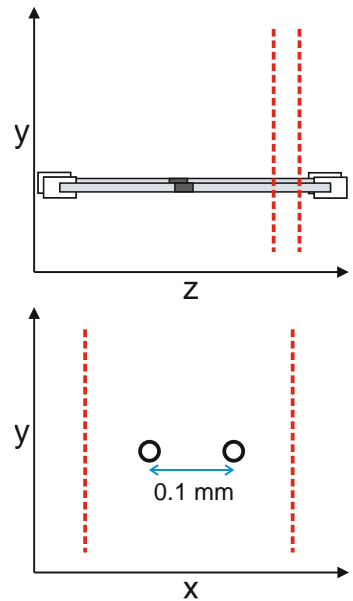

(a)

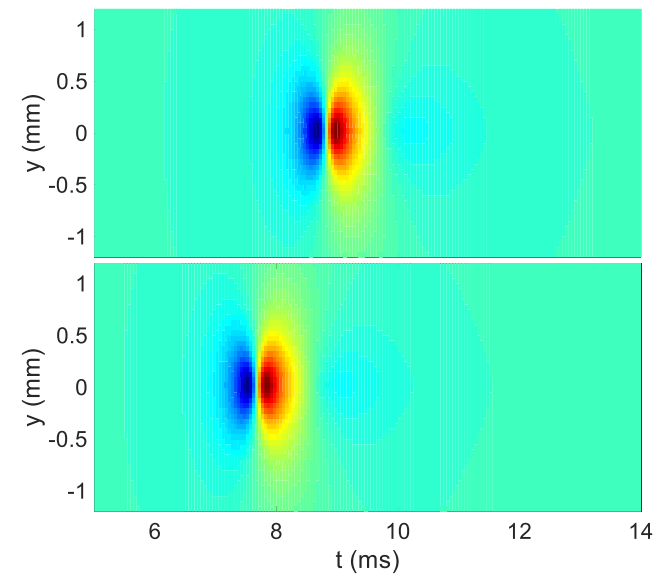

(b)

Fig. 3-20 (a) Schematic representation of the two-needle scanning-EMG recording for a disposition of two fibers separated $0.1 \mathrm{~mm}$ in the $\mathrm{x}$-axis. The recording positions along the two corridors (each corridor associated to one needle) are represented by two red dashed lines; (b) corresponding reference signal. Note that this is a double scanning-EMG signal, where each signal corresponds to one needle.

\section{Superimposed SFAPs and two-needle scanning-EMG recording}

In this last case, the dispersion region has been calculated for the two-needle scanning-EMG recording configuration, and for the same reference fiber arrangement than in the last cases (Fig. 3-20 (a)), which ensures that the SFAPs of the fibers are superimposed both in the time and spatial dimension (Fig. 3-20 (b)).

The dispersion region obtained in this case is shown in Fig. 3-21. Note that this dispersion region spreads over a relatively small region around the reference fiber parameters. This region is noticeably smaller than that obtained for the standard scanning-EMG recording for the same fiber arrangement (Fig. 3-19). Remember that the two-needle configuration records the MUP along two different corridors, which are separated both in the longitudinal (the $\mathrm{z}$-axis) and perpendicular (the $\mathrm{x}$-axis) direction respect to the fibers. As already explained for the one fiber arrangement, the use of an additional needle electrode helps to noticeably reduce the number of parameter combinations lying within the dispersion region. The fact that the dispersion region is relatively small for the present case suggests that the two-needle scanning-EMG could estimate the fiber parameters even in cases in which the fiber SFAPs fall in the same space-time region, something that does not seem possible with the standard scanningEMG, let alone with the single-point recording. 


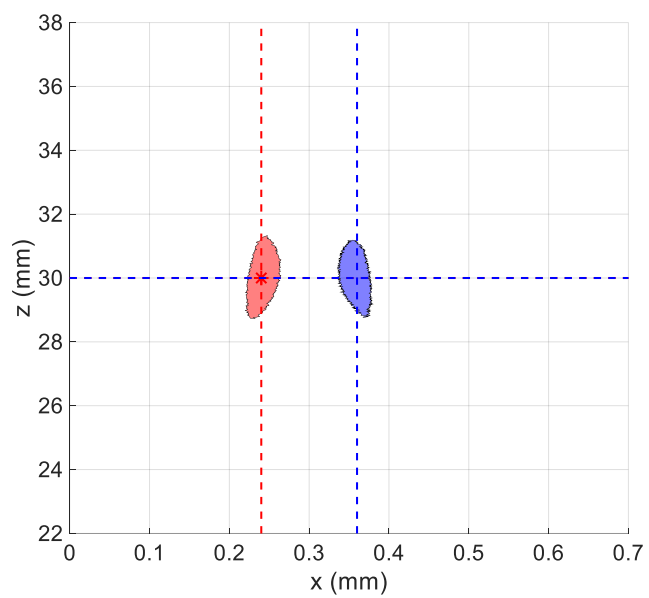

(a)

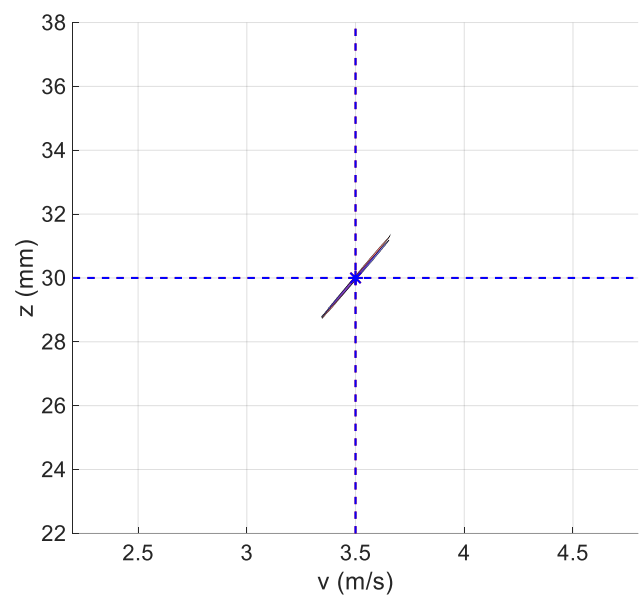

(c)

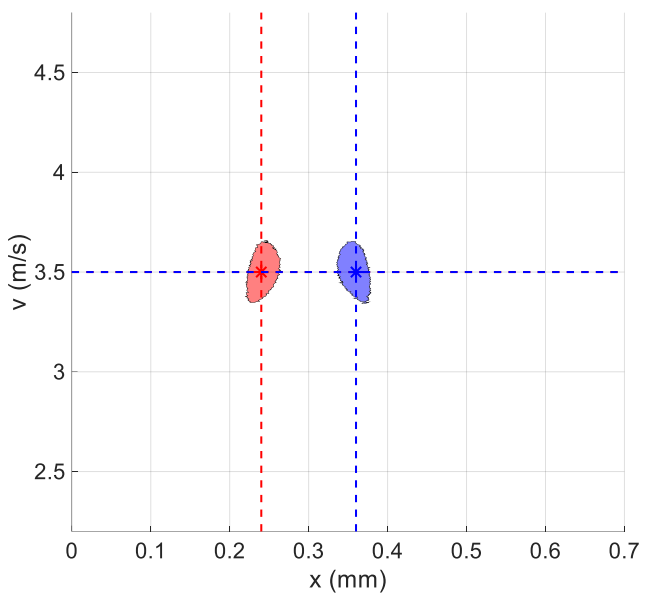

(b)

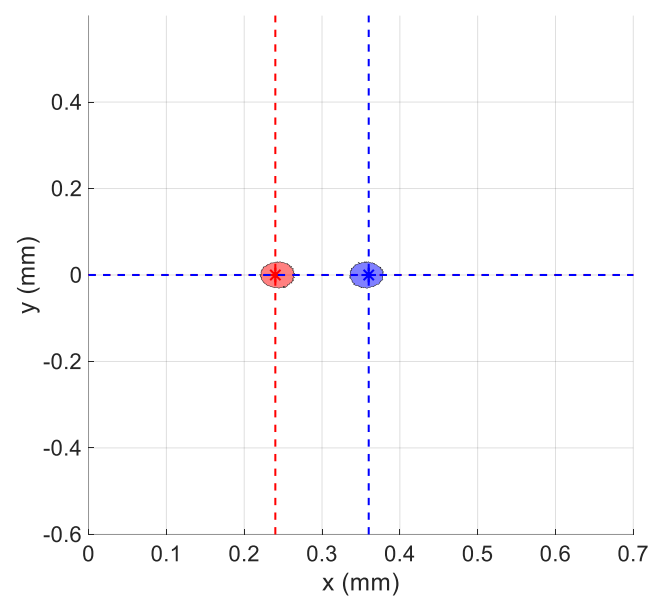

(d)

Fig. 3-21. Dispersion region obtained for the two-needle scanning-EMG recording configuration, and for two fibers with SFAPs superimposed in the same region. The colored area corresponds to the dispersion region, and the dashed lines and asterisk in which they intersect indicates the reference fiber parameters. The red color is corresponded with one fiber, and the blue one with the other: (a) projection in the z-x plane; (b) projection in the v-x plane; (c) projection in the $\mathrm{z}-\mathrm{v}$ plane; (d) projection in the $\mathrm{y}$-x plane (muscle cross-section).

\subsubsection{Summary}

The most relevant conclusions about the dispersion region analysis performed in this section are the following: 
- The larger the superimposition degree between the fiber SFAPs, the wider the dispersion regions, and therefore, the greater the expected difficulty to estimate the parameters.

- The single-point recording configuration presents wide dispersion regions, suggesting that its ability to estimate the MU fiber parameters is very poor.

- The dispersion regions are significantly reduced for the standard scanning-EMG recording configuration. As long as the fiber SFAPs do not fall in the same spacetime region, it seems possible to estimate the MU fiber parameters.

- The dispersion regions are further reduced for the two-needle scanning-EMG recording configuration. This configuration seems able to estimate the MU fiber parameters even in cases in which the different fiber SFAPs fall in the same spacetime region.

This forward problem study has served to analyze the limitations of the different recording configurations for very simple fiber arrangements. With a larger number of fibers, it is expected that new sources of dispersion may appear. Therefore, the conclusions drawn from this study about the potential ability to estimate the MU fiber parameters, although useful for the design of estimation systems, should be taken with caution. 


\subsection{Analysis of the MUP inverse problem}

This section describes the basis of the estimation system developed during this thesis to solve the inverse problem. It should be remembered that the goal of this system is to estimate the MU fiber parameters, i.e., the parameters characterizing each muscle fiber of the MU under study. These parameters are

$$
\boldsymbol{M}=\left\{\boldsymbol{m}_{j}\right\} ; 1 \leq j \leq J
$$

where $J$ is the number of MU fibers, and $\boldsymbol{m}_{j}=\left(x_{j}, y_{j}, z_{j}, v_{j}\right)$ are the parameters of the $j$ th fiber, where $\left(x_{j}, y_{j}\right)$ is the position in the muscle cross-section, $z_{j}$ is the position of the neuromuscular junction, and $v_{j}$ is the MFCV.

Before understanding how the estimation system works, it is necessary to understand the context in which it is applied. Fig. 3-22 shows a block diagram describing this scenario. We will focus on a particular MU of a certain muscle. For our purposes, this MU is completely characterized by a set of parameters, $\boldsymbol{M}$, that is intended to be estimated. In the first step, the electromyographic signals (scanningEMG and surface-MUP) are recorded using the suitable recording setup in the muscle and MU under study, as described in Fig. 3-23. These signals are used as inputs of the estimation system. The output of this system is the set of estimated MU parameters, $\widehat{\boldsymbol{M}}$.

The estimation system will be explained in the following sections, but first, the proposed recording montage to obtain the inputs of the system is described.

\subsubsection{Recording setup}

The proposed recording setup aims to simultaneously record a scanning-EMG and a surface-EMG signal (Fig. 3-23). Note that, although the fiber parameter estimation is mainly based on the information provided by the scanning-EMG recording, a correct estimation requires to obtain complementary information to characterize the MU (estimations of the average MFCV, and the average IZ position) which is provided by the surface-MUP recording.

\section{Scanning-EMG recording montage}

Three different scanning-EMG recording configurations have been proposed. They differ in the number of scanning needles employed to record the MUP, and in the number of SF ports that each needle has. Note that all the ports are assumed to be SFlike. This is because they have a very high selectivity, which is expected to favor the correct estimation of the MU fiber parameters. The three proposed configurations are: a single scanning needle with a single port (1-port configuration), a single scanning needle with two ports (2-port configuration); and two scanning needles, each one with 


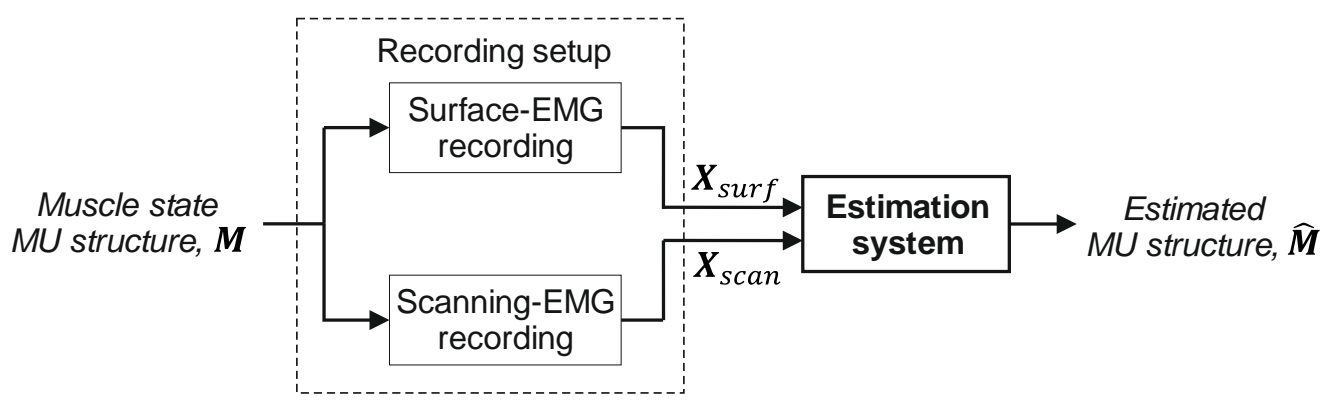

Fig. 3-22. Block diagram describing the context in which the estimation system is used. The muscle state and MU structure (described by the MU parameters, $\boldsymbol{M}$ ), in addition to the recording montage, determine the recorded surface-EMG and scanning-EMG signals (represented by $\boldsymbol{X}_{\text {surf }}$ and $\boldsymbol{X}_{\text {scan }}$ respectively). These signals are used as inputs of the estimation system. The output of the system is the estimated MU structure (described by the MU parameters, $\widehat{\boldsymbol{M}})$.

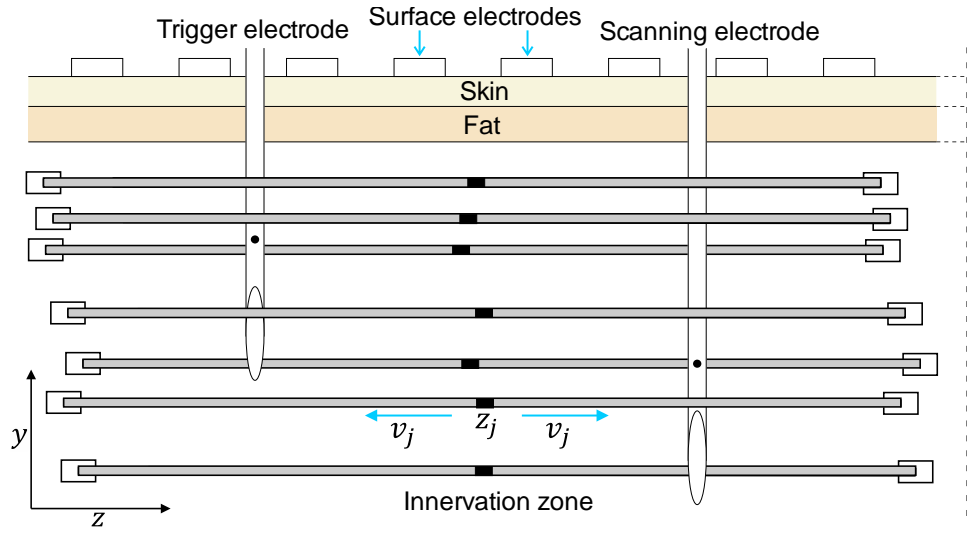

(a)

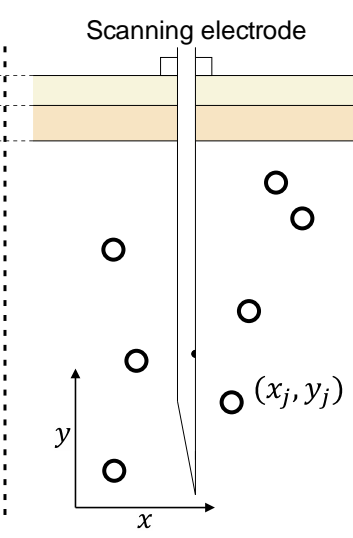

(b)

Fig. 3-23. Schematic representation of the simultaneous recording montage of scanning-EMG and surface-EMG, showing the parameters of the fibers to be estimated $\left(x_{j}, y_{j}, z_{j}, v_{j}\right)$; (a) cross-section of the muscle; (b) longitudinal section of the muscle.

two ports (4-port configuration). The recording configuration used determines the sites in which the MUP is recorded. Therefore, the ability to estimate the MU parameters will vary accordingly from one configuration to another. The three recording configurations are described in detail in the following sections.

\section{1-port scanning-EMG recording}

This configuration is the standard scanning-EMG (see section 1.2.1 for a general description), which has already been described in section 3.2.2, when it came to analyze the dispersion region for different recording configurations. 


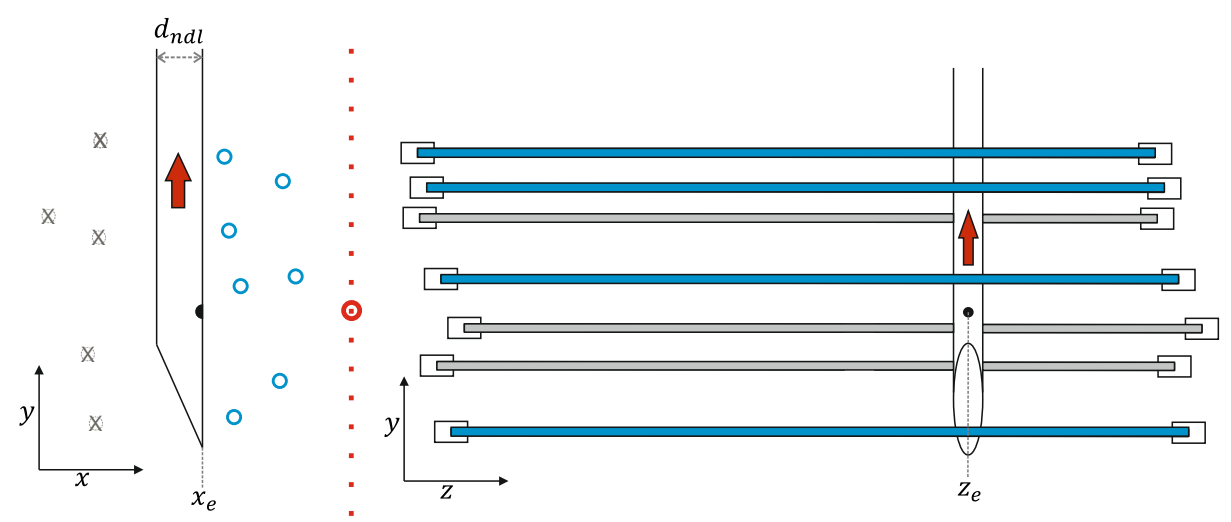

Fig. 3-24. Schematic representation of the 1-port scanning-EMG recording configuration. This configuration employs a single scanning needle, with a single port. The red arrow represents the direction along which the needle moves during the recording. The red points represent the steps along the y-axis that the needle takes during the recording, with a circle marking the position of the current step; (a) representation of the scanning needle in the muscle cross-section plane, $x-y$. The port is represented by a black filled semi-circle, which is located at a side of the needle. The position of this port in the $\mathrm{x}$-axis is $x_{e}$. The MU fibers that the port can detect (those located at the right side of the needle) are represented by holed blue circles. The not detected fibers (those located at the left) are represented by gray crosses; (b) representation of the scanning needle in the longitudinal section plane, z-y. The port is represented by a filled black circle. The position of this port in the z-axis is $z_{e}$. The blue and gray color represent the detected and not detected fibers, respectively.

The recording positions of this configuration are obtained as follows. It is assumed that the recording of this signal is performed with the scanning needle inserted along the $\mathrm{y}$-axis, and with the port located at $x_{e}$ in the x-axis, and at $z_{e}$ in the z-axis (Fig. 3-24). It is also assumed that the port records the MUP along the y-axis, from $y=$ $y_{\min }$ to $y=y_{\max }$, in steps of $0.05 \mathrm{~mm}$, where $y_{\min }$ and $y_{\max }$ are the deepest and the most superficial muscle positions, respectively. Mathematically, these recording positions can be expressed as the set of points $E=\left\{\left(x_{e_{k, p},} y_{\left.e_{k, p}, z_{e_{k, p}}\right)}\right) ; 1 \leq k \leq\right.$ $K ; 1 \leq p \leq P$, where

$$
\begin{aligned}
& x_{e_{k, 1}}=x_{e}, \quad \forall k \\
& y_{e_{k, 1}}=y_{\min }+0.05 \cdot(k-1) \\
& z_{e_{k, 1}}=z_{e} \quad \forall k
\end{aligned}
$$

where the number of scanning steps is $K=1+\frac{y_{\max }-y_{\min }}{0.05}$, and the number of ports is $P=1$.

This configuration has the advantage of being simple to implement, but it also has an important disadvantage. Due to the hemispherical uptake area of the SF electrode, only those fibers located at the side of the needle at which the port is pointing can be 
detected (Fig. 3-24). This implies that only the fibers at one side of the scanning electrode can be estimated.

\section{2-port scanning-EMG recording}

To overcome the above-mentioned limitation of the 1-port configuration, an alternative configuration is proposed here, that consists on incorporating a second port to the scanning needle. In this way, the scanning needle would be composed by two ports, located at opposite sides, and therefore pointing in opposite directions (Fig. 3-25). This modification is an attempt to estimate the parameters of the MU fibers located at both sides of the scanning needle. Note that, in this configuration, the MUP is simultaneously recorded along two parallel linear corridors, which are spaced by the needle diameter in the $\mathrm{x}$-axis (the direction perpendicular to the longitudinal fiber axis and the scanning-corridors direction).

The recording positions of this configuration are defined as follows. It is assumed that the scanning needle is inserted along the y-axis. In the x-axis, the two ports are positioned at $x_{e 1}=x_{e}-d_{n d l}$ and $x_{e 2}=x_{e}$ respectively, where $d_{n d l}=0.55 \mathrm{~mm}$ is the diameter of the scanning needle (Fig. 3-25). In the z-axis, both ports are positioned at $z_{e}$. It is also assumed that both ports record the MUP along the y-axis, from $y=$ $y_{\min }$ to $y=y_{\max }$, in steps of $0.05 \mathrm{~mm}$, where $y_{\min }$ and $y_{\max }$ are the deepest and the most superficial muscle positions, respectively. Mathematically, the complete set of

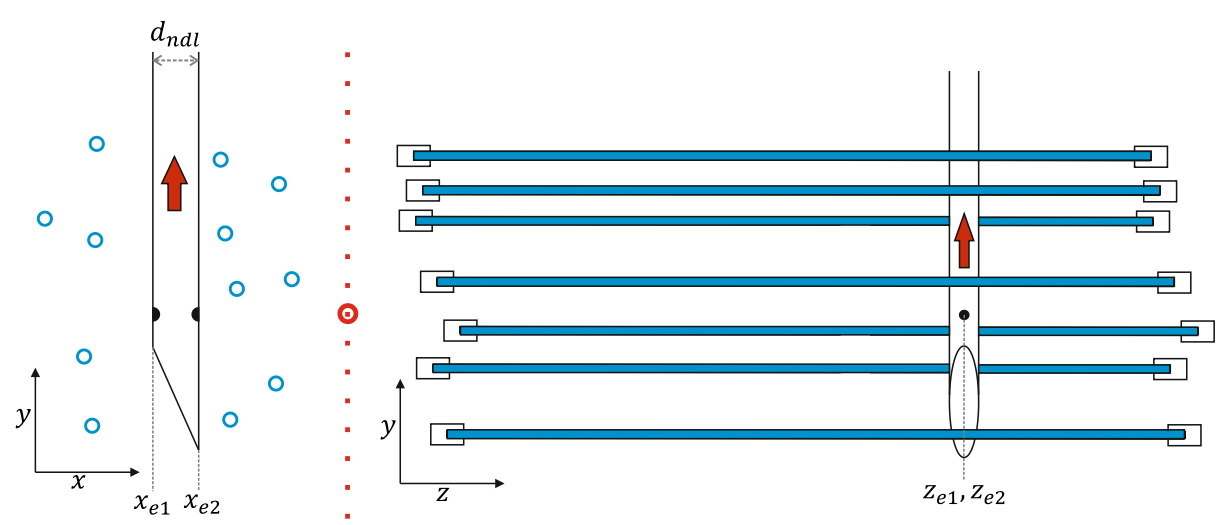

Fig. 3-25. Schematic representation of the 2-port scanning-EMG recording configuration. This configuration employs a single scanning needle with two ports. The red arrow represents the direction along which the needle moves during the recording. The red points represent the steps along the $y$-axis that the needle takes during the recording, with a circle marking the position of the current step; (a) representation of the scanning needle in the muscle cross-section plane, $\mathrm{x}-\mathrm{y}$. The ports are represented by black filled semi-circles. The position of the ports in the $\mathrm{x}-$ axis are $x_{a}=x_{e}-d_{n d l}$ and $x_{b}=x_{e}$. The MU fibers are represented by blue circles. Note that all MU fibers can be recorded, either by one port or by the other one; (b) representation of the scanning needle in the muscle longitudinal section plane, $z-y$. The ports are represented by filled black circles. The position of the ports in the z-axis are $z_{a}=z_{e}$ and $z_{b}=z_{e}$. Note that in this plane one of the ports is behind the needle, and therefore it is not visible. 
recording positions can be expressed as $E=\left\{\left(x_{e_{k, p}}, y_{e_{k, p}}, z_{e_{k, p}}\right)\right\}$, where

$$
\begin{aligned}
& x_{e_{k, 1}}=x_{e 1}, \quad \forall k \\
& x_{e_{k, 2}}=x_{e 2}, \quad \forall k \\
& y_{e_{k, 1}}=y_{\min }+0.05 \cdot(k-1) \\
& y_{e_{k, 2}}=y_{\min }+0.05 \cdot(k-1) \\
& z_{e_{k, 1}}=z_{e 1} \quad \forall k \\
& z_{e_{k, 2}}=z_{e 2} \quad \forall k
\end{aligned}
$$

where the number of scanning steps is $K=1+\frac{y_{\max }-y_{\min }}{0.05}$, and the number of ports is $P=2$.

\section{4-port scanning-EMG recording}

A simplified version of this recording configuration has already been described in section 3.2.2. The one proposed here consists on recording the scanning-EMG signal using two different scanning needles, where each needle contains two ports located at opposite sides of the needle (Fig. 3-26). The implementation of this configuration would consist on a rigid assembly of two needles, physically attached in such a way that that there is a certain distance between them. The needle assembly should be inserted in the muscle with the suitable angle respect to the fiber direction, so that the desired spacing between the two scanning needles is obtained, both in the longitudinal and transverse fiber directions (Fig. 3-26). The recording protocol would be similar to that of the standard scanning-EMG. The needle assembly is inserted within the muscle, and is moved step by step, recording the four versions of the MUP at each step.

Note that, in this configuration, the MUP is simultaneously recorded along four different linear corridors, with two corridors corresponding to each of the scanning needles. This is especially advantageous for the MU fibers located between both needles, as these are observed at the same time by two different ports (one from each needle). In this way, this configuration will provide much more information than the previous ones.

The recording positions of this configuration are defined as follows. It is assumed that the two scanning needles are inserted along the $y$-axis, with a spacing of $\Delta x=$ $1.15 \mathrm{~mm}$ in the $\mathrm{x}$-axis, and $\Delta z=4 \mathrm{~mm}$ in the z-axis. In the $\mathrm{x}$-axis, the four ports are located at $x_{e 1}=x_{e}-\Delta x / 2-d_{n d l} / 2, \quad x_{e 2}=x_{e}-\Delta x / 2+d_{n d l} / 2, \quad x_{e 3}=x_{e}+$ $\Delta x / 2-d_{n d l} / 2$, and $x_{e 4}=x_{e}+\Delta x / 2+d_{n d l} / 2$ respectively, where $d_{n d l}$ is the diameter of the scanning needle (Fig. 3-26). In the z-axis, the four ports are located at $z_{e 1}=z_{e}-\Delta z / 2, \quad z_{e 2}=z_{e}-\Delta z / 2, \quad z_{e 3}=z_{e}+\Delta z / 2, \quad$ and $\quad z_{e 4}=z_{e}+\Delta z / 2$ respectively (Fig. 3-26). Note that the two first ports correspond to the first needle, while the last two ports correspond to the second needle. It is also assumed that the 

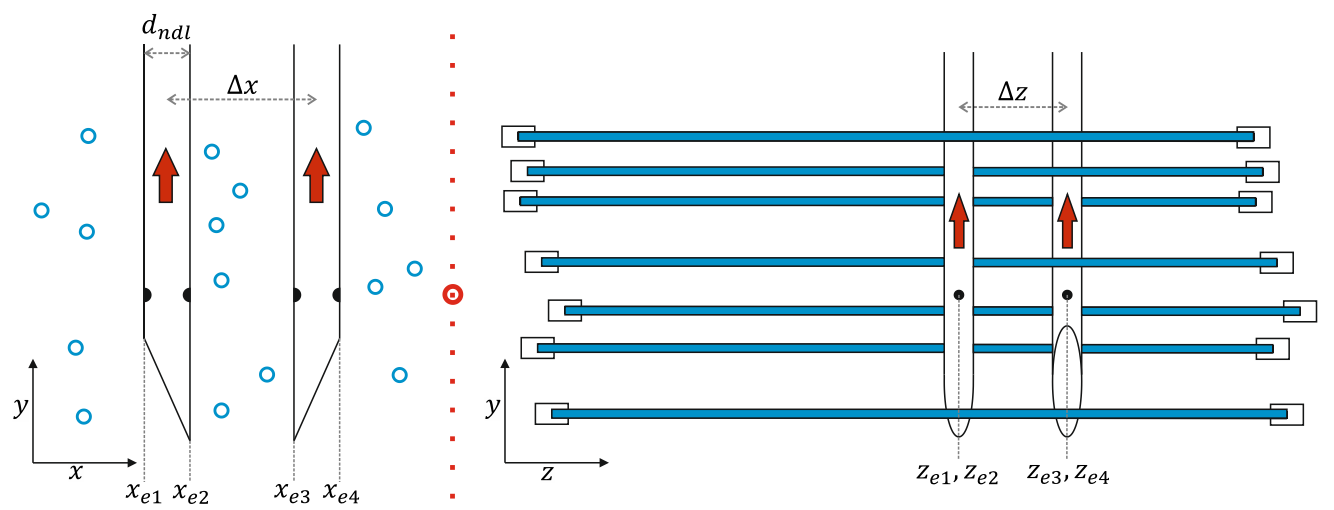

Fig. 3-26. Schematic representation of the 4-port scanning-EMG recording configuration. This configuration employs two scanning needles, each one with two ports. The red arrows represent the direction along which the needles move during recording. The red points represent the steps along the $y$-axis that the needles take during the recording, with a circle marking the position of the current step; (a) representation of the two scanning needles in the muscle cross-section plane, $\mathrm{x}-\mathrm{y}$. The ports are represented by black semi-circles. The position of the ports in the $\mathrm{x}-$ axis are $x_{e 1}=x_{e}-\Delta x / 2-d_{n d l} / 2, x_{e 2}=x_{e}-\Delta x / 2+d_{n d l} / 2, x_{e 3}=x_{e}+\Delta x / 2-d_{n d l} / 2$, and $x_{e 4}=x_{e}+\Delta x / 2+d_{n d l} / 2$. The MU fibers are represented by blue circles. Note that all MU fibers can be recorded at least by one port; (b) representation of the two scanning needles in the muscle longitudinal section plane, $z-y$. The ports are represented by black circles. The position of the ports in the z-axis are $z_{e 1}=z_{e}-\Delta z / 2, z_{e 2}=z_{e}-\Delta z / 2, z_{e 3}=z_{e}+\Delta z / 2$, and $z_{e 4}=z_{e}+\Delta z / 2$. Note that in this plane two of the ports are behind the needle, and therefore they are not visible.

ports record the MUP along the y-axis, from $y=y_{\min }$ to $y=y_{\max }$, in steps of 0.05 $\mathrm{mm}$, where $y_{\min }$ and $y_{\max }$ are the deepest and the most superficial muscle positions, respectively. Under these assumptions, the recording positions can be mathematically expressed as $E=\left\{\left(x_{e_{k, p}}, y_{e_{k, p}}, z_{e_{k, p}}\right)\right\}$, where

$$
\begin{aligned}
& x_{e_{k, 1}}=x_{e 1}, \quad \forall k \\
& x_{e_{k, 2}}=x_{e 2}, \quad \forall k \\
& x_{e_{k, 3}}=x_{e 3}, \quad \forall k \\
& x_{e_{k, 4}}=x_{e 4}, \quad \forall k \\
& y_{e_{k, 1}}=y_{\min }+0.05 \cdot(k-1) \\
& y_{e_{k, 2}}=y_{\min }+0.05 \cdot(k-1) \\
& y_{e_{k, 3}}=y_{\min }+0.05 \cdot(k-1) \\
& y_{e_{k, 4}}=y_{\min }+0.05 \cdot(k-1) \\
& z_{e_{k, 1}}=z_{e 1} \quad \forall k \\
& z_{e_{k, 2}}=z_{e 2} \quad \forall k \\
& z_{e_{k, 3}}=z_{e 3} \quad \forall k \\
& z_{e_{k, 4}}=z_{e 4} \quad \forall k
\end{aligned}
$$


where the number of scanning steps is $K=1+\frac{y_{\max }-y_{\min }}{0.05}$, and the number of ports is $P=4$.

To summarize, three different configurations have been proposed as candidates to be incorporated in the recording setup: the 1-port, the 2-port, and the 4-port scanningEMG recording. Note that, to the best of our knowledge, both the 2-port, and the 4port configurations have never been physically implemented. In this thesis, these recording configurations, along with the 1-port one, are analyzed from a theoretical point of view using simulation experiments, in order to assess its ability to estimate the MU fiber parameters. This will allow to identify which of them is the best option to be used as the input of the estimation system.

\section{Surface-MUP recording setup}

The entire recording setup includes, besides the scanning-EMG recording, an additional setup aimed to record a surface-EMG signal (Fig. 3-23). This second recording setup consists on a linear array of surface electrodes placed on the skin along the longitudinal direction of the muscle fibers (i.e., along the z-axis), so that the IZ lies approximately in the middle of the array. The electrode array positions comprise from $z=z_{\min }$ to $z=z_{\max }$, where the inter-electrode distance is $I E D$. Thus, the array is constituted by $K_{S}$ electrodes, where $K_{S}=1+\frac{z_{\max }-z_{\min }}{I E D}$. In the direction perpendicular to the muscle fibers (the x-axis), the electrodes should be placed above the center of the muscle belly, approximately. The surface electrodes are circular in shape, with diameter $d_{s}$.

The electrode array included in the montage records the raw surface-EMG pattern, which in turn is used to extract the surface-MUP of the MU under study. In order to do it, spike trigger averaging techniques are employed (see more details in section 2.2.1). It is important to keep in mind that the scanning-EMG and the surface-EMG signals must be synchronized in time, hence it is required that the recording of both signals is performed simultaneously. This allows the use of the MU firing pattern extracted from the trigger needle electrode of the scanning-EMG setup in the spike trigger averaging procedure.

\subsubsection{Probabilistic interpretation of the MUP inverse problem}

The most general approach to solve an inverse problem like the one at hand, is to describe the information available about the parameters and the recorded signals by using probability densities [133]. Before solving the inverse problem, we have three different sources of information. The first one is some a priori knowledge about the MU fiber parameters, $\boldsymbol{M}$, described by the probability density $\rho_{M}(\boldsymbol{M})$. The second one is the information provided by the recorded signal itself, $\boldsymbol{X}$, described by a probability density $\rho_{X}(\boldsymbol{X})$. Note that it is interesting to characterize the information about this 
signal using a probability density, as it allows to deal with uncertainties associated to the recording procedure. The third source of information is the theoretical model, which establishes a connection between the parameters $\boldsymbol{M}$ and the predicted recorded signal, $\widehat{\boldsymbol{X}}(\boldsymbol{M})$. Under this probabilistic approach, the general solution of the inverse problem involves the calculation of the probability density representing the $a$ posteriori distribution of the parameters $\boldsymbol{M}$. This probability density, $\sigma_{M}(\boldsymbol{M})$, is obtained as follows [133]

$$
\sigma_{M}(\boldsymbol{M})=C \cdot \rho_{M}(\boldsymbol{M}) \rho_{X}(\widehat{\boldsymbol{X}}(\boldsymbol{M}))
$$

where $C$ is a normalization constant.

Let's see how this approach applies to our particular problem. As it is already known, in our case, $\boldsymbol{M}$ represents the parameters of the MU fibers. In the first instance, we assume that these parameters are located within a certain region $\mathfrak{M}_{R}$ of the parameter space. This region is roughly obtained from the general knowledge about the physiology of the MUs, and from a preliminary analysis of the scanning-EMG and surface-MUP signals. This will be detailed in the later section. Under this consideration, the a priori probability density of the parameters is modeled by a uniform distribution

$$
\rho_{M}(\boldsymbol{M})= \begin{cases}C^{\prime} & \boldsymbol{M} \in \mathfrak{M}_{R} \\ 0 & \boldsymbol{M} \notin \mathfrak{M}_{R}\end{cases}
$$

where $C^{\prime}$ is a normalization constant.

In our problem, the recorded scanning-EMG signal is $\boldsymbol{X}=\left\{x_{n, k, p}\right\}$, where $n$ and $k$ denote the samples along time and along the different scanning steps, respectively, and $p$ denotes the port in which the MUP is recorded. Note that the actual value of the signal samples is unknown, as the recording procedure always introduces uncertainty in the form of noise. The probability density $\rho_{X}(\boldsymbol{X})$, representing the information available about $\boldsymbol{X}$, is approximated from the raw recorded scanning-EMG signal $\boldsymbol{X}_{\text {scan }}$, under the assumption that such signal has been contaminated with AWGN of variance $\sigma^{2}$. In this way, the probability density representing the information about the signal $\boldsymbol{X}$ can be modeled by a Gaussian distribution

$$
\rho_{X}(\boldsymbol{X})=C^{\prime \prime} \cdot e^{-\frac{1}{2 \sigma^{2}}\left\|\boldsymbol{X}-\boldsymbol{X}_{\text {scan }}\right\|^{2}}
$$

where $C^{\prime \prime}$ is a normalization constant, and

$$
\left\|\boldsymbol{X}-\boldsymbol{X}_{\text {scan }}\right\|^{2}=\sum_{n=1}^{N} \sum_{k=1}^{K} \sum_{p=1}^{P}\left(x_{n, k, p}-x_{\text {scan }_{n, k, p}}\right)^{2}
$$


Note that, modeling the noise as AWGN is motivated by the simplicity of the mathematics that derive from such assumption. In general the noise introduced by the recording procedure is complex (see section 2.1.1 for a detailed description of such noise), so a specific approach to deal with this noise is left for later chapters.

In our problem, the model predicts the sample values of the recorded scanningEMG signal $\widehat{\boldsymbol{X}}$, from the MU fiber parameters $\boldsymbol{M}$

$$
\widehat{\boldsymbol{X}}(\boldsymbol{M})=\left\{\hat{x}_{n, k, p}(\boldsymbol{M})\right\}
$$

Particularizing equation (3-15) for the considerations of our problem, it follows that the probability density representing the a posteriori information about the MU fiber parameters can be calculated as

$$
\sigma_{M}(\boldsymbol{M})=\left\{\begin{array}{cc}
C^{\prime \prime \prime} \cdot e^{-\frac{1}{2 \sigma^{2}} \varepsilon(\boldsymbol{M})} & \boldsymbol{M} \in \mathfrak{M}_{R} \\
0 & \boldsymbol{M} \notin \mathfrak{M}_{R}
\end{array}\right.
$$

where $\varepsilon(\boldsymbol{M})$ is the error function

$$
\varepsilon(\boldsymbol{M})=\left\|\widehat{\boldsymbol{X}}(\boldsymbol{M})-\boldsymbol{X}_{\text {scan }}\right\|^{2}=\sum_{n=1}^{N} \sum_{k=1}^{K} \sum_{p=1}^{P}\left(\hat{x}_{n, k, p}(\boldsymbol{M})-x_{\text {scan }_{n, k, p}}\right)^{2}
$$

and $C^{\prime \prime \prime}$ is a normalization constant.

\subsubsection{General outline of the estimation system}

The full computation of the a posteriori probability density $\sigma_{M}(\boldsymbol{M})$ is extremely expensive. It requires to evaluate the expression (3-20), and therefore the model, in a sufficiently dense grid of points within the region $\mathfrak{M}_{R}$. If the dimensionality of the parameter space is very large, as it is our case, this task becomes impracticable. For instance, suppose that we want to solve the inverse problem for a MU of 20 muscle fibers (let's assume here that the fiber number is known), then, the number of dimensions of the parameter space will be 80 ( 20 fibers and 4 parameters per fiber). If we assume that we want to cover each dimension with a resolution of 100 points, then the number of times the model should be evaluated is $100^{80}$.

Instead of the complete computation of the a posteriori probability density, a much less expensive approach is to find the MU fiber parameters $\widehat{M}$, corresponding to the maximum value of the a posteriori probability density. These parameters are the best estimation of $\boldsymbol{M}$, in the sense that it is the point in the parameter space that most probably have generated the recorded signal. Maximizing $\sigma_{M}(\boldsymbol{M})$ is equivalent to minimizing the error function $\varepsilon(\boldsymbol{M})$ in the region $\mathfrak{M}_{R}$. Therefore, the goal of the 
estimation system (Fig. 3-27) is to find in the region $\mathfrak{M}_{R}$ of the parameter space, the point (set of parameters $\widehat{\boldsymbol{M}}$ ) that minimizes the error function $\varepsilon(\boldsymbol{M})$ when the simulation model $\widehat{\boldsymbol{X}}(\boldsymbol{M})$ is applied. This goal can be achieved by means of optimization techniques. This minimization procedure can be understood as the search for the set of parameters that makes the simulated signal $\widehat{\boldsymbol{X}}(\boldsymbol{M})$, to be as similar as possible to the recorded one $\boldsymbol{X}_{\text {scan }}$, which means that the discrepancies between both signals, quantified by the error function, have to be minimal.

In the following sections the main steps of the estimation system will be detailed. A scheme representing these steps is shown in Fig. 3-27.

\section{Optimization procedure}

To estimate the MU fiber parameters, different points in the parameter space (each corresponding to a specific MU fiber parameters) are generated iteratively in an

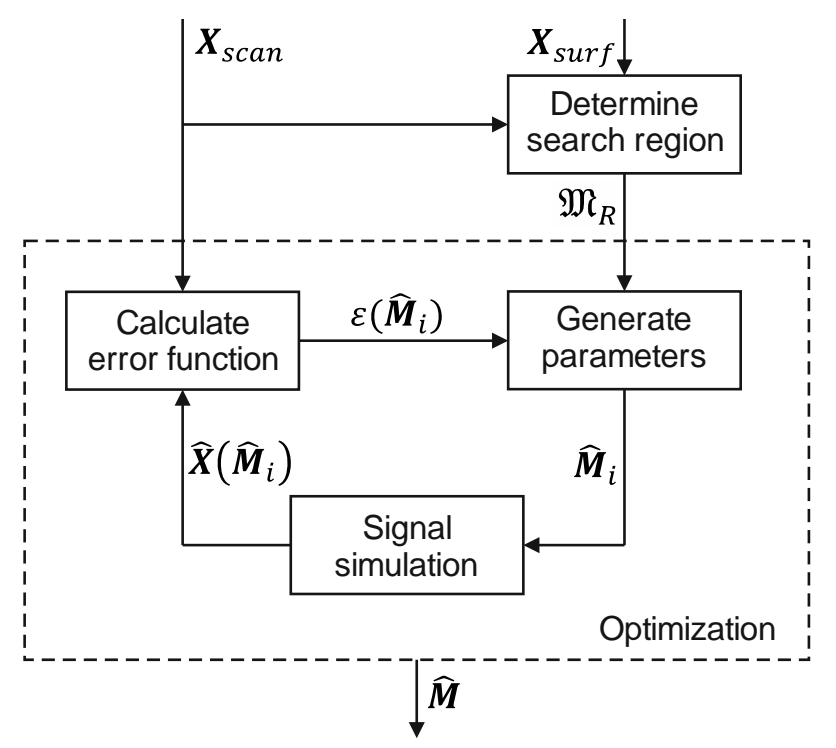

Fig. 3-27. Block diagram outlining the estimation system. The inputs of the system are the raw recorded scanning-EMG signal $\boldsymbol{X}_{\text {scan }}$, and the surface-MUP signal $\boldsymbol{X}_{\text {surf }}$. Both signals are used to determine the region of the parameter space $\mathfrak{M}_{R}$ in which the estimated parameters will be sought. The optimization procedure used to estimate the parameters is boxed with a dashed line. The optimization method iteratively generates and evaluates different solutions (sets of parameters) $\widehat{\boldsymbol{M}}_{i}$. These sets of parameters are used to generate the simulated signals $\widehat{\boldsymbol{X}}\left(\widehat{\boldsymbol{M}}_{i}\right)$, which in turn are used to compute the error function values $\varepsilon\left(\widehat{\boldsymbol{M}}_{i}\right)$. Note that the calculation of the error function requires both the simulated signal $\widehat{\boldsymbol{X}}\left(\widehat{\boldsymbol{M}}_{i}\right)$, and the recorded one $\boldsymbol{X}_{\text {scan }}$. The error function values of previous iterations are used to adequately generate the new sets of parameters. The output of the optimization algorithm is supposed to be the optimal set of parameters $\widehat{\boldsymbol{M}}$; i.e., the set of parameters for which the error is minimum. 
optimization procedure. Each of them is used to simulate its corresponding scanningEMG signal, and then to compute the error function. The final estimated parameter set $\widehat{\boldsymbol{M}}$, corresponds to the point for which the calculated error has the lowest value. The specific criteria used to generate at each iteration new points in the parameter space is determined by the optimization algorithm employed.

Simulation of the scanning-EMG signal

Given a certain point in the parameter space, $\widehat{\boldsymbol{M}}_{i}$, where the sub-index $i$ denotes the different points iteratively generated by the optimization procedure, the simulated scanning-EMG signal is calculated as

$$
\widehat{\boldsymbol{X}}\left(\widehat{\boldsymbol{M}}_{i}\right)=\left\{\widehat{x}_{n, k, p}\left(\widehat{\boldsymbol{M}}_{i}\right)\right\}
$$

where $n$ and $k$ denote the samples along time and along the different scanning steps respectively, $p$ denotes the port in which the MUP is recorded, and each sample value is calculated using the Dimitrov model [21] (see sections 1.3.1 and 1.4.2 for a detailed description of the model)

$$
\widehat{x}_{n, k, p}\left(\widehat{\boldsymbol{M}}_{i}\right)=\operatorname{MUP}{\widehat{M}_{i}}_{i}\left(t_{n}, x_{e_{k, p},} y_{e_{k, p},} z_{e_{k, p}}\right)
$$

Note that each SF port can only detect the activity of the fibers located at one side of the needle. This effect has been included in the MUP model by zeroing the SFAP contributions of those fibers located on the opposite side to the recording port.

\section{Calculation of the error function}

The error function $\varepsilon\left(\widehat{\boldsymbol{M}}_{i}\right)$ to be minimized is calculated from the simulated and recorded scanning-EMG signals, $\widehat{\boldsymbol{X}}\left(\widehat{\boldsymbol{M}}_{i}\right)$ and $\boldsymbol{X}_{\text {scan }}$ respectively, as follows

$$
\varepsilon\left(\widehat{\boldsymbol{M}}_{i}\right)=\sum_{n=1}^{N} \sum_{k=1}^{K} \sum_{p=1}^{P}\left(\widehat{x}_{n, k, p}\left(\widehat{\boldsymbol{M}}_{i}\right)-x_{s c a n_{n, k, p}}\right)^{2}
$$

\section{Optimization scheme}

The optimization procedure used to minimize the error function has two steps. In the first step, a genetic algorithm [41] is used to find an approximate solution. Genetic algorithms are suitable to deal with high dimensionality parameter spaces, as in our problem. The implementation used for the genetic algorithm is the ga function provided by MATLAB. In the second step, an interior point minimization method [15] is applied, using as starting point the solution obtained at the output of the genetic algorithm. The aim of this second step is to refine the solution, closing the gap to the 
actual minimum of the error function. The implementation used for the interior point method is the fmincon function provided by MATLAB. In order to avoid falling into local minima, this optimization scheme can be repeated several times, and the solution for which the error is minimum can be chosen as final estimation. The specific operating parameters of the algorithm scheme associated to the different recording configurations will be detailed in the next sections.

\section{Determination of the number of MU fibers}

When solving the inverse problem, neither the total number of MU fibers nor the number of MU fibers significantly contributing to the recorded signal are known beforehand. This fact implies an added difficulty in solving the MUP inverse problem. Note that, in order to simulate the scanning-EMG signal during the optimization procedure, a specific number of MU fibers must be assumed by the model. The choice of a suitable number of MU fibers can significantly affect the estimation performance. Two strategies have been implemented to address this problem. As it will be explained in following sections, the selection of the most adequate strategy will depend on the recording configuration used.

The first strategy is to assign a number of simulated fibers large enough as to ensure that it is larger than the number of MU fibers actually contributing to the real scanning-EMG signal. In this way, the optimization procedure should be able to find a parameter solution that adequately reproduces the waveform of the recorded signal using only part of these simulated MU fibers. Those fibers that the method does not need in order to reproduce the signal waveform will be gradually moved away from the needle corridor during the optimization procedure, in such a way their contributions to the signal can be considered negligible. A similar approach has been used in [140] to solve the inverse problem.

The second strategy consists on repeating the optimization procedure for different values of the number of simulated MU fibers, and chose as definitive parameter solution the one with the lowest error. This second strategy, similar to a brute-force approach, is computationally more expensive than the first one. For this reason, it is used only when the first one cannot be applied, as it occurs when estimating within the region located between two recording needles.

\section{Determination of the search region in the parameter space}

A correct parameter estimation requires to properly establish the region of the parameter space $\mathfrak{M}_{R}$, in which the optimization algorithm has to find the estimated MU fiber parameters. Note that our problem deals with a parameter space with a high dimensionality, so it is convenient to limit this search region as much as possible. A search region too wide could make it difficult for the optimization algorithm to reach the minimum of the error function, and therefore, imprecise estimations would be achieved. On the other hand, care must be taken not to make the region too small, as 
this may cause that the point corresponding to the real MU fiber parameters to be outside that region, which would inevitably imply an incorrect parameter estimation.

The method implemented to determine the search region $\mathfrak{M}_{R}$ parts, on the one hand from the general knowledge about the anatomy and physiology of the MUs, and on the other hand from the analysis of both the scanning-EMG and the surface-MUP signals.

The search region $\mathfrak{M}_{R}$ is defined by establishing the search interval associated to each of the dimensions of the parameter space; i.e., to each of the parameters characterizing the MU fibers of the solution. Let $\widehat{\boldsymbol{M}}=\left\{\widehat{\boldsymbol{m}}_{j}\right\} ; 1 \leq j \leq \hat{J}$ be a point in the parameter space, with $\widehat{\boldsymbol{m}}_{j}=\left(\hat{x}_{j}, \hat{y}_{j}, \hat{z}_{j}, \hat{v}_{j}\right)$, and $\hat{J}$ the number of simulated MU fibers, then $\widehat{\boldsymbol{M}} \in \mathfrak{M}_{R}$ if and only if

$$
\begin{aligned}
& \hat{x}_{j} \in \bigcup_{c=1}^{C}\left[x_{L}^{c}, x_{H}^{c}\right] \\
& \hat{y}_{j} \in\left[y_{L}, y_{H}\right] \\
& \hat{z}_{j} \in\left[z_{L}, z_{H}\right] \\
& \hat{v}_{j} \in\left[v_{L}, v_{H}\right] \\
& \forall 1 \leq j \leq \hat{J}
\end{aligned}
$$

where $x_{L}^{c}$ and $x_{H}^{c}$ are the bounds of the search region along de x-axis; $y_{L}$ and $y_{H}$ the bounds along the y-axis; $z_{L}$ and $z_{H}$ the bounds along the z-axis; and $v_{L}$ and $v_{H}$ the bounds along the v-axis.

Note that, as stated in the first of these equations, the overall search region $\mathfrak{M}_{R}$ is actually constituted by the union of $C$ different search regions, each of them associated to one different search interval along the x-axis (Fig. 3-28). These intervals are determined by the physical limits established by the scanning needles. This, in turn, implies that the intervals are determined by the recording configuration (Fig. 3-28). The criterion to obtain the intervals along the different parameter axes is described below.

\section{Determination of the $\boldsymbol{x}_{\boldsymbol{L}}^{\boldsymbol{c}}$ and $\boldsymbol{x}_{\boldsymbol{H}}^{\boldsymbol{c}}$ bounds}

When the 1-port scanning-EMG recording configuration is used, only the MU fibers located at one side of the scanning needle can be recorded, and therefore estimated. Accordingly, a unique search interval is defined ( $C=1$ ) (Fig. 3-28 (a)). Such interval $\left[x_{L}, x_{H}\right]$ extends from the port position to a distance $R_{x}$ such that the MU fibers barely contributes to the simulated signal. Therefore, the bounds are 


$$
\begin{aligned}
& x_{L}=x_{e} \\
& x_{H}=x_{e}+R_{x}
\end{aligned}
$$

When the 2-port scanning-EMG recording configuration is used, a similar criterion is applied to obtain the bounds. The difference is that, for this configuration, there are two search intervals $(C=2)$ (Fig. 3-28 (b)): $\left[x_{L}^{1}, x_{R}^{1}\right]$ and $\left[x_{L}^{2}, x_{R}^{2}\right]$, corresponding to the left and right side of the scanning needle, respectively. In this case the bounds are

$$
\begin{aligned}
& x_{L}^{1}=x_{e}-d_{n d l}-R_{x} \\
& x_{H}^{1}=x_{e}-d_{n d l} \\
& x_{L}^{2}=x_{e} \\
& x_{H}^{2}=x_{e}+R_{x}
\end{aligned}
$$

where $d_{n d l}$ is the diameter of the scanning needle.

When the 4-port scanning-EMG recording procedure is used, three search intervals are defined $(C=3)$ (Fig. 3-28 (c)): $\left[x_{L}^{1}, x_{H}^{1}\right],\left[x_{L}^{2}, x_{H}^{2}\right]$ and $\left[x_{L}^{3}, x_{H}^{3}\right]$, associated to left side of the leftmost needle, the region between the needles, and the right side of the rightmost needle, respectively. In this case the bounds are

$$
\begin{aligned}
& x_{L}^{1}=x_{e}-\Delta x / 2-d_{n d l} / 2-R_{x} \\
& x_{H}^{1}=x_{e}-\Delta x / 2-d_{n d l} / 2 \\
& x_{L}^{2}=x_{e}-\Delta x / 2+d_{n d l} / 2 \\
& x_{H}^{2}=x_{e}+\Delta x / 2-d_{n d l} / 2 \\
& x_{L}^{3}=x_{e}+\Delta x / 2+d_{n d l} / 2 \\
& x_{H}^{3}=x_{e}+\Delta x / 2+d_{n d l} / 2+R_{x}
\end{aligned}
$$

where $\Delta x$ is the separation between the two scanning needles, along the $\mathrm{x}$-axis.

The specific values of $R_{x}$ will depend on the recording configuration, and on the stage of the optimization scheme in which the limits are applied. These values will be detailed in the next section, where the particularities of the estimation system associated with each recording configuration will be described.

\section{Determination of the $\boldsymbol{y}_{\boldsymbol{L}}$ and $\boldsymbol{y}_{\boldsymbol{H}}$ bounds}

The $y_{L}$ and $y_{H}$ bounds are determined as follows. In a first stage, the active MUP region of the recorded scanning-EMG signal is obtained using the algorithm described in section 2.1.3 (Algorithm 2). The output of this algorithm is a mask set to 1 within the active region, and 0 otherwise (Fig. 3-29 (b)). The bounds are determined as the first and last spatial positions of the mask for which there is at least one temporal sample set to 1 (i.e., within the active region) (Fig. 3-29 (b)). 


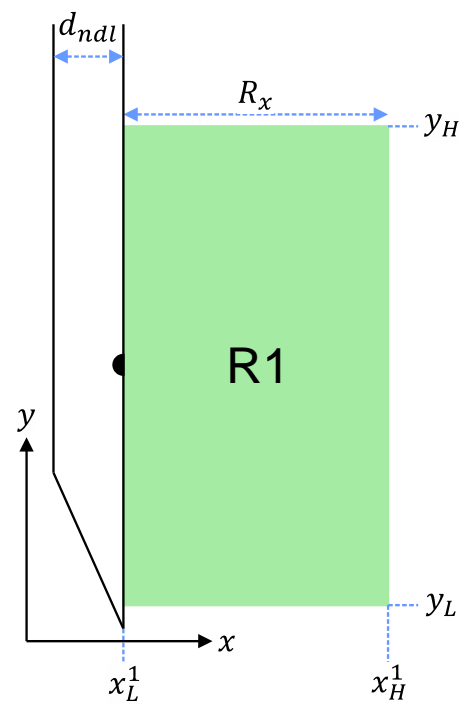

(a)

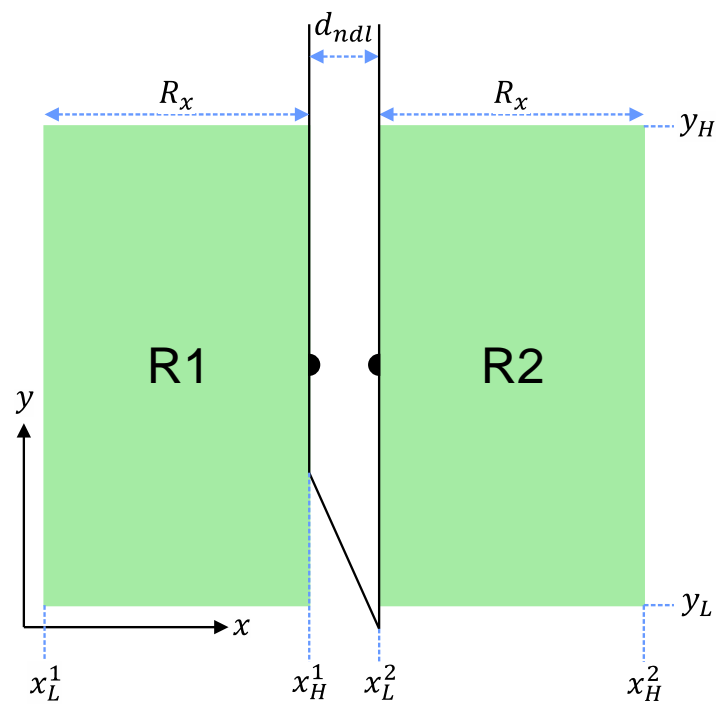

(b)

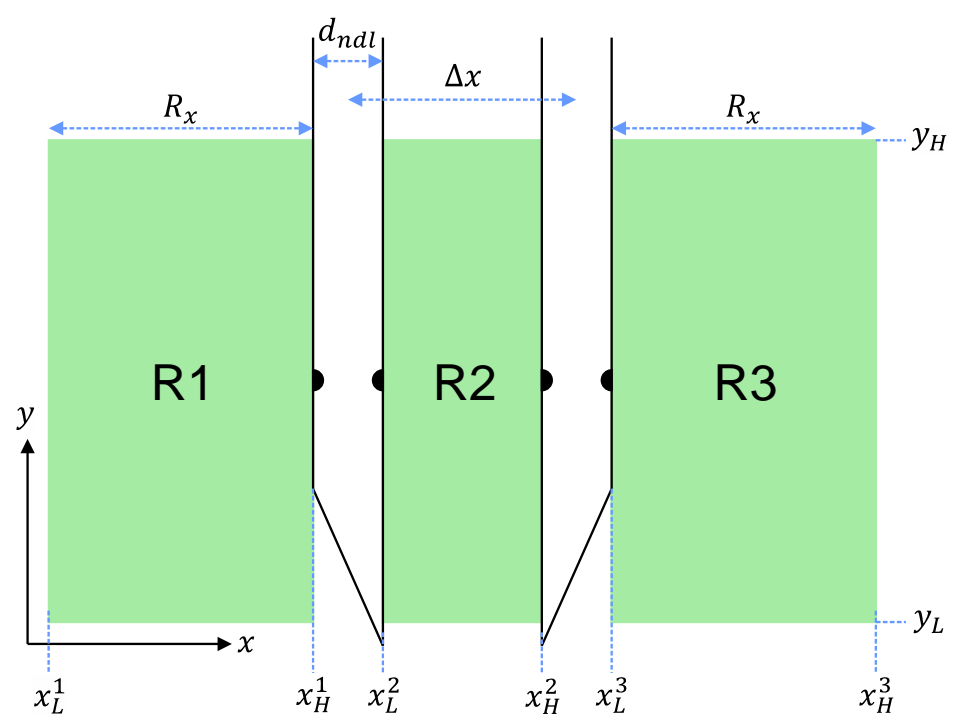

(c)

Fig. 3-28. Schematic representation of the search regions in the muscle cross-section plane corresponding to each recording configuration: (a) search regions of the 1-port scanning-EMG recording configuration, where there is only one search region (R1); (b) search regions of the 2-port scanning-EMG recording configuration, where there are two search regions (R1 and R2) separated by the scanning needle; (c) search regions of the 4-port scanning-EMG recording configuration, where there are three search regions (R1, R2, and R3) separated by the two scanning needles. 


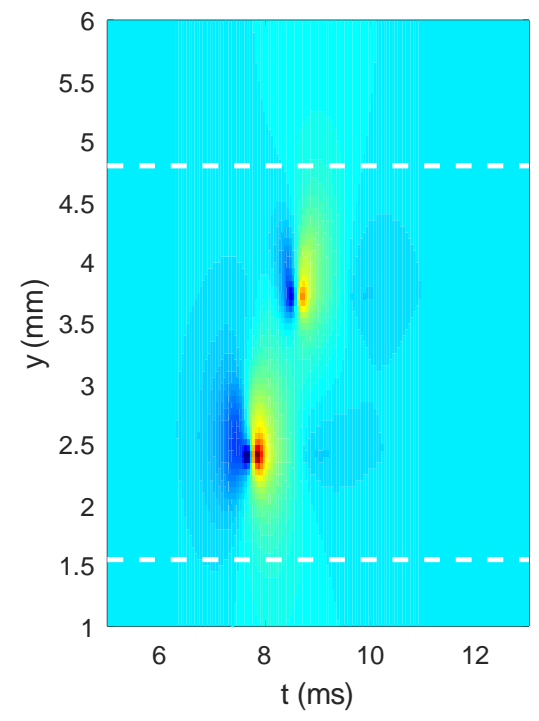

(a)

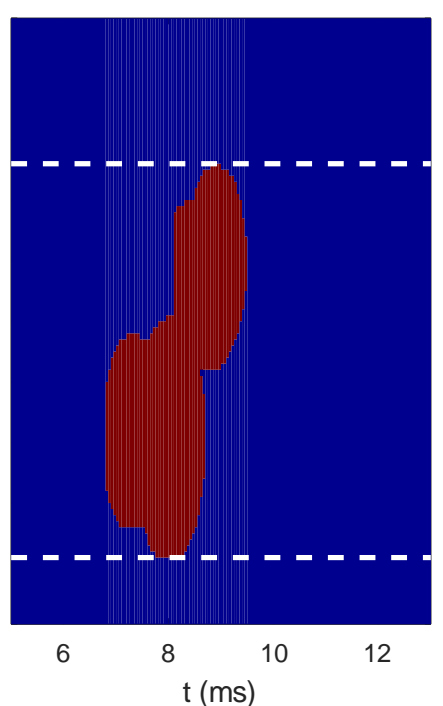

(b)

Fig. 3-29. Example of how the $y_{L}$ and $y_{H}$ bounds are determined; (a) recorded scanning-EMG signal used to calculate the bounds; (b) detection mask obtained from the scanning-EMG signal, using the algorithm to detect the active MUP region. The red color corresponds to the ones in the mask, and the blue one to the zeros. The bounds are the first and last spatial position in which there are temporal samples with the mask set tol (white dashed lines).

Note that, if the recording configuration in use implies the existence of more than one recording port, this procedure is applied separately to the signal recorded from each of the ports. In this way, specific limits $\left(y_{L_{p}}\right.$ and $\left.y_{H_{p}}\right)$ are obtained for each port $p$, and the final bounds are given by the outer bounds of these limits

$$
\begin{aligned}
& y_{L}=\min \left(y_{L_{1}}, \ldots, y_{L_{P}}\right) \\
& y_{H}=\max \left(y_{H_{1}}, \ldots, y_{H_{P}}\right)
\end{aligned}
$$

where $P$ is the total number of ports.

\section{Determination of the $\mathbf{z}_{\mathbf{L}}$ and $\mathbf{z}_{\boldsymbol{H}}$ bounds}

The bounds of the $\mathrm{z}$ - and $\mathrm{v}$-axis are extracted from the surface-MUP. From this signal, two important parameters are calculated: the average MFCV of the MU, $V_{M U}$; and the approximate IZ position of the MU, $Z_{M U}$. To calculate these two parameters, the algorithm described in section 2.2.2 is used. Once these parameters are obtained, the determination of the bounds simply consists on defining an interval around these parameters. The $z_{L}$ and $z_{H}$ bounds are calculated as 


$$
\begin{aligned}
& z_{L}=Z_{M U}-R_{Z} \\
& z_{H}=Z_{M U}+R_{z}
\end{aligned}
$$

where $R_{Z}$ must be set so that the search interval is wide enough to ensure that the entire IZ of the MU are within it. For instance, in the biceps brachii muscle, the IZ comprises a region about $6-10 \mathrm{~mm}$ width $[4,3]$, and therefore, a suitable choice of this parameter can be $R_{z}=8 \mathrm{~mm}$.

\section{Determination of the $\boldsymbol{v}_{\boldsymbol{L}}$ and $\boldsymbol{v}_{\boldsymbol{H}}$ bounds}

The $v_{L}$ and $v_{H}$ bounds are calculated as

$$
\begin{aligned}
& v_{L}=V_{M U}-R_{v} \\
& v_{H}=V_{M U}+R_{v}
\end{aligned}
$$

where $R_{v}$ must be set so that the search interval is wider than the dispersion of the MFCVs within the MU. In non-pathological MUs, the dispersion of the MFCV's within the MU is much narrower than the dispersion in the entire muscle. In [87], the authors found that, in the biceps brachii muscle, a standard deviation of $0.15 \mathrm{~m} / \mathrm{s}$ in the MFCVs of the MU is enough to explain the observed MUP complexity. Therefore, a suitable choice of this parameter can be $R_{v}=0.6 \mathrm{~m} / \mathrm{s}$.

\section{Adaptations of the estimation system to each recording configuration}

This section describes how the estimation system is adapted to the different recording configurations (1-port, 2-port, 4-port).

\section{1-port scanning-EMG}

For the 1-port scanning-EMG recording configuration, a single search region is defined, corresponding to the side of the scanning needle at which the port is pointing (Fig. 3-28 (a)). The estimation procedure, in this case, consists on executing the optimization scheme (genetic algorithm + interior point algorithm), for the defined search region. This is performed for 5 independent runs, and the one for which the error in (3-24) is minimum is chosen as the output of the estimation. The operating parameters used for this configuration are shown in Table 3-2.

Note that there are two different values for the maximum fiber-port distance allowed along the x-axis, $R_{x}$. These two values correspond to the two optimization stages: the genetic and the interior-point algorithms, respectively. The reason why the interior point algorithm is allowed to move the fibers further away is to ensure that the unnecessary fibers are far away enough so that their contribution to the signal is negligible. 
Table 3-2. Operating parameters used in the estimation system for the 1- and 2-port configurations.

\begin{tabular}{ll}
\hline Parameter & Value \\
\hline \multicolumn{1}{c}{ Genetic algorithm } \\
\hline Maximum distance, $R_{x}$ & 0.8 \\
Number of generations & 250 \\
Population size & 80 \\
Crossover fraction & 0.2 \\
Elite count & $0.05 \cdot$ Population size \\
Mutation scale & 1 \\
Mutation shrink & 1 \\
\hline \multicolumn{2}{c}{ Interior point } \\
\hline Maximum distance, $R_{x}$ & 1.5 \\
Number of iterations & 10000 \\
\hline \multicolumn{2}{c}{ General } \\
\hline Interval semi-width in $\mathrm{z}, R_{z}$ & $8 \mathrm{~mm}$ \\
Interval semi-width in $\mathrm{v}, R_{v}$ & $0.6 \mathrm{~m} / \mathrm{s}$ \\
Number of repetitions & 5 \\
Number of simulated fibers & 18 \\
\hline
\end{tabular}

\section{2-port scanning-EMG}

For the 2-port scanning-EMG recording configuration there are two search regions associated to both sides of the scanning needle (Fig. 3-28 (b)). In this case, the estimation procedure is identical to that of the previous configuration, but it is applied separately to both regions. Note that this approach is feasible, given that each port only detects exclusively the fibers belonging to one of the regions.

\section{4-port scanning-EMG}

For the 4-port scanning-EMG recording configuration there are three search regions, one between both scanning needles, and two at the external sides (Fig. 3-28 (c)). An important remark about this configuration must be done. Let's name the different ports, from left to right, P1, P2, P3, and P4. Let's also name the different search regions, from left to right, R1, R2, and R3 (Fig. 3-28 (c)). Remember that each port can only detect the activity of those fibers located in front of it. Then, it turns out that P1 and P4 can only detect the fibers within a single region, namely, R1 and R3 respectively. However, P2 and P3 can detect fibers in two different regions. P2 can 
Table 3-3. Operating parameters used in the estimation system for the 4-port configuration.

\begin{tabular}{|c|c|}
\hline Parameter & Value \\
\hline \multicolumn{2}{|c|}{ Genetic algorithm } \\
\hline Maximum distance, $R_{x}$, in $\mathrm{R} 1 / \mathrm{R} 3$ & 0.8 \\
\hline Number of generations & 250 \\
\hline Population size & 80 \\
\hline Crossover fraction & 0.2 \\
\hline Elite count & 0.05. Population size \\
\hline Mutation scale & 1 \\
\hline Mutation shrink & 1 \\
\hline \multicolumn{2}{|l|}{ Interior point } \\
\hline Maximum distance, $R_{x}$, in $\mathrm{R} 1 / \mathrm{R} 3$ & 1.5 \\
\hline Number of iterations in R1 / R3 & 10000 \\
\hline Number of iterations in R2 & 5000 \\
\hline \multicolumn{2}{|l|}{ General } \\
\hline Interval semi-width in z, $R_{z}$ & $8 \mathrm{~mm}$ \\
\hline Interval semi-width in $\mathrm{v}, R_{v}$ & $0.6 \mathrm{~m} / \mathrm{s}$ \\
\hline Number of repetitions & 5 \\
\hline Number of simulated fibers in R1 / R3 & 18 \\
\hline Number of simulated fibers in R2 & 1 to 20 \\
\hline
\end{tabular}

detect the fibers in R2 and R3, and P3 can detect those in R2 and R1. This implies that, for this configuration it is not possible to apply the optimization scheme to each region independently. A possible approach would be to make a simultaneous estimation in the three regions, but this solution has been discarded due to its high requirement in terms of computation cost. The approach that has been adopted is somewhat more complex, but less demanding in terms of computation cost. The solution essentially consists on performing the estimation in the different regions in a sequential approach. Firstly, the MU fibers located in the external regions are estimated from the recordings of the external ports; and secondly, taking into account the result of this estimation, the fibers located in the internal region are estimated from the recording of the internal ports. The estimation procedure is performed as follows:

1- The estimation is performed independently in region R1 and R3 from the sections of the signal $\boldsymbol{X}_{\text {scan }}$ recorded in P1 and P4, respectively. The optimization scheme based in a genetic algorithm and an interior point algorithm is applied. 
2- Once the fibers located in R1 and R3 ( $\widehat{\boldsymbol{M}}_{R 1}$ and $\widehat{\boldsymbol{M}}_{R 3}$, respectively) are estimated, the signal contribution corresponding to the fibers of R3 as it would be recorded from P2, and the signal contribution corresponding to the fibers of R1 as it would be recorded from $\mathrm{P} 3$, are calculated.

3- The signal contribution of $\mathrm{R} 3$ simulated in $\mathrm{P} 2$ is subtracted from the section of the signal $\boldsymbol{X}_{\text {scan }}$ recorded in P2. Analogously, the signal contribution of R1 simulated in P3 is subtracted from the section of the signal $\boldsymbol{X}_{\text {scan }}$ recorded in P3. We will call the signal resulting from subtracting these two contributions $\boldsymbol{X}_{\text {scan }}{ }^{\prime}$.

4- The fibers located in R2 ( $\left.\widehat{\boldsymbol{M}}_{R 2}\right)$ are estimated from the sections of the signal $\boldsymbol{X}_{\text {scan }}{ }^{\prime}$ in P2 and P3. An important consideration to keep in mind is that, in this case, the fibers of the solution cannot be moved during the optimization beyond the physical limits imposed by the two needles (in the x-axis). Therefore, those fibers within R2 that are unnecessary cannot be distanced from the ports as for their contributions to be negligible. Thus, a correct estimation cannot be performed using a single value of the number of simulated fibers. Instead, the estimation is repeated for different values of the number of fibers (between 1 and 20), and the solution corresponding to the best fit (minimum value of the error) is chosen.

These four steps are repeated in 5 independent runs, and the solution for which the error is minimum is selected as the final estimation. The operating parameters of this estimation procedure are shown in Table 3-3. 


\subsection{Evaluation framework of the estimation system.}

In this section, the strategies used to evaluate the performance of the estimation system are presented. An important consideration to keep in mind is that the evaluation is possible because we are using a simulation environment that provides the exact $a$ priori knowledge about the muscle and the MU fiber parameters used to generate the signals, so that the estimated MU fiber parameters can be compared with the real ones. This allows to objectively evaluate the estimation performance. A detailed description of the simulation environment used has been presented in section 1.4.

The procedure used to evaluate the estimation system can be divided in the following steps (Fig. 3-30):

1- Simulation of the muscle and MU: using the simulation environment, the parameters describing the fibers of the MU to be estimated, and the ones describing the rest of MUs of the muscle are generated.

2- Simulation of the recorded signals: using the simulation environment, the scanning-EMG and surface-MUP recordings are simulated for the parameters obtained in step 1.

3- Estimation: the estimation system is applied to obtain an estimation of the MU fiber parameters from the scanning-EMG and surface-MUP signals.

4- Evaluation: the error between the actual MU fiber parameters (obtained in step 1) and the estimated MU fiber parameters (obtained in step 3) is calculated.

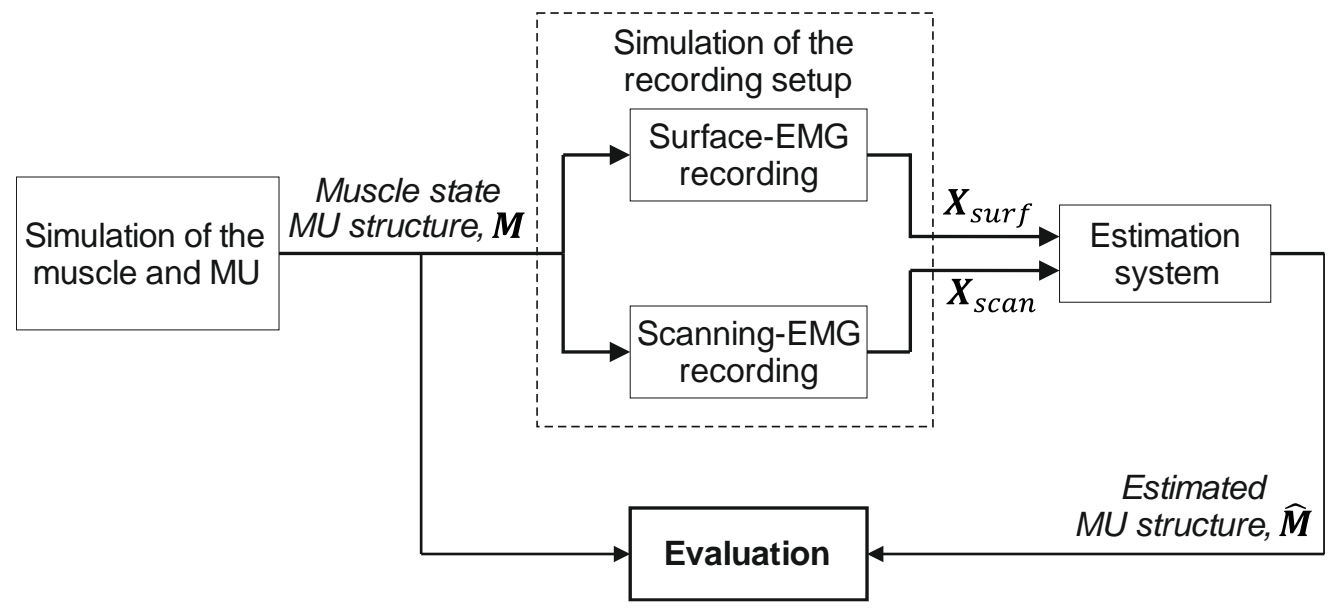

Fig. 3-30. Block diagram showing the main steps of the procedure used to evaluate the estimation system. The structure of the MU (described by the parameters, $\boldsymbol{M}$ ), and the state of the muscle are simulated. This, in turn, allows to simulate the recording of the scanning-EMG and surface-MUP signals ( $\boldsymbol{X}_{\text {scan }}$ and $\boldsymbol{X}_{\text {surf }}$ respectively). The simulated signals are used to estimate the MU structure (described by the parameters, $\widehat{\boldsymbol{M}}$ ). The evaluation itself is performed by comparing the real and the estimated MU fiber parameters. 
This procedure can be repeated in a large enough number of independent runs, so that a statistical analysis of the estimation error can be performed. In the following sections, different figures of merit quantifying the estimation performance will be described.

\subsubsection{Error of the individual MU fiber parameters}

The estimation performance can be quantified by calculating a set of errors between the real parameters of the different MU fibers and the estimated ones. Given the different nature of each parameter that characterizes the muscle fibers, a specific figure of merit has been developed for each one, taking into account their particularities.

\section{Fiber matching}

In order to quantify these errors, it is necessary to establish a correspondence between estimated and real fibers beforehand, so that the individual parameters of each real fiber can be compared with that of the corresponding estimated fiber. Estimated fibers are associated to real fibers following this procedure:

1- For each real and estimated MU fiber, the scanning-EMG SFAP is calculated. The SFAP of a MU fiber $\boldsymbol{m}$, is calculated as

$$
\boldsymbol{X}(\boldsymbol{m})=\left\{x_{n, k, p}(\boldsymbol{m})\right\}
$$

where $n$ and $k$ denote the samples along time and along the different scanning steps respectively, $p$ denote the port at which the SFAP is recorded, and where each sample value is calculated using the Dimitrov model [21] (see sections 1.3.1 and 1.4.2)

$$
x_{n, k, p}(\boldsymbol{m})=\operatorname{SFAP} \boldsymbol{m}_{\boldsymbol{m}}\left(t_{n}, x_{e_{k, p}}, y_{e_{k, p}}, z_{e_{k, p}}\right)
$$

2- Estimated-real fiber pairs are determined iteratively. The fibers to be associated at each iteration, $\{\widehat{\boldsymbol{m}}, \boldsymbol{m}\}$, are the pair for which the NMSE between their SFAPs is minimum (among those fibers that have not yet been associated), where the NMSE is calculated as

$$
\varepsilon_{a}=\frac{\sum_{n=1}^{N} \sum_{k=1}^{K} \sum_{p=1}^{P}\left(\widehat{x}_{n, k, p}(\widehat{\boldsymbol{m}})-x_{n, k, p}(\boldsymbol{m})\right)^{2}}{\sum_{n=1}^{N} \sum_{k=1}^{K} \sum_{p=1}^{P} x_{n, k, p}(\boldsymbol{m})^{2}}
$$

The iterative procedure finishes when there are no real or no estimated fibers to associate (Fig. 3-31 (a)). 
3- The fibers that remain unassociated after the previous step, are associated with an already associated fiber (Fig. 3-31 (b)), under the criterion of minimum error energy $\varepsilon_{a}$.

\section{Evaluation regions}

An important consideration to keep in mind is that the estimation error obtained for a certain association will be strongly correlated with the distance between the recording points and the MU fibers involved. MU fibers located close to the recording corridors corresponding to the different ports will be estimated with more precision than those that are farther away. For this reason, the analysis of the errors should be distance-dependent. In order to do this, the evaluation regions are defined by establishing certain limits along the $\mathrm{x}$-axis. The errors are only taken into account for those associations such that its two constituent MU fibers are located within the defined region. This allows to study the estimation quality as a function of the distance to the recording points (Fig. 3-32).

The evaluation regions are defined for the different recording configuration as follows. For the 1-port scanning-EMG, each evaluation region is defined by an interval of length $W_{x}$ measured from the port position (Fig. 3-32 (a)). In this way, the evaluation region is defined as $I_{x} \equiv\left[x_{e}, x_{e}+W_{x}\right]$. Different values of the distance $W_{x}$ define different evaluation regions, allowing a distance-dependent analysis of the estimator performance.

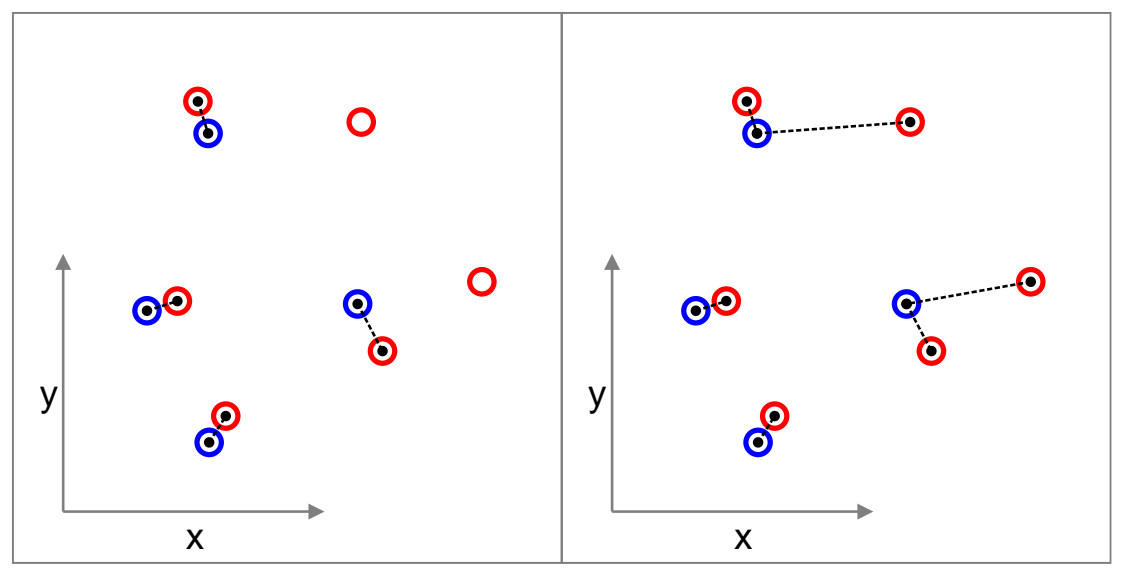

Fig. 3-31. Schematic representation of the fiber pair associations between real and estimated MU fibers. Real fibers are represented by blue circles, estimated fibers by red circles, and the fiber pair associations by black dashed lines; (a) associations formed after the step 2 of the fiber matching procedure. Note that all associations have been made without any fiber being used more than once. In this example two estimated fibers remain unassociated after this step; (b) associations formed after the step 3 of the fiber matching procedure. The unassociated fibers are associated with an already associated fiber. 
For the 2-port scanning-EMG recording configuration, each evaluation region is defined by the union of two intervals, each one corresponding to one side of the scanning needle (Fig. 3-32 (b)). Extending the intervals to both sides of the needle, each evaluation region is defined as $I_{x} \equiv\left[x_{e}-d_{n d l}-W_{x}, x_{e}-d_{n d l}\right] \cup\left[x_{e}, x_{e}+\right.$ $\left.W_{x}\right]$. Note that the positions of the two ports are $x_{e}-d_{n d l}$ and $x_{e}$ respectively, where $d_{n d l}$ is the diameter of the scanning needle.

For the 4-port scanning-EMG recording configuration, each evaluation region is defined by the union of three intervals, each of them corresponding to the left side, the middle and the right side of the scanning needles, respectively. Extending the region to these three intervals, each evaluation region is defined as $I_{x} \equiv\left[x_{e}-\Delta x / 2-\right.$ $\left.d_{n d l} / 2-W_{x}, x_{e}-\Delta x / 2-d_{n d l} / 2\right] \cup\left[x_{e}-\Delta x / 2+d_{n d l} / 2, \quad x_{e}+\Delta x / 2-d_{n d l} /\right.$ 2] $\cup\left[x_{e}+\Delta x / 2+d_{n d l} / 2, x_{e}+\Delta x / 2+d_{n d l} / 2+W_{x}\right]$. Note that, the position of the four ports are $x_{e}-\Delta x / 2-d_{n d l} / 2, x_{e}-\Delta x / 2+d_{n d l} / 2, x_{e}+\Delta x / 2-d_{n d l} / 2$, and $x_{e}+\Delta x / 2+d_{n d l} / 2$ respectively, where $\Delta x$ is the distance between the scanning needles along the $\mathrm{x}$-axis. The values of $W_{x}$ used throughout the experiments range, for all three recording configurations, between $0.05 \mathrm{~mm}$ and $1.2 \mathrm{~mm}$ in steps of $0.025 \mathrm{~mm}$.

It is important to keep in mind that, when it comes to evaluate the estimation system, we are interested in comparing the three recording configurations (the 1-port, 2-port, and 4-port scanning-EMG configurations) with the case in which the MUP has been recorded in a single position (single-point recording). The evaluation regions defined for this fourth recording configuration have an important particularity: when the MUP is recorded at a single recording position the signal has radial symmetry respect to such position. Therefore, the evaluation regions have been defined not along the $\mathrm{x}$-axis, as occurred with the rest of configurations, but as a function of the radial distance. In this way, each evaluation region corresponds to the muscle cross-section zone located within a circle of radius $W_{r}$, centered at the electrode position $\left(x_{e}, y_{e}\right)$ (Fig. 3-32 (d)). The values of $W_{r}$ range between $0.05 \mathrm{~mm}$ and $1.2 \mathrm{~mm}$ in steps of 0.025 $\mathrm{mm}$.

\section{Estimation errors}

As stated above, the estimation error of the different MU parameters is calculated for each pair of associated fibers only if these two fibers are within the evaluation region. Let $\left\{\widehat{\boldsymbol{m}}_{j_{e}}, \boldsymbol{m}_{j_{r}}\right\}$ be an estimated-real fiber pair association, with $j_{e}$ and $j_{r}$ being the indices of the estimated and real MU fiber respectively, the estimation errors corresponding to this association are

$$
\begin{aligned}
& e_{x}^{j_{e}, j_{r}}=\hat{x}_{j_{e}}-x_{j_{r}} \\
& e_{y}^{j_{e}, j_{r}}=\hat{y}_{j_{e}}-y_{j_{r}} \\
& e_{z}^{j_{e}, j_{r}}=\hat{z}_{j_{e}}-z_{j_{r}} \\
& e_{v}^{j_{e}, j_{r}}=\hat{v}_{j_{e}}-v_{j_{r}}
\end{aligned}
$$




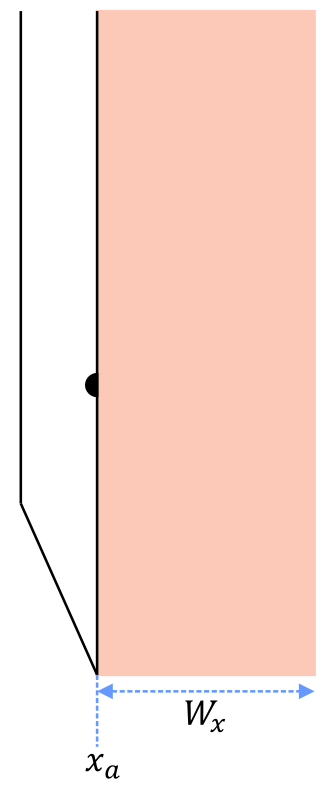

(a)

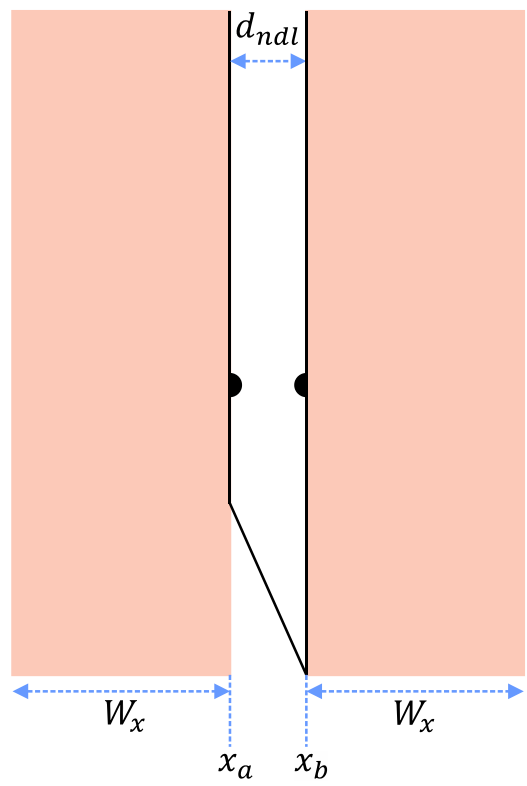

(b)

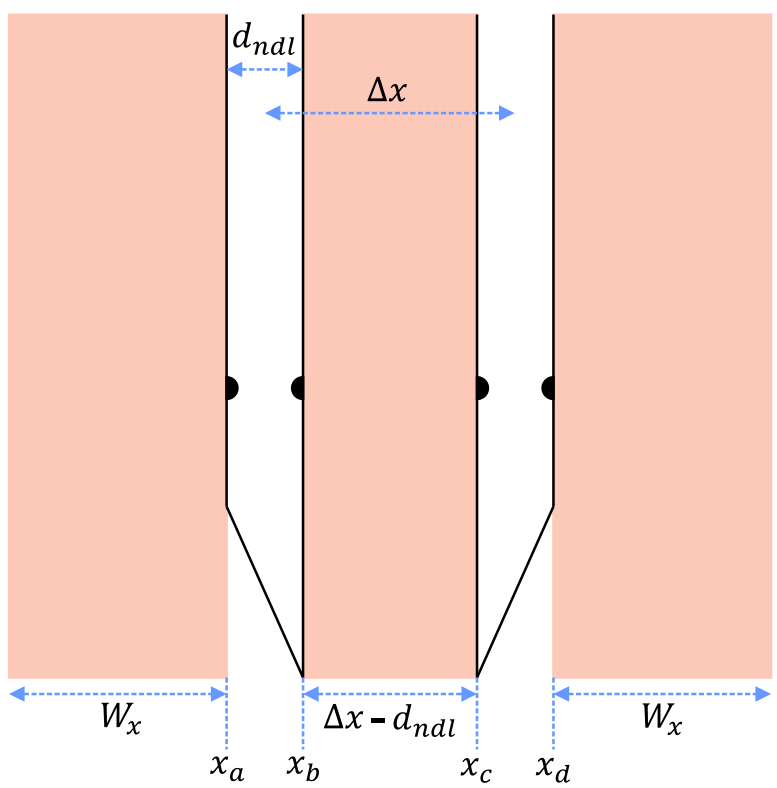

(c)

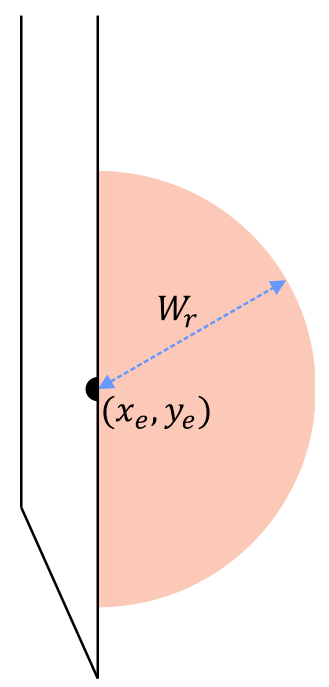

(d)

Fig. 3-32. Schematic representation of the evaluation region in the muscle cross-section plane for each recording configuration (colored area); (a) evaluation region defined for the 1-port scanning-EMG recording configuration; (b) evaluation region defined for the 2-port scanningEMG recording configuration; (c) evaluation region defined for the 4-port scanning-EMG recording configuration; (d) evaluation region defined for the single-point recording configuration. 
where $x_{j_{r}}, y_{j_{r}}, z_{j_{r}}$ and $v_{j_{r}}$, are the parameters of the real MU fiber $\boldsymbol{m}_{j_{r}} ; \hat{x}_{j_{e}}, \hat{y}_{j_{e}}, \hat{z}_{j_{e}}$ and $\hat{v}_{j_{e}}$, the parameters of the estimated MU fiber $\widehat{\boldsymbol{m}}_{j_{e}}$; and $e_{x}^{j_{e}, j_{r}}, e_{y}^{j_{e}, j_{r}}, e_{z}^{j_{e}, j_{r}}$ and $e_{v}^{j_{e}, j_{r}}$, their corresponding estimation errors.

In addition to these four estimation errors, we are also interested in quantifying the error in the neuromuscular junction positions, but regardless the error component associated to an incorrect estimation of the IZ average position. In order to achieve this, we define the parameter $\zeta$ as the neuromuscular junction position, once the bias of all $z$ positions within the evaluation region is subtracted. Given the fiber association $\left\{\widehat{\boldsymbol{m}}_{j_{e}}, \boldsymbol{m}_{j_{r}}\right\}$, the actual and estimated parameter $\zeta$ are

$$
\begin{aligned}
& \zeta_{j_{r}}=z_{j_{r}}-\mu_{z} \\
& \hat{\zeta}_{j_{e}}=\hat{z}_{j_{e}}-\mu_{\hat{z}}
\end{aligned}
$$

respectively, where $\mu_{z}$ and $\mu_{\hat{z}}$ are the bias of the $z$ positions for the actual and the estimated MU respectively, and can be calculated as

$$
\begin{gathered}
\mu_{z}=\frac{\sum_{\forall\left\{\widehat{\boldsymbol{m}}_{j_{e}}, \boldsymbol{m}_{j_{r}}\right\} \mid \hat{x}_{j_{e}} \in I_{x} \wedge x_{j_{r}} \in I_{x}} z_{j_{r}}}{\sum_{\forall\left\{\hat{\boldsymbol{m}}_{j_{e}}, \boldsymbol{m}_{j_{r}}\right\} \mid \hat{x}_{j_{e}} \in I_{x} \wedge x_{j_{r}} \in I_{x}}} \\
\mu_{\hat{z}}=\frac{\sum_{\forall\left\{\hat{\boldsymbol{m}}_{j_{e}}, \boldsymbol{m}_{j_{r}}\right\} \mid \hat{x}_{j_{e}} \in I_{x} \wedge x_{j_{r}} \in I_{x}} \hat{z}_{j_{e}}}{\sum_{\forall\left\{\hat{\boldsymbol{m}}_{j_{e}}, \boldsymbol{m}_{j_{r}}\right\} \mid \hat{x}_{j_{e}} \in I_{x} \wedge x_{j_{r}} \in I_{x}}}
\end{gathered}
$$

where $I_{x}$ is the evaluation region. Note that the bias is computed using only the values of $z$ corresponding to those association pairs $\left\{\widehat{\boldsymbol{m}}_{j_{e}}, \boldsymbol{m}_{j_{r}}\right\}$ for which its two constituent fibers are within $I_{x}$. The estimation error of the parameter $\zeta$ is calculated, for the association $\left\{\widehat{\boldsymbol{m}}_{j_{e}}, \boldsymbol{m}_{j_{r}}\right\}$, as

$$
e_{\zeta}^{j_{e}, j_{r}}=\hat{\zeta}_{j_{e}}-\zeta_{j_{r}}
$$

A last figure of merit has been defined, which is the error in the estimation of the radial distance between the fibers and the recording point. We are interested in calculating this error specifically for the single-point recording configuration. This is because the potential recorded in a single position does not provide enough information to determine univocally the position of the muscle fibers. As stated in previous sections, only the radial distance can be potentially determined (see section 3.2.3). The so called radial distance estimation error is calculated as

$$
e_{r}^{j_{e}, j_{r}}=\hat{r}_{j_{e}}-r_{j_{r}}
$$


where $r_{j_{r}}$ is the radial distance between the real fiber and the recording point $\left(x_{e}, y_{e}\right)$

$$
r_{j_{r}}=\sqrt{\left(x_{j_{r}}-x_{e}\right)^{2}+\left(y_{j_{r}}-y_{e}\right)^{2}}
$$

and $\hat{r}_{j_{e}}$ is the radial distance between the estimated fiber and the recording point

$$
\hat{r}_{j_{e}}=\sqrt{\left(\hat{x}_{j_{e}}-x_{e}\right)^{2}+\left(\hat{y}_{j_{e}}-y_{e}\right)^{2}}
$$

where $x_{j_{r}}$ and $y_{j_{r}}$ are the positions along the $\mathrm{x}$ - and $\mathrm{y}$-axis of the real MU fiber $\boldsymbol{m}_{j_{r}}$; and $\hat{x}_{j_{e}}$ and $\hat{y}_{j_{e}}$ that of the estimated MU fiber $\widehat{\boldsymbol{m}}_{j_{e}}$.

\subsubsection{Error of MU global parameters}

Another useful way to analyze the estimation performance is based on the characterization of certain global parameters of the MU, such as the distribution of the MFCV's, the distribution of the neuromuscular junction positions in the IZ, or the MU fiber density. These global parameters can be extracted for both the estimated and the real MU, so that the estimation performance can be assessed by quantifying the discrepancies between these two cases. The interest of using these global parameters lies in their clinical relevance. Note that an abnormal fiber density, an abnormal distribution of the MFCV's, or an abnormal distribution of the neuromuscular junctions may be indicative of a pathological condition [127].

An important consideration is that, in the estimation case, the calculation of the global MU parameters must not be performed using the entire MU, but a certain region of it, so that the estimated parameters can be considered to be reliable. Note that the parameters of the fibers located far away from the recording points are expected to be estimated with very low accuracy, so taking them into account for the calculation will lead to a larger error in the MU global parameters. The region in which the MU global parameters are calculated, $I_{x}$, is defined by the union of several intervals along the xaxis, as described in section 3.4.1.

\section{MFCV distribution}

The distribution of the MFCV's of the MU can be characterized by its mean and its standard deviation [87]. For the real MU, the mean and the standard deviation of the MFCV's (denoted by $V_{m}$ and $V_{s}$ respectively) are calculated as

$$
V_{m}=\frac{1}{J} \sum_{j=1}^{J} v_{j}
$$




$$
V_{s}=\sqrt{\frac{1}{J} \sum_{j=1}^{J}\left(v_{j}-V_{m}\right)^{2}}
$$

where $J$ is the total number of real MU fibers. For the estimated MU, the mean and the standard deviation of the MFCV's (denoted by $\widehat{V}_{m}$ and $\widehat{V}_{s}$ respectively) are calculated as

$$
\begin{aligned}
& \hat{V}_{m}\left(W_{x}\right)=\frac{1}{\hat{J}\left(W_{x}\right)} \sum_{j \mid x_{j} \in I_{x}\left(W_{x}\right)} \hat{v}_{j} \\
& \hat{V}_{s}\left(W_{x}\right)=\sqrt{\frac{1}{\hat{J}\left(W_{x}\right)} \sum_{j \mid x_{j} \in I_{x}\left(W_{x}\right)}\left(\hat{v}_{j}-\hat{V}_{m}\left(W_{x}\right)\right)^{2}}
\end{aligned}
$$

where $\hat{J}$ is the total number of estimated fibers within $I_{x}$. Note that, for the real MU all fibers of the MU are used in the calculation, while for the estimated MU, only those located within the region $I_{x}$ are used. In this way, the estimated mean and standard deviation are a function of the region $I_{x}$, which in turn is function of $W_{x}$ (see section 3.4.1). The estimation error of the MFCV mean is defined as

$$
e_{V m}\left(W_{x}\right)=\widehat{V}_{m}\left(W_{x}\right)-V_{m}
$$

and the estimation error of the MFCV standard deviation is defined as

$$
e_{V S}\left(W_{x}\right)=\widehat{V}_{s}\left(W_{x}\right)-V_{S}
$$

\section{Neuromuscular junctions distribution}

The distribution of the neuromuscular junction positions in the IZ can be characterized by identifying the sub-bands within the IZ where the neuromuscular junctions are located. This characterization is justified by studies suggesting the organization of the IZ in different sub-bands $[120,122,87]$. To characterize the IZ subbands, an indexing function, $I(z)$, is calculated, indicating the positions along the $\mathrm{z}$ axis that are considered as part of a sub-band $(I(z)=1)$, and the positions that are not $(I(z)=0)$ (Fig. 3-33).

To calculate the sub-band function $I(z)$, the individual neuromuscular positions are convolved with a rectangular function of width $w_{z}=0.8 \mathrm{~mm}$. The value that $I(z)$ takes at each position $z$ is calculated from the result of this convolution. For positions where the result is larger than $0, I(z)=1$; otherwise, $I(z)=0$. Mathematically, the calculation of the sub-band function for the real MU is described as 


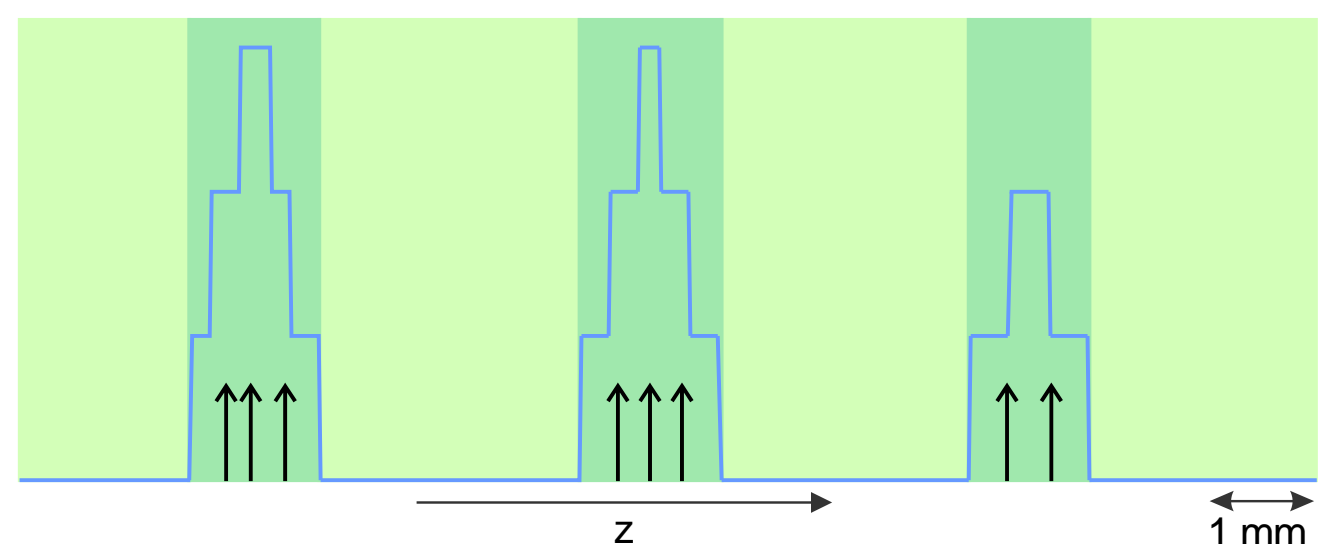

Fig. 3-33. Schematic representation of how the IZ sub-band function $I(z)$ is calculated. The black arrows represent the positions of the neuromuscular junctions along the $z$-axis. These positions are convolved by a rectangular function, resulting in the function depicted with a gray line. The sub-bands, $I(z)$, are calculated from the result of this convolution. The $z$ positions in which this result is higher than 0 are considered to be within a sub-band, and therefore $I(z)=$ 1 (dark green); otherwise, the $z$ positions are considered outside the sub-bands, and therefore $I(z)=0$ (light green)

$$
I(z)=\mathrm{H}\left(\Pi\left(\frac{z}{w_{z}}\right) * \sum_{j=1}^{J} \delta\left(z-z_{j}\right)\right)
$$

where

$$
\Pi\left(\frac{z}{w_{z}}\right)= \begin{cases}1 & |z| \leq \frac{w_{z}}{2} \\ 0 & |z|>\frac{w_{z}}{2}\end{cases}
$$

is the rectangular function, and $\mathrm{H}(\cdot)$ is the Heaviside step function

$$
\mathrm{H}(a)= \begin{cases}1 & a>0 \\ 0 & a \leq 0\end{cases}
$$

In the case of the estimated MU, the sub-band function is calculated as

$$
\hat{I}\left(z, W_{x}\right)=\mathrm{H}\left(\Pi\left(\frac{z}{w_{z}}\right) * \sum_{j \mid x_{j} \in I_{x}\left(W_{x}\right)} \delta\left(z-\hat{z}_{j}\right)\right)
$$


The discrepancies between the real and the estimated sub-band functions can be quantified by means of two merit figures. The first figure, the IZ excess, is the proportion of the IZ that has been wrongly considered inside the sub-bands

$$
E_{I}\left(W_{x}\right)=\frac{\int_{Z \mid I(z)=0 \wedge \hat{I}\left(z, W_{x}\right)=1} d z}{\int_{Z \mid I(z)=1} d z}
$$

And the second figure, the IZ defect, is the proportion of the IZ that has been wrongly considered outside of the sub-bands

$$
D_{I}\left(W_{x}\right)=\frac{\int_{z \mid I(z)=1 \wedge \hat{I}\left(z, W_{x}\right)=0} d z}{\int_{Z \mid I(z)=1} d z}
$$

\section{Fiber density}

The global MU fiber density, defined for our purposes as the average number of MU fibers per $\mathrm{mm}^{2}$, can be calculated as follows. The first step is to calculate a local fiber density in the muscle cross-section as the convolution of the fiber positions $\left(x_{j}, y_{j}\right)$ with a two-dimensional Gaussian kernel $K_{m d f}(x, y)$, where this kernel is symmetrical with a standard deviation of $0.141 \mathrm{~mm}$, and an amplitude such that its integral in the muscle cross-section plane is 1 . For the real MU, the local fiber density can be mathematically expressed as

$$
f_{D}(x, y)=K_{m d f}(x, y) * \sum_{j=1}^{J} \delta\left(x-x_{j}\right) \cdot \delta\left(y-y_{j}\right)
$$

and for the estimated MU as

$$
\widehat{f_{D}}(x, y)=K_{m d f}(x, y) * \sum_{j=1}^{\hat{\jmath}} \delta\left(x-\hat{x}_{j}\right) \cdot \delta\left(y-\hat{y}_{j}\right)
$$

Note that all fibers of the MU are taken into account to calculate the local fiber density, both in the real and the estimated case.

The local fiber density provides a density value in each position of the muscle cross-section. However, we are interested in a global measure of such density. To obtain this global measure, the local density is averaged within a region of the musclecross-section where the estimation is considered to be reliable. This region is the one already defined in section 3.4.1, but delimited with the bounds of the MU territory (Fig. 3-34). Then, for the real MU, the region used to compute the global fiber density is 


$$
\mathfrak{I}_{I_{x}}\left(W_{x}\right) \equiv\left(I_{x}\left(W_{x}\right) \cap\left[x_{M U T}^{\min }, x_{M U T}^{\max }\right]\right) \times\left(\left[y_{M U T}^{\min }\left(W_{x}\right), y_{M U T}^{\max }\left(W_{x}\right)\right]\right)
$$

where $x_{M U T}^{\min }$ and $x_{M U T}^{\max }$ are the territory bounds of the real MU along the $\mathrm{x}$-axis, and $y_{M U T}^{\min }\left(W_{x}\right)$ and $\hat{y}_{M U T}^{\max }\left(W_{x}\right)$ that of along the y-axis. The territory bounds $x_{M U T}^{\min }$ and $x_{M U T}^{\max }$ are calculated as the minimum and maximum $x$ position for which there is any $y$ position satisfying

$$
f_{D}(x, y) \geq \frac{\max \left(K_{m d f}(x, y)\right)}{2}
$$

The territory bounds $y_{M U T}^{\min }\left(W_{x}\right)$ and $y_{M U T}^{\max }\left(W_{x}\right)$ are calculated similarly, but considering only the region defined by $I_{x}\left(W_{x}\right)$ (Fig. 3-34). These bounds are calculated as the minimum and maximum $y$ position for which there is any $x$ position within $I_{x}\left(W_{x}\right)$, satisfying (3-57).

Analogously, for the estimated MU, the evaluation region is

$$
\widehat{\mathfrak{I}}_{I_{x}}\left(W_{x}\right) \equiv\left(I_{x}\left(W_{x}\right) \cap\left[\hat{x}_{M U T}^{\min }, \hat{x}_{M U T}^{\max }\right]\right) \times\left(\left[\hat{y}_{M U T}^{\min }\left(W_{x}\right), \hat{y}_{M U T}^{\max }\left(W_{x}\right)\right]\right)
$$

where $\hat{x}_{M U T}^{\min }, \hat{x}_{M U T}^{\max }, \hat{y}_{M U T}^{\min }\left(W_{x}\right)$ and $\hat{y}_{M U T}^{\max }\left(W_{x}\right)$ are the territory bounds of the estimated $\mathrm{MU}$, and where these bounds are calculated analogously to the case of the real MU, under the criterion

$$
\widehat{f_{D}}(x, y) \geq \frac{\max \left(K_{m d f}(x, y)\right)}{2}
$$

After calculating $\widetilde{I}_{I_{x}}\left(W_{x}\right)$ and $\widehat{\mathfrak{I}}_{I_{x}}\left(W_{x}\right)$, the global fiber density is obtained by averaging the local fiber density in the region $\mathfrak{J}_{I_{x}}\left(W_{x}\right)$ for the real MU, and in the region $\widehat{\mathfrak{I}}_{I_{x}}\left(W_{x}\right)$ for the estimated one. Mathematically, the global fiber density is expressed for the real MU as

$$
D\left(W_{x}\right)=\frac{\int_{\widetilde{I}_{I_{x}}\left(W_{x}\right)} f_{D}(x, y) d x d y}{\int_{\mathfrak{I}_{I_{x}}\left(W_{x}\right)} d x d y}
$$

where $\int_{\mathfrak{I}_{I_{x}}\left(W_{x}\right)} d x d y$ is the area of the region $\mathfrak{I}_{I_{x}}\left(W_{x}\right)$. For the estimated MU the global fiber density is expressed as

$$
\widehat{D}\left(W_{x}\right)=\frac{\int_{\widetilde{\mathfrak{I}}_{I_{x}}\left(W_{x}\right)} \widehat{f_{D}}(x, y) d x d y}{\int_{\widetilde{\mathfrak{I}}_{I_{x}}\left(W_{x}\right)} d x d y}
$$


where $\int_{\widetilde{\mathfrak{I}}_{I_{x}}\left(W_{x}\right)} d x d y$ is the area of the region $\widehat{\mathfrak{I}}_{I_{x}}\left(W_{x}\right)$. Finally, the error in the estimation of the global MU fiber density is calculated as

$$
e_{D}\left(W_{x}\right)=\widehat{D}\left(W_{x}\right)-D\left(W_{x}\right)
$$

It is important to remark that for the calculation of the global density in the real MU, an averaging region $\mathfrak{I}_{I_{x}}\left(W_{x}\right)$ that varies as a function of $W_{x}$ has been chosen instead of the entire MU territory. This is because the local fiber density will vary in a notable and systematic way along the $\mathrm{x}$-axis, as a consequence of the uneven distribution of the MU fibers and the ploughing effect caused by the needle (see section 1.4.3). In this way, there is no point in computing an error between the real and the estimated fiber density, unless the densities are computed under the same restrictions in the $\mathrm{x}$-axis.

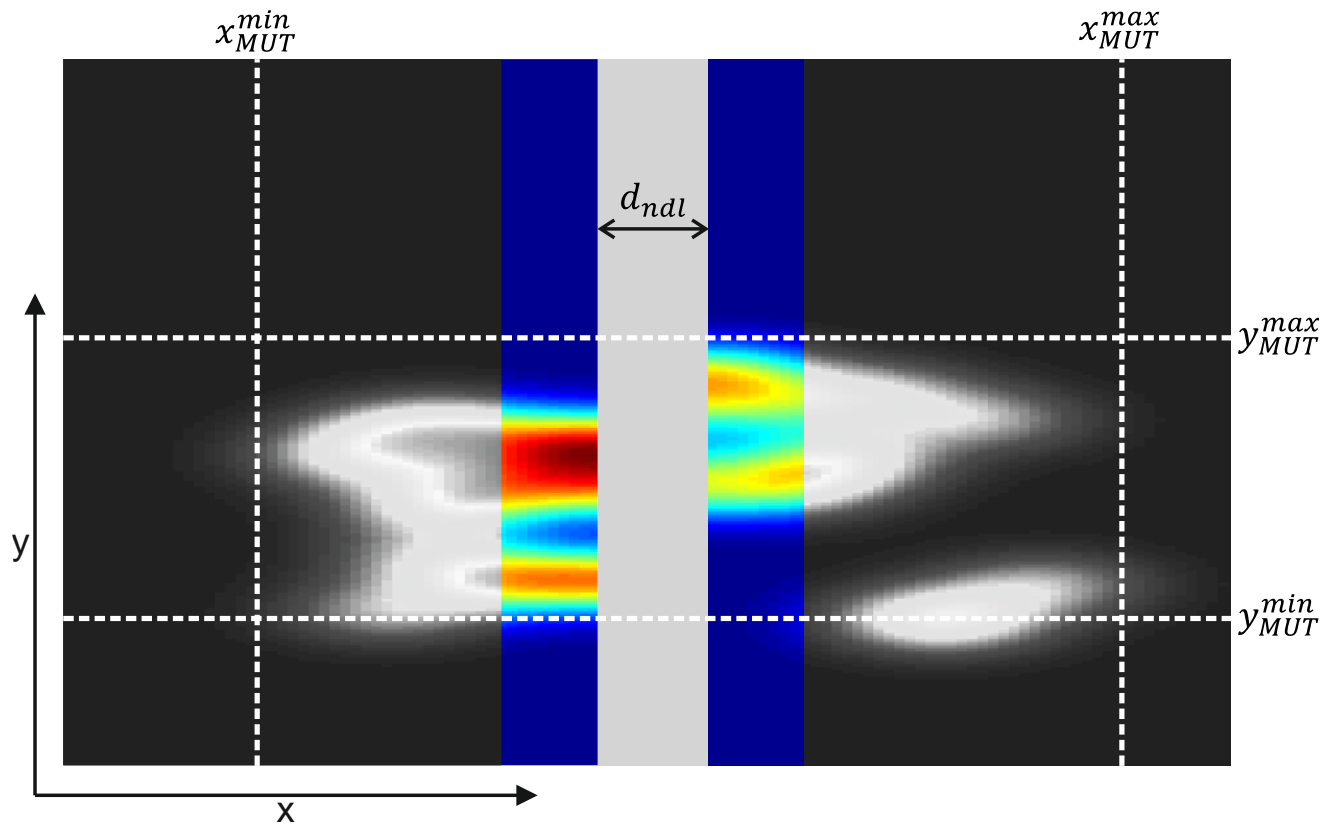

Fig. 3-34. Example of how the region to calculate the global fiber density $\mathfrak{I}_{I_{x}}$, is determined for a 2-port scanning-EMG recording configuration. The determination of $\mathfrak{J}_{I_{x}}$ is performed from a previously calculated local fiber density, and from the region $I_{x}$. The local fiber density is represented by a color map, which is colored in a grayscale outside $I_{x}$, and in color inside of it. The horizontal dashed lines represent the bounds of the territory along the y-axis, $y_{M U T}^{\min }$ and $y_{M U T}^{\max }$, which are calculated taking into account the local fiber density within $I_{x}$. The region $\mathfrak{J}_{I_{x}}$ is the part of the MU territory located inside $I_{x}$, and delimited by $y_{M U T}^{\min }$ and $y_{M U T}^{\max }$. The vertical dashed lines represent the bounds of the territory along the $\mathrm{x}$-axis, $x_{M U T}^{\min }$ and $x_{M U T}^{\max }$. In this example, as these bounds are outside $I_{x}$, they do not delimit the region $\widetilde{J}_{I_{x}}$. 
upna

Universidad Púbilica de Navara
Natarmaako Unibersitate Publikoa 


\section{Design of the estimation system}

So far, the system proposed to estimate the MU fiber parameters has been described for an ideal situation, without considering many of the particular problems that occur in a more realistic scenario. In this chapter, the most relevant of these problems are analyzed, and the pertinent modifications of the system are described. The particular scenarios taken into account include: the effect of the indetermination of the potential initiation instant; the effect of noise in the recording procedure of the scanning-EMG signal; the effect of the cannula in such recording; and the effect of using concentric needle electrodes, instead of single fiber ones. 
upna

Universidad Púbilica de Navara
Natarmaako Unibersitate Publikoa 


\subsection{The indetermination of the potential initiation instant}

The starting time of a real scanning-EMG signal is chosen arbitrarily, depending on the recording window used to capture each MUP trace during the recording procedure. This starting time does not necessarily coincide with the time of the firing of the MU, i.e., with the time instant in which the potentials of the MU SFAPs have been generated at the neuromuscular junctions. This fact implies an additional difficulty to solve the inverse problem, since knowing this time instant is a necessary condition for a correct estimation of some of the MU fiber parameters.

Note that, in the model used to simulate the scanning-EMG signal, the starting time does coincide with the instant in which the potential is initiated. In order for the estimation procedure to compare the recorded scanning-EMG signal with the one simulated with the model, it is necessary to align the recorded signal so that its starting time also coincides with the potential initiation instant. The problem is that this instant cannot be deduced by analyzing the scanning-EMG signal, at least not for the types of electrodes (SF ports) proposed for the estimation system.

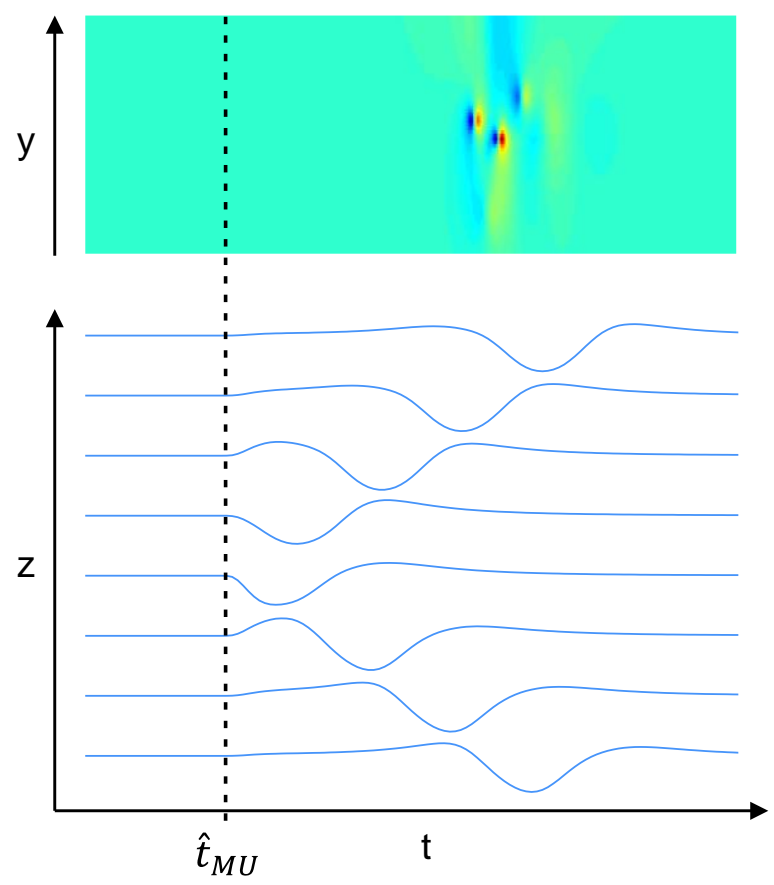

Fig. 4-1. Representation of the scanning-EMG and surface-MUP signals (upper and lower plot respectively) along a common time axis. The potential initiation instant (dashed vertical line), is estimated from the surface-MUP signal, and is used as the starting time of the scanningEMG signal. 
A possible way to estimate the potential initiation instant could be the use monopolar needle electrodes [61] to record the scanning-EMG signal (see section 1.2.1), instead of SF electrodes. The reason for this is that the monopolar needle electrode uses a separate surface electrode as reference, instead of the cannula. This allows more distant events like the potential initiation to be detected in the recorded signal. Given a recorded MUP, a change in the baseline slope can be observed at the potential initiation instant, which allows a precise identification [61]. However, the monopolar electrode has also important limitations. This kind of electrode has very low selectivity, and also usually introduces a large amount of noise in the recorded signal. To reduce the noise, the MUPs recorded with a monopolar needle are usually averaged across a very large number of discharges. Note that this would make the recording procedure of the scanning-EMG signal to take too long time to be viable in a real scenario. Taking this in mind, we consider that the monopolar needle is not suitable for solving the inverse problem. Therefore, the use of this type of electrode as a solution of the problem exposed here is discarded.

The solution adopted to determine the potential initiation instant is to use the recording of the surface-MUP for this purpose. Remember that this signal is recorded by using a linear array of surface electrodes, placed on the skin along the direction of the muscle fibers (see section 3.3.1). To obtain the potential initiation instant from the recorded surface-MUP, the algorithm described in section 2.2.2 is used. The potential initiation instant determined for the surface-MUP recording can be set for the scanning-EMG signal, since the recordings of the scanning-EMG and surface-MUP signals are synchronized (Fig. 4-1).

\subsubsection{Estimation error analysis of the potential initiation instant}

The method used to determine the potential initiation instant introduces some amount of error, which in turn implies an error in the estimation of the MU fiber parameters. For this reason, it is important to characterize the error of the potential initiation estimation. With this characterization in mind, the following simulation experiment is executed in 100 independent runs:

1- A muscle and its constituent MUs are simulated using the model described in section 1.4.1, with the parameters in Table 1-2. The MU chosen to estimate the potential initiation is the smallest one whose territory is traversed by a hypothetical scanning corridor located on the middle of the muscle.

2- The recording of the surface-EMG signal is simulated from a linear array of surface electrodes disposed along the direction of the muscle fibers (the z-axis). The model described in section 1.4.7 is used for this purpose. The electrodes of the array are placed from $z=-35 \mathrm{~mm}$ to $z=35 \mathrm{~mm}$ along the z-axis, with an inter-electrode distance of $10 \mathrm{~mm}$; the IZ is located at $z=0 \mathrm{~mm}$. In the direction perpendicular to the muscle fibers (the $\mathrm{x}$-axis), the electrode array is positioned in the middle of the muscle. The surface electrodes are circular in shape, with a 
diameter of $5 \mathrm{~mm}$. The surface-EMG signal is simulated for a force level of 3.5\% MVC.

3- The surface-MUP of the MU under analysis is obtained by applying spike-trigger averaging to the surface-EMG signal, as described in section 2.2.1.

4- The potential initiation instant is estimated from the surface-MUP using the algorithm described in section 2.2.2.

5- The estimation error is calculated as the difference between the estimated potential initiation instant and the actual one.

Fig. 4-2 shows the histogram of the error in the estimation of the potential initiation obtained from the experiments. The error distribution can be characterized by its mean (estimation bias of $-0.492 \mathrm{~ms}$ ) and its standard deviation (standard error of $0.402 \mathrm{~ms}$ ).

\subsubsection{Effect of the potential initiation error on the fiber parameter estimation}

The next step is to analyze how an error in the estimated potential initiation instant produces an error in the estimated fiber parameters. This analysis of the error propagation assumes that the only source of error is the misestimation of the potential initiation. Therefore, the analysis assumes that the estimation procedure is able to perfectly reproduce the signal waveform of each fiber contribution, and that this waveform has enough information to univocally estimate the MU fiber parameters. Remember also that, in order to solve the inverse problem, we are assuming that all the MU fibers start to depolarize at the same time.

Given a recorded scanning-EMG signal for which the MU fiber parameters are being estimated, and a simulated signal corresponding to the estimation of these parameters, let $t_{M U}$ and $\hat{t}_{M U}$ be the actual and estimated potential initiation instant of the recorded signal, respectively. Then, for the recorded signal, the propagation delay corresponding to the $j$ th MU fiber is

$$
\delta t_{j}=t_{j}-\hat{t}_{M U}
$$

where $t_{j}$ is the time instant at which the propagation front of the $j$ th SFAP arrives to the electrode position in the recorded signal. For the simulated signal, the propagation delay is

$$
\widehat{\delta t_{j}}=\hat{t}_{j}-t_{M U}
$$

where $\hat{t}_{j}$ is the instant at which the $j$ th SFAP arrives to the electrode in the simulated signal. Since it is assumed that each fiber contribution is perfectly adjusted in the estimation procedure, the time instant at which a certain signal contribution arrives to 


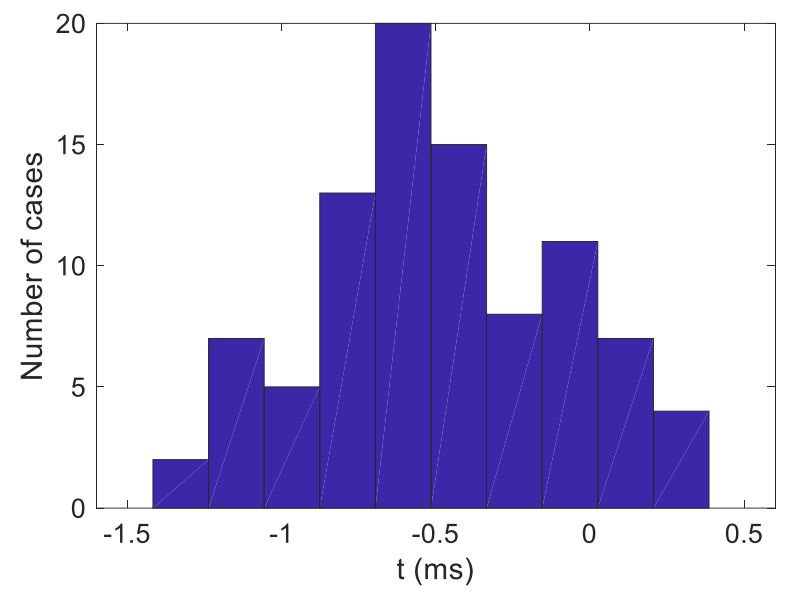

Fig. 4-2. Histogram of the error in the estimation of the potential initiation

the electrode must be the same for the real and the simulated case. Therefore $\hat{t}_{j}=t_{j}$ is fulfilled, so it follows that

$$
\hat{t}_{M U}+\delta t_{j}=t_{M U}+\widehat{\delta} t_{j}
$$

If we assume that an error $\varepsilon_{t}=\hat{t}_{M U}-t_{M U}$ has been made in the estimation of the potential initiation, the previous expression results in

$$
\delta t_{j}=\widehat{\delta t}_{j}-\varepsilon_{t}
$$

which means that the propagation delays associated with the actual and estimated case differ in $\varepsilon_{t}$. The propagation delay of a certain fiber contribution can be related to the MFCV and the neuromuscular junction position of such fiber. In this way, (4-4) can be expressed as

$$
\frac{z_{e}-z_{j}}{v_{j}}=\frac{z_{e}-\hat{z}_{j}}{\hat{v}_{j}}-\varepsilon_{t}
$$

where $z_{e}$ is the position of the electrode; $z_{j}$ and $\hat{z}_{j}$ are the actual and estimated neuromuscular junction position of the $j$ th MU fiber, respectively; and, $v_{j}$ and $\hat{v}_{j}$ are the actual and estimated MFCV of the $j$ th MU fiber, respectively. At this point, it is important to keep in mind that $z_{j}$ only affects to the time delay of its corresponding signal contribution (the effect in the shape of the signal contribution is negigible), while $v_{j}$ affects not only to the time delay, but also to the shape of such contribution. Here we assume that the shape of each fiber contribution has been perfectly fitted in the estimation procedure, hence allowing a perfect estimation of the parameter $v_{j}$; i.e., 
we have that $v_{j}=\hat{v}_{j}$. Under this assumption, (4-5) can be simplified, resulting in

$$
\hat{z}_{j}=z_{j}-\varepsilon_{t} v_{j}
$$

Therefore, when the only source of error is that corresponding to the estimation of the potential initiation, an error of this parameter necessarily implies an error in the estimation of the neuromuscular junction positions, which is also proportional to the MFCV.

To understand the magnitude of the error in the estimation of $z_{j}$, we can give specific values to (4-6). Suppose that $v_{j}=4 \mathrm{~m} / \mathrm{s}$, and that the error made in the estimation of the potential initiation is $\varepsilon_{t}=-1 \mathrm{~ms}$. Note that this is a particularly unfavorable case, given the distribution of the error characterized in the previous section (Fig. 4-2). Then, the error made in the estimation of the neuromuscular junction position is $\hat{z}_{j}-z_{j}=4 \mathrm{~mm}$. In the biceps brachii, the IZ has a width ranged between 6 and $10 \mathrm{~mm}[4,3]$. This IZ is, in turn, considered to be formed by several narrow subbands that could be of a width around $1 \mathrm{~mm}$ [87]. Therefore, the error in the estimation of $z_{j}$ is very large when compared to those physiological values.

Although it cannot be guaranteed that the absolute position of the neuromuscular junctions can be accurately estimated, it is possible that the relative position within the IZ can be estimated with sufficient precision. Note that the physiological information regarding the distribution of the neuromuscular junctions within the IZ is, in fact, more valuable than the specific distances between these neuromuscular junctions and the electrode. We are therefore interested in analyzing to what extent the error in the estimated potential initiation produces an error in estimating the distance between two neuromuscular junctions. Let $\hat{z}_{j}$ and $\hat{z}_{i}$ be the estimated neuromuscular junction positions of the $j$ th and $i$ th MU fiber respectively, then the estimated distance between both neuromuscular junctions is

$$
\widehat{\delta z}_{j, i}=\hat{z}_{j}-\hat{z}_{i}
$$

Using (4-6), we have that

$$
\widehat{\delta z}_{j, i}=\left(z_{j}-z_{i}\right)-\varepsilon_{t}\left(v_{j}-v_{i}\right)
$$

which can be expressed as

$$
\widehat{\delta z}_{j, i}=\delta z_{j, i}-\varepsilon_{t} \delta v_{j, i}
$$

where $\delta z_{j, i}$ is the actual distance between the neuromuscular junctions, and $\delta v_{j, i}$ the difference between the MFCV of the $j$ th and $i$ th MU fiber. We can give specific values to this expression. Suppose again that $\varepsilon_{t}=-1 \mathrm{~ms}$, and that the difference between the 
MFCVs is $\delta v_{j, i}=0.5 \mathrm{~m} / \mathrm{s}$. Note that this MFCV difference corresponds to an unfavorable case, as in non-pathological MUs the distribution of the MFCVs within the MU presents a small coefficient of variation [87]. For these values, the error when estimating the distance between the two neuromuscular junctions is $\widehat{\delta z}_{j, i}-\delta z_{j, i}=0.5$ $\mathrm{mm}$, which is below the width of the sub-bands of the IZ. This example suggests that an imperfect estimation of the potential initiation instant does not in itself prevent an estimation of the neuromuscular junction positions accurate enough to characterize the IZ in its constituent sub-bands. The performance of the estimation system, when considering not only the error associated to a misestimation of the potential initiation but also other error sources, will be analyzed in the next chapter when the overall experiment results will be presented. 


\subsection{Noise and processing in the estimation system}

When it comes to estimate the parameters of the MU fibers from the recorded scanning-EMG signal, the presence of noise and interference in such signals will adversely affect the estimation. To mitigate the effect of noise, different processing steps can be applied (see section 2.1.1 for a general overview). In this section, the influence of noise and processing stages in the parameter estimation is analyzed from a theoretical and experimental point of view, and an overall processing scheme to be integrated in the estimation system is proposed.

\subsubsection{Metric adapted to the noise present in the scanning-EMG signal}

As explained in the previous chapter, the estimation system estimates the MU fiber parameters by minimizing the following error function:

$$
\varepsilon(\widehat{\boldsymbol{M}})=\sum_{n=1}^{N} \sum_{k=1}^{K} \sum_{p=1}^{P}\left(\hat{x}_{n, k, p}(\widehat{\boldsymbol{M}})-x_{\text {scan }_{n, k, p}}\right)^{2}
$$

where $\widehat{\boldsymbol{X}}(\widehat{\boldsymbol{M}})=\left\{\hat{x}_{n, k, p}(\widehat{\boldsymbol{M}})\right\}$, and $\boldsymbol{X}_{\text {scan }}=\left\{x_{\text {scan }_{n, k, p}}\right\}$ are the simulated and recorded scanning-EMG signals respectively, with $n$ and $k$ denoting the samples along time and along the different scanning steps respectively, and $p$ denoting the port at which the MUP is recorded; and $\widehat{M}$ is the MU fiber parameters used to simulate the scanning-EMG signal. This error function corresponds to the squared distance in a Euclidean metric space, which is optimal when the noise present in the recorded scanning-EMG signal is AWGN. An optimal metric means that the minimization of the distance between the actual and the estimated signal implies that the estimated MU parameters maximize the a posteriori probability density of the MU parameters (3-20). For a detailed explanation of how the Euclidean metric is derived from the assumption that the noise follows an AWGN distribution see sections 3.3.2 and 3.3.3.

However, the Euclidean metric is no longer optimal as soon as the statistical properties of the noise differ from those of the AWGN, as occurs in real scanningEMG recordings. The noise in the recorded signal is not Gaussian, and also presents correlations between different samples of the recording. Remember that three main noise sources are present in scanning-EMG recordings. First, a slow time varying baseline noise caused by needle or muscle movements [42, 44, 90]; second, high frequency noise introduced by the instrumentation system; and third, artifacts derived from the coactivation of nearby MUs during the recording of the MUP being tracked $[42,44,90]$. An optimal estimation of the MU parameters would imply replacing the Euclidean metric with a metric adapted to the statistical properties of the afore mentioned sources of noise. However, a full characterization of such noise is very difficult to achieve in practice, as it requires to describe the noise uncertainties as a 
multivariate probability density in a signal space of $N K P$ dimensions. Moreover, the characterization will strongly depend on the muscle, insertion point, activation level, etc. Instead, the properties of noise can be roughly described by assuming that such probability density is a multivariate Gaussian distribution with a covariance matrix, $\boldsymbol{C}_{\sigma}$. Under this assumption, the noise is still considered to be Gaussian, but taking into account the possible correlations that may exist between the different spatio-temporal samples of the recording. In this way, the error function to be minimized would be [133]:

$$
\varepsilon(\widehat{\boldsymbol{M}})=\left(\widehat{X}(\widehat{\boldsymbol{M}})-X_{\text {scan }}\right)^{T} \boldsymbol{C}_{\sigma}^{-1}\left(\widehat{X}(\widehat{\boldsymbol{M}})-X_{\text {scan }}\right)
$$

where $\hat{X}(\widehat{M})$ and $X_{\text {scan }}$ are the simulated and the recorded scanning-EMG signals respectively, both structured as column vectors of NKP length. Note that these signals are calculated as $\widehat{X}(\widehat{\boldsymbol{M}})=\operatorname{vec}(\widehat{\boldsymbol{X}}(\widehat{\boldsymbol{M}}))$ and $X_{\text {scan }}=\operatorname{vec}\left(\boldsymbol{X}_{\text {scan }}\right)$, where $\operatorname{vec}(\cdot)$ is the operation that transforms a tridimensional matrix of $N \times K \times P$ size into a column vector of length $N K P$. Note that this error function corresponds to the squared distance in a Mahalanobis metric space, which is optimal when the noise present in the recorded scanning-EMG signal is Gaussian with correlations between the different samples. When no correlations exist, the covariance matrix $\boldsymbol{C}_{\sigma}$ simplifies to the identity matrix, the multivariate Gaussian distribution converges to the AWGN distribution, and the Mahalanobis metric converges to the Euclidean one. It is important to keep in mind that the Mahalanobis metric is also not optimal for the type of noise actually present in the scanning signal; however, it can be closer to the optimal than the Euclidean one, if the covariance matrix $\boldsymbol{C}_{\sigma}$ is properly characterized.

\subsubsection{Relationship between the Mahalanobis metric and the signal filtering}

Assume that, to estimate the MU parameters, the squared Euclidean distance is used as an error function, but it is applied not to the raw recorded scanning-EMG signal, but to a filtered version of it. Under this assumption, the error function would be

$$
\varepsilon(\widehat{\boldsymbol{M}})=\left(\widehat{Y}(\widehat{\boldsymbol{M}})-Y_{\text {scan }}\right)^{T}\left(\widehat{Y}(\widehat{\boldsymbol{M}})-Y_{\text {scan }}\right)
$$

where $\hat{Y}(\widehat{M})$ and $Y_{\text {scan }}$ are filtered versions of $\hat{X}(\widehat{M})$ and $X_{\text {scan }}$ respectively, both being structured as column vectors. The linear filtering applied over the signals can be mathematically expressed as a matrix product between an impulse response matrix $\boldsymbol{H}$ and the involved signals

$$
\begin{aligned}
& \widehat{Y}(\widehat{\boldsymbol{M}})=\boldsymbol{H} \hat{X}(\widehat{\boldsymbol{M}}) \\
& Y_{\text {scan }}=\boldsymbol{H} X_{\text {scan }}
\end{aligned}
$$


Note that the size of the column vectors $\hat{Y}(\widehat{\boldsymbol{M}}), \hat{X}(\widehat{\boldsymbol{M}}), Y_{\text {scan }}$, and $X_{\text {scan }}$ is $N K P \times 1$, and the size of the matrix $\boldsymbol{H}$ is $N K P \times N K P$. Therefore, the error function in (4-12) can be expressed as

$$
\begin{array}{r}
\varepsilon(\widehat{\boldsymbol{M}})=\left(\boldsymbol{H} \hat{X}(\widehat{\boldsymbol{M}})-\boldsymbol{H} X_{\text {scan }}\right)^{T}\left(\boldsymbol{H} \hat{X}(\widehat{\boldsymbol{M}})-\boldsymbol{H} X_{\text {scan }}\right) \\
=\left(\widehat{X}(\widehat{\boldsymbol{M}})-X_{\text {scan }}\right)^{T}\left(\boldsymbol{H}^{T} \boldsymbol{H}\right)\left(\widehat{X}(\widehat{\boldsymbol{M}})-X_{\text {scan }}\right)
\end{array}
$$

Therefore, it can be concluded that the use of the Euclidean metric in filtered signals is equivalent to the use of a Mahalanobis metric with covariance matrix $\boldsymbol{C}_{\sigma}=$ $\left(\boldsymbol{H}^{T} \boldsymbol{H}\right)^{-1}$ in the non-filtered ones. This is an important conclusion, as it allows to operate with the Euclidean metric, even if the noise presents correlations between different spatio-temporal samples, as long as these correlations are eliminated by applying an adequate filter to the recorded scanning-EMG signal.

\subsubsection{Processing of the scanning-EMG signal noise}

The strategy used to address the problem of noise in the estimation of the MU parameters is to maintain the Euclidean metric as error function, while different filtering steps, adapted to the different noise sources, are applied to the recorded scanning-EMG signal. These steps try to transform the noise properties so that they resemble those of the AWGN as much as possible. The objective is to obtain a set of estimated MU parameters closer to the maximum of the a posteriori probability density (3-20).

\section{Processing of the instrumentation noise}

The scanning-EMG signal includes instrumentation noise caused by the electronics of the recording equipment. This noise is adequately modeled as AWGN, so it does not seem necessary to introduce a specific filter to deal with this type of noise. To support this hypothesis, a simple simulation experiment has been designed. The experiment consists on estimating the parameters of a single MU fiber for two different cases: one in which the instrumentation noise is low-pass filtered before carrying out the estimation procedure, and another in which noise is not filtered. In both cases the experiment scheme has the following steps:

1. The noise-free scanning-EMG signal is simulated from the parameters of the MU fiber: $x_{1}=0.2 \mathrm{~mm} ; y_{1}=0 \mathrm{~mm} ; z_{1}=30 \mathrm{~mm} ; v_{1}=3.3 \mathrm{~m} / \mathrm{s}$. The recording configuration used for this purpose is the 1-port scanning-EMG signal, whose unique port is located at $x_{e}=0 \mathrm{~mm}$ and $z_{e}=0 \mathrm{~mm}$. The MUP is recorded at several points along the y-axis from $y_{\min }=-1 \mathrm{~mm}$ to $y_{\max }=1 \mathrm{~mm}$, in steps of $0.05 \mathrm{~mm}$. More details about this configuration have been presented in section 3.3.1. 


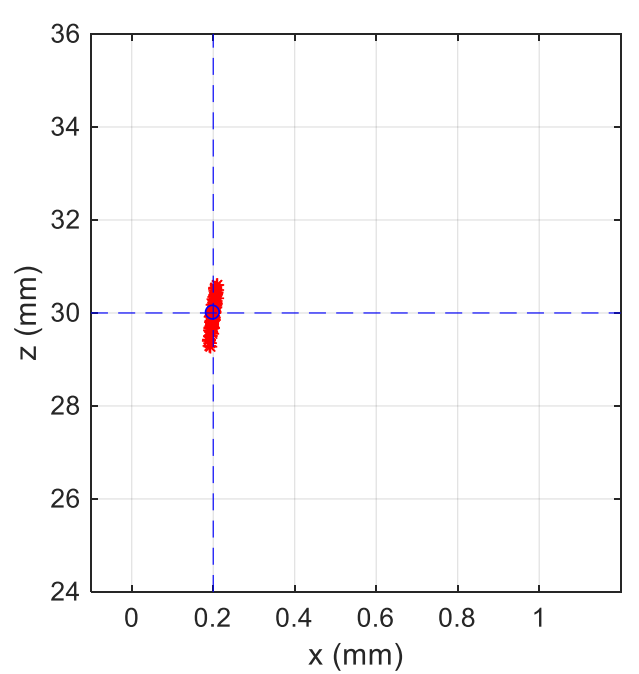

(a)

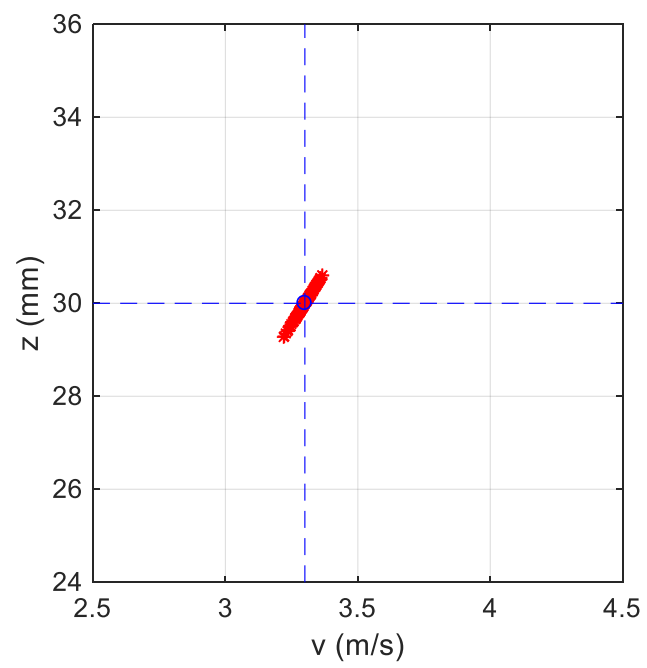

(c)

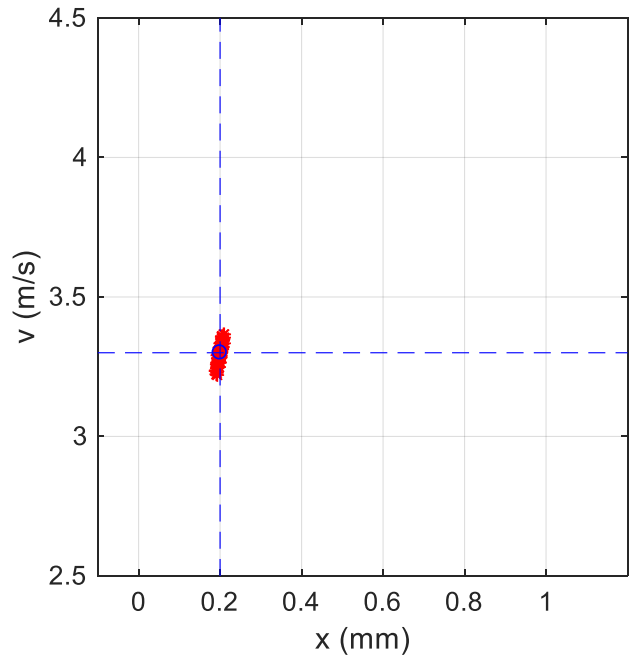

(b)

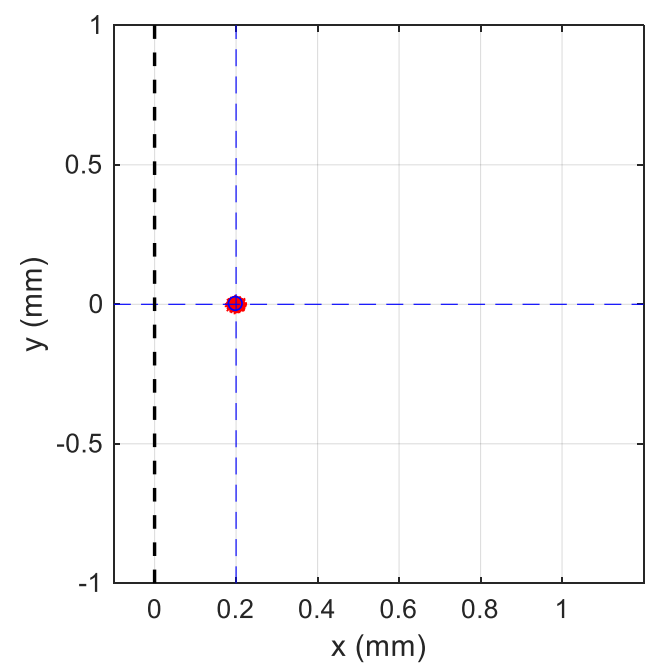

(d)

Fig. 4-3. Results of the estimated fiber parameters when the scanning-EMG signal has been contaminated with instrumentation noise. Red asterisks represent the estimated fiber parameters for the 100 runs executed. The dashed blue lines and the circle in which they intersect indicates the actual fiber parameters: (a) projection of the fiber parameters in the $\mathrm{z}$-x plane; (b) projection of the fiber parameters in the $\mathrm{V}-\mathrm{x}$ plane; (c) projection of the fiber parameters in the $\mathrm{z}-\mathrm{v}$ plane; (d) projection of the fiber parameters in the $\mathrm{y}-\mathrm{x}$ plane. The black dashed line represents the recording corridor.

\section{For 100 independent runs}

2.1. AWGN noise of $0.2 \mathrm{mV}$ standard deviation is added to the noise-free scanning-EMG signal to simulate the instrumentation noise. For the filtered case, an ideal low-pass filter with a cut-off frequency of $5000 \mathrm{~Hz}$ is applied 
to the noisy scanning-EMG signal along the time dimension.

2.2. The scanning-EMG signal obtained in the previous step (reference signal) is used as input of the estimation procedure, to obtain the estimated fiber parameters.

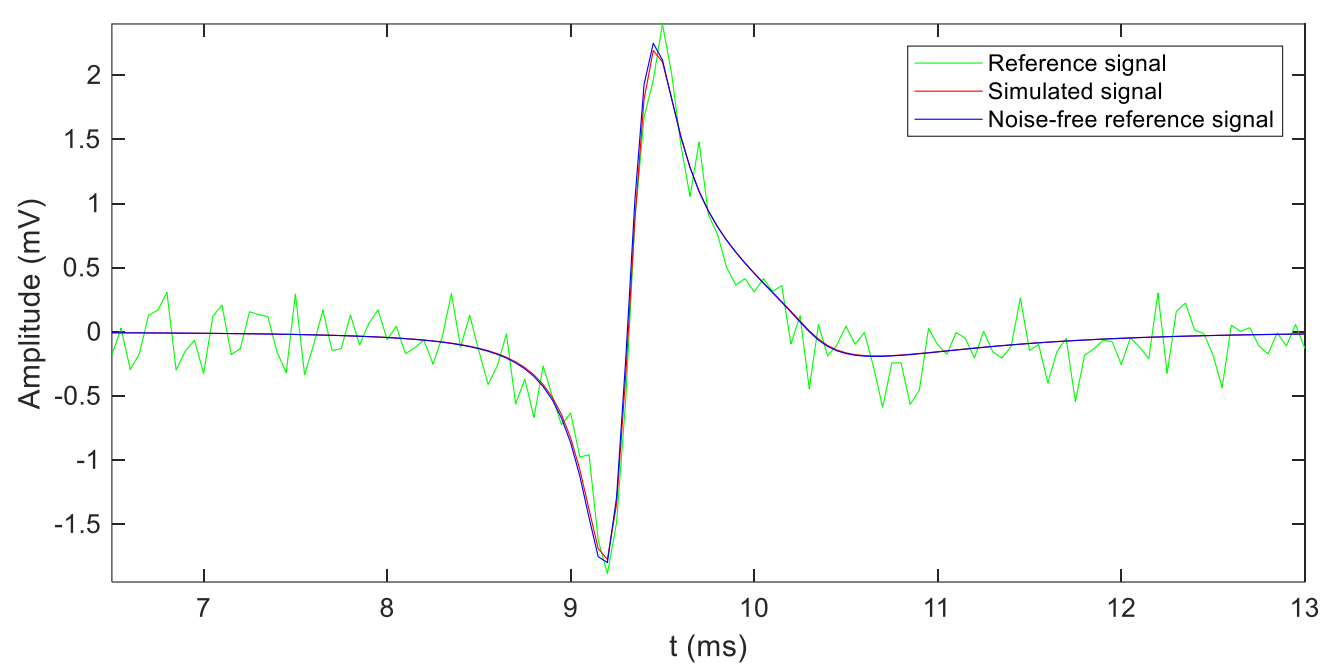

(a)

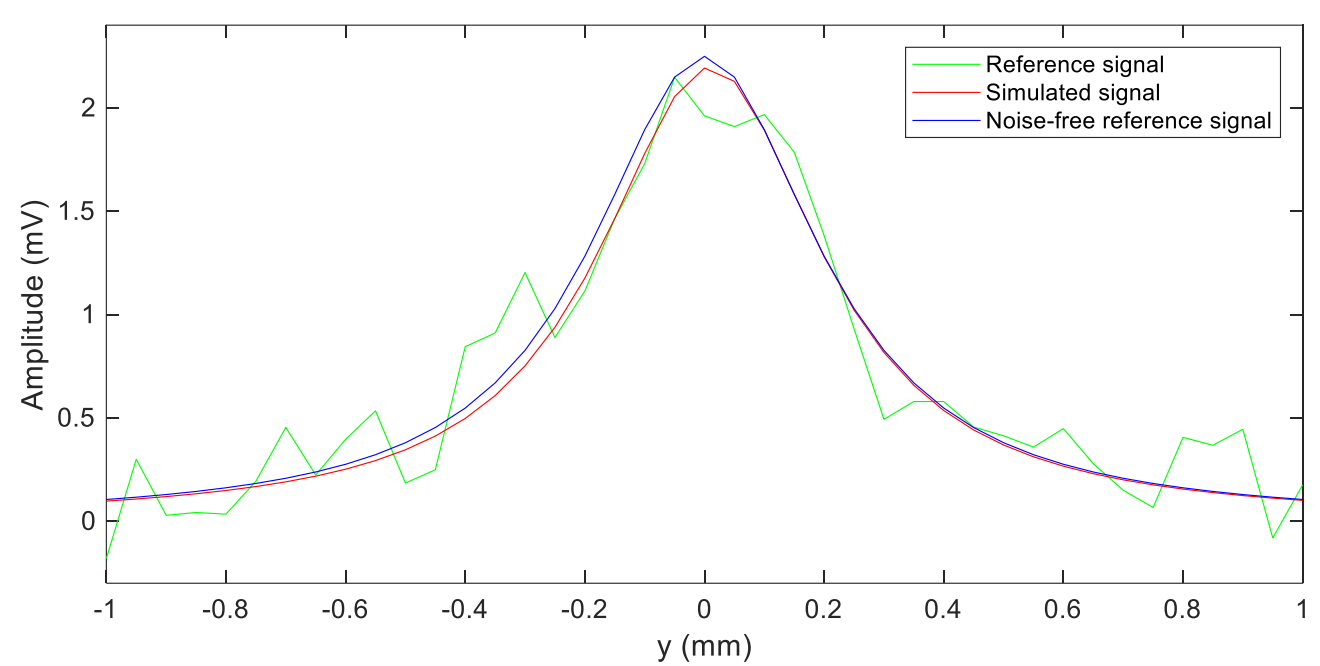

(b)

Fig. 4-4. Representation of the scanning-EMG signals involved in the estimation for one of the runs of the experiment in the unfiltered case. In green, the reference signal; in red, the simulated signal; and in blue the noise-free reference signal; (a) MUP trace corresponding to the recording position in which the signal passes through its maximum; (b) trace along the spatial dimension corresponding to the time instant for which the signal passes through its maximum. 


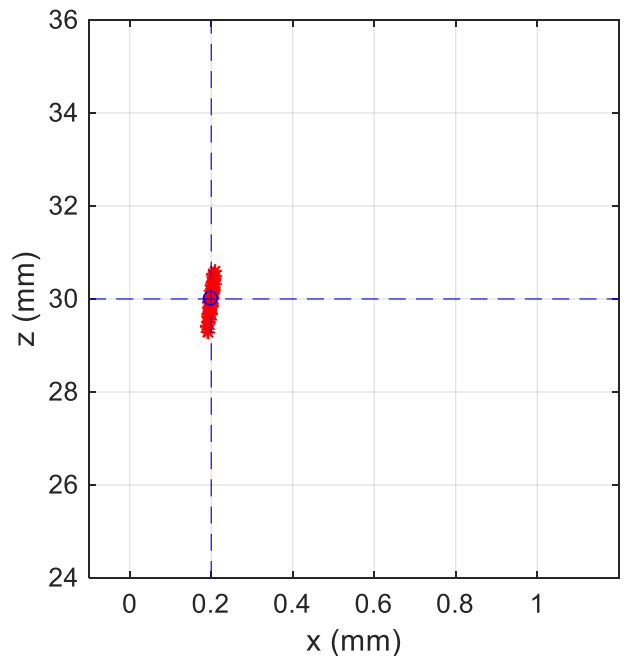

(a)

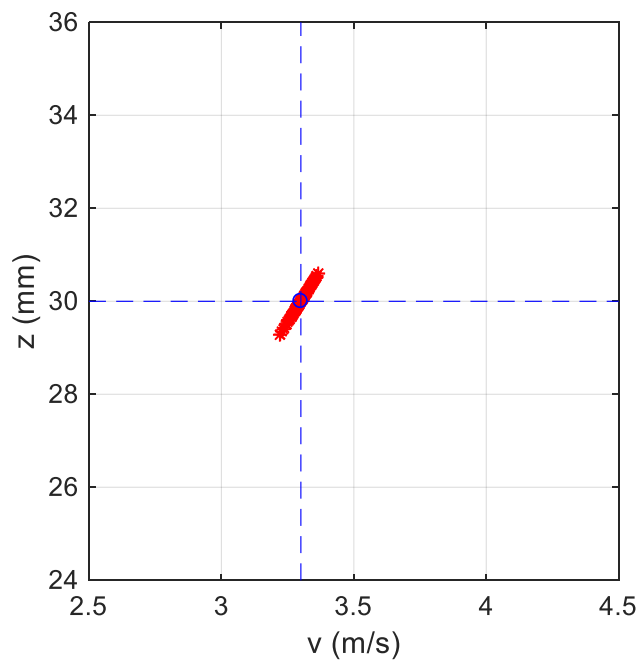

(c)

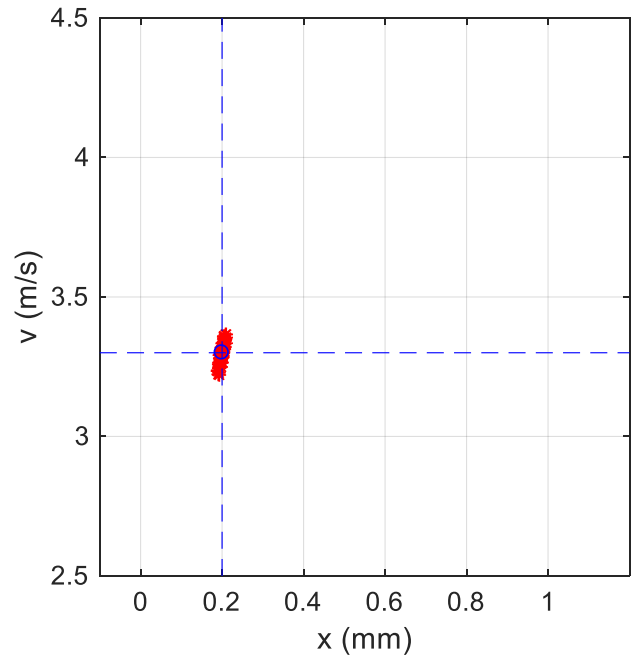

(b)

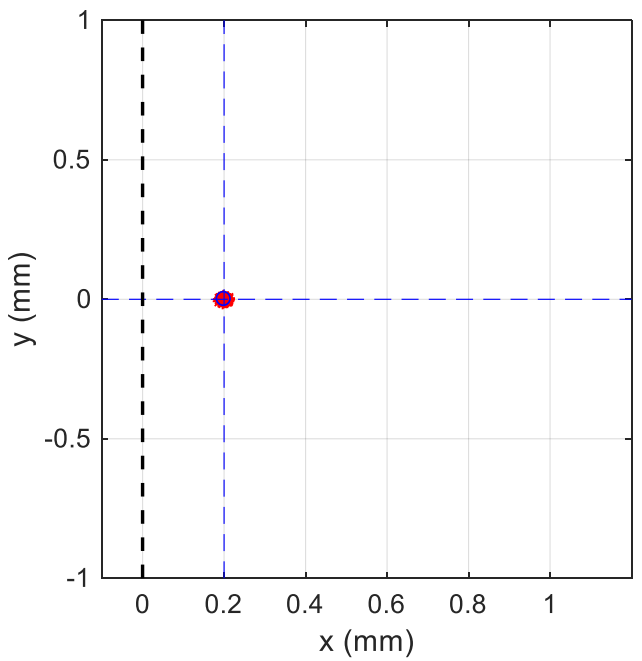

(d)

Fig. 4-5. Results of the estimated fiber parameters when the scanning-EMG signal has been contaminated with instrumentation noise, and then low-pass filtered. Red asterisks represent the estimated fiber parameters for the 100 runs executed. The dashed blue lines and the circle in which they intersect indicates the actual fiber parameters: (a) projection of the fiber parameters in the z-x plane; (b) projection of the fiber parameters in the v-x plane; (c) projection of the fiber parameters in the $\mathrm{z}-\mathrm{v}$ plane; (d) projection of the fiber parameters in the $\mathrm{y}-\mathrm{x}$ plane. The black dashed line represents the recording corridor.

The results obtained for the estimated fiber parameters in the unfiltered case are shown in Fig. 4-3. It seems evident from the results that in presence of noise, the estimated fiber parameters are not exactly the same as the actual fiber parameters, but they are dispersed within a bounded region of the parameter space. In Fig. 4-4, the 
signals involved in one of the runs are depicted. Note that the simulated scanningEMG signal (in red in Fig. 4-4) tends to adjust as much as possible to the reference signal (in green in Fig. 4-4), which is contaminated by noise. Due to this noise, the best fit does not perfectly coincide with the noise-free reference signal generated with the actual fiber parameters (in blue in Fig. 4-4). This is the reason why the estimated fiber parameters also differ from the actual ones.

The results obtained in the filtered case seem to be identical to that of the unfiltered case (Fig. 4-5). Hence, including the filter in the estimation does not imply a better estimation. The reason is that the metric used, i.e., the squared Euclidean distance, is optimal for the AWGN; therefore, in the best-case scenario, filtering will not affect to the estimation performance, and in the worst-case scenario it could negatively affect to it. This can occur, for instance, if the low-pass filter employed has a cut-off frequency low enough to eliminate useful physiological information from the scanning-EMG signal.

\section{Processing of the baseline noise}

During the recording of the scanning-EMG signal, the movement of the needle or the muscle itself can cause a slow time-varying baseline noise, affecting to the MUP at each recording step. An example of a scanning-EMG signal contaminated with this kind of noise can be observed in Fig. 4-6. Note that the baseline noise presents a very slow variation along the temporal dimension, and thus, a very high autocorrelation along it. This kind of noise is, therefore, far from being AWGN, for which the autocorrelation is a Dirac delta function. Therefore, an adequate high-pass time filter that eliminates the slowest frequency components of the signal should help to mitigate the potentially harmful effect that this noise may have on the estimation procedure. For this purpose, the use of the temporal filter for baseline and instrumentation noise described in section 2.1.1 is proposed.

To briefly analyze the effect that the baseline noise and its filtering has in the estimation procedure, the same simulation experiment already done for the instrumentation noise has been performed. Remember that this experiment consists on estimating the parameters of a single MU fiber for two different cases: one in which the noise is filtered before carrying out the estimation procedure, and another one in which the noise remains unfiltered. The only difference between the experiment for the instrumentation noise and the one for baseline noise concerns to the step 2.1. In the current experiment, this step consists on adding baseline noise to the noise-free scanning-EMG signal. This baseline noise is modeled as an ARMA process [99] obtained by filtering AWGN of $4.5 \mathrm{mV}$ standard deviation. The filter used for this purpose is a 5th-order Butterworth low-pass filter with a 3-dB cut-off frequency of 50 $\mathrm{Hz}$. For the case in which the signal is filtered, the temporal filter for baseline and instrumentation noise described in section 2.1.1 is used, with the operating parameters: $N_{a d d}=400$ samples; $N_{a v}=50$ samples; $f_{H P F}=33.3 \mathrm{~Hz} ;$ and $f_{L P F}=\infty$. 


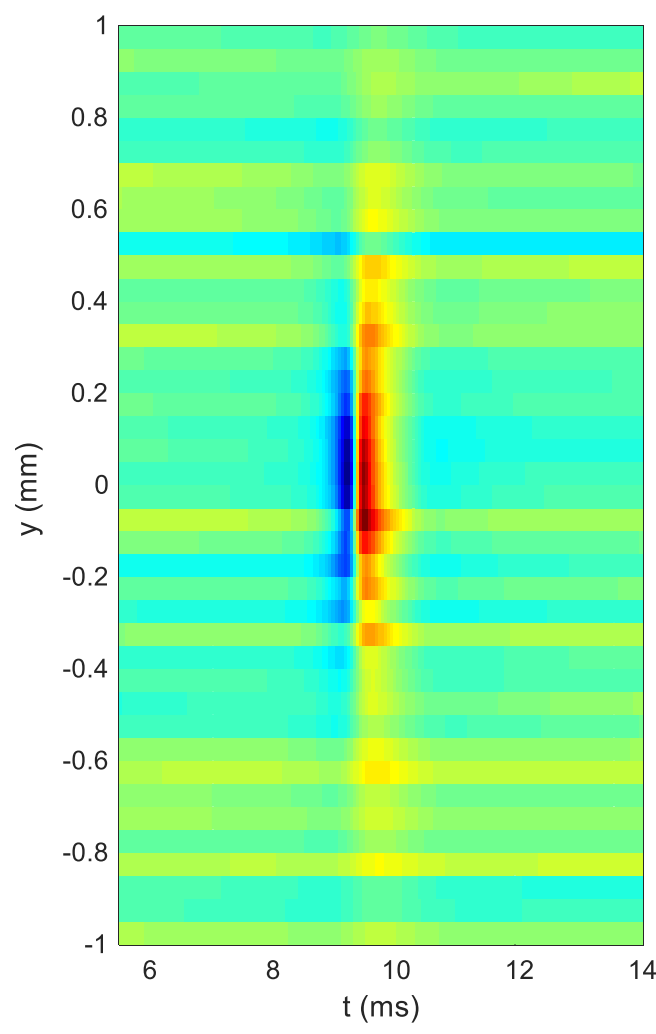

(a)

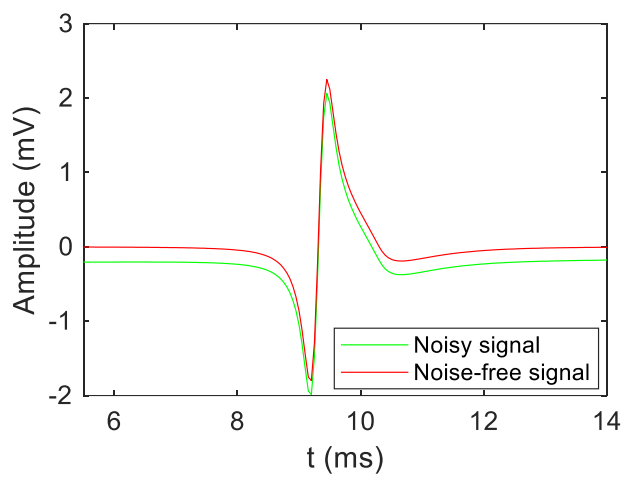

(b)

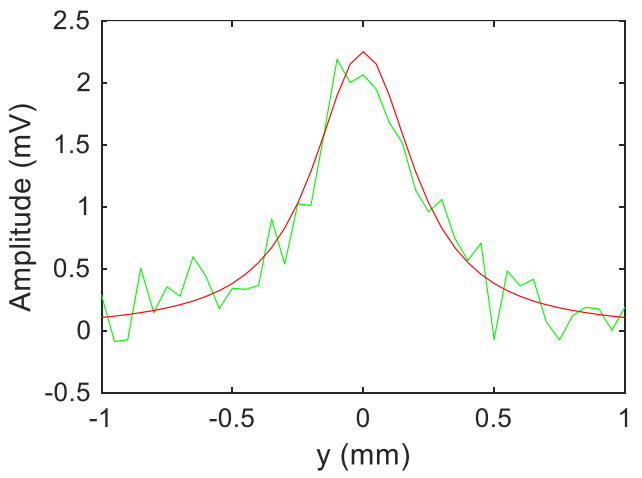

(c)

Fig. 4-6. Simulation example of a scanning-EMG signal contaminated with baseline noise; (a) color plot representing the amplitude of the scanning-EMG signal; (b) MUP trace corresponding to the recording position in which the signal passes through its maximum. In green, the signal contaminated with baseline noise; in red, the noise-free version of this signal. Note that, the baseline corresponds to a very slow variation noise superimposed to the MUP; (c) trace along the spatial dimension corresponding to the time instant for which the signal passes through its maximum; in green, the signal contaminated with baseline noise; in red, the noise-free version of this signal. Note that the baseline noise is uncorrelated along the spatial dimension.

The results obtained for the estimated fiber parameters in the unfiltered case are shown in Fig. 4-7. Note that the estimated parameters match exactly the actual fiber parameters in all runs, which means that the presence of the baseline noise does not negatively affect to the estimation performance, at least not in the context of the experiment. This result suggests that the simulated signal obtained from the estimation is not able to reproduce the slow variations of the baseline noise, and therefore the presence of such noise does not condition to any degree the waveform of the simulated signal. This can be interpreted as if the simulated signal would be exclusively focused on reproducing the waveform of the noise-free reference signal (i.e., excluding the baseline). 


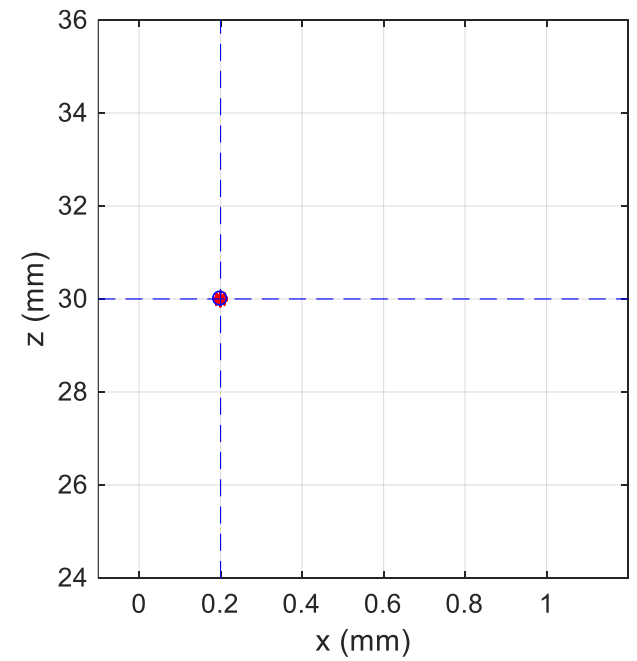

(a)

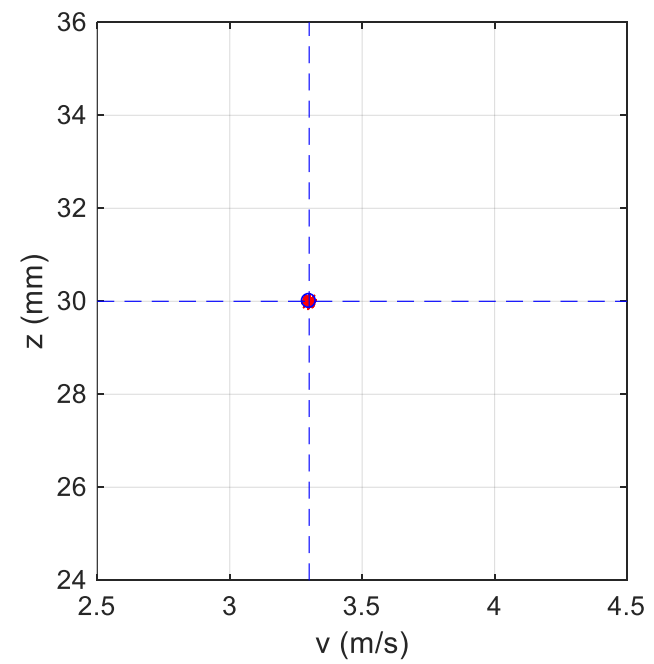

(c)

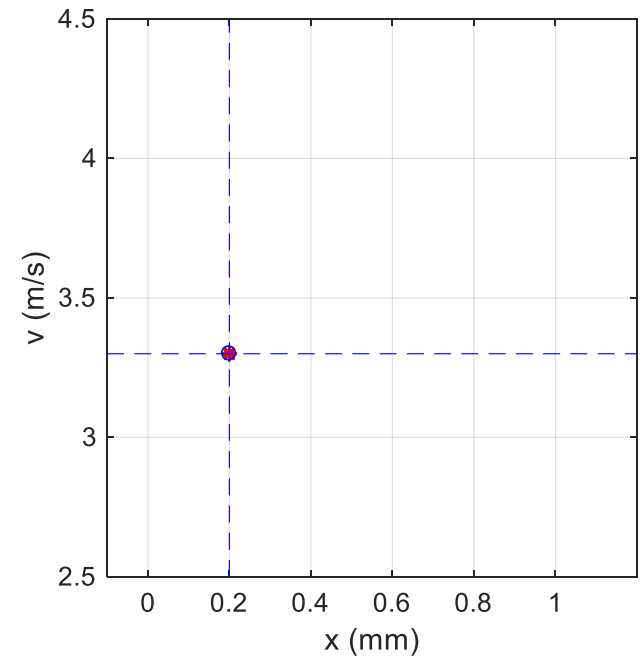

(b)

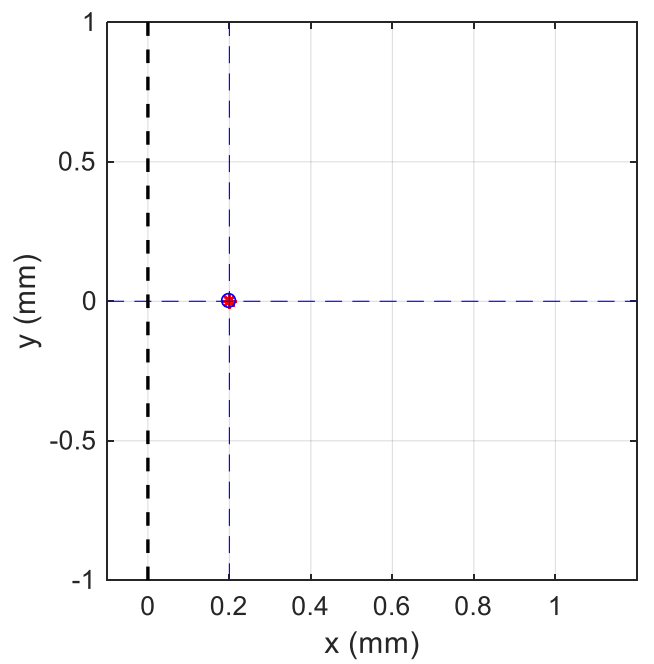

(d)

Fig. 4-7. Results of the estimated fiber parameters when the scanning-EMG signal has been contaminated with baseline noise. Red asterisks represent the estimated fiber parameters for the 100 runs executed. The dashed blue lines and the circle in which they intersect indicates the actual fiber parameters: (a) projection of the fiber parameters in the z-x plane; (b) projection of the fiber parameters in the v-x plane; (c) projection of the fiber parameters in the $\mathrm{z}-\mathrm{v}$ plane; (d) projection of the fiber parameters in the $y-x$ plane. The black dashed line represents the recording corridor.

The results obtained for the estimated fiber parameters in the case in which the noisy signal is high pass filtered are shown in Fig. 4-8. As in the non-filtered case, the estimated parameters match exactly with the actual fiber parameters. Obviously, the 


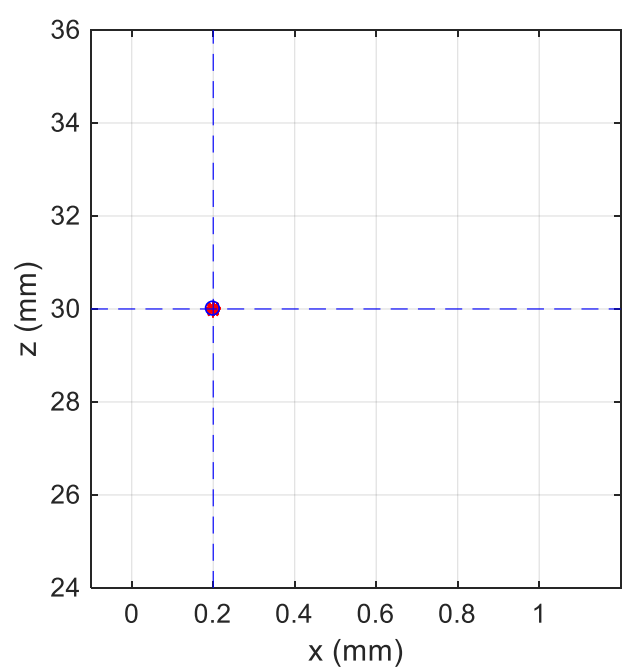

(a)

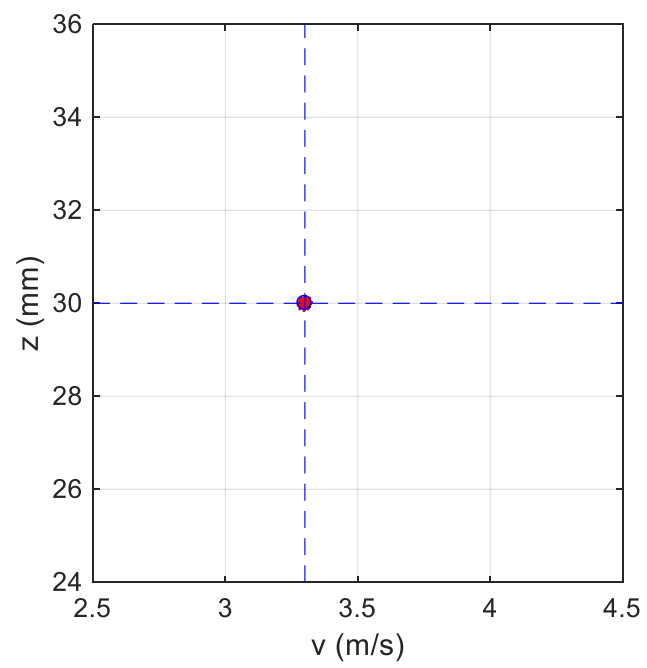

(c)

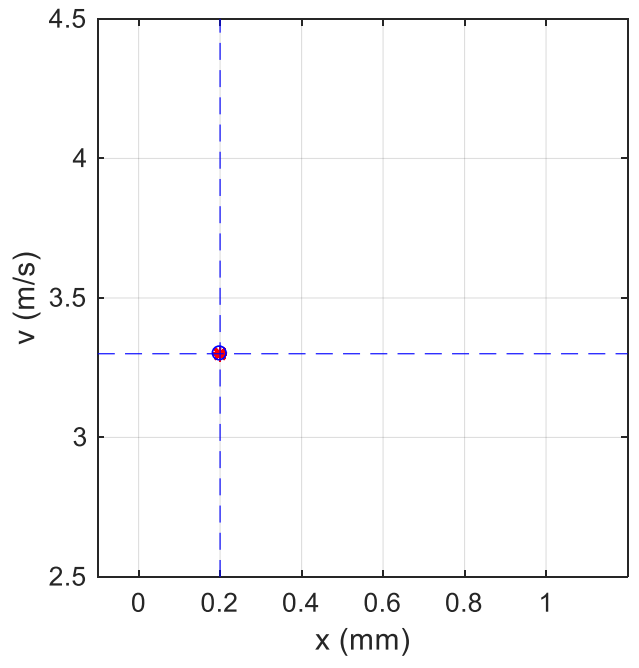

(b)

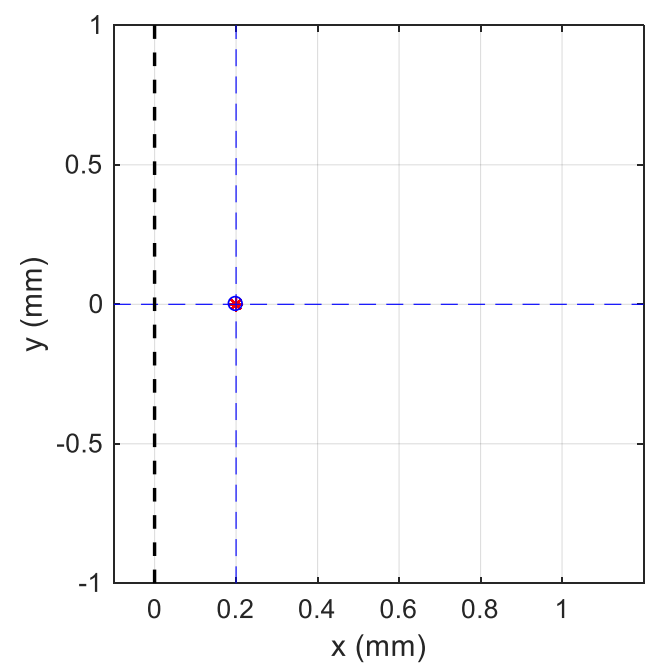

(d)

Fig. 4-8. Results of the estimated fiber parameters when the scanning-EMG signal has been contaminated with baseline noise, and then high-pass filtered. Red asterisks represent the estimated fiber parameters for the 100 runs executed. The dashed blue lines and the circle in which they intersect indicates the actual fiber parameters: (a) projection of the fiber parameters in the z-x plane; (b) projection of the fiber parameters in the v-x plane; (c) projection of the fiber parameters in the $\mathrm{z}-\mathrm{v}$ plane; (d) projection of the fiber parameters in the $\mathrm{y}-\mathrm{x}$ plane. The black dashed line represents the recording corridor.

filter cannot improve the estimation performance because the estimation was already perfect without filtering. This makes this filtering step also redundant, as was already the case with the instrumentation noise, at least in the simulated conditions given by the experiment setup. 


\section{Processing of the artifact noise}

The raw scanning-EMG signal usually presents interference derived from the coactivation of nearby MUs $[42,44,90]$. This interference consists on a certain number of MUP artifacts not synchronized with the firing of the MU being tracked, that are superimposed with the MUP at each recording step [90]. An example of a scanningEMG signal contaminated with artifact noise can be observed in Fig. 4-9. Note that, the interfering MUPs (the artifacts) present a smooth variation along the spatial dimension, although not as smooth as in the baseline noise. This implies that artifact noise is highly autocorrelated along the time dimension. As it occurred with the baseline noise, the statistical properties of the artifact noise are far from being those of the AWGN. This implies that the Euclidean metric used in the estimation procedure is again not optimal. For this reason, it is expected that, if the artifact noise is removed with a proper processing technique, the negative effects it may provoke in the estimation can be partially mitigated.

To eliminate the artifact noise, the MMLSS algorithm is used. This algorithm consists of detecting in which samples the scanning-EMG signal has been contaminated with artifacts, and replacing each sample value of the signal using a least-squares smoothing applied along the spatial dimension, taking into account only those samples that are not contaminated with artifacts. The MMLSS algorithm is described in detail in section 2.1.1. The values of MMLSS parameters used here are: median filter order, $L=5$ samples; artifact detection threshold, $U=0.04$; polynomial order, $Q=6$; and window semi-length, $W=3$ samples. These values have been chosen to ensure that only the samples in which the presence of an artefact has been detected are recalculated, while the rest retains the original amplitude values. This aims to prevent the MMLSS algorithm from eliminating useful physiological information from the scanning-EMG signal.

An important consideration is that the MMLSS algorithm must be applied separately to the different sections of the scanning-EMG signal, if the signal is simultaneously recorded from different SF ports.

To analyze the effect that the artifact noise and its processing may have in the estimation procedure, the same simulation experiment performed for the previous noise sources has been performed here. The estimation of the fiber parameters is again performed in two cases: one in which the artifact noise is eliminated before carrying out the estimation, and another in which not. For the current experiment, the step 2.1 of the scheme consists on adding simulated artifact noise to the noise-free scanningEMG signal. This artifact noise has been generated by simulating the recording procedure in a realistic way in which MUPs from other active MUs are interfering (see section 1.4.6 for more details about the simulation of the scanning-EMG recording procedure). The muscle contraction used for this experiment is $2 \% \mathrm{MVC}$. For the case in which the signal is filtered, the MMLSS algorithm is applied with the previously indicated parameters. 


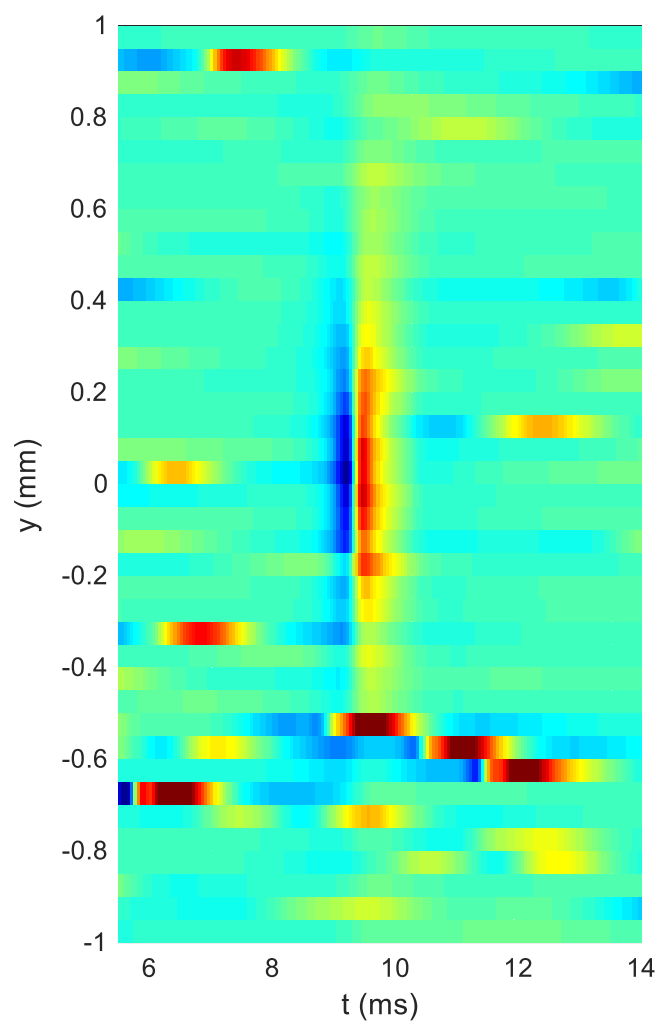

(a)

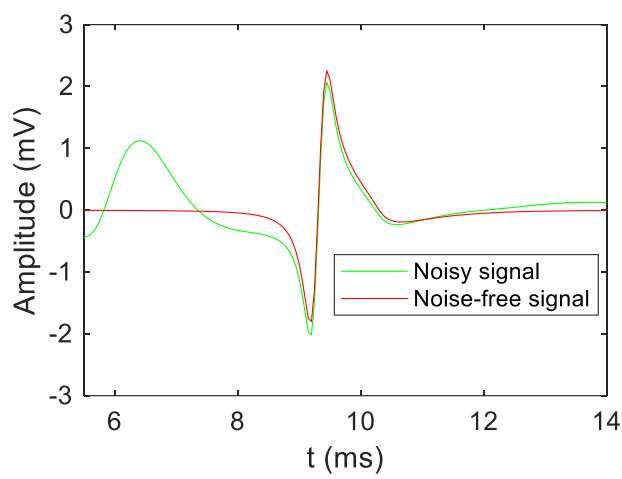

(b)

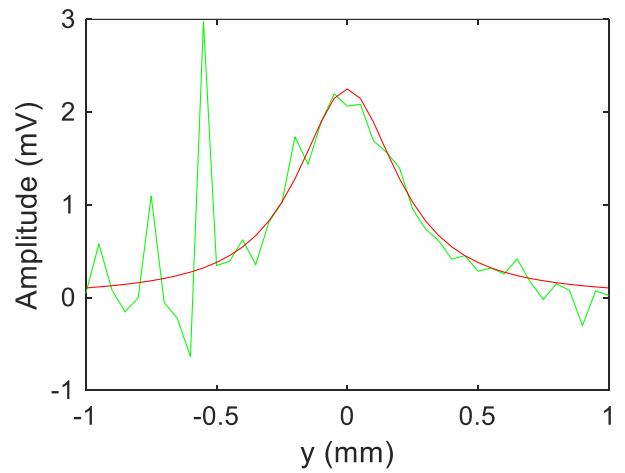

(c)

Fig. 4-9. Simulation example of a scanning-EMG signal contaminated with artifact noise; (a) color plot representing the amplitude of the scanning-EMG signal; (b) MUP trace corresponding to the recording position in which the signal passes through its maximum; in green, the signal contaminated with artifact noise; in red, the noise-free version of this signal. The artifacts are associated to activity from other MUs that are superimposed to the MUP of the MU being tracked; (c) trace along the spatial dimension corresponding to the time instant for which the signal passes through its maximum; in green, the signal contaminated with artifact noise; in red, the noise-free version of this signal. Note that, the artifact noise is uncorrelated along the spatial dimension.

The results obtained for the estimated fiber parameters when artifacts have not been removed are shown in Fig. 4-10. Note that the estimated fiber parameters present a very high dispersion respect to the actual fiber parameters, which means that the estimation performance is drastically reduced by the presence of artifacts. This may be due to the fact that the artifacts present, along the time dimension, waveforms that are similar to those that the simulation model can generate (note that, artifacts are in fact MUPs from other MUs). Note that when artifacts are superimpossed to the noise-free reference signal, the resulting noisy signal can be, at least partially, reproducible with the model. This might induce that, during the estimation procedure, the simulated 


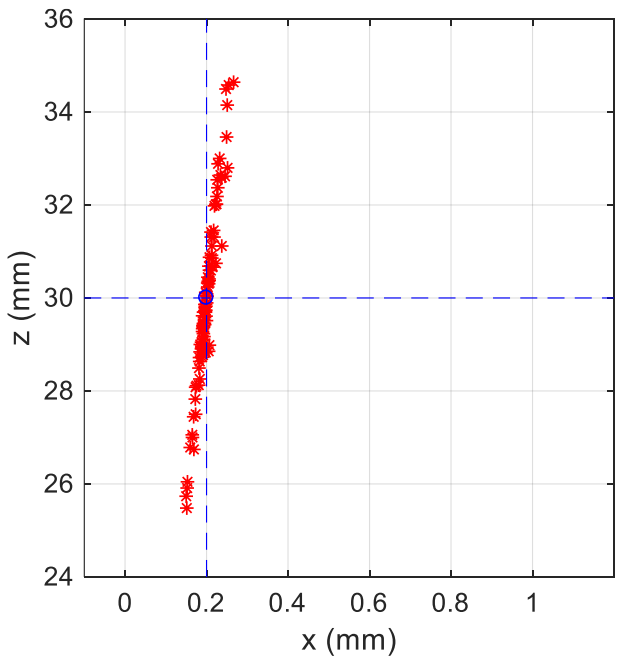

(a)

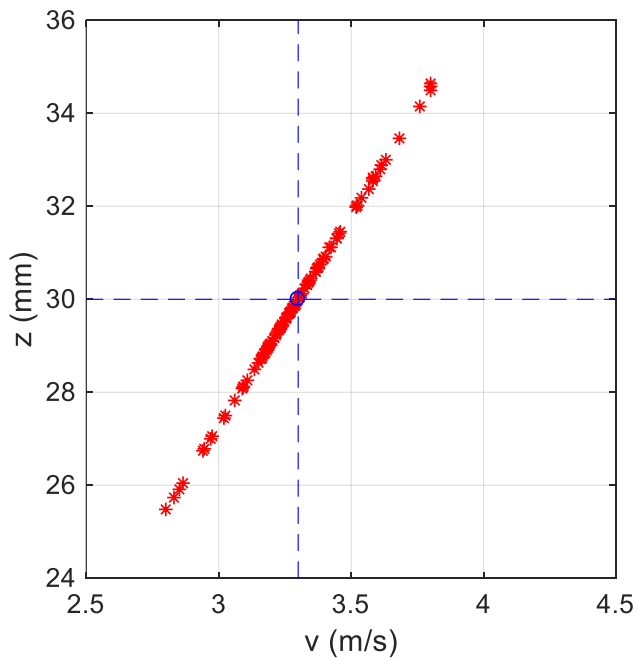

(c)

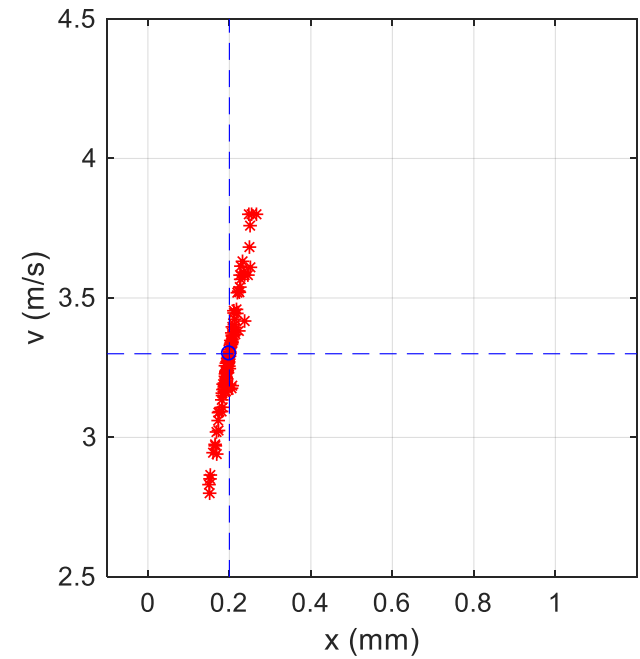

(b)

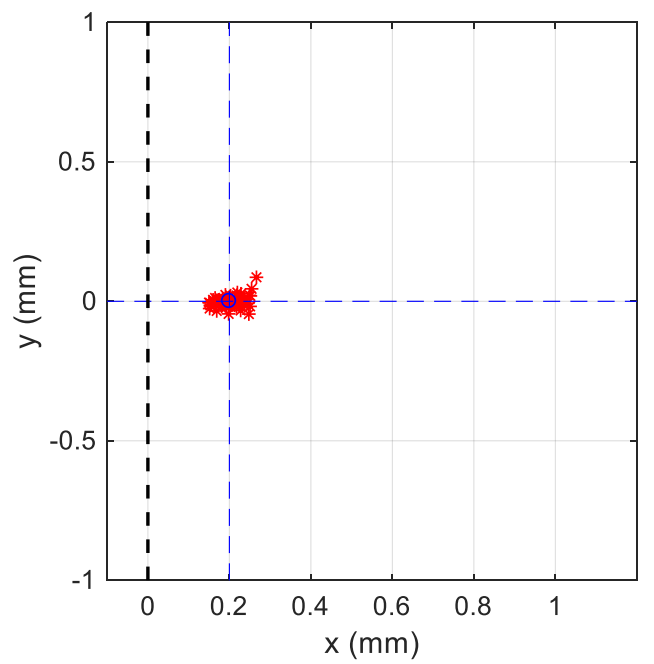

(d)

Fig. 4-10. Results of the estimated fiber parameters when the scanning-EMG signal has been contaminated with artifact noise. Red asterisks represent the estimated fiber parameters for the 100 runs executed. The dashed blue lines and the circle in which they intersect indicates the actual fiber parameters: (a) projection of the fiber parameters in the z-x plane; (b) projection of the fiber parameters in the $\mathrm{v}-\mathrm{x}$ plane; (c) projection of the fiber parameters in the $\mathrm{z}-\mathrm{v}$ plane; (d) projection of the fiber parameters in the $y$-x plane. The black dashed line represents the recording corridor.

signal tries to reproduce not only the noise-free reference signal, but also to some extent the superimposed artifacts, what results in a large error when estimating the fiber parameters. 


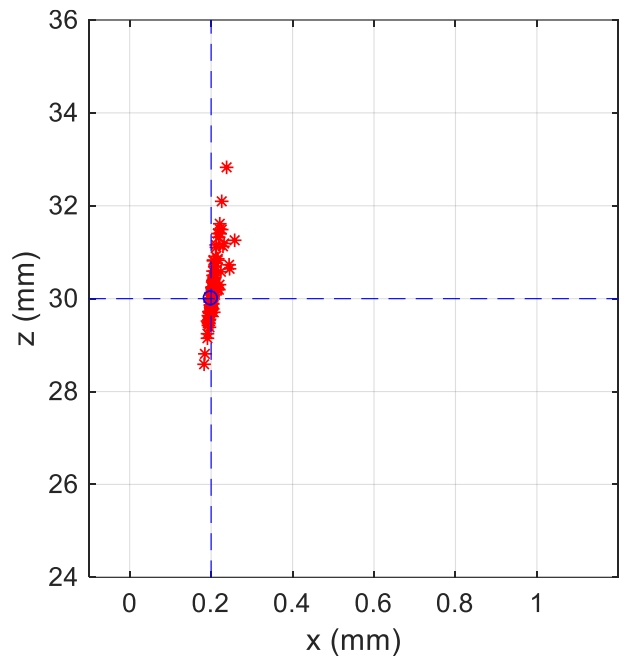

(a)

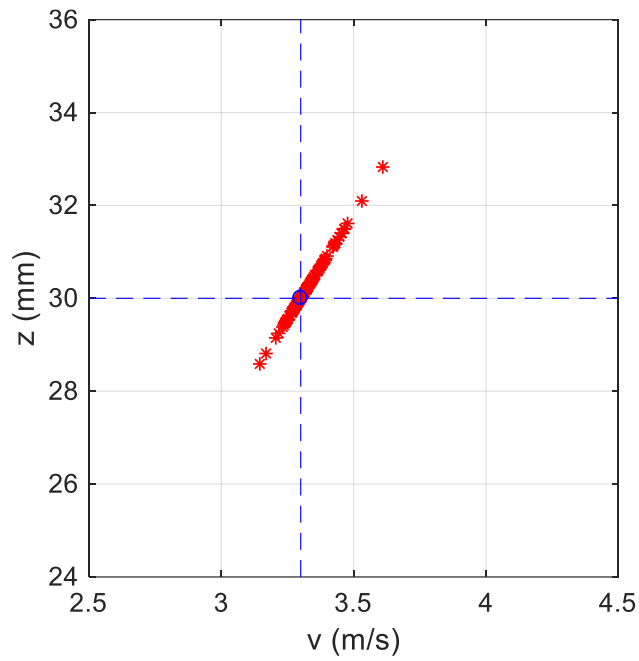

(c)

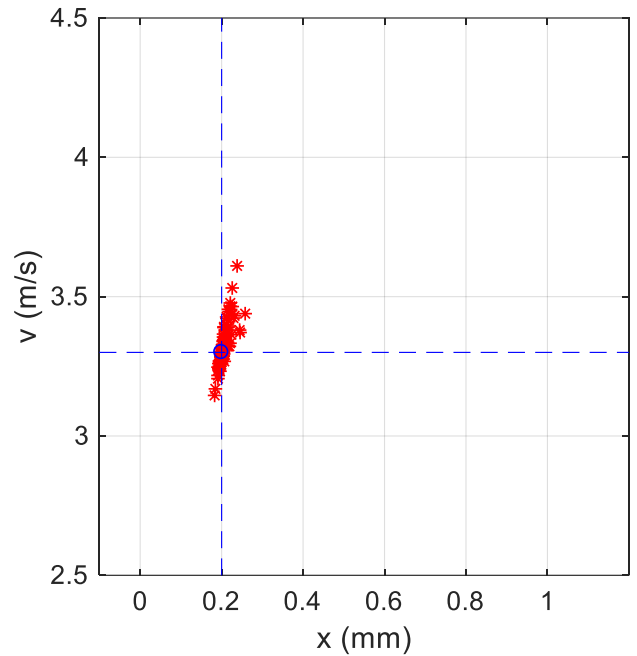

(b)

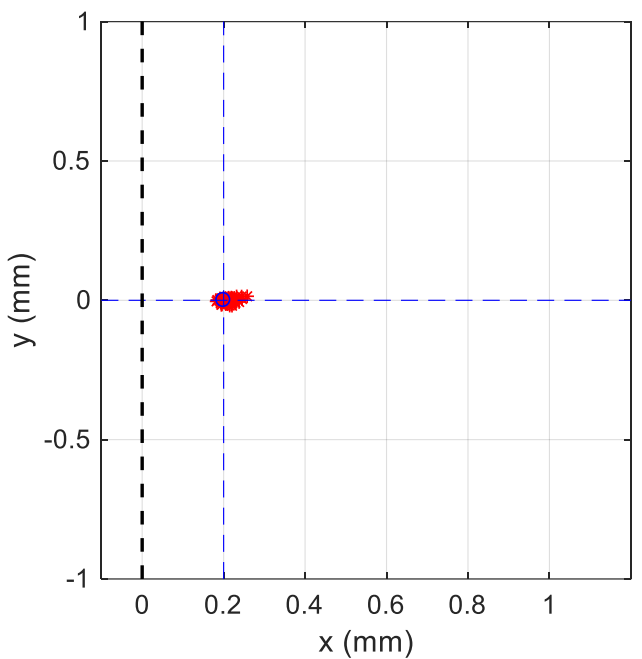

(d)

Fig. 4-11. Results of the estimated fiber parameters when the scanning-EMG signal has been contaminated with artifact noise, and then the MMLSS algorithm has been applied. Red asterisks represent the estimated fiber parameters for the 100 runs executed. The dashed blue lines and the circle in which they intersect indicates the actual fiber parameters: (a) projection of the fiber parameters in the z-x plane; (b) projection of the fiber parameters in the $v$-x plane; (c) projection of the fiber parameters in the $\mathrm{z}-\mathrm{v}$ plane; (d) projection of the fiber parameters in the $y$-x plane. The black dashed line represents the recording corridor.

The results obtained for the estimated fiber parameters, when artifacts have been removed with the MMLSS algorithm are shown in Fig. 4-11. It can be observed that the dispersion of the estimated fiber parameters has been noticeably reduced respect to the case in which artifacts are not removed. For instance, the results of the parameter 
$z$ ranges between $28.6 \mathrm{~mm}$ and $32.8 \mathrm{~mm}$ when the MMLSS is applied; and between $25.4 \mathrm{~mm}$ and $34.7 \mathrm{~mm}$ when it is not applied. This means that, as expected, removing the artifact noise by using the MMLSS algorithm improves the estimation performance. As already pointed out, this is because the Euclidean metric used in optimization is not optimal for the artifact noise. The MMLSS algorithm considerably reduces the level of this type of noise, and therefore improves the estimation of the MU fiber parameters.

\subsubsection{Complete noise processing scheme}

Once we have studied the effect in the estimation of the different noise sources, and that of the filters designed to remove them, the overall processing scheme introduced in the estimation system is now presented. It is necessary to keep in mind the following conclusions from the previous sections:

- The most important piece of the processing scheme is the MMLSS algorithm, since it has demonstrated to be effective in reducing the harmful effect that the artifact noise produces in the estimation.

- The filters used to remove the instrumentation and baseline noise have turned out not to improve the estimation performance, at least not when analyzed independently. However, this cannot exclude the existence of possible cross effects when considering all the noise sources and the corresponding filtering steps at the same time.

For example, the artifact detection performed by the MMLSS algorithm could be adversely affected by the presence of baseline or instrumentation noise, which in turn, could undermine the estimation procedure. For this reason, the final decision has been to incorporate both the low- and the high-pass filter into the processing scheme, as a previous step to the MMLSS algorithm.

In order to further improve the performance of the estimation, the recording of the scanning-EMG signal can be configured so that more than one MUP discharge is recorded at each position of the scanning corridor. By combining the information of the different discharges, the amount of noise present in the scanning-EMG signal is drastically reduced. Note that the MMLSS algorithm is capable of dealing with more than one MUP discharge per recording position (see section 2.1.1).

The definitive processing scheme to be incorporated to the estimation system takes into account these considerations. Let $\boldsymbol{X}_{\text {scan }}=\left\{x_{\text {scan }}^{l}, k, p\right\}$, be the raw recorded scanning-EMG signal, where $1 \leq n \leq N$ denote the temporal samples, $1 \leq k \leq K$ the different scanning steps, $1 \leq p \leq P$ the ports in which the signal is recorded, and $1 \leq$ $l \leq L$ the different discharges. The overall processing scheme consists on the two following steps: 
1- Band-pass time filtering. Instrumentation and baseline noise are removed from the raw recorded scanning-EMG signal by applying a band pass-filter along the time dimension to each of the MUP discharges at each recording position. The filter used for this purpose is the one described in section 2.1.1, with the parameters: $N_{a d d}=400$ samples; $N_{a v}=50$ samples; $f_{H P F}=33.3 \mathrm{~Hz} ; f_{L P F}=5000 \mathrm{~Hz}$. Note that this filter eliminates the low frequencies to reduce the baseline noise, and the high frequencies to attenuate part of the instrumentation noise.

2- MMLSS algorithm. This algorithm eliminates the artifacts, and combines the different MUP discharges. The values of MMLSS parameters used here are: median filter order, $L=5$ samples; artifact detection threshold, $U=0.04$; polynomial order, $Q=6$; and window semi-length, $W=3$ samples.

This processing scheme is incorporated in the estimation system in the way described in Fig. 4-12. Note that the processing is applied not only to the raw scanningEMG signal, but also to all the signals simulated during the estimation procedure, in such a way the distortion produced by the processing also affects to them. This consideration is important when it comes to properly quantify the error function. Another consideration is that in the case of the simulated signal, it is not necessary to apply the MMLSS algorithm, since such algorithm has been configured to modify only those samples contaminated with artifacts, which implies that its effect on the simulated signal, which obviously does not present any type of noise, is null.

A simple experiment has been done to study how the estimation performance improves when more than one MUP discharge is recorded at each corridor position. This experiment is analogous to those of the previous sections. It consists on estimating the parameters of a single fiber when the scanning-EMG signal is contaminated with all noise sources, and then it is processed with the processing scheme. Two cases are studied: a first one in which the scanning-EMG signal consists on a single MUP discharge per recording position, and a second one in which 5 MUP discharges per position are acquired. In both cases the experiment scheme consists con the following steps:

1. The noise-free scanning-EMG signal is simulated from the parameters of the MU fiber: $x_{1}=0.2 \mathrm{~mm} ; y_{1}=0 \mathrm{~mm} ; z_{1}=30 \mathrm{~mm} ; v_{1}=3.3 \mathrm{~m} / \mathrm{s}$. The recording configuration used for this purpose is the 1-port scanning-EMG signal, whose unique port is located at $x_{e}=0 \mathrm{~mm}$ and $z_{e}=0 \mathrm{~mm}$. The MUP is recorded at several points along the y-axis from $y_{\min }=-1 \mathrm{~mm}$ to $y_{\max }=1 \mathrm{~mm}$, in steps of $0.05 \mathrm{~mm}$.

2. For 100 independent runs

2.1. Noise from the three sources is added to the noise-free scanning-EMG signal. AWGN noise of $0.2 \mathrm{mV}$ standard deviation is added to simulate the instrumentation noise. Baseline noise is also added to the signal; this baseline 


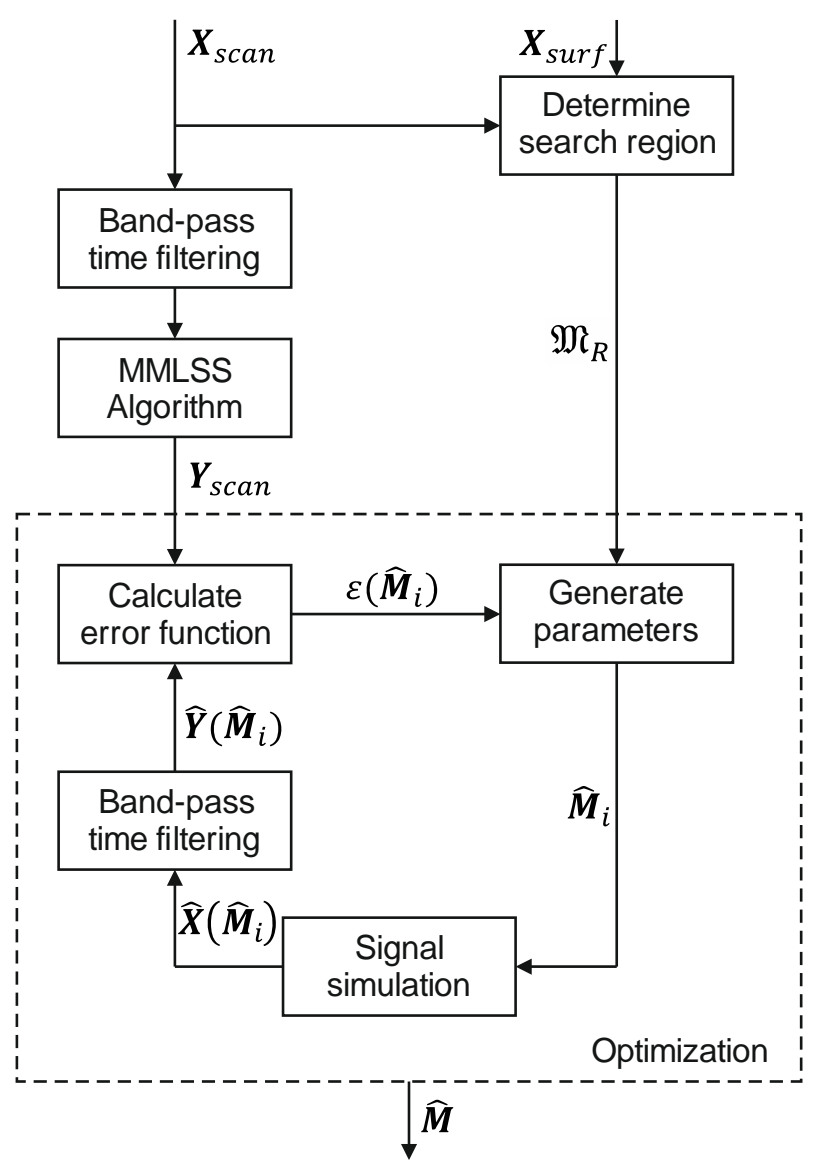

Fig. 4-12. Block diagram describing the estimation system after incorporating the processing scheme. The inputs of the system are the raw recorded scanning-EMG signal $\boldsymbol{X}_{\text {scan }}$, and the surface-MUP signal $\boldsymbol{X}_{\text {surf }}$. Both signals are used to determine the region of the parameter space $\mathfrak{M}_{R}$ in which the estimated parameters will be sought. The scanning-EMG signal $\boldsymbol{X}_{\text {scan }}$, is processed by applying: firstly, a band pass filter along the time dimension; and secondly the MMLSS algorithm. The output of the processing is the scanning-EMG signal $\boldsymbol{Y}_{\text {scan }}$. The optimization procedure used to estimate the parameters are boxed with a dashed line. Such procedure iteratively generates different sets of parameters $\widehat{\boldsymbol{M}}_{i}$. These sets of parameters are used to generate the simulated signals $\widehat{\boldsymbol{X}}\left(\widehat{\boldsymbol{M}}_{i}\right)$, which are subjected to the same band-pass filter than the one applied to the raw recorded signal. The resulting signals $\widehat{\boldsymbol{Y}}\left(\widehat{\boldsymbol{M}}_{i}\right)$, are used to compute the error function values $\varepsilon\left(\widehat{\boldsymbol{M}}_{i}\right)$. Note that the calculation of the error function requires both the simulated signal $\widehat{\boldsymbol{Y}}\left(\widehat{\boldsymbol{M}}_{i}\right)$, and the recorded one $\boldsymbol{Y}_{\text {scan }}$. The error function values of previous iterations are used to adequately generate new sets of parameters. The output of the optimization algorithm, and of the estimation system are the optimal estimated parameters $\widehat{\boldsymbol{M}}$, that is, the set of parameters for which the error function has reached a minimum. 


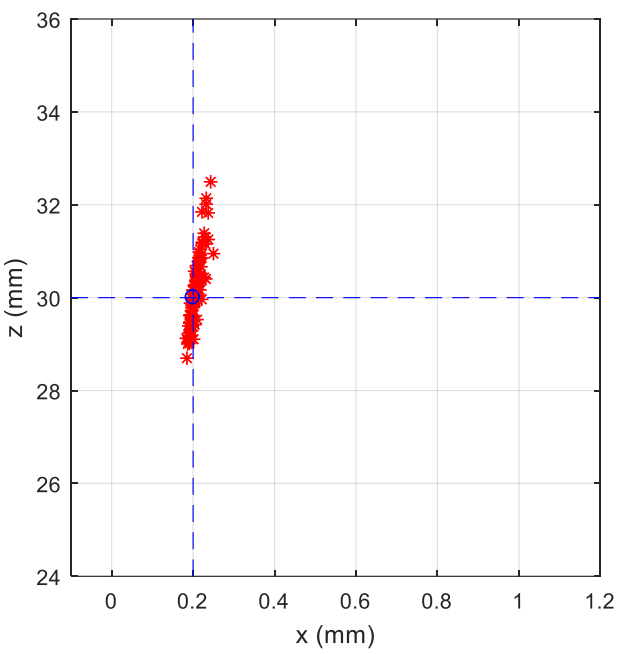

(a)

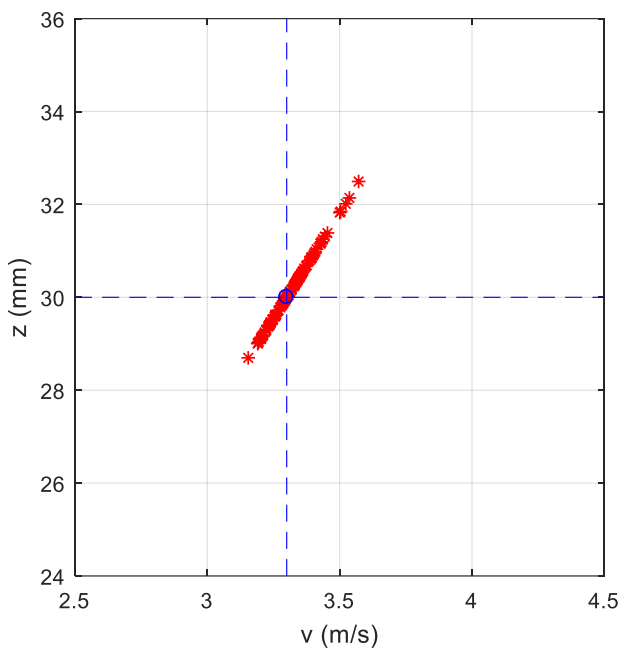

(c)

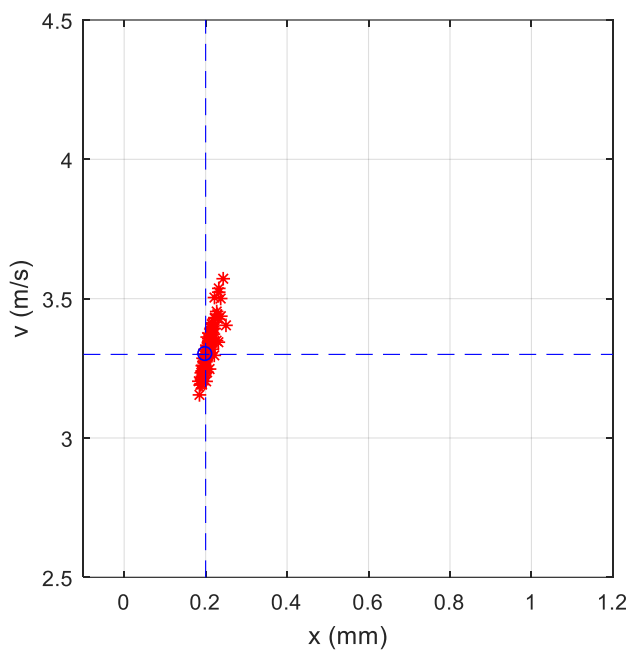

(b)

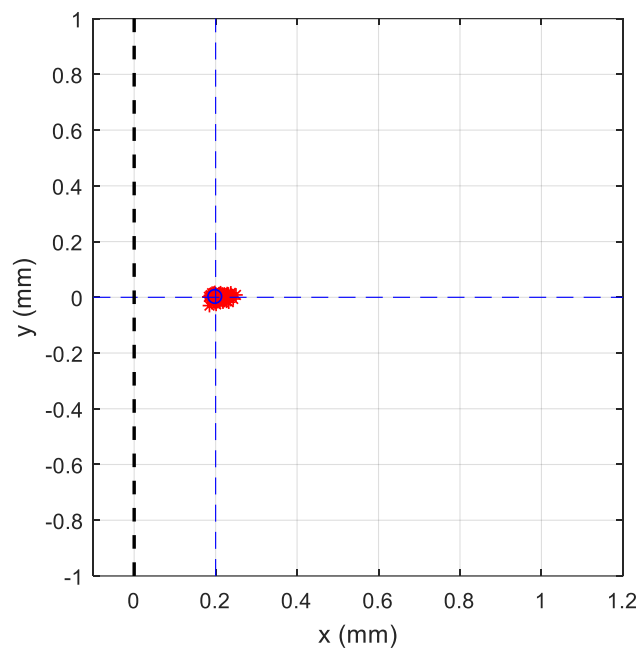

(d)

Fig. 4-13. Results of the estimated fiber parameters when a single-discharge scanning-EMG signal has been contaminated noise of all sources, and then the overall processing scheme has been applied. Red asterisks represent the estimated fiber parameters for the 100 runs executed. The dashed blue lines and the circle in which they intersect indicates the actual fiber parameters: (a) projection of the fiber parameters in the z-x plane; (b) projection of the fiber parameters in the $\mathrm{v}$-x plane; (c) projection of the fiber parameters in the $\mathrm{z}$ - $\mathrm{v}$ plane; (d) projection of the fiber parameters in the $y$-x plane. The black dashed line represents the recording corridor. 


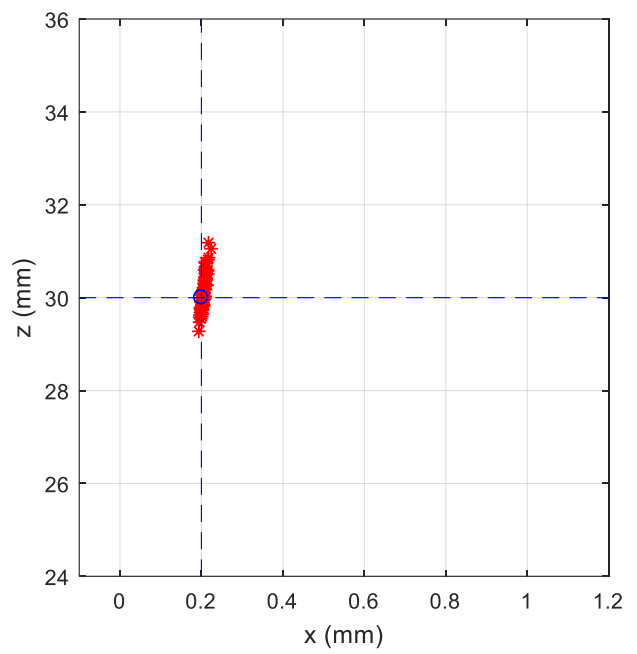

(a)

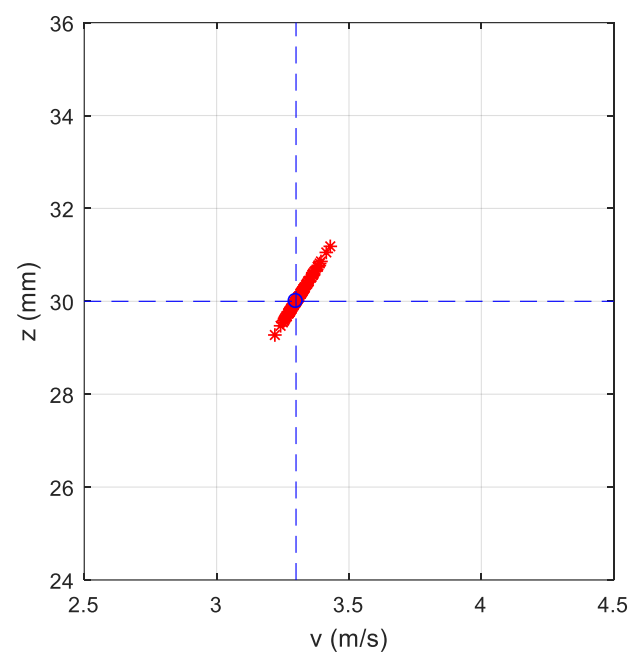

(c)

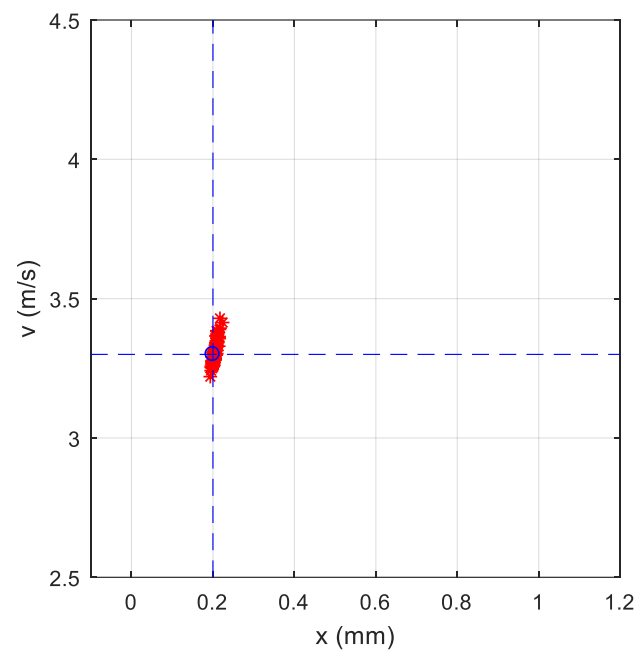

(b)

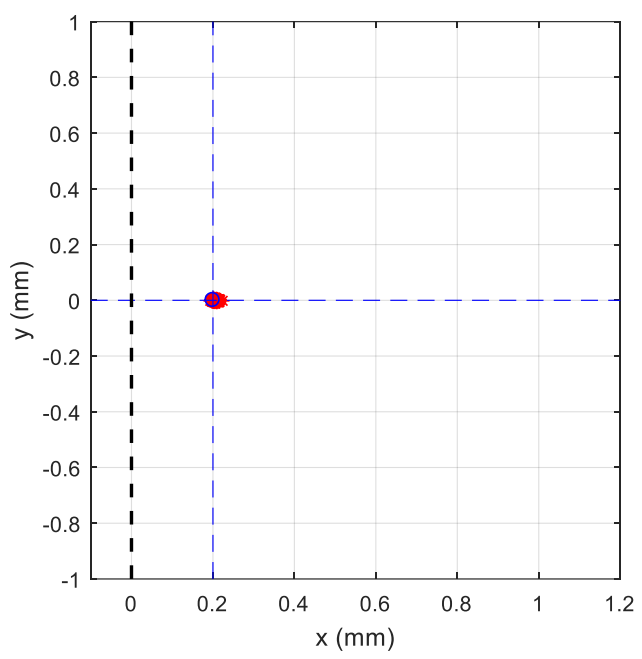

(d)

Fig. 4-14. Results of the estimated fiber parameters when a five-discharge scanning-EMG signal has been contaminated noise of all sources, and then the overall processing scheme has been applied. Red asterisks represent the estimated fiber parameters for the 100 runs executed. The dashed blue lines and the circle in which they intersect indicates the actual fiber parameters: (a) projection of the fiber parameters in the $\mathrm{z}$-x plane; (b) projection of the fiber parameters in the $\mathrm{v}$-x plane; (c) projection of the fiber parameters in the $\mathrm{z}-\mathrm{v}$ plane; (d) projection of the fiber parameters in the $y$-x plane. The black dashed line represents the recording corridor. 
noise is modeled as an ARMA process [99] obtained by filtering AWGN of $4.5 \mathrm{mV}$ standard deviation. The filter used for this purpose is a 5th-order Butterworth low-pass filter with a 3-dB cut-off frequency of $50 \mathrm{~Hz}$. Simulated artifact noise is added; this noise has been generated by simulating the signal recording procedure within a fully simulated muscle, so that MUPs from other active MUs are interfering with the MUP of the MU being tracked. The muscle contraction used for this experiment is $2 \%$ of the MVC.

2.2. The processing scheme is applied to the noisy scanning-EMG signal.

2.3. The scanning-EMG signal obtained in the previous step (reference signal) is used as input of the estimation procedure. Note that the processing scheme is also applied (excluding the MMLSS algorithm) to each simulated signal generated during the optimization. The output of this procedure is the estimated fiber parameters.

The results of the experiments are shown in Fig. 4-13 and Fig. 4-14 for the cases in which the number of MUP discharges recorded at each position are one and five, respectively. It can be observed that the dispersion in the parameter space of the points representing the fiber parameter estimations is noticeability lower when five MUP discharges per position are recorded. This means, as it would be expected, that having multiple discharges per position ensures a better parameter estimation. Therefore, in the view of the final design of the estimation system, it seems very convenient to configure the recording of the scanning-EMG signal to record several MUP discharges per position. Note that, although the use of multiple discharges involves longer times in the scanning-EMG recording procedure, it will result in a better estimation of the MU fiber parameters. 


\subsection{Effect of the cannula in the estimation}

For the purposes of this thesis, the scanning-EMG signal is recorded using SF needle electrodes. This kind of needles record the signal at each port (remember that the needle may be composed by more than one port) by subtracting from the potential detected in the port itself that of detected in the cannula. In a scanning-EMG signal, the effect of the cannula can be easily recognized by the appearance of an inverted potential in the deepest scanning positions that remains almost constant along the spatial dimension [117].

So far, the simulation model used in the estimation procedure has not taken into account the effect of the cannula, i.e., it is assumed that the potential recorded by the cannula is zero. However, it is necessary to incorporate the cannula effect into the model, so that the estimation can be successful in a real environment. Given a needle with a SF port located at a certain position, the MUP recorded by the port including the cannula effect is calculated by subtracting a simulated cannula MUP from the MUP simulated in the port position (see section 1.4.3). The cannula MUP is obtained as the result of calculating and averaging the MUP at all points defining the cannula: a set of points arranged in a straight line along the central axis of the needle.

In the first instance, it may seem that the effect of the cannula is not a severe problem for the MU parameter estimation, as it should suffice to incorporate the effect of the cannula in the simulation model. However, the estimation performance would not remain unchanged. So far, it has been assumed that the potential recorded at a certain port is only affected by those MU fibers located at one side of the needle (the side to which the port is pointing at). However, once the cannula potential is considered this assumption is not correct, as the cannula detects MU fibers located at both sides of the needle. In consequence, although the recorded potential is mainly determined by the fibers located at the side to which the port is pointing, due to the cannula effect, this one is also affected to some extent by the fibers located at the other side of the needle. This is an important consequence, as the estimation procedure designed for each recording configuration (see section 3.3.3) assumes that each port is only affected by the fibers at one side of the needle. Here, the estimation procedure is revised to make it compatible with the effect of the cannula, i.e., with the fact that the fibers at the opposite side of the port have some influence on the recorded potential.

\subsubsection{Effect of the cannula in the 1-port scanning-EMG configuration}

As previously explained, this configuration consists on a single scanning needle with a single port. In this way, only the parameters of the MU fibers located at the side at which the port is pointing are estimated. The problem of this configuration is that the MU fibers located at the opposite side, although they are not detected by the port, they are detected by the cannula. Since these fibers cannot be identified during the estimation procedure, their contribution to the recorded scanning-EMG signal can be 
considered as an unwanted interference that will impair the performance of the estimation. In order to illustrate this situation, a simulation experiment in which the cannula effect is considered is performed. This experiment consists on estimating the parameters of single fiber located at the same side as the port, when the interfering fibers located at the opposite side are present in the recorded scanning-EMG signal. The experiment steps are:

1. The noise-free scanning-EMG signal is simulated from the parameters of the MU fiber: $x_{1}=0.15 \mathrm{~mm} ; y_{1}=1 \mathrm{~mm} ; z_{1}=30 \mathrm{~mm} ; v_{1}=3.3 \mathrm{~m} / \mathrm{s}$. The recording configuration used for this purpose is the 1-port scanning-EMG signal, whose unique port is located at $x_{e}=0 \mathrm{~mm}$ and $z_{e}=0 \mathrm{~mm}$. The MUP is recorded at several locations along the $y$-axis from $y_{\min }=0 \mathrm{~mm}$ to $y_{\max }=3 \mathrm{~mm}$, in steps of $0.05 \mathrm{~mm}$. The cannula points are those corresponding to the central axis of the scanning-needle, whose position is $x_{e}=-d_{n d l} / 2$ and $z_{e}=0 \mathrm{~mm}$, where $d_{n d l}=$ $0.55 \mathrm{~mm}$ is the needle diameter.

2. For 100 independent runs:

2.1. The contribution of three fibers located at the opposite needle side is simulated, where the fiber positions are randomly drawn between $-0.8 \mathrm{~mm}$ and $-0.6 \mathrm{~mm}$ in the x-axis, and between $0 \mathrm{~mm}$ and $2 \mathrm{~mm}$ in the y-axis. This contribution is added to the noise-free scanning-EMG signal.

2.2. The scanning-EMG signal obtained in the previous step (reference signal) is used as input of the estimation procedure to obtain the estimated fiber parameters.

The results obtained for the estimated fiber parameters of the experiment are shown in Fig. 4-15. Note that the estimated fiber parameters not only present a moderate dispersion in the parameter space, but also, they present a large bias respect to the actual fiber parameters. This suggests that the presence of fibers at the opposite side of the needle provokes, due to the cannula effect, a systematic estimation error. This evidences the need of reducing the interference caused by the fibers at the opposite side of the needle.

This can be achieved by removing the lowest frequencies along the spatial dimension, by applying a proper spatial filter. The rationale is that the signal contribution recorded by the cannula barely varies along the spatial dimension, and therefore such contribution can be largely reduced if the lowest frequencies are eliminated. Reducing the signal contribution of the cannula also implies reducing the influence of the fibers at the opposite needle side. The proposed filtering consists on a moving average of 7 samples, followed by a first-order difference, both applied along the spatial dimension. The effect of this filter in a simulated scanning-EMG signal can be observed in Fig. 4-16. The figure shows two different cases, one in which the scanning-EMG signal has been simulated including the cannula effect (in red), and another in which such effect has not been included (in blue). When filtering is not 


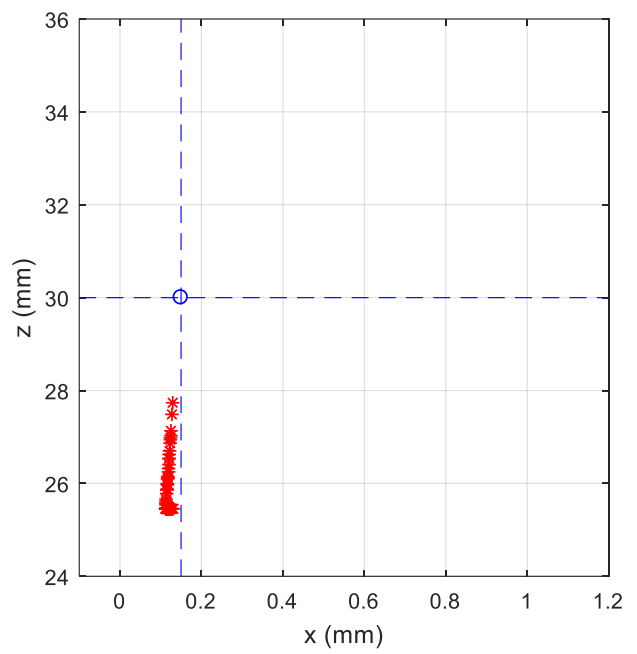

(a)

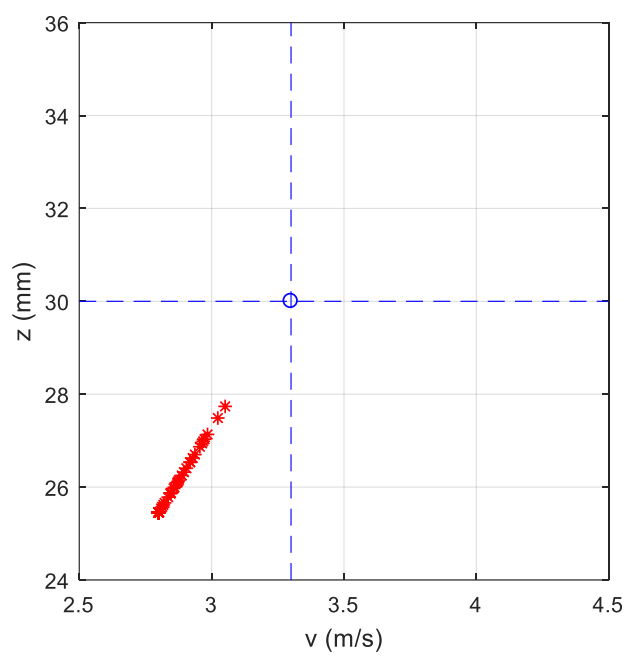

(c)

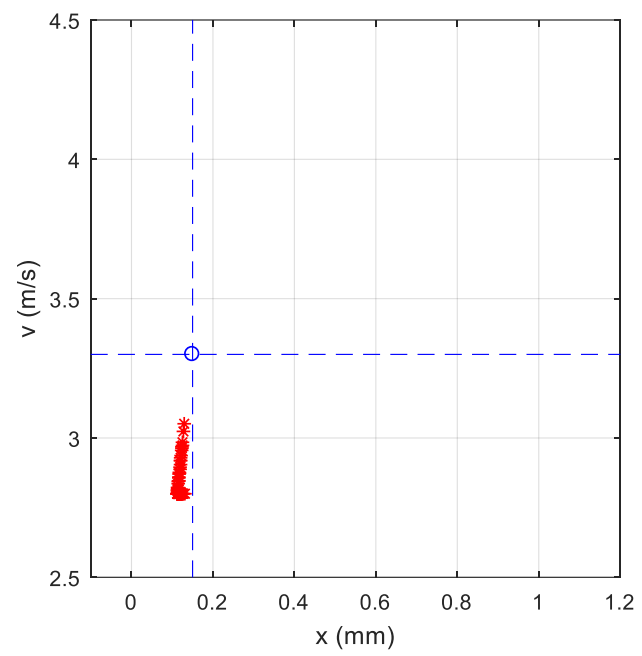

(b)

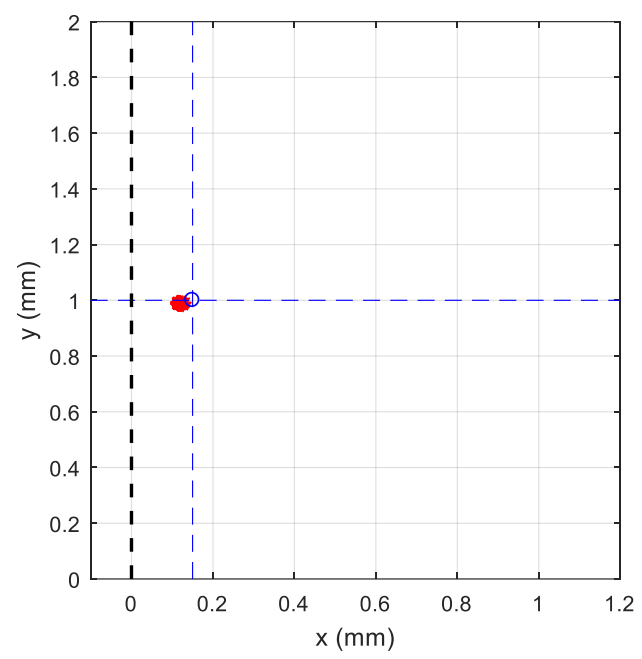

(d)

Fig. 4-15. Results of the estimated fiber parameters when the scanning-EMG signal has been contaminated with interference from fibers located at the opposite side of the needle. Red asterisks represent the estimated fiber parameters for the 100 runs executed. The dashed blue lines and the circle in which they intersect indicates the actual fiber parameters: (a) projection of the fiber parameters in the $\mathrm{z}-\mathrm{x}$ plane; (b) projection of the fiber parameters in the $\mathrm{V}-\mathrm{x}$ plane; (c) projection of the fiber parameters in the $\mathrm{z}-\mathrm{v}$ plane; (d) projection of the fiber parameters in the $y-x$ plane. The black dashed line represents the recording corridor.

applied, the signals with and without the cannula effect significantly differ in its waveform. However, when filtering is applied, these differences practically disappear. This suggests that filtering is effective in eliminating the effect of the cannula, and therefore also the effect of the fibers located at the opposite side of the port. 

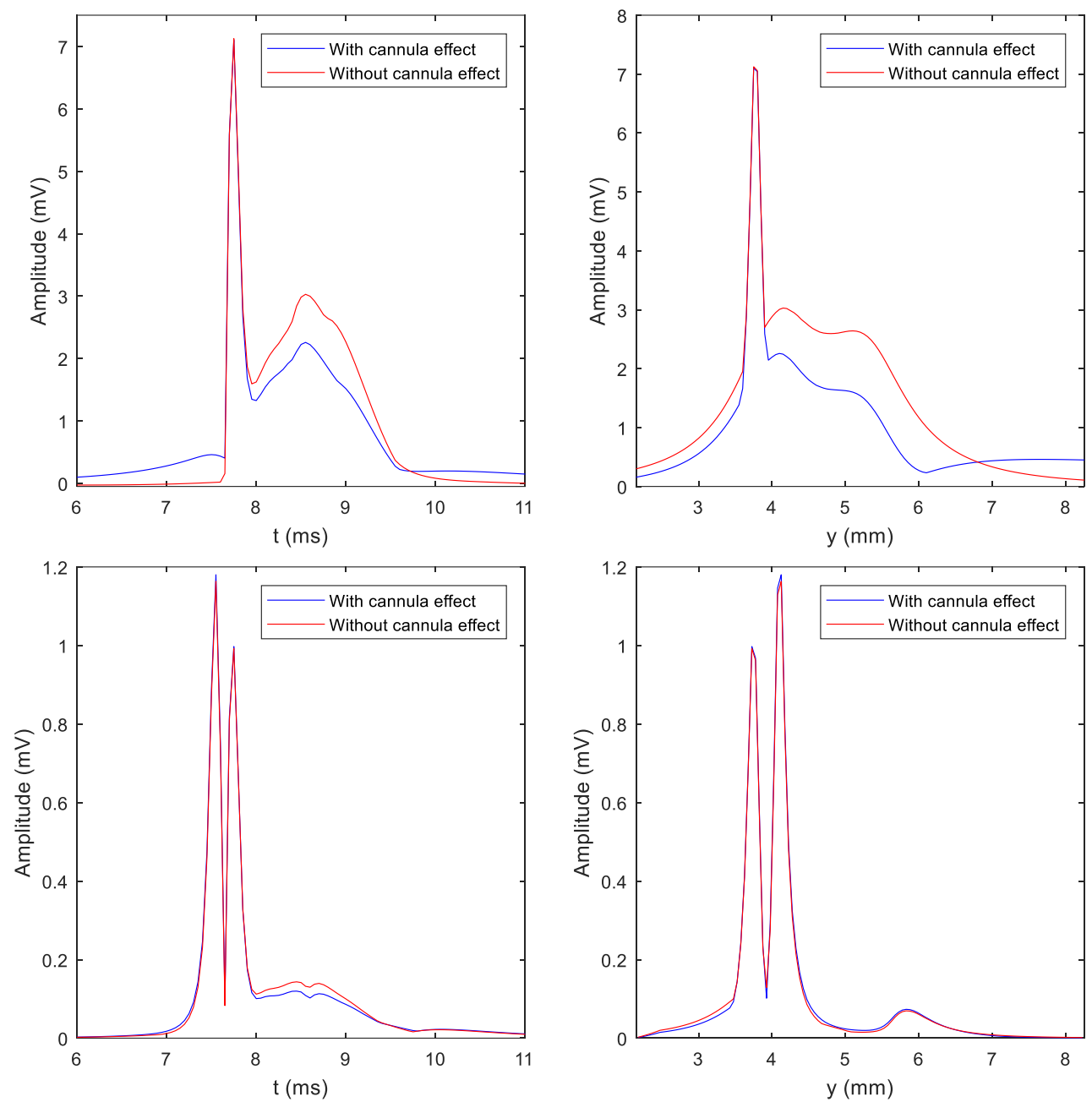

Fig. 4-16. Scanning-EMG signal simulated in two different scenarios: one in which the cannula effect is taken into account (blue), and another one in which such effect is not taken into account (red). The upper sub-figures show the simulated scanning-EMG signal (for the two cases) when no filtering is applied. The lower sub-figures show the scanning-EMG signal when the spatial filtering is applied. The subfigures on the left show the maximum amplitude profile along the time dimension, and that of the right show the profile along the spatial dimension.

To assess whether the proposed filtering improves the estimation performance or not, the previously described experiment has been modified to incorporate the spatial filtering of the scanning-EMG signal before performing the estimation procedure. The results obtained for the estimated fiber parameters are shown in Fig. 4-17. Note that the dispersion of estimated fiber parameters in the parameter space persists, but the bias respect to the actual fiber parameters has been completely removed. That is, filtering has demonstrated to be effective in eliminating the systematical error produced in the estimated parameters. However, the interference of the fibers located 
at the opposite side of the port is still introducing certain error dispersion in the parameter estimation.

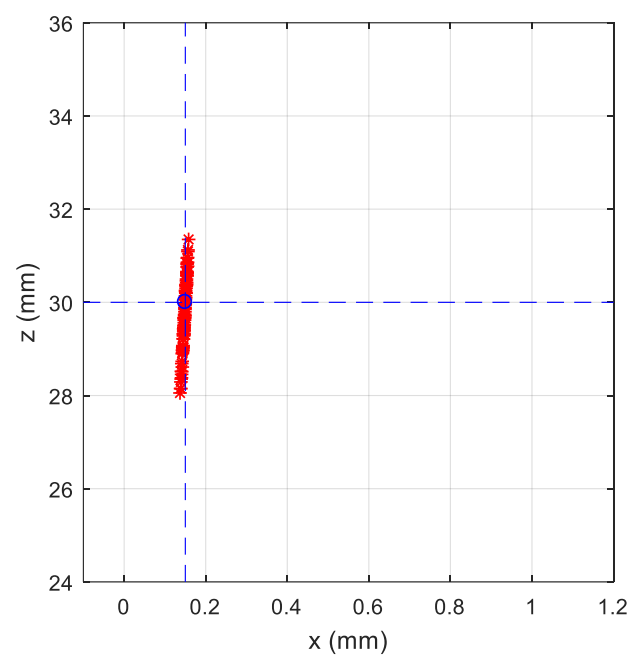

(a)

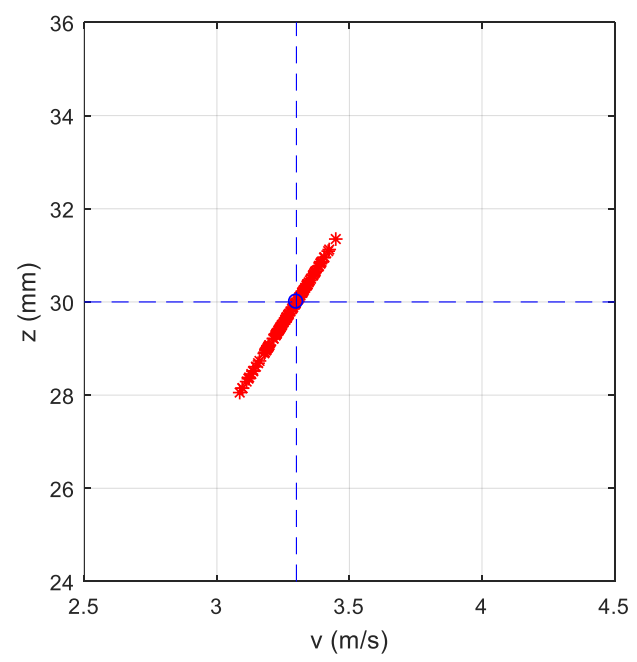

(c)

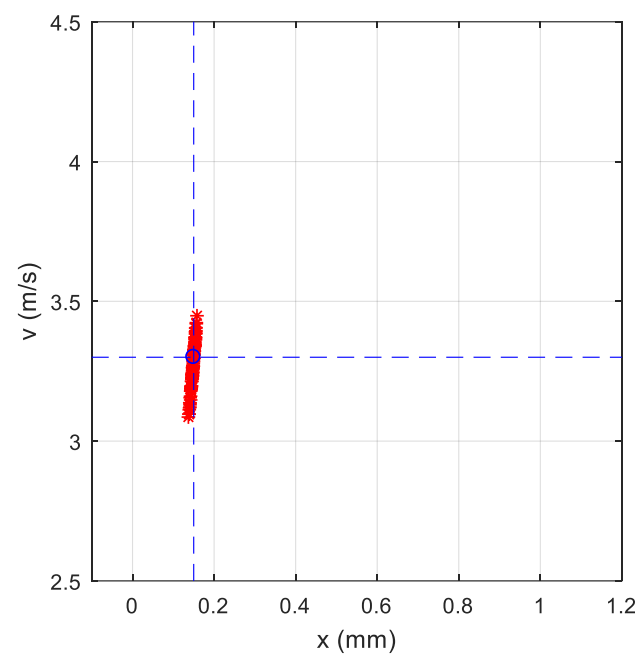

(b)

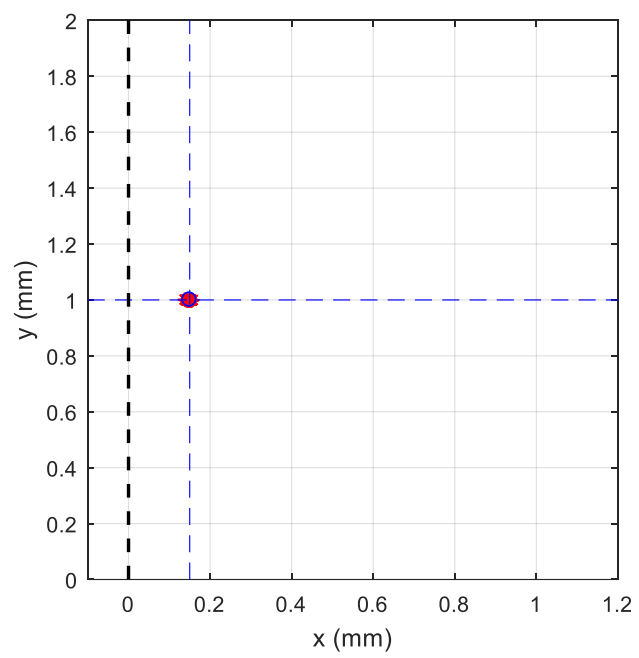

(d)

Fig. 4-17 Results of the estimated fiber parameters when the scanning-EMG signal has been contaminated with interference from fibers located at the opposite side of the needle, and then the spatial filtering has been applied. Red asterisks represent the estimated fiber parameters for the 100 runs executed. The dashed blue lines and the circle in which they intersect indicates the actual fiber parameters: (a) projection of the fiber parameters in the $\mathrm{z}-\mathrm{x}$ plane; (b) projection of the fiber parameters in the $\mathrm{v}-\mathrm{x}$ plane; (c) projection of the fiber parameters in the $\mathrm{z}-\mathrm{v}$ plane; (d) projection of the fiber parameters in the $y$-x plane. The black dashed line represents the recording corridor. 


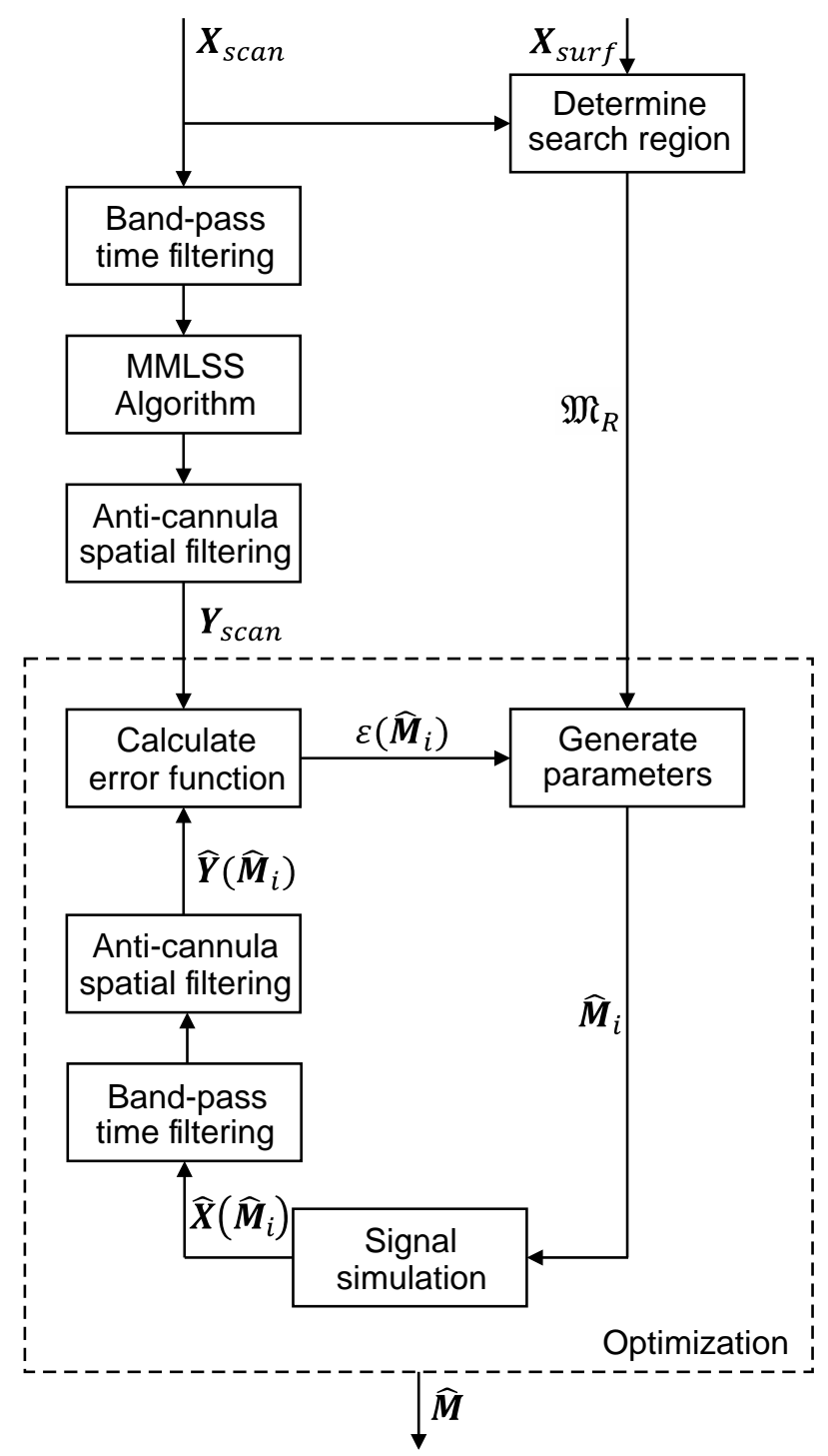

Fig. 4-18. Block diagram describing the estimation system after incorporating the anti-cannula filter. The inputs of the system are the raw recorded scanning-EMG signal $\boldsymbol{X}_{\text {scan }}$, and the surface-MUP signal $\boldsymbol{X}_{\text {surf }}$. Both signals are used to determine the region of the parameter space $\mathfrak{M}_{R}$ in which the estimated parameters will be sought. The scanning-EMG signal $\boldsymbol{X}_{\text {scan }}$, is processed to remove noise by applying: firstly, a band pass filter along the time dimension; and secondly the MMLSS algorithm. Then, the resulting signal is filtered in the spatial dimension to reduce the influence of the cannula. The output of these processing steps is the scanning-EMG signal $\boldsymbol{Y}_{\text {scan }}$. The optimization procedure used to estimate the parameters is boxed with a dashed line. This procedure iteratively generates different sets of parameters $\widehat{\boldsymbol{M}}_{i}$. These sets of parameters are used to generate the simulated signals $\widehat{\boldsymbol{X}}\left(\widehat{\boldsymbol{M}}_{i}\right)$, which are subjected to the same filters (excluding the MMLSS) applied to the raw recorded signal. The resulting signals $\widehat{\boldsymbol{Y}}\left(\widehat{\boldsymbol{M}}_{i}\right)$, are used to compute the error function values $\varepsilon\left(\widehat{\boldsymbol{M}}_{i}\right)$. Note that the calculation 
of the error function requires both the simulated signal $\widehat{\boldsymbol{Y}}\left(\widehat{\boldsymbol{M}}_{i}\right)$, and the recorded one $\boldsymbol{Y}_{\text {scan }}$. The error function values of previous iterations are used to adequately generate new sets of parameters. The output of the optimization algorithm, and of the estimation system are the estimated parameters $\widehat{\boldsymbol{M}}$, that is, the set of parameters for which the error function value is minimum. Note that usage of the anti-cannula filter depends on the recording configuration. For the 1-port scanning-EMG configuration, this filter must be applied, not so for the 2-port configuration. In the case of the 4-port scanning-EMG configuration, the anti-cannula filter must be applied only to the sections of the scanning-EMG signal corresponding to the ports pointing outwards.

Table 4-1. Operating parameters used in the estimation system for the 2-port configuration (simultaneous approach).

\begin{tabular}{ll}
\hline Parameter & Value \\
\hline Maximum distance, $R_{x}$ & 0.8 \\
Number of generations & 350 \\
Population size & 120 \\
Crossover fraction & 0.2 \\
Elite count & $0.05 \cdot$ Population size \\
Mutation scale & 1 \\
Mutation shrink & 1 \\
\hline \multicolumn{1}{c}{ Interior point } \\
\hline Maximum distance, $R_{x}$ & 1.5 \\
Number of iterations & 10000 \\
\hline & \\
\hline Interval semi-width in $\mathrm{z}, R_{z}$ & $8 \mathrm{~mm}$ \\
Interval semi-width in $\mathrm{v}, R_{v}$ & $0.6 \mathrm{~m} / \mathrm{s}$ \\
Number of repetitions & 5 \\
Number of simulated fibers & $18 \mathrm{at}$ each side \\
\hline
\end{tabular}

In conclusion, to reduce the detrimental effect produced by the cannula in the estimation, the 1-port scanning-EMG recording configuration has been modified by including a spatial filter in the estimation system (Fig. 4-18).

\subsubsection{Effect of the cannula in the 2-port scanning-EMG configuration}

This recording configuration consists on a single scanning needle with two ports located at opposite sides of the needle. In this way, the parameter of the MU fibers located at both sides of the scanning needle can be estimated. This fact can be exploited 
when it comes to deal with the cannula effect and the influence of the fibers on the opposite side. By allowing the MU fibers to be estimated on both sides of the needle, the estimation procedure can take into account the contribution of the fibers located at the opposite side of each port. In this way, such contribution is no longer an unwanted interference, as it occurred for the 1-port scanning-EMG configuration; instead, it is a contribution that the simulated scanning-EMG signal can adjust as the estimation of the fibers at the opposite side is refined during the estimation process. To achieve this, it is necessary to modify the estimation procedure associated to this configuration, which is described in section 3.3.3, so that the estimation is carried out simultaneously in the two search regions corresponding to both sides of the scanning needle (Fig. 3-28 (b)). It is important to recall that, until now, the parameters of the MU fibers in each of these two regions could be estimated independently. The operating parameters corresponding to this simultaneous estimation procedure are shown in Table 4-1.

By implementing this modification in the estimation procedure, the 2-port scanning-EMG configuration is expected not to be negatively affected by the presence of the cannula. Therefore, unlike the previous configuration, it seems not necessary to apply the anti-cannula spatial filtering.

\subsubsection{Effect of the cannula in the 4-port scanning-EMG configuration}

This recording configuration consists of two scanning needles, where each needle is composed by two ports located at opposite needle sides. Let's call these ports, from left to right: P1, P2, P3 and P4 (Fig. 3-28 (c)). For this configuration, the estimation is performed in three different search regions: one at one side of the needle arrangement (R1), another one between both scanning needles (R2), and the last one at the other side of the needle arrangement (R3) (Fig. 3-28 (c)).

The estimation procedure used so far for this configuration is complex (see section 3.3.3), but it is essentially based on applying the estimation procedure in the different regions in a sequential approach. Firstly, the MU fibers located in the external regions are independently estimated from the sections of the scanning-EMG signal recorded in the external ports (R1 from P1, and R2 from P4); and secondly, taking into account the result of this estimation, the fibers located in the internal region (R2) are estimated from the signal sections recorded in the internal ports (P2 and P3). As long as the effect of the cannula is not taken into account, this scheme is valid, as the external ports (P1 and P4) only detect the fibers of its corresponding external region (R1 and R3, respectively) (Fig. 3-28 (c)). However, when the cannula effect is considered, the external ports are affected by the fibers of the other regions. The simplest solution to this problem would be to apply a simultaneous estimation in the three regions, using the signal sections recorded in all ports at the same time. However, this alternative has been discarded due to its high computational load, mainly because it requires a very large number of iterations for the optimization algorithms to converge. Therefore, the adopted solution has been to keep the estimation scheme used so far, but applying the 
previously described anti-cannula spatial filtering to the sections of the scanning-EMG signal associated to the external ports (that is, $\mathrm{P} 1$ and $\mathrm{P} 4$ ), in such a way that the effect of the cannula, and therefore that of the fibers on the opposite side, is mitigated. The resulting estimation system once such filter has been incorporated is shown in Fig. 4-18. 


\subsection{Considerations about the type of electrode used in the estimation}

The estimation system designed in this thesis assumes the use of SF needle electrodes to record the scanning-EMG signal. As explained in section 1.2.1, this kind of electrode consists on a needle of about $0.5 \mathrm{~mm}$ diameter, with one or several ports, in which a platinum surface is exposed. This surface has a diameter of about $25 \mu \mathrm{m}$. The very small size of the port surface is the main reason why this type of electrode has been chosen for the estimation. Note that this small size implies a very pronounced radial decline, which in turn implies a very high spatial resolution. This characteristic allows to distinguish individual fiber contributions in the recorded scanning-EMG signal much easier than with other needle electrodes. This, in turn, could result in an enhanced estimation of the MU fiber parameters.

There is another important advantage of using SF needle electrodes. It is possible to manufacture them with more than one port surface $[12,13,113]$. In this way, the needle can be designed to have a port at each side, which allows that MU fibers can be detected and estimated at each side of the needle. This point has been crucial when it came to raise the different recording configurations proposed in this thesis.

Despite these advantages, the SF needle electrode has an important drawback, as this type of electrode is very expensive. This led to consider using other type of needle electrodes to record the scanning-EMG signal: the $\mathrm{MN}$ and the $\mathrm{CN}$ electrode. Although these alternative electrodes were eventually discarded, its feasibility as a part of the estimation system is briefly analyzed here.

\subsubsection{The monopolar needle electrode}

The MN electrode, as explained in section 1.2.1, is composed by an insulated needle with a conical recording surface of $0.25 \mathrm{~mm}^{2}$ area. In this electrode, the potential is derived between such surface and a separate surface electrode that acts as reference. Due to its design, this electrode has several drawbacks when used in the estimation system:

- As a consequence of a very large recording surface, the spatial resolution of the electrode is very low. This would probably make difficult to separate the signal contribution of each MU fiber during the estimation procedure.

- The fact that, instead of the cannula, a separate surface electrode is used as reference implies that much more noise and distant interference is introduced during the recording procedure, which would possibly imply an inaccurate parameter estimation. 
These two important drawbacks make this electrode inadequate to be used in the estimation system.

\subsubsection{The concentric needle electrode}

In its standard version, the $\mathrm{CN}$ electrode has an elliptical recording surface of 0.07 $\mathrm{mm}^{2}$, but there are also smaller versions of this electrode, like the facial CN electrode, whose recording surface is about $0.02 \mathrm{~mm}^{2}$. Note that for the purpose of this thesis, the facial version is the most suitable option because of its smaller dimensions. Even so, the facial electrode still has a large recording surface compared to the SF electrode. The great advantage of the facial $\mathrm{CN}$ electrode is that it is much cheaper. Note that, a single SF needle electrode costs between 300 and 600 euros in its reusable version, and about 60 euros in its disposable version. In the case of the $\mathrm{CN}$ electrode, a batch of 25 disposable needles costs around 60 euros (January 2020 prices).

All these characteristics make the facial $\mathrm{CN}$ electrode the best alternative to the SF electrode. To assess whether or not this electrode is actually suitable to be used in the estimation system, a few trials of a complete MU estimation were performed. These trials consist on estimating the MU fiber parameters from a scanning-EMG signal recorded with a single scanning electrode. The simulation of the recording consists on the following steps:

1- The muscle and MUs are simulated as described in section 1.4.1.

2- The scanning needle electrode is inserted in the middle of the muscle cross-section, along the y-axis; and $30 \mathrm{~mm}$ away from the innervation zone along the z-axis (the direction parallel to the fibers).

3- The MU selected to perform the estimation is the smallest one whose territory is traversed by the corridor.

4- The recording procedure of scanning-EMG signal is simulated for this MU. During the recording, the needle is moved along the y-axis in steps of $0.05 \mathrm{~mm}$, traversing the muscle completely from the deepest to the most superficial part. No noise has been simulated in the recording of the scanning-EMG signal.

At each trial, the recording of the scanning-EMG signal is simulated both for the $\mathrm{CN}$ and the SF electrode. This allows to perform the estimation of the same MU with both electrodes, so that these can be compared in equal terms. An important consideration about the signal recorded with the $\mathrm{CN}$ electrode is that the effect of recording surface must be simulated (see section 1.4.3). In this way, the MUP is calculated and averaged for a grid of points over the $\mathrm{CN}$ recording surface [84]. This surface, for the facial version, consists on an elliptical recording surface of $0.02 \mathrm{~mm}^{2}$, with a $300 \mu \mathrm{m}$ mayor axis and $80 \mu \mathrm{m}$ minor axis.

Both for the CN and the SF needle electrode, the MU fibers located at both sides of the needle were estimated. Note that, we assume that the $\mathrm{CN}$ electrode detects the MU fibers lying both in front of and behind the electrode, which is in acordance with 


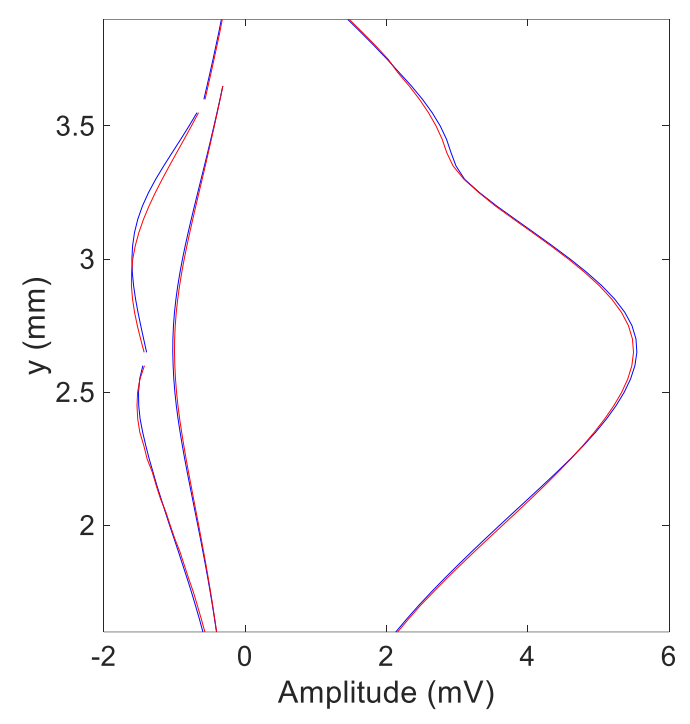

(a)

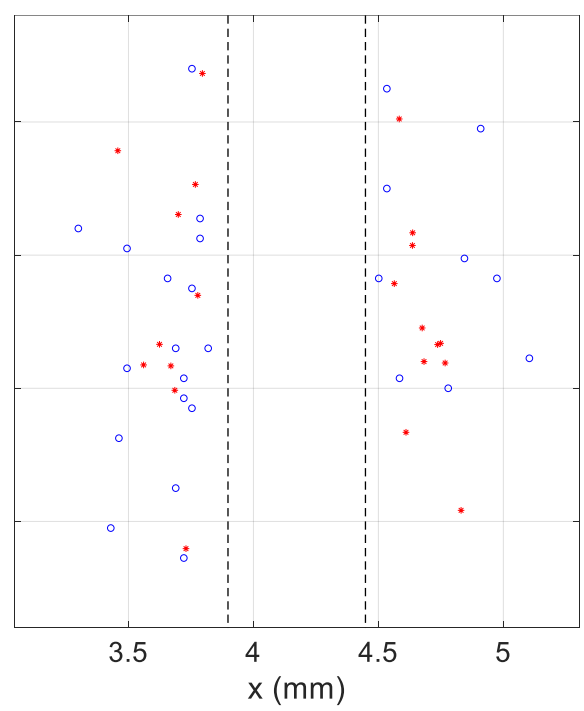

(b)

Fig. 4-19. Estimation results of the first example for the scanning-EMG signal recorded with the $\mathrm{CN}$ needle electrode; (a) amplitude of the MU profile in the spatial dimension of the estimated (red lines) and reference (blue lines) scanning-EMG signals; (b) estimated (red asterisks) and real (blue circles) MU fibers in the $x-y$ plane. The two vertical dashed lines represent the limits of the scanning needle, and the needle corridor is the space between them.

$[50,57]$. Hence, a correct parameter estimation requires that the estimation procedure is performed simultaneously at both sides of the needle. In the case of the SF needle electrode, the 2-port recording configuration is used, so that the estimation is also performed at both sides of the needle.

Two specific cases extracted from the set of trials are presented here as examples. Fig. 4-19 and Fig. 4-20 show the estimation results corresponding to the first example for the $\mathrm{CN}$ and the SF needle electrode, respectively. Analogously, Fig. 4-21 and Fig. 4-22 show the results of the second example. Note that each figure shows, in addition to the estimated and real parameters, the MU profiles of the estimated and reference scanning-EMG signals, so that the degree of waveform fitting can be visually assessed. See section 2.1.2 for a description of how the MU profile is extracted from the scanning-EMG signal.

In both estimation examples, both for the $\mathrm{CN}$ and the $\mathrm{SF}$ needle electrode, the estimated scanning-EMG signal has been fitted to the reference scanning-EMG signal with great precision. For the estimations with the SF needle electrode, this good fit corresponds to a correct estimation of the MU fibers, at least for those fibers close to the scanning corridor. This is not the case for the estimation with the $\mathrm{CN}$ electrode, for which the parameter estimation has been incorrect, despite the good fitting of the signal 
waveform. This behavior has been observed, not only in the examples shown here, but in almost all the estimation trials. The fact that a good waveform fit does not lead to a correct estimation implies that the scanning-EMG signal recorded with the $\mathrm{CN}$ electrode does not provide enough information to solve the inverse problem, at least not under these experimental conditions. This lack of information means that there are several parameter solutions that give rise to a simulated signal waveform almost identical to that of the recorded signal, and that the estimation procedure is unable to distinguish which of these solutions actually corresponds to the real MU parameters. Given the good fit of the waveform, and the fact that no noise source has been introduced in the recorded signal, it is clear that the misestimation of the MU fiber parameters is exclusively due to the lack of information inherent to the recording with the $\mathrm{CN}$ electrode. This suggests that, in general, it will be difficult to correctly estimate the MU fiber parameters from a $\mathrm{CN}$ electrode scanning-EMG recording.

This apparent increased difficulty to estimate the MU fiber parameters for the $\mathrm{CN}$ electrode when compared to the SF electrode, may be due to several reasons. The first one is because of the radial decline. As a consequence of the size of the recording surface, the radial decline is much less pronounced for the $\mathrm{CN}$ electrode than for the SF. This fact implies that the individual signal contributions of the different MU fibers

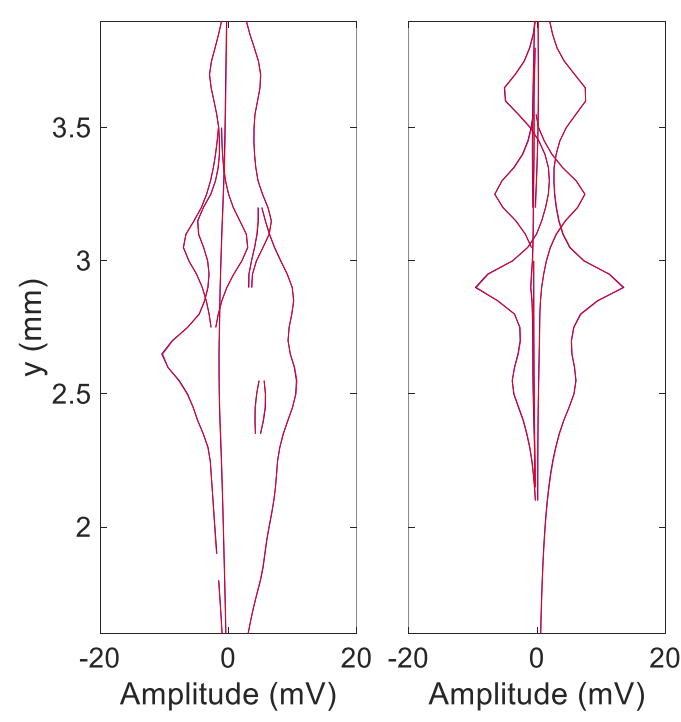

(a)

(b)

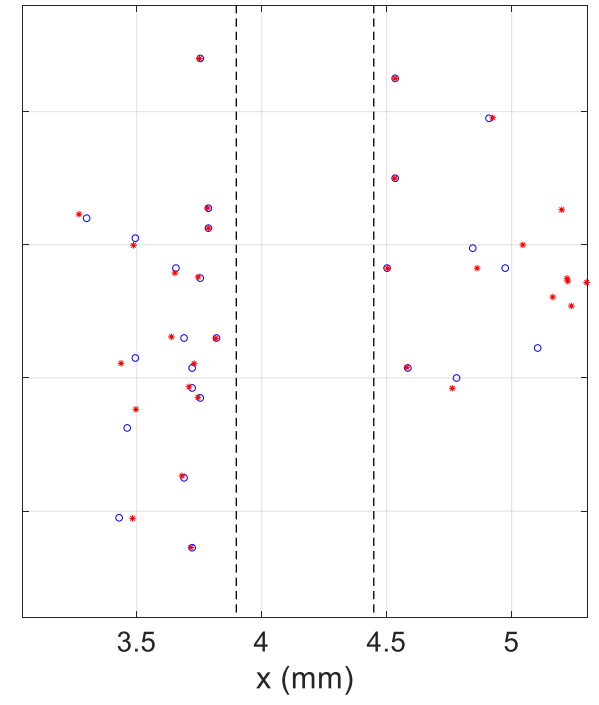

(c)

Fig. 4-20. Estimation results of the first example for the scanning-EMG signal recorded with the 2-port SF needle electrode; (a) and (b) amplitude of the MU profile in the spatial dimension of the estimated (red lines) and reference (blue lines) scanning-EMG signals, where (a) corresponds to the scanning-EMG signal section recorded in the left port, and (b) corresponds to that of recorded in the right port; (c) estimated (red asterisks) and real (blue circles) MU fibers in the $x-y$ plane. The two vertical dashed lines represent the limits of the scanning needle, and the needle corridor is the space between them. 


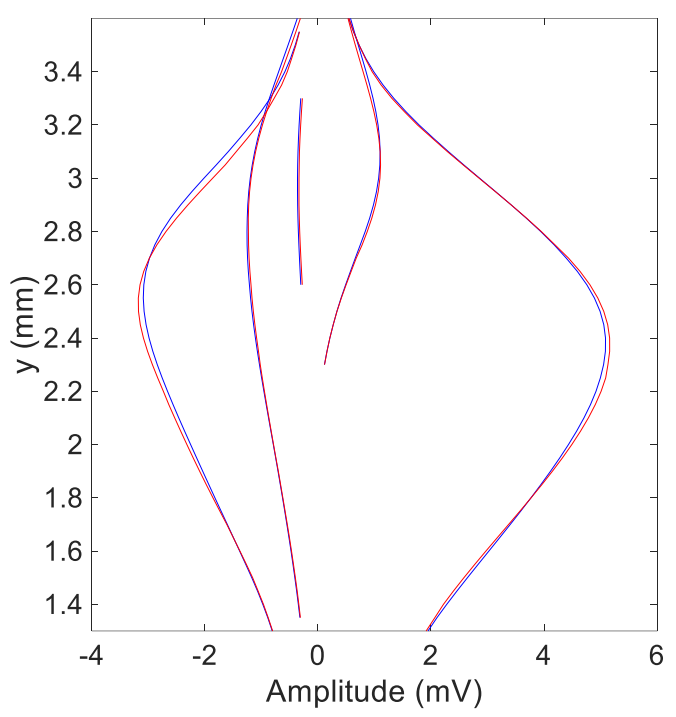

(a)

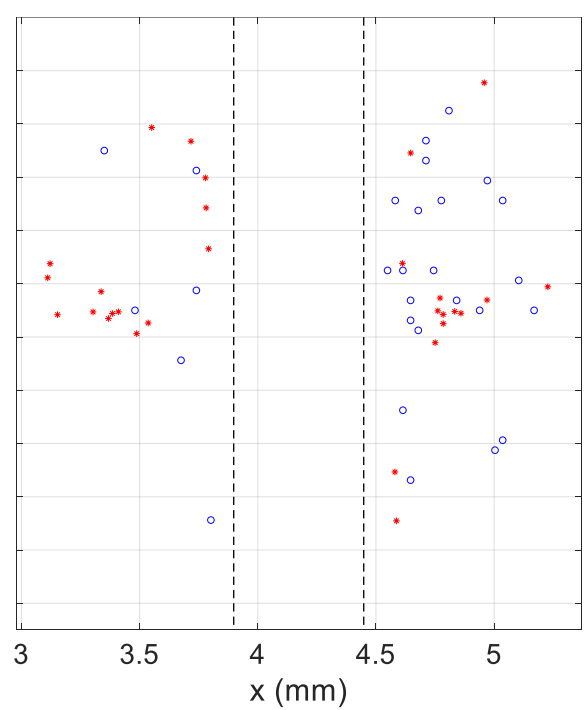

(b)

Fig. 4-21. Estimation results of the second example for the scanning-EMG signal recorded with the $\mathrm{CN}$ needle electrode; (a) amplitude of the MU profile in the spatial dimension of the estimated (red lines) and reference (blue lines) scanning-EMG signals; (b) estimated (red asterisks) and real (blue circles) MU fibers in the $x-y$ plane. The two vertical dashed lines represent the limits of the scanning needle, and the needle corridor is the space between them.

are much wider for the $\mathrm{CN}$ electrode recordings than for the SF ones. This can be visually corroborated in the signals of the previous examples. For the signals recorded with the SF needle (Fig. 4-20 (a) and Fig. 4-22 (a)), it can be observed that the profile along the spatial dimension presents several sharp peaks associated to one or very few MU fibers. This is not the case for the $\mathrm{CN}$, for which the recorded signals present a profile in the spatial dimension in which a large number of fiber contributions are mixed in the same spatial region (Fig. 4-19 (a) and Fig. 4-21 (a)). In this context, many fiber combinations originating a simulated signal very similar to the recorded one may exist. This lack of uniqueness of the solution means that after the estimation there is a high uncertainty about the actual value of the MU fiber parameters. In addition, the less pronounced radial decline associated to the $\mathrm{CN}$ electrode also implies that the distant fibers contribute to the scanning-EMG signal with higher relative amplitude than in the case of the SF needle electrode. This may also negatively affect to the estimation, as, in practice, it implies that a larger number of MU fibers are contributing to the potential, which in turn could further increase the number of valid solutions (that is, with a good waveform fit).

Another important factor to understand why the MU fiber parameters are not correctly estimated with the $\mathrm{CN}$ electrode is the shape of its uptake area. Note that, in the estimation examples, we assume that the $\mathrm{CN}$ recording surface detects the $\mathrm{MU}$ 


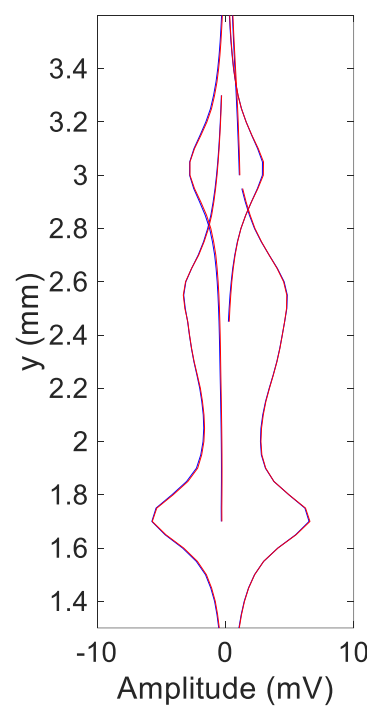

(a)

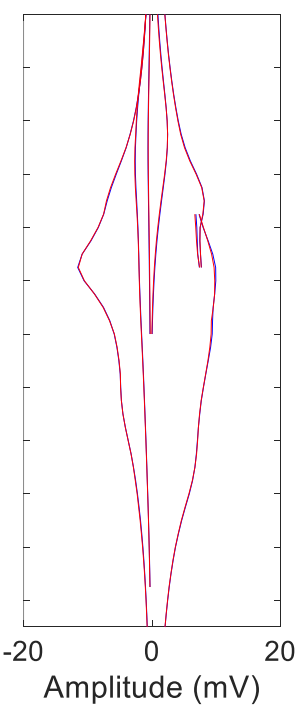

(b)

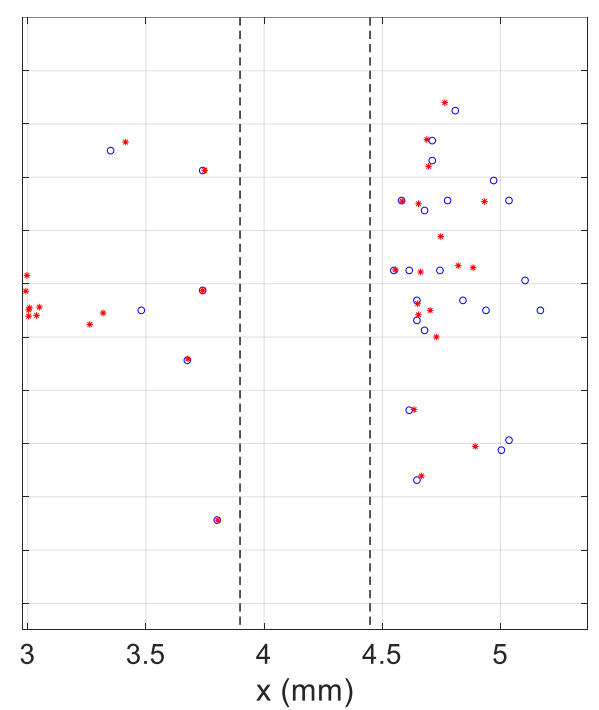

(c)

Fig. 4-22. Estimation results of the second example for the scanning-EMG signal recorded with the 2-port SF needle electrode; (a) and (b) amplitude of the MU profile in the spatial dimension of the estimated (red lines) and reference (blue lines) scanning-EMG signals, where (a) corresponds to the scanning-EMG signal section recorded in the left port, and (b) corresponds to that of recorded in the right port; (c) estimated (red asterisks) and real (blue circles) MU fibers in the $x-y$ plane. The two vertical dashed lines represent the limits of the scanning needle, and the needle corridor is the space between them.

fibers located at both sides of the needle $[50,57]$, unlike the SF needle electrode, for which each port mainly detects the fibers located in front of it (only the cannula can partially detect activity from fibers located behind the port). In this way, for the 2-port SF needle electrode used in the trial examples, the fiber signal contributions of one side of the needle barely interacts with those of the other side. However, for the $\mathrm{CN}$ electrode, all these contributions are recorded with the same recording surface, and therefore they may appear superimposed in the same region of the recorded scanningEMG signal. This, in turn implies a greater difficulty to correctly estimate the MU fiber parameters. Another consequence of the $\mathrm{CN}$ electrode detecting the fibers on both sides of the needle is that a fiber located at one side of the needle can lead to an estimated fiber located at the opposite needle side. An evident case of this phenomenon has occurred in the second example (Fig. 4-21 (b)), with the MU fiber located at $y=$ $1.72 \mathrm{~mm}$. This phenomenon is therefore, another source of error in the estimation of the MU fiber parameters.

In conclusion, the results from the estimation trials suggest that the $\mathrm{CN}$ electrode, when used in the estimation system, leads to an incorrect parameter estimation. The analysis of these results suggests that this is due to a loss of information inherent to the 
recording with this kind of electrode. After these considerations the use of the $\mathrm{CN}$ electrode to record the scanning-EMG signal was discarded. The SF needle electrode, which according to these preliminary experiments is able to achieve a correct parameter estimation, would be used instead. 


\section{Evaluation of the estimation system}

In this chapter, the ability of the estimation system to correctly estimate the MU fiber parameters is evaluated. This evaluation is mostly based on simulation experiments, which consist on estimating the MU fiber parameters for multiple realizations of the muscle, the MU under study, and the signal recording procedure. The experiments have been performed for the three recording configurations proposed to be incorporated in the estimation system (the 1-port scanning-EMG, the 2-port scanning-EMG, and the 4-port scanning-EMG), and also for a fourth configuration consisting on recording the MUP at a single-point. Furthermore, different scenarios in which the estimation performance may decrease have been studied. These scenarios comprise: increased muscle voluntary contraction; discrepancies between the IAP model used in the estimation system, and the actual IAP of the MU fibers; increased MU fiber density; increased variance of the MFCV's; and increased IZ width. In addition to the evaluation based on simulation, the chapter also includes a brief analysis of a few cases in which the estimation system has been applied to real muscles. The chapter starts describing the different sets of simulation experiments, and continues showing and analyzing the results of these experiments. Then, tests performed with real muscles are described and analyzed. The chapter ends with a general discussion about the estimation system performance. 
upna

Universidad Púbilica de Navara
Natarmaako Unibersitate Publikoa 


\subsection{Description of the simulation experiments}

\subsubsection{General experimental setup}

The performance of the estimation system has been evaluated by executing different sets of experiments under a realistic simulation environment, which takes into account many of the possible effects that would occur in a real scenario, such as the noise introduced in the signal recording, the ploughing effect of the scanning needles, and the cannula effect. Different experiments correspond to different scenarios (different recording configurations, different muscle contraction levels, etc.). Each run of a simulation experiment comprises the following steps:

1. Generation of the simulation environment

1.1. Generation of the muscle and MUs: the parameters describing the different fibers of each MU of the entire muscle are obtained as described in section 1.4.1, with the set of simulation parameters indicated in Table 1-2.

1.2. Placement of the surface electrode array: a linear array of surface electrodes is simulated, where the electrode positions comprise from $z=-35 \mathrm{~mm}$ to $z=35 \mathrm{~mm}$ along the z-axis, with an inter-electrode distance of $10 \mathrm{~mm}$. Note that the IZ is located at $z=0 \mathrm{~mm}$. In the $\mathrm{x}$-axis, the electrode array is placed in the middle of the muscle. The surface electrodes are circular in shape, with a diameter of $5 \mathrm{~mm}$.

1.3. Insertion of the scanning needles: the number of scanning needles inserted in the muscle, their positions, and the number the number of ports of each needle, determine the set of points at which the MUP will be recorded. All these aspects will depend on the recording configuration used, and are simulated according to the description of the scanning-EMG montage explained in section 3.3.1. In all configurations $x_{e}$ corresponds to the center of the muscle along the $\mathrm{x}$-axis, and $z_{e}=30 \mathrm{~mm}$. The ploughing effect that the scanning needles cause in the muscle fibers is simulated according to the ploughing model described in section 1.4.3.

1.4. Selection of the MU under analysis: the MU selected to perform the estimation is the smallest one whose territory is traversed by the needle corridors.

1.5. Generation of the firing patterns: the firing patterns of the MU pool are simulated for a 3.5\% MVC. The model described in section 1.4.4 has been used, with the parameters indicated in Table 1-4.

1.6. Simulation of the recorded surface-MUP: the surface-EMG pattern is 
simulated according to the model described in section 1.4.7, by convoluting the (noise-free) simulated surface-MUPs of all active MU with their corresponding firing patterns, and summing all contributions. The (noisy) recorded surface-MUP corresponding to the MU under analysis is obtained by applying spike-trigger averaging to the surface-EMG pattern.

1.7. Simulation of the recorded scanning-EMG signal: the recording of scanningEMG corresponding to the MU under analysis is simulated as described in section 1.4.6, with the parameters indicated in Table 1-5. Note that the entire scanning-EMG recording procedure is simulated so that the resulting signal is contaminated with artifacts. This requires to simulate the (noise-free) MUP of the active MUs at each recording position, with the model described in section 1.4.2, using the parameters indicated in Table 1-3. Note that the cannula effect is included in the modeling of the MUP. Instrumentation and baseline noise are also added to the scanning-EMG signal.

2. Processing of the scanning-EMG signal: the processing scheme described in section 4.2.4 is applied to the scanning-EMG signal to reduce the effect of noise. Furthermore, depending on the recording configuration used (see details in section 4.3), a spatial filter is applied to the scanning-EMG signal to mitigate the effect of the cannula.

3. Estimation of the MU fiber parameters: the estimation system obtains the estimated MU fiber parameters, from the surface-MUP and scanning-EMG signals. Note that the estimation system presents certain variations depending on the recording configuration used (as detailed in section 3.3.3). An important consideration is that the potential initiation instant is estimated following the algorithm explained in section 4.1 .

4. Calculation of the errors: different figures of merit are calculated to quantify the discrepancies between the estimated MU (obtained in step 3) and the actual one (obtained in step 1.4). The calculation of these errors is described in detail in sections 3.4.1 and 3.4.2. Note that the errors are calculated as a function of the distance to the electrode (i.e., of the evaluation region), as detailed in section 3.4.1.

An experiment is composed by the execution of these steps for 100 independent runs, so that it allows to perform a statistical analysis of the errors. Given a certain evaluation region that covers up to a certain distance from the electrode, this statistical analysis consists on calculating the 10th, 25th, 50th, 75th and 90th percentiles, and the median absolute error (MAE) of the different estimation errors, from the total of runs executed. In this way, for each experiment, the following statistics can be obtained:

- Percentiles and MAE of the estimation error of the individual MU fiber parameters (see section 3.4.1). Note that these percentiles are calculated from the set of all the 
fiber associations within the evaluation region from all the available runs.

- Percentiles of the estimation error of the MFCV mean, $e_{V m}$ (see section 3.4.2).

- Percentiles of the estimation error of the MFCV standard deviation, $e_{V s}$ (see section 3.4.2).

- Percentiles of the IZ excess, $E_{I}$ (see section 3.4.2).

- Percentiles of the IZ defect, $D_{I}$ (see section 3.4.2).

- Percentiles of the estimation error of the global fiber density, $e_{D}$ (see section 3.4.2).

\subsubsection{Estimation with the different recording configurations}

In order to analyze and compare the estimation performance for the different recording configurations, the previously described experiment setup is executed independently for all these configurations, i.e., for the 1-port scanning-EMG, the 2port scanning-EMG, and the 4-port scanning-EMG recording.

We are also interested in comparing these three configurations with the case in which the MUP is recorded in a single position, as this case would be equivalent to the previous attempts to solve the inverse problem [67, 68, 140] (see sections 1.3.3 and 1.3.4). Therefore, the experiment scheme is also executed for a fourth recording configuration, the single-point recording, where the recording point is located in the center of the muscle.

Note that the general experiment scheme allows to study the general performance of each configuration by carrying out the estimation for different simulated MUs, each of them generated independently. However, we are also interested in the comparing the estimation results when the four methods deal with the estimation of the same MU. In this case, an additional visual comparison about the estimated parameters of its constituting fibers can be obtained. In this way, a different experiment scheme has been executed, also for the four recording configurations studied. This single MU experimental setup consists on the following steps:

1. Generation of a single MU, applying the steps 1.1 to 1.4 of the general experiment scheme.

2. For 35 independent runs:

2.1. Generation of a random realization of the recorded signals, applying the steps 1.5 to 1.7 of the general experiment scheme.

2.2. Processing of the scanning-EMG signal, applying the step 2 of the general experiment scheme.

2.3. Estimation of the MU fiber parameters, applying the step 3 of the general experiment.

2.4. Association between the estimated MU fibers and the actual ones, applying the criterion explained in section 3.4.1. 


\subsubsection{Estimation with increased muscle contraction}

In order to assess to what extent a lower signal quality negatively affects to the estimation performance, some experiments have been performed at a higher level of muscle voluntary contraction. Note that, the larger the contraction level, the larger the number of superimposed artifacts in the recorded scanning-EMG signal. In this way, the general experiment scheme has been executed for the 2-port recording configuration, for a $5.5 \% \mathrm{MVC}$.

Note that, from now on, only the 2-port scanning-EMG configuration is going to be studied. The reason why this configuration has been chosen is because it presents an adequate balance between simplicity and estimation performance, as it will be discussed throughout this chapter. Among the two configurations that use a single scanning-needle (the 1-port and 2-port scanning-EMG), the 2-port scanning-EMG configuration is the one with the best performance, as it will be seen in the results.

\subsubsection{Estimation with variability in the IAP parameters}

Another important scenario in which the estimation performance has been studied is the one in which there are discrepancies between the MUP model used in the estimation system to estimate the MU parameters, and that used to generate the recorded signal in the simulation experiments. Note that the MUP model employed in this thesis, as it occurs with all models, is a simplification of the reality. Therefore, the fact of correctly estimating the MU parameters in a simulation environment does not necessarily imply a correct estimation in a real-world scenario. Thus, introducing discrepancies between the estimation and simulation MUP models allows to evaluate the robustness of the estimation procedure against model limitations or inaccuracies.

Keeping these considerations in mind, a new experiment has been designed. In essence, the IAP parameters used to generate the MUPs (see section 1.4.2) have been made to vary randomly from one fiber to another in the MU under study. In this way, the waveform of the recorded scanning-EMG signal is different than it would be in the case that the IAP parameters remained unaltered. On the other hand, the IAP parameters used in the estimation system remain constant at the values used in the previous experiments (default IAP parameters), as these parameters are not subject to estimation.

For each fiber of the MU under study, the IAP parameters are randomly generated following a Gaussian distribution whose mean is given by the default parameters, and whose standard deviation is indicated in Table 5-1. These standard deviation values have been chosen so that the corresponding random samples produce a noticeable variability in the waveform of the IAP (Fig. 5-1), and consequently, in the waveform of the recorded scanning-EMG signal. 


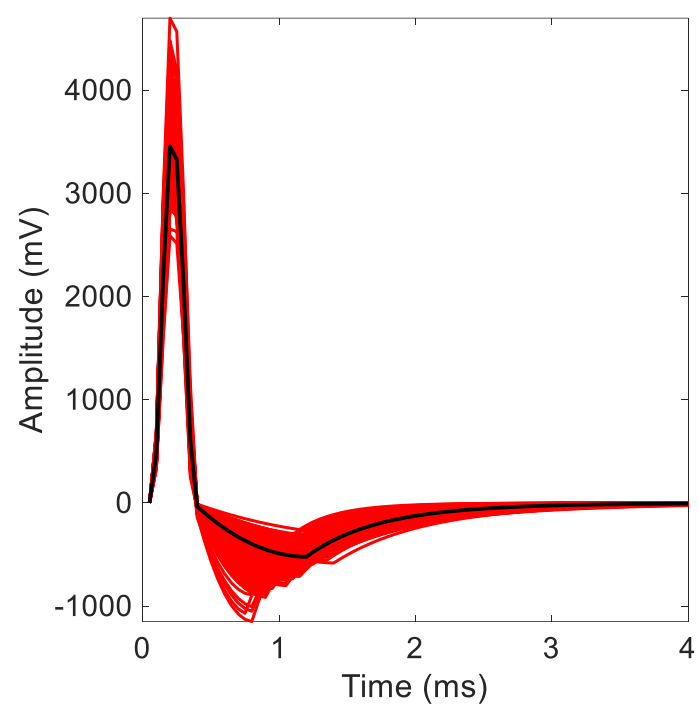

(a)

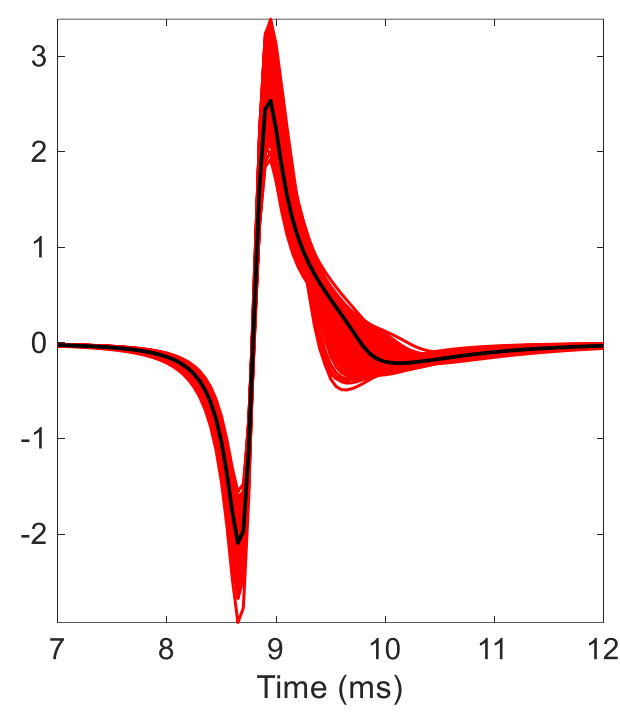

(b)

Fig. 5-1. (a) IAPs obtained in 500 independent simulations, using a specific realization of the IAP parameters (red lines) for each of the cases. Notice how the parameter distribution used implies a high IAP waveform variability; (b) SFAPs generated with the different IAPs, for a muscle fiber located at $0.2 \mathrm{~mm}$ respect to the electrode. Note how IAP variability involves variability also in the SFAP waveform. The black lines represent the waveform generated from the default IAP parameters.

Table 5-1. Mean and standard deviation of the different IAP parameters

\begin{tabular}{lll}
\hline IAP parameter & Mean & Std \\
\hline$A_{1}$ & 9000000 & 317540 \\
$A_{2}$ & 4,5 & 0.0462 \\
$A_{3}$ & 14 & 0.1732 \\
$T_{s p l}$ & 2 & 0.3 \\
\hline
\end{tabular}

Two new experiments including IAP variability have been performed. These two experiments follow the general scheme described in section 5.1.1, for the 2-port recording configuration. In one of the experiments, no noise has been introduced into the recorded scanning signal, so that the effect of the IAP variability on the estimation can be studied in isolation. In the other experiment, both the variability of the IAP and the noise have been included. 


\subsubsection{Estimation varying the global properties of the MUs}

The experiments proposed so far restrict the study of the estimation performance to scenarios in which the MUs and its constituent fibers have been generated according to specific global MU parameters (a specific MU fiber density, MFCV variability, and IZ width). We are interested in studying scenarios in which the value of these global parameters is modified. The interest of this study is twofold. Firstly, in a real scenario, the global MU parameters may vary as a function of the patient, the type of muscle analyzed, or even as a function of the MU analyzed within a specific muscle. In this way, analyzing the estimation performance when the global parameters are modified will give some information about the robustness of the estimation system. Secondly, it is important to assess the potential applicability in a medical context, where the MUs may suffer significant structural changes due to different pathologies. Note that, under neuropathy, reinnervation increases the MU fiber density [58], the variability of the MFCV's, and the dispersion of the neuromuscular junctions within the IZ [128]. Under myopathy, as a result of a loss of muscle fibers, the MU fiber density use to decrease [53], furthermore, fiber atrophy and hypertrophy use to increase the variability of the MFCV's [128].

Keeping these considerations in mind, several experiments have been designed to study the effect of increasing the MU fiber density, the coefficient of variation of the MFCV's, and the width of the overall IZ.

The increase of the fiber density has been studied by performing two new experiments. These experiments follow the general setup described in section 5.1.1, for the 2-port recording configuration. In the first experiment, the fiber density has been increased, while the rest of global MU parameters remain with the default values. In the second, all global MU parameters remain with the default values. In this way, the estimation performance can be compared between the increased and default case in the same experimental conditions. The default and increased values of the fiber density are indicated in Table 5-2.

Table 5-2. Default and increased values for the global MU parameters used in the simulation experiments

\begin{tabular}{lll}
\hline Global MU parameter & Default value & Increased value \\
\hline MU fiber density & 8 fibers $/ \mathrm{mm}^{2}$ & 16 fibers $/ \mathrm{mm}^{2}$ \\
Coefficient of variation of the MFCV's & 0.03 & 0.06 \\
Width of the overall IZ & $10 \mathrm{~mm}$ & $20 \mathrm{~mm}$ \\
\hline
\end{tabular}

In order to allow the estimation algorithm to infer the increased variability, some operating parameters of the algorithm should be chosen in a less restrictive way; e.g., 
in order to study the effect of the increased fiber density, it is necessary to increase the number of simulated fibers used in the estimation. In this way, the number of simulated fibers has been increased for these two experiments from 18 (default value) to 32 on each side of the needle.

The increase of the MFCV coefficient of variation, and the increase of the IZ width have been studied by performing three new experiments. These experiments also follow the general experimental setup for the 2-port recording configuration. In the first experiment, the MFCV coefficient of variation has been increased, while the rest of global MU parameters remain at its default values. In the second experiment, the IZ width has been increased, while the rest of global MU parameters remain at its default values. Finally, in the third experiment, all global MU parameters remain at its default values. In this way, the estimation performance of the increased-parameters cases can be compared with that of the default values in the same experimental conditions. The default and increased values of the MFCV coefficient of variation, and the IZ width are indicated in Table 5-2.

For these three experiments, the parameter search region $\mathfrak{M}_{R}$ has been expanded respect to the previous experiments. This has been done to avoid the actual fiber parameters from being located outside the bounds of the search region, hence out of reach of the estimation algorithm. More specifically the search interval bounds in $z$ and in $v$ have been expanded (see details about the calculation of these bounds in section 3.3.3). So far, the semi-width of the search intervals in $z$ and in $v$ are $R_{z}=8$ $\mathrm{mm}$ and $R_{v}=0.6 \mathrm{~m} / \mathrm{s}$, respectively. These two parameters have been increased for these three experiments to $R_{z}=15 \mathrm{~mm}$ and $R_{v}=1.2 \mathrm{~m} / \mathrm{s}$.

An important consideration to keep in mind is that, for all experiments described in this section, the number of generations of the genetic algorithm has been increased from 350 to 450 , and the number of iterations of the interior point algorithm has been increased from 10000 to 13000 . This increase in the number of iterations aims to compensate the increased difficulty for the estimation algorithm due to the increase in the search region hypervolume. 


\subsection{Results and discussion}

\subsubsection{Estimation with different recording configurations}

This first study presents and discusses the results about the experiments described in section 5.1.2, concerning to the different recording configurations under analysis.

\section{Results}

In Fig. 5-2, Fig. 5-4, Fig. 5-6, and Fig. 5-8, the results of the single MU experiment setup (described in section 5.1.2) are presented for the four recording configurations: the single-point, the 1-port scanning-EMG, the 2-port scanning-EMG and the 4-port scanning-EMG, respectively. In these figures, the estimated fiber parameters of a single MU are shown for different realizations of the noisy recorded signal. Note that, each color corresponds to the estimated parameters of a single MU fiber, so that the dispersion of the estimated parameters of each fiber can be independently analyzed. The estimated fibers colored in gray correspond to associations for which the normalized error energy (given by (3-34)) is larger than 0.5 . For these fibers, the corresponding SFAP waveforms do not resemble any of the actual fibers. Therefore, for the purpose of this analysis, these fibers are considered as not associated fibers.

In Fig. 5-3, Fig. 5-5, Fig. 5-7 and Fig. 5-9, the results of a particular realization of the experiment are shown for the single-point, the 1-port scanning-EMG, the 2-port scanning-EMG and the 4-port scanning-EMG recording configuration, respectively. Note that, in addition to the estimation results, the signal waveform adjustment achieved in the estimation is depicted. The figures show the signal for three different cases: the recorded noisy signal used as reference in the estimation procedure (in green), a noise free version of the reference signal (in blue), and the estimated signal resulting from simulating the signal with the estimated parameters (in red).

Fig. 5-10, Fig. 5-11, Fig. 5-12 and Fig. 5-13 show the results of the individual parameter estimation errors obtained from executing the general experiment scheme for the four analyzed recording configurations. In these figures, the percentile errors associated to the different fiber parameters are shown, where these parameters are, the position of the fiber along the x-axis, $x$ (for the single-point configuration, the radial distance, $r$ ); the position along the y-axis, $y$; the position of the fiber neuromuscular junction, $z$; the MFCV, $v$; and the position of the fiber neuromuscular junction once the bias has been extracted, $\zeta$. These percentiles are presented in the figures as a function of the evaluation region. Remember that the evaluation regions are defined so that they comprise from the position of the ports to a certain distance (see section 3.4.1). For each figure, the subfigures on the left show the percentile errors as a function of the distance to the ports covered by the evaluation region $\left(W_{r}\right.$ for the singlepoint, and $W_{x}$ for the rest of recording configurations). 


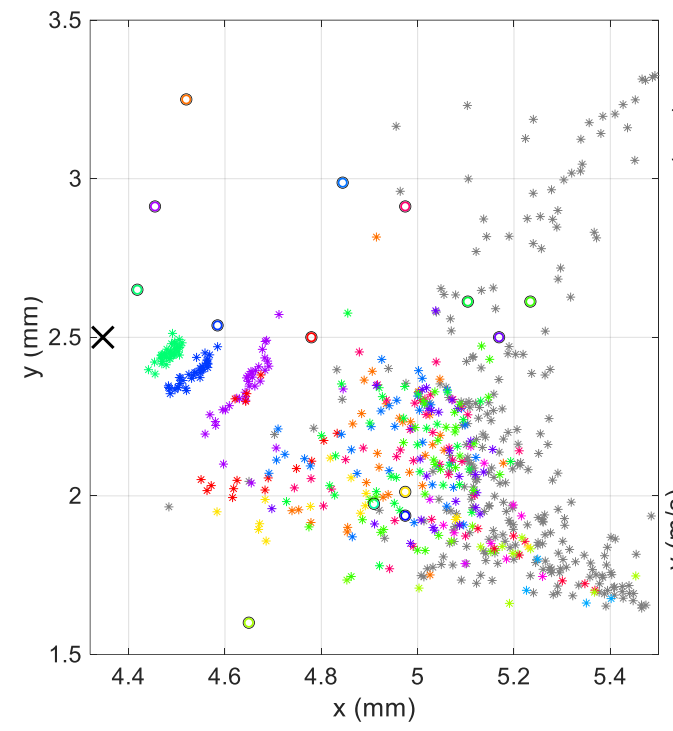

(a)

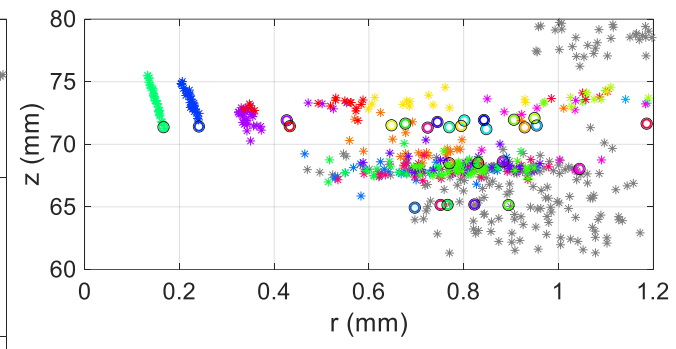

(b)

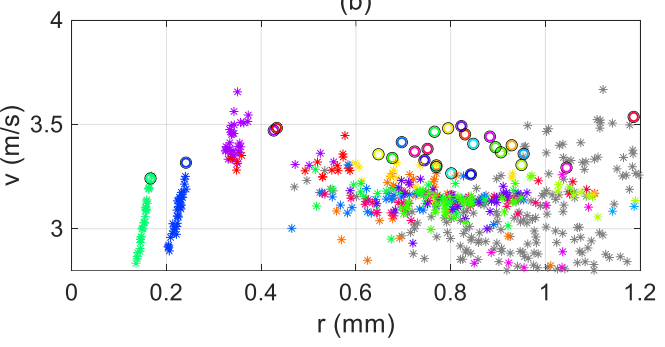

(c)

Fig. 5-2. Estimated MU fiber parameters obtained for all the realizations of the single MU experiment scheme described in section 5.1.2, when the single-point recording configuration is used. The actual MU fiber parameters are represented by circles, and the estimated ones by asterisks of different colors, where each color represents the estimated fibers associated to a single actual MU fiber; (a) estimated and actual MU fibers in the muscle cross-section plane, $\mathrm{x}-\mathrm{y}$. The black cross represents the position of the port; (b) estimated and actual MU fibers in the radial distance - neuromuscular junction position plane, r-z; (c) estimated and actual MU fibers in the radial distance - MFCV plane, $\mathrm{r}-\mathrm{v}$.

The subfigures on the right show the error percentiles as a function of the percentage of fibers of the MU that covers on average a certain evaluation region. Note that, each evaluation region covers, on average, a certain proportion of the MU fibers, which is determined by the maximum distance to the ports covered by this region. The larger the distance, the larger the proportion of MU fibers covered. In this way, a mapping between the distance and the percentage of covered MU fibers can be established. The percentiles are presented as a function of this percentage of MU fibers. These subfigures aim to show how the error varies as a function of the portion of the MU territory considered for the analysis.

Fig. 5-14 shows the MAE of the individual estimation errors versus the percentage of MU fibers covered by the evaluation regions, obtained for the different recording configurations. 


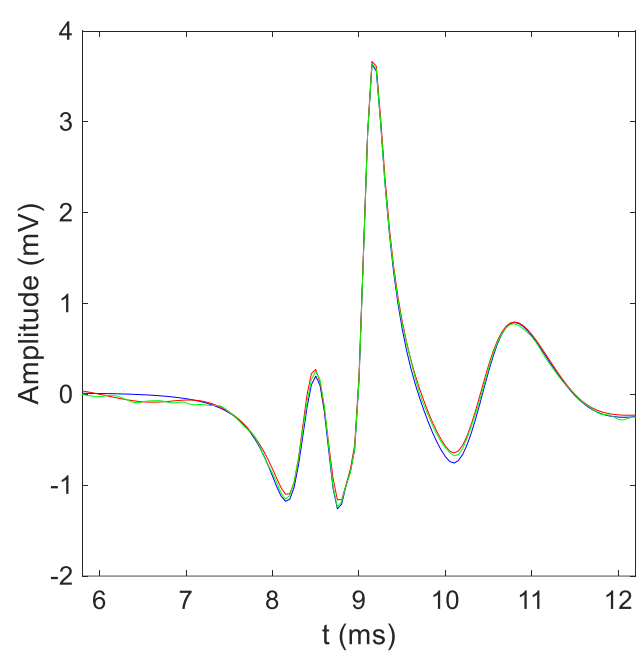

(a)

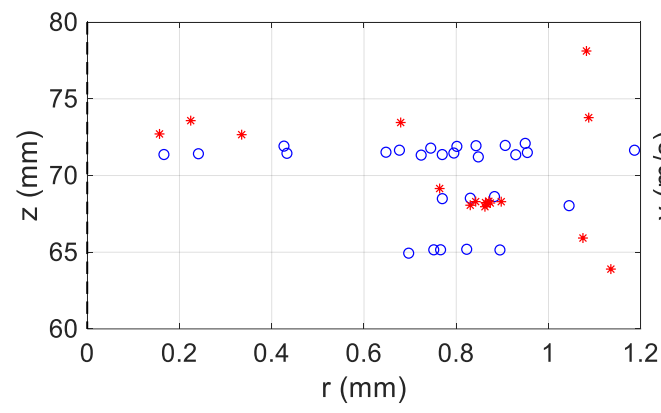

(c)

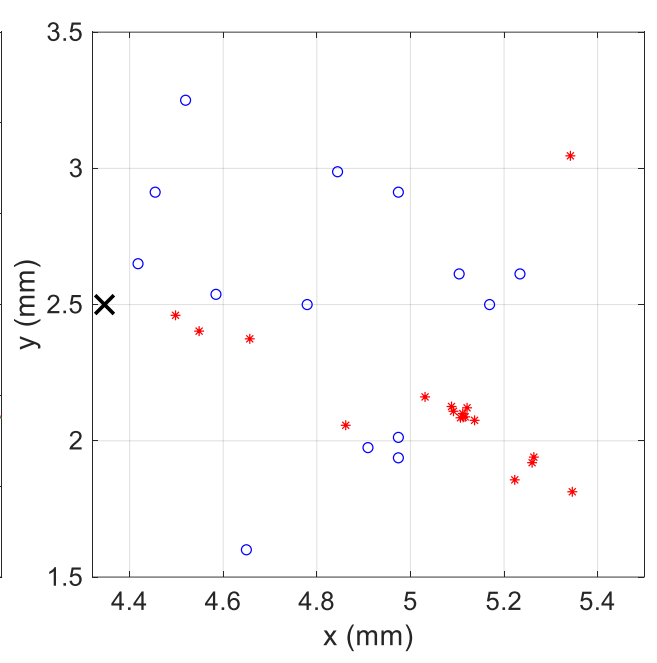

(b)

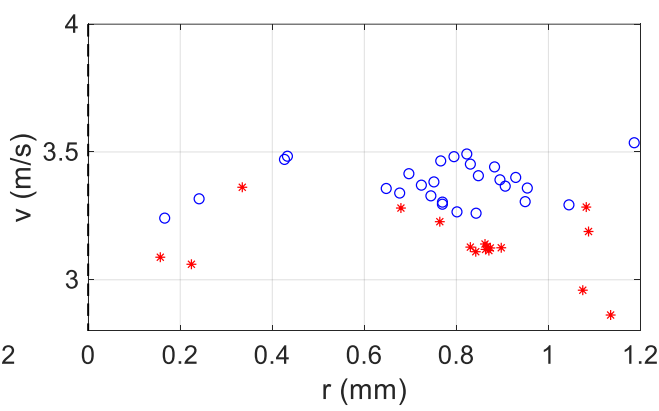

(d)

Fig. 5-3 Estimation results of a single experiment realization, for a MUP signal recorded with the single-point recording configuration; (a) MUP amplitude of the estimated (red lines), reference (green lines), and noise-free reference (blue lines) signals; (b) estimated (red asterisks) and actual (blue circles) MU fibers in the $x-y$ plane. The black cross represents the position of the port; (c) estimated (red asterisks) and actual (blue circles) MU fibers in the r-z plane; (d) estimated (red asterisks) and actual (blue circles) MU fibers in the r-v plane.

\section{Discussion of the results}

\section{General results}

A first observation to be made is that there is a strong dependence between the distance of the MU fibers to the ports and the estimation performance. This occurs for all the recording configurations. For instance, observing the estimations of a single 


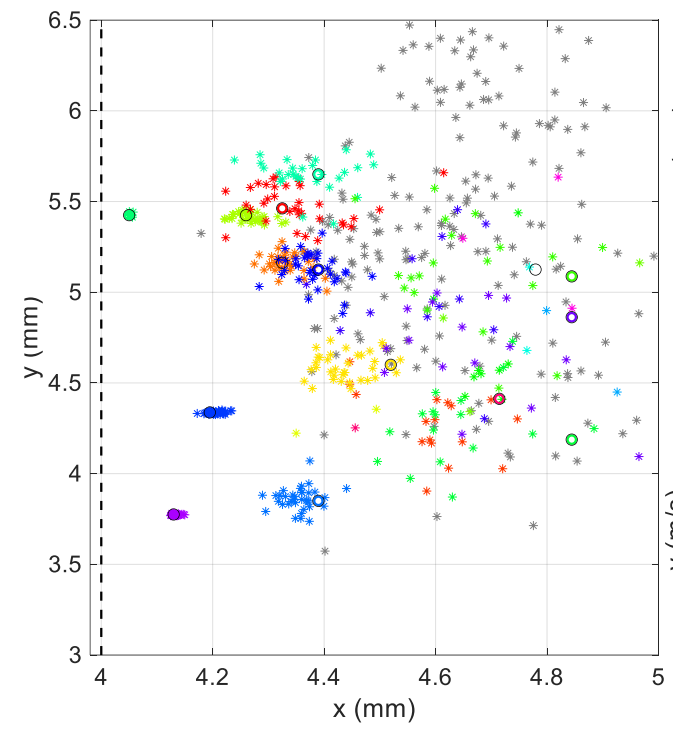

(a)

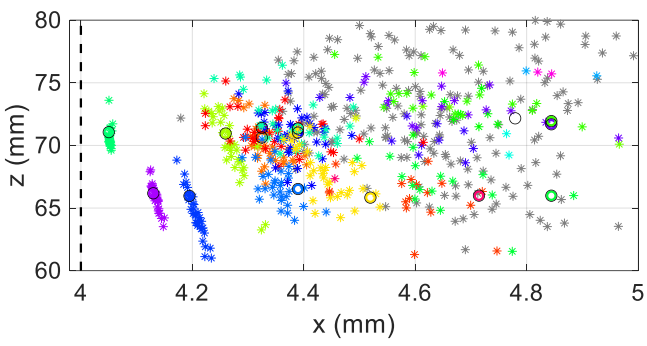

(b)

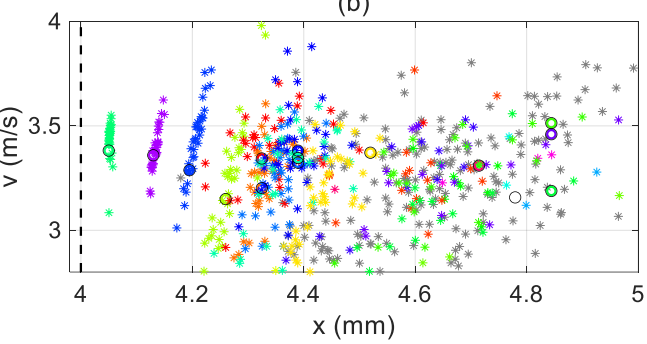

(c)

Fig. 5-4. Estimated MU fiber parameters obtained for all the realizations of the single MU experiment scheme described in section 5.1.2, when the 1-port recording configuration is used. The actual MU fiber parameters are represented by circles, and the estimated ones by asterisks of different colors, where each color represents the estimated fibers associated to a single actual MU fiber; (a) estimated and actual MU fibers in the x-y plane; (b) estimated and actual MU fibers in the x-z plane; (c) estimated and actual MU fibers in the X-v plane. The vertical dashed line represents the port position (x-axis) and corridor (y-axis).

MU obtained for the 1-port recording configuration (Fig. 5-4), it is clear that the estimations corresponding to the fibers located near to the port position corridor present a very lower dispersion in the parameter space compared to fibers locater further. The further the fiber is from the port corridor the larger the dispersion. So much so that for distant fibers, the corresponding estimated points in the parameter space are so dispersed that all of them are intermingled. The same dependency between the estimation performance and the distance can be observed in the results of the experiments of multiple MUs (Fig. 5-10, Fig. 5-11, Fig. 5-12 and Fig. 5-13), where the wider the evaluation region the higher the dispersion of the errors.

If we focus in the particular case concerning the estimations of the parameter $x$, for the 1-port recording configuration (Fig. 5-11 (a)), we observe that, for a region covering a distance of $0.2 \mathrm{~mm}$ to the port corridor, $80 \%$ of the errors in the parameter $x$ range from $-10 \cdot 10^{-3}$ to $6 \cdot 10^{-3} \mathrm{~mm}$, while for a distance of $0.7 \mathrm{~mm}$ the errors range from $-0.124 \mathrm{~mm}$ to $0.333 \mathrm{~mm}$. To understand the magnitude of these errors, note that the territory of the simulated MUs has a radius around $1 \mathrm{~mm}$, which means that for a distance of $0.7 \mathrm{~mm}$, the error dispersion occupies a range whose size is almost half the 
radius of the territory. The reason of this behavior is that the fibers located far from the recording points are detected with very low amplitude, so that their potential contribution to the signal is small. Furthermore, the corresponding waveforms are very smooth both in time and in space, which implies that there is a wide range of parameters of the estimated fibers that may give rise to a waveform contribution similar to that of such distant fibers. Both aspects make the precision of the estimation to decrease the further the fibers are from the ports.

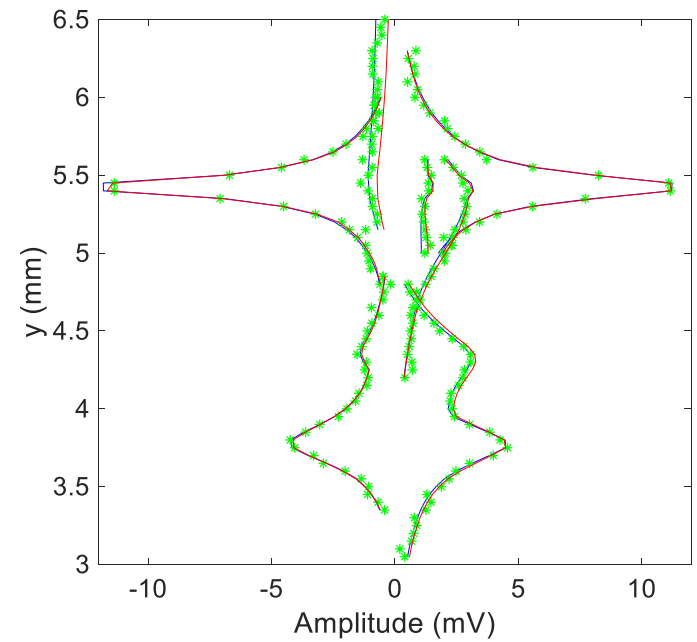

(a)

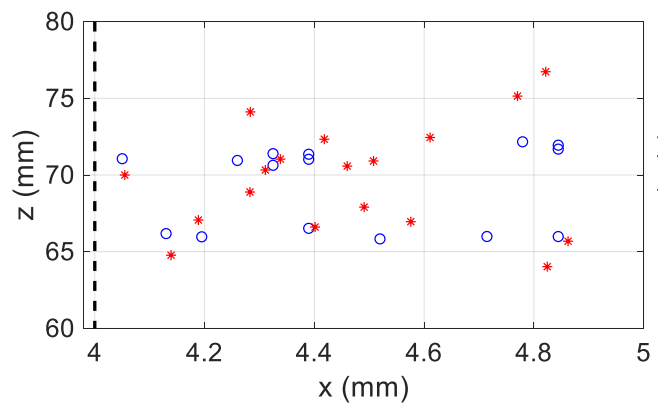

(c)

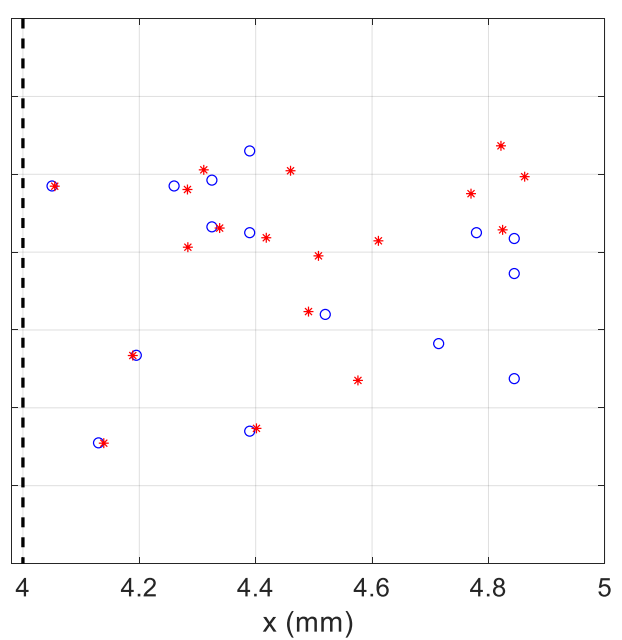

(b)

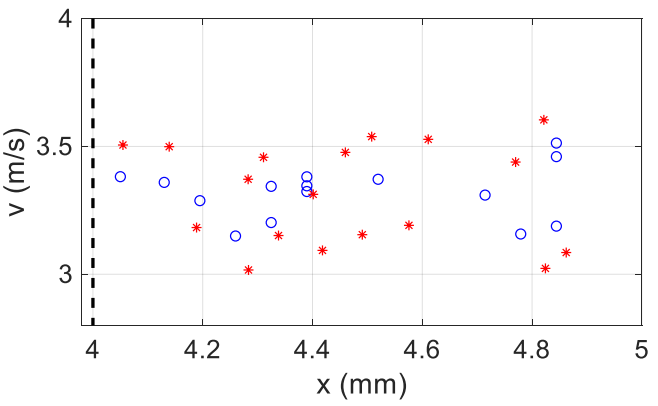

(d)

Fig. 5-5. Estimation results of a unique experiment realization, for a scanning-EMG signal recorded with the 1-port recording configuration; (a) amplitude of the MU profile in the spatial dimension of the estimated (red lines), reference (green asterisks), and noise-free reference (blue lines) scanning-EMG signals; (b) estimated (red asterisks) and actual (blue circles) MU fibers in the x-y plane; (c) estimated (red asterisks) and actual (blue circles) MU fibers in the $\mathrm{x}-\mathrm{z}$ plane; (d) estimated (red asterisks) and actual (blue circles) MU fibers in the $\mathrm{x}-\mathrm{v}$ plane. The vertical dashed line represents the port position (x-axis) and corridor (y-axis). 


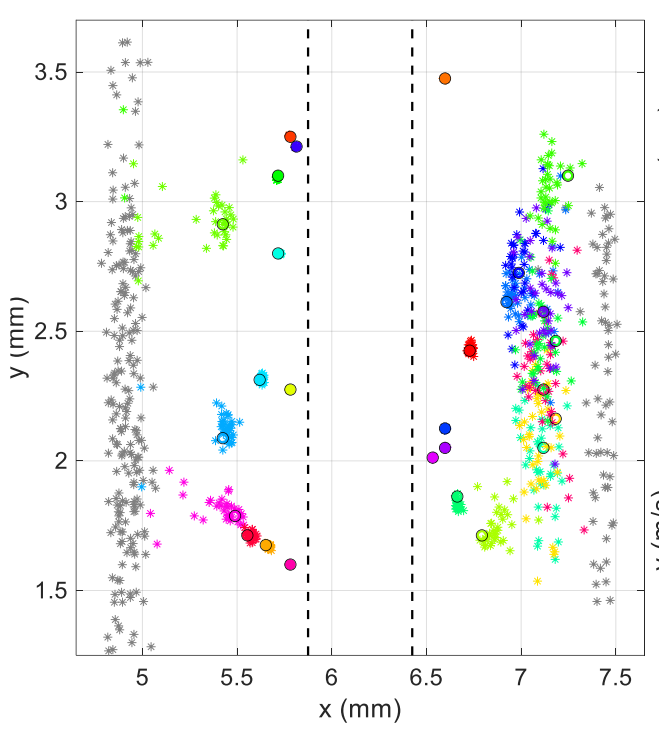

(a)
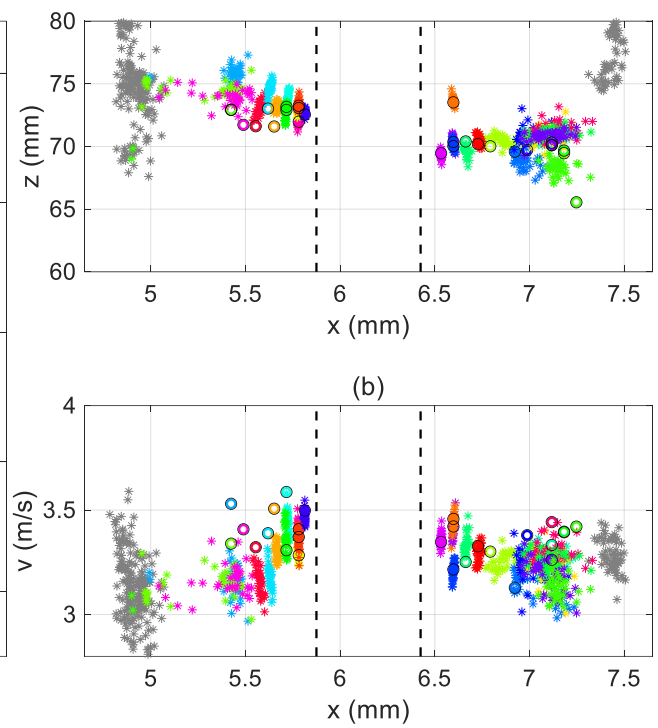

(c)

Fig. 5-6. Estimated MU fiber parameters obtained for all the realizations of the single MU experiment scheme described in section 5.1.2, when the 2-port recording configuration is used. The actual MU fiber parameters are represented by circles, and the estimated ones by asterisks of different colors, where each color represents the estimated fibers associated to a single actual MU fiber; (a) estimated and actual MU fibers in the x-y plane; (b) estimated and actual MU fibers in the X-z plane; (c) estimated and actual MU fibers in the X-v plane. The two vertical dashed lines represent the corridors of the two ports of the needle.

\section{Single-point recording}

Among the different recording configurations, the worst estimation results correspond to the single-point recording configuration. As we know from the analysis of the forward problem (section 3.2), when only a single position is used to record the MUP, the muscle cross-section position coordinates $x$ and $y$ cannot be correctly estimated, because of the radial symmetry of the problem. This is the reason why, in Fig. 5-2 (a), it is evident that the positions $x$ and $y$ do not match with that of the actual MU fibers. The results of the experiments of multiple MUs show the same behavior. In Fig. 5-10 (g) it can be observed that for an evaluation region covering only $20 \%$ of the MU fibers, $80 \%$ of the estimation errors in the parameter $y$ range between -0.56 $\mathrm{mm}$ and $0.19 \mathrm{~mm}$. This error range is wide enough to consider that this parameter has been completely misestimated. Although the position of the fibers in the muscle crosssection cannot be estimated, it is assumed that the radial distance between the fibers and the port position should be estimated to some extent, at least in the most favorable cases [140]. However, the results contradict this hypothesis. In Fig. 5-10 (f) it can be 


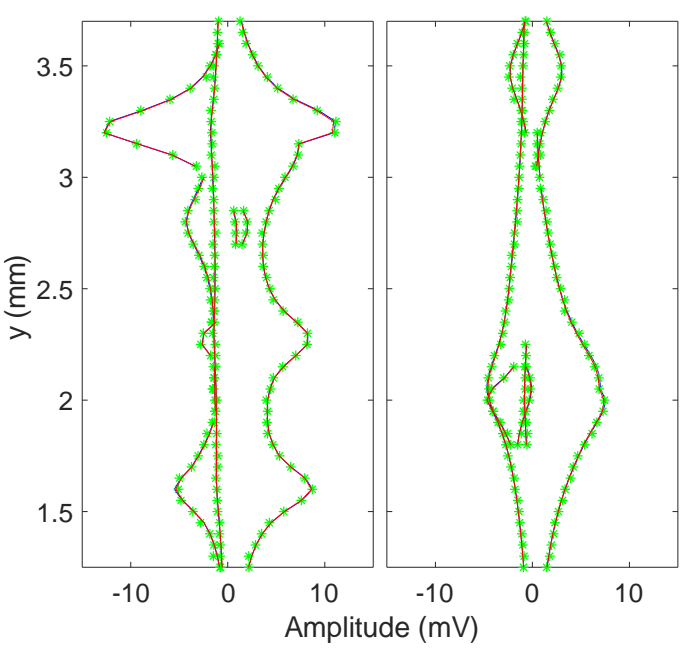

(a)

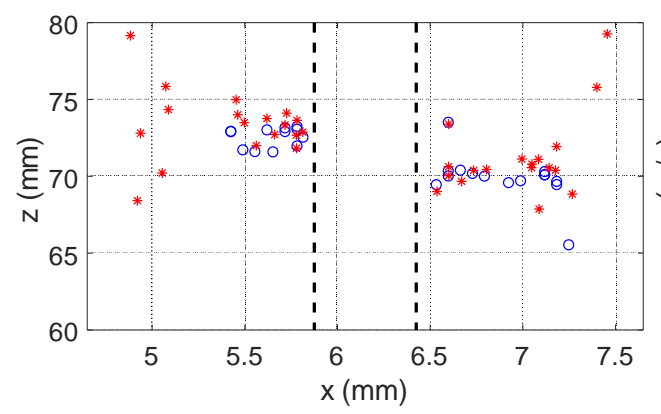

(d)

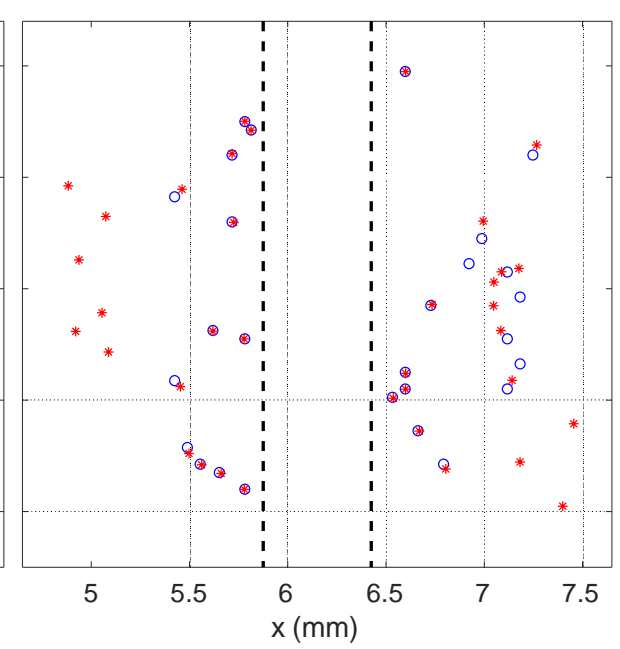

(c)

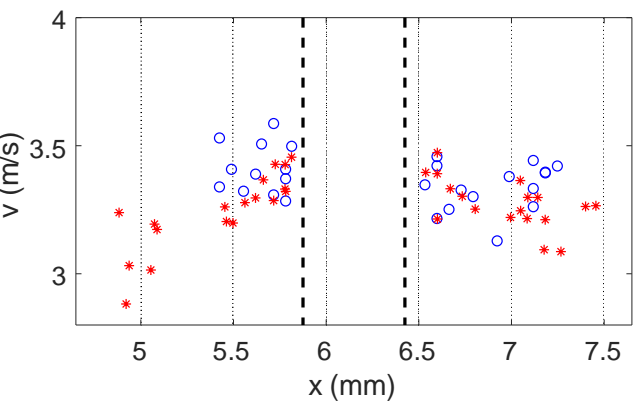

(e)

Fig. 5-7. Estimation results of a single experiment realization, for a scanning-EMG signal recorded with the 2-port recording configuration; (a) and (b), amplitude of the MU profile in the spatial dimension of the estimated (red lines), reference (green asterisks), and noise-free reference (blue lines) scanning-EMG signals, where (a) corresponds to the scanning-EMG signal section recorded in the left port, and (b) to that of the right port; (c) estimated (red asterisks) and actual (blue circles) MU fibers in the $x-y$ plane; (d) estimated (red asterisks) and actual (blue circles) MU fibers in the X-Z plane; (e) estimated (red asterisks) and actual (blue circles) MU fibers in the $\mathrm{x}-\mathrm{v}$ plane. The two vertical dashed lines represent the corridors of the two ports of the needle.

observed that for a region covering $20 \%$ of the fibers, $80 \%$ of the estimation errors in the radial distance $r$ range between $-0.11 \mathrm{~mm}$ and $0.25 \mathrm{~mm}$. Furthermore, when a percentage of MU fibers higher than $20 \%$ is considered in the analysis, the error dispersion grows noticeably. For a region covering $40 \%$ of the fibers, $80 \%$ of the estimation errors in the radial distance $r$ range between $-0.19 \mathrm{~mm}$ and $0.36 \mathrm{~mm}$. The fact that the radial distance cannot be successfully estimated may be due to the 
compensation effects that occur when only a single position is used to record the MUP. This phenomenon has been studied in detail in section 3.2: even in the simplest case in which a single MU fiber is considered, there are many combinations of the radial distance $r$, the MFCV $v$, and the neuromuscular junction position $z$ of the fibers that may result in almost the same simulated signal waveform. Furthermore, as soon as more fibers are considered, more compensation effects arise, making it more difficult or even impossible to achieve a correct parameter estimation. In conclusion, the results presented here demonstrate that a correct parameter estimation is not possible when only one recording position is considered. Since the previous attempts to address the estimation of the MU fiber parameters $[67,68,140]$ are based on a single MUP recording, from our point of view it is questionable that they could achieve a correct fiber parameter estimation.

When the single-point configuration is compared to the other configurations, the latter show a clearly better performance. In Fig. 5-14 (a-b) it can be observed that the MAE of the single-point configuration is higher than that of any other configuration

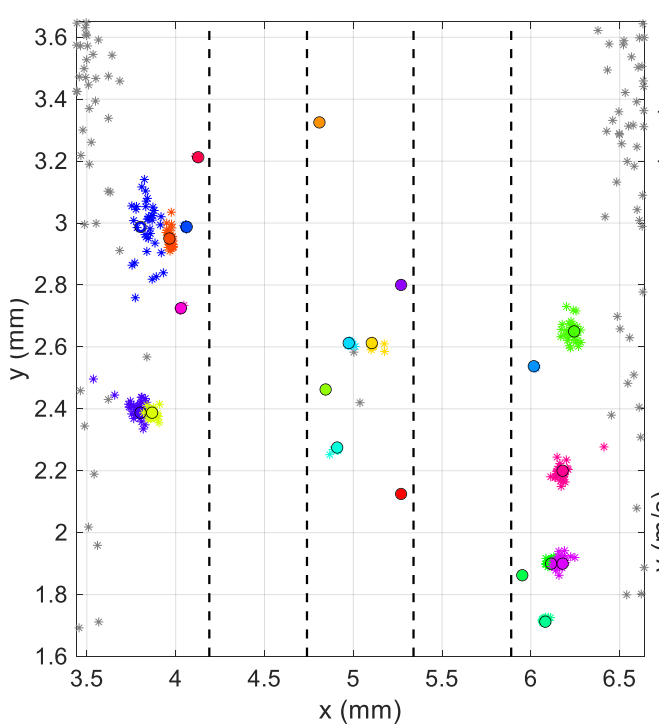

(a)

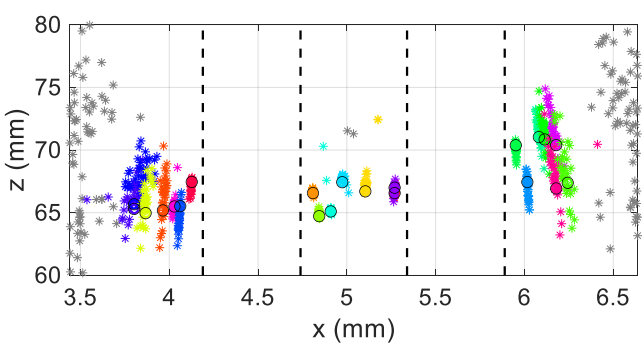

(b)

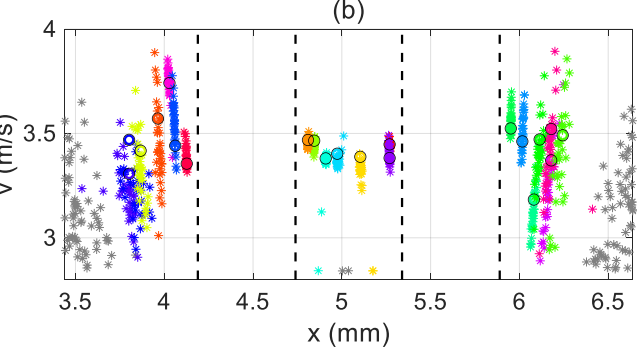

(c)

Fig. 5-8. Estimated MU fiber parameters obtained for all the realizations of the single MU experiment scheme described in section 5.1.2, when the 4-port recording configuration is used. The actual MU fiber parameters are represented by circles, and the estimated ones by asterisks of different colors, where each color represents the estimated fibers associated to a single actual MU fiber; (a) estimated and actual MU fibers in the $\mathrm{x}-\mathrm{y}$ plane; (b) estimated and actual MU fibers in the x-z plane; (c) estimated and actual MU fibers in the $x-v$ plane. The vertical dashed lines represent the port corridors, where the two leftmost corresponding with the needle located on the left, and the two rightmost corresponding with the needle located on the right. 
for the $x$ and $y$ parameters. Regarding the parameter $\zeta$ ( $z$ without bias), and the parameter $v$ (Fig. 5-14 (c-d)), when considering evaluation regions covering low percentages of MU fibers, the single-point recording configuration is the one that presents higher MAEs. This is not the case when considering regions with higher

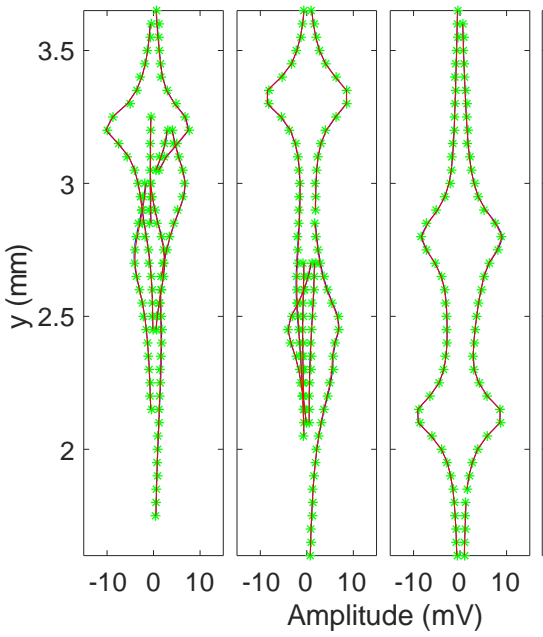

(a) (b)

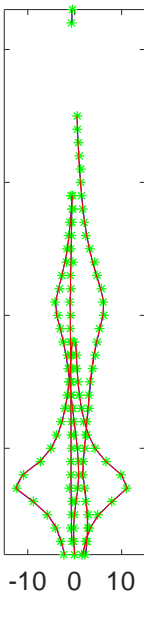

(d)

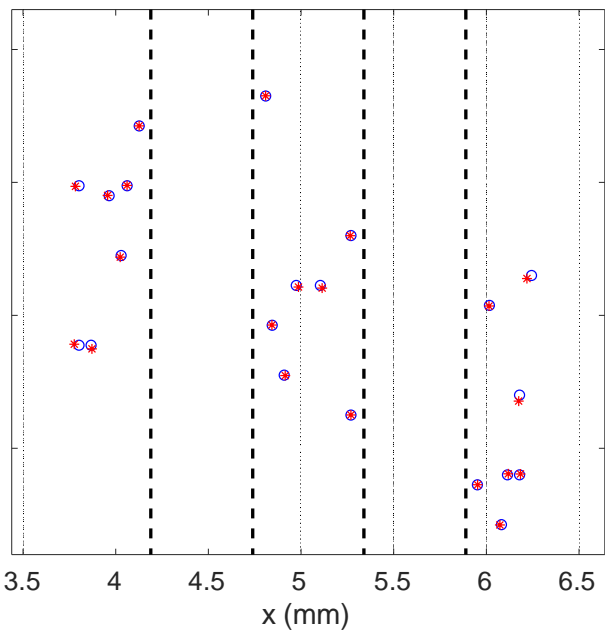

(e)

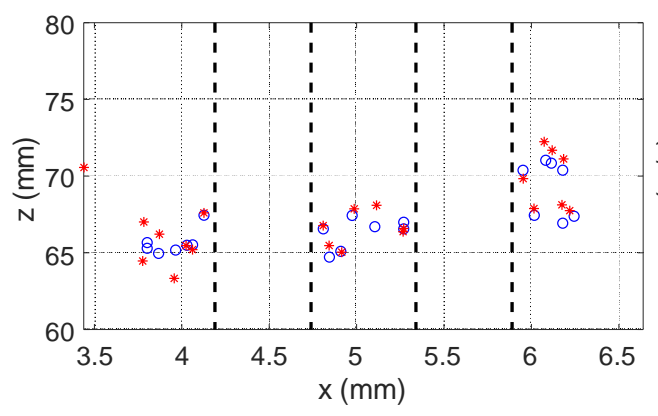

(f)

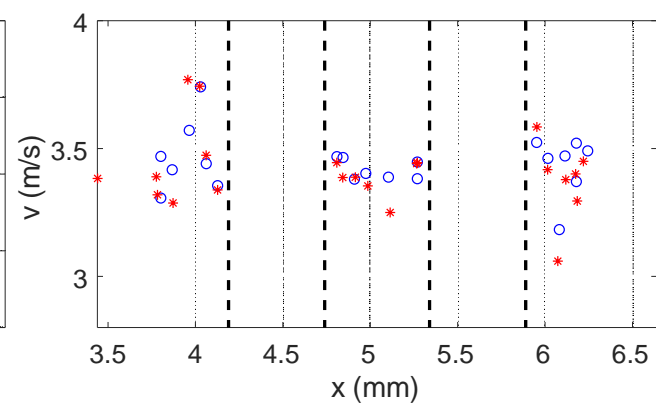

(g)

Fig. 5-9. Estimation results of a single experiment realization, for a scanning-EMG signal recorded with the 4-port recording configuration; (a), (b), (c) and (d), amplitude of the MU profile in the spatial dimension of the estimated (red lines), reference (green asterisks), and noise-free reference (blue lines) scanning-EMG signals, where (a) corresponds to the scanningEMG signal section recorded in the left port of the needle located in the left, (b) to that of the right port of the left needle, (c) to that of the left port of the right needle, and (d) to that of the right port of the right needle; (e) estimated (red asterisks) and actual (blue circles) MU fibers in the $\mathrm{x}-\mathrm{y}$ plane; (f) estimated (red asterisks) and actual (blue circles) MU fibers in the $\mathrm{x}-\mathrm{Z}$ plane; (g) estimated (red asterisks) and actual (blue circles) MU fibers in the $\mathrm{X}-\mathrm{v}$ plane. The vertical dashed lines represent the port corridors, where the two leftmost corresponding with the needle located on the left, and the two rightmost corresponding with the needle located on the right. 
percentage of fibers, for which the 1-port recording configuration presents higher MAEs than the single-point configuration. The apparent better performance of the single-point configuration respect the 1-port one in these particular cases is believed to be reflecting the tendency of the parameters to remain in the middle of the parameter search regions, than to a tendency to individually adjust the actual MU fiber parameters. This is supported by the following rationale. The dispersion region in the parameter space is very large, as long as more than one fiber is considered in a singlepoint configuration (see in section 3.2.4, Fig. 3-16). Thus, during the estimation procedure, the optimization algorithm has to deal with a very wide region in which the error function is very small, and in this context, it seems that the optimization tends to provide solutions that are found approximately in the middle of the search regions established for the optimization.

\section{Multi-point recordings}

The three recording configurations proposed to be incorporated in the estimation system are now analyzed together. The parameters $x$ and $y$ are estimated with high precision for fibers located near to the corridor (approximately up to $0.4 \mathrm{~mm}$ distance), as can be corroborated both for the experiments with a single MU (Fig. 5-4, Fig. 5-6 and Fig. 5-8), and multiple MUs (Fig. 5-11, Fig. 5-12 and Fig. 5-13). The neuromuscular junction positions $z$ are not correctly estimated. For instance, for the 2port configuration, $80 \%$ of the errors in $z$ range between $-3.1 \mathrm{~mm}$ and $1 \mathrm{~mm}$, for a region covering a distance of up to $0.4 \mathrm{~mm}$ from the port (Fig. 5-12 (c)). Such range is very wide if we consider that the sub-bands are approximately about $1 \mathrm{~mm}$ width [87]. One of the reasons why this error is so high is because the potential initiation instant is also estimated, and its error affects directly to the estimation of the neuromuscular junction positions (see section 4.1). In this analysis it was concluded that, although the parameter $z$ cannot be estimated accurately, its position relative to the IZ position could be estimated with acceptable accuracy. For this reason, the estimation error of the neuromuscular junction positions once the $z$ bias has been eliminated (i.e., of the parameter $\zeta$ ), has also been analyzed. The error in $\zeta$ presents systematically a noticeably lower dispersion than $z$ for the three configurations considered. For example, in the 2-port configuration, $80 \%$ of the errors range between $-1.1 \mathrm{~mm}$ and $1.2 \mathrm{~mm}$, for a region covering a distance of up to $0.4 \mathrm{~mm}$ from the port (Fig. 5-12 (e)), which is a range noticeably narrower than in the case of the parameter $z$ (Fig. 5-12 (c)). Even so, this error is still large compared to the width of the sub-bands, so the information obtained about the sub-band's organization of the IZ will be quite imprecise. Regarding the fiber MFCV, $v$, the error dispersions (Fig. 5-11 (d), Fig. 5-12 (d) and Fig. 5-13 (d)) are in general low when compared to the physiological range of MFCV's (between $2 \mathrm{~m} / \mathrm{s}$ and $6.5 \mathrm{~m} / \mathrm{s}$ approximately), but high when compared to the dispersion of MFCV's that a normal MU can have (in our experiments the standard deviation of the MFCV's varies from one MU to another, but on average this value is about $0.1 \mathrm{~m} / \mathrm{s}$ ). In conclusion, the general tendency of all recording configurations is that the parameters that are estimated with better precision are the positions $x$ and $y$; 


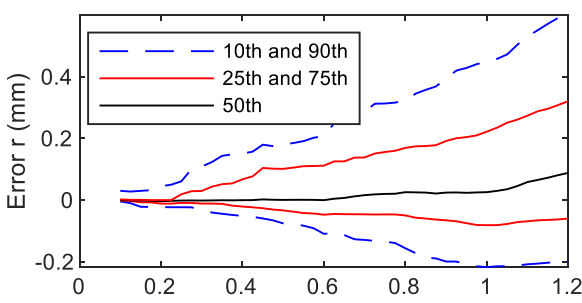

(a)

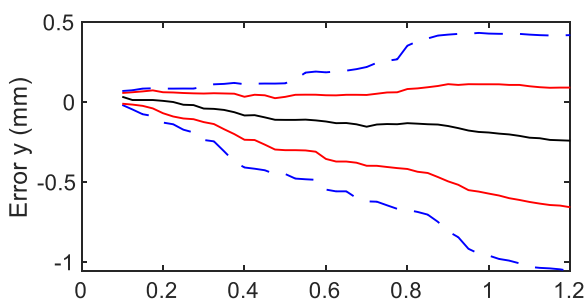

(b)

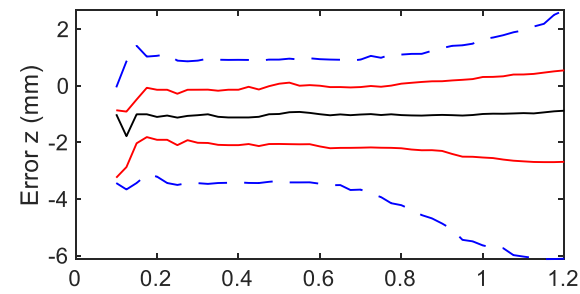

(c)

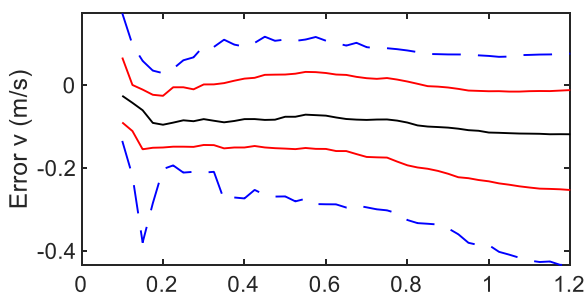

(d)

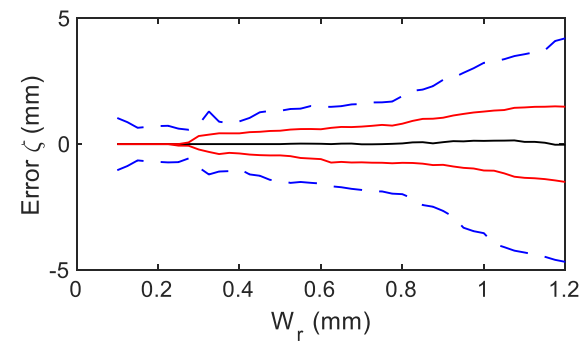

(e)

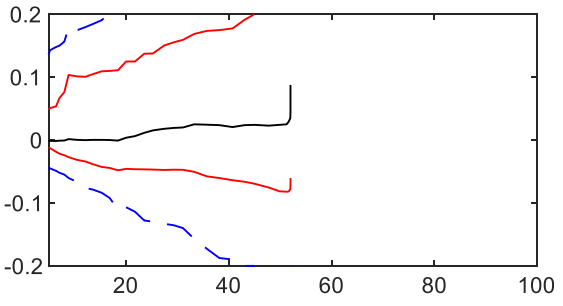

(f)

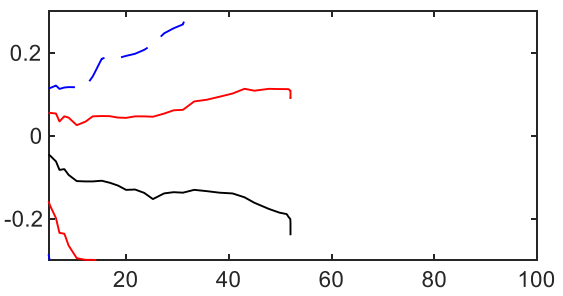

(g)

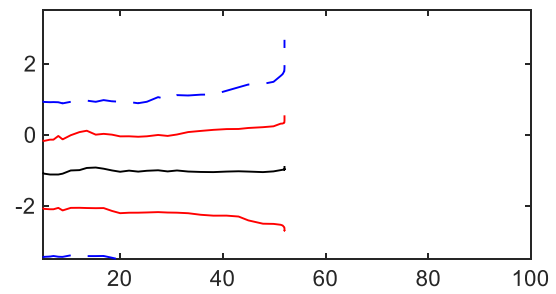

(h)

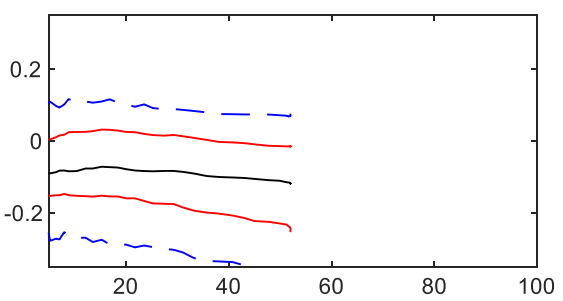

(i)

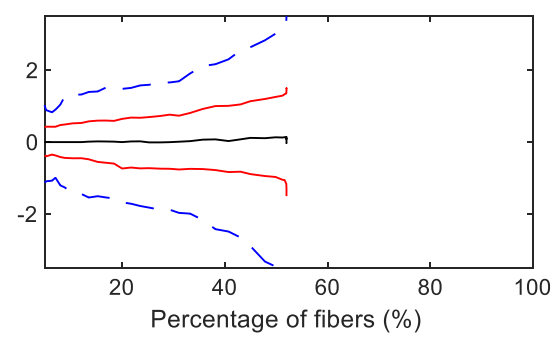

(j) 
Fig. 5-10 [left page]. 10th, 25th, 50th, 75th and 90th percentiles, of the individual estimation errors obtained for the single-point recording configuration. Error percentiles against the distance to the port, $W_{r}$ (a-e); (a) estimation error in the radial distance $r$; (b) estimation error in the position $y$; (c) estimation error in the neuromuscular junction position, $z$; (d) estimation error in the MFCV, $v$; (e) estimation error in the parameter $\zeta$ (the neuromuscular junction position after removing the bias, see section 3.4.1). Error percentiles against the percentage of MU fibers evaluated (f-j); (f) estimation error in the radial distance $r$; $(\mathrm{g}$ ) estimation error in the position $y$; (h) estimation error in the neuromuscular junction position, $z$; (i) estimation error in the MFCV, $v$; (j) estimation error in the parameter $\zeta$.

MFCV, $v$ is also estimated with a relatively high precision; and the neuromuscular junction position $z$, is the parameter that presents a greater difficulty to be accurately estimated.

\section{1-port recording}

Let's analyze the specific estimation behavior of each of the three recording configurations. The 1-port recording configuration is only able to correctly estimate the parameters of the MU fibers that are located very close to the scanning needle (Fig. $5-11$ ): for a region covering a distance of $0.2 \mathrm{~mm}, 80 \%$ of the estimation errors in the position $x$ range between $-0.0058 \mathrm{~mm}$ and $0.01 \mathrm{~mm} ; 80 \%$ of the estimation errors in the position $y$ range between $-0.0046 \mathrm{~mm}$ and $0.0051 \mathrm{~mm} ; 80 \%$ of the estimation errors in the neuromuscular junction position $z$ range between $-3.09 \mathrm{~mm}$ and $0.98 \mathrm{~mm} ; 80 \%$ of the estimation errors in the MFCV $v$ range between $-0.114 \mathrm{~mm}$ and $0.144 \mathrm{~mm}$; and $80 \%$ of the estimation errors in the parameter $\zeta$ range between $-1.07 \mathrm{~mm}$ and $1.05 \mathrm{~mm}$. Furthermore, only the fibers located at one side of the needle can be estimated. These considerations imply that only a very small portion of the MU territory can be correctly characterized. For instance, if we observe the error in the parameter $x, 80 \%$ of its values range between $-0.018 \mathrm{~mm}$ and $0.014 \mathrm{~mm}$ for a region covering $20 \%$ of the MU fibers (Fig. 5-11 (f)). When the region is wider, the error range increases drastically; e.g., for a region covering $40 \%$ of the MU fibers, the error values range between -0.12 $\mathrm{mm}$ and $0.2 \mathrm{~mm}$ (Fig. 5-11 (f)).

\section{2-port recording}

By comparing the 1-port with the 2-port configuration, the performance of the latter is noticeably better (Fig. 5-12). For instance, for the error in the parameter $x$, $80 \%$ of its values range between $-0.006 \mathrm{~mm}$ and $0.007 \mathrm{~mm}$ for a region covering $20 \%$ of the MU fibers, and range between $-0.01 \mathrm{~mm}$ and $0.011 \mathrm{~mm}$ for a region covering $40 \%$ of the MU fibers. The fact that this configuration has a noticeably better estimation performance is mainly due to two factors. Firstly, if we consider the fibers located closer than a given distance respect to the needle, the 2-port configuration identifies approximately twice as many MU fibers than the 1-port configuration, due to the fact that it detects fibers on both sides of the needle. This implies that, for the 


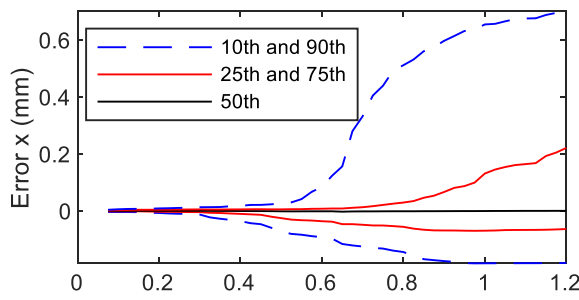

(a)

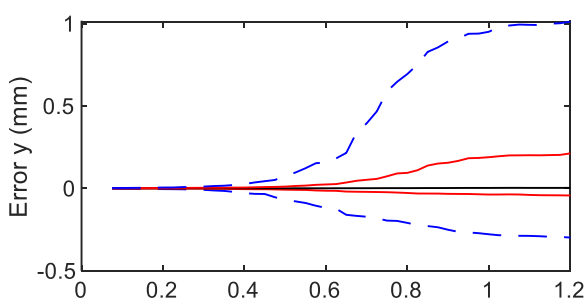

(b)

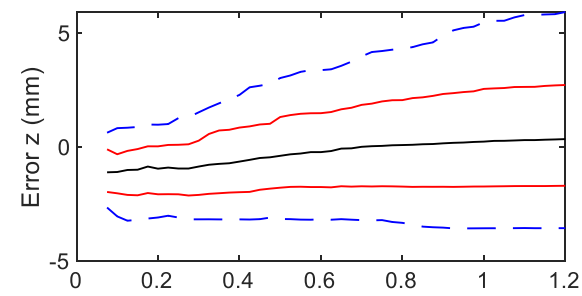

(c)

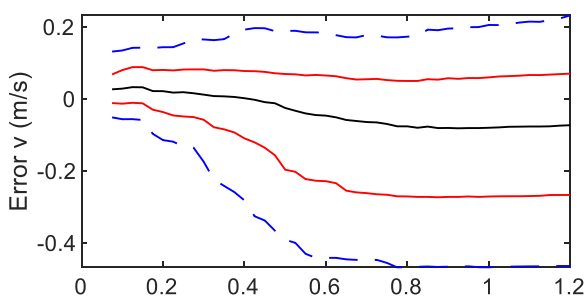

(d)

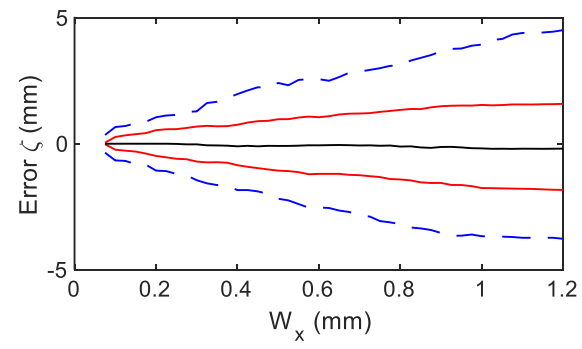

(e)

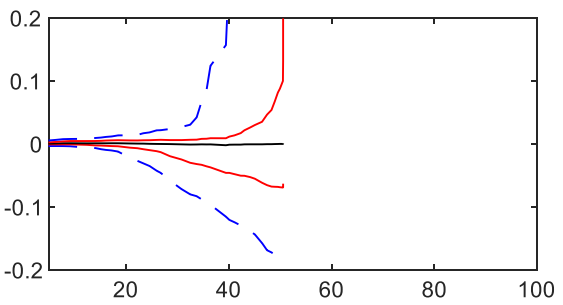

(f)

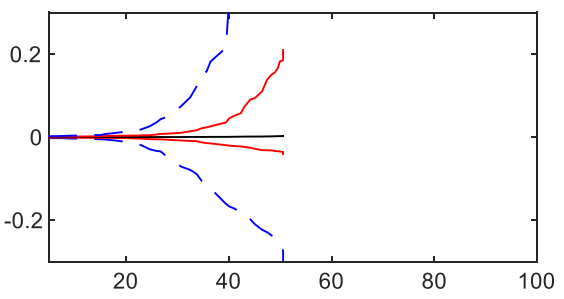

(g)

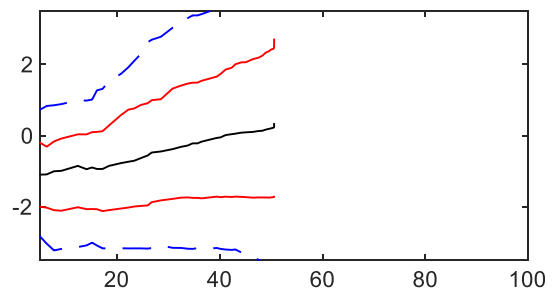

(h)

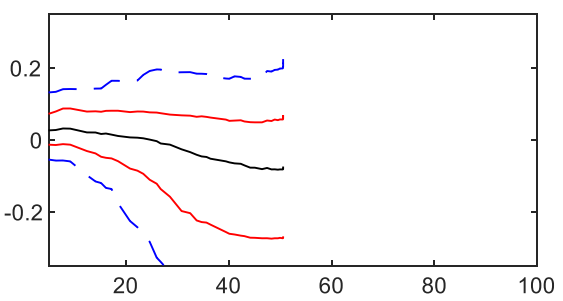

(i)

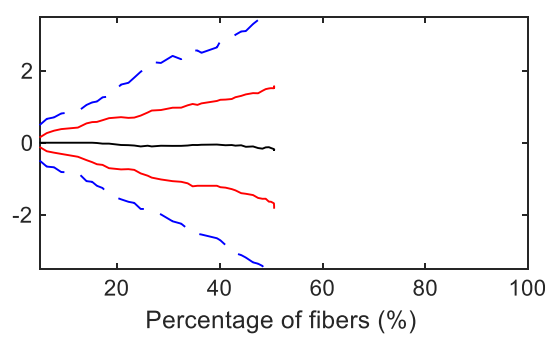

(j) 
Fig. 5-11 [left page]. 10th, 25th, 50th, 75th and 90th percentiles, of the individual estimation errors obtained for the 1-port recording configuration. Error percentiles against the distance to the port, $W_{x}$ (a-e); (a) estimation error in the position, $x$; (b) estimation error in the position, $y$; (c) estimation error in the neuromuscular junction position, $z$; (d) estimation error in the MFCV, $v$; (e) estimation error in the parameter $\zeta$ (the neuromuscular junction position after removing the bias, see section 3.4.1). Error percentiles against the percentage of MU fibers evaluated (f$\mathrm{j}$ ); (f) estimation error in the position $x$; (g) estimation error in the position $y$; (h) estimation error in the neuromuscular junction position, $z$; (i) estimation error in the MFCV, $v$; (j) estimation error in the parameter $\zeta$ after removing the MU bias.

same evaluation distance, this configuration is identifying a larger percentage of MU fibers of the territory (approximately double). Secondly, because if we consider the fibers located closer than a certain distance, the corresponding estimation parameter errors are lower for the 2-port configuration than for the 1-port one; e.g., if we consider all MU fibers located at a distance less than $0.5 \mathrm{~mm}$, the estimation errors of the parameter $x$ range between $-0.071 \mathrm{~mm}$ and 0.028 for the 1 -port configuration (Fig. $5-11$ (a)), and range between $-0.027 \mathrm{~mm}$ and $0.031 \mathrm{~mm}$ for the 2-port configuration (Fig. 5-12 (a)).

This second factor is most probably a consequence of the cannula effect. Remember that the 1-port configuration is affected by the fibers located at the opposite side of the port, as the cannula detects to some extent the contributions of these MU fibers. This fact negatively affects to the estimation performance. On the other hand, in the 2-port configuration this negative effect does not occur, as the fibers at both sides of the needle are estimated simultaneously, so that the contribution of all MU fibers can be taken into account during the optimization procedure (see section 4.3).

\section{4-port recording}

Regarding the 4-port recoding configuration, it generally presents low estimation errors (Fig. 5-13). When compared to the other configurations, Fig. 5-14 shows that the 4-port configuration is the one that presents a better performance for all percentages of the fibers of the MU. This occurs especially in the case of the parameters $x$ and $y$, when a high percentage of MU fibers is considered. In that case, the MAE of the errors is very low compared to that of the other configurations. The fact that for the 4-port configuration, the MAE grows respect to the percentage of fibers more slowly than for the rest of configurations is due to the use of two needles to record the scanning-EMG signal. Note that when two needles are used, each with two ports, a larger portion of the fibers of the MU is being detected with good amplitude. In other words, there is a larger number of MU fibers whose distances to the ports are small enough to be properly detected, which implies that there is a larger number of fibers that can be correctly estimated. 


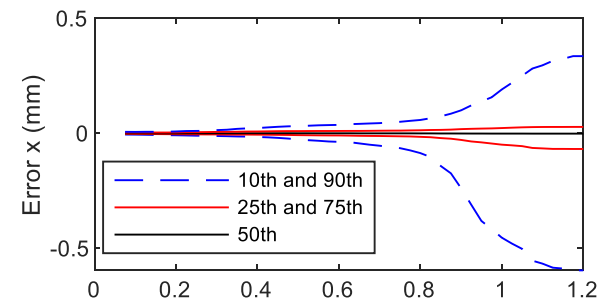

(a)

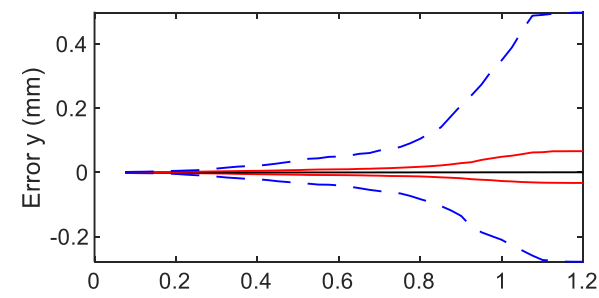

(b)

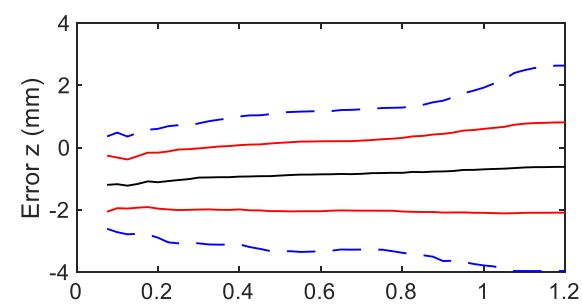

(c)

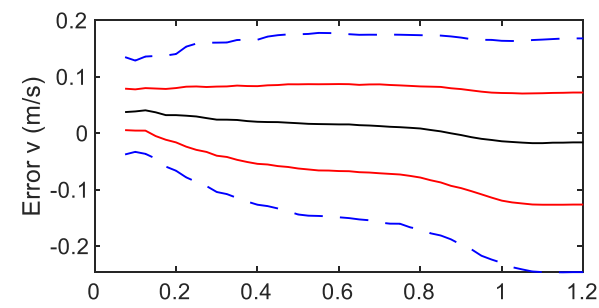

(d)

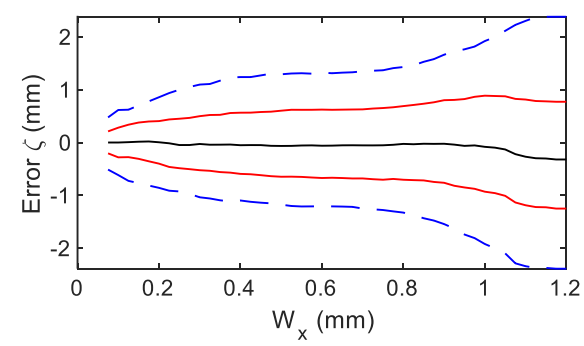

(e)

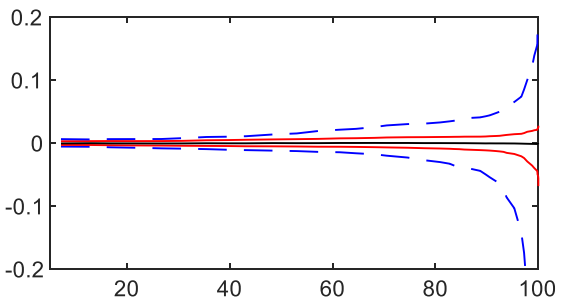

(f)

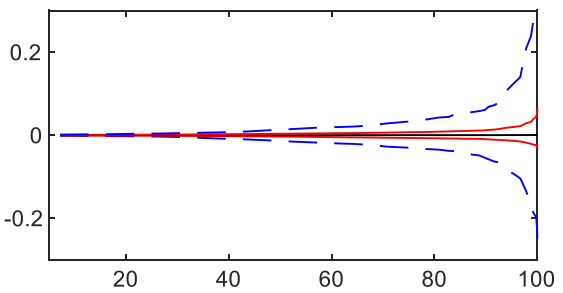

(g)

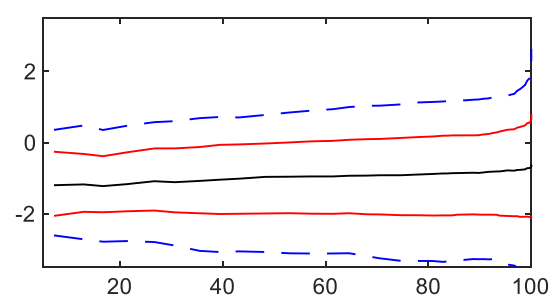

(h)

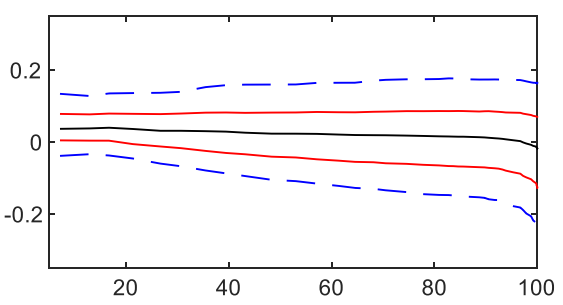

(i)

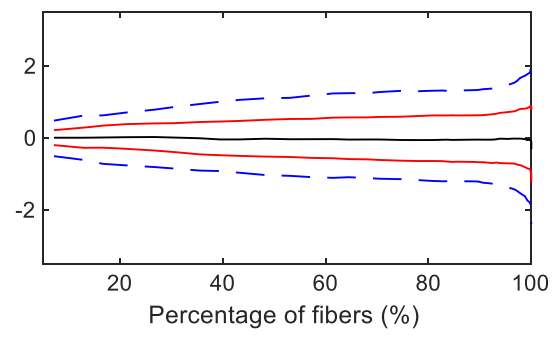

(j) 
Fig. 5-12 [left page]. 10th, 25th, 50th, 75th and 90th percentiles, of the individual estimation errors obtained for the 2-port recording configuration. Error percentiles against the distance to the ports, $W_{x}$ (a-e); (a) estimation error in the position, $x$; (b) estimation error in the position, $y$; (c) estimation error in the neuromuscular junction position, $z$; (d) estimation error in the MFCV, $v$; (e) estimation error in the parameter $\zeta$ (the neuromuscular junction position after removing the MU bias, see section 3.4.1). Error percentiles against the percentage of MU fibers evaluated (f-j); (f) estimation error in the position $x ;(\mathrm{g})$ estimation error in the position $y$; (h) estimation error in the neuromuscular junction position, $z$; (i) estimation error in the MFCV, $v$; (j) estimation error in the parameter $\zeta$.

When smaller regions that cover lower percentage of MU fibers are considered, the 4-port recording configuration also presents lower MAE than the rest of configurations. Note that the lowest value of the percentage of MU fibers corresponds, for the 4-port configuration, to the region located between both scanning needles. The fact that for this region the MAE has resulted to be lower than for the other configurations is in agreement to the conclusions obtained in the study of the forward problem (section 3.2.2). Remember that in that study, it was concluded that, when considering the space between two needles, the parameter dispersion region is noticeably smaller than when a single needle is used.

\section{Summary of the estimation performance}

A summary of the estimation performance corresponding to each recording configuration is shown in Table 5-3. Among the recording configurations proposed to be incorporated in the estimation system, the one with better performance is the 4-port configuration, followed by the 2-port configuration. However, apart from the estimation performance, there is another important factor to keep in mind when it comes to choose the best option, which is the complexity of the recording configuration. The simplest one is the 1-port scanning-EMG configuration, for which the type of needle electrode required (a SF needle electrode, with a single port located at a side of the needle) is the only one which is commercially available to this day. A step forward in complexity is taken by the 2-port scanning-EMG configuration, for which only one scanning needle is required but it has two ports, pointing to opposite directions. However, this small increase in complexity means a notable increase in the estimation performance. The most complex recording configuration is, by far, the 4port scanning-EMG configuration. Note that this configuration requires two needles properly assembled, which in addition should be inserted in the muscle with the suitable angle respect to the fiber direction, in such a way that the spacing between the two scanning needles in the longitudinal and perpendicular fiber direction is the desired one. This makes this configuration difficult to implement in practice.

Following this rationale, we concluded that the best option to be incorporated in the estimation system is the 2-port recording configuration, as it keeps an optimal balance between being relatively simple to implement, and being able to achieve a good estimation of the MU fiber parameters at the same time. 


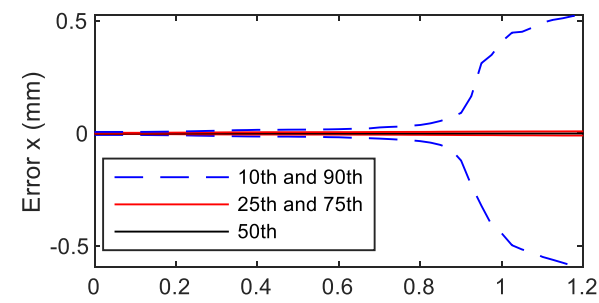

(a)

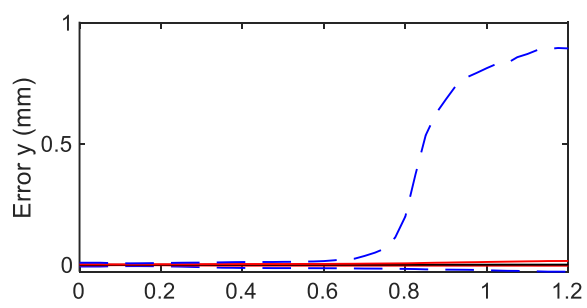

(b)

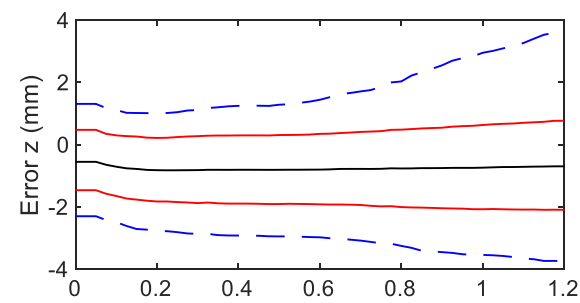

(c)

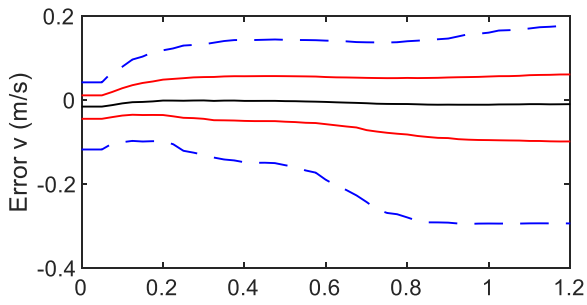

(d)

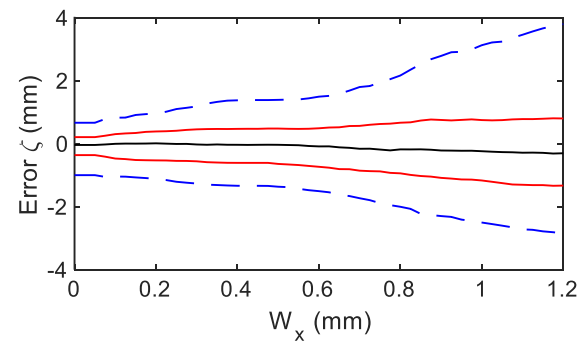

(e)

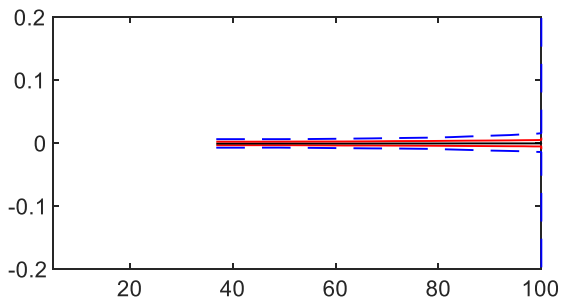

(f)

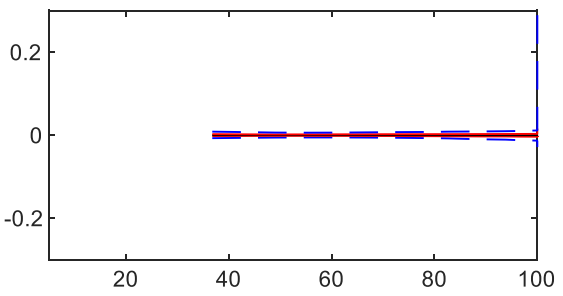

(g)

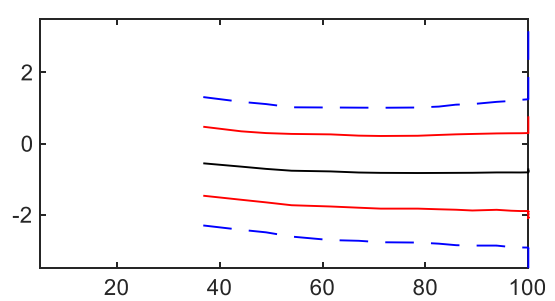

(h)

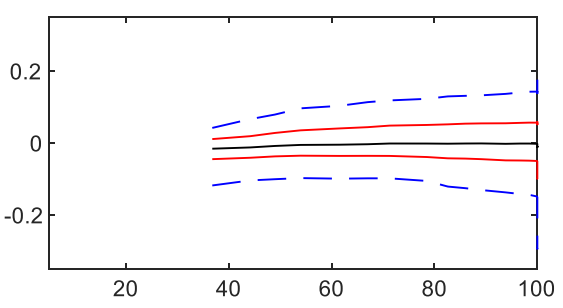

(i)

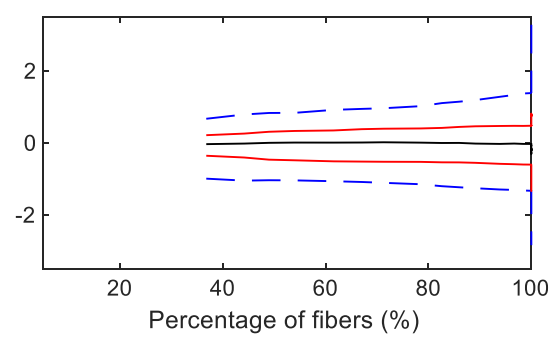

(j) 
Fig. 5-13 [left page]. 10th, 25th, 50th, 75th and 90th percentiles, of the individual estimation errors obtained for the 4-port recording configuration. Error percentiles against the distance to the ports, $W_{x}$ (a-e); (a) estimation error in the position, $x$; (b) estimation error in the position, $y$; (c) estimation error in the neuromuscular junction position, $z$; (d) estimation error in the MFCV, $v$; (e) estimation error in the parameter $\zeta$ (the neuromuscular junction position after removing the MU bias, see section 3.4.1). Error percentiles against the percentage of MU fibers evaluated (f-j); (f) estimation error in the position $x ;(\mathrm{g})$ estimation error in the position $y$; (h) estimation error in the neuromuscular junction position, $z$; (i) estimation error in the MFCV, $v$; (j) estimation error in the parameter $\zeta$.

Table 5-3. MAE of the MU fiber parameters for the different recording configurations.

\begin{tabular}{|c|c|c|c|c|c|c|c|c|}
\cline { 2 - 9 } \multicolumn{1}{c|}{} & \multicolumn{2}{c|}{ Single-point } & \multicolumn{2}{c|}{ 1-port } & \multicolumn{2}{c|}{ 2-port } & \multicolumn{2}{c|}{ 4-port } \\
\hline$\%$ of MU fibers & $30 \%$ & $60 \%$ & $30 \%$ & $60 \%$ & $30 \%$ & $60 \%$ & $30 \%$ & $60 \%$ \\
\hline$x(\mathrm{~mm})$ & $\mathbf{0 . 1 6 4 4}$ & - & 0.0115 & - & 0.0040 & 0.0067 & - & 0.0031 \\
\hline$y(\mathrm{~mm})$ & $\mathbf{0 . 2 4 8 9}$ & - & 0.0094 & - & 0.0016 & 0.0048 & - & 0.0018 \\
\hline$\zeta(\mathrm{mm})$ & 0.7456 & - & 1.0042 & - & 0.4020 & 0.5647 & - & 0.4106 \\
\hline$v(\mathrm{~m} / \mathrm{s})$ & 0.1111 & - & 0.1129 & - & 0.0536 & 0.0694 & - & 0.0379 \\
\hline
\end{tabular}

MAE of the parameters characterizing the MU fibers, for the different recording configurations, and for two different percentages of MU fibers covered by the evaluation region. Note that a color has been assigned to each MAE value in order to give a qualitative interpretation of the quality of the estimation. These colors are: red (very low quality), orange (moderately low quality), lime (moderately high quality) and green (very high quality).

\section{Considerations about the nature of the estimation error}

From a theoretical point of view, the precision with which an inverse MUP modeling procedure, like the one used in the estimation system estimates the MU fiber parameters, will be determined by several factors. The most important one is concerned to what extent the problem has an ill-posed nature, that is to say, to what extent different MU fiber parameters generate in simulation very similar signal waveforms. If this is the case, the inverse modeling will have difficulties to determine what are the parameters actually corresponding to the recorded signal waveform, so that the parameter estimation will be imprecise, or even incorrect. How much of illposed is the inverse MUP modeling is strongly conditioned by the disposition of the positions in which the MUP is recorded, that is, by the recording configuration used (see the study of the forward problem in section 3.2).

The consequences of an ill-posed character are aggravated as soon as the presence of noise in the recorded signal is considered. In an ideal scenario in which there is no noise, and a perfect optimization has achieved, the estimated signal waveform will perfectly match with the waveform of the reference recorded signal. In this case, the 


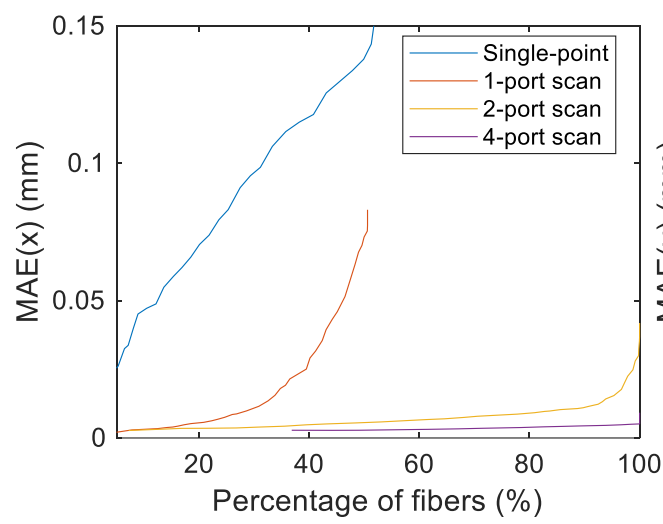

(a)

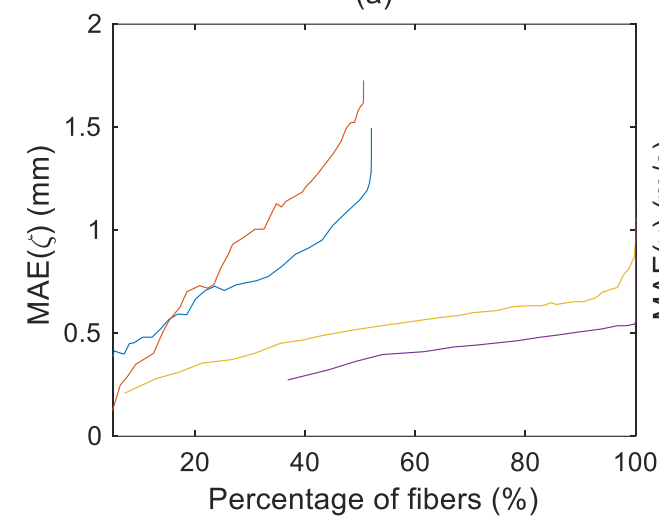

(c)

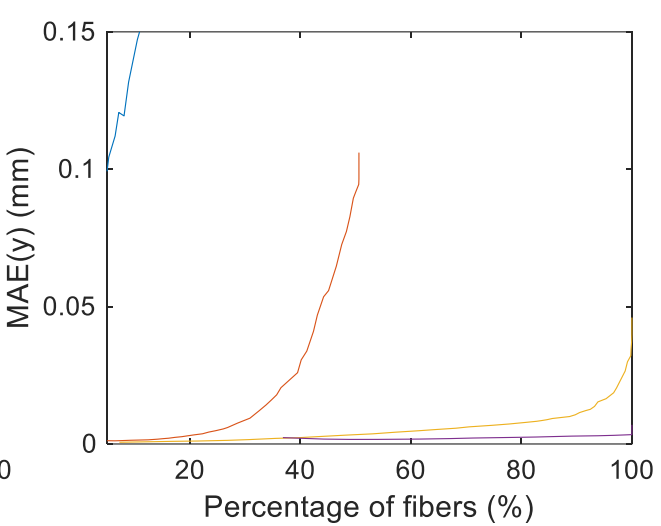

(b)

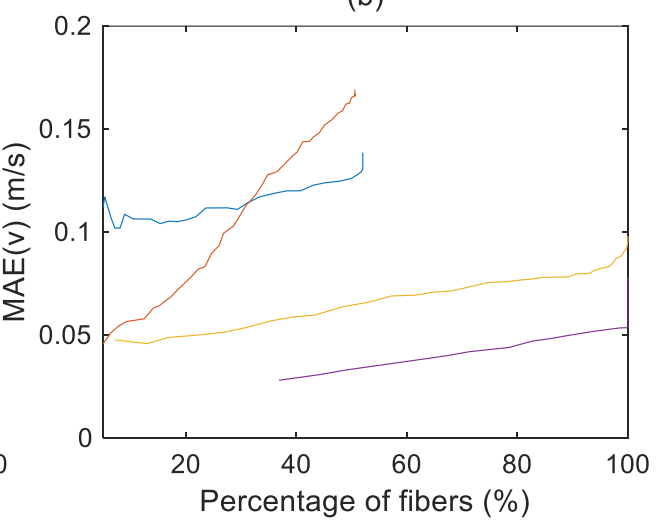

(d)

Fig. 5-14. MAE of the individual estimation errors as a function of the percentage of MU fibers evaluated, comparing the different recording configurations: the single-point (blue), the 1-port (red), the 2-port (yellow), and the 4-port (purple) recording configuration: (a) MAE of the position $x$. Note that, in the case of the single-point configuration, the MAE of the radial distance $r$ is represented instead; (b) MAE of the position $y$; (c) MAE of the parameter $\zeta$; (d) MAE of the MFCV, $v$.

global minimum solution (assuming that there is only one) coincides with the actual MU fiber parameters, and therefore the inverse modeling will correctly estimate the MU fiber parameters. The presence of noise affects the fitting of the signal waveform, so that the estimated waveform tends to follow the noise variations, in such a way that the estimated signal waveform is no longer equal to the signal generated by the actual MU fiber parameters, i.e., the noise free reference signal (for an example of this behavior see Fig. 4-4 in section 4.2.3). Therefore, the global minimum no longer coincides with the actual MU fiber parameters, but it is displaced to another point of the parameter space. Whether or not the global minimum solution is near to the actual 
MU fiber parameters point will depend, apart from the amount of noise, on the degree of ill-posed of the problem. If the problem is very ill-posed, a little amount of noise will produce that the global minimum to be displaced far from the point corresponding to the actual MU fiber parameters, otherwise, the global minimum will remain close to such point.

We have just seen that a source of the estimation error is due to the displacement of the global minimum from the point where the actual MU fiber parameters are located. Another important error source concerns to what extent this global minimum is properly reached by the optimization algorithm. The severity of this second error source will also depend, apart from the effectiveness of the optimization, on the degree of ill-posed of the problem. If the problem is very ill-posed, a small mismatch in the estimated waveform will cause that the solution given by the optimization algorithm will be located far from the global minimum in the parameter space. Otherwise such solution will remain close to the global minimum.

Regarding this second error source, note that the optimization procedure incorporated in the estimation system has been designed to make it as small as possible. In such procedure, a large enough number of iterations has been set so as to achieve a refined solution, and a large enough number of repetitions has also been set, as to avoid to fall into local minima (see section 3.3.3). The fact that in the experiments the estimated signal waveform has been fitted with precision to the reference signal (examples of this can be found in Fig. 5-3, Fig. 5-5, Fig. 5-7 and Fig. 5-9) suggests that the optimization procedure has been successful. In this way, the analysis can be more focused on the error caused by the first source (displacement of the global minimum caused by the noise in the recording), which is mainly determined by recording (the configuration used, and the noise introduced) and the MUP modeling to be inverted.

\subsubsection{Estimation with an increased muscle contraction}

This study presents and discusses the results of the experiments described in section 5.1.3, analyzing the influence of an increased muscle contraction in the estimation performance. It is important to recall that, from now on, only the 2-port configuration will be analyzed.

\section{Results}

Fig. 5-15 shows the percentiles of the individual parameter estimation errors, obtained from executing the general experiment scheme for the 2-port recording configuration for a 5.5\% MVC. Fig. 5-16 shows the MAE of the individual estimation errors, obtained for the 2-port recording configuration, for two different MVC percentages: the default value, $3.5 \%$ (results in section 5.2.1); and the increased value, $5.5 \%$. 


\section{Discussion of the results}

There is a noticeable performance degradation between the estimations obtained in the $3.5 \%$ MVC case (default case), and that corresponding to $5.5 \%$ MVC. Note that the MAE is always lower for the $3.5 \% \mathrm{MVC}$ case when compared to the $5.5 \% \mathrm{MVC}$ case; this is consistently found for all the MU fiber parameters, and for all percentages of MU fibers covered by the evaluation region (Fig. 5-16). For example, considering a region covering $50 \%$ of the MU fibers, the MAE of the parameter $x$ is $5.6 \cdot 10^{-3} \mathrm{~mm}$ and $9.3 \cdot 10^{-3} \mathrm{~mm}$ for an MVC of $3.5 \%$ and $5.5 \%$ respectively; the MAE of the parameter $y$ is $3.4 \cdot 10^{-3} \mathrm{~mm}$ and $4.6 \cdot 10^{-3} \mathrm{~mm}(3.5 \%$ and $5.5 \%$ MVC respectively); the MAE of the parameter $\zeta$ is $0.52 \mathrm{~mm}$ and $0.57 \mathrm{~mm}$ ( $3.5 \%$ and $5.5 \%$ MVC respectively); and the MAE of the parameter $v$ is $0.065 \mathrm{~m} / \mathrm{s}$ and $0.1 \mathrm{~m} / \mathrm{s}(3.5 \%$ and $5.5 \% \mathrm{MVC}$ respectively). A summary of the estimation performance comparing the two MVC percentages is shown in Table 5-4.

The increase of the muscle contraction implies that a larger number of MUP artifacts are superimposed to the recorded scanning-EMG signal, making it noisier. This implies that the waveform fitting between the estimated signal and the ideal

Table 5-4. MAE of the MU fiber parameters for the two evaluated MVC percentages.

\begin{tabular}{|c|c|c|c|c|}
\cline { 2 - 5 } \multicolumn{1}{c|}{} & \multicolumn{2}{c|}{ MVC 5.5\% } & \multicolumn{2}{c|}{ MVC 3.5\% } \\
\hline$\%$ of MU fibers & $30 \%$ & $60 \%$ & $30 \%$ & $60 \%$ \\
\hline$x(\mathrm{~mm})$ & 0.0076 & 0.0101 & 0.0040 & 0.0067 \\
\hline$y(\mathrm{~mm})$ & 0.0024 & 0.0059 & 0.0016 & 0.0048 \\
\hline$\zeta(\mathrm{mm})$ & 0.5286 & 0.5932 & 0.4020 & 0.5647 \\
\hline$v(\mathrm{~m} / \mathrm{s})$ & 0.0979 & 0.1034 & 0.0536 & 0.0694 \\
\hline
\end{tabular}

MAE of the parameters characterizing the MU fibers, for the two evaluated MVC percentages, and for two different percentages of MU fibers covered by the evaluation region. Note that a color has been assigned to each MAE value in order to give a qualitative interpretation of the quality of the estimation. These colors are: red (very low quality), orange (moderately low quality), lime (moderately high quality) and green (very high quality).

Fig. 5-15 [right page]. 10th, 25th, 50th, 75th and 90th percentiles, of the individual estimation errors obtained for the 2-port recording configuration under an increased muscle contraction $(5.5 \% \mathrm{MVC})$. Error percentiles against the distance to the ports, $W_{x}$ (a-e); (a) estimation error in the position $x$; (b) estimation error in the position $y$; (c) estimation error in the neuromuscular junction position $z$; (d) estimation error in the MFCV, $v$; (e) estimation error in the parameter $\zeta$. Error percentiles against the percentage of MU fibers inside the evaluation region (f-j); (f) estimation error in the position $x$; (g) estimation error in the position $y$; (h) estimation error in the neuromuscular junction position, $z$; (i) estimation error in the MFCV, $v$; (j) estimation error in the parameter $\zeta$. 

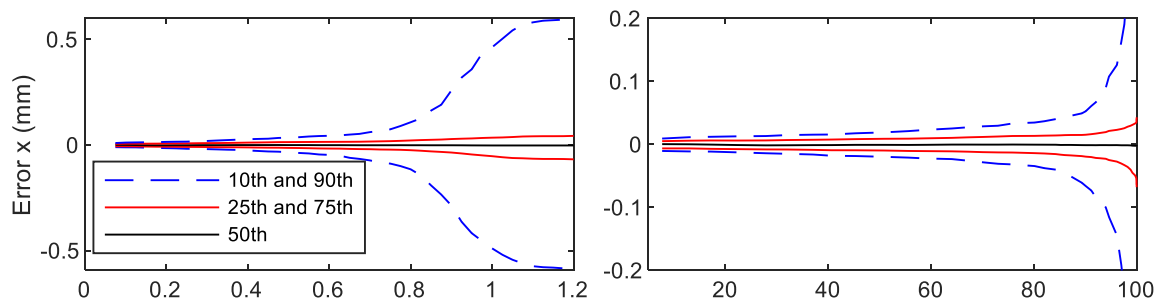

(a)

(f)
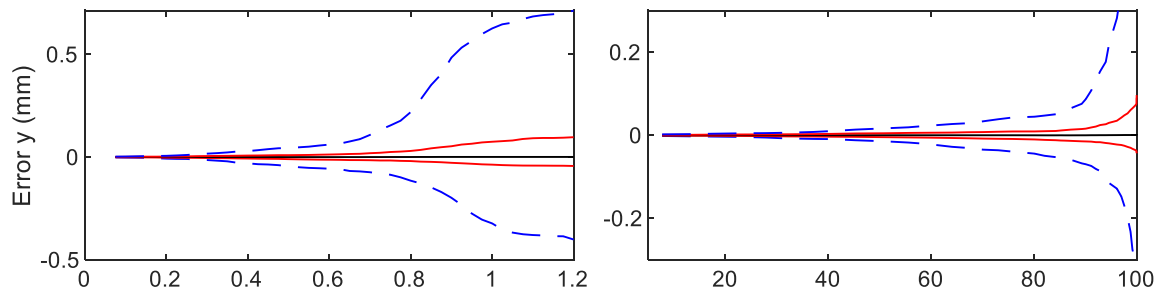

(b)

(g)
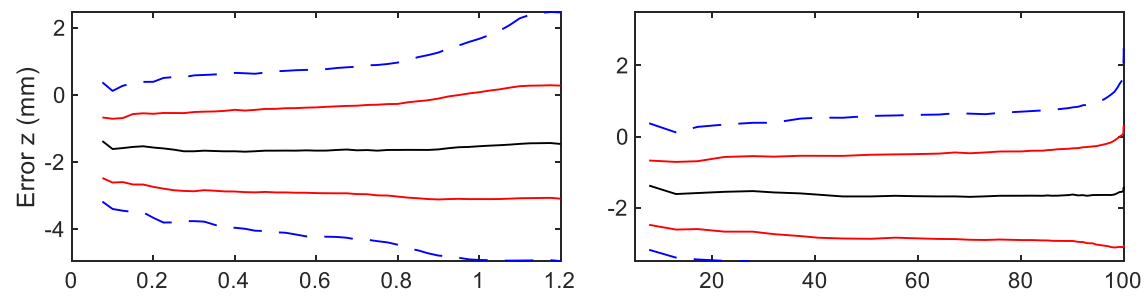

(c)

(h)
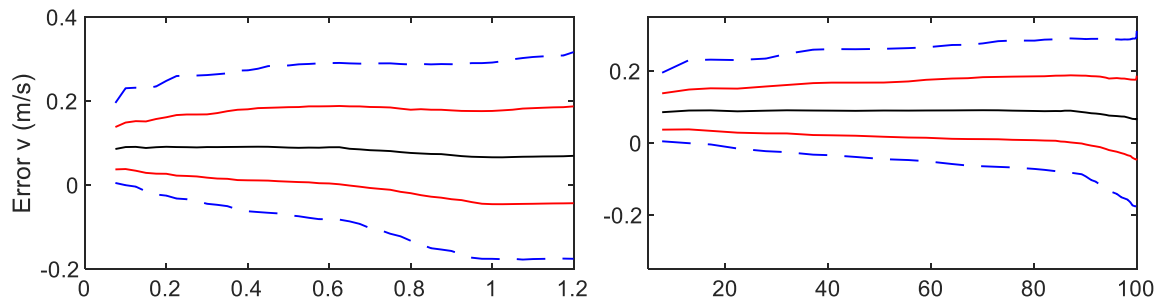

(d)

(i)
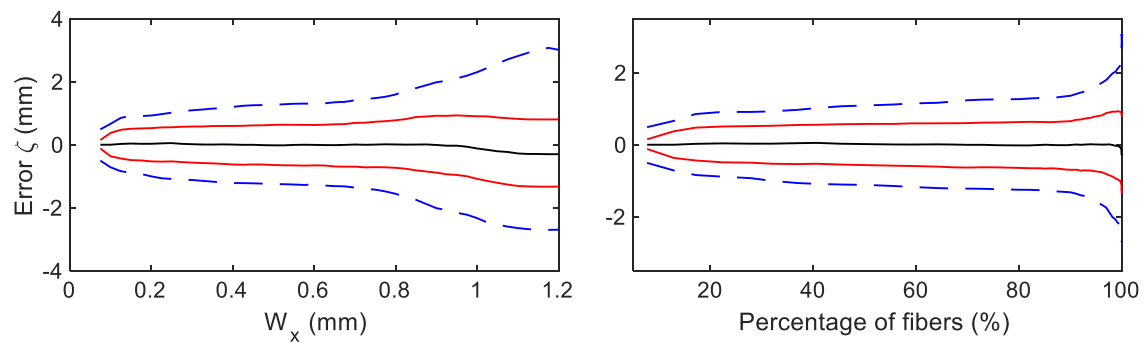

(e)

(j) 
(noise-free) recorded one is more imprecise. As a consequence, the global minimum in the parameter space tends to be farther from the point corresponding to the actual fiber parameters, making the error in the estimated parameters to increase. Remember that artifact noise is produced by the activation of other MUs during muscle contraction, and that this type of noise is, of those present in the scanning-EMG recordings, the most harmful for the estimation performance (see section 4.2.3). As a larger contraction implies a lower estimation quality, it is recommended that the scanning-EMG signal is recorded at the lowest possible contraction. This is in agreement with standard recommendations for scanning-EMG recording of individual MUs [90].

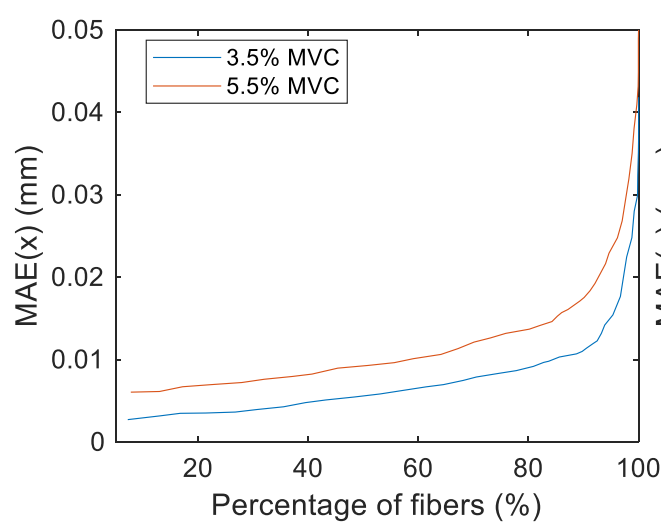

(a)

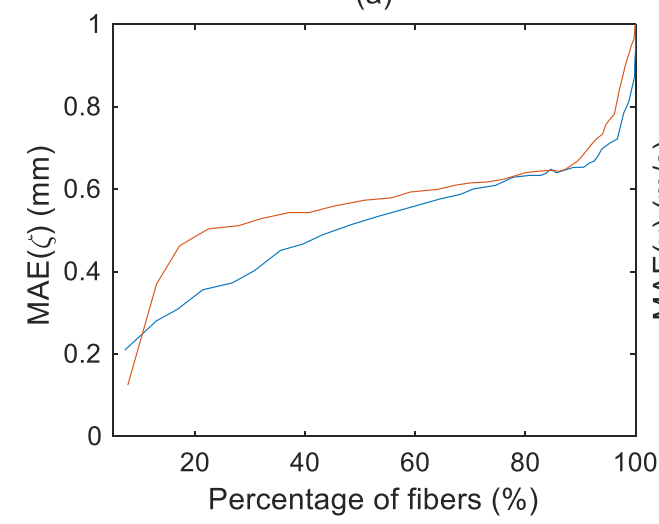

(c)

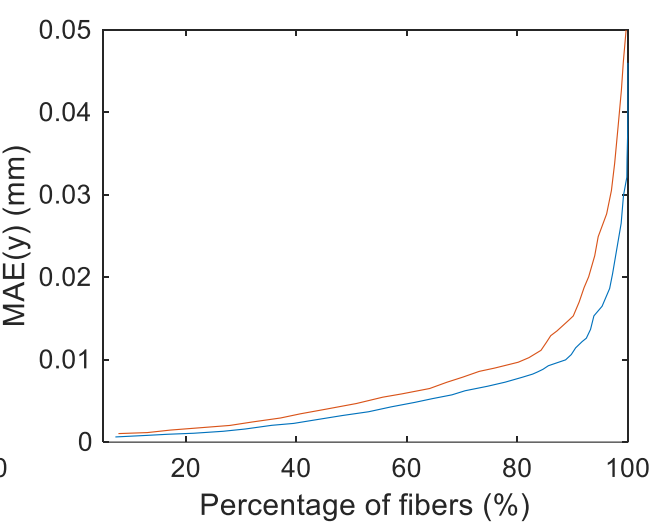

(b)

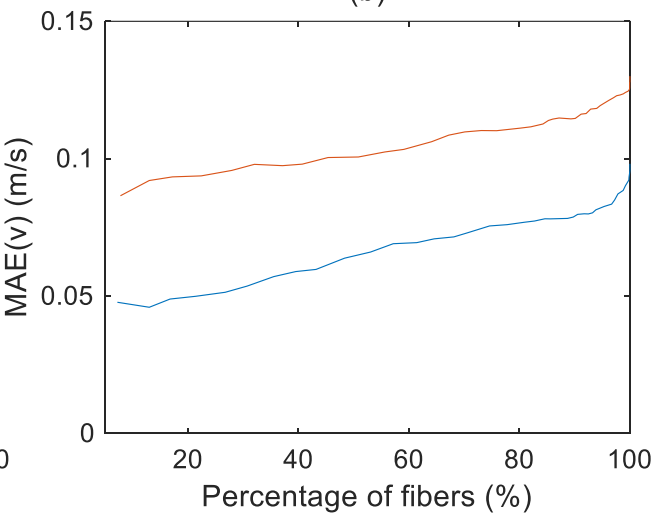

(d)

Fig. 5-16. MAE of the individual estimation errors against the percentage of MU fibers inside the evaluation region, obtained for two different levels of muscle voluntary contraction: $3.5 \%$ (blue) and 5.5\% MVC (red): (a) MAE in the position $x$; (b) MAE in the position $y$; (c) MAE in the parameter $\zeta$; (d) MAE in the MFCV, $v$. 


\subsubsection{Estimation with variability in the IAP parameters}

This study presents and discusses the results about the experiments described in section 5.1.4, analyzing the influence in the estimation of introducing IAP waveform variability in the recorded scanning-EMG signal, while keeping a fixed IAP in the estimation system.

\section{Results}

Fig. 5-17 and Fig. 5-18 show the percentiles of the individual parameter estimation errors, obtained from executing the general experiment scheme for the 2-port recording configuration, under two different scenarios: one in which the recorded signal has been generated with IAP variability among the MU fibers but without noise (Fig. 5-17); and another one in which the signal has been generated including both IAP variability and noise (Fig. 5-18).

Fig. 5-19 shows the MAE of the individual estimation errors, obtained for the 2port recording configuration, under three different scenarios: including only IAP variability; including IAP variability and noise; and the default scenario (results in section 5.2.1), including only noise, to be used as a reference for the discussion.

\section{Discussion of the results}

In Fig. 5-19, the lowest MAE occurs for the case in which only noise is included in the recorded signal, and no variability exists in the IAP parameters of the fibers. When IAP variability is implemented, the case with noise presents higher MAE than the one without it. These tendencies have been consistently observed for all the MU fiber parameters.

It seems clear that when the recorded signal is generated with IAP parameters that vary from one MU fiber to another, the MU fiber parameters are estimated with lower accuracy. In fact, the negative effect produced in the estimation by a variable IAP is dominant respect that produced by the noise present in the recorded signal. This is true at least in the recording conditions in which the experiments have been performed. Although the effect of IAP variability on the estimation is noticeable, the estimation is still precise for evaluation regions that involve low percentages of MU fibers. If we consider the case in which there is IAP variability and noise (Fig. 5-18), for an evaluation region covering $40 \%$ of the MU fibers, $80 \%$ of the estimation errors are between $-0.012 \mathrm{~mm}$ and $0.013 \mathrm{~mm}$ for the parameter $x$; between $-0.01 \mathrm{~mm}$ and 0.011 $\mathrm{mm}$ for the parameter $y$; between $-0.114 \mathrm{~m} / \mathrm{s}$ and $0.185 \mathrm{~m} / \mathrm{s}$ for the parameter $v$; and between $-1.19 \mathrm{~mm}$ and $1.19 \mathrm{~mm}$ for the parameter $\zeta$. This has been the case, even though the IAP variability was large enough to assume a considerable change in the waveform of the IAP. A summary of the estimation performance corresponding to the three scenarios is shown in Table 5-5. 


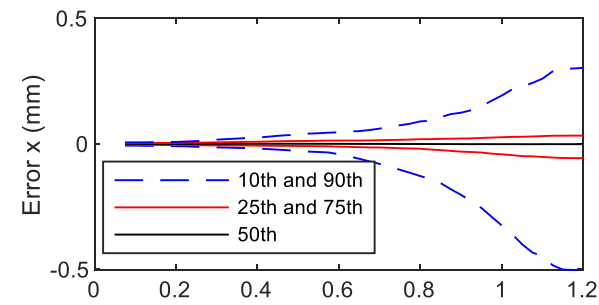

(a)

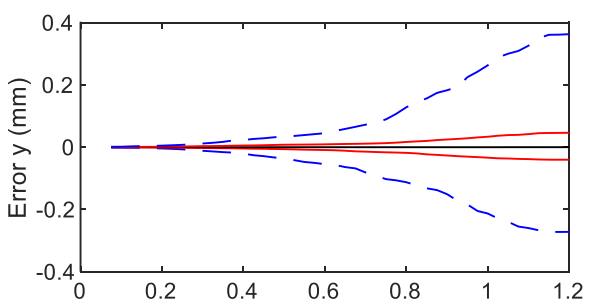

(b)

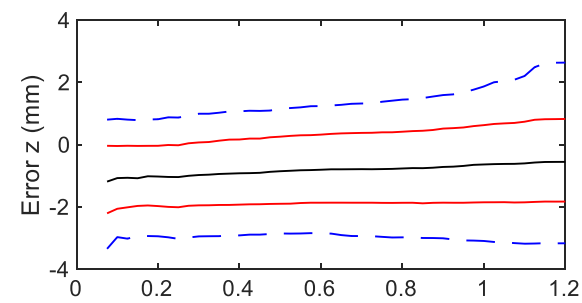

(c)

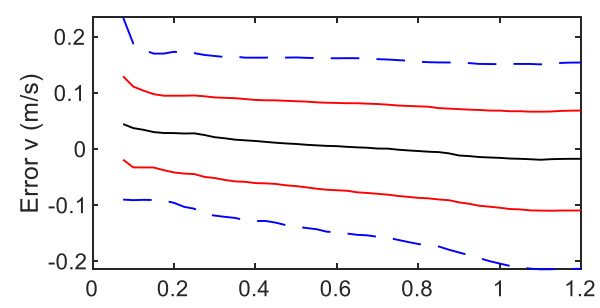

(d)

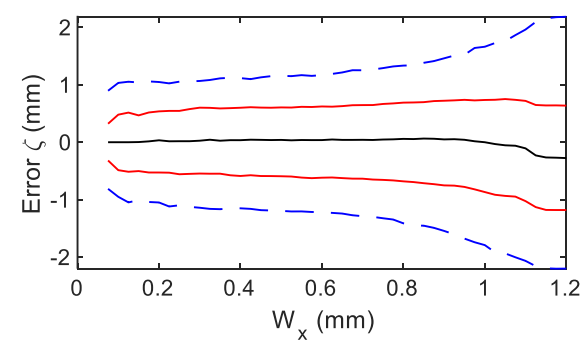

(e)

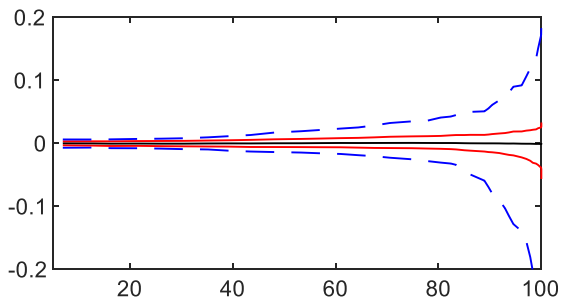

(f)

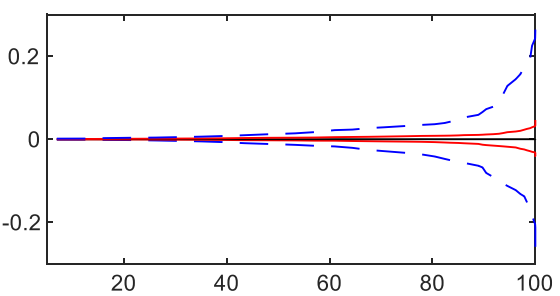

(g)

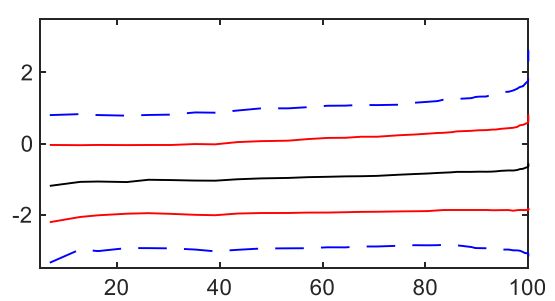

(h)

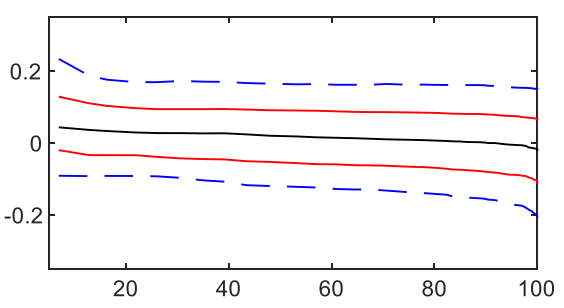

(i)

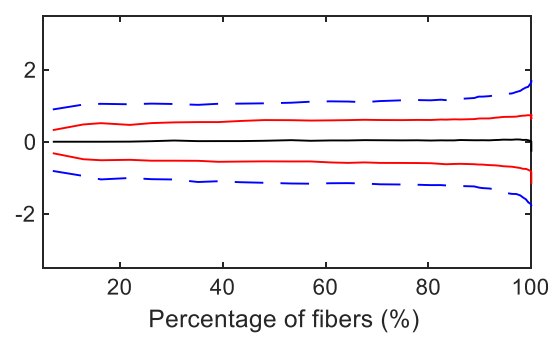

(j) 
Fig. 5-17 [left page]. 10th, 25th, 50th, 75th and 90th percentiles, of the individual estimation errors obtained for the 2-port recording configuration, when no noise exists in the recorded signal and the MU fibers present variability in the IAP parameters. Error percentiles against the distance to the ports, $W_{x}$ (a-e); (a) estimation error in the position, $x$; (b) estimation error in the position, $y$; (c) estimation error in the neuromuscular junction position, $z$; (d) estimation error in the MFCV, $v$; (e) estimation error in the parameter $\zeta$. Error percentiles against the percentage of MU fibers evaluated (f-j); (f) estimation error in the position $x$; $(\mathrm{g})$ estimation error in the position $y$; (h) estimation error in the neuromuscular junction position, $z$; (i) estimation error in the MFCV, $v$; (j) estimation error in the parameter $\zeta$.

Table 5-5. MAE of the MU fiber parameters for the three analyzed scenarios.

\begin{tabular}{|c|c|c|c|c|c|c|}
\cline { 2 - 7 } \multicolumn{1}{c|}{} & \multicolumn{2}{c|}{ Noise } & \multicolumn{2}{c|}{ IAP var } & \multicolumn{2}{c|}{ Noise + IAP var } \\
\hline$\%$ of MU fibers & $30 \%$ & $60 \%$ & $30 \%$ & $60 \%$ & $30 \%$ & $60 \%$ \\
\hline$x(\mathrm{~mm})$ & 0.0040 & 0.0067 & 0.0044 & 0.0074 & 0.0047 & 0.0084 \\
\hline$y(\mathrm{~mm})$ & 0.0016 & 0.0048 & 0.0013 & 0.0042 & 0.0023 & 0.0058 \\
\hline$\zeta(\mathrm{mm})$ & 0.4020 & 0.5647 & 0.5303 & 0.5860 & 0.5576 & 0.6169 \\
\hline$v(\mathrm{~m} / \mathrm{s})$ & 0.0536 & 0.0694 & 0.0710 & 0.0758 & 0.0770 & 0.0818 \\
\hline
\end{tabular}

MAE of the parameters characterizing the MU fibers, for the three considered scenarios (including only IAP variability, including IAP variability and noise, and only including noise), and for two different percentages of MU fibers covered by the evaluation region. Note that a color has been assigned to each MAE value in order to give a qualitative interpretation of the quality of the estimation. These colors are: red (very low quality), orange (moderately low quality), lime (moderately high quality) and green (very high quality).

These results suggest that the estimation system is robust enough to deal with discrepancies between the invariant IAP model used in the estimation procedure, and the IAP variability that may be found in the real world.

Another important consideration to keep in mind is that having developed an estimation system that correctly estimates the MU fiber parameters in a simulation environment, does not necessary imply that such estimation system will work properly in a real environment, no matter how complete the simulation environment may be. However, the robustness of the estimation system to changes in the IAP model suggests that the system could also succeed in a real environment, even when the discrepancies between the model and reality are significant.

\subsubsection{Estimation varying the global properties of the MUs}

This study presents and discusses the results about the experiments described in section 5.1.5, analyzing the influence in the estimation of changing some of the global 


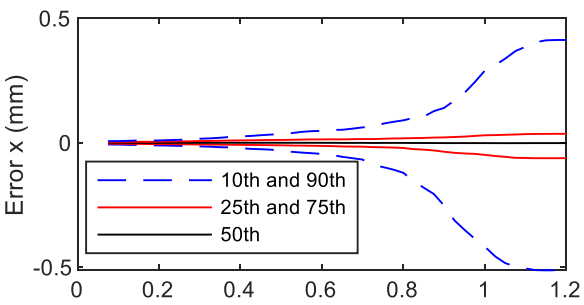

(a)

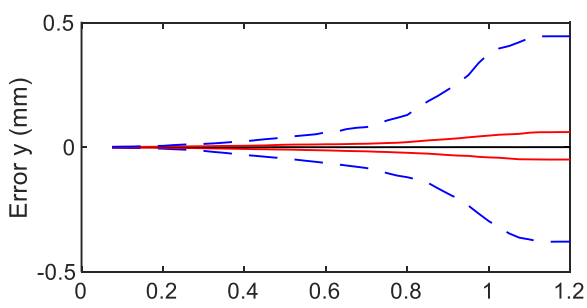

(b)

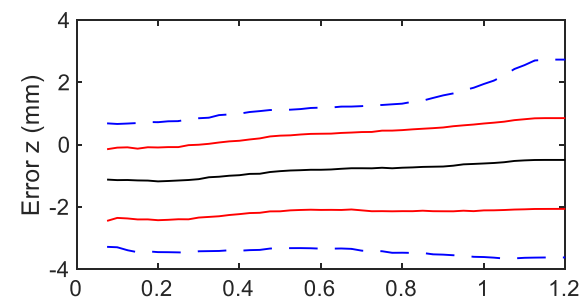

(c)

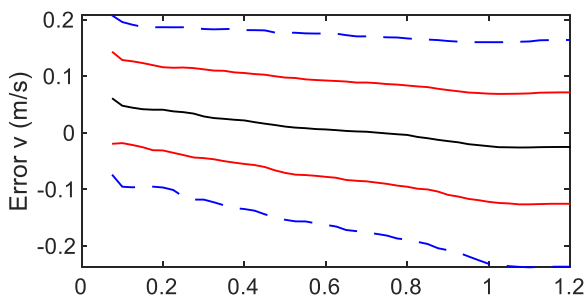

(d)

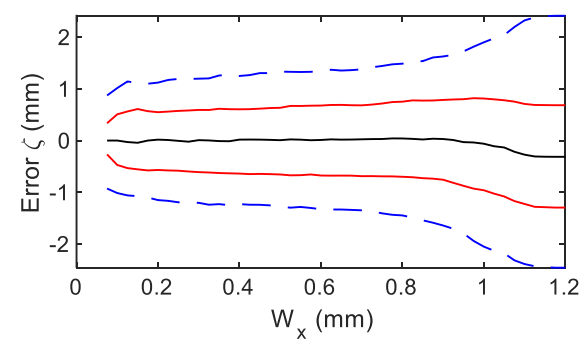

(e)

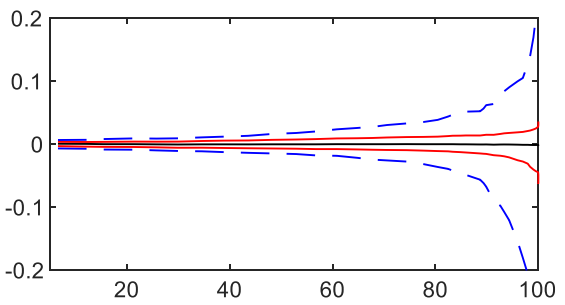

(f)

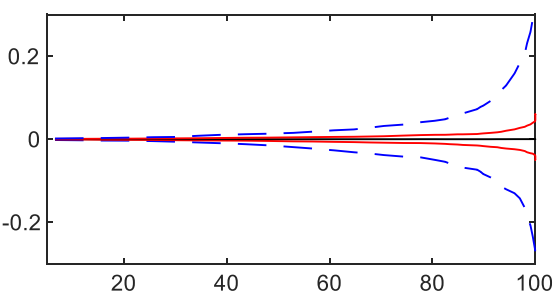

(g)

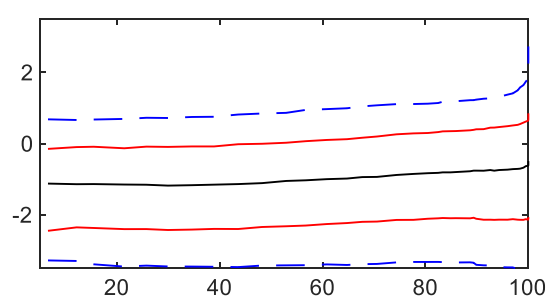

(h)

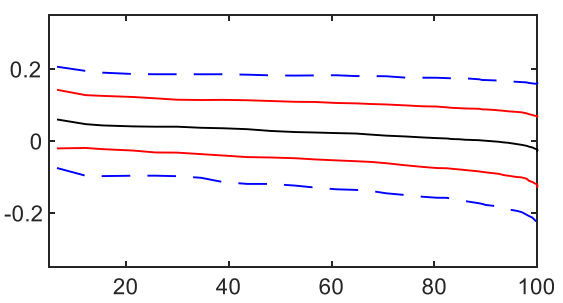

(i)

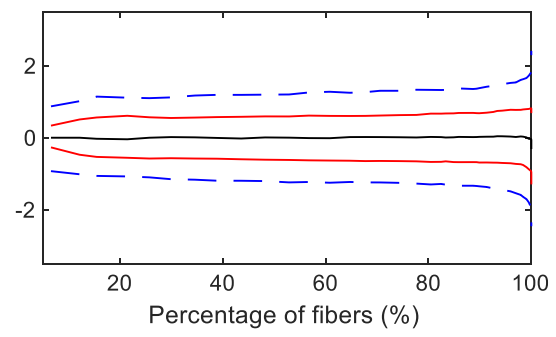

(j) 
Fig. 5-18 [left page]. 10th, 25th, 50th, 75th and 90th percentiles, of the individual estimation errors obtained for the 2-port recording configuration, when noise does exist in the recorded signal and the MU fibers present variability in the IAP parameters. Error percentiles against the distance to the ports, $W_{x}$ (a-e); (a) estimation error in the position, $x$; (b) estimation error in the position, $y$; (c) estimation error in the neuromuscular junction position, $z$; (d) estimation error in the MFCV, $v$; (e) estimation error in the parameter $\zeta$. Error percentiles against the percentage of MU fibers evaluated (f-j); (f) estimation error in the position $x$; (g) estimation error in the position $y$; (h) estimation error in the neuromuscular junction position, $z$; (i) estimation error in the MFCV, $v$; (j) estimation error in the parameter $\zeta$.

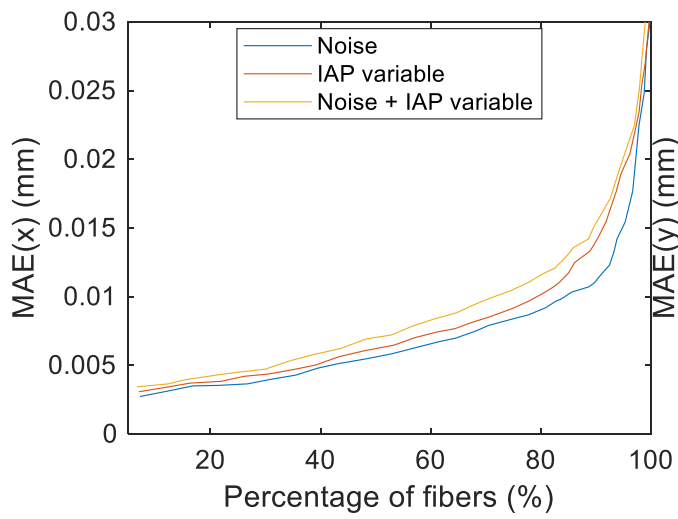

(a)

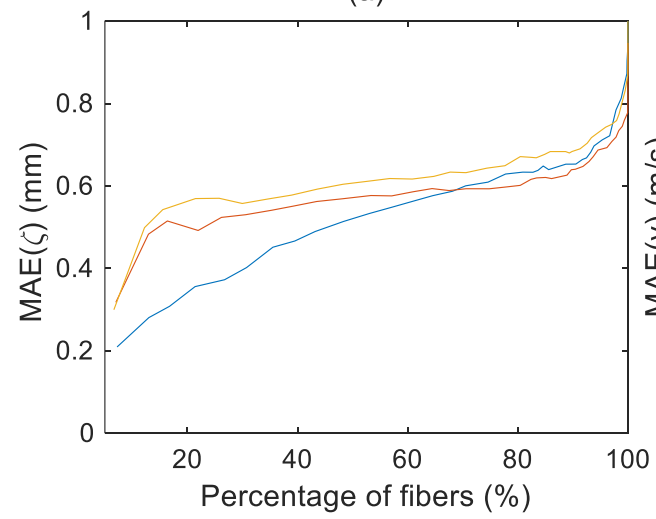

(c)

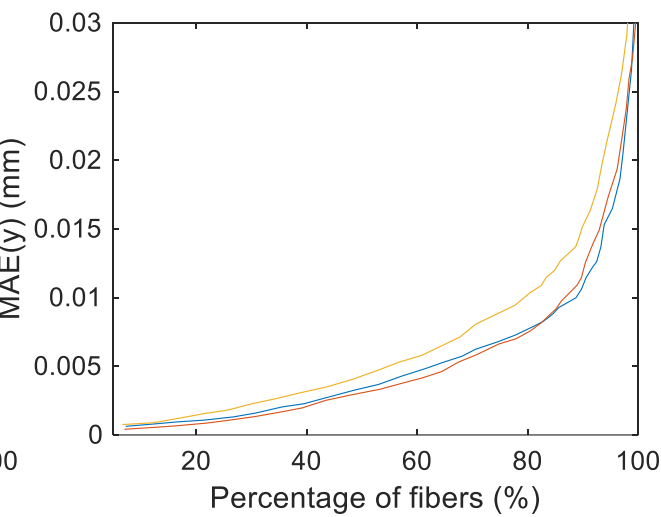

(b)

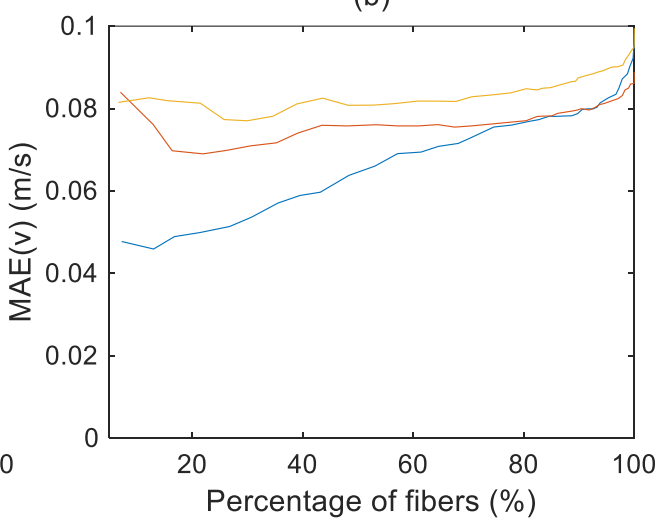

(d)

Fig. 5-19. MAE of the individual estimation errors against the percentage of MU fibers in the evaluation region, obtained for three different cases: when noise is introduced to the recorded signal (blue), when variability is introduced to the IAP parameters of the MU fibers (red), and when noise and variability in the IAP parameters are both introduced (yellow); (a) MAE of the position $x$; (b) MAE of the position $y$; (c) MAE of the parameter $\zeta$; (d) MAE of the MFCV, $v$. 
parameters of the MU. In this regard, in addition to studying the estimation of the individual MU fiber parameters, the estimation of the global parameters of the MU is also analyzed.

\section{Studying the error in the estimated individual MU fiber parameters}

Results

Fig. 5-20 and Fig. 5-21 show the percentiles of the individual parameter estimation errors, obtained after executing the general experiment scheme for the 2-port recording configuration, under two different scenarios: default, and increased MU fiber density (8 and 16 fibers per $\mathrm{mm}^{2}$, respectively). Fig. 5-22 shows the MAE of the individual estimation errors for these two scenarios.

Fig. 5-23, Fig. 5-24 and Fig. 5-25 show the percentiles of the individual parameter estimation errors, obtained after executing the general experiment scheme for the 2port recording configuration, under three different scenarios: default values for the overall IZ width and MFCV coefficient of variation ( $10 \mathrm{~mm}$ and 0.03 , respectively); IZ width increased to $20 \mathrm{~mm}$; and MFCV coefficient of variation increased to 0.06 . Fig. 5-26 shows the MAE of the individual estimation errors for these three scenarios.

\section{Discussion}

Before analyzing the results of this section, the following consideration must be taken into account. Note that the experiments using the default global MU parameters have been repeated twice, applying certain modifications to the operating parameters of the estimation procedure (see section 5.1.5): the first time, using the same operating parameters than that used in the increased fiber density experiment; and the second time, using the same operating parameters than that of used in the increased IZ width and increased MFCV coefficient of variation experiments. This has been done to compare the results between increased and default global MU parameters on equal terms. In the study varying the fiber density, the number of fibers used by the optimization procedure has been increased respect to previous experiments, so that it is sufficiently large as to ensure that it is larger than the number of actual fibers contributing to the recorded signal. In the study varying the MFCV coefficient of variation and the IZ width, the search region used in the optimization has been expanded so as to ensure it always covers the increased parameter range taken by the actual MU fibers.

It is reasonable to think that setting the estimation procedure with less restrictive operating parameters (e.g., with a larger search region, or with an increased number of fibers used in the optimization) can difficult the optimization process, and therefore can worsen the performance of the estimation. However, results indicate that the performance decrease is small. This can be corroborated by comparing the results of 
the experiments with default global parameters, for three different cases: that corresponding to the experiments of previous sections, i.e., without modifying the operating parameters of the estimation process (Fig. 5-12); that in which the number of fibers used in the optimization has been increased (Fig. 5-20); and that in which the bounds of the search region have been expanded (Fig. 5-23). Note that the error dispersion in the individual fiber parameters is similar in all cases, although somewhat higher in the case in which the number of simulated fibers is increased. The fact that the estimation performance has not decreased significantly when using less restrictive estimation operating parameters may be due to the larger number of iterations in the optimization set (see the description of the experiments in section 5.1.5), so that the possible performance degradation has been compensated. This is an important result, as it implies that it is possible to set the estimation operating parameters to cover a wide range of MU conditions without implying a large decrease in the quality of the estimation. Note that this is especially useful when considering its application as a medical diagnostic assistance tool, since different pathologies can make some global MU parameters to be significantly altered, and hence the least assumptions as possible about these parameters should be done before applying the estimation procedure.

Let's analyze to what extent increasing the value of the global MU parameters affects to the estimation performance. Regarding the experiments varying the global MU fiber density, note that the dispersion of the errors in the estimated fiber parameters is somewhat higher for the case in which the fiber density has been increased (Fig. 5-21) than for the default fiber density case (Fig. 5-20), although in both cases the error dispersion is acceptably low. For the increased density, covering $40 \%$ of the MU fibers, $80 \%$ of the estimation errors are between $-0.022 \mathrm{~mm}$ and $0.02 \mathrm{~mm}$ for the parameter $x$; between $-0.016 \mathrm{~mm}$ and $0.02 \mathrm{~mm}$ for the parameter $y$; between $-0.078 \mathrm{~m} / \mathrm{s}$ and 0.22 $\mathrm{m} / \mathrm{s}$ for the parameter $v$; and between $-1 \mathrm{~mm}$ and $1 \mathrm{~mm}$ for the parameter $\zeta$. For the default density, covering $40 \%$ of the MU fibers, $80 \%$ of the estimation errors are between $-0.012 \mathrm{~mm}$ and $0.012 \mathrm{~mm}$ for the parameter $x$; between $-0.007 \mathrm{~mm}$ and 0.09 $\mathrm{mm}$ for the parameter $y$; between $-0.079 \mathrm{~m} / \mathrm{s}$ and $0.17 \mathrm{~m} / \mathrm{s}$ for the parameter $v$; and between $-0.91 \mathrm{~mm}$ and $0.9 \mathrm{~mm}$ for the parameter $\zeta$. A summary of the comparison in the estimation performance between the increased and the default fiber density cases is shown in Table 5-6.

The fact that the estimation is less precise for an increased MU fiber density may be due to the following reason. For a MU with a high fiber density, many fibers may be significantly contributing to the scanning-EMG signal, and therefore the degrees of freedom of the problem may be larger than in MUs with lower fiber density. In this context, the number of combinations of estimated parameters that give rise to a simulated waveform similar to the recorded one may have increased noticeably, which makes the estimation error to increase. In Fig. 5-27, an example of estimation with increased fiber density is shown. It seems that the estimation tends to reconstruct the waveform of the signal using fewer fibers than the actual number, as at medium 


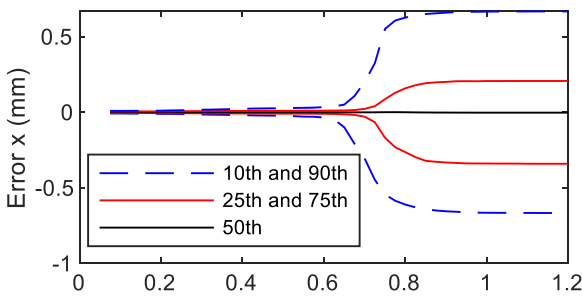

(a)

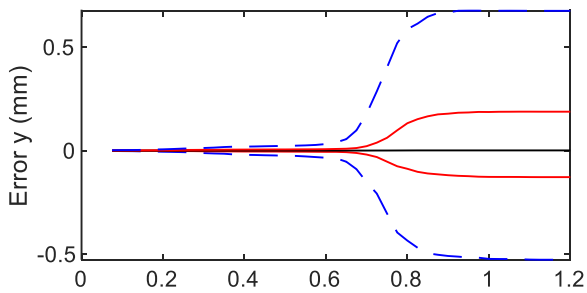

(b)

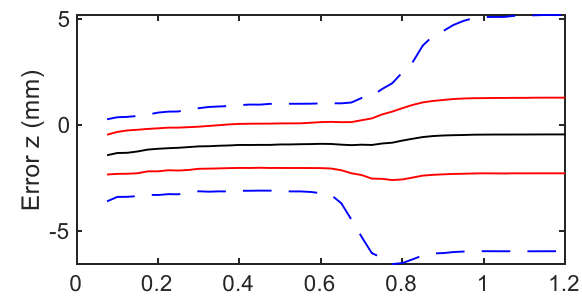

(c)

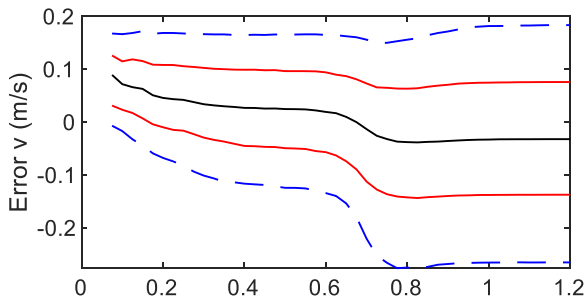

(d)

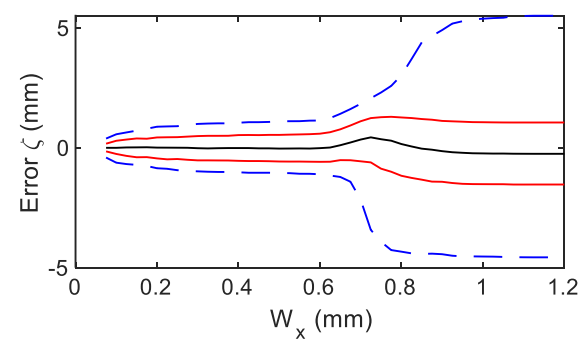

(e)

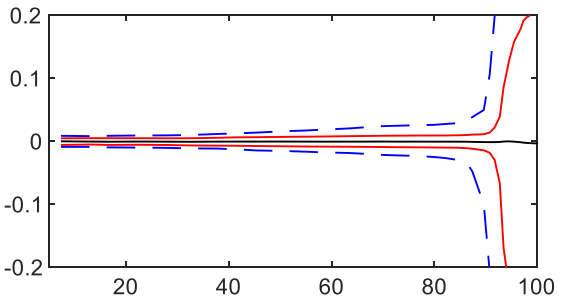

(f)

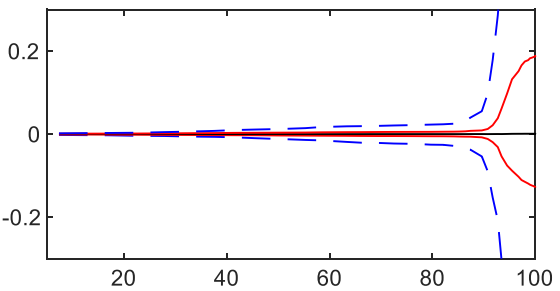

(g)

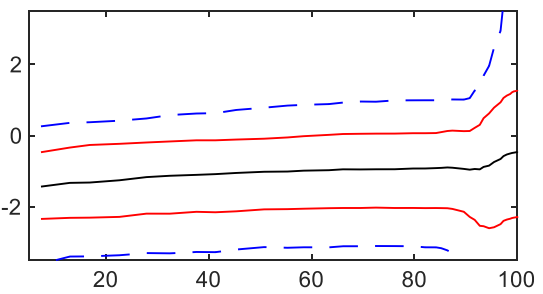

(h)

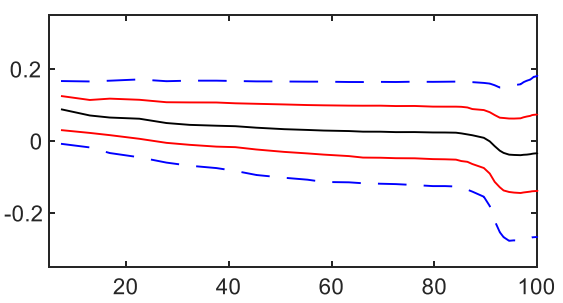

(i)

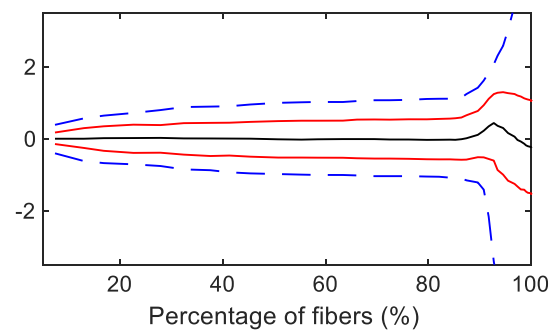

(j) 
Fig. 5-20 [left page]. 10th, 25th, 50th, 75th and 90th percentiles, of the individual estimation errors obtained for the 2-port recording configuration, when the MU fiber density has been set with the default value. Error percentiles against the distance to the ports, $W_{x}$ (a-e); (a) estimation error in the position, $x$; (b) estimation error in the position, $y$; (c) estimation error in the neuromuscular junction position, $z$; (d) estimation error in the MFCV, $v$; (e) estimation error in the parameter $\zeta$. Error percentiles against the percentage of MU fibers evaluated (f-j); (f) estimation error in the position $x$; (g) estimation error in the position $y$; (h) estimation error in the neuromuscular junction position, $z$; (i) estimation error in the MFCV, $v$; (j) estimation error in the parameter $\zeta$.

Table 5-6. MAE of the MU fiber parameters for two values of the global MU fiber density.

\begin{tabular}{|c|c|c|c|c|}
\cline { 2 - 5 } \multicolumn{1}{c|}{} & \multicolumn{2}{c|}{ Default density } & \multicolumn{2}{c|}{ Increased density } \\
\hline$\%$ of MU fibers & $30 \%$ & $60 \%$ & $30 \%$ & $60 \%$ \\
\hline$x(\mathrm{~mm})$ & 0.0052 & 0.0081 & 0.0103 & 0.0137 \\
\hline$y(\mathrm{~mm})$ & 0.0017 & 0.0040 & 0.0042 & 0.0086 \\
\hline$\zeta(\mathrm{mm})$ & 0.3842 & 0.5141 & 0.4952 & 0.5563 \\
\hline$v(\mathrm{~m} / \mathrm{s})$ & 0.0688 & 0.0740 & 0.0946 & 0.0890 \\
\hline
\end{tabular}

MAE of the parameters characterizing the MU fibers, for two values of the global MU fiber density (default and increased value), and for two different percentages of MU fibers covered by the evaluation region. Note that a color has been assigned to each MAE value in order to give a qualitative interpretation of the quality of the estimation. These colors are: red (very low quality), orange (moderately low quality), lime (moderately high quality) and green (very high quality).

Table 5-7. MAE of the MU fiber parameters for different values of the IZ width and the MFCV coefficient of variation.

\begin{tabular}{|c|c|c|c|c|c|c|}
\cline { 2 - 7 } \multicolumn{1}{c|}{} & \multicolumn{2}{c|}{ Default values } & \multicolumn{2}{c|}{ Increased IZ width } & \multicolumn{2}{c|}{ Increased MFCV cov } \\
\hline$\%$ of MU fibers & $30 \%$ & $60 \%$ & $30 \%$ & $60 \%$ & $30 \%$ & $60 \%$ \\
\hline $\mathrm{x}(\mathrm{mm})$ & 0.0043 & 0.0065 & 0.0046 & 0.0077 & 0.0038 & 0.0067 \\
\hline $\mathrm{y}(\mathrm{mm})$ & 0.0014 & 0.0037 & 0.0016 & 0.0046 & 0.0011 & 0.0034 \\
\hline $\mathrm{z}(\mathrm{no} \mathrm{bias})(\mathrm{mm})$ & 0.4434 & 0.6155 & 0.4814 & 0.7733 & 0.3898 & 0.6535 \\
\hline $\mathrm{v}(\mathrm{m} / \mathrm{s})$ & 0.0601 & 0.0816 & 0.0613 & 0.0907 & 0.0523 & 0.0756 \\
\hline
\end{tabular}

MAE of the parameters characterizing the MU fibers, for different values of the IZ width and the MFCV coefficient of variation (default values, increased IZ width, and increased MFCV coefficient of variation), and for two different percentages of MU fibers covered by the evaluation region. Note that a color has been assigned to each MAE value in order to give a qualitative interpretation of the quality of the estimation. These colors are: red (very low quality), orange (moderately low quality), lime (moderately high quality) and green (very high quality). 


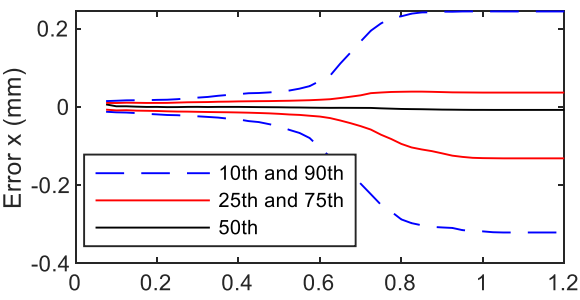

(a)

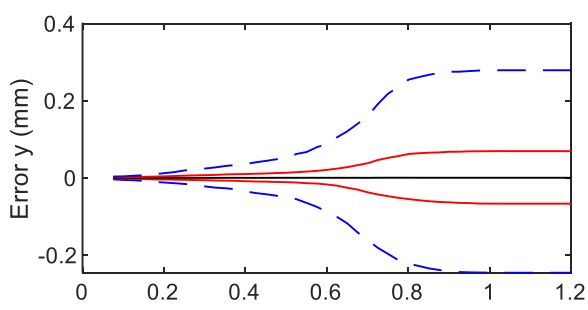

(b)

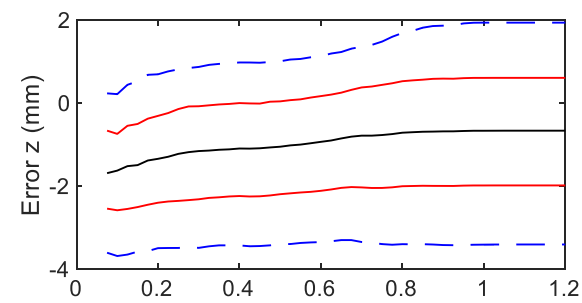

(c)

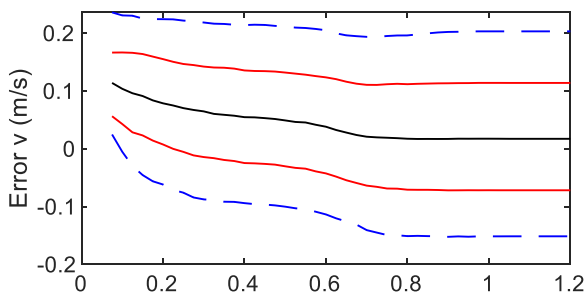

(d)

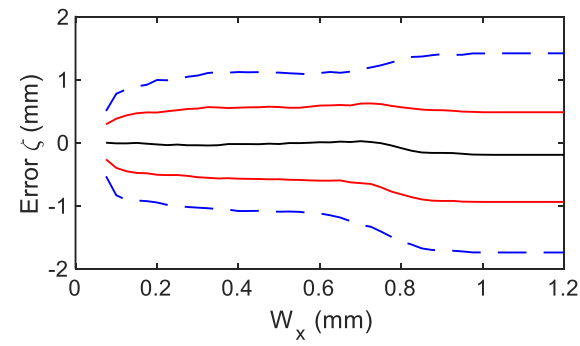

(e)

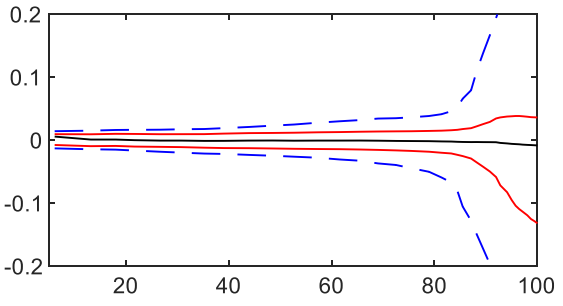

(f)

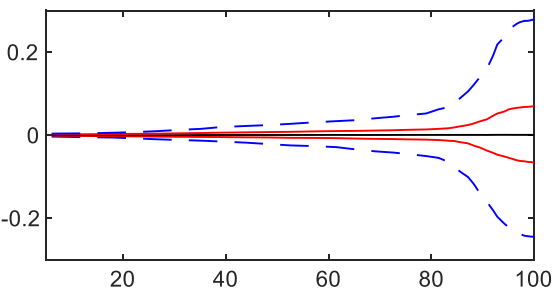

(g)

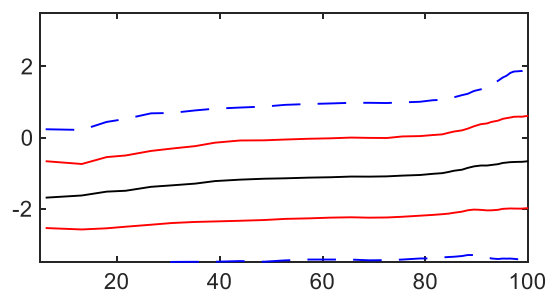

(h)

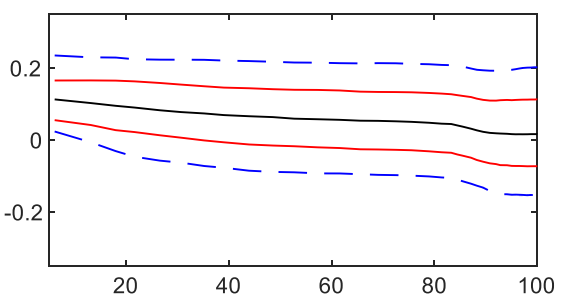

(i)

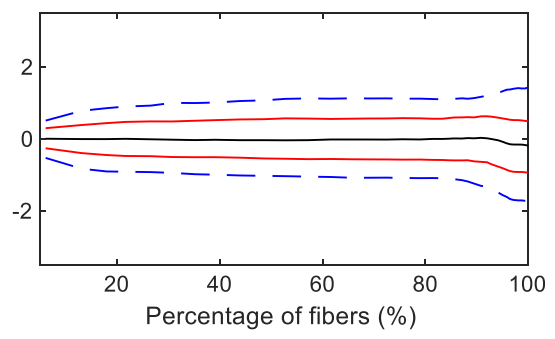

(j) 
Fig. 5-21 [left page]. 10th, 25th, 50th, 75th and 90th percentiles, of the individual estimation errors obtained for the 2-port recording configuration, when the MU fiber density has been increased. Error percentiles against the distance to the ports, $W_{x}$ (a-e); (a) estimation error in the position, $x$; (b) estimation error in the position, $y$; (c) estimation error in the neuromuscular junction position, $z$; (d) estimation error in the MFCV, $v$; (e) estimation error in the parameter $\zeta$. Error percentiles against the percentage of MU fibers evaluated (f-j); (f) estimation error in the position $x$; $(\mathrm{g})$ estimation error in the position $y$; (h) estimation error in the neuromuscular junction position, $z$; (i) estimation error in the MFCV, $v$; (j) estimation error in the parameter $\zeta$.

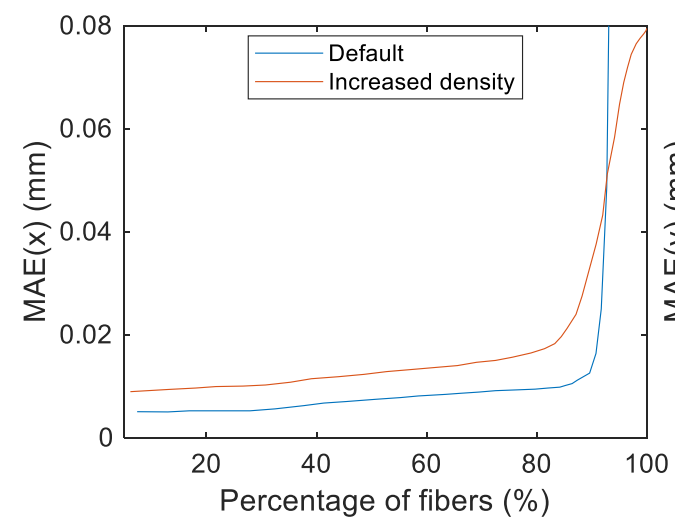

(a)

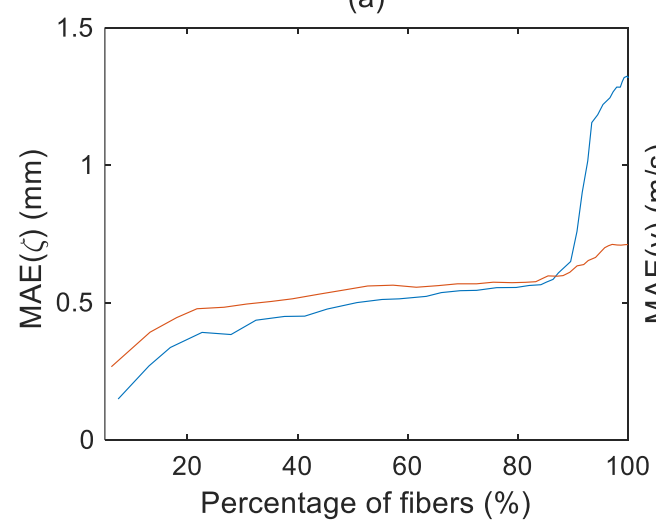

(c)

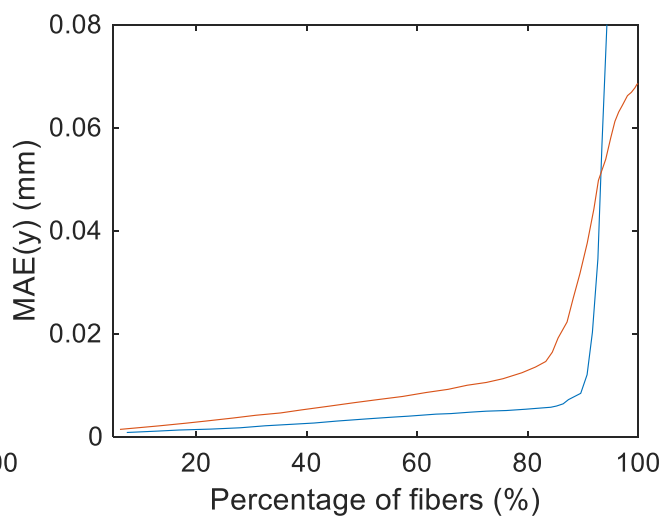

(b)

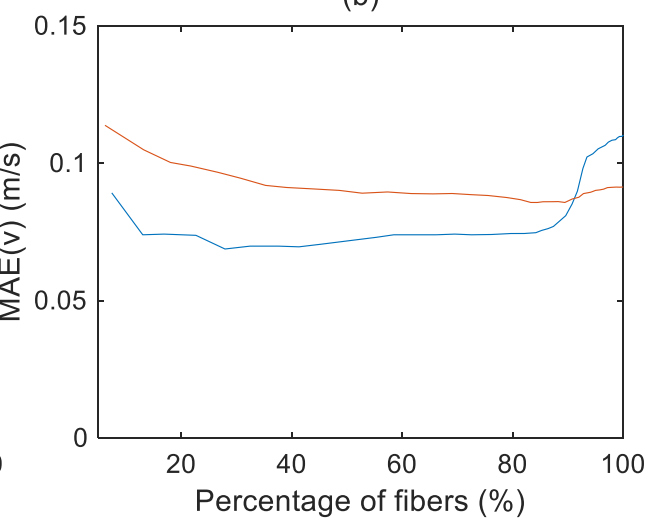

(d)

Fig. 5-22. MAE of the individual estimation errors against the percentage of MU fibers in the evaluation region, obtained for the default (blue) and increased (red) values of the global MU fiber density; (a) MAE of the position $x$; (b) MAE of the position $y$; (c) MAE of the parameter $\zeta$; (d) MAE of the MFCV, $v$. 


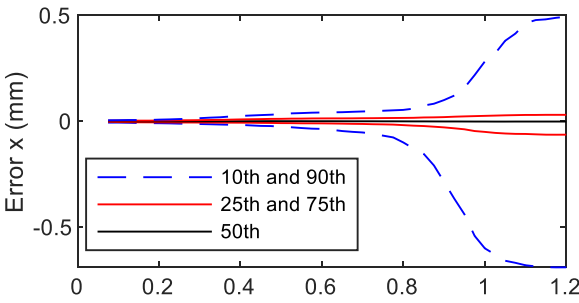

(a)

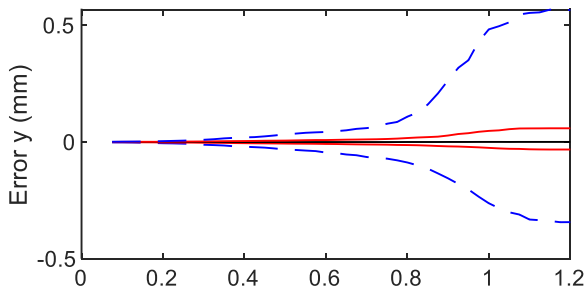

(b)

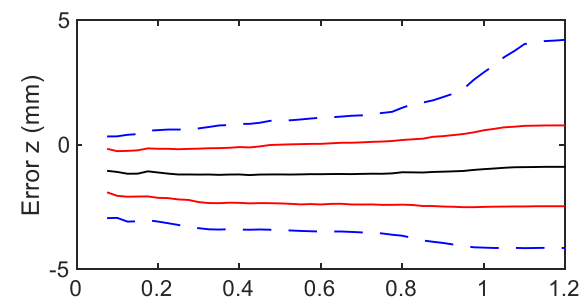

(c)

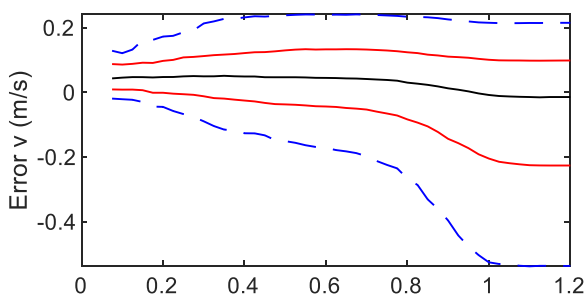

(d)

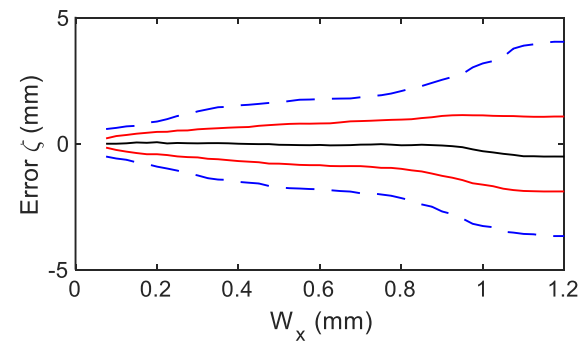

(e)

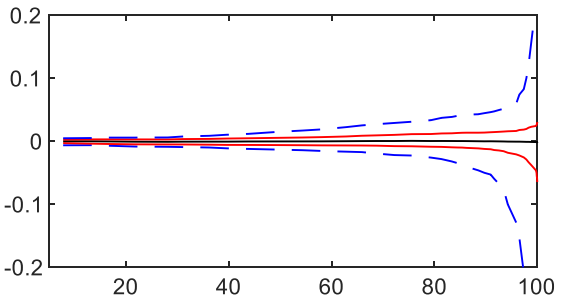

(f)

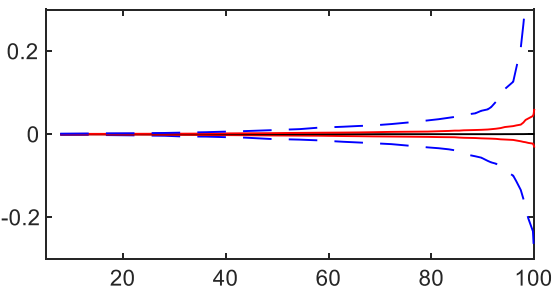

(g)

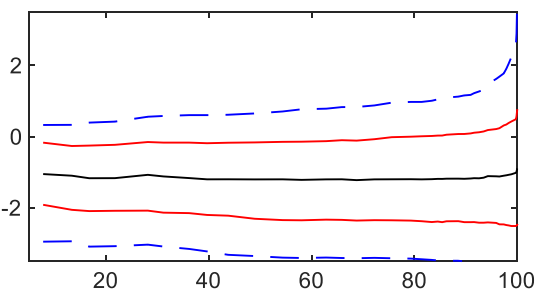

(h)

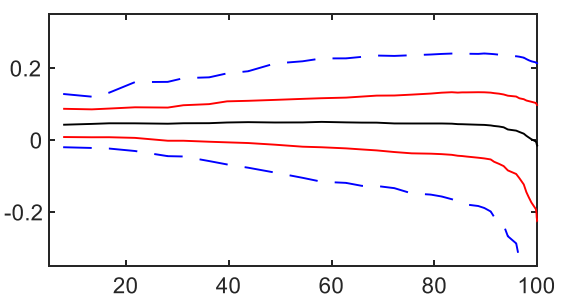

(i)

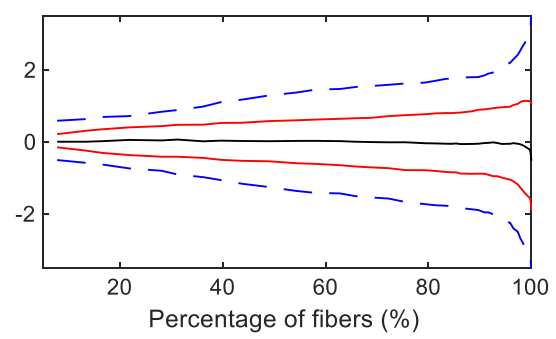

(j) 
Fig. 5-23 [left page]. 10th, 25th, 50th, 75th and 90th percentiles, of the individual estimation errors obtained for the 2-port recording configuration, when the IZ width and the MFCV coefficient of variation have been set with the default values. Error percentiles against the distance to the ports, $W_{x}$ (a-e); (a) estimation error in the position, $x$; (b) estimation error in the position, $y$; (c) estimation error in the neuromuscular junction position, $z$; (d) estimation error in the MFCV, $v$; (e) estimation error in the parameter $\zeta$. Error percentiles against the percentage of MU fibers evaluated (f-j); (f) estimation error in the position $x$; $(\mathrm{g})$ estimation error in the position $y$; (h) estimation error in the neuromuscular junction position, $z$; (i) estimation error in the MFCV, $v$; (j) estimation error in $\zeta$.

distances respect to the port corridors there is a clear lack of fibers in the solution (Fig. 5-27 (c)). This behavior has been observed in many of the experiment realizations executed with increased fiber density.

Regarding the experiments changing the IZ width and the MFCV coefficient of variation, a similar MAE is obtained in the three cases studied (Fig. 5-26), i.e., for the default global parameter values, for the increased IZ width, and for the increased MFCV coefficient of variation. This indicates that a good estimation quality can also be achieved also in MUs with an abnormally high IZ width, or an abnormally high variability of the MFCV's. A summary of the estimation performance for the three studied scenarios is shown in Table 5-7.

\section{Studying the error in the estimated global MU parameters}

Results

Fig. 5-28 shows the percentile errors of the estimated global MU parameters respect to the percentage of the MU fibers covered by the evaluation regions, for the 2-port recording configuration, under two different scenarios: default and increased value of the theoretical MU fiber density parameters ( 8 and 16 fibers per $\mathrm{mm}^{2}$, respectively).

Fig. 5-29 shows the estimation error of the estimated global MU parameters, for the 2-port recording configuration, under three different scenarios: default values for the theoretical overall IZ width and MFCV coefficient of variation $(10 \mathrm{~mm}$ and 0.03 , respectively); increased IZ width to $20 \mathrm{~mm}$; and MFCV coefficient of variation increased to 0.06 .

\section{Discussion}

The ability to estimate the global parameters of the MU, under both default and increased values of the global MU parameters is now discussed. Regarding the estimation of the global MU fiber density, it is of special interest to compare the following cases: default values of the actual global MU parameters (Fig. 5-29 (a)); 


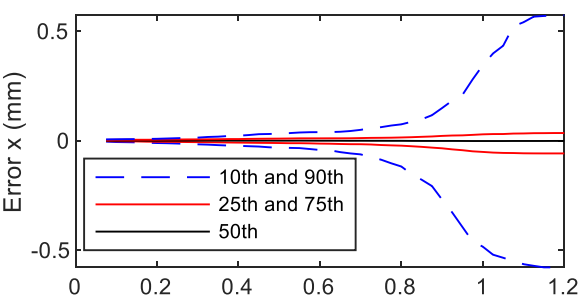

(a)

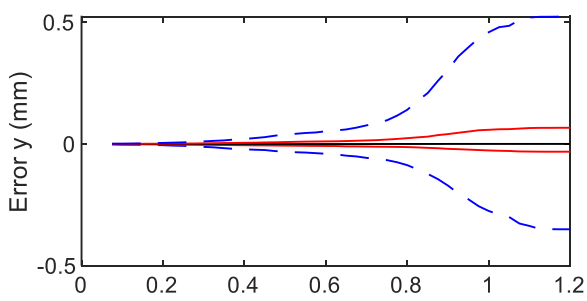

(b)

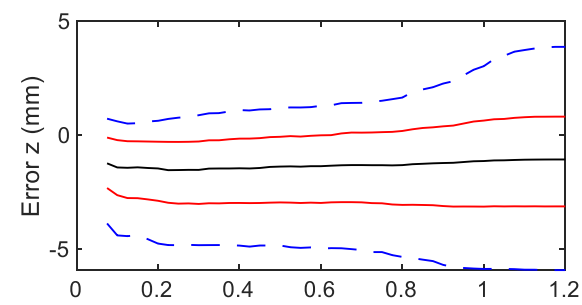

(c)

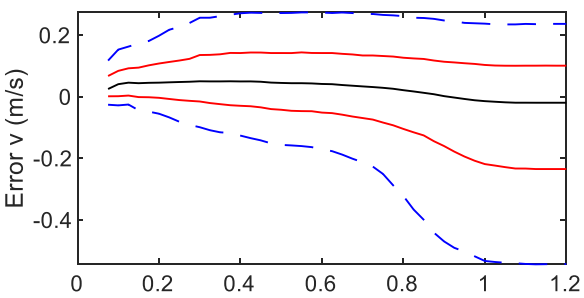

(d)

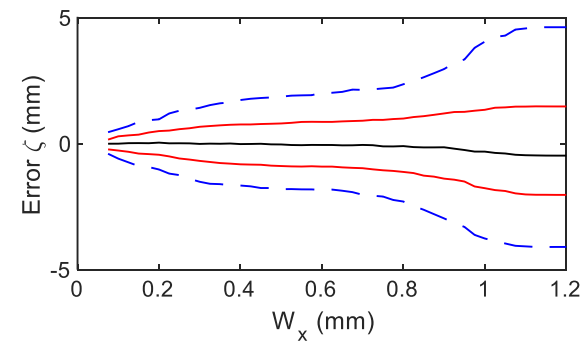

(e)

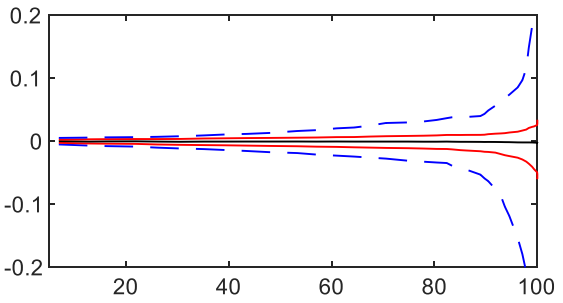

(f)

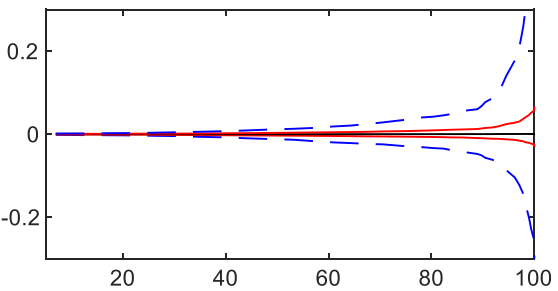

(g)

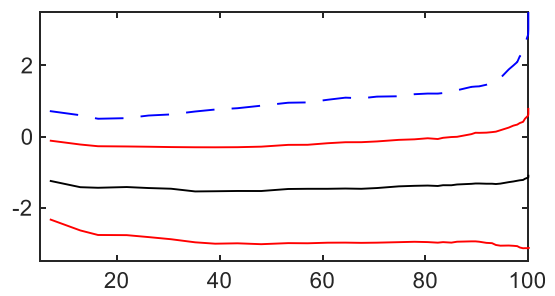

(h)

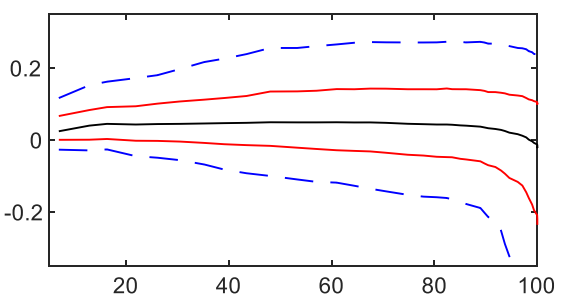

(i)

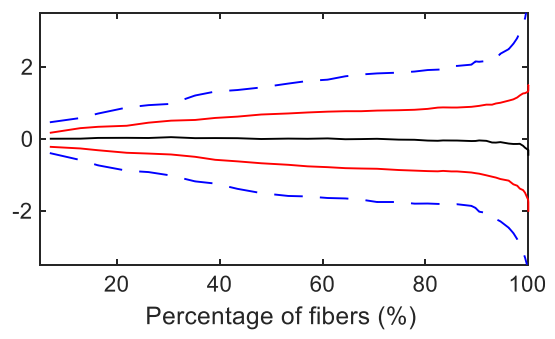

(j) 
Fig. 5-24 [left page]. 10th, 25th, 50th, 75th and 90th percentiles, of the individual estimation errors obtained for the 2-port recording configuration, when the IZ width has been increased. Error percentiles against the distance to the ports, $W_{x}$ (a-e); (a) estimation error in the position, $x$; (b) estimation error in the position, $y$; (c) estimation error in the neuromuscular junction position, $z$; (d) estimation error in the MFCV, $v$; (e) estimation error in the parameter $\zeta$. Error percentiles against the percentage of MU fibers evaluated (f-j); (f) estimation error in the position $x$; $(\mathrm{g})$ estimation error in the position $y$; (h) estimation error in the neuromuscular junction position, $z$; (i) estimation error in the MFCV, $v$; (j) estimation error in the parameter $\zeta$.

increased value of the actual fiber density (Fig. 5-28 (b)); and another case with default value of the actual global MU parameters (Fig. 5-28 (a)) but with an increased number of simulated fibers used by the optimization. This value has been increased to deal with possible cases in which the MU has an increased fiber density. It can be observed that for the default global parameters with an increased number of simulated fibers (Fig. 5-28 (a)) $80 \%$ of the estimation errors range between -1.7 fibers $/ \mathrm{mm}^{2}$ to 0.3 fibers $/ \mathrm{mm}^{2}$, for an evaluation region (in which the global MU fiber density is calculated) that covers $50 \%$ of the MU fibers. For the case with increased fiber density (Fig. 5-28 (b)), 80\% of the estimation errors range approximately between -4.2 fibers $/ \mathrm{mm}^{2}$ to -1.7 fibers $/ \mathrm{mm}^{2}$, for the same evaluation region as before. And for the default global parameters without increasing the number of simulated fibers (Fig. 5-29 (a)) $80 \%$ of the estimation errors range approximately between -0.7 fibers $/ \mathrm{mm}^{2}$ to 0.37 fibers $/ \mathrm{mm}^{2}$, again for the same evaluation region as before.

The best performance in the estimation of the global MU fiber density is achieved for the case in which the number of simulated fibers has not been increased. Note that, setting the optimization with an increased number of simulated fibers may also imply an increased difficulty to find the global minimum due the increased dimensionality, and therefore a certain quality decrease in the estimation. Specifically, the estimated positions in the muscle cross-section, $x$ and $y$ (which are used to calculate fiber density), are estimated with lower precision, which in turn implies a more imprecise estimation of the global MU fiber density. For this reason, ideally, if we wanted to achieve the best possible estimation of the MU fiber density, we should set the number of simulated fibers as small as possible. However, this is not always easy, since the suitable choice of the number of simulated fibers will depend on the MU fiber density, which is precisely the unknown parameter that we want to estimate. For this reason, in practice a large number of simulated fibers must be set, so that the estimation procedure can deal also with the cases in which the fiber density is high, i.e., with cases in which there is a high number of fibers actually contributing to the recorded signal. In this regard, although the estimation of the fiber density is somewhat more imprecise when the number of simulated fibers is high (Fig. 5-28 (a) and Fig. 5-28 (b), for the default and the increased fiber density, respectively), the amount of error can be considered acceptable. Note that, for the default fiber density, whose value is 8 


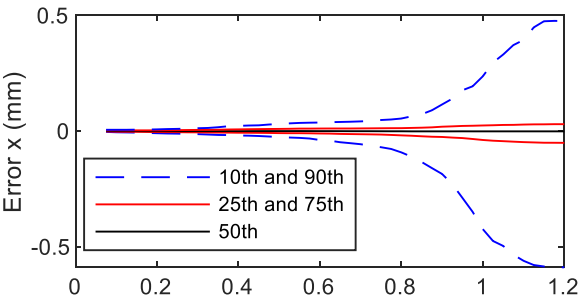

(a)

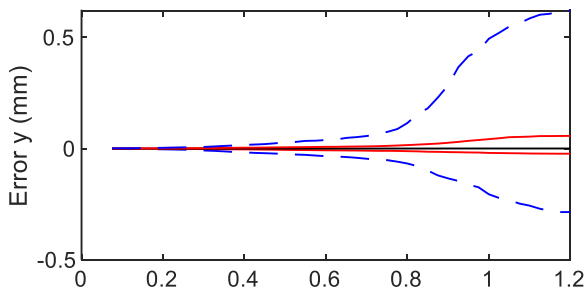

(b)

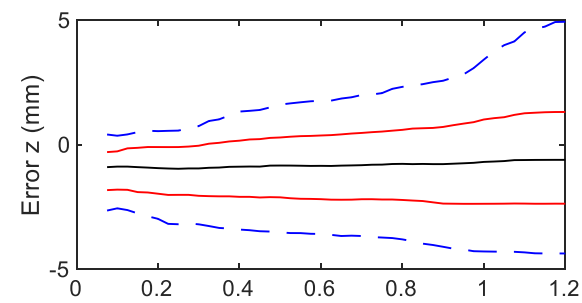

(c)

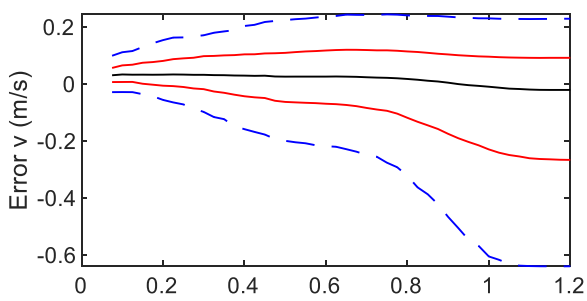

(d)

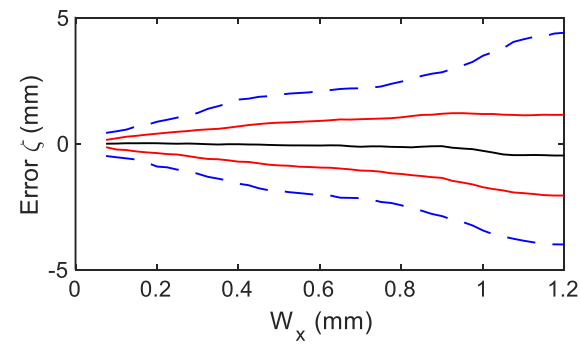

(e)

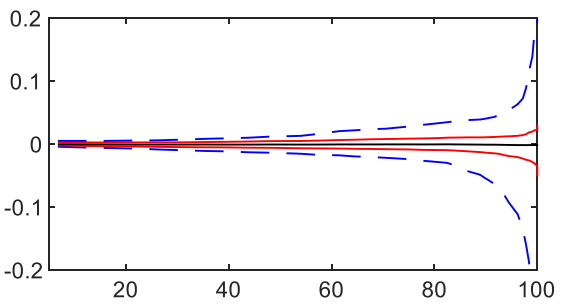

(f)

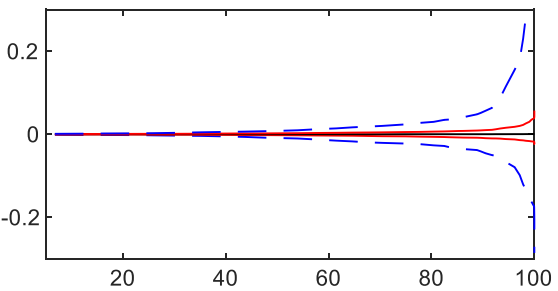

(g)

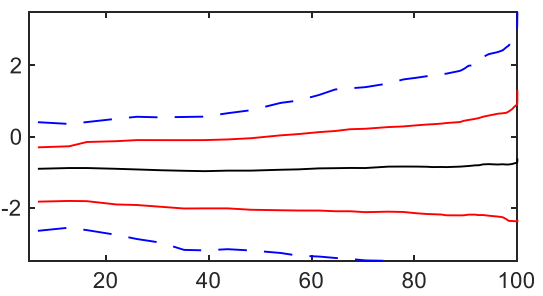

(h)

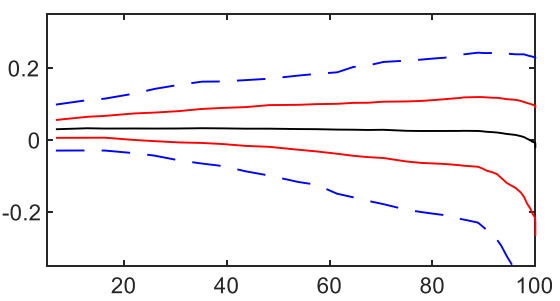

(i)

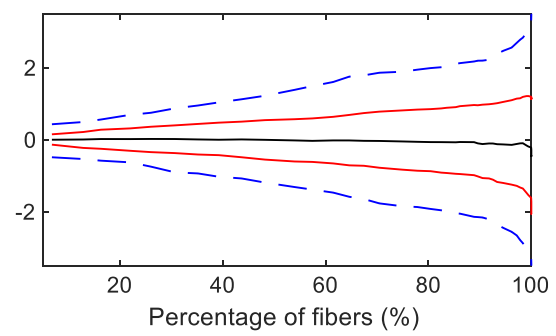

(j) 
Fig. 5-25 [left page]. 10th, 25th, 50th, 75th and 90th percentiles, of the individual estimation errors obtained for the 2-port recording configuration, when the MFCV coefficient of variation has been increased. Error percentiles against the distance to the ports, $W_{x}$ (a-e); (a) estimation error in the position, $x$; (b) estimation error in the position, $y$; (c) estimation error in the neuromuscular junction position, $z$; (d) estimation error in the MFCV, $v$; (e) estimation error in the parameter $\zeta$. Error percentiles against the percentage of MU fibers evaluated (f-j); (f) estimation error in the position $x$; (g) estimation error in the position $y$; (h) estimation error in the neuromuscular junction position, $z$; (i) estimation error in the MFCV, $v$; (j) estimation error in the parameter $\zeta$.
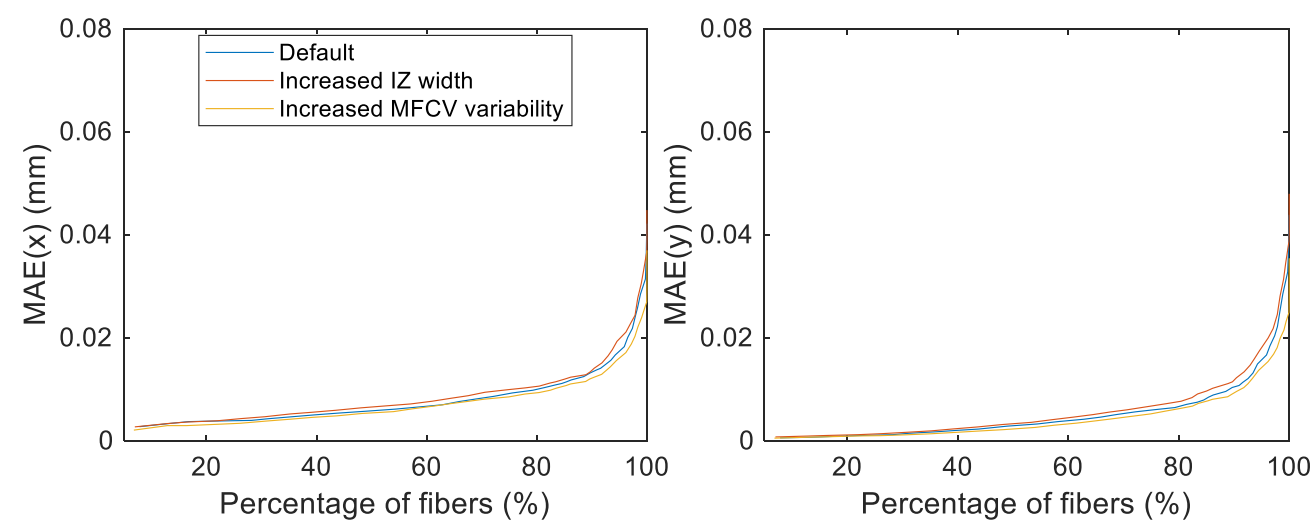

(a)

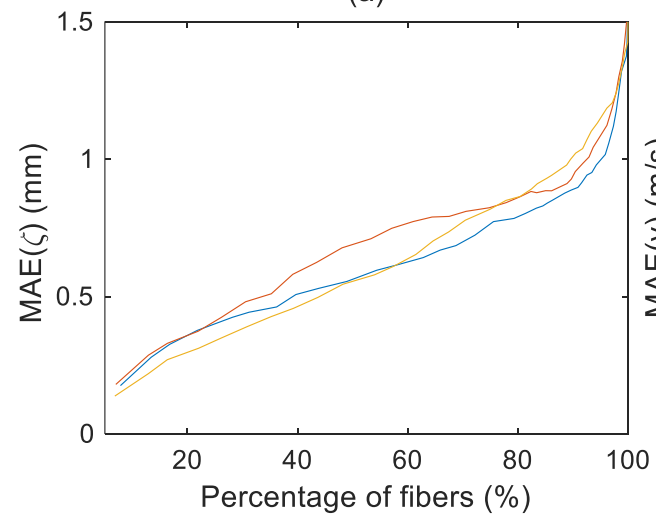

(c) (b)

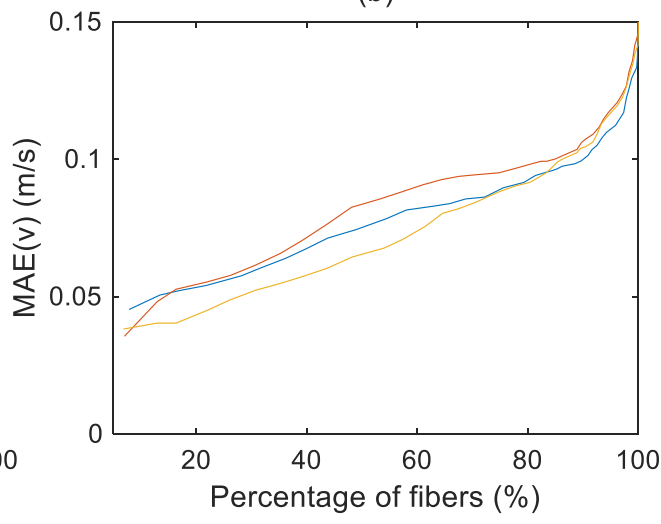

(d)

Fig. 5-26. MAE of the individual estimation errors against the percentage of MU fibers in the valuation region, obtained for different values of the IZ width, and the MFCV coefficient of variation: default values (blue), increased IZ width (red), and increased MFCV coefficient of variation (yellow); (a) MAE of the position $x$; (b) MAE of the position $y$; (c) MAE of the parameter $\zeta$; (d) MAE of the MFCV, $v$. 


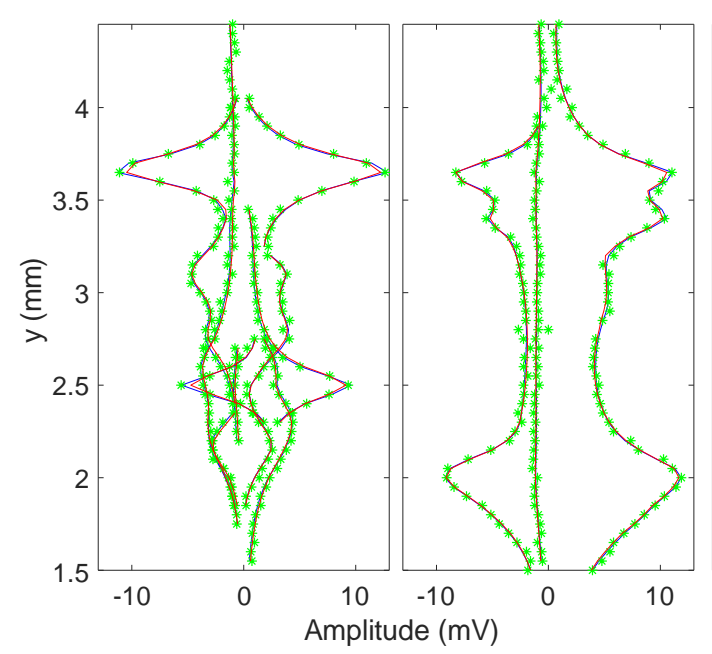

(a)

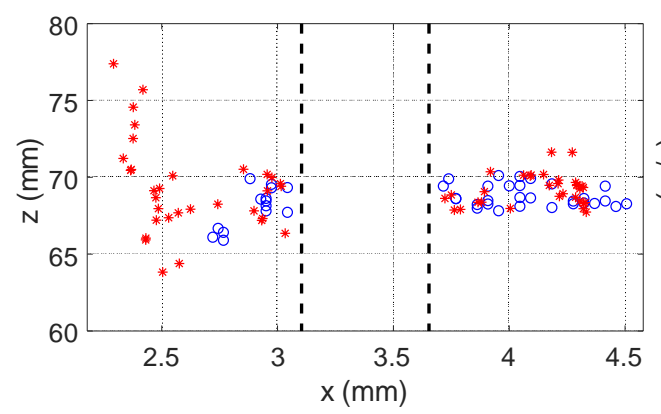

(d)

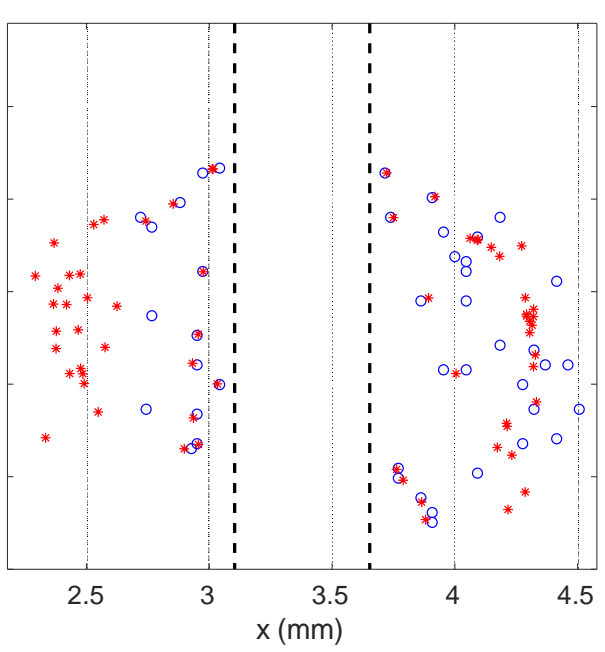

(c)

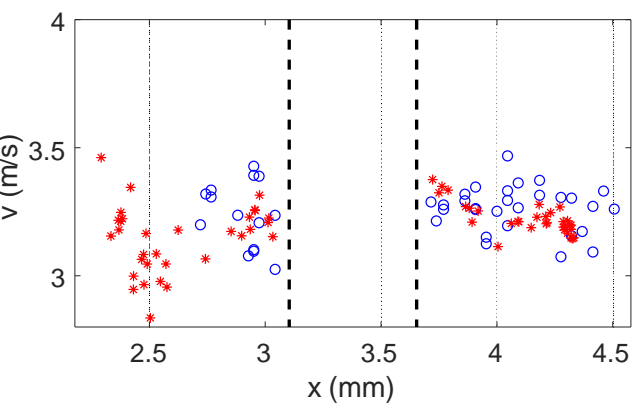

(e)

Fig. 5-27. Estimation results of a single experiment realization, for a scanning-EMG signal recorded with the 2-port recording configuration, when the MU fiber density has been increased: (a) and (b), amplitude of the profile in the spatial dimension of the estimated (red lines), reference (green asterisks), and noise-free reference (blue lines) scanning-EMG signals, where (a) corresponds to the scanning-EMG signal section recorded in the left port, and (b) to that of recorded in the right port; (c) estimated (red asterisks) and actual (blue circles) MU fibers in the $x-y$ plane; (d) estimated (red asterisks) and actual (blue circles) MU fibers in the $\mathrm{x}-\mathrm{z}$ plane; (e) estimated (red asterisks) and actual (blue circles) MU fibers in the $\mathrm{x}-\mathrm{v}$ plane. The two vertical dashed lines represent the corridors of the two ports of the needle.

Fig. 5-28 [left page]. 10th, 25th, 50th, 75th and 90th percentiles of the errors in the estimation of the global MU parameters vs the percentage of MU fibers of evaluated, for two different values of the global fiber density: default value, and increased value. From the top to the bottom: fiber density estimation error $e_{D}$; IZ excess, $E_{I}$; IZ defect, $D_{I}$; MFCV mean, $e_{V m}$; and MFCV standard deviation, $e_{V s}$. 


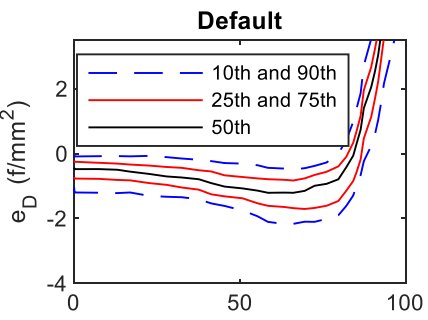

(a)

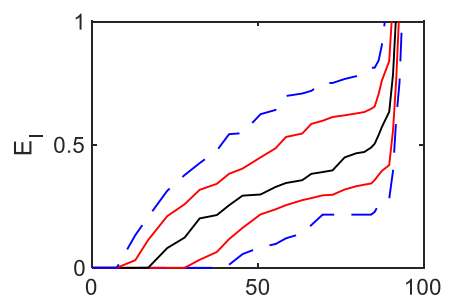

(c)

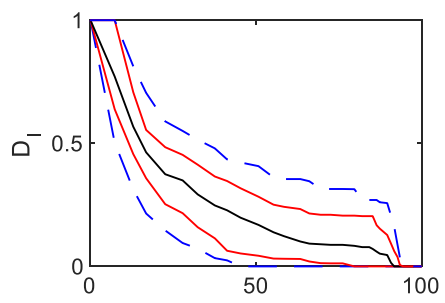

(e)

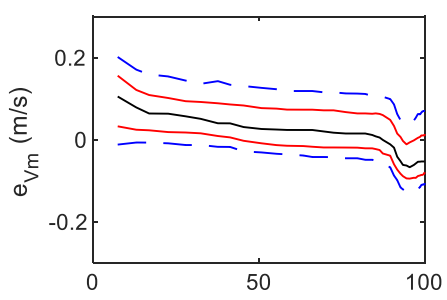

(g)

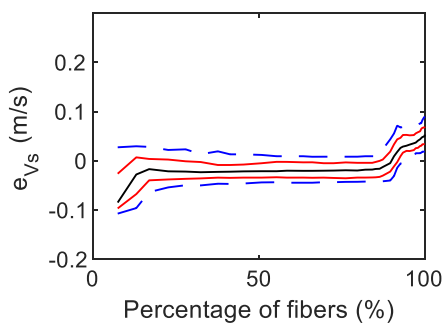

(i)

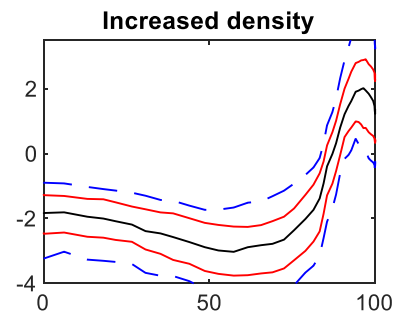

(b)

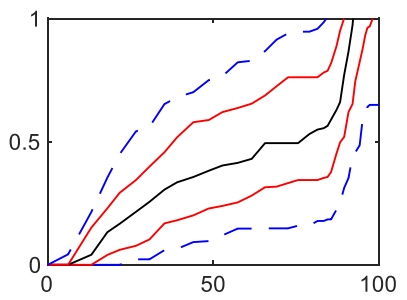

(d)

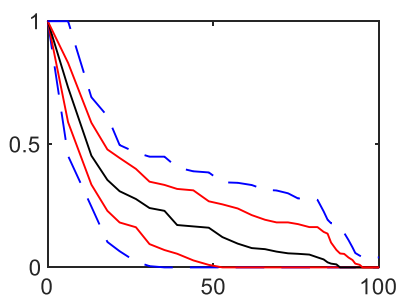

(f)

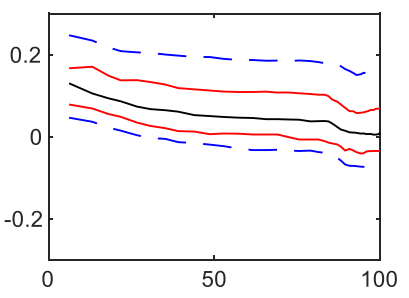

(h)

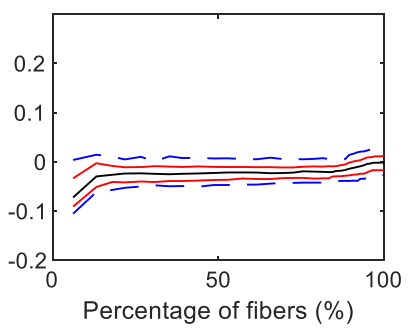

(j) 


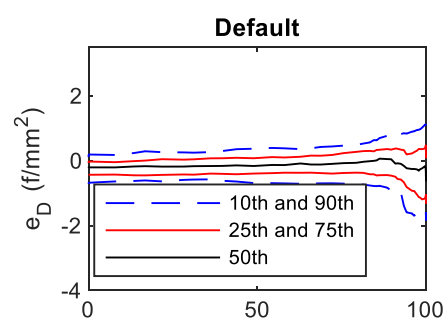

(a)

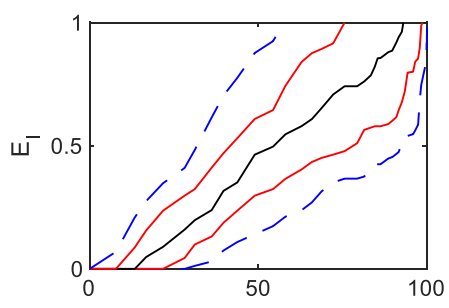

(d)

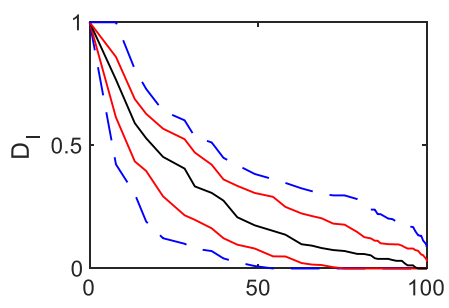

(g)

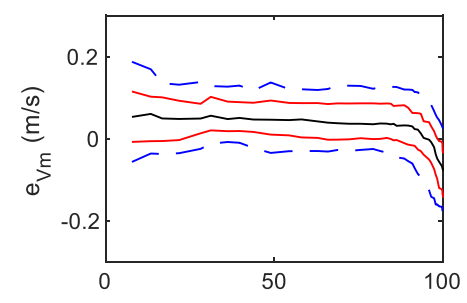

(j)

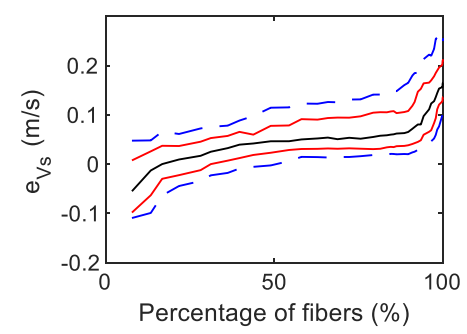

(m)

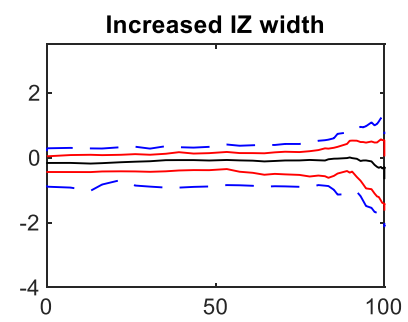

(b)

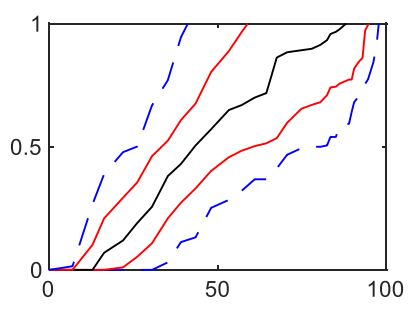

(e)

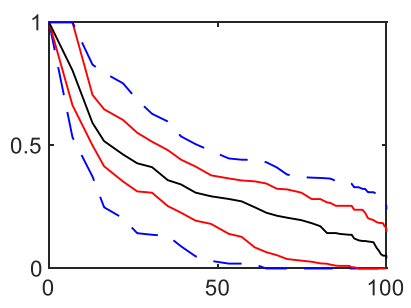

(h)

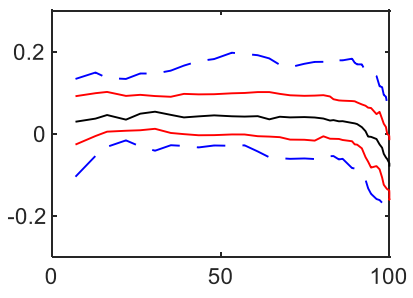

(k)

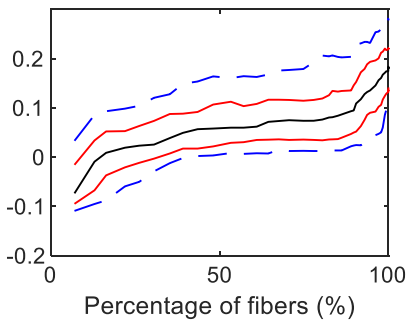

(n)

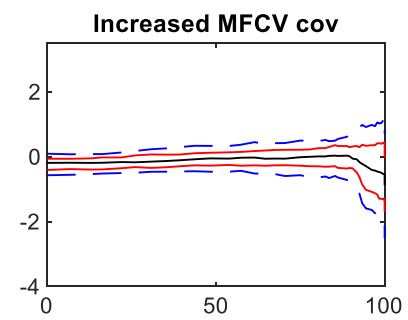

(c)

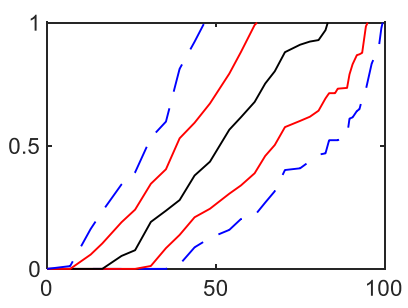

(f)

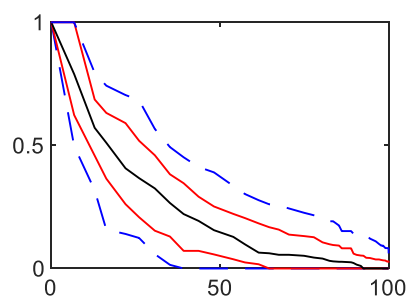

(i)

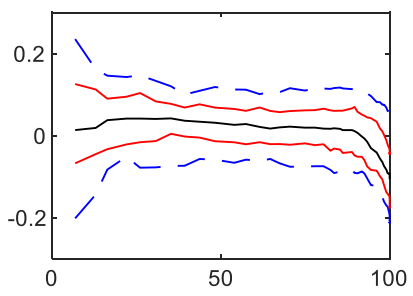

(I)

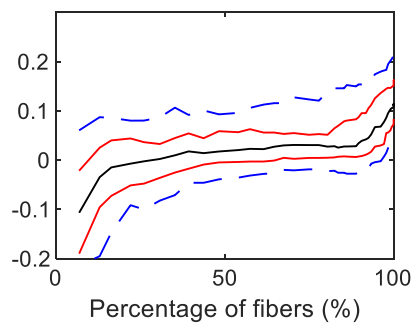

(o) 
Fig. 5-29 [left page]. 10th, 25th, 50th, 75th and 90th percentiles of the errors in the estimation of the global MU parameters vs the percentage of MU fibers of evaluated, for three different scenarios: default values for the IZ width and MFCV coefficient of variation, increased IZ width, and increased MFCV coefficient. From the top to the bottom: fiber density estimation error $e_{D}$; IZ excess, $E_{I}$; IZ defect, $D_{I}$; MFCV mean, $e_{V m}$; and MFCV standard deviation, $e_{V s}$.

fibers $/ \mathrm{mm}^{2}$, the error ranges between -1.7 and 0.3 fibers $/ \mathrm{mm}^{2}$, and for the increased fiber density, whose value is 16 fibers $/ \mathrm{mm}^{2}$, the error ranges between -4.2 and 0.7 fibers $/ \mathrm{mm}^{2}$. This implies that, even with a certain bias, fiber density can be estimated reasonably well. This is not surprising if we take into account that the positions in the muscle cross-section, $x$ and $y$ are estimated correctly, at least in a certain region close to the scanning needle (Fig. 5-20 (a) (b) (f) and (g), and Fig. 5-21 (a) (b) (f) and (g)).

An important consideration that has to be made regarding the estimation of the global MU fiber density, is that there is a trade-off when choosing the suitable size of the region in which the fiber density is calculated. On the one hand, it should not be very large, so as not to include distant fibers in the calculation, whose estimation is most likely going to be erroneous. But, on the other hand, it is important that it covers the larger proportion of the MU as possible, so as to give an accurate measure of the actual MU fiber density.

In conclusion, the method proposed to calculate the global MU fiber density from the estimated MU fiber parameters is working properly, so its possible practical application can be very interesting from a diagnostic point of view. It has two main advantages respect to the electrophysiological measure of the fiber density, routinely used in clinical neurophysiology $[115,39,40]$ (see sections 1.2.1 and 1.3.2). The first is that the estimated fiber density proposed here provides a more precise approximation to the actual fiber density of the MU, as its calculation parts from a more exhaustive identification of the individual MU fibers. And the second is that the proposed estimation is specific to a single MU, unlike the electrophysiological measure of the fiber density, which gives an average result of several MUs of the same muscle.

Regarding the estimation of the IZ sub-bands (Fig. 5-28 (c-f), and Fig. 5-29 (d-i)), the larger the region chosen to calculate it, the lower the defect, and the larger the excess of the estimated sub-bands. This means that increasing the size of the evaluation region implies, on one hand, that there is a lower proportion of the IZ sub-bands that has not been identified as such; and on the other hand, that there is a larger proportion of the IZ that has been erroneously detected as part of the sub-bands. This is even more evident if we take into account that larger evaluation regions involve considering fibers further from the scanning needle, for which the corresponding estimation error is higher. Therefore, it is difficult to choose an optimal region size to estimate the subbands, as there is a compromise relationship between subestimating and overestimating the sub-bands. For a region covering $40 \%$ of the fibers, in the case corresponding to the default global MU parameters, the median excess is 0.32 (Fig. 
5-29 (d)), and the median defect is 0.27 (Fig. 5-29 (g)), which means that the IZ subbans are estimated with a considerable error (note that the excess and defect are merit figures normalized to the IZ sub-band size). By increasing the width of the overall IZ for the same evaluation region, the error gets higher: the median excess is 0.45 (Fig. 5-29 (e)), and the median defect 0.33 (Fig. 5-29 (h)). Not surprisingly, the estimation sub-bands are estimated with a notable error, taking into account that the neuromuscular junction position, $z$, which is the fiber parameter used for the calculation of the sub-bands, has resulted to be estimated with a fairly notable error, even without considering the bias for the analysis (parameter $\zeta$ ).

Regarding the estimation of the MFCV mean (Fig. 5-29 (j-1)), the dispersion ranges of the estimation error present similar values for the different sizes of the evaluation regions. Keep in mind that here come into play two opposing effects. On the one hand, there is a tendency of the error to increase as larger regions are considered, as they include farther fibers, with higher estimation errors. But, on the other hand, the more fibers considered the larger the sample size used to compute the mean, which makes the error dispersion to decrease. Note that, when it comes to compute the error in the MFCV mean, the reference with which the estimated MFCV mean is compared is the one calculated from the overall MU. According to the results, these two effects seem to be compensated. In general, the estimation of the MFCV mean is precise for both the default global MU parameters and for case in which the MFCV coefficient of variation has been increased. Note that, for an evaluation region covering $40 \%$ of the MU fibers, $80 \%$ of the errors range between $-0.01 \mathrm{~m} / \mathrm{s}$ and 0.13 $\mathrm{m} / \mathrm{s}$ for the default case (Fig. $5-29(\mathrm{j})$ ), and between $-0.07 \mathrm{~m} / \mathrm{s}$ and $0.1 \mathrm{~m} / \mathrm{s}$ for the increased case (Fig. 5-29 (1)). The mean MFCV of the MUs generated in the experiments is $3.33 \mathrm{~m} / \mathrm{s}$. Thus, the dispersion of the errors obtained is small, compared to that value. Therefore, the results seem to indicate that the average MFCV is a parameter that can be estimated correctly.

Regarding the estimation of the MFCV standard deviation (Fig. 5-29 (m-o)), the same two opposing effects are present. The best estimation results are obtained for regions covering intermediate MU fiber percentages, where there are enough fibers so that the sample size is adequate, and where the fibers used in the calculation have estimation errors that are not excessively high. Note that, for an evaluation region covering $50 \%$ of the MU fibers, $80 \%$ of the errors range between $0 \mathrm{~m} / \mathrm{s}$ and $0.11 \mathrm{~m} / \mathrm{s}$ for the default case (Fig. $5-29(\mathrm{~m})$ ), and between $-0.04 \mathrm{~m} / \mathrm{s}$ and $0.09 \mathrm{~m} / \mathrm{s}$ for the increased case (Fig. 5-29 (o)). The error ranges in the estimation of the MFCV standard deviation are large, if we take into account that the actual value of the standard deviation of the MUs generated in the experiments is, on average, of $0.1 \mathrm{~m} / \mathrm{s}$ for the default case, and of $0.2 \mathrm{~m} / \mathrm{s}$ for the increased case. It can be concluded that the MFCV standard deviation is estimated with limited accuracy. Remembering that a high variability of the diameters of the MU fibers may be indicative of pathology. MFCV standard deviation has a considerable diagnostic usefulness, as it is directly related to the variability of the MFCV's, and thus also directly related to the variability of the MU fiber diameters. 


\subsection{Testing the estimation system with real EMG signals}

The evaluation of the estimation system is based in results obtained from the decomposition of synthetic signals; i.e., it is developed in a completely simulated environment. In this way, exact information about the actual MU fiber parameters is available, which allows to objectively quantify the estimation quality. Since the values of these parameters are not available when dealing with real muscles, it is not possible to develop an objective evaluation of the performance of the system based on real signals. Even so, the estimation system has been applied to signals recorded from real muscles, in order to have a few estimation cases as examples. This allows to briefly analyze two important aspects. On the one hand, we can observe to what extent the simulated signals can reproduce the waveform of the real signals. On the other hand, we can check whether or not the estimated parameters are consistent with the expected parameter ranges, given the prior physiological knowledge available.

\subsubsection{Experiment set up}

The procedure applied to test the estimation system in real muscles consisted in the following steps:

1. Recording of the scanning-EMG signals. A set of 20 scanning-EMG signals were recorded from the biceps brachii of five normal subjects ( 3 male and 2 female) aged between 24 and 54 years. Informed consent was obtained from all subjects, and the study was approved by the ethics committee of Public University of Navarra. The scanning-EMG recordings were obtained following the recording protocol described in [117], with sampling frequency of $20 \mathrm{kHz}$ and scanning steps of $50 \mu \mathrm{m}$. A standard SF electrode was used as scanning electrode, and a facial concentric needle was used as trigger electrode. The scanning electrode was inserted $30 \mathrm{~mm}$ away from the trigger electrode along the longitudinal axis of the muscle. Note that the recording configuration that has been used for these recordings is the 1-port scanning-EMG configuration.

2. Processing of the scanning-EMG signals. The processing scheme described in section 4.2.4 is applied to each recorded scanning-EMG signal to reduce the effect of noise.

3. Estimation of the MU fiber parameters. The estimation system has been applied to each scanning-EMG signal to obtain the MU fiber parameters.

It should be noted that the procedure presents an important difference with respect to the experiments carried out in simulation: no surface-EMG signal has been recorded. This is so because, at the moment of the recordings, the experimental equipment was only available for the recording of the scanning-EMG signal, and not for that of the surface-EMG signal. Note that the objective of the thesis was the design of the 
estimation system, that is, of the algorithmic solution to the problem at hand. The setup of the experimental equipment for the application of the system in real muscles was out of the scope of the thesis objectives.

Not recording the surface-EMG signal has important implications that must be taken into account. First of all, remember that the estimation system uses the surfaceEMG signal to estimate the potential initiation instant (see section 4.1). It is not possible to estimate this parameter without recording the surface-EMG signal. In turn, not knowing the potential initiation instant implies that the positions of neuromuscular junctions, $z$, of the MU fibers also remain unknown. A detailed rationale can be found in section 4.1.2. Broadly speaking, the reason is that the values of the $z$ parameters exclusively affect to the propagation delay of the generated SFAP contributions, and not to their shape (as long as the electrode is at a certain distance from the neuromuscular junction, something that in practice always occurs). This means that the estimation procedure can only use the information about the temporal delay of the signal to estimate the values of the $z$ parameters. The problem is that, as long as the potential initiation instant is unknown, so is the delay, and therefore the $z$ parameters are also undetermined. Regarding the rest of fiber parameters, that is, the position $x$ and $y$, and the MFCV $v$ of the MU fibers, they have influence in the shape of the generated SFAPs. So, these parameters can be estimated, at least theoretically, even if the potential initiation instant remains unknown. Keeping in mind these considerations, in the tests performed in this section, only the parameters $x, y$ and $v$, are estimated, disregarding the estimation of the parameter $z$.

The estimation system also uses the recorded surface-EMG signal to estimate the average MFCV of the MU, $V_{M U}$. Since this signal is not available, this parameter cannot be estimated either. Remember that $V_{M U}$ is used to establish the bounds of the search region, $v_{L}$ and $v_{H}$, in which the optimization procedure operates to find the values of the $v$ parameters (given by (3-31)). As in the tests of this section $V_{M U}$ cannot be estimated, the bounds $v_{L}$ and $v_{H}$ are configured so that they cover an interval wide enough as to include the entire range of MFCV values physiologically plausible. These bounds are $v_{L}=2.2 \mathrm{~m} / \mathrm{s}$ and $v_{H}=5.8 \mathrm{~m} / \mathrm{s}$.

\subsubsection{Results and discussion}

The results of the estimation applied to real recorded scanning-EMG signals are shown for three particular cases (selected from all the recorded signals). These three cases are shown in Fig. 5-30, Fig. 5-31, and Fig. 5-32, respectively. Note that in general, the estimated signal waveform has been adjusted to the recorded signal with a relative success. For instance, if we observe the case of Fig. 5-30, it can be noticed that the crests and valleys of the estimated and reference signals match spatiotemporarily (Fig. 5-30 (c) and (d)). However, there are significant differences in the amplitude profile of such crests and valleys between both signals (Fig. 5-30 (e)). This behavior also occurs for the other two presented cases, as well as for most of the 
estimations made with real scanning-EMG signals. Remember that this was not the case for the experiments performed with simulated signals, for which the estimated signal was fitted to the recorded one with outstanding precision.

This imprecise waveform adjustment may be due to different factors. One of these factors may be that, in the present experiments with real recorded signals, it can be more difficult for the optimization algorithm to reach the global minimum than in the

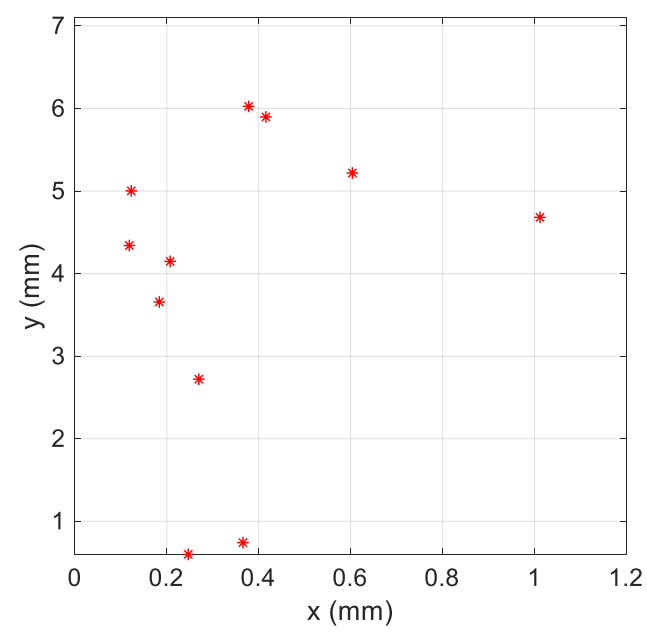

(a)

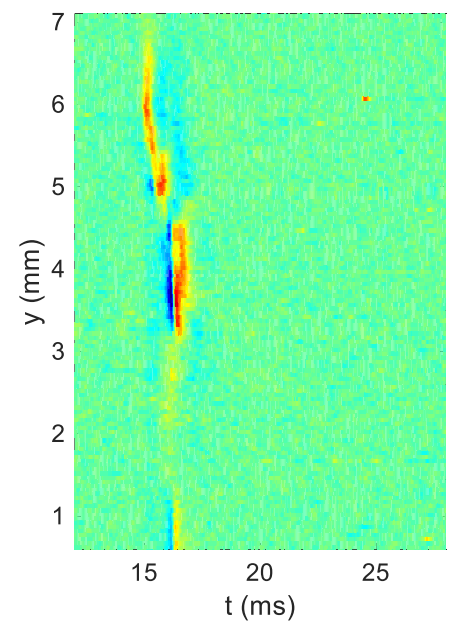

(c)

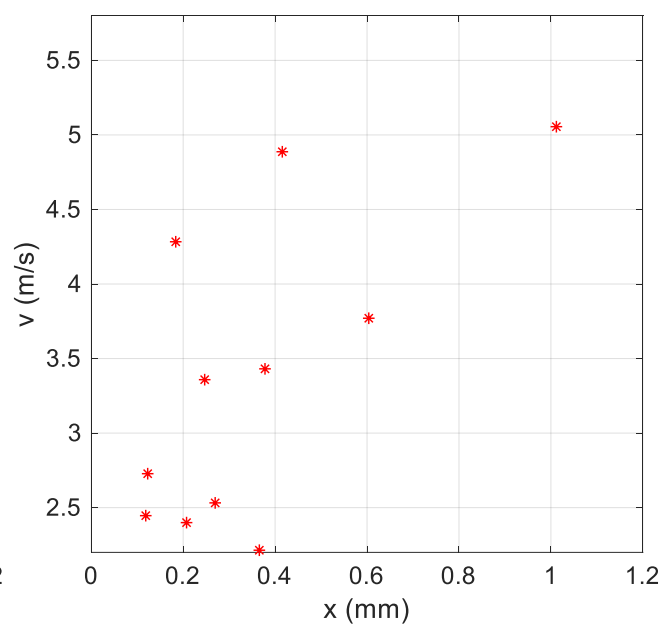

(b)

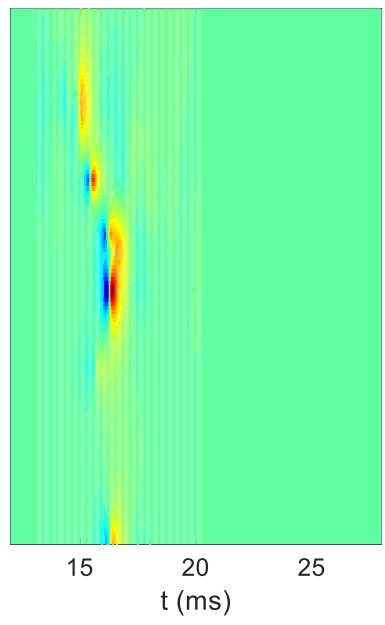

(d)

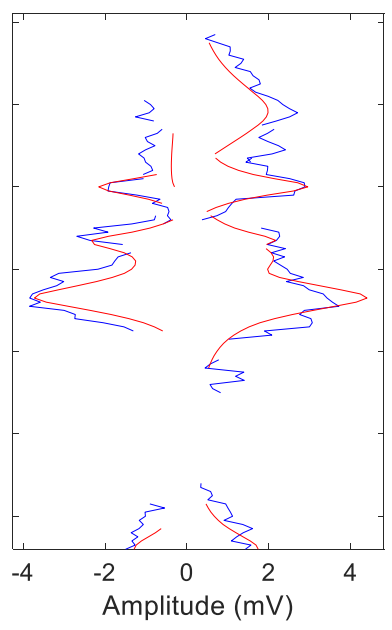

(e)

Fig. 5-30. Estimation results for a real recorded scanning-EMG signal; (a) estimated MU fibers (red asterisks) in the X-y plane; (b) estimated MU fibers (red asterisks) in the X-V plane; (c) color map representing the recorded (reference) scanning-EMG signal; (d) color map representing the estimated scanning-EMG signal; (e) amplitude of the MU profile in the spatial dimension of the estimated (red) and reference (blue) scanning-EMG signals. 
simulation experiments. In this regard, it may be also determinant that a much larger search interval has been used for the MFCV's. On the other hand, it does not seem that this is the main reason to explain the mismatch in the waveform fitting, since the experiments with simulated signals suggested that an increase in the range of this interval does not imply a significant degradation of the quality of the estimation, and hence neither a worse waveform fitting.

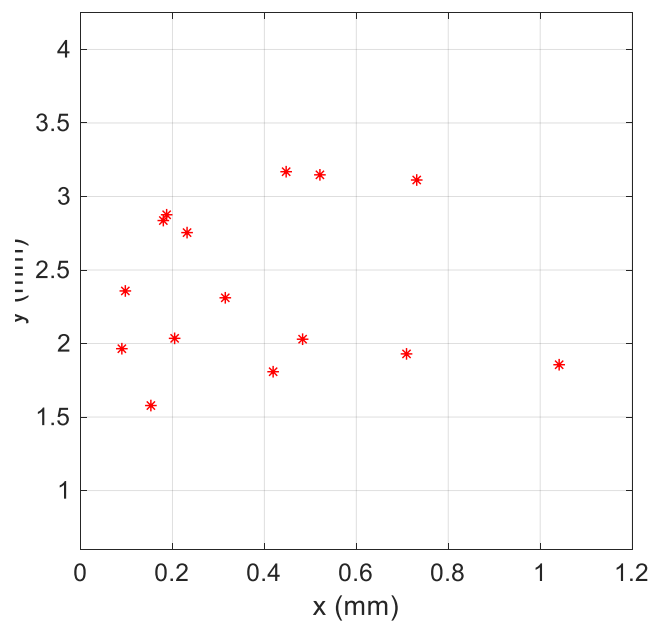

(a)

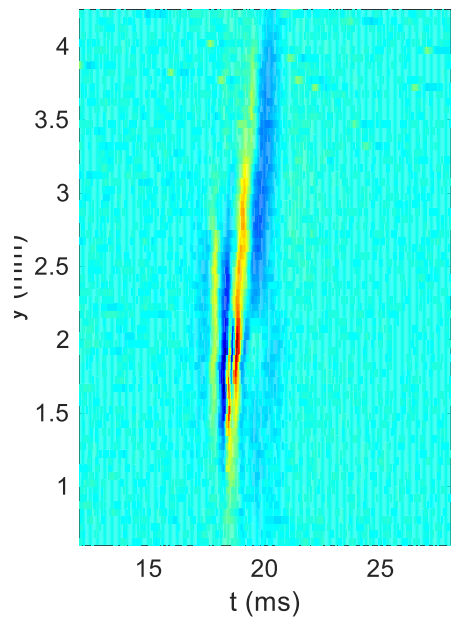

(c)

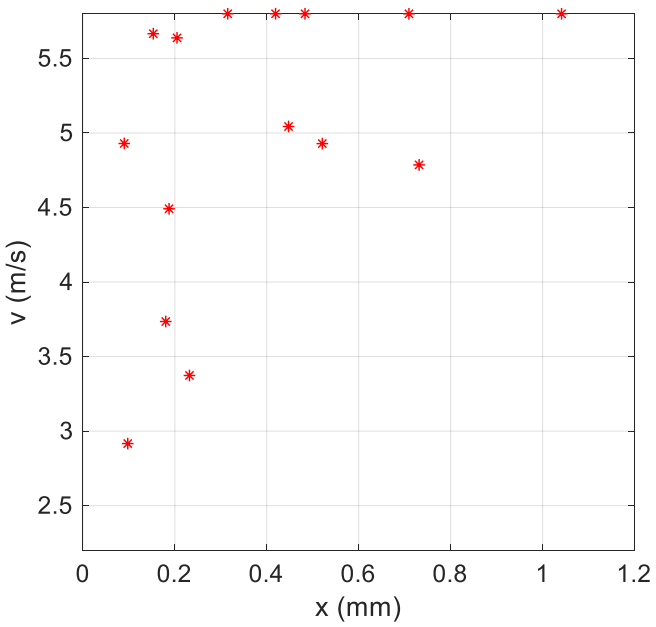

(b)

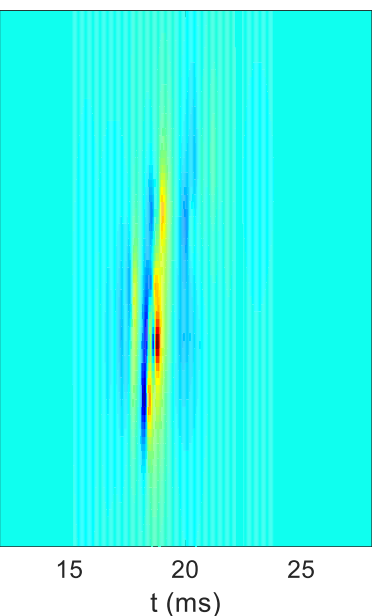

(d)

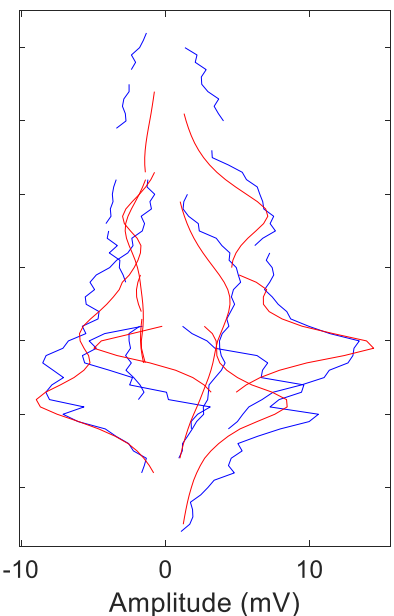

(e)

Fig. 5-31. Estimation results for a real recorded scanning-EMG signal; (a) estimated MU fibers (red asterisks) in the x-y plane; (b) estimated MU fibers (red asterisks) in the X-v plane; (c) color map plot representing the recorded (reference) scanning-EMG signal; (d) color map plot representing the estimated scanning-EMG signal; (e) amplitude of the MU profile in the spatial dimension of the estimated (red) and reference (blue) scanning-EMG signals. 
Another important factor that may negatively influence in the waveform fitting is the poor signal-noise-ratio of the recorded scanning-EMG signals. Note that the equipment used to record the signals is set to record a single discharge at each recording position, while in the simulated experiments the signals are recorded with five discharges per recording position. It will be necessary to fine-tune the experimental equipment, so that in future experiments more than one discharge per position can be recorded. New recording techniques as the multi-scanning [136, 92,

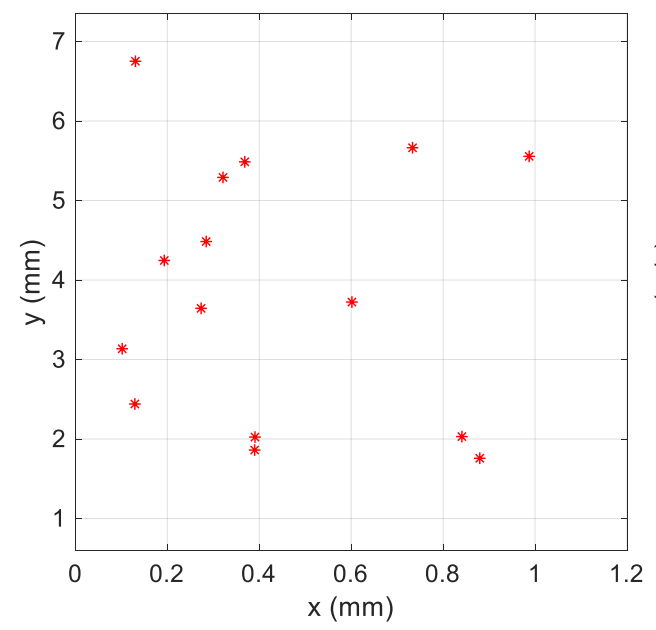

(a)

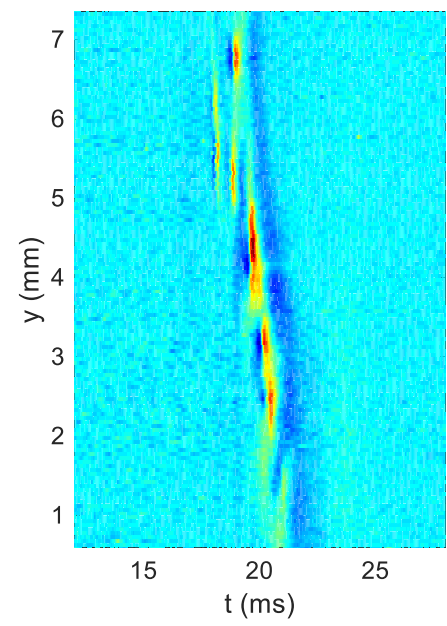

(c)

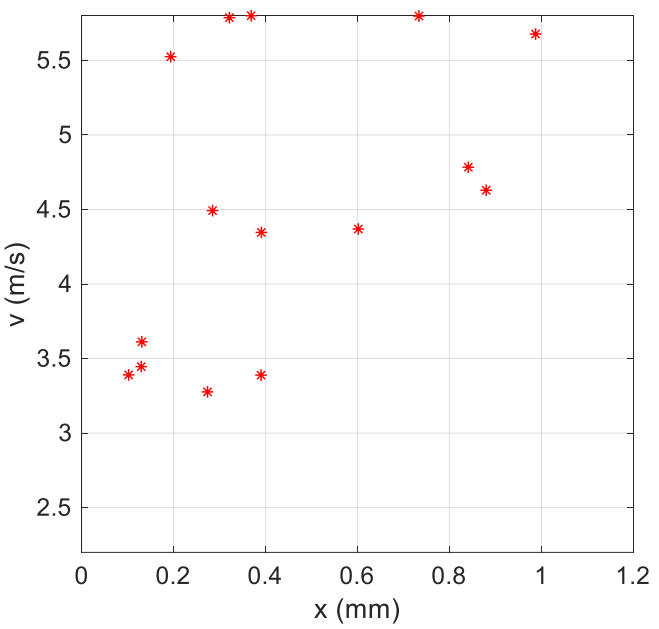

(b)

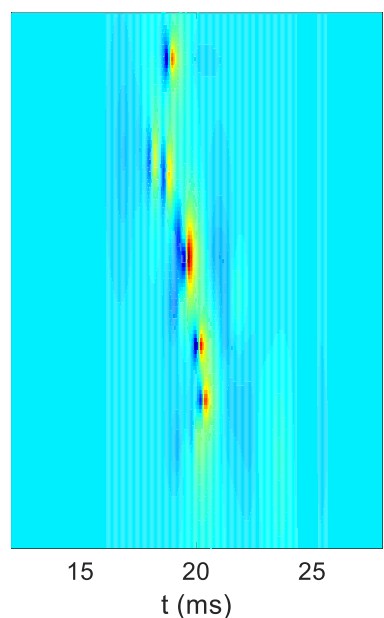

(d)

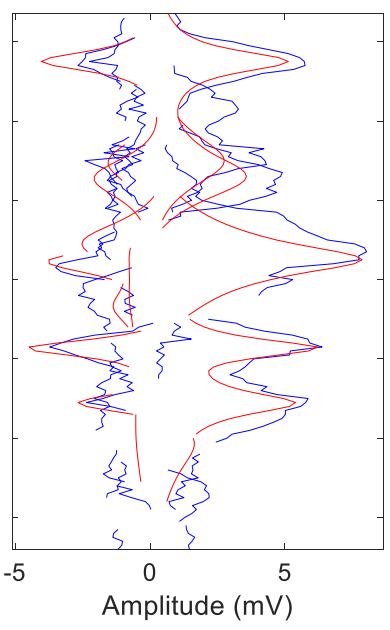

(e)

Fig. 5-32. Estimation results for a real recorded scanning-EMG signal; (a) estimated MU fibers (red asterisks) in the X-y plane; (b) estimated MU fibers (red asterisks) in the X-v plane; (c) color map representing the recorded (reference) scanning-EMG signal; (d) color map representing the estimated scanning-EMG signal; (e) amplitude of the MU profile in the spatial dimension of the estimated (red) and reference (blue) scanning-EMG signals. 
60] may indirectly solve this lack of discharges per position.

Another factor that can contribute to the inaccuracy of the waveform fitting are possible discrepancies between the simulation model used in the estimation, and reality. That is, when generating the electric potential in real muscles there can be many effects that significantly influence the signal waveform, and that are not taken into account by the model. If this was the case, it could be necessary to improve the simulation model so that it incorporates the most relevant of these effects.

Regarding the estimated parameters, as already mentioned, it is not easy to establish to what extent the estimation is being correct, as the actual MU fiber parameters are not available. If we observe the dispersion of the estimated MFCV's (Fig. 5-30 (b), Fig. 5-31 (b) and Fig. 5-32 (b)), it seems to be large compared to the expected dispersion for a normal MU; for instance, in [87] it was concluded that the standard deviation of the MFCV's in the biceps brachii muscle must be about $0.15 \mathrm{~m} / \mathrm{s}$. This may be indicative that the estimation is not being accurate, which would make sense if we consider that the waveform is adjusting with certain degree of inaccuracy.

In short, in order to apply the estimation system in a real environment, it would be necessary to carry out more exhaustive experiments to analyze the causes by which the waveform is not being adjusted as accurately as in a simulated environment, and to try improve this adjustment. Prior to that, the recording system should be completed, so that it includes the recording of the surface-EMG signal, and that it is able to record several discharges at each scanning position. Taking these aspects into account, the margin of improvement for the waveform fitting is still considerably high. 


\subsection{General discussion}

In this thesis, a complete system that estimates the parameters of the fibers of the MU from EMG signals has been developed. To achieve this, the system applies an inverse MUP modeling to the recorded scanning-EMG signal. The parameters that the system estimates are, for each MU fiber, its position in the muscle cross-section along the $\mathrm{x}$ - and $\mathrm{y}$-axis, $x$ and $y$; its neuromuscular junction position, $z$; and its MFCV, $v$. Furthermore, as there is a strong correlation (which is considered to be linear (1-7) [81]) between the MFCV and the diameter of the fibers, the latter can also be indirectly estimated from the obtained parameters. According to the results, the parameters $x$ and $y$ are estimated with high accuracy, at least for the fibers located within a certain region close to the needle. The parameter $v$ is also estimated with acceptably high accuracy. In the case of the parameter $z$, although it can also be estimated, the estimation accuracy is lower than for the other parameters.

The key of the developed system to achieve a correct parameter estimation is that the estimation is based on the recording of the MUP in multiple positions, and using small-size recording ports (SF ports). For this purpose, the scanning-EMG technique has been employed. This provides much more information than in the case in which a single position is used to record the MUP, as occurred in the previous attempts to estimate the MU fiber parameters [67, 68, 140]. Thus, while in these attempts, the estimation of the radial distance between the port and the fibers was addressed, in the system developed here the position of the fibers in the muscle cross-section is estimated. The most complete of the previous attempts is the one proposed by Zalewska [140], for which, in addition to the radial distance, the estimation of the fiber diameters was addressed. However, this previous attempt lacks of a rigorous evaluation of the estimation error. Given the results obtained for the single-point configuration (with very high estimation errors (Fig. 5-10)), it is reasonable to think that the Zalewska estimation method (also based on a single-point MUP recording) cannot obtain an accurate parameter estimation. Another limitation of the Zalewska method with respect to the proposed system is that it does not allow estimating the positions of the neuromuscular junctions within the IZ, since in this method it is assumed that all these positions are equal.

The estimation system, not only provides information about the parameters of the individual MU fibers, but also it allows to perform a characterization of some global parameters of the MU, such as the fiber density, the organization in sub-bands of the IZ, and the distribution of the MFCV's of the MU fibers. In short, the proposed system allows, by means of a subsequent analysis of the estimated fiber parameters, to obtain relevant physiological information about the MU. In this way, the estimation system is a promising tool when it comes to analyze the MU, not only in normal conditions, but also in the detection of structural changes in the MU parameters associated to certain conditions, such as myopathies, neuropathies, aging, etc. 
Regarding the limitations of the developed system, first of all, it should be taken into account that an accurate estimation is only achieved in a limited region close to the scanning needle. Therefore, when making any subsequent analysis, such as the calculation of the aforementioned global MU parameters, this fact must be taken into account. The calculation of the global parameters should be done after an appropriate evaluation region, in which the estimation of the MU fiber parameters is considered to be correct, is established.

Another important limitation of the estimation system, is that, as any method based on inverse modeling, the quality of the estimations is subordinated to the accuracy of the simulation model representing the real world. With regard to this limitation, the model makes some assumptions that should be taken into account. Firstly, inhomogeneities of the volume conductor (apart from the anisotropy constant) have not been considered in the model. Secondly, it has been assumed that the muscle fibers of the MU have infinite length, which neglects the possible effect of the extinction of the potential in the tendon endings. This effect, on the other hand, is very small in SF recordings. And thirdly, the depolarization instant of all MU fibers is assumed to be identical, which implies that all SFAPs are initiated at the same instant, and also that the presence of jitter in the signals is neglected.

Other phenomena that occur in real recordings and that have not been considered when it comes to carrying out the estimation process are: firstly, possible movements of the needle electrode or the muscle fibers as a consequence of the muscle contraction; secondly, a non entirely correct needle insertion during the recording, such as not inserting the scanning needle completely perpendicular to the direction of the muscle fibers (i.e., along the y-axis), or not aligning the SF-ports of the needles completely perpendicular to the fibers (i.e., along the $\mathrm{x}$-axis); and thirdly, the displacement (ploughing) and possible cutting of the muscle fibers produced by the insertion of the needle in the muscle. In this regard, remember that the model used in this thesis to simulate the recorded signals does include the ploughing (although not the estimation procedure). However, it is not clear to what extent this ploughing model is accurate when reproducing the mechanical effect produced by the needle in the fibers.

Another limitation of the system is that the computation time required to estimate the parameters is large. In a conventional computer, the system developed takes about 4 hours to estimate the parameters of a single MU, something that has limited the number of experiments that have been performed when it came to evaluate the performed estimation system. However, no specific effort to write a computationally efficient code or to apply the parallel computing paradigm has been made, hence there is a lot of room for improvement. On the other hand, the continuous progress of computing capacity means that this time will always tend to decrease. Related to this problem is the trade-off existing between the computational time required to estimate the MU fiber parameters, and the quality of the estimation achieved. The higher the number of iterations used by the optimization method, the longer the computation time, 
but the better the quality of the estimation. However, this relationship is only fulfilled as long as the algorithm is not able to reach the global minimum satisfactorily. Otherwise, an increase in the number of iterations will entail a higher computational cost, but not a better estimation performance. The trade-off between computational cost and estimation quality is also manifested in regard to the MUP model used. In general, the more complex the model of the MUP, the more accurate the generation of the MUP. In turn, the more accurate the MUP modeling, the greater the correctness with which the parameters are estimated in a real environment. In return, a more complex model will also imply a higher computation time.

Another limitation that has the system is that not all muscles are suitable to be subject to the estimation. Note that, when it comes to properly place the needle and the surface electrodes to record the signals, it is necessary to know the direction of the muscle fibers. For this reason, the fusiform muscles are the most appropriate to carry out the recording procedure, as in these muscles the fibers lie parallel to the muscle length. This is the case of the biceps brachii muscle. If we want to perform the recording in a pennated muscle instead, in which the fibers lie with a certain angle (pennation) respect to the fiber length, it would be necessary to determine this angle before placing the recording electrodes, which would make the recording protocol more complex. In the case of multipennate muscles, in which the muscle fibers are arranged along several directions, it seems difficult that the estimation system can be applied satisfactorily.

Finally, it is important to keep in mind that the estimation system has been evaluated in a simulated environment, and that only a small set of estimation examples has been performed in real muscles. Note that, performing the evaluation in a simulated environment has the great advantage that it provides the exact a priori knowledge about the muscle and the MU fiber parameters, so that the estimated MU fiber parameters can be compared with the real ones, allowing to objectively evaluate the estimation performance. But, on the other hand, this has the disadvantage that, like any simulation environment, it is a simplified description of reality, in which, although many of the possible problems that can occur in a real environment have been simulated (noise, cannula effect, fiber ploughing, etc.), there will always be other effects not taken into account. 
upna

Universidad Púbilica de Navara
Natarmaako Unibersitate Publikoa 


\section{Final conclusions and future lines}

\subsection{Final conclusions}

- A system that estimates the parameters of the fibers of the MU from EMG signals has been developed. The system applies an inverse MUP modeling to the scanningEMG signal recorded from the MU under study. The parameters to be estimated for each MU fiber are: the position in the muscle cross-section along the $\mathrm{x}-$ and $\mathrm{y}-$ axis, $x$ and $y$; the neuromuscular junction position, $z$; and the MFCV, $v$.

- The design of the system is grounded in the fact that it is not possible to achieve a correct parameter estimation by recording the signal at a single position. This is because different mechanisms of waveform compensation between the parameters of the MU fibers exist, making that different parameter combinations give rise to very similar MUP waveforms.

- The estimation system, keeping in mind this idea, is based on the recording the MUP at different positions, hence it is based on the scanning-EMG technique. Several recording configurations, which differ in the number of scanning needles and ports used to record the signal have been proposed: the 1-port recording configuration, based on one scanning needle with a single port placed at one side of the needle; the 2-port recording configuration, based on a single scanning needle, with two ports placed at opposing sides of the needle; and the 4-port recording configuration, based on two scanning-needles, each one with two ports.

- Each of the ports in the recording configurations acts as a SF needle electrode to record the scanning-EMG signal. The choice of this kind of electrode has been motivated by its very high spatial and temporal resolution, which favors the correct estimation of the MU fiber parameters. The $\mathrm{CN}$ electrode was discarded in preliminary tests, due to its low spatial and temporal resolution.

- The system, apart from using the scanning-EMG recording, also uses a surfaceEMG recording, which provides complementary information about the MU. From this recording, the average IZ position, and the average MFCV can be estimated. These two values allow to establish the boundaries of the region in which the estimation algorithm searches the fiber parameters. Furthermore, the surface recording provides a way to determine the potential initiation instant, which is critical and difficult to obtain by other means.

- The estimation system has been evaluated in a simulated environment in which the muscle, the MUs, and the recording of the involved signals are simulated in detail. This provides a controlled environment that allows to objectively quantify the 
performance of the estimation. Specific merit figures for all the estimated parameters have been developed. These merit figures are evaluated both as a function of the distance to the electrode and as a function of the ratio of MU fibers within the evaluation region. Global MU parameters have been defined, along with the merit figures to evaluate the ability of the system to estimate them.

- The results show that the fiber location parameters $x, y$, and the MFCV $v$ are accurately estimated for the fibers within a certain region close to the needle (approximately $0.3 \mathrm{~mm}$ away from the scanning corridor).

- The neuromuscular junction position parameter $z$ can also be estimated, but less accurately. The estimation of this parameter is impaired by the estimation error of the potential initiation instant. This error implies that only the parameter $\zeta$ can be estimated with acceptable precision, that is, once the bias of the parameter $z$ has been subtracted.

- The ability of the system to estimate the MU fiber parameters is, for the three recording configurations, very superior than that of the configuration based on the recording of the MUP in a single position. In the latter, the fiber parameters are incorrectly estimated, even at close distances of the port position. This suggests that the previous attempts to estimate the MU fiber parameters [67, 68, 140], which are based on a single-point MUP recording, cannot achieve a correct parameter estimation. A recording configuration with multiple recording points, as the ones proposed here, is required for this purpose.

- Among the three proposed recording configurations, the easiest to implement, and also the one for which the worst estimation results are obtained is the 1-port configuration. The 2-port configuration is a little more complex, but in return it gives much better results; in addition, it allows to detect and estimate fibers on both sides of the needle. The 4-port configuration is, by far, the hardest one to implement, but it is also the one for which the estimation achieves the best performance.

- The 2-port recording configuration is the one proposed to be incorporated in the estimation system, as it provides an optimal trade between a good estimation performance and a not excessively high implementation difficulty.

- From the estimated parameters of the individual MU fibers, several global MU parameters can be obtained, which can provide relevant physiological information clinically useful in the diagnosis and follow up of certain pathological conditions. The global MU parameters that can be calculated are: the global MU fiber density, the IZ sub-bands, and the mean and standard deviation of the MFCVs.

- The precision with which the parameters of a certain MU fiber are estimated 
strongly depends on the distance of the fiber to the needle. This must be taken into account when it comes to perform any subsequent analysis from the estimated parameters, as it will be necessary to restrict the analysis to a certain region around the scanning needle corridor, in which the estimation is considered to be reliable.

- The estimation system has been tested in real muscles for a small set of scanningEMG recordings. These preliminary tests suggest that, although there is an increased difficulty to fit the waveform of the recorded signal in real scanningEMG recordings than in simulated ones, the waveform is fitted with relative success. Furthermore, there is still a huge margin for improvement, as the recording setup needs to be completed to fully incorporate the surface-EMG recording, and to deal with multiple discharges of the scanning-EMG signal. 


\subsection{Future lines}

- Although some preliminary tests in which the estimation is applied to real muscles have already been done, it will be necessary to carry out more exhaustive experiments to validate the applicability of the estimation system in a real environment. Note that an objective testing is not possible using real recorded scanning-EMG signals, as the exact a priori information about the actual MU fiber parameters is not available. However, it is possible to make an indirect validation of the system by obtaining parameter estimations for a certain muscle, and comparing the statistical distribution of the estimated parameters, with normal physiological ranges previously established for the muscle under study.

- The estimation system can be applied both in normal and in pathologic patients, so that a data base can be generated that allows to assess correlations between certain pathologies and the estimated MU structure.

- A promising line will be to test the ability of the global MU parameters calculated in this thesis (the fiber density, the IZ sub-bands, and the mean and standard deviation of the MFCV's) to discriminate between pathological and normal conditions, and to compare such ability with that of corresponding to the typical parameters used routinely in quantitative EMG. This can be done both in simulation and in real scenarios. If successful, the method would provide a diagnostic tool sensitive to structural changes that may occur in MUs under certain pathologies.

- Multi-scanning-EMG technique (see section 1.2.1) could be employed to perform the recording process, so that, in a single needle insertion, the scanning-EMG signals of several MUs of the muscle can be simultaneously recorded. This would not only allow to estimate the parameters of the fibers of several MUs at the same time, but would also give a more detailed image of the muscular section (in an environment close to the needle), allowing to analyze how the fibers of the different MUs are intermingled. Another important advantage of using the multiscanning-EMG technique is that the trigger needle can be replaced by an array of surface electrodes to extract the firing pattern of the MUs [60]. In this way, the recording setup would be simplified, since the same surface electrode array could be used both to extract the MU firing patterns and the rest of physiological information required by the estimation system.

- We could delve deeper into the possible incorporation of the concentric electrode in the estimation system, because although the preliminary results obtained with this electrode suggest that the potential performance of the estimation is lower than in the case of SF, the use of spatial and temporal high pass filters has not been tested enough to discard its usability. Note that the $\mathrm{CN}$ needle electrode due to its low price and being disposable, is taking over some of the previously SF-exclusive techniques, such as neuromuscular jitter measurement [129]. 


\section{Bibliography}

[1]. Akaboshi, K., Masakado, Y., \& Chino, N. (2000). Quantitative EMG and motor unit recruitment threshold using a concentric needle with quadrifilar electrode. Muscle \& Nerve: Official Journal of the American Association of Electrodiagnostic Medicine, 23(3), 361-367.

[2]. Andreassen, S., \& Rosenfalck, A. (1981). Relationship of intracellular and extracellular action potentials of skeletal muscle fibers. Critical reviews in bioengineering, 6(4), 267.

[3]. Amirali, A., Mu, L., Gracies, J. M., \& Simpson, D. M. (2007). Anatomical localization of motor endplate bands in the human biceps brachii. Journal of clinical neuromuscular disease, 9(2), 306-312.

[4]. Aquilonius, S. M., Arvidsson, B., Askmark, H., \& Gillberg, P. G. (1982). Topographical localization of end-plates in cryosections of whole human biceps muscle. Muscle \& nerve, 5(5), 418.

[5]. Arendt-Nielsen, L., \& Zwarts, M. (1989). Measurement of muscle fiber conduction velocity in humans: techniques and applications. Journal of clinical neurophysiology: official publication of the American Electroencephalographic Society, 6(2), 173-190.

[6]. Artuğ, N. T., Goker, I., Bolat, B., Osman, O., Orhan, E. K., \& Baslo, M. B. (2018). New features for scanned bioelectrical activity of motor unit in health and disease. Biomedical Signal Processing and Control, 41, 109-128.

[7]. Bodine, S., Roy, R. R., Eldred, E. A. R. L., \& Edgerton, V. R. (1987). Maximal force as a function of anatomical features of motor units in the cat tibialis anterior. Journal of neurophysiology, 57(6), 1730-1745.

[8]. Bodine, S. C., Garfinkel, A., Roy, R. R., \& Edgerton, V. R. (1988). Spatial distribution of motor unit fibers in the cat soleus and tibialis anterior muscles: local interactions. journal of Neuroscience, 8(6), 2142-2152.

[9]. Bodine-Fowler, S., Garfinkel, A., Roy, R. R., \& Edgerton, V. R. (1990). Spatial distribution of muscle fibers within the territory of a motor unit. Muscle \& Nerve: Official Journal of the American Association of Electrodiagnostic Medicine, 13(12), 1133-1145.

[10]. Broman, H., Bilotto, G., \& De Luca, C. J. (1985). A note on the noninvasive estimation of muscle fiber conduction velocity. IEEE transactions on biomedical engineering, (5), 341-344. 
[11]. Brooke, M. H., \& Engel, W. K. (1969). The histographic analysis of human muscle biopsies with regard to fiber types: I. Adult male and female. Neurology, 19(3), 221-221.

[12]. Buchthal, F., Erminio, F., \& Rosenfalck, P. (1959). Motor unit territory in different human muscles. Acta Physiologica Scandinavica, 45(1), 72-87.

[13]. Buchthal, F., Guld, C., \& Rosenfalck, P. (1957). Multielectrode study of the territory of a motor unit. Acta Physiologica Scandinavica, 39(1), 83-104.

[14]. Burke, R. E., Levine, D. N., Salcman, M., \& Tsairis, P. (1974). Motor units in cat soleus muscle: physiological, histochemical and morphological characteristics. The Journal of physiology, 238(3), 503-514.

[15]. Byrd, R. H., Gilbert, J. C., \& Nocedal, J. (2000). A trust region method based on interior point techniques for nonlinear programming. Mathematical programming, 89(1), 149-185.

[16]. Corera, Í., Malanda, A., Rodriguez-Falces, J., Porta, S., \& Navallas, J. (2017). Motor unit profile: a new way to describe the scanning-EMG potential. Biomedical Signal Processing and Control, 34, 64-73.

[17]. Corera, Í., Eciolaza, A., Rubio, O., Malanda, A., Rodríguez-Falces, J., \& Navallas, J. (2018). A masked least-squares smoothing procedure for artifact reduction in scanning-EMG recordings. Medical \& biological engineering \& computing, 56(8), 1391-1402.

[18]. Corera, Í., Malanda, A., Rodríguez-Falces, J., \& Navallas, J. (2020). Masked least-squares averaging in processing of scanning-EMG recordings with multiple discharges. Medical \& Biological Engineering \& Computing, 58(12), 3063-3073.

[19]. De Luca, C. J., \& Adam, A. (1999). Decomposition and analysis of intramuscular electromyographic signals. In Modern Techniques in Neuroscience Research (pp. 757-776). Springer, Berlin, Heidelberg.

[20]. De Luca, C. J., \& Hostage, E. C. (2010). Relationship between firing rate and recruitment threshold of motoneurons in voluntary isometric contractions. Journal of neurophysiology, 104(2), 1034-1046.

[21]. Dimitrov, G. V., \& Dimitrova, N. A. (1998). Precise and fast calculation of the motor unit potentials detected by a point and rectangular plate electrode. Medical engineering \& physics, 20(5), 374-381.

[22]. Diószeghy, P. (2002). Scanning electromyography. Muscle \& Nerve: Official Journal of the American Association of Electrodiagnostic Medicine, 25(S11), S66-S71. 
[23]. Eciolaza Ferrando, A. (2018). Multiscannning-EMG con una aguja.

[24]. Enoka, R. M. (1995). Morphological features and activation patterns of motor units. Journal of clinical neurophysiology: official publication of the American Electroencephalographic Society, 12(6), 538-559.

[25]. Enoka, R. M., \& Fuglevand, A. J. (2001). Motor unit physiology: some unresolved issues. Muscle \& Nerve: Official Journal of the American Association of Electrodiagnostic Medicine, 24(1), 4-17.

[26]. Ekstedt, J. (1964). Human single muscle fiber action potentials. Extracellular recording during voluntary and chemical activation. With some comments on end-plate physiology and on the fiber arrangement of the motor unit. Acta physiologica Scandinavica. Supplementum, SUPPL-226.

[27]. Ekstedt, J. (1963). A method of recording extracellular action potentials of single muscle fibres and measuring their propagation velocity in voluntarity activated human muscles. Bull Am Assoc Electromyogr Electrodiagn, 10, 16.

[28]. Falck, B., Alanen, A., Halonen, P., Jokinen, K., Kalimo, H., \& Karsikas, R. (1993). Diagnostic utility of MUP parameters in chronic neuropathy. Electroencephalography and Clinical Neurophysiology, 87(2), S99.

[29]. Falla, D., Dall'Alba, P., Rainoldi, A., Merletti, R., \& Jull, G. (2002). Location of innervation zones of sternocleidomastoid and scalene muscles-a basis for clinical and research electromyography applications. Clinical Neurophysiology, 113(1), 57-63.

[30]. Farina, D., Fortunato, E., \& Merletti, R. (2000). Noninvasive estimation of motor unit conduction velocity distribution using linear electrode arrays. IEEE Transactions on Biomedical Engineering, 47(3), 380-388.

[31]. Farina, D., Muhammad, W., Fortunato, E., Meste, O., Merletti, R., \& Rix, H. (2001). Estimation of single motor unit conduction velocity from surface electromyogram signals detected with linear electrode arrays. Medical and Biological Engineering and Computing, 39(2), 225-236.

[32]. Farina, D., \& Merletti, R. (2001). A novel approach for precise simulation of the EMG signal detected by surface electrodes. IEEE Transactions on Biomedical Engineering, 48(6), 637-646.

[33]. Farina, D., Arendt-Nielsen, L., Merletti, R., \& Graven-Nielsen, T. (2002). Assessment of single motor unit conduction velocity during sustained contractions of the tibialis anterior muscle with advanced spike triggered averaging. Journal of neuroscience methods, 115(1), 1-12. 
[34]. Feinstein, B., Lindegård, B., Nyman, E., \& Wohlfart, G. (1955). Morphologic studies of motor units in normal human muscles. Cells Tissues Organs, 23(2), 127-142.

[35]. Fuglevand, A. J., Winter, D. A., \& Patla, A. E. (1993). Models of recruitment and rate coding organization in motor-unit pools. Journal of neurophysiology, 70(6), 2470-2488.

[36]. Gates, H. J., \& Betz, W. J. (1993). Spatial distribution of muscle fibers in a lumbrical muscle of the rat. The Anatomical Record, 236(2), 381-389.

[37]. Gath, I., \& Stålberg, E. (1977). On the volume conduction in human skeletal muscle: in situ measurements. Electroencephalography and clinical neurophysiology, 43(1), 106-110.

[38]. Gath, I., \& Stålberg, E. (1979). Measurements of the uptake area of small-size electromyographic electrodes. IEEE Transactions on Biomedical Engineering, (6), 374-376.

[39]. Gath, I., \& Stålberg, E. (1981). In situ measurement of the innervation ratio of motor units in human muscles. Experimental brain research, 43(3-4), 377-382.

[40]. Gath, I., \& Stålberg, E. (1982). On the measurement of fibre density in human muscles. Electroencephalography and clinical neurophysiology, 54(6), 699706.

[41]. Goldberg, D. E. (1989). Genetic Algorithms in Search, Optimization, and Machine Learning, Addison Wesley, Reading, MA. Summary the applications of ga-genetic algorithm for dealing with some optimal calculations in economics.

[42]. Gootzen, T. H. (1990). Electrophysiological investigation of motor unit structure by means of scanning EMG. Muscle Fiber and Motor Unit Action Potentials. Katholieke Universiteit te Nijmegen, 89-106.

[43]. Gootzen, T. H. J. M., Stegeman, D. F., \& Van Oosterom, A. (1991). Finite limb dimensions and finite muscle length in a model for the generation of electromyographic signals. Electroencephalography and Clinical Neurophysiology/Evoked Potentials Section, 81(2), 152-162.

[44]. Gootzen, T. H., Vingerhoets, D. J., \& Stegeman, D. F. (1992). A study of motor unit structure by means of scanning EMG. Muscle \& Nerve: Official Journal of the American Association of Electrodiagnostic Medicine, 15(3), 349-357.

[45]. Gootzen, T. H. J. M., Stegeman, D. F., \& Van Oosterom, A. (1993). Muscle electric activity II: On the feasibility of model-based estimation of experimental 
conditions in electromyography. Annals of biomedical engineering, 21(4), 391399.

[46]. Grönlund, C., Östlund, N., Roeleveld, K., \& Karlsson, J. S. (2005). Simultaneous estimation of muscle fibre conduction velocity and muscle fibre orientation using 2D multichannel surface electromyogram. Medical and Biological Engineering and Computing, 43(1), 63-70.

[47]. Hakansson, C. H. (1956). Conduction velocity and amplitude of the action potential as related to circumference in the isolated fibre of frog muscle. Acta Physiologica Scandinavica, 37(1), 14-34.

[48]. Hamilton-Wright, A., \& Stashuk, D. W. (2005). Physiologically based simulation of clinical EMG signals. IEEE Transactions on biomedical engineering, 52(2), 171-183.

[49]. Hastings, W. K. (1970). Monte Carlo sampling methods using Markov chains and their applications.

[50]. Henneberg, K. A., \& Plonsey, R. (1993). Boundary element analysis of the directional sensitivity of the concentric EMG electrode. IEEE transactions on biomedical engineering, 40(7), 621-631.

[51]. Henneman, E., Somjen, G., \& Carpenter, D. O. (1965). Excitability and inhibitibility of motoneurons of different sizes. Journal of neurophysiology, 28(3), 599-620.

[52]. Henneman, E., Somjen, G., \& Carpenter, D. O. (1965). Functional significance of cell size in spinal motoneurons. Journal of neurophysiology, 28(3), 560-580.

[53]. Hilton-Brown, P., \& Stålberg, E. (1983). The motor unit in muscular dystrophy, a single fibre EMG and scanning EMG study. Journal of Neurology, Neurosurgery \& Psychiatry, 46(11), 981-995.

[54]. Kakuda, N., Nagaoka, M., \& Tanaka, R. (1991). Discrimination of different motor units by spike-triggered averaging of surface electromyograms. Neuroscience letters, 122(2), 237-240.

[55]. Kanda, K. E. N. R. O., \& Hashizume, K. (1992). Factors causing difference in force output among motor units in the rat medial gastrocnemius muscle. The Journal of physiology, 448(1), 677-695.

[56]. Keenan, K. G., \& Valero-Cuevas, F. J. (2007). Experimentally valid predictions of muscle force and EMG in models of motor-unit function are most sensitive to neural properties. Journal of neurophysiology, 98(3), 1581-1590. 
[57]. King, J. C., Dumitru, D., \& Nandedkar, S. (1997). Concentric and single fiber electrode spatial recording characteristics. Muscle \& Nerve: Official Journal of the American Association of Electrodiagnostic Medicine, 20(12), 1525-1533.

[58]. Kugelberg, E., Edström, L., \& Abbruzzese, M. (1970). Mapping of motor units in experimentally reinnervated rat muscle: Interpretation of histochemical and atrophic fibre patterns in neurogenic lesions. Journal of Neurology, Neurosurgery \& Psychiatry, 33(3), 319-329.

[59]. Kukulka, C. G., \& Clamann, H. P. (1981). Comparison of the recruitment and discharge properties of motor units in human brachial biceps and adductor pollicis during isometric contractions. Brain research, 219(1), 45-55.

[60]. Lapatki, B. G., Eiglsperger, U., Schindler, H. J., Radeke, J., Holobar, A., \& van Dijk, J. P. (2019). Three-dimensional amplitude characteristics of masseter motor units and representativeness of extracted motor unit samples. Clinical Neurophysiology, 130(3), 388-395.

[61]. Lateva, Z. C., \& McGill, K. C. (2001). Estimating motor-unit architectural properties by analyzing motor-unit action potential morphology. Clinical neurophysiology, 112(1), 127-135.

[62]. Lawrence, J. H., \& De Luca, C. J. (1983). Myoelectric signal versus force relationship in different human muscles. Journal of Applied Physiology, 54(6), 1653-1659.

[63]. LeFever, R. S., \& De Luca, C. J. (1982). A procedure for decomposing the myoelectric signal into its constituent action potentials-part I: technique, theory, and implementation. IEEE transactions on biomedical engineering, (3), 149157.

[64]. LeFever, R. S., Xenakis, A. P., \& De Luca, C. J. (1982). A procedure for decomposing the myoelectric signal into its constituent action potentials-part II: execution and test for accuracy. IEEE transactions on biomedical engineering, (3), 158-164.

[65]. Lieber, R. L. (2002). Skeletal muscle structure, function, and plasticity. Lippincott Williams \& Wilkins.

[66]. Lorente de Nó, R. (1947). A study of nerve physiology. Studies from Rockefeller Institute, 132, Ch16.

[67]. Lööf, Y. (1986). Model for decomposition of the motor unit action potential 1 The algorithm. Medical and Biological Engineering and Computing, 24(5), 506-512. 
[68]. Lööf, Y. (1986). Model for decomposition of the motor unit action potential 2 Analysis of simulated motor unit action potentials. Medical and Biological Engineering and Computing, 24(5), 513-520.

[69]. Masuda, T., Miyano, H., \& Sadoyama, T. (1983). The propagation of motor unit action potential and the location of neuromuscular junction investigated by surface electrode arrays. Electroencephalography and clinical neurophysiology, 55(5), 594-600.

[70]. Masuda, T., Miyano, H., \& Sadoyama, T. (1985). The position of innervation zones in the biceps brachii investigated by surface electromyography. IEEE Transactions on Biomedical Engineering, (1), 36-42.

[71]. Masuda, T., \& Sadoyama, T. (1986). The propagation of single motor unit action potentials detected by a surface electrode array. Electroencephalography and clinical Neurophysiology, 63(6), 590-598.

[72]. McComas, A. J., Fawcett, P. R. W., Campbell, M. J., \& Sica, R. E. P. (1971). Electrophysiological estimation of the number of motor units within a human muscle. Journal of Neurology, Neurosurgery \& Psychiatry, 34(2), 121-131.

[73]. McGill, K. C., \& Dorfman, L. J. (1984). High-resolution alignment of sampled waveforms. IEEE transactions on biomedical engineering, (6), 462-468.

[74]. MacIntosh, B. R., Gardiner, P. F., \& McComas, A. J. (2006). Skeletal muscle: form and function. Human Kinetics.

[75]. Merletti, R., Farina, D., \& Granata, A. (1999). Non-invasive assessment of motor unit properties with linear electrode arrays. Electroencephalography and clinical neurophysiology. Supplement, 50, 293-300.

[76]. Merletti, R., Farina, D., \& Gazzoni, M. (2003). The linear electrode array: a useful tool with many applications. Journal of Electromyography and Kinesiology, 13(1), 37-47.

[77]. Merletti, R., Parker, P. A., \& Parker, P. J. (Eds.). (2004). Electromyography: physiology, engineering, and non-invasive applications (Vol. 11). John Wiley $\&$ Sons.

[78]. Milner-Brown, H. S., Stein, R. B., \& Yemm, R. (1973). The orderly recruitment of human motor units during voluntary isometric contractions. The Journal of physiology, 230(2), 359-370.

[79]. Miller-Larsson, A. (1980). A model of spatial distribution of muscle fibres of a motor unit in normal human limb muscles. Electromyogr. Clin. Neurophysiol., 20, 281-298. 
[80]. Moritani, T., \& Muro, M. (1987). Motor unit activity and surface electromyogram power spectrum during increasing force of contraction. European journal of applied physiology and occupational physiology, 56(3), 260-265.

[81]. Nandedkar, S. D., \& St, E. (1983). Simulation of single muscle fibre action potentials. Medical and Biological Engineering and Computing, 21(2), 158165.

[82]. Nandedkar, S., \& Stålberg, E. (1983). Simulation of macro EMG motor unit potentials. Electroencephalography and clinical Neurophysiology, 56(1), 5262.

[83]. Nandedkar, S. D., Sigl, J. C., Kim, Y. I., \& Stålberg, E. V. (1984). Radial decline of the extracellular action potential. Medical and Biological Engineering and Computing, 22(6), 564-568.

[84]. Nandedkar, S. D., Sanders, D. B., Stälberg, E. V., \& Andreassen, S. (1988). Simulation of concentric needle EMG motor unit action potentials. Muscle \& Nerve: Official Journal of the American Association of Electrodiagnostic Medicine, 11(2), 151-159.

[85]. Nandedkar, S. D., Sanders, D. B., \& Stålberg, E. V. (1988). EMG of reinnervated motor units: a simulation study. Electroencephalography and clinical Neurophysiology, 70(2), 177-184.

[86]. Nandedkar, S. D., \& Sanders, D. B. (1989). Simulation of myopathic motor unit action potentials. Muscle \& Nerve: Official Journal of the American Association of Electrodiagnostic Medicine, 12(3), 197-202.

[87]. Navallas, J., \& Stålberg, E. (2009). Studying motor end-plate topography by means of scanning-electromyography. Clinical Neurophysiology, 120(7), 13351341.

[88]. Navallas, J., Malanda, A., Gila, L., Rodriguez, J., \& Rodriguez, I. (2009). Comparative evaluation of motor unit architecture models. Medical \& biological engineering \& computing, 47(11), 1131.

[89]. Navallas, J., Malanda, A., Gila, L., Rodríguez, J., \& Rodríguez, I. (2010). A muscle architecture model offering control over motor unit fiber density distributions. Medical \& biological engineering \& computing, 48(9), 875-886.

[90]. Navallas Irujo, J., Rodríguez Falces, J., \& Stålberg, E. (2012). Scanning electromyography. EMG methods for evaluating muscle and nerve function, Mr. Mark Schwartz (Ed.). InTech, 2012. ISBN: 978-953-307-793-2. 
[91]. Navallas, J., Malanda, A., \& Rodriguez-Falces, J. (2014). Maximum likelihood estimation of motor unit firing pattern statistics. IEEE Transactions on Neural Systems and Rehabilitation Engineering, 22(3), 460-469.

[92]. Navallas, J., Eciolaza, A., Rubio, O., Corera, Í., Rodríguez-Falces, J. \& Malanda, A. (2018). Single-needle multiscanning-EMG. Proceedings of the XXII Congress of the International Society of Electrophysiology and Kinesiology (ISEK).

[93]. Okabe, A., Boots, B., Sugihara, K., \& Chiu, S. N. (2009). Spatial tessellations: concepts and applications of Voronoi diagrams (Vol. 501). John Wiley \& Sons.

[94]. Pfeiffer, G., \& Friede, R. L. (1985). The localization of axon branchings in two muscle nerves of the rat. Anatomy and embryology, 172(2), 177-182.

[95]. Plonsey, R. (1964). Volume conductor fields of action currents. Biophysical journal, 4(4), 317.

[96]. Plonsey, R. (1974). The active fiber in a volume conductor. IEEE transactions on biomedical engineering, (5), 371-381.

[97]. Plonsey, R. (1977). Action potential sources and their volume conductor fields. Proceedings of the IEEE, 65(5), 601-611.

[98]. Press, W. H., Teukolsky, S. A., Vetterling, W. T., \& Flannery, B. P. (1992). Numerical recipes in $\mathrm{C}++$. The art of scientific computing, 2, 1002.

[99]. Rodríguez-Carreño, I., Malanda-Trigueros, A., Gila-Useros, L., Navallas-Irujo, J., \& Rodríguez-Falces, J. (2006). Filter design for cancellation of baselinefluctuation in needle EMG recordings. Computer methods and programs in biomedicine, 81(1), 79-93.

[100]. Rodríguez-Falces, J. R., Trigueros, A. M., Useros, L. G., Carreño, I. R., \& Irujo, J. N. (2006). Modeling fibrillation potentials-a new analytical description for the muscle intracellular action potential. IEEE transactions on biomedical engineering, 53(4), 581-592.

[101]. Rodríguez-Falces, J. R., Trigueros, A. M., Useros, L. G., Carreño, I. R., \& Irujo, J. N. (2007). Modelling Fibrillation Potentials-Analysis of Time Parameters in the Muscle Intracellular Action Potential. IEEE transactions on biomedical engineering, 54(8), 1361-1370.

[102]. Rodríguez-Falces, J., Malanda, A., Gila, L., Rodríguez, I., \& Navallas, J. (2010). Identification Procedure in a model of single fibre action potential-Part I: Estimation of fibre diameter and radial distance. Journal of Electromyography and Kinesiology, 20(2), 264-273. 
[103]. Rodríguez-Falces, J., Malanda, A., Gila, L., Rodríguez, I., \& Navallas, J. (2010). Identification procedure in a model of single fibre action potential-Part II: Global approach and experimental results. Journal of Electromyography and Kinesiology, 20(2), 274-283.

[104]. Roeleveld, K., Stegeman, D. F., Vingerhoets, H. M., \& Oosterom, A. V. (1997). The motor unit potential distribution over the skin surface and its use in estimating the motor unit location. Acta physiologica scandinavica, 161(4), 465-472.

[105]. Roeleveld, K., Stegeman, D. F., Falck, B., \& Stålberg, E. V. (1997). Motor unit size estimation: confrontation of surface EMG with macro EMG. Electroencephalography and Clinical Neurophysiology/Electromyography and Motor Control, 105(3), 181-188.

[106]. Roeleveld, K., Sandberg, A., Stålberg, E. V., \& Stegeman, D. F. (1998). Motor unit size estimation of enlarged motor units with surface electromyography. Muscle \& Nerve: Official Journal of the American Association of Electrodiagnostic Medicine, 21(7), 878-886.

[107]. Rosenfalck, P. (1969). Intra-and extracellular potential fields of active nerve and muscle fibres. Acta Phisiologica Scandinavica, 321.

[108]. Salari, V., \& Sethi, I. K. (1990). Feature point correspondence in the presence of occlusion. IEEE Transactions on Pattern Analysis and Machine Intelligence, 12(1), 87-91.

[109]. Santo-Neto, H., de Carvalho, V. C., \& Marques, M. J. (1998). Estimation of the number and size of human flexor digiti minimi muscle motor units using histological methods. Muscle \& Nerve: Official Journal of the American Association of Electrodiagnostic Medicine, 21(1), 112-114.

[110]. Schnetzer, M. A., Ruegg, D. G., Baltensperger, R., \& Gabriel, J. P. (2001, October). Three-dimensional model of a muscle and simulation of its surface EMG. In 2001 Conference Proceedings of the 23rd Annual International Conference of the IEEE Engineering in Medicine and Biology Society (Vol. 2, pp. 1038-1043). IEEE.

[111]. Sethi, I. K., \& Jain, R. (1987). Finding trajectories of feature points in a monocular image sequence. IEEE Transactions on pattern analysis and machine intelligence, (1), 56-73.

[112]. Sherrington, C. S \& Eccles, J. C. (1930). Numbers and contraction-values of individual motor-units examined in some muscles of the limb. Proceedings of the Royal Society of London. Series B, Containing Papers of a Biological Character, 106(745), 326-357. 
[113]. Stålberg, E. (1966). Propagation velocity in human muscle fibers in situ. Acta Physiol Scand, 287, 3-112.

[114]. Stålberg, E., \& Trontelj, J. V. (1970). Demonstration of axon reflexes in human motor nerve fibers. J Neurol Neurosurg Psychiatry, 33, 571-579.

[115]. Stålberg, E., \& Thiele, B. A. R. B. A. R. A. (1975). Motor unit fibre density in the extensor digitorum communis muscle. J Neurol Neurosurg Psychiatry, 38, $874-80$.

[116]. Stålberg, E., Schwartz, M. S., Thiele, B., \& Schiller, H. H. (1976). The normal motor unit in man: A single fibre EMG multielectrode investigation. Journal of the neurological sciences, 27(3), 291-301.

[117]. Stålberg, E., \& Antoni, L. (1980). Electrophysiological cross section of the motor unit. Journal of Neurology, Neurosurgery \& Psychiatry, 43(6), 469-474.

[118]. Stålberg, E. (1980). Macro EMG, a new recording technique. Journal of Neurology, Neurosurgery \& Psychiatry, 43(6), 475-482.

[119]. Stålberg, E. (1986). Single fiber EMG, macro EMG, and scanning EMG. New ways of looking at the motor unit. CRC critical reviews in clinical neurobiology, 2(2), 125-167.

[120]. Stålberg, E. (1986). Single fiber EMG, macro EMG, and scanning EMG. New ways of looking at the motor unit. CRC critical reviews in clinical neurobiology, 2(2), 125-167.

[121]. Stålberg, E., Andreassen, S., Falck, B., Lang, H., Rosenfalck, A., \& Trojaborg, W. (1986). Quantitative analysis of individual motor unit potentials: a proposition for standardized terminology and criteria for measurement. Journal of clinical neurophysiology: official publication of the American Electroencephalographic Society, 3(4), 313-348.

[122]. Stålberg, E., \& Dioszeghy, P. (1991). Scanning EMG in normal muscle and in neuromuscular disorders. Electroencephalography and Clinical Neurophysiology/Evoked Potentials Section, 81(6), 403-416.

[123]. Stålberg, E., \& Trontelj, J. O. Z. E. (1992). Clinical neurophysiology: the motor unit in myopathy. Handbook of clinical neurology, 18, 49-84.

[124]. Stålberg, E., Nandedkar, S. D., Sanders, D. B., \& Falck, B. (1996). Quantitative motor unit potential analysis. Journal of clinical neurophysiology, 13(5), 401-422.

[125]. Stålberg, E., \& Falck, B. (1997). The role of electromyography in neurology. Electroencephalography and clinical Neurophysiology, 103(6), 579-598. 
[126]. Stålberg, E., \& Karlsson, L. (2001). Simulation of the normal concentric needle electromyogram by using a muscle model. Clinical Neurophysiology, 112(3), 464-471.

[127]. Stålberg, E., \& Karlsson, L. (2001). Simulation of EMG in pathological situations. Clinical Neurophysiology, 112(5), 869-878.

[128]. Stålberg, E. (2004). Clinical Neurophysiology of disorders of muscle and neuromuscular junction, including fatigue.

[129]. Stålberg, E., \& Sanders, D. (2009). Jitter recordings with concentric needle electrodes. Muscle \& Nerve: Official Journal of the American Association of Electrodiagnostic Medicine, 40(3), 331-339.

[130]. Stashuk, D. W. (1999). Detecting single fiber contributions to motor unit action potentials. Muscle \& Nerve: Official Journal of the American Association of Electrodiagnostic Medicine, 22(2), 218-229.

[131]. Stashuk, D. (2001). EMG signal decomposition: how can it be accomplished and used?. Journal of Electromyography and Kinesiology, 11(3), 151-173.

[132]. Strutz, T. (2010). Data fitting and uncertainty: A practical introduction to weighted least squares and beyond. Vieweg and Teubner.

[133]. Tarantola, A. (2005). Inverse problem theory and methods for model parameter estimation (Vol. 89). siam.

[134]. Theeuwen, M. M. H. J., Gootzen, T. H. J. M., \& Stegeman, D. F. (1993). Muscle electric activity I: A model study on the effect of needle electrodes on single fiber action potentials. Annals of biomedical engineering, 21(4), 377-389.

[135]. Van Dijk, J. P., Stegeman, D. F., \& Zwarts, M. J. (1999). Possibilities and limitations of conduction velocity distribution estimation. In The State of the Art on Signal Processing Methods for Surface Electromyography. European concerted action (BIOMED II, SENIAM) (pp. 211-217). Roessingh Enschede.

[136]. van Dijk, J. P., Eiglsperger, U., Hellmann, D., Giannakopoulos, N. N., McGill, K. C., Schindler, H. J., \& Lapatki, B. G. (2016). Motor unit activity within the depth of the masseter characterized by an adapted scanning EMG technique. Clinical Neurophysiology, 127(9), 3198-3204.

[137]. Willison, R. G. (1980). Arrangement of muscle fibers of a single motor unit in mammalian muscles. Muscle \& nerve, 3(4), 360.

[138]. Zalewska, E., \& Hausmanowa-Petrusewicz, I. (1995). Evaluation of MUAP shape irregularity-a new concept of quantification. IEEE transactions on biomedical engineering, 42(6), 616-620. 
[139]. Zalewska, E., Hausmanowa-Petrusewicz, I., \& Stålberg, E. (2004). Modeling studies on irregular motor unit potentials. Clinical neurophysiology, 115(3), 543-556.

[140]. Zalewska, E., \& Hausmanowa-Petrusewicz, I. (2008). Approximation of motor unit structure from the analysis of motor unit potential. Clinical Neurophysiology, 119(11), 2501-2506.

[141]. Zalewska, E., Rowinska-Marcinska, K., Gawel, M., \& HausmanowaPetrusewicz, I. (2012). Simulation studies on the motor unit potentials with satellite components in amyotrophic lateral sclerosis and spinal muscle atrophy. Muscle \& nerve, 45(4), 514-521.

[142]. Zalewska, E., Nandedkar, S. D., \& Hausmanowa-Petrusewicz, I. (2012). A method for determination of muscle fiber diameter using single fiber potential (SFP) analysis. Medical \& biological engineering \& computing, 50(12), 13091314.

[143]. Zalewska, E., Szmidt-Salkowska, E., Rowinska-Marcinska, K., Kaminska, A., \& Hausmanowa-Petrusewicz, I. (2013). Motor unit potentials with satellites in dystrophinopathies. Journal of Electromyography and Kinesiology, 23(3), 580586.

[144]. Zalewska, E. (2017). Deriving muscle fiber diameter from recorded single fiber potential. Neurophysiologie Clinique, 47(5-6), 413-417.

[145]. Zalewska, E., \& Hausmanowa-Petrusewicz, I. (2018). Correlating motor unit morphology with bioelectrical activity-A simulation study. Clinical Neurophysiology, 129(1), 271-279.

[146]. Zalewska, E., Kaminska, A., Kierdaszuk, B., Szmidt-Salkowska, E., \& Gawel, M. (2018). Comprehensive evaluation of EMG and biopsy findings supported by computer simulations-A preliminary study. Clinical Neurophysiology, 129(8), 1595-1604.

[147]. Zalewska, E., \& Gawel, M. (2019). Identification of components from distant fibers in a recorded single muscle fiber potential (SFP)-a new approach to the SFP criteria. Neurophysiologie Clinique, 49(1), 69-80.

[148]. Zwarts, M. J., \& Van Dijk, J. P. (1998). Methods to determine muscle fiber conduction velocity. State of the Art on Modelling Methods for Surface Electromyography, European Concerted Action (BIOMED II, SENIAM), edited by Hermens HJ, Stegeman D, Blok JH, and Freriks B. Enschede, The Netherlands: Roessingh, 85-89. 
[149]. Zwarts, M. J., \& Stegeman, D. F. (2003). Multichannel surface EMG: basic aspects and clinical utility. Muscle \& Nerve: Official Journal of the American Association of Electrodiagnostic Medicine, 28(1), 1-17. 


\section{List of Abbreviations}

$\begin{array}{ll}\text { ALS } & \text { Amyotrophic lateral sclerosis } \\ \text { AWGN } & \text { Additive white Gaussian noise } \\ \text { CN } & \text { Concentric needle } \\ \text { DFT } & \text { Discrete Fourier Transform } \\ \text { EMG } & \text { Electromyography } \\ \text { IAP } & \text { Intracellular action potential } \\ \text { IDFT } & \text { Inverse Discrete Fourier Transform } \\ \text { IZ } & \text { Innervation zone } \\ \text { MAE } & \text { Median absolute error } \\ \text { MFCV } & \text { Muscle fiber conduction velocity } \\ \text { MLSS } & \text { Masked least-squares smoothing } \\ \text { MMLSS } & \text { Multi MLSS } \\ \text { MN } & \text { Monopolar needle } \\ \text { MU } & \text { Motor unit } \\ \text { MUP } & \text { Motor unit potential } \\ \text { MVC } & \text { Muscle voluntary contraction } \\ \text { NMSE } & \text { Normalized mean squared error } \\ \text { QEMG } & \text { Quantitative EMG } \\ \text { SER } & \text { Signal to error ratio } \\ \text { SF } & \text { Single fiber } \\ \text { SFAP } & \text { Single fiber action potential } \\ \text { SMA } & \text { Spinal muscular atrophy } \\ \text { STSR } & \text { Squared time shift reduction }\end{array}$




\section{List of Publications}

\section{International Journals}

- Corera, Í., Malanda, A., Rodriguez-Falces, J., Porta, S., \& Navallas, J. (2017). Motor unit profile: a new way to describe the scanning-EMG potential. Biomedical Signal Processing and Control, 34, 64-73.

- Corera, Í., Eciolaza, A., Rubio, O., Malanda, A., Rodríguez-Falces, J., \& Navallas, J. (2018). A masked least-squares smoothing procedure for artifact reduction in scanning-EMG recordings. Medical \& biological engineering \& computing, 56(8), 1391-1402.

- Corera, Í., Malanda, A., Rodríguez-Falces, J., \& Navallas, J. (2020). Masked leastsquares averaging in processing of scanning-EMG recordings with multiple discharges. Medical \& Biological Engineering \& Computing, 58(12), 3063-3073.

\section{International Congresses}

- Corera, Í., Eciolaza, A., Rubio, O., Rodríguez-Falces, J., Malanda, A., \& Navallas, J. (2018). Scanning-EMG masked least-squares averaging from multipledischarges recordings. Proceedings of the XXII Congress of the International Society of Electrophysiology and Kinesiology (ISEK).

\section{In progress}

- Corera, Í., \& Navallas, J. Estimation of the structure of the MU from scanningEMG recordings. [in preparation] 